\title{
Die fehleranalytische Relevanz der prädominanten Spracherwerbshypothesen
}

\author{
Untersuchung des Fehlererklärungspotentials \\ der Kontrastiv-, der Identitäts- und der Interlanguagehypothese \\ auf Grundlage einer Analyse linguistischer Fehlleistungen \\ deutscher Muttersprachler beim Erwerb des Englischen
}

\begin{abstract}
Dissertation
zur Erlangung des philosophischen Doktorgrades

an der Philosophischen Fakultät der Georg-August-Universität Göttingen
\end{abstract}

vorgelegt von

Michael Achten

aus Linnich

Göttingen 2005 



\title{
Die fehleranalytische Relevanz der prädominanten Spracherwerbshypothesen
}

\author{
Untersuchung des Fehlererklärungspotentials \\ der Kontrastiv-, der Identitäts- und der Interlanguagehypothese \\ auf Grundlage einer Analyse linguistischer Fehlleistungen \\ deutscher Muttersprachler beim Erwerb des Englischen
}


Ich möchte mich an dieser Stelle bei allen Probanden bedanken, die sich an der Studie, die der vorliegenden Arbeit zugrunde liegt, beteiligt haben, sowie bei den Rektoren und Lehrkräften der teilnehmenden Schulen für ihre Bereitschaft, die Studie an ihren Schulen durchführen zu lassen.

Danken möchte ich auch Herrn Prof. Dr. Thomas Gardner für die Betreuung der Arbeit. 


\section{Inhaltsverzeichnis}

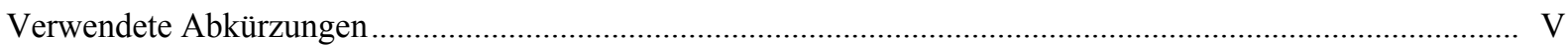

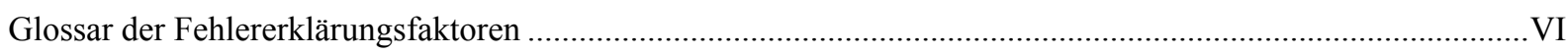

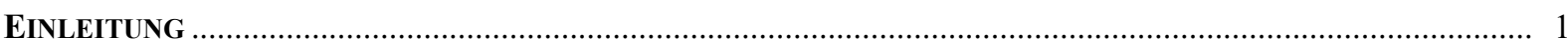

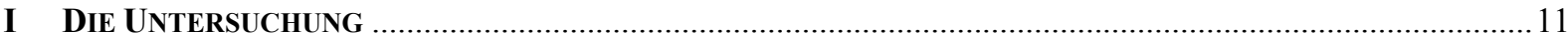

1 Grundlagen und Voraussetzungen der Untersuchung.................................................................... 11

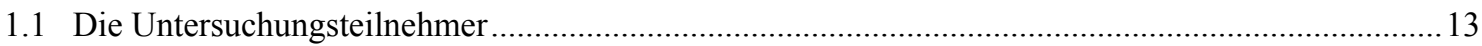

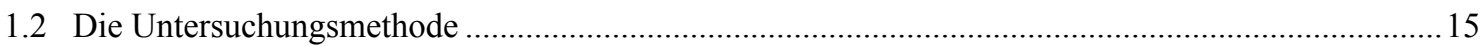

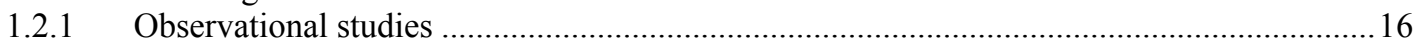

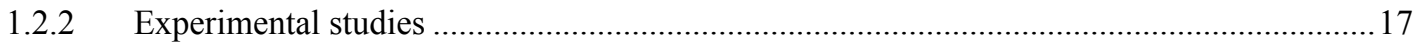

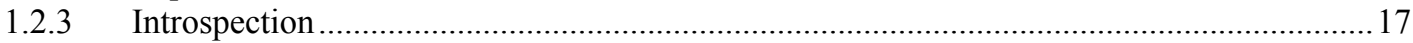

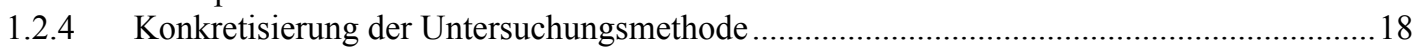

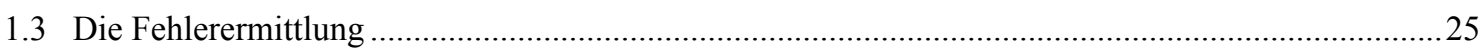

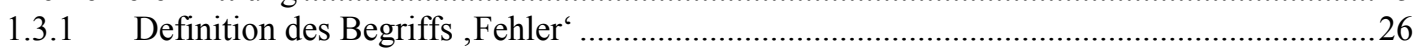

1.3.2 Linguistische Abweichungsformen......................................................................... 31

1.3.3 Die Bedeutung des Begriffs ,Fehler` für das Untersuchungsverfahren .............................33

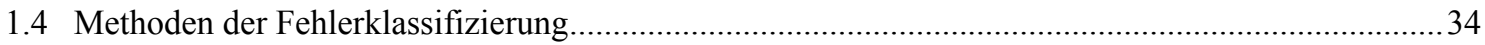

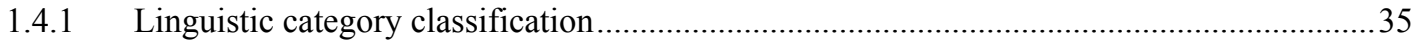

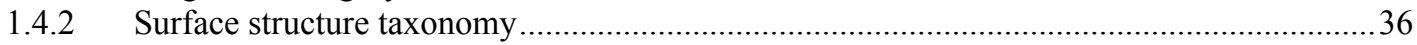

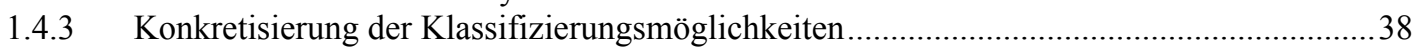

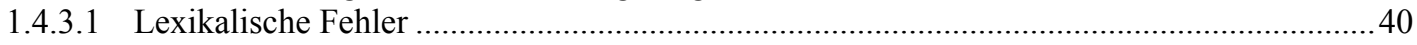

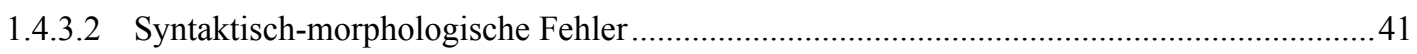

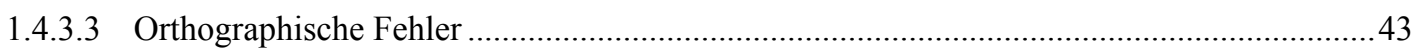

2 Linguistische Abweichungen deutscher Englischlernender: Gegenwärtiger Kenntnisstand ....................44

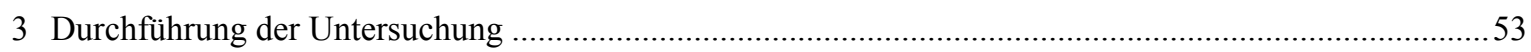

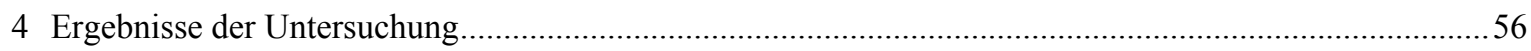

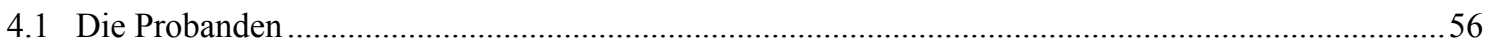

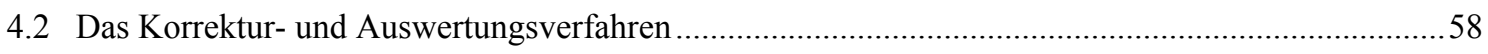

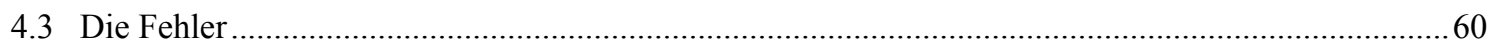

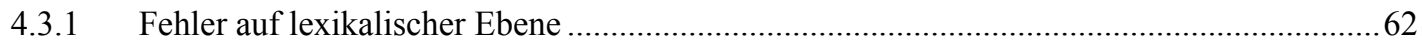

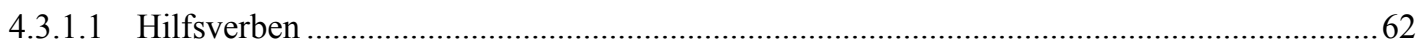

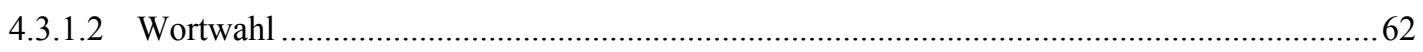

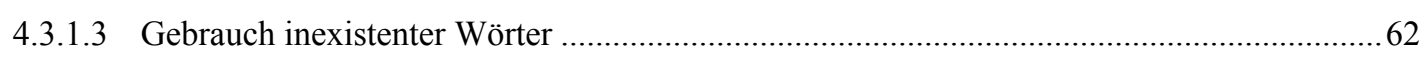

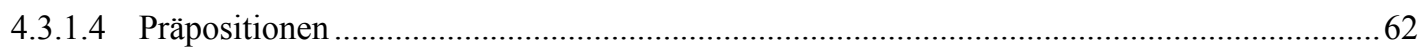

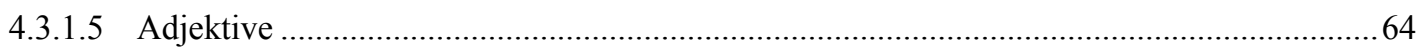

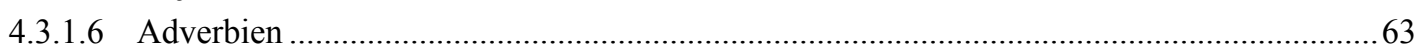

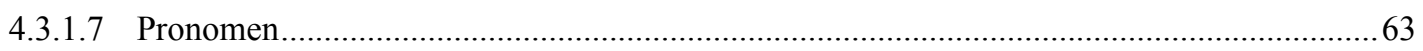

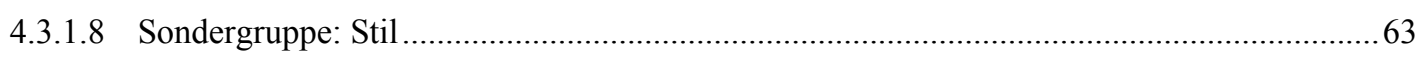

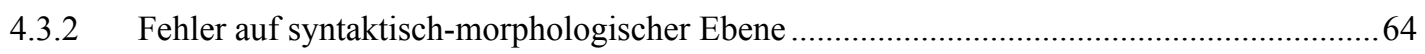

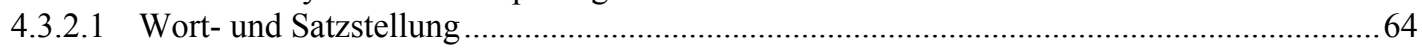

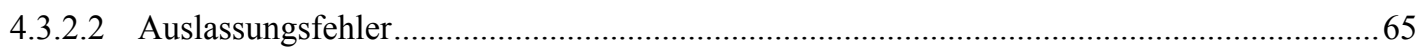

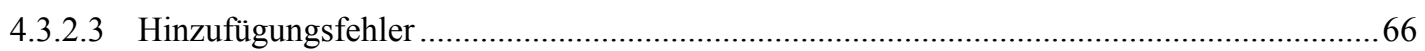

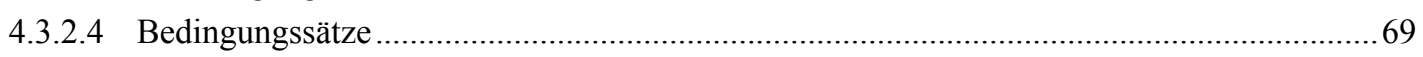

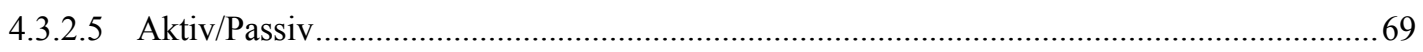

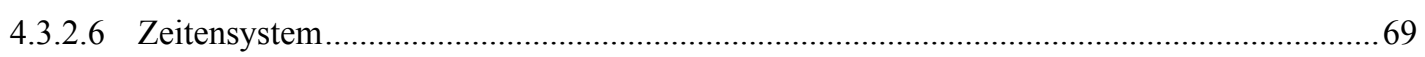

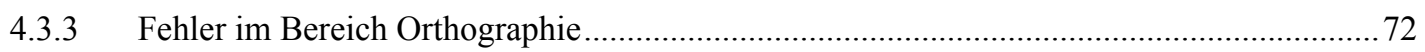

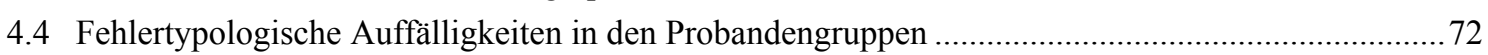

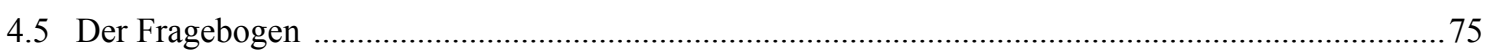


II DIE ZWEITSPRACHENERWERBSHYPOTHESEN UND FEHLERENTSTEHUNGSFAKTOREN........................... 87

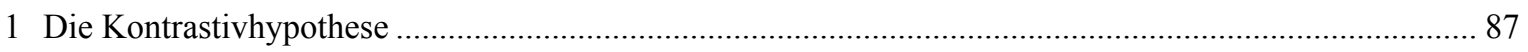

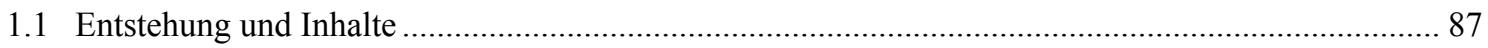

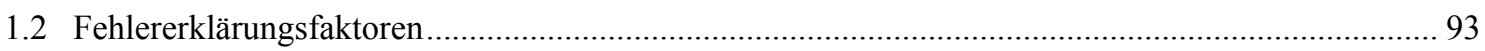

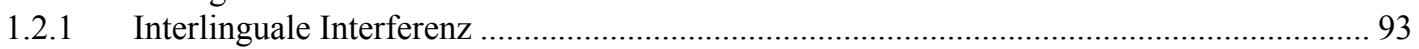

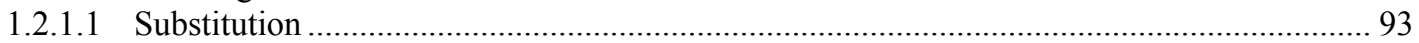

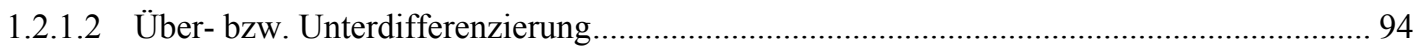

1.2.1.3 Über- bzw. Unterrepräsentation............................................................................. 94

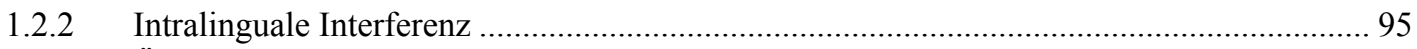

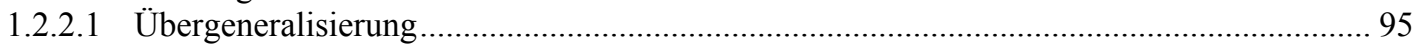

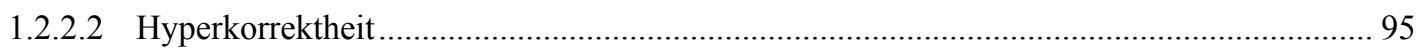

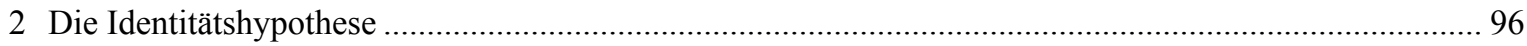

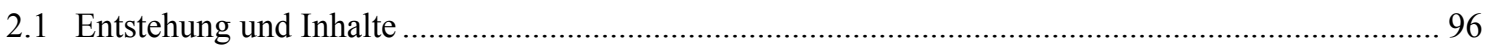

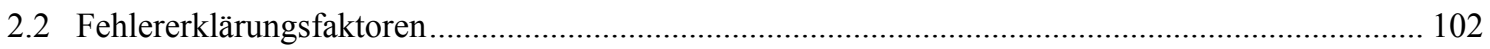

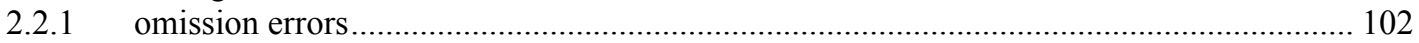

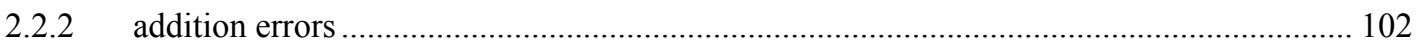

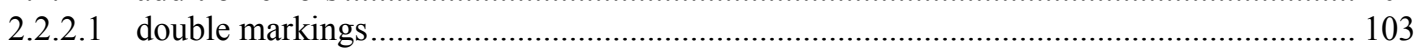

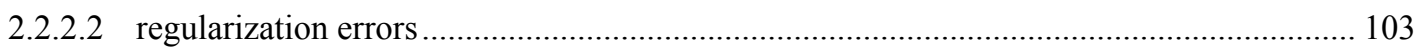

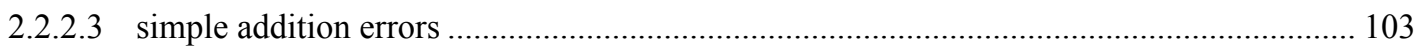

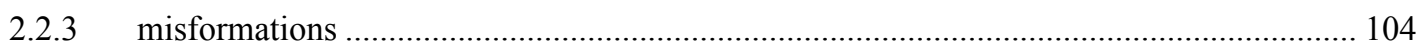

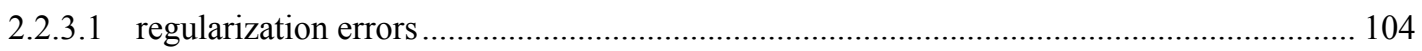

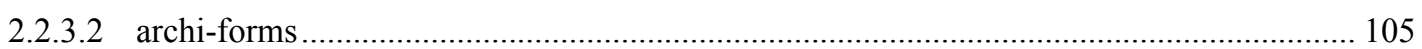

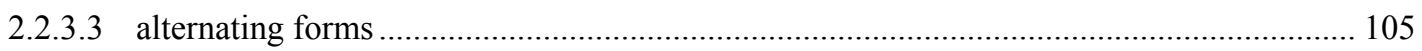

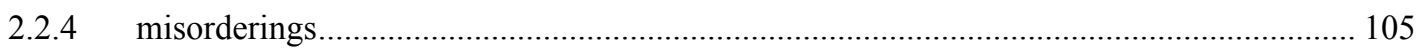

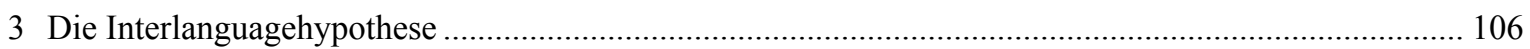

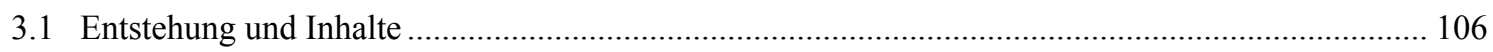

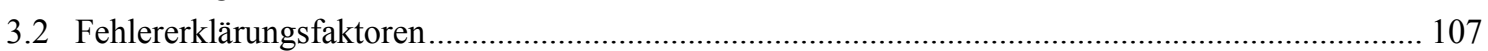

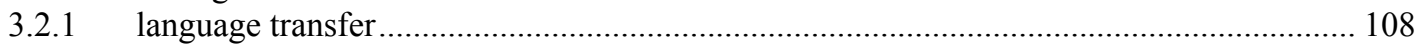

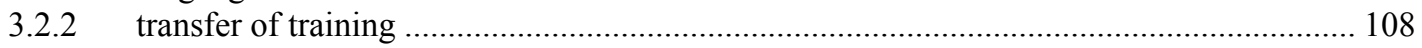

3.2.3 strategies of second language learning .................................................................. 108

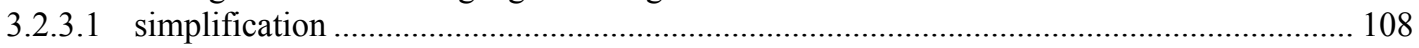

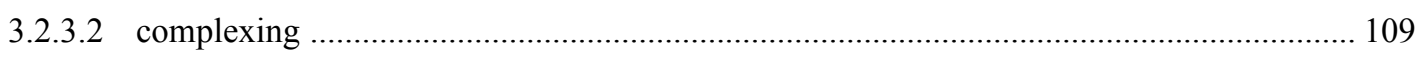

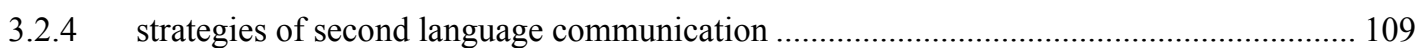

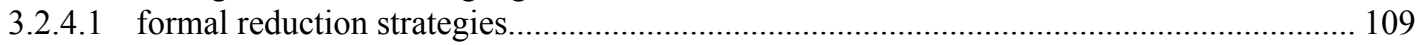

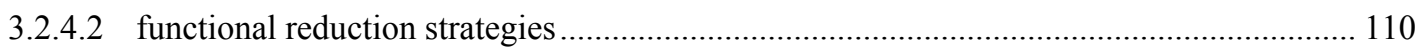

3.2.4.3 productive achievement strategies ............................................................................... 111

3.2.4.4 receptive achievement strategies .............................................................................. 114

3.2.5 overgeneralization of target language material ..................................................... 116

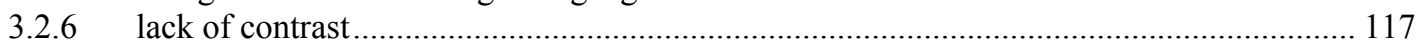

3.3 Exkurs: Differenzierung der Begriffe ,Prozesse' und ,Strategien' ............................................. 117

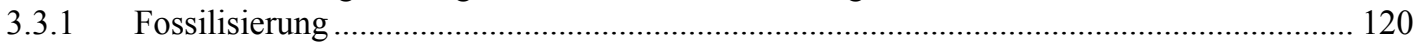

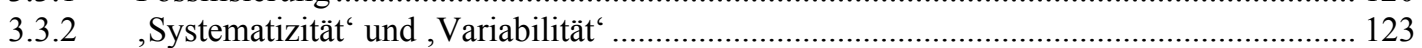




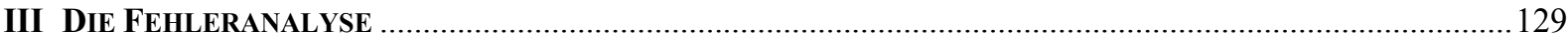

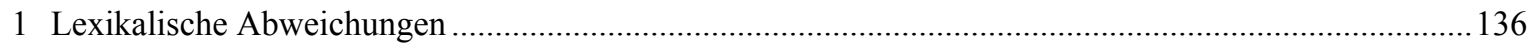

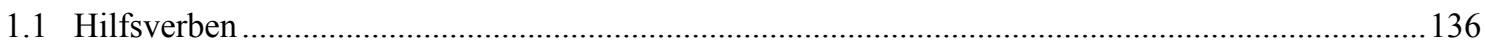

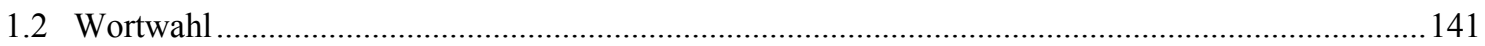

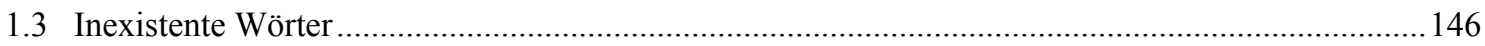

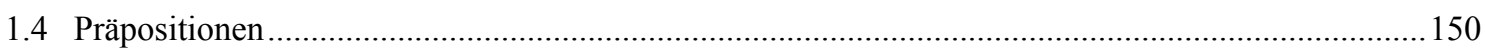

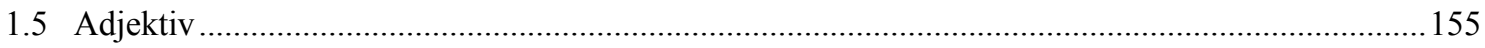

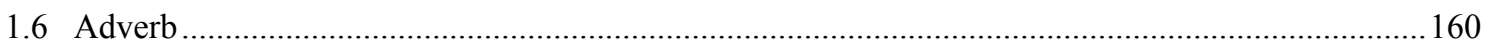

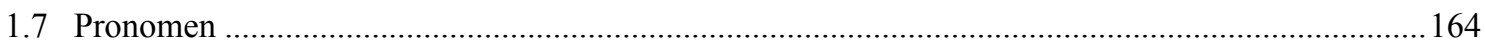

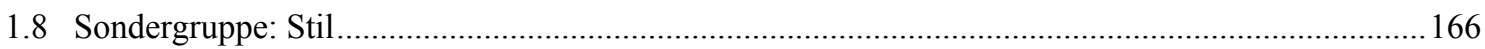

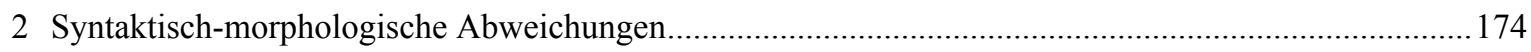

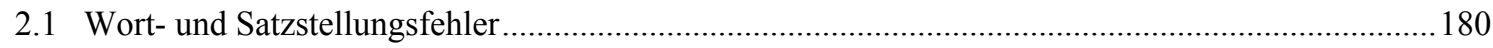

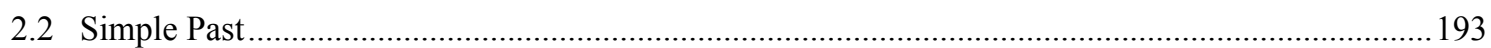

2.3 Hinzufügen und Auslassen sonstiger Wortarten und Bestandteile .................................................. 197

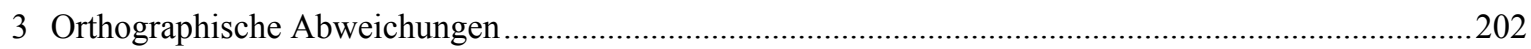

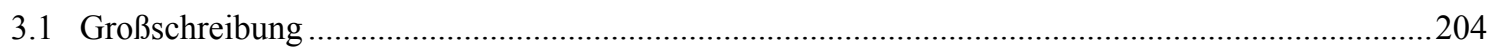

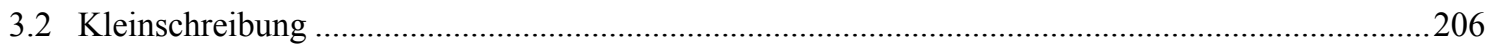

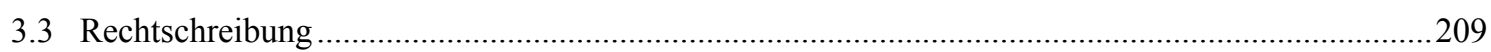

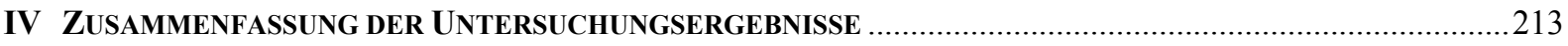

1 Vergleich der Untersuchungsergebnisse mit den Ergebnissen früherer Studien zur Fehlertypologie

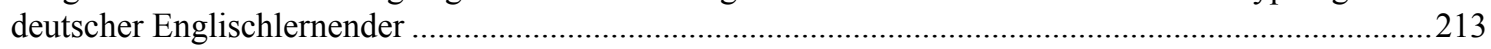

2 Die Anwendbarkeit der Fehlererklärungsfaktoren der Kontrastiv-, der Identitäts- und der

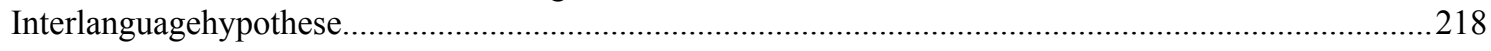

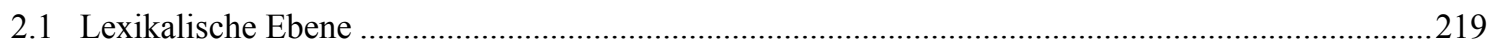

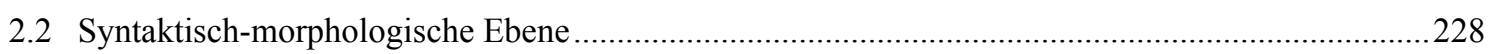

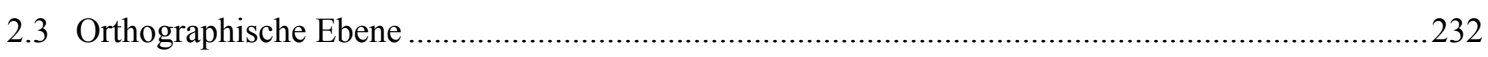

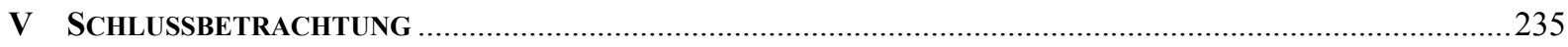

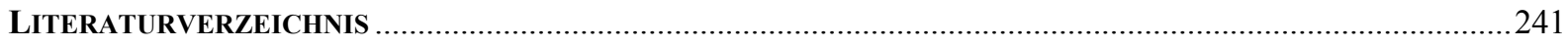

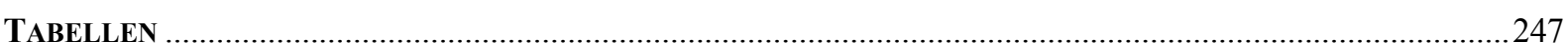

\section{Abbildungen}

Abbildung 1: Anteil der lexikalischen, syntaktisch-morphologischen und orthographischen Fehler ..................60

Abbildung 2: prozentualer Anteil der lexikalischen Fehler je Schulform ................................................... 74

Abbildung 3: prozentualer Anteil der syntaktisch-morphologischen Fehler je Schulform ..................................74

Abbildung 4: prozentualer Anteil der orthographischen Fehler je Schulform...............................................74

Abbildung 5: Fragebogen und Aufgabenstellung des Briefes ..............................................................275 



\section{Verwendete Abkürzungen}

FB

$=$ Fehlerbeispiel

HS

$=$ Hauptschule

$10 \mathrm{E}=$ Erweiterungskurs der Jahrgangsstufe 10

$10 \mathrm{G}=$ Grundkurs der Jahrgangsstufe 10

RS

$=$ Realschule

10spra. = Jahrgangsstufe 10, sprachlich orientiert

10sowi. = Jahrgangsstufe 10, sozialwissenschaftlich orientiert

10natw. $=$ Jahrgangsstufe 10, naturwissenschaftlich orientiert

GY

$=$ Gymnasium

$12 \mathrm{G}=$ Grundkurs der Jahrgangsstufe 12

$12 \mathrm{~L}=$ Leistungskurs der Jahrgangsstufe 12

EB

$=$ Erwachsenenbildung

$\begin{array}{ll}\text { beg. } & =\text { beginners } \\ \text { int. } & =\text { intermediate } \\ \text { adv. } & =\text { advanced }\end{array}$

PmeilAb $=$ Probanden mit den meisten lexikalischen Abweichungen (RS 10natw.)

PwenlAb $=$ Probanden mit den wenigsten lexikalischen Abweichungen (EB adv.)

$\mathrm{PmeioAb}=$ Probanden mit den meisten orthographischen Abweichungen (HS 10G)

PwenoAb $=$ Probanden mit den wenigsten orthographischen Abweichungen (GY 12L)

PmeismAb $=$ Probanden mit den meisten syntaktisch-morphologischen Abweichungen (HS 10E)

PwensmAb $=$ Probanden mit den wenigsten syntaktisch-morphologischen Abweichungen (EB adv.) 


\section{Glossar der Fehlererklärungsfaktoren der Erwerbshypothesen}

Im folgenden werden die Fehlererklärungsfaktoren der drei Erwerbshypothesen zur Übersicht aufgeführt. Die Darstellung der Fehlererklärungsfaktoren der Kontrastivhypothese erfolgt in deutscher Sprache, da diese auch in der deutschen Fachliteratur in der Regel in die deutsche Sprache übertragen werden. In bezug auf die Identitätshypothese und die Interlanguagehypothese werden hingegen die englischen Termini verwendet, weil diese zum Teil nicht sachgerecht ins Deutsche zu übertragen sind.

\section{Kontrastivhypothese}

1. Starke Version - interlinguale Interferenz

1.1 Substitutionen

1.2 Über- bzw. Unterdifferenzierung

1.3 Über- bzw. Unterrepräsentation

2. Schwache Version - intralinguale Interferenz

2.1 Übergeneralisierung

2.2 Hyperkorrektheit

\section{Identitätshypothese}

1. omission errors

2. addition errors

2.1 double markings

2.2 regularization errors

2.3 simple addition errors

3. misformations

Einheiten

3.1 regularization errors

3.2 archi-forms

3.3 alternating forms

4. misorderings

\section{Interlanguagehypothese}

1. language transfer

2. transfer of training

3. strategies of second language learning

3.1 simplification

3.2 complexing

4. strategies of second language communication
$=$ Auslassungsfehler

$=$ Hinzufügungsfehler

$=$ Doppelmarkierungen

$=$ Anwendung von Markierungselementen regelmäßiger Formen bei unregelmäßigen Formen

$=$ einfache Hinzufügungsfehler

$=$ Strukturverletzungen innerhalb grammatischer

$=$ Austausch von Formen zwischen Lexemen, die sich in ihrer grammatischen Funktion partiell entsprechen

$=$ Übertragung der Funktion von Lexemen einer Wortart auf andere Lexeme derselben Wortart

= Substitution lexikalischer Elemente einer grammatischen Kategorie durch andere Elemente dieser Kategorie

$=$ Elementvertauschungen

$=$ Übertragungen aus der Erstsprache oder anderen Zweitsprachen

$=$ Sprachtransfer bedingt durch Variablen des Fremdsprachenunterrichts

$=$ Lernstrategien

$=$ Simplifizierung, Vereinfachungsstrategie

$=$ Komplexierung

$=$ Kommunikationsstrategien 
4.1 formal reduction strategies

4.2 functional reduction strategies
a) topic avoidance
b) message abandonment
c) meaning replacement
d) modality reduction
e) personality reduction

4.3 productive achievement strategies
a) language switch
b) interlingual transfer
c) borrowing
d) inter-/intralingual transfer
e) generalization of interlanguage rules / items in new contexts
f) paraphrase
g) word coinage
h) restructuring
i) discourse related strategy

4.4 receptive achievement strategies
a) inferencing

b) monitoring

5. overgeneralization of target language material

6. lack of contrast
$=$ formale Reduktionsstrategien

$=$ funktionale Reduktionsstrategien

$=$ Themenvermeidung

$=$ Aufgabe der Äußerungsabsicht

$=$ Bedeutungsveränderung

$=$ Modalitätsreduktion

$=$ Persönlichkeitsreduktion

$=$ produktive Erweiterungsstrategien

$=$ bewußter Rückgriff auf die Erst- oder auf eine andere Zweitsprache

$=$ interlingualer Transfer

$=$ Entlehnung aus anderen Sprachen

$=$ inter-/intralingualer Transfer

$=$ bewußte Generalisierung von Interlanguage-Regeln / -Elementen

$=$ Paraphrasierung

$=$ Wortprägung

$=$ Umstrukturierung

$=$ diskurs-bezogene Strategie

$=$ rezeptive Erweiterungsstrategien

$=$ inferenzieren

$=$ Aktivierung der eigenen sprachlichen Kontrollinstanz

$=$ unbewußte Übergeneralisierung zielsprachlicher Regeln oder Elemente

$=$ Kontrastmangel 



\section{Einleitung}

Das Erlernen einer Sprache, sei es die Erstsprache oder eine sogenannte Fremdsprache, führt bei Anwendung des Erlernten zu Äußerungen, die der syntaktisch-semantischen bzw. lautlichen Norm dieser Sprache entsprechen, aber auch zu Äußerungen, die von dieser Norm partiell oder gänzlich abweichen. Während normentsprechende Äußerungen in der Regel willkommen sind, hat der Stellenwert normabweichender Sprachformen im Laufe der Geschichte des Fremdsprachenunterrichts insbesondere auch des deutschen Fremdsprachenunterrichts - entscheidende Veränderungen erfahren. Dies betrifft zum einen die fachliche Einstellung zu sprachlichen Abweichungen und zum anderen die Erkenntnisse, die in bezug auf die Entstehungsursachen sprachlicher Fehler gewonnen wurden. ${ }^{1}$

Das Streben nach sprachlicher Korrektheit, das unter anderem aus den Prinzipien der Aufklärung resultierte, führte dazu, daß sprachliche Fehler in der Frühphase des Fremdsprachenunterrichts und damit auch im deutschen Englischunterricht als negative Randerscheinungen des Spracherwerbs betrachtet wurden, die der Lernende zu vermeiden hatte. ${ }^{2}$ Dabei wurden die Ursachen sprachlicher Fehler in der Regel nicht eingehend untersucht, sondern nahezu ausschließlich auf mangelnden Einsatz der Lernenden zurückgeführt. Versuche, die Ursachen sprachlicher Fehler zu erkunden und deren Entstehung auf diesem Wege vorzubeugen, sind bis Ende des 19. Jahrhunderts nur sporadisch verzeichnet. ${ }^{3}$

Die Entwicklung des Englischen zu einer Weltsprache erforderte zu dieser Zeit auch in Deutschland eine Optimierung des Englischunterrichts. Dies führte dazu, daß die normabweichenden Sprachbestandteile deutscher Englischlernender mehr und mehr zum Gegenstand linguistischer Untersuchungen wurden, weil das bislang praktizierte Korrekturverhalten die Art und Anzahl der Fehlleistungen nicht zu reduzieren vermochte.

Die Entwicklung behavioristischer Lerntheorien und deren Erprobung im fremdsprachlichen Unterricht führten in den fünfziger Jahren des 20. Jahrhunderts schließlich zu einer verstärkten Beachtung der sogenannten Fehlergenese. Neben der bloßen Konstatierung eines Sprachfehlers sollten die fehlerevozierenden Faktoren künftig untersucht und Anleitungen zu deren Vermeidung gegeben werden. Somit stand erstmals der Weg, der zur Entstehung von Abweichungen geführt hatte, im Mittelpunkt der Forschung und nicht mehr ausschließlich das Produkt: die sprachliche Abweichung. In der Folge wurde eine Vielzahl von Untersuchungen durchgeführt, mit dem Ziel, die Entstehungsursachen

\footnotetext{
vgl. Raabe 1980, $61-68$

vgl. Palmer 1921, $64 \mathrm{f}$

Die englische Sprache unterscheidet zwischen den Lexemen mistake und error. Vgl. hierzu Corder 1967, 161 - 170.
} 
sprachlicher Fehler zu ergründen. Die Ergebnisse machten deutlich, daß Fehler das Resultat bestimmter sprachlicher Verhaltensweisen sind, die bei der Anwendung der Zielsprache eingesetzt werden und, wenngleich individuell variierend, bei allen Lernenden $\mathrm{zu}$ beobachten sind. Diese Verhaltensweisen zeigten, daß die Lernenden einer Fremdsprache beim Sprechen oder Schreiben dieser Sprache Strategien verfolgen, um in der Fremdsprache kommunizieren zu können.

Die zunehmende Bedeutung der behavioristischen Lerntheorie führte dazu, daß auch im Englischunterricht deutscher Schulen Studien durchgeführt wurden, die auf dem Konzept der Behavioristen basierten. In deren Rahmen wurde deutlich, daß Fehler bei deutschen Muttersprachlern, die Englisch lernen, dadurch entstehen, daß Lernende zur Kommunikation mit englischen Muttersprachlern häufig sprachliche Elemente des Englischen durch erstsprachliche Elemente ersetzen. ${ }^{4}$ Dabei glaubte man zu erkennen, daß diese Ersatzformen in der Regel zu Fehlern führen, weil die Struktur der englischen Sprache anderen Regeln unterliegt als die der deutschen. Zudem gewann man aus der Analyse dieser Verhaltensweisen die bis heute unangefochtene Erkenntnis, daß linguistische Abweichungen einen Einblick in die Mechanismen des Zweitspracherwerbs ${ }^{5}$ geben können. Hierdurch wurde die Fremdsprachendidaktik um eine den Spracherwerb maßgeblich beeinflussende Komponente erweitert: die Integration sprachpsychologischer Untersuchungen. ${ }^{6}$ Fehler beim Erlernen und Anwenden einer Fremdsprache wurden daher immer weniger als störende Nebenprodukte des Zweitspracherwerbs betrachtet, die einer unmittelbaren Korrektur bedürfen. Vielmehr galten sie forthin als Elemente, die einen Einblick in die Denkweise der Zweitsprachenlernenden ermöglichen und dem Lehrenden damit die Möglichkeit bieten, Fehlervermeidungsmodelle zu erstellen.

Die Studien, die im Englischunterricht an deutschen Schulen durchgeführt wurden, verdeutlichten dabei auch, daß die Fehler deutscher Muttersprachler beim Erlernen der englischen Sprache einander ähneln. Dies gilt sowohl für den schriftlichen Gebrauch der Sprache als auch für die mündliche Sprachproduktion, bei der die Ähnlichkeit zwischen den einzelnen Lautfehlern so deutlich ist, daß man von einem typisch deutschen Akzent sprechen kann. ${ }^{7}$

Diese Erkenntnisse führten zu der Auffassung, daß Fehler, die beim Erwerb einer Fremdsprache entstehen, ausschließlich sprachlich bedingt seien. Dem Prinzip behavioristischer Lerntheorien entsprechend, wurde das Erlernen einer Fremdsprache zunächst vereinfacht als reines input-output-

4 vgl. Heindrichs / Gester / Kelz 1980, $112-116$

5 Zur Unterscheidung der Begriffe Zweitsprache und Fremdsprache siehe insbesondere Edmondson 1999, 3 ff.

6 Wolff hatte aufgezeigt, daß im Zeitraum von 1894 bis 1970 in der Zeitschrift „Die Neueren Sprachen“ nur vier Beiträge zum Thema Sprachpsychologie und Zweitspracherwerb verzeichnet sind. Vgl. hierzu Wolff 1994, 103 - 123.

7 vgl. Weiher 1974, $14 \mathrm{f}$ 
Verhalten betrachtet. ${ }^{8}$ Die nach heutigem Wissensstand als erwiesen geltende Komplexität fehlergenetischer Faktoren blieb in den fünfziger Jahren weitgehend unerkannt. Die damaligen Forschungsansätze zur Fehlergenese beschränkten sich im wesentlichen auf einen Faktor, den Kontrast zwischen Erst- und Zweitsprache.

Als Folgerung aus einer Vielzahl kontrastiv-linguistischer Untersuchungen wurde die allzu einseitige Behauptung aufgestellt, lexikalische und syntaktisch-morphologische Identität zwischen Erst- und Zweitsprache vereinfachten das Erlernen der Zielsprache, während Differenzen auf diesen Gebieten den Prozeß erschwerten. ${ }^{9}$ Im Vordergrund der Untersuchungen stand folglich der kontrastive Vergleich zwischen Erst- und Zielsprache.

Die auf dieser Theorie begründete „Kontrastive Erwerbshypothese ${ }^{\text {“10 }}$ wurde bereits kurz nach ihrer Entstehung heftig kritisiert. Spätestens jedoch mit der Veröffentlichung von N. Chomskys Abhandlung „Rezension von Skinner, B. (1957): Verbal Behavior“"11 entwickelte sich ein linguistisches Streitgespräch, das zu einem völligen Umdenken innerhalb der Fremdsprachendidaktik führte. Ausgehend von Untersuchungen zum Erstspracherwerb setzte sich zunächst parallel zur Kontrastivhypothese die auf Chomskys Ergebnissen beruhende, in erster Linie von H. C. Dulay und M. K. Burt ${ }^{12}$ geprägte „Identitätshypothese“13 ${ }^{\text {“13 }}$ durch. Im Mittelpunkt dieser Theorie steht die Annahme, daß der Erwerb einer Zweitsprache nach universalen Prinzipien verläuft, die es jedem Sprecher ermöglichen, in der Fremdsprache eine Kompetenz zu erlangen, die seiner erstsprachlichen Kompetenz gleicht. Entwickelt in Anlehnung an Chomskys ,Language Acquisition Device' und die damit verbundene Prädeterminierung des Spracherwerbs, führte die Hypothese zu eingreifenden Veränderungen in der Fremdsprachendidaktik und zu einer Schwerpunktverlagerung innerhalb der Angewandten Sprachwissenschaft. Verstärkt wurde fortan der Versuch unternommen, die Verhaltensweisen, die Kinder beim Erwerb ihrer eigenen Erstsprache zeigen, auch für den Fremdsprachenunterricht nutzbar zu machen. ${ }^{14}$

Entgegen der einseitig orientierten sprachpsychologischen Betrachtung der Behavioristen verdeutlichten die Suche nach Parallelen zwischen der Methodik des Erwerbs der Erst- und der Zweitsprache sowie

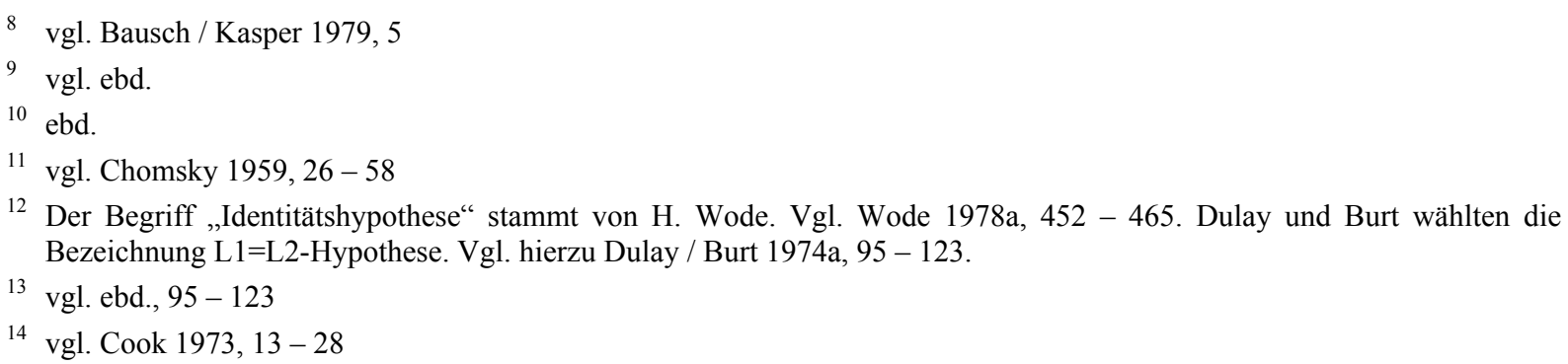


das Verständnis von Spracherwerb als creative construction ${ }^{15}$, daß Spracherwerb keineswegs bloß als input-output-Vorgang nach dem Prinzip trial und error verstanden werden darf. Vielmehr ist Spracherwerb als ein komplexer Vorgang sich gegenseitig beeinflussender Prozesse zu betrachten, deren Kenntnis zur Optimierung des Zweitspracherwerbs beitragen kann. Anstelle der objektiven Beobachtung von Sprache als Produkt eines durch Konditionierung gelernten Verhaltens stellt die generative Linguistik die dem Spracherwerb zugrundeliegenden Prozesse, die sich in Form einer Zwischensprache als der Annäherungsstufe zur target language manifestieren, in den Mittelpunkt ihrer Untersuchungen. ${ }^{16}$

Auf der Grundlage dieser Theorie entstand die Fehlerlinguistik als Teilgebiet der Angewandten Sprachwissenschaft. An die Stelle eines Vergleichs von Erstsprache und Zielsprache trat bei dem methodischen Ansatz der Fehlerlinguistik der Vergleich der Zwischensprache des Lernenden mit der erstrebten Zielsprache, nachdem vorab beide Sprachsysteme objektiv und unabhängig voneinander beschrieben wurden.

Das Konzept der generativen Linguistik und die davon geprägte Fehlerlinguistik in ihrer ursprünglichen Form, die erstsprachliche Interferenz als Fehlerfaktor ausschloß, konnte jedoch keine permanente Anwendung in der Fremdsprachendidaktik erzielen. Untersuchungen von Zweitspracherwerbsprozessen verdeutlichten immer wieder, daß der Einfluß der Erstsprache auf den Erwerbsprozeß einer Zweitsprache nicht zu ignorieren war. Gleichzeitig zeigten die Studien, daß der Prozeß des Zweitspracherwerbs individuell erheblich variiert und keineswegs linear verläuft. ${ }^{17}$ Letzteres gilt selbst dann, wenn unterschiedliche Individuen der Zweitsprache in bezug auf Dauer und Intensität in identischer Form ausgesetzt sind. Für die Zweitspracherwerbsforschung implizierte dies eine intensivere Betrachtung des Lernenden als Produzent zweitsprachlicher Äußerungen sowie eine verstärkte Untersuchung der Sprachform, die der Lernende auf dem Weg des Zielsprachenerwerbs benutzt. ${ }^{18}$

Die Einsicht, daß erstsprachliche Interferenz als Fehlerentstehungsfaktor nicht ausgeschlossen werden kann, führte in den späten sechziger Jahren schließlich zur Integration dieses Faktors in das Verfahren der Fehlerlinguistik.

Aus den Erkenntnissen, die im Rahmen der Untersuchungen zum Zweitspracherwerb gewonnen wurden, resultierte in den siebziger Jahren eine Vielzahl von Erwerbstheorien, deren Grundgedanken

\footnotetext{
15 vgl. Dulay / Burt 1977, 95 - 126

16 vgl. Bausch / Kasper 1979, 10

17 vgl. Young-Scholten / Archibald 2000, 64 - 101

18 vgl. ebd. $95 \mathrm{f}$
} 
als nahezu kongruent beschrieben werden können. Zu den bekanntesten Hypothesen gehört die 1972 von L. Selinker nach ihrem Forschungsschwerpunkt benannte „Interlanguagehypothese“ ${ }^{\text {“19 }}$, die bis heute ihre Gültigkeit bewahren konnte, jedoch insbesondere seit den achtziger Jahren entscheidend ergänzt wurde. ${ }^{20}$ Im Mittelpunkt dieser Theorie steht die Vorstellung, daß der Erwerb einer Zweitsprache von psychologischen Lernprozessen und Anwendungsstrategien bestimmt wird, die zu einem Teil unbewußt verlaufen, zum anderen Teil jedoch von den Lernenden bewußt angewandt werden. Damit wird Spracherwerb - entgegen der Theorie der Kontrastivhypothese - zu einem aktiven Vorgang, der zumindest teilweise der Steuerung des Lernenden unterliegt. Entscheidenden Einfluß auf den Lernprozeß haben der Interlanguagehypothese zufolge Persönlichkeitsmerkmale des Lernenden, d. h. die individuell unterschiedliche Art und Weise der Verarbeitung und Anwendung zweitsprachlicher Regeln und Strukturen. Sprachliche Faktoren - etwa der Einfluß der Erstsprache - bilden nach Selinker nur Teilaspekte der zweitsprachlichen Entwicklung. Ebenso wichtig sind das Lernumfeld, in dem die Zweitsprache erworben wird, und die Kommunikationserfahrungen, die der Lernende bei Anwendung der Zweitsprache sammelt.

Die der Identitätshypothese zugrundeliegende Vorstellung, der Verlauf des Zweitspracherwerbs sei aufgrund der universal principles vorbestimmt, ${ }^{21}$ gilt der Interlanguagehypothese zufolge nur für Sprachbereiche, bei deren Entwicklung anatomische Faktoren zum Tragen kommen. Dazu gehören u. a. phonetische Komponenten. Wenngleich die Interlanguagehypothese bis zum heutigen Tag nicht an Bedeutung verloren hat, erschwerten jedoch die Unterschiede in der Persönlichkeit der Lernenden und die Tatsache, daß Lernprozesse und Lernstrategien nur partiell überprüft werden können, die Umsetzung dieser Hypothese im Fremdsprachenunterricht. Zudem erkennt die Interlanguagehypothese die Konzepte der Kontrastivhypothese und der Identitätshypothese partiell an und stellt damit die wissenschaftliche Berechtigung dieser Hypothesen nicht grundsätzlich in Frage.

Die neu gewonnenen Erkenntnisse zur Fehlergenese führten seit den siebziger Jahren zu einem Wiederaufleben der schon die vorhergehenden Jahrzehnte beherrschenden linguistischen Debatten um die Frage, welche Theorie letztendlich die beste Grundlage für eine Optimierung der Fremdsprachendidaktik bieten könne. Zur Unterstützung der einzelnen Hypothesen wurde seither eine Vielzahl fehlerlinguistischer Untersuchungen durchgeführt, deren Aussagefähigkeit jedoch bereits zum damaligen Zeitpunkt in Frage gestellt wurde und bis heute einen zentralen Aspekt fehlerlinguistischer Diskussionen bildet. Die Zweifel an der wissenschaftlichen Fundiertheit der Untersuchungen betreffen zunächst die Untersuchungsmethoden, mit deren Hilfe die sprachlichen Fehler ermittelt wurden und die geringe Zahl der Probanden, die an den einzelnen Studien teilnahmen. Entscheidend für die

\footnotetext{
19 vgl. Selinker 1972, $209-231$

20 Einen allgemeinen Überblick über neuere Ergebnisse in der Interlanguage-Forschung bietet Selinker 1992.
} 
heftigen Kontroversen sind jedoch die Konzeption der Analyseverfahren, die der Untersuchung der Fehlerentstehungsursachen zugrunde gelegt wurden, und die damit verbundene Intention:

Die meisten seit Beginn der modernen Zweitspracherwerbsforschung durchgeführten Studien ${ }^{22}$ beruhen auf dem Bestreben, die Validität eines Zweitspracherwerbsmodells bzw. die Übertragbarkeit dieses Modells auf die Gestaltung des Fremdsprachenunterrichts zu überprüfen. Vordergründig dienen diese Studien dem Ziel, die Bedeutung der betreffenden Theorie bzw. des daraus abgeleiteten Zweitspracherwerbsmodells zu bestätigen oder zu widerlegen - und das Modell in letzterem Fall durch ein Konzept zu substituieren, welches auf einer Theorie basiert, die für den Fremdsprachenunterricht relevanter zu sein scheint. Die Analyse der Fehlerentstehungsursachen orientiert sich demnach in erster Linie an den Fehlerentstehungsfaktoren des Zweitspracherwerbsmodells, das beurteilt werden soll. Dabei wird bei der Mehrzahl der Studien ${ }^{23}$ jedoch die wirkliche Intention der Untersuchung - eine vorgefaßte Meinung zu bestätigen - bereits durch die Gestaltung des Testverfahrens impliziert: Die Aufgabenstellungen evozieren Fehlertypen, die die Vermutung nahelegen, daß die Zweitspracherwerbshypothese, deren Validität bestätigt oder widerlegt werden soll, in der Tat fremdsprachendidaktisch geeignet bzw. nicht geeignet ist. ${ }^{24}$

So werden zum Beispiel in der kontrastiven Linguistik zur Unterstützung der kontrastiven Erwerbshypothese vornehmlich phonetische Untersuchungen ${ }^{25}$ durchgeführt, weil die lautliche Ebene bei der Mehrzahl der Zweitsprachenlernenden erstsprachlichen Einfluß eindeutig erkennen läßt. Ebenso bezeichnend ist die Tatsache, daß Vertreter der Identitätshypothese syntaktisch-morphologische Untersuchungen durchführen, ${ }^{26}$ weil die Internalisierung sprachlicher Strukturen beim Zweitspracherwerb zweifellos Parallelen zum Erstspracherwerb verdeutlicht, während diese Übereinstimmungen im lexikalischen und phonetischen Bereich nicht vorbehaltlos nachgewiesen werden können. ${ }^{27}$ Weitere Beispiele bieten die im Rahmen der kontrastiven Linguistik häufig verwendeten Übersetzungsaufgaben, welche durch die Gestaltung der erstsprachlichen Vorlage oftmals die Entstehung interlingualer Interferenzen provozieren - beispielsweise durch Einbeziehung einer hohen Anzahl der sogenannten false friends ${ }^{28}$.

\footnotetext{
21 vgl. Kaltenbacher 2001a, $11-17$

22 vgl. Christ / Hüllen 1995, $1-7$

${ }^{23}$ vgl. Weller 1991, $710-732$. Zu Veröffentlichungen, die über das Jahr 1991 hinausgehen, siehe insbesondere James $1998,278-299$.

24 vgl. u. a. Burgschmidt / Götz 1974

25 vgl. u. a. Rein 1983, 35 ff

${ }^{26}$ vgl. Dulay / Burt 1974b, $37-53$

27 vgl. Libben 2000, 228 - 249; vgl. Weiher 1974, 5 ff

28 vgl. u. a. Helliwell 1989 u. Barnickel, 1992
} 
Eine weitere $\mathrm{zu}$ beobachtende Methode, die intendierte Befürwortung oder Ablehnung eines bestimmten Modells schon vorab zu gewährleisten, besteht darin, die Testgrundlagen inhaltlich zu begrenzen. So vergleichen Befürworter der Identitätshypothese häufig die Problemlaute deutscher Englischlernender mit den Problemlauten von Kindern, die Englisch als Erstsprache erwerben: Dabei soll durch den Nachweis von Übereinstimmungen die Theorie der Identitätshypothese unterstützt werden, ohne daß jedoch beachtet wird, daß phonetische Probleme beim Erstspracherwerb von begrenzter Dauer sind. ${ }^{29}$

Aufgrund der Vielzahl der deutschen Englischlernenden und der unterschiedlichen psycho- und soziolinguistischen Faktoren, die den Spracherwerb begleiten, sind die zuvor genannten Studien nur äußerst begrenzt aussagefähig in bezug auf die Aspekte, die zur Entstehung der einzelnen Fehler geführt haben. Die Ergebnisse, deren Repräsentativität aus obengenannten Gründen bereits im Vorfeld zu bezweifeln ist, beziehen sich zudem jeweils nur auf einen sehr eingeschränkten Personenkreis: die ausgewählte Probandengruppe in ihrer spezifischen Lernsituation. So führen diese Verfahren nicht nur zu unterschiedlichen Meinungen hinsichtlich der Faktoren, die die Entstehung der Fehler verursacht haben, sondern auch zu differierenden Vorstellungen über die Art der Fehler, die von deutschen Englischlernenden produziert werden.

Zur Vermeidung solch tendenziöser Ergebnisse bedarf es folglich einer Ergänzung der traditionellen Untersuchungsmethoden, außerdem der Zusammenstellung einer heterogenen und quantitativ äußerst umfangreichen Probandengruppe sowie der Entwicklung eines objektiven Analyseverfahrens zur Begründung der Fehlergenese.

\section{Ziele der Arbeit}

Die vorliegende Arbeit basiert auf dem Bemühen, die Ergebnisse, die im Rahmen fehlerlinguistischer Untersuchungen zum Thema ,Fehler deutscher Englischlernender ${ }^{\circ 30}$ gewonnen wurden, zu ergänzen und zu erweitern. Die Ausführungen beruhen hierbei auf einer empirischen Studie, die an deutschen Bildungseinrichtungen durchgeführt wurde.

Ziel der vorliegenden Arbeit ist es, die Entstehung linguistischer Fehler deutscher Muttersprachler beim Erwerb des Englischen durch ein neu zu entwickelndes Verfahren zu erklären, das die Theorien und Fehlererklärungsfaktoren der zuvor genannten Hypothesen miteinander verbindet. Auf dieser Grundlage werden die Hypothesen einander nicht konkurrierend gegenübergestellt, sondern als

29 vgl. u. a. Wode 1988, 188 - 191

30 Hierbei werden vornehmlich Fehlleistungen berücksichtigt, die in gesteuerten Erwerbssituationen zu beobachten sind. 
Spracherwerbsmodelle betrachtet, die sich fehleranalytisch ergänzen. Dies bedeutet, daß bei der Analyse der Fehler sowohl die Fehlererklärungsfaktoren der Interlanguagehypothese als auch die Fehlererklärungsfaktoren, die aus der Kontrastiv- und Identitätshypothese abgeleitet wurden, in Betracht gezogen werden.

Während traditionelle Fehleranalysen in der Regel auf einem Erwerbsmodell basieren, das widerlegt oder bestätigt werden soll, ${ }^{31}$ beruht das Analyseverfahren, das im Mittelpunkt dieser Arbeit steht, auf dem Bemühen, sprachliche, entwicklungsbedingte und strategisch-prozedurale Faktoren gemeinsam zu berücksichtigen. Die Untersuchungsmethodik unterscheidet sich daher in erster Linie durch die Entwicklung eines ganzheitlichen Analysemodells von den Methoden vorhergehender Fehleranalysen. Ausgehend von diesem Bestreben wird ein Modell entwickelt, das auf einer Kombination der Fehlererklärungsfaktoren der zuvor genannten Erwerbshypothesen basiert. Strukturell ist das Modell mit einem Katalog zu vergleichen, der inhaltlich aus den Fehlererklärungsfaktoren der Kontrastiv-, Identitäts- und Interlanguagehypothese besteht. Die Analyse der Fehler konzentriert sich dabei auf die Probandengruppen, die innerhalb einer bestimmten linguistischen Kategorie die meisten bzw. die wenigsten Fehler produzieren. Auf der Grundlage dieser Gegenüberstellung soll nachgewiesen werden, welche Faktoren in der einen Probandengruppe zu einer äußerst hohen Fehlerzahl beitragen und in der entgegengesetzten Gruppe dazu führen, daß die Fehlerzahl in der betreffenden Kategorie sehr gering ist.

Alle Fehler, die bei der Korrektur der Probandenbeiträge ermittelt werden, durchlaufen hierzu den gesamten Katalog, um auf diese Weise zu gewährleisten, daß die Fehler aus drei Sichtweisen analysiert werden. Zusätzlich unterscheidet sich das Verfahren dadurch von bereits bekannten Fehleranalysen, daß nicht nur die Fehler der Lernenden berücksichtigt werden, sondern auch das sprachliche Umfeld der betreffenden Abweichungen und die psycho- und soziolinguistischen Erfahrungen der Fehlerproduzenten. Der Begriff, sprachliches Umfeld‘ bezieht sich hier darauf, daß die Fehler nicht isoliert betrachtet werden, sondern immer in Beziehung zu dem Text gesetzt werden, in dem sie ermittelt wurden. Der Begriff ,psycho- und soziolinguistische Erfahrungen“ umfaßt das linguistische Wissen der Fehlerproduzenten, wie etwa Kenntnisse in anderen Zweitsprachen, das schulische Umfeld, in dem die Zweitsprache erworben wurde, Auslandserfahrungen in englischsprachigen Ländern und die sogenannten affektiven Faktoren. Hierzu zählen die emotionale Einstellung der Lernenden zur Zielsprache und die Gründe, die zum Erwerb der Zielsprache geführt haben.

Bei dieser Art der Fehleranalyse wird vermieden, bereits im Vorfeld eine der genannten Erwerbshypothesen zu bevorzugen: Das neue Verfahren beruht auf der Erkenntnis, daß die Komplexität

31 vgl. u. a. Hellinger 1977, 4 ff u. James 1998, $4-6$ 
sprachlicher Fehler bzw. ihrer Entstehung nur dann ausreichend analysiert werden kann, wenn alle Faktoren, die Sprachfehler erzeugen können, d. h. sowohl linguistische als auch außersprachliche, untersucht werden. Die Ergebnisse der Untersuchungen der vergangenen Jahre haben gezeigt, daß die Faktoren, die sprachliche Fehler verursachen, äußerst komplex sind. Sprachfehler basieren in der Regel nicht nur auf den Unterschieden zwischen der Erstsprache des Lernenden und der Zielsprache. Vielmehr sind linguistische Abweichungen in der Regel gleichzeitig auch auf eine Vielzahl entwicklungsbedingter, anatomischer, sozialer und psychologischer Faktoren zurückzuführen. Hierzu gehören u. a. das Alter des Lernenden, die Einstellung des Lernenden zur Zielsprache, die Beschaffenheit der Artikulationsorgane, Erfahrungen mit anderen Fremdsprachen etc. ${ }^{32}$

Da bei nahezu allen Fehlleistungen eine Kombination unterschiedlicher Entstehungsfaktoren zu konstatieren ist, soll mit Hilfe vorliegender Arbeit gleichzeitig bestätigt werden, daß weder die wissenschaftliche Gültigkeit der Kontrastivhypothese noch die der Identitäts- oder der Interlanguagehypothese grundsätzlich zu widerlegen ist. Vielmehr möchte die Arbeit dazu beitragen zu zeigen, bei welchen Fehlertypen die einzelnen Erwerbshypothesen besonders deutlich bzw. weniger deutlich zum Tragen kommen. Auf diese Weise sollen die Anwendungsmöglichkeiten der einzelnen Erwerbshypothesen in bezug auf die Bereiche Lexik, Syntax, Morphologie und Orthographie konkretisiert werden. Auch sollen Anregungen für zukünftige Fehlervermeidungsmodelle gewonnen werden. Der Schwerpunkt der Arbeit liegt dabei auf Fehlern des schriftlichen Sprachgebrauchs. Mündliche Fehler werden dann berücksichtigt, wenn sie zur Unterstützung einer getroffenen Aussage beitragen können.

Die Untersuchung der Fehler, die aus der empirischen Studie hervorgehen, dient darüber hinaus dem Ziel zu überprüfen, ob die ermittelten Fehler mit den Ergebnissen früherer Studien vergleichbar sind, oder ob beim Vergleich der Studien fehlertypologische Veränderungen zu beobachten sind. Es gilt daher zu überprüfen, inwieweit sich die Art der Fehler, die in der Fachliteratur als charakteristische Abweichungen deutscher Englischlernender bezeichnet werden, verändert hat - etwa aufgrund neuer Lehr- und Lernmethoden. Insgesamt betrachtet ist die vorliegende Arbeit als sprachwissenschaftlicher Beitrag zu sehen. Der didaktische Akzent, der dabei sichtbar wird, entsteht durch den Bezug der beschriebenen Fehlertypen und ihrer Analyse zu didaktischen Lehrmaterialien. In dieser Arbeit werden Fehler jedoch objektiv als sprachliche Erscheinungen betrachtet, die den Spracherwerb ebenso begleiten wie andere Erscheinungen, die in der Linguistik untersucht werden, etwa die Entwicklung der Artikulationsorgane. Der direkte Bezug zum Unterricht ist damit von sekundärer Bedeutung. Die didaktische Seite der Ausführungen kann - wie zuvor erwähnt - die Entwicklung von Unterrichtskonzepten allenfalls unterstützen, die Umsetzung der Ergebnisse für schulische Zwecke hingegen fällt in den Bereich fachdidaktischer Arbeiten.

32 vgl. Vogel 1990, $122-154$ 



\section{Die Untersuchung}

\section{Grundlagen und Voraussetzungen der Untersuchung}

Die Untersuchung, die in den nachfolgenden Ausführungen beschrieben wird, bildet die Grundlage der Fehleranalyse, die im Zentrum der vorliegenden Arbeit steht. Die Durchführung der Untersuchung erfolgte 1995. Wissenschaftliche Ergebnisse, die nach Abschluß der empirischen Studie publiziert wurden und für die Auswertung fehlerlinguistischer Untersuchungen von Bedeutung sind, wurden nachträglich berücksichtigt. Die Studie wurde an nordrhein-westfälischen und rheinland-pfälzischen Schulen und Sprachinstituten durchgeführt.

Da zielsprachliche Fehler auch durch den regionalen Dialekt des Lernenden verursacht werden können, wurden bei der anschließenden Analyse der Fehler auch Informationen über Lernende miteinbezogen, die aus anderen deutschsprachigen Regionen stammen, ${ }^{33}$ um somit ein objektiviertes Bild der Fehler deutscher Muttersprachler beim Erwerb des Englischen zu erhalten. ${ }^{34}$

Im Vordergrund des Untersuchungsaufbaus stand das Bestreben, eine Untersuchungsmethode anzuwenden, die den zuvor genannten Zielsetzungen der Arbeit sowohl in bezug auf die fehlertypologischen als auch auf die spracherwerbsspezifischen Aspekte gerecht wird. Folgende Voraussetzungen waren demnach zu berücksichtigen: Zum einen sollte die Untersuchungsgrundlage einen objektiven Einblick in die zielsprachlichen Kenntnisse der Teilnehmer ermöglichen, um die Vergleichbarkeit mit anderen Fehleruntersuchungen zu gewährleisten. Zu diesem Zweck sollten die Testmethode bzw. die dabei zugrundeliegenden Aufgaben ein breites Spektrum unterschiedlicher Abweichungsformen erzielen, nicht aber das gehäufte Auftreten ausgewählter Fehlertypen fördern. Zum anderen sollte bei Anwendung der Testmethode das strategisch-prozedurale Verhalten der Probanden deutlich werden. Es war daher wichtig, die Aufgaben so zu formulieren, daß die Abweichungen nicht in isolierten sprachlichen Äußerungen auftreten, sondern in einem größeren, mehrere Sätze umfassenden Zusammenhang.

Da im Zentrum der Arbeit die Analyse linguistischer Abweichungen des schriftlichen Sprachgebrauchs steht und dabei alle Formen schriftlicher Abweichungen berücksichtigt werden sollten, mußte die Untersuchungsmethode auf der Erarbeitung eines geschriebenen Textes beruhen. Der Untersuchungsaufbau sollte dabei gewährleisten, daß im Rahmen der Texterarbeitung alle Bereiche der schriftlichen Sprache, d. h. sowohl die lexikalische als auch die syntaktisch-morphologische und

33 Diese Informationen wurden der Fachliteratur zu Fehlern deutscher Englischlernender entnommen. Vgl. u. a. Hecht / Green 1983. 
die orthographische Ebene, zur Anwendung kommen. Zudem sollte die Untersuchungsgrundlage für alle Lernertypen geeignet sein und daher nur Inhalte berücksichtigen, die bereits im Englischunterricht der Probanden behandelt worden waren. Thematisch sollte der Test für die Probanden interessant sein, um dadurch die Bereitschaft zur Teilnahme am Untersuchungsverfahren zu fördern. Von Bedeutung war dabei auch, daß das gesamte Untersuchungsverfahren für alle Beteiligten, $d$. h. sowohl für die Probanden als auch für die Untersuchungsleiter und die Korrektoren, verständlich war. Hierdurch sollte zum einen ausgeschlossen werden, daß während der Untersuchung Fehler entstehen, die darauf beruhen, daß das Testverfahren bzw. die dabei zugrundeliegenden Aufgaben mißverstanden wurden. Für die Untersuchungsleiter und Korrektoren mußte der inhaltliche Hintergrund der Aufgabenstellung nachvollziehbar sein, damit die Untersuchungsleiter bei eventuell auftretenden Rückfragen während des Testverfahrens Erklärungen bieten konnten und die Korrektoren schneller in der Lage waren zu verstehen, auf welchem sprachlichen Hintergrund die Äußerungen der Probanden basierten. Darüber hinaus war es wichtig, das Testverfahren allgemein verständlich zu gestalten, weil sich die Untersuchung nicht nur an prüfungserfahrene Teilnehmer richten sollte, sondern auch an Probanden, die weniger Erfahrungen mit sprachlichen Tests gesammelt hatten. Schließlich sollte das Untersuchungsverfahren einen Einblick auch in die Faktoren des Spracherwerbs ermöglichen, die nicht ausschließlich mit dem linguistischen System einer Sprache verknüpft sind und zu den zuvor erwähnten psycho- und soziolinguistischen Komponenten gehören.

Auch in bezug auf die Probanden waren, ausgehend von der Zielsetzung dieser Arbeit, wichtige Voraussetzungen zu berücksichtigen. Zunächst war zu beachten, daß die Konstellation der Teilnehmer in bezug auf die Faktoren Alter, Geschlecht, schulische Ausbildung sowie auf die sogenannten affektiven Faktoren möglichst heterogen zu gestalten war. Letztere umfassen u. a. die Motive, die die Teilnehmer dazu veranlaßt haben, Englisch zu lernen, und die emotionale Einstellung zur englischen Sprache. Wie zuvor verdeutlicht, soll durch Gewährleistung der Heterogenität der Probandengruppe ausgeschlossen werden, daß die Studie sich auf einen festgelegten Teilnehmerkreis konzentriert, wodurch in erster Linie solche Fehler berücksichtigt würden, die für diesen Kreis charakteristisch sind.

Daneben mußte die Studie eine repräsentative Teilnehmerzahl umfassen, um die Aussagekraft der Ergebnisse zu gewährleisten. Zunächst war in diesem Zusammenhang festzulegen, welche Teilnehmerzahl als repräsentativ zu betrachten ist. Da im Rahmen der Untersuchung unterschiedliche Gruppen deutscher Muttersprachler beim Erwerb des Englischen betrachtet werden sollten, mußte die Gestaltung der Untersuchung sicherstellen, daß jede dieser Einzelgruppen aus einer aussagekräftigen Teilnehmerzahl bestand. Die Betrachtung vorhergehender Fehleranalysen hatte gezeigt, daß die einzelnen Untersuchungsgruppen dieser Studien in der Regel eine Teilnehmerzahl analog einer Schulklasse mittlerer

\footnotetext{
${ }^{34}$ Zum Problem Muttersprache / Dialekte - Fremdsprache vgl. u. a. Burgschmidt / Götz 1972, 209 - 225.
} 
Größe besaßen. ${ }^{35} \mathrm{Da}$ ein wesentliches Ziel der Untersuchung, die der vorliegenden Arbeit zugrunde liegt, darin besteht, die Erkenntnisse aus früheren Fehleranalysen zu ergänzen, war bei der Planung der Untersuchung zu beachten, daß die Größe der einzelnen Probandengruppen mindestens der Gruppengröße vorhergehender Studien entsprach bzw. sie möglichst überstieg.

\subsection{Die Untersuchungsteilnehmer}

Die geschilderten Voraussetzungen, die in bezug auf die Zusammensetzung der Probandengruppen zu berücksichtigen waren, führten $\mathrm{zu}$ der Entscheidung, die Untersuchung vornehmlich an Schulen durchzuführen. Dabei sollten sowohl unterschiedliche Schulformen und Jahrgangsstufen als auch Klassen bzw. Kurse mit unterschiedlichen fachlichen oder qualifikationsbezogenen Schwerpunkten berücksichtigt werden. Die Entscheidung, die Untersuchung in Schulen durchzuführen, beruhte auf folgenden Faktoren: Zum ersten bieten Schulen die Möglichkeit, einer Vielzahl deutscher Englischlernender zu begegnen, so daß dadurch eine Probandengruppe zugrunde gelegt werden konnte, die den quantitativen Voraussetzungen der Untersuchung entsprach. Die Berücksichtigung beider Geschlechter wird durch den geschlechtsgemischten Unterricht an deutschen Schulen automatisch gewährleistet. Zudem ermöglichte die Durchführung der Untersuchung in unterschiedlichen Schulformen und verschiedenen schulinternen Kursformen die Zusammenstellung von heterogenen Probandengruppen, weil sich die psycho- und soziolinguistischen Faktoren der Englischlernenden der einzelnen Schulformen erheblich unterscheiden und auch innerhalb der Kursformen eines bestimmten Schultyps heterogene Verhältnisse vorliegen. So haben die Lernenden vor dem Besuch einer bestimmten Schulform die englische Sprache möglicherweise zuvor schon an einer anderen Schulform erlernt. Auch die Regel, daß Lernende bei nicht ausreichenden schulischen Leistungen eine Jahrgangsstufe wiederholen müssen, führt zu einer Heterogenität innerhalb der einzelnen Schulklassen, weil die sprachlichen und außersprachlichen Erfahrungen der Lernenden, die nicht versetzt wurden und daher eine andere Klasse besuchen mußten, nicht immer mit den sprachlichen und außersprachlichen Erfahrungen der anderen Lernenden dieser Klasse übereinstimmen.

Unterschiede in den einzelnen Klassen werden in der Regel auch beim Vergleich der affektiven Faktoren deutlich: In jeder Klasse sind Lernende vertreten, die Englisch sowohl aus extrinsischen als auch aus intrinsischen Motiven erlernen, sowie Lernende, bei denen die extrinsische Motivation eindeutig überwiegt. Eine homogene Verteilung ist in den meisten Schulen nur in den sogenannten Wahlfächern zu beobachten, d. h. in den Kursen, die von den Lernenden besucht werden, weil sie sich - wie etwa bei den Leistungskursen der gymnasialen Oberstufe - ohne feste Vorgaben der Schule bewußt für diesen Kurs entschieden haben. Innerhalb der einzelnen Klassen bzw. Kurse einer bestimmten

35 vgl. u. a. Grabowski 1985, 452 - 456 u. Havranek 2001, 10 
Schulform unterscheiden sich oftmals auch die Englischkenntnisse der Lernenden, so daß neben den außersprachlichen Differenzen auch sprachliche Unterschiede deutlich werden. Dabei fördert die Berücksichtigung unterschiedlicher Schulformen und Jahrgangsstufen das Auftreten psycho- und soziolinguistischer Differenzen zusätzlich, weil sich die Probanden auch in bezug auf die Aspekte Alter und Lebenserfahrung unterscheiden. Daneben kommen auch linguistische Unterschiede wesentlich deutlicher zum Tragen, weil der Englischunterricht zwischen den einzelnen Schulformen bzw. Jahrgangsstufen inhaltliche und quantitative Unterschiede aufweist.

Bei der Überlegung, welche Schulformen berücksichtigt werden sollten, war folgender Aspekt zu beachten: Die Probanden, die die traditionellen Schulformen Hauptschule, Realschule und Gymnasium besuchen, sind in der Regel nicht älter als 18 - 20 Jahre, wenn sie die Schule verlassen. Um im Rahmen der Untersuchung unterschiedliche Altersstufen berücksichtigen zu können, mußte die Untersuchung auch an Schulen durchgeführt werden, die dem Bereich Erwachsenenbildung zuzuordnen sind. Neben den erstgenannten Schultypen sollte das Verfahren daher auch an Volkshochschulen, Sprachschulen sowie an sogenannten Abendgymnasien durchgeführt werden. In bezug auf die Jahrgangsstufen der einzelnen Schultypen war dabei ein weiterer Aspekt zu beachten: Wie die Richtlinien der Hauptschulen, Realschulen und Gymnasien verdeutlichen, werden in den ersten drei Lernjahren des Englischunterrichts die Grundlagen der englischen Sprache vermittelt. ${ }^{36}$ Wenngleich die meisten Lernenden bereits innerhalb dieses Zeitraums dazu fähig sind, elementare kommunikative Aufgaben zu bewältigen, entwickelt sich eine wirkliche Kommunikationsfähigkeit erst mit Beginn der Mittelstufe. Sprachliche Fehler, die vor dem Eintritt in die Mittelstufe zu beobachten sind, gehen daher oftmals darauf zurück, daß den Lernenden die sprachlichen Mittel fehlen, die sie zu einer normgerechten Kommunikationsform benötigen. Die Untersuchung sollte daher nur solche Klassen berücksichtigen, die die ersten drei Lernjahre bereits absolviert hatten. So sollte vermieden werden, daß bei der Untersuchung Fehler ermittelt werden, die auf Unkenntnis der zweitsprachlichen Regeln und Strukturen zurückzuführen sind und daher nicht wirklich verdeutlichen, inwieweit die Lernenden in der Lage sind, zielsprachliche Strukturen normgerecht anzuwenden. Da zum Zeitpunkt der Untersuchung in den meisten Schulen Englisch erst mit Beginn der 5. Klasse erlernt werden konnte, war davon auszugehen, daß die meisten Lernenden erst nach Abschluß der 7. Klasse über eine elementare Kommunikationsfähigkeit verfügen, so daß die Untersuchung erst in der Mittelstufe durchgeführt werden konnte. Jedoch mußte auch hier berücksichtigt werden, daß an vielen Schulen Latein als erste Fremdsprache erlernt werden kann und daher viele Schüler erst mit Beginn der 7. Klasse Englisch als Fremdsprache erlernen. Jahrgangsstufen, die davon betroffen waren, mußten daher gesondert untersucht werden.

36 vgl. Kultusminister des Landes Nordrhein-Westfalen (Hrsg.) 1989, 50 - 86; Kultusminister des Landes NordrheinWestfalen (Hrsg.) 1993, 67 - 98; Kultusminister des Landes Nordrhein-Westfalen (Hrsg.) 1994, 99 - 154 
Insgesamt sollten von jeder Jahrgangsstufe möglichst zwei Klassen berücksichtigt werden, um dadurch die Repräsentativität der Ergebnisse zu erhöhen. Da die Schulen, die dem Bereich Erwachsenenbildung zuzuordnen sind - abgesehen von den Abendgymnasien - Lernende in der Regel nicht einzelnen Jahrgangsstufen zuordnen, sondern sich bei der Konstellation der Gruppen an den Sprachkenntnissen der Teilnehmer orientieren, ${ }^{37}$ sollten bei der Durchführung der Untersuchungen im Bereich Erwachsenenbildung jeweils zwei Lernergruppen eines bestimmten Sprachniveaus berücksichtigt werden.

\subsection{Die Untersuchungsmethode}

In der Zweitspracherwerbsforschung werden je nach Untersuchungsschwerpunkt zwei Untersuchungsansätze verwendet, auf deren Grundlage das sprachliche Verhalten der Lernenden analysiert werden kann. Dies sind zum einen der produkt- und zum anderen der prozeßorientierte Ansatz. ${ }^{38} \mathrm{Im}$ Vordergrund des produktorientierten Ansatzes steht das Ergebnis der schriftlichen und mündlichen Sprachanwendung. Das Ziel produktorientierter Fehleruntersuchungen besteht in der Regel entweder darin zu ermitteln, inwieweit die Lernenden vorgegebene Elemente normgerecht anwenden können, oder darin zu untersuchen, welche Fehlertypen in den einzelnen Erwerbsstadien zu beobachten sind. ${ }^{39}$

Im Mittelpunkt prozeßorientierter Untersuchungen hingegen steht die Analyse des Wegs, der zu bestimmten sprachlichen Äußerungen geführt hat. Dieser Ansatz beruht auf Erkenntnissen der kognitiven Psychologie. Mit Hilfe prozeßorientierter Studien können die mentalen Verarbeitungsprozesse und die bewußten Strategien und unbewußten Prozesse, die bei Anwendung zielsprachlicher Strukturen zu erkennen sind, zurückverfolgt werden. ${ }^{40}$ Zur Untersuchung der zweitsprachlichen Produkte und Prozesse wurden in der Zweitspracherwerbsforschung verschiedene Untersuchungsformen entwickelt, die entweder nur bei produkt- oder nur bei prozeßorientierten Ansätzen zur Anwendung kommen, oder auch bei beiden Ansätzen gleichzeitig. Zu den wichtigsten Untersuchungsformen gehören hier die sogenannten observational studies, die experimental studies und die introspection, die als jüngste Untersuchungsmethode zu betrachten ist. ${ }^{41}$

\footnotetext{
37 vgl. Bianchi / Busch / Sommet 1987, 5 - 9

38 vgl. Herrmann 1994, 566 - 584 u. Lightbrown 1990, 82 - 92

39 vgl. Timm 1996a, 271

40 vgl. Multhaup / Wolff 1992, 7 - 13

41 vgl. James 1998, 20
} 


\subsubsection{Observational studies}

Wie die Bezeichnung observational bereits impliziert, liegt der Schwerpunkt der Untersuchungsformen dieser Kategorie auf dem Bemühen, die sprachlichen Ergebnisse der Lernenden objektiv zu betrachten. Diese Form der Sprachuntersuchung wird bei produkt- und bei prozeßorientierten Ansätzen verwendet. ${ }^{42}$ Observational studies werden entweder in Form von longitudinal studies oder in Form von cross-sectional studies durchgeführt. ${ }^{43}$

Longitudinal studies werden zugrunde gelegt, wenn die sprachliche Entwicklung einer bestimmten Lernergruppe über einen längerfristigen, d. h. mehrere Erwerbsstadien umfassenden Zeitraum hinweg untersucht werden soll. Untersuchungsformen dieser Art werden häufig bei Studien zum ungesteuerten Spracherwerb verwendet. ${ }^{44}$ Bei dieser Beobachtungsform müssen die Probanden meist keine sprachlichen Aufgaben bearbeiten. Im Vordergrund steht vielmehr die natürliche Interaktion. Das Sprachverhalten der Probanden wird dabei in der Regel mittels Tonträgern oder schriftlichen Aufzeichnungen festgehalten. ${ }^{45}$

Die Bezeichnung cross-sectional wurde für Untersuchungen geprägt, die punktuell, zu einem festgelegten Zeitpunkt, durchgeführt werden. In der Regel werden dabei mehrere Probandengruppen gleichzeitig untersucht, um den zweitsprachlichen Entwicklungsstand der einzelnen Probandengruppen vergleichen zu können. ${ }^{46}$ Bei dieser Untersuchungsform werden häufig sprachliche Aufgaben zugrunde gelegt, die dem Ziel der Untersuchung entsprechend entweder prozeß- oder produktorientiert sind ${ }^{47}$, oder auch beide Ansätze miteinander verbinden. Zu den bekanntesten Untersuchungsmethoden gehören dabei die sogenannten information-gap activities, die als Kombination von produkt- und prozeßorientierten Untersuchungsformen zu betrachten sind. ${ }^{48}$ Inhaltlich basiert die Methode darauf, daß die Probanden Informationen erfragen und weitergeben müssen.

\footnotetext{
42 vgl. u. a. Schneider 1992, $34-40$

43 vgl. James 1998, 20

44 vgl. hierzu u. a. Vogel 1989, $123-147$

45 vgl. ebd., $124 \mathrm{f}$

46 vgl. James 1998, 20

47 vgl. ebd.

48 vgl. Timm 1996a, 274
} 


\subsubsection{Experimental studies}

Untersuchungen der Kategorie experimental studies ${ }^{49}$ unterscheiden sich von den observational studies dadurch, daß sie das sprachliche Verhalten der Probanden durch die Vorgabe festgelegter Aufgaben beeinflussen, während - wie erwähnt - observational studies eine objektive Betrachtungsweise anstreben.$^{50}$ Experimental studies bezeichnen Untersuchungsformen, mit deren Hilfe überprüft werden soll, ob die Probanden eine bestimmte Kategorie zielsprachlicher Elemente normgerecht anwenden können. ${ }^{51}$ Diese Untersuchungsformen können weder eindeutig als prozeß- noch als produktorientiert bezeichnet werden, weil sie zum einen nur beschränkten Einblick in das strategischprozedurale Verhalten der Lernenden bieten und zum anderen kaum zur Produktion von selbstverfaßten zielsprachlichen Äußerungen beitragen. Zu eigenständigen Äußerungen führen experimental studies in der Regel nur dann, wenn sie mit Elementen anderer Untersuchungsformen kombiniert werden. $\mathrm{Zu}$ den klassischen Formen der experimental studies zählen u. a. cloze tests, Diktate und multiple-choiceAufgaben.

\subsubsection{Introspection}

Testmethoden dieser Kategorie beruhen auf dem Bemühen, Einblicke in die sogenannte black box der Lernenden zu erhalten, in der das Sprachwissen gespeichert ist. Auf diese Weise sollen die Prozesse, die zu bestimmten sprachlichen Äußerungen geführt haben, nachvollzogen werden. ${ }^{52}$ Testmethoden dieser Art sind demnach eindeutig als prozeßorientiert zu bezeichnen. ${ }^{53}$ Sie unterscheiden sich jedoch von den zuvor genannten Ansätzen dadurch, daß die Lernenden thematisch im Mittelpunkt der Testmethoden stehen und dadurch, daß sie die Kooperation der Lernenden erfordern. Diese Testmethoden basieren auf der Annahme, daß der Lernende Zugang zu den mentalen Prozessen hat, die seine Sprachproduktion steuern. ${ }^{54}$ Dieser Zugang ermöglicht es ihm, die mentalen Prozesse zu beobachten und befähigt ihn damit, über diese Abläufe in Form einer sogenannten metalanguage zu sprechen. ${ }^{55}$ Da der Lernende thematisch im Zentrum dieser Testmethoden steht, gehören Beschreibungen des eigenen Sprachverhaltens in Form von self-reports oder self-observations und der Gebrauch von

\footnotetext{
49 In der literarisch ausgerichteten Fremdsprachendidaktik hat der Begriff experimental studies eine vollständig andere Bedeutung: Er bezeichnet hier spielerische Untersuchungsmethoden, wie etwa die Anregung zum kreativen Umgang mit zielsprachlichen Texten. Vgl. hierzu u. a. Kaspari 1995, 345 - 353.

50 vgl. James 1998, 20

51 vgl. Lado 1965

52 vgl. Faerch / Kasper (Hrsg.) 1987

53 vgl. Herrmann 1994, 566

54 vgl. James 1998, 21 u. Faerch / Kasper 1987, 5 - 23

55 vgl. Timm 1996b, 177
} 
Fragebögen, die psycho- und soziolinguistische Fragen enthalten, zu den charakteristischsten Merkmalen dieser Untersuchungsform. ${ }^{56}$

\subsubsection{Konkretisierung der Untersuchungsmethode}

Der Vergleich der unterschiedlichen Untersuchungsmethoden hat vor dem Hintergrund der Untersuchungsziele und der damit verbundenen Voraussetzungen gezeigt, daß bei der Konzeption der hier $\mathrm{zu}$ verwendenden Untersuchungsmethode sowohl Aspekte der observational und der experimental studies als auch Aspekte der introspection zu berücksichtigen waren. Darüber hinaus sollte die Untersuchung auf einem Verfahren basieren, welches prozeß- und produktorientierte Ansätze miteinander verbindet. Aspekte der observational studies waren von Bedeutung, weil die Ermittlung objektiver Fehlerergebnisse zu den wichtigsten Grundvoraussetzungen der Studie zählten. Da die Fehler der einzelnen Probandengruppen nach Abschluß des Korrekturverfahrens miteinander verglichen werden sollten und es dazu einer Vergleichsgrundlage bedurfte, mußte die Untersuchung jedoch auch auf der Erarbeitung sprachlicher Aufgaben, d. h. auf der Verwendung von Elementen der experimental studies beruhen, die die quantitative Überschaubarkeit und die thematische Vergleichbarkeit der Ergebnisse gewährleisten sollten. In diesem Zusammenhang war es zudem wichtig, methodische Verfahren der introspection $\mathrm{zu}$ berücksichtigen sowie prozeß- und produktorientierte Ansätze miteinander $\mathrm{zu}$ verbinden, weil im Rahmen der Untersuchung auch psycho- und soziolinguistische Aspekte ${ }^{57}$ betrachtet werden sollten und dabei nicht nur das Ergebnis sprachlicher Äußerungen im Vordergrund stand, sondern auch der Weg, der zur Entstehung dieser Äußerungen geführt hatte.

Die Überlegung, welche methodischen Ansätze der einzelnen Untersuchungsformen dabei konkret angewandt werden sollten, führte zunächst in bezug auf die Kategorie der observational studies zu der Entscheidung, die Untersuchung in Form einer cross-sectional study durchzuführen. Als Untersuchungsgrundlage war dabei die Aufgabe vorgesehen, einen Brief in englischer Sprache zu verfassen. Zur Berücksichtigung methodischer Elemente der experimental studies sollten die Briefe auf thematischen Vorgaben beruhen. Die Anwendung einer Methode der introspection schließlich sollte in Form eines Fragebogens ${ }^{58}$ erfolgen, der von den Probanden auszufüllen sein würde.

Die Entscheidung, die Untersuchung in Form einer cross-sectional study durchzuführen, lag darin begründet, daß zum einen der Zeitraum, der zur Durchführung der Untersuchung zur Verfügung stand, begrenzt war und daher keine longitudinal study durchgeführt werden konnte und daß es zum anderen

\footnotetext{
56 vgl. Faerch / Kasper in: Faerch / Kasper, 5 - 23

57 vgl. Vogel 1990, 121 - 166

58 vgl. Abb. 5 im Anhang
} 
schwierig ist, eine Vielzahl unterschiedlicher Lernertypen über einen Zeitraum hinweg zu beobachten, der mehrere Erwerbsstadien umfaßt. Hinzu kommt, daß cross-sectional studies aufgrund der Tatsache, daß hierbei mehrere Probandengruppen zur gleichen Zeit und unter gleichen Bedingungen untersucht werden, objektiver sind als longitudinal studies, weil sich bei Langzeitstudien die Untersuchungsbedingungen im Laufe des Untersuchungszeitraums ändern können. ${ }^{59}$

Den Anstoß dazu, die Kommunikationsform Brief als Testgrundlage zu verwenden, gab eine 1983 von K. Hecht und P. S. Green durchgeführte Untersuchung, in der das Korrekturverhalten englischer und deutscher Englischlehrer bei der Korrektur von Schülerbriefen miteinander verglichen wurde. ${ }^{60}$ Das Verfassen von Briefen erschien auch in bezug auf die Ziele der vorliegenden Arbeit geeignet, weil Briefe zum einen zu den schriftlichen Kommunikationsformen gehören und daher geeignet sind, Abweichungen der Schriftsprache zu ermitteln. Zum anderen kommen beim Verfassen eines Briefes in der Regel alle Bereiche der Schriftsprache zur Anwendung und die Textgestaltung erfordert den Gebrauch unterschiedlicher lexikalischer Elemente und grammatischer Strukturen. ${ }^{61}$ Sprachliche Fehler, die in diesem Zusammenhang entstehen, treten nicht in isolierten Einzelsätzen auf, sondern in einem größeren sprachlichen Kontext. Damit ermöglicht diese Testform nicht nur die Untersuchung sprachlicher Ergebnisse, sondern auch die Analyse des Wegs, der zur Entstehung von Abweichungen geführt hat, weil das sprachliche Umfeld, das den Fehler umgibt, in die Untersuchung miteinbezogen werden kann. Die Aufgabenstellung, einen Brief zu verfassen, gehört demnach zu den Untersuchungsmethoden, die sowohl prozeß- als auch produktorientiert sind. Daneben zählen Briefe zu den lebensnahen Kommunikationsformen, die trotz der zunehmenden Kommunikationsmöglichkeiten auch in der heutigen Zeit von Menschen unterschiedlichen Alters genutzt werden. Somit war die Untersuchungsgrundlage realitätsbezogen und für alle Beteiligten des Untersuchungsverfahrens verständlich. Hinzu kommt, daß bei Anwendung dieser Untersuchungsgrundlage keine Altersunterschiede zu berücksichtigen waren und aufgrund der Realitätsbezogenheit der Methode eine erhöhte Kooperationsbereitschaft der Probanden zu erwarten war. Darüber hinaus gehört das Thema Briefeschreiben in allen Schulformen zu den elementaren Inhalten des Englischunterrichts ${ }^{62}$ und wird darüber hinaus auch im Deutschunterricht behandelt. Die Testmethode war daher den Probanden demnach bekannt und sollte keiner eingehenden Erläuterung bedürfen. Gleichzeitig war diese Untersuchungsmethode damit für alle Lernertypen geeignet.

Eine weitere Funktion der verwendeten Elemente der experimental studies, d. h. der thematischen Vorgaben, die dem Verfassen der Briefe zugrunde gelegt wurden, besteht darin, den Probanden zu

\footnotetext{
59 vgl. Vogel 1989, 123 - 147

${ }^{60}$ vgl. Hecht / Green 1983, $11-59$

61 vgl. ebd., 12

62 vgl. ebd.
} 
ermöglichen, die Briefe ohne thematische Vorüberlegungen zu schreiben. Auf diese Weise sollte verhindert werden, daß sie mangels eines Themas, über das sie schreiben konnten, nicht oder nur partiell an dem Untersuchungsverfahren teilnehmen. Gleichzeitig sollten diese Vorgaben die Korrektoren dazu befähigen, die Redeabsicht der Probanden bei schwerwiegenden Normabweichungen, die das Verständnis der sprachlichen Aussage erheblich beeinträchtigen, zu verstehen und entsprechende Korrekturbeispiele zu geben.

Die Entscheidung, die psycho- und soziolinguistischen Faktoren des Spracherwerbs mit Hilfe eines Fragebogens zu ermitteln, basierte - ähnlich wie die Auswahl der Aufgabenstellung, einen Brief zu verfassen - auf der Tatsache, daß das Ausfüllen von Fragebögen allen Beteiligten des Untersuchungsverfahrens aus dem täglichen Leben bekannt ist. Auch diese Testmethode mußte daher vor der Durchführung der Untersuchung nicht näher erläutert werden. Hinzu kommt, daß Fragebögen zu den zeitsparenden Testmethoden zählen und von nahezu jeder Altersgruppe ausgefüllt werden können. Darüber hinaus gehören psycho- und soziolinguistische Aspekte zu den Bereichen der Sprachwissenschaft, die die Privatsphäre der Lernenden berühren. ${ }^{63}$ Fragen, die sich auf diesen Bereich beziehen, werden daher verständlicherweise nicht von allen Probanden bereitwillig beantwortet. Mit Hilfe der Testform ,Fragebogen“ konnten die Probanden jedoch zu diesen Fragen anonym Stellung nehmen, ohne sich dabei in elaborierter Form zu den einzelnen Aspekten äußern zu müssen.

Bei der Konzeption der inhaltlichen Vorgaben der Briefe und der Gestaltung des Fragebogens waren folgende Kriterien zu beachten: Die inhaltlichen Vorgaben waren so zu gestalten, daß die Aufgaben einerseits einen großen sprachlichen Freiraum eröffneten, der zur Entstehung unterschiedlicher Abweichungsformen beitragen sollte, andererseits jedoch das sprachliche Verhalten der Probanden steuerten und damit zu vergleichbaren inhaltlichen Ergebnissen führten. Gleichzeitig sollten die Themen, die den Vorgaben zugrunde lagen, die Kooperationsbereitschaft der Probanden fördern und realitätsbezogen sein. Auch war zu beachten, daß die Vorgaben gleichermaßen für Probanden geeignet sein sollten, die über geringe Englischkenntnisse verfügen wie auch für Probanden, deren Englischkenntnisse sich auf mittlerem oder fortgeschrittenem Niveau befinden. Daneben waren bei der thematischen Auswahl Motive zu wählen, die sowohl inhaltlich als auch grammatisch in unterschiedlichen Altersgruppen angewandt werden konnten.

Die Betrachtung der Richtlinien der einzelnen Schulformen in den unterschiedlichen Bundesländern und der Materialien, die dem Unterricht zugrunde gelegt werden, zeigte, daß im Englischunterricht

${ }^{63}$ Hierzu zählt insbesondere die Frage nach dem psychischen und physischen Befinden der Probanden. 
deutscher Schulen einige Themen zum Basisstoff zählen und in allen Schultypen vertreten sind. ${ }^{64}$ Diese Themen betreffen Aspekte, die dem Alltagsleben der Lernenden zuzuordnen und nicht an den Schultyp gebunden sind, an dem der Englischunterricht stattfindet. Übereinstimmend ist dabei festzustellen, daß diese Themen vornehmlich zu den Inhalten der ersten Lernjahre gehören, ${ }^{65}$ jedoch in fortgeschrittenen Phasen des Englischunterrichts wieder aufgegriffen werden. ${ }^{66} \mathrm{Zu}$ diesen Motiven gehören beispielsweise die Beschreibung des Wohnorts, die Freizeitgestaltung oder die Planung und Durchführung eines Schüleraustauschs.

Auch auf grammatikalischer Ebene sind beim Vergleich der unterschiedlichen Schulformen Übereinstimmungen zu erkennen. Hier zeigte sich, daß bis zur Mittelstufe an allen deutschen Schulformen die elementaren Zeitformen der Gegenwart, Zukunft und Vergangenheit sowie die if-clauses und der Gebrauch des Aktivs und des Passivs vermittelt werden. ${ }^{67}$ Dies gilt mit der Einschränkung, daß im Falle der if-clauses zum Teil nur der sogenannte erste und zweite Typ gelehrt werden. Aufgrund dieser Unterrichtsinhalte sollten jedenfalls alle Probanden, die die Unterstufe absolviert haben, in der Lage sein, ihre gegenwärtige Situation zu beschreiben, über vergangene und zukünftige Ereignisse zu berichten und eventuell eintretende Ereignisse zum Ausdruck zu bringen. Ebenfalls sollten sie über die sprachlichen Mittel verfügen, über Dinge und Personen in passivischer Form zu schreiben.

Auf der Basis der somit vorauszusetzenden sprachlichen Grundlagen wurden nachfolgend die inhaltlichen Vorgaben für die von den Probanden zu verfassenden Briefe erarbeitet. Diese sollten dazu führen, daß die sprachlichen Aspekte, die die Probanden den Richtlinien entsprechend bereits internalisiert haben müßten, in möglichst umfassender Form zur Anwendung kommen. Zu diesem Zweck wurden Themen ausgewählt, die in allen Schulformen behandelt werden und gleichzeitig zu den Inhalten der ersten und der späteren Lernjahre gehören. Ausgehend von dieser Entscheidung wurden fünf thematische Motive erarbeitet, die als inhaltliche Vorgaben der Briefe dienen sollten. ${ }^{68}$ Diese Themen wurden sodann auf einem Aufgabenblatt festgehalten, das die Probanden zu Beginn der Durchführung des Testverfahrens erhielten. Vorgabe war, die Themen in der Reihenfolge zu bearbeiten, in der sie auf dem Aufgabenblatt erschienen. Als thematische Grundlage, auf der die fünf Motive ausgearbeitet werden sollten, diente die Aufgabe, einen Brief an einen fiktiven Brieffreund in englischer Sprache zu schreiben. Bei der Bearbeitung der Themen war es den Verfassern freigestellt, ob sie sich auf reale oder erfundene Ereignisse beziehen wollten.

\footnotetext{
64 vgl. Kultusminister des Landes Nordrhein-Westfalen (Hrsg.) 1989, 50 - 86; Kultusminister des Landes NordrheinWestfalen (Hrsg.) 1993, 67 - 98; Kultusminister des Landes Nordrhein-Westfalen (Hrsg.) 1994, 99 - 154

65 vgl. ebd.

${ }^{66}$ vgl. Kultusminister des Landes Nordrhein-Westfalen (Hrsg.) 1989, 67 - 112; Kultusminister des Landes NordrheinWestfalen (Hrsg.) 1993, 98 - 167; Kultusminister des Landes Nordrhein-Westfalen (Hrsg.) 1994, 174 - 304

${ }^{67}$ vgl. ebd.

68 vgl. Abb. 5 im Anhang
} 
Das Motiv der ersten inhaltlichen Vorgabe war die Schilderung eines Urlaubs, den die Verfasser mit dem Brieffreund verbracht hatten. In einem zweiten Schritt sollte ein weiterer gemeinsamer Urlaub geplant werden. Die dritte Vorgabe bestand darin, über die derzeitige Lebenssituation des Verfassers zu schreiben und die Dinge zu beschreiben, die sich in der letzten Zeit ereignet hatten. Das Motiv der vierten thematischen Vorgabe bestand in der Beschreibung eines Diebstahls, von dem der Verfasser selber betroffen war. Im letzten Teil des Briefes sollte schließlich ein persönlicher Besuch beim Briefpartner angekündigt werden.

Die Anordnung und der konkrete Inhalt der Vorgaben beruhen auf folgenden Gründen: Die Vorgaben wurden so angeordnet, daß die zeitliche Abfolge der Ereignisse den Probanden nachvollziehbar war. Dem Ablauf des täglichen Lebens entsprechend führten die Vorgaben thematisch von der Vergangenheit in die Zukunft. Wie vorher festgelegt, kamen dabei nahezu alle sprachlichen Bereiche zur Anwendung, die die Probanden mit Beginn der Mittelstufe internalisiert haben sollten. Gleichzeitig wurden die Vorgaben jedoch so formuliert, daß die Probanden innerhalb des thematischen Rahmens sprachlichen Freiraum hatten.

Ziel der ersten Aufgabe war es, die Tempora der Vergangenheit sowie das Vokabular, das im Zusammenhang mit dem Thema Urlaub erlernt wurde, zu aktivieren. Die zweite Aufgabe sollte zur Anwendung der Zukunftstempora anregen ${ }^{69}$ und dabei gleichzeitig klären, inwieweit die Probanden sprachliche Idiome, die bei Vorschlägen verwendet werden, ${ }^{70}$ normgerecht anwenden können. Die dritte Vorgabe sollte dazu führen, daß die Zeitformen simple present, present progressive, simple past und present perfect benutzt werden. Hierdurch sollte ermittelt werden, welche Abweichungsformen beim Gebrauch dieser Tempora auftreten. In diesem Zusammenhang sollten die Probanden das Vokabular, das mit den Themen Schule, Freizeitgestaltung und Freundschaft verbunden ist, einsetzen. Intention der vierten Vorgabe war es, die Verwendung der Zustandsform Passiv und der unterschiedlichen Vergangenheitstempora anzuregen ${ }^{71}$. Ziel der fünften Vorgabe war der Gebrauch der Zukunftstempora. Die Wahl der Themen geht dabei auf die Absicht zurück, Vorgaben zugrunde zu legen, die jeweils unabhängig voneinander sind und dennoch eine inhaltliche Verbindung erkennen lassen. Dies sollte dadurch gewährleistet werden, daß alle Vorgaben mit dem Thema Freizeit verknüpft waren und bei nahezu allen Handlungen beide Kommunikationspartner beteiligt waren. Darüber hinaus basiert die Auswahl der Themen Urlaub, Diebstahl und Besuch eines Freundes auf der Überlegung, daß es sich hier um Motive handelt, die Menschen unterschiedlichen Alters und unterschiedlicher schulischer Ausbildung in gleicher Weise ansprechen und in der aktuellen Lebenssituation aller Probanden auftreten könnten.

${ }^{69}$ Dazu zählen das will-future und das going to-future sowie das future progressive.

${ }^{70}$ Hierzu zählen u. a. Ausdrücke wie what about, how about etc. 
Bei der Konzeption des Fragebogens stand die Intention im Vordergrund, möglichst viele der psychound soziolinguistischen Faktoren, die den Erwerb einer Zweitsprache beeinflussen, ${ }^{72}$ zu berücksichtigen, die Fragen jedoch so zu formulieren, daß die Art der Fragestellung nicht auf Ablehnung der Probanden stößt. Aus zeitlichen Gründen und um ausführliche Stellungnahmen zu vermeiden, sollten die Fragen durch das Ankreuzen vorgegebener Aspekte oder durch eine kurze Stellungnahme von ein bis zwei Wörtern oder Sätzen beantwortet werden können. Es sollten folgende Informationen ermittelt werden, die den Ergebnissen der Zweitspracherwerbsforschung zufolge zu den wichtigsten Einflußgrößen des Zweitspracherwerbs gehören: ${ }^{73}$

1. Alter und Geschlecht der Lernenden

2. schulische und berufliche Ausbildung

3. die Form des Zweitspracherwerbs (gesteuert/ungesteuert)

4. Beginn, Kontinuität und Intensität des Zweitspracherwerbs

5. linguistische Form der Zweitsprache, d. h. in bezug auf das Englische:

Britisches / Amerikanisches Englisch oder eine andere Ausprägung der englischen Sprache

6. Erstsprache des Lernenden

7. Kenntnisse in anderen Zweitsprachen

8. extrinsische und intrinsische Motivation der Lernenden ${ }^{74}$

9. körperliche und seelische Verfassung der Lernenden

Die Fragen sollten so angeordnet werden, daß die exogenen Faktoren, d. h. die Aspekte, die den Spracherwerb von außen beeinflussen, zuerst erfragt werden und die endogenen Faktoren, d. h. die Aspekte, die eng mit der Persönlichkeit des Lernenden verbunden sind, im letzten Teil des Fragebogens behandelt werden. Gleichzeitig war zu berücksichtigen, daß der Anteil der Fragen, der sich auf exogene bzw. endogene Aspekte bezieht, ${ }^{75}$ gleich gewichtet war. Dieser Aufbau sollte zum einen dazu beitragen, daß die Probanden nicht bereits zu Beginn eine ablehnende Haltung gegenüber dem Fragebogen entwickeln, weil ihnen die Fragen zu persönlich erscheinen. Zum anderen sollten die Probanden dadurch erkennen, daß der Fragebogen ausschließlich dazu dient, einen Einblick in die unterschiedlichen Faktoren des Zweitspracherwerbs zu erhalten, zu denen persönliche Informationen ebenso gehören wie allgemeine linguistische Informationen.

Bei der Formulierung der Fragen war außerdem zu beachten, daß linguistische Informationen zum Teil nicht direkt, sondern nur indirekt erfragt werden können. Dies betrifft beispielsweise die Frage,

\footnotetext{
$71 \mathrm{Zu}$ diesen Zeitformen zählen insbesondere das simple past, das past progressive sowie das past perfect.

72 vgl. Apeltauer 1987, $11-43$

73 vgl. ebd.

74 Zum Begriff der Motivation vgl. insbesondere Vogel 1990, 143 - 148.

75 vgl. ebd., 121 - 166
} 
ob der Zweitspracherwerb der einzelnen Probanden als gesteuert oder ungesteuert zu bezeichnen ist. ${ }^{76}$ Die Problematik in der Formulierung dieser Frage besteht einerseits darin, daß die Probanden den Unterschied zwischen den beiden Aspekten in der Regel nicht kennen und somit keine eindeutige Antwort geben können und andererseits in der Tatsache, daß die Frage aufgrund der Komplexität der Faktoren, die bei manchen Probanden auf den Zweitspracherwerb eingewirkt haben, nicht oder nur partiell beantwortet werden kann.

Damit die Erwerbsbedingungen dennoch ermittelt werden konnten, mußte die Frage, die sich auf diesen Aspekt bezieht, relativ allgemein formuliert werden, so daß unterschiedliche Antworten möglich waren und damit möglichst vielfältige Einblicke in die Erwerbsbedingungen gewonnen werden konnten. Ausgehend von diesen Überlegungen sollten die Probanden daher lediglich beantworten, ob sie außerhalb des schulischen Unterrichts Erfahrungen mit der englischen Sprache gesammelt hatten. Anhand von Beispielen wurde dabei verdeutlicht, welche Erfahrungen u. a. dazu zählen könnten. Diese Art der Fragestellung ermöglichte es den Probanden, bei der Beantwortung zu erkennen zu geben, ob der Zweitspracherwerb teilweise unter natürlichen Bedingungen verlaufen ist, ohne dabei die Begriffe ,gesteuert‘ und ,ungesteuert‘ verwenden zu müssen.

Während die Ermittlung der Erwerbsbedingungen damit einer sorgfältig überlegten Fragestellung bedurfte, konnten die anderen exo- und endogenen Faktoren des Spracherwerbs, ${ }^{77}$ die im Zentrum des Fragebogens standen, durch konkrete Benennung der linguistischen Aspekte, die untersucht werden sollten, ermittelt werden - etwa indem die Probanden direkt gefragt wurden, ob neben dem Englischen noch andere Zweitsprachen erlernt wurden. Somit waren in bezug auf die Formulierung der Fragen keine weiteren Besonderheiten zu berücksichtigen.

Neben den methodischen und thematischen Aspekten waren bei der Planung der Untersuchung auch formale Kriterien zu berücksichtigen: Die Untersuchungsbedingungen sollten die inhaltlichen Ergebnisse nicht beeinträchtigten und für alle Probanden identisch sein. Hierzu war es erforderlich, daß die Untersuchungsleiter - d. h. diejenigen Fachlehrer, die der Durchführung der Untersuchung in ihrem Kurs zugestimmt hatten - während der Bearbeitung der Testgrundlagen anwesend waren, um gegebenenfalls auftretende Mißverständnisse inhaltlicher oder formaler Art zu klären und um den Informationsaustausch zwischen den Probanden zu verhindern. Um die objektive Beurteilung der Sprachkenntnisse der Probanden zu gewährleisten, sollten Hilfsmittel wie Wörterbücher bei der Bearbeitung der Aufgaben nicht benutzt werden.

\footnotetext{
76 Zur Unterscheidung der beiden Formen der Sprachaneignung siehe u. a. Apeltauer 2003, 13 - 15.

77 vgl. Vogel 1990, $121-166$
} 
Aus Gründen der quantitativen Überschaubarkeit sollten die zu verfassenden Briefe nach Möglichkeit nicht mehr als 250 Wörter beinhalten. Diese Begrenzung war jedoch nicht als strikte Vorlage zu betrachten, sondern als quantitative Orientierung. Der Umgang mit Briefen im Fremdsprachenunterricht hat gezeigt, daß diese Wortanzahl den sprachlichen Freiraum der Probanden nicht einschränkt, weil Schülerbriefe - wie etwa die Untersuchungsergebnisse von Hecht/Green verdeutlichen ${ }^{78}-$ selten mehr als 200 Wörter umfassen. Dies gilt selbst dann, wenn die Briefe von fortgeschrittenen Lernenden verfaßt werden und die Anzahl der zu schreibenden Wörter nicht vorab begrenzt wird.

Zur Bearbeitung der Untersuchungsgrundlagen sollten jeder Probandengruppe 90 Minuten, d. h. zwei Unterrichtseinheiten, zur Verfügung stehen. Beide Untersuchungsgrundlagen, sowohl die Briefe als auch die Fragebögen, sollten dabei innerhalb eines Durchgangs bearbeitet werden. Diese Entscheidung beruhte darauf, daß die Probanden mit dieser Zeitspanne aus dem Unterrichtsalltag vertraut sind: Sowohl die Unterrichtssequenzen als auch die schulischen Prüfungen, insbesondere in der Mittelstufe, umfassen zwei zusammenhängende Unterrichtseinheiten. Darüber hinaus ist aus den Erfahrungen des fremdsprachlichen Unterrichts bekannt, daß Fremdsprachenlernende zum Verfassen eines Briefes, der etwa 200 Wörter enthält und auf thematischen Vorgaben beruht, im allgemeinen mindestens eine Unterrichtseinheit benötigen. ${ }^{79}$ Da neben dem Verfassen der Briefe auch noch der Fragebogen auszufüllen war und den Probanden Gelegenheit gegeben werden sollte, die Briefe vor deren Abgabe nochmals gründlich durchzulesen, wurden insgesamt zwei Unterrichtseinheiten zur Durchführung des Testverfahrens eingeräumt.

Abschließend war bei der Planung zu berücksichtigen, daß die Untersuchung in einem Zeitraum stattfinden sollte, in dem die Probanden keine weiteren schulischen Prüfungen zu erwarten hatten, um die Teilnehmer nicht zu überlasten und damit eine Beeinflussung der Ergebnisse zu vermeiden.

\subsection{Die Fehlerermittlung}

Zur Ermittlung sprachlicher Fehler bedarf es zunächst einer konkreten Definition des Begriffs ,Fehler sowie einer Beschreibung der Fehlertypen, die im Rahmen des Analyseverfahrens untersucht werden sollen. Diese Vorgehensweise soll zum einen dazu beitragen, daß die Konstellation des Fehlerkorpus, der dem Analyseverfahren zugrunde liegt, nachvollzogen werden kann. Zum anderen soll sie gewährleisten, daß der Fehlerkorpus nur Abweichungen ${ }^{80}$ enthält, deren Fehlerhaftigkeit eindeutig ist.

\footnotetext{
78 vgl. Hecht / Green 1983, 12

79 Diese Aussage beruht auf Erfahrungen des Autors der vorliegenden Arbeit, die während des Referendariats im Englischunterricht gewonnen wurden.

${ }^{80}$ Zur Unterscheidung zwischen den unterschiedlichen Abweichungsformen siehe u. a. Norrish 1983
} 
Gleichzeitig dienen die Erläuterung der Definition und die Entscheidung, welche Fehler beim Analyseverfahren berücksichtigt werden sollen, den Korrektoren als Leitfaden während des Korrekturverfahrens. Zusätzlich verdeutlichen die nachfolgenden Ausführungen, daß Fehler beim Erwerb des Englischen aufgrund der Komplexität der englischen Sprache eine besondere Stellung in der Fehlerlinguistik einnehmen.

\subsubsection{Definition des Begriffs ,Fehler'}

Die Definition des Begriffs ,Fehler‘ gehört zu den wichtigsten Inhalten der Fehlerlinguistik und hat seit der Gründung dieser Teildisziplin der Sprachwissenschaft immer wieder zu kontroversen Auffassungen geführt. ${ }^{81}$ Die definitorischen Schwierigkeiten beruhen dabei zum einen darauf, daß Sprachen ständigen Veränderungen unterliegen, die sich auf allen linguistischen Ebenen äußern. Daher kann der Gebrauch sprachlicher Strukturen, der in der Vergangenheit als normabweichend bezeichnet wurde, heute durchaus als sprachlich korrekt gelten. Auch ist es möglich, daß Elemente, die früher in einem sprachlichen Zusammenhang verwendet wurden, heute in diesem Kontext nicht mehr auftreten. So werden beispielsweise im heutigen Englisch zukünftige Handlungen in der Regel nicht mehr durch das Hilfsverb shall verdeutlicht, sondern durch das Lexem will. ${ }^{82}$ Ebenso war es bis vor wenigen Jahren im Britischen Englisch inkorrekt, zur Verdeutlichung einer Zeitspanne beim present perfect die Präposition in zu benutzen, während das Wort im heutigen Britischen Englisch in diesem sprachlichen Zusammenhang ebenso akzeptiert wird wie das Lexem for $^{83}$

Neben den definitorischen Problemen, die durch den Sprachwandel begründet sind, erschweren auch die regionalen Varianten einer Sprache die Definition des Begriffs ,Fehler', weil zum Teil linguistische Strukturen, die in einer Region sprachlich korrekt sind, in einer anderen Region, in der dieselbe Sprache benutzt wird, als Normabweichungen betrachtet werden. ${ }^{84}$ Während beispielsweise im Amerikanischen Englisch in Verbindung mit dem Adverb just durchaus das simple past benutzt werden kann - wie etwa in der Äußerung I just saw her - entspricht diese Struktur nicht der Norm des Britischen Englisch. ${ }^{85}$ Hier wäre stattdessen das present perfect zu erwarten, so daß die normgerechte Äußerung in dieser Form der englischen Sprache I have just seen her lauten müßte. Daher kann die in der Fehlerlinguistik partiell vertretene Auffassung, eine normgerechte Äußerung unterscheide sich dadurch von einer Normabweichung, daß sie in einem bestimmten Kontext „von einem educated

\footnotetext{
81 vgl. Raabe 1980, 65-68

82 vgl. Swan 1990, Textstelle 546, o. S.

83 vgl. Pons Großwörterbuch 1988, 321

84 vgl. Trudgill / Hannah 1982

85 vgl. Swan 1990, Textstelle 352, o. S.
} 
native speaker"86 geäußert würde und „von anderen educated speakers als zu der betreffenden Sprache gehörig akzeptiert würde ${ }^{\star 87}$ nur ansatzweise befürwortet werden: Beide der vorgenannten Beispiele werden von educated native speakers des Englischen verwendet und beide Beispiele gehören zu den Elementen der englischen Sprache. Festzustellen ist zudem, daß die Schwierigkeiten, den Begriff ,Fehler' zu definieren, die auf unterschiedlichen Varianten einer Sprache basieren, in bezug auf das Englische besonders stark zum Tragen kommen, weil Englisch von 350 - 400 Mio. Menschen als Erstsprache gesprochen wird ${ }^{88}$ und darüber hinaus in vielen asiatischen und afrikanischen Ländern die Funktion einer Verkehrssprache übernommen hat. ${ }^{89}$ Hinzu kommt, daß eine große Anzahl der sogenannten lingua francas auf das Englische zurückgeht, ${ }^{90}$ welches die Anzahl unterschiedlicher Formen der englischen Sprache zusätzlich erhöht. ${ }^{91}$ Wie die zuvor dargestellten Beispiele verdeutlichten, unterscheiden sich diese Formen dadurch voneinander, daß sie jeweils auf einem eigenständigen, offiziell anerkannten Modell der englischen Sprache beruhen und sich dabei sowohl durch die Bedeutung einzelner Wörter als auch durch ihre grammatische Struktur und ihre Aussprache von anderen Formen des Englischen unterscheiden. ${ }^{92}$ Hinzu kommt, daß selbst innerhalb von Ländern, in denen eine einzige Form der englischen Sprache benutzt wird, sprachliche Differenzen zu erkennen sind. ${ }^{93}$

Es sind jedoch nicht nur linguistische Faktoren, die dazu beitragen, daß die semantische Bedeutung des Begriffs ,sprachlicher Fehler' nicht eindeutig geklärt werden kann. Vielmehr wird die Fehlerdefinition auch erheblich durch das individuelle Normverständnis der einzelnen englischen Muttersprachler innerhalb einer bestimmten Region erschwert. Untersuchungen haben gezeigt, daß Muttersprachler einer bestimmten Sprache bei dem Versuch zu beurteilen, ob eine Äußerung eines Lernenden ihrer Erstsprache als richtig oder falsch einzustufen ist, teilweise zu unterschiedlichen Ergebnissen kommen, auch wenn sie keinerlei Unterschiede in bezug auf die Spracherwerbsbedingungen, ihre Ausbildung und die linguistische Form ihrer Erstsprache aufweisen ${ }^{94}$ Dieses Problem, das in der Regel als ,interpersonelle Inkonsistenz ${ }^{6}$ bezeichnet wird, ${ }^{95}$ beruht zum einen darauf, daß Muttersprachler die sprachliche Intention eines Lernenden ihrer Zielsprache nicht immer richtig deuten und zum anderen darauf, daß viele sprachliche Äußerungen selbst bei Vorlage einer bestimmten Norm

\footnotetext{
86 vgl. Legenhausen 1975, 16

${ }^{87}$ vgl. Legenhausen 1975, 16

88 vgl. Strang 1970, 17

${ }^{89}$ vgl. Kachru 1985, $11-30$

90 vgl. Samarin 1962

91 vgl. Quirk / Widdowson 1985, 16

92 vgl. Trudgill / Hannah 1982

93 vgl. ebd.

94 vgl. Davies 1991

95 vgl. Raabe 1980, 68
} 
weder eindeutig als normgerecht noch als normabweichend bezeichnet werden können. ${ }^{96}$ Ein Beispiel für sprachliche Äußerungen, die zu Fehldeutungen führen könnten, bietet die bei James verzeichnete Aussage „I came to London to study the English“"97. Dieser Satz wurde von den englischen und amerikanischen Korrektoren, die sich an der Untersuchung der vorliegenden Arbeit beteiligten, zum Teil als inkorrekt bezeichnet, weil dem Lexem English kein weiteres Lexem wie etwa language folgt. Dabei wurde jedoch nicht berücksichtigt, daß die Aussage als normgerecht zu bezeichnen wäre, wenn der Autor dieser Aussage ein anthropologisches Ziel verfolgt hätte und der Grund seines LondonAufenthalts darin bestand, die Lebensweise der Briten zu untersuchen. ${ }^{98}$

Ein typisches Beispiel dafür, daß auch Muttersprachler nicht immer in der Lage sind, eine sprachliche Aussage zweifelsfrei als richtig oder falsch zu bewerten, selbst wenn bei der Entscheidung nur eine Norm zugrunde gelegt wurde, stellt der folgende Satz dar: ,, Who did you meet at the zoo? " 99 Etwa die Hälfte der oben erwähnten Korrektoren bezeichnete die Aussage als Normabweichung, weil statt des Lexems who das Wort whom zu erwarten gewesen wäre. Dies bestätigt F. Gieras Auffassung: „Meinungen zur Adäquatheit (Normgerechtheit) einer Äußerung können sehr subjektiv sein, aber auch auf dem relativ sicheren Gebiet der Akzeptabilität (Systemgerechtheit) können die Auffassungen von native speakers auseinandergehen.“ ${ }^{100}$ Das Problem der interpersonellen Inkonsistenz ist jedoch nicht nur bei Muttersprachlern einer bestimmten Sprache zu beobachten, sondern auch bei Unterrichtenden einer bestimmten Zielsprache, die diese Zielsprache nicht als Erstsprache erworben haben. ${ }^{101}$ In bezug auf die englische Sprache ist das Problem besonders ausgeprägt, weil an den Schulen in der Regel nicht nur eine Variante der englischen Sprache unterrichtet wird. Insbesondere in Deutschland sind an nahezu allen Schulformen Englischlehrer vertreten, die entweder Britisches oder Amerikanisches Englisch oder beide Formen der englischen Sprache vermitteln. ${ }^{102}$ Darüber hinaus hat in den vergangenen Jahren auch die Anzahl von Lehrern zugenommen, die eine andere Form der englischen Sprache, etwa das Englisch der ehemaligen Kolonien, internalisiert haben und auch im Unterricht anwenden. ${ }^{103}$ Folglich kommen auch bei den Lernenden unterschiedliche Formen der englischen Sprache zur Anwendung, so daß dadurch die Fehlerbewertung zunehmend erschwert wird. Wie bei englischen Muttersprachlern ist jedoch auch bei deutschen Muttersprachlern, die Englisch unterrichten, interpersonelle Inkonsistenz auch dann zu beobachten, wenn sich die Unter-

\footnotetext{
96 vgl. Giera 1980, 356

97 James 1998, 67

98 vgl. ebd.

99 ebd., 74

100 vgl. Giera 1980, 356

101 vgl. Legenhausen 1975

102 Dies beruht auf Erfahrungen des Autors dieser Arbeit während seines Referendariats und während der Planung und Durchführung der vorliegenden Studie.

103 dto.
} 
richtenden in bezug auf die Form des Englischen, die sie erlernt haben und im Unterricht vermitteln, nicht unterscheiden. ${ }^{104}$

Trotz aller zuvor dargestellten Schwierigkeiten, die mit der Definition des Begriffs ,sprachlicher Fehler' verbunden sind, kommen allerdings immer wieder Sprachelemente und Strukturen zur Anwendung, die sowohl von unterschiedlichen Muttersprachlern einer bestimmten Zielsprache als auch von Unterrichtenden, die diese Zielsprache selbst als Zweitsprache erworben haben, ohne Beurteilungsdifferenzen eindeutig als Abweichungen beurteilt werden. ${ }^{105}$ Somit ist die Existenz sprachlicher Fehler grundsätzlich nicht zu bezweifeln. Zur Lösung der zuvor beschriebenen Definitionsprobleme wurden in der Fehlerlinguistik unterschiedliche Beurteilungskriterien entwickelt, mit deren Hilfe entschieden werden sollte, ob eine sprachliche Äußerung als normgerecht oder als normabweichend zu bezeichnen ist. ${ }^{106} \mathrm{Zu}$ den wichtigsten Beurteilungskriterien gehören die sogenannte ,Grammatikalität‘ und die ,Akzeptabilität ${ }^{\star}{ }^{107}$ Der Begriff Grammatikalität geht auf N. Chomsky zurück $^{108}$ und dient als Synonym für die Bezeichnung, grammatische Wohlgeformtheit ${ }^{\text {t109 }}$. Charakteristisch für dieses Beurteilungskriterium ist die Vorgabe, daß nicht die Sprecher einer bestimmten Sprache entscheiden, ob eine linguistische Form richtig oder falsch ist, sondern ein grammatisches Modell ${ }^{110}$. So wird der sprachliche Kontext, in dem eine bestimmte Äußerung auftritt, nicht in die Fehlerbeurteilung miteinbezogen, sondern es wird lediglich das sprachliche Element betrachtet, das als normgerecht oder als normabweichend zu bezeichnen ist. ${ }^{111}$ Diesem Kriterium zufolge gilt ein Element immer dann als inkorrekt, wenn das Element gegen die Regeln des Modells verstößt, das bei der Fehlerbeurteilung zugrunde gelegt wird ${ }^{112}$ - selbst wenn dieses Element bei näherer Betrachtung des sprachlichen Kontextes, in dem es ermittelt wurde, nicht gegen die Regeln der betreffenden Sprache verstößt. ${ }^{113}$

Wie der Begriff ,Grammatikalität‘ bereits impliziert, wurde dieses Beurteilungskriterium ursprünglich nur für syntaktisch-morphologische Abweichungen konzipiert. ${ }^{114} \mathrm{Da}$ es jedoch eines Kriteriums bedurfte, das auch semantische und phonetische Abweichungen berücksichtigt, wurde es in der heuti-

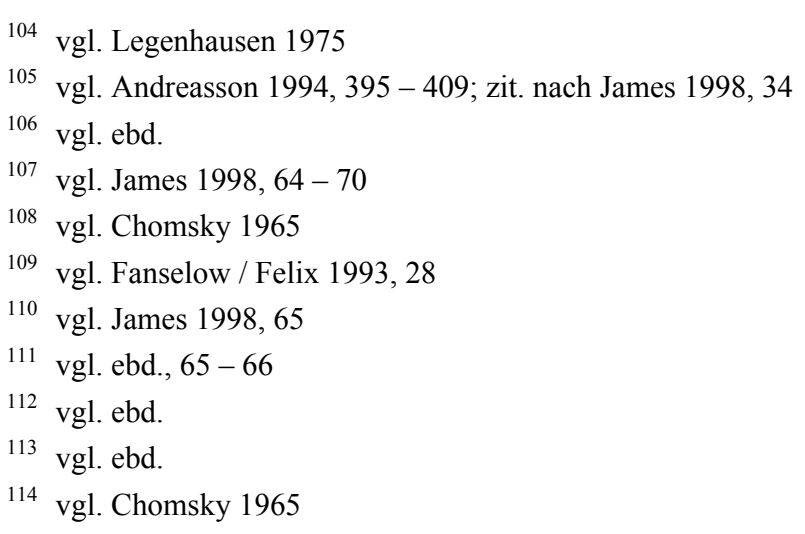


gen Fehlerlinguistik auf diese Felder erweitert. ${ }^{115}$ Diese Begriffserweiterung beruht dabei auch auf der Erkenntnis, daß ein sprachliches Beurteilungskriterium, das sprachliche Normeinhaltung daran überprüft, ob ein Element korrigiert werden kann oder nicht, die Bedeutungs- und Lautebene einer Sprache nicht ausgrenzen darf, weil semantische und phonetische Verstöße ebenso korrigiert werden können wie syntaktisch-morphologische Fehler. ${ }^{116}$ Jedoch bleibt auch in dieser erweiterten Version des Grammatikalitätsbegriffs der sprachliche Kontext, in dem die scheinbare Abweichung ermittelt wurde, unberücksichtigt. ${ }^{117}$

Ein weiteres Problem dieses Begriffs besteht darin, daß bei Anwendung des Kriteriums, Grammatikalität' in der Regel nur eine Norm zugrunde gelegt wird und sich die so gewonnenen Ergebnisse deutlich von denen unterscheiden, die möglicherweise bei Anwendung einer anderen Norm gewonnen worden wären. ${ }^{118}$ Dies bedeutet, mit Hilfe des Begriffs ,Grammatikalität‘ können nur solche Sprachelemente eindeutig als falsch beurteilt werden, die als eklatante Verstöße gegen die englische Sprache zu verstehen sind und bei jeder Norm dieser Sprache als falsch zu betrachten wären. Zur Beurteilung sogenannter borderline cases $^{119}$, d. h. von Elementen, deren Normgerechtigkeit nur unter Zugrundelegung unterschiedlicher Normen beurteilt werden kann, sowie von Elementen, die zunächst als richtig oder falsch bezeichnet wurden, bei Berücksichtigung des sprachlichen Kontextes jedoch in einer anderen Weise zu beurteilen wären, wird bei der Bewertung linguistischer Strukturen in der Regel auch das Kriterium ,Akzeptabilität' ${ }^{\star}$ zugrunde gelegt. ${ }^{120}$ Auch dieser Begriff wurde im Zusammenhang mit der Darstellung der, sprachlichen Wohlgeformtheit‘ von Chomsky eingeführt, er ist der Ebene der Performanz, d.h. der praktischen Sprachanwendung zuzuordnen. ${ }^{121}$ Das Beurteilungskriterium ,Akzeptabilität' basiert auf der Theorie, daß sprachliche Elemente nur dann als falsch bezeichnet werden können, wenn die Sprecher unterschiedlicher Formen einer Sprache übereinstimmend feststellen, daß ein sprachliches Element nicht in den Kontext einer bestimmten sprachlichen Äußerung paßt. Der Schwerpunkt dieses Beurteilungskriteriums liegt demnach auf der pragmatischen Ebene. ${ }^{122}$ In bezug auf das linguistische System wäre etwa die Äußerung She decided to answer the telephone call in allen Formen der englischen Sprache grammatisch als korrekt zu bezeichnen, wenn der Satz isoliert auftreten würde und zur Beschreibung eines vergangenen Ereignisses benutzt würde. Die Äußerung wäre jedoch als normabweichend zu betrachten, wenn aus dem Kontext hervorgehen würde, daß der Ausdruck to answer the telephone call als Synonym für die

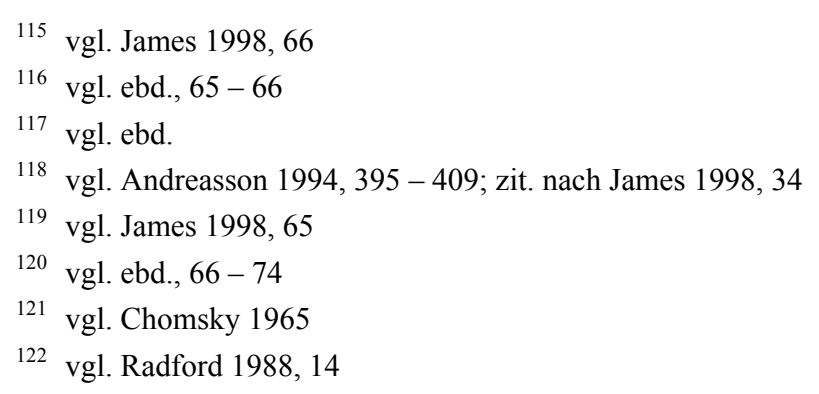


Äußerung to pick up the receiver while the phone is ringing zu betrachten wäre. ${ }^{123}$ Zusammenfassend ist also festzustellen, daß sprachliche Elemente nur dann als ,wirkliche Fehler ${ }^{6}$ bezeichnet werden können, wenn sie sowohl gegen das Kriterium der ,Grammatikalität‘ als auch gegen das Kriterium der ,Akzeptabilität‘ verstoßen. ${ }^{124}$

\subsubsection{Linguistische Abweichungsformen}

In der Fehlerlinguistik wird zwischen verschiedenen Abweichungsformen unterschieden. Zu den Hauptkategorien zählen die Fehlergruppen errors, mistakes, slips bzw. lapses und solecisms. ${ }^{125}$ Die Unterscheidung zwischen errors und mistakes geht auf S. P. Corder zurück. ${ }^{126}$ Zur Kategorie errors zählen Abweichungen, die unbeabsichtigt entstehen und von den Lernenden nicht selbst korrigiert werden können, weil ihnen das sprachliche Wissen, das sie zur Selbstkorrektur benötigen, nicht zur Verfügung steht. ${ }^{127}$ Diese Selbstkorrektur kann auch dann nicht erfolgen, wenn die Betreffenden auf den Fehler aufmerksam gemacht werden. ${ }^{128}$ Die Kategorie der mistakes hingegen umfaßt Abweichungen, die die Lernenden selbst korrigieren können, nachdem sie darauf hingewiesen wurden, daß ihre sprachliche Äußerung eine Normabweichung enthält. ${ }^{129}$ Man unterscheidet hierbei zwischen first und second order mistakes. ${ }^{130}$ Eine Abweichung wird als first order mistake bezeichnet, wenn die Lernenden die Abweichung lokalisieren und korrigieren können, ohne zuvor erfahren zu haben, an welcher Stelle in ihrer Äußerung die Abweichung zu beobachten war. ${ }^{131}$ Second order mistakes sind dadurch gekennzeichnet, daß die Lernenden erst dann fähig waren, den Fehler selbst zu korrigieren, wenn die Art der Abweichung und die Stelle des Auftretens konkret benannt wurden. ${ }^{132}$

Fehler der Kategorie first order mistakes werden manchmal auch intentional eingesetzt, um einen bestimmten Effekt hervorzurufen, wie etwa in der Werbung, in der diese Abweichungsformen mitunter dazu benutzt werden, die Aufmerksamkeit auf ein bestimmtes Produkt zu lenken. ${ }^{133}$ Nach Corder stellen errors ein größeres sprachliches Problem dar als mistakes, weil errors das tatsächliche Wissen über eine Sprache reflektieren, während mistakes nur verdeutlichen, inwieweit der Lernende

\footnotetext{
123 vgl. James 1998, 68

124 vgl. ebd., $65-74$

125 vgl. ebd., $83-84$

126 vgl. Corder 1967, $161-170$

127 vgl. James 1998, 83

128 vgl. Edge 1989, 10

129 vgl. James 1998, 83

130 vgl. ebd., 83

131 vgl. ebd.

132 vgl. ebd.

133 vgl. ebd., 31
} 
zu einem bestimmten Zeitpunkt in der Lage ist, das bereits verinnerlichte Wissen anzuwenden. ${ }^{134}$ Corder orientiert sich bei seiner Unterscheidung zwischen den beiden Fehlertypen an der von Chomsky postulierten Dichotomie zwischen der allgemeinen Sprachfähigkeit, der competence $^{135}$ und der individuellen Sprachverwendung, der sogenannten performance. ${ }^{136}$ Dieser Unterscheidung entsprechend führt Corder errors auf eine Störung der competence zurück, während mistakes auf einer Beeinträchtigung der performance basieren. ${ }^{137}$

Die Bezeichnungen slips und lapses wurden für Fehler geprägt, die auf Müdigkeit, Konzentrationsschwächen oder Unaufmerksamkeit zurückzuführen sind. ${ }^{138}$ Dazu zählen u. a. die sogenannten spoonerisms ${ }^{139}$, die beispielsweise dadurch gekennzeichnet sind, daß der Anfangsbuchstabe eines Wortes durch den Anfangsbuchstaben eines später folgenden Wortes ersetzt wird, wie in folgendem Beispiel:

Fehler: $\quad$ Bake my bike. ${ }^{140}$

Korrektur: Take my bike.

Slips und lapses unterscheiden sich von den zuvor genannten Fehlertypen dadurch, daß die Betreffenden die Fehler schnell und ohne jegliche Hilfe selbst korrigieren können. Die Kategorie der solecisms umfaßt Abweichungsformen, die häufig auch bei Muttersprachlern der betreffenden Zielsprache zu beobachten sind. ${ }^{141}$ Fehler dieser Art treten insbesondere in umgangssprachlichen Situationen auf und werden aufgrund ihrer Gebrauchsfrequenz häufig nicht als Fehler betrachtet, sondern als Bestandteile der informellen Sprechweise. ${ }^{142}$ Dennoch weichen Äußerungen, die der Kategorie solecisms zuzuordnen sind, bei genauerer Betrachtung von der grammatischen Norm der betreffenden Sprache ab, so daß sie bei Anwendung des Beurteilungskriteriums Grammatikalität nicht als normgerecht bezeichnet werden können, obwohl sie aus der Sicht des Kriteriums Akzeptabilität möglicherweise korrekt wären. ${ }^{143} \mathrm{Zu}$ den Fehlern dieser Kategorie gehören u. a. die sogenannten split infinitives ${ }^{144}$, die dadurch gekennzeichnet sind, daß zwischen dem Lexem to und dem nachfolgenden Verb ein anderes Wort eingefügt wird - wie etwa in folgender Äußerung:

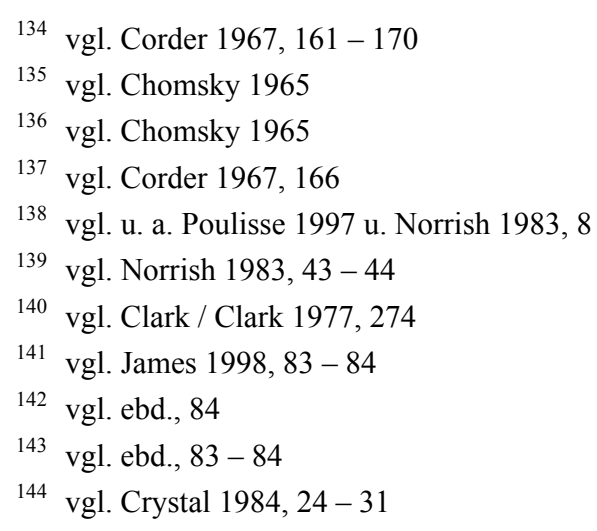


Fehler: $\quad$ I want to quickly run up to Boots to pick up my prescription. ${ }^{145}$

Korrektur: I quickly want to run up to Boots to pick up my prescription.

Solecisms treten bei Nicht-Muttersprachlern seltener auf als bei native speakers, weil Fremdsprachenlehrer sich in der Regel an dem theoretischen Modell der Zweitsprache orientieren und auch die Lernenden daher dazu tendieren, das theoretische Modell der betreffenden Sprache zu benutzen. Häufig entsteht dadurch bei Muttersprachlern der Eindruck, daß Lernende ihrer Sprache die betreffende Sprache besser beherrschen als sie selber. ${ }^{146}$

\subsubsection{Die Bedeutung des Begriffs ,Fehler' für das Untersuchungsverfahren}

Die vorhergehenden Ausführungen haben gezeigt, daß die Normgerechtheit sprachlicher Elemente insbesondere englischsprachiger Elemente - nur unter Berücksichtigung regionaler, grammatischer und pragmatischer Aspekte beurteilt werden kann. Auch wurde deutlich, daß Fehler ihrer Entstehungsursache entsprechend unterschiedlichen Kategorien zugeordnet werden können. Ausgehend von diesen Erkenntnissen sollten bei der Fehlerermittlung, d. h. bei der Entscheidung, ob ein sprachliches Element als normgerecht oder normabweichend zu bezeichnen ist, und bei der Entscheidung, welche der dabei ermittelten Abweichungskategorien in den Fehlerkorpus aufgenommen werden, folgende Aspekte berücksichtigt werden: Grundsätzlich sollten bei der Fehlerermittlung alle Elemente miteinbezogen werden, die gegen die Kriterien der Grammatikalität und der Akzeptabilität verstoßen. Somit waren auch Abweichungsformen zu berücksichtigen, die zur Gruppe der solicisms gehören. Da zum Zeitpunkt der Fehlerermittlung nicht immer eindeutig zu erkennen war, ob die Abweichungen auf Störungen der Kompetenz- oder der Performanzebene basierten, sollten Fehler, die sich nachträglich als lapses oder slips erwiesen, ebenso berücksichtigt werden wie Fehler der Kategorien errors und mistakes.

Die Normunterschiede, die in bezug auf das Englische zu beachten sind, führten darüber hinaus zu der Entscheidung, bei der Ermittlung der Abweichungen sowohl die Norm des Britischen Englisch, das sogenannte Southern British Standard ${ }^{147}$, als auch die Norm des Amerikanischen Englisch, die durch das sogenannte Network English festgelegt wird, ${ }^{148}$ zugrunde zu legen. Diese Entscheidung beruht auf der Tatsache, daß - wie oben geschildert - trotz der Entwicklung anderer Normen das Britische und das Amerikanische Englisch unverändert die Hauptformen der englischen Sprache bilden und beide Sprachformen an deutschen Schulen unterrichtet werden, wenngleich dabei das Britische Englisch aus

\footnotetext{
145 vgl. ebd., 26

146 vgl. James 1998, 84

147 vgl. Weiher 1982, $19-20$

148 vgl. Brenni 1964
} 
historischen Gründen überwiegt. ${ }^{149}$ Dabei sollten auch dann beide Normen bei der Korrektur berücksichtigt werden, wenn die Probanden in ihrem Fragebogen vermerkten, nur eine Form des Englischen erlernt zu haben, weil viele Englischlernende nicht zwischen dem Britischen und dem Amerikanischen Englisch differenzieren können. ${ }^{150}$ Andere Normen sollten nur dann zugrunde gelegt werden, wenn aus dem Fragebogen des Probanden hervorging, daß der Lernende weder das Britische noch das Amerikanische Englisch erlernt hat und zudem deutlich wurde, auf welcher Sprachform die Englischkenntnisse des Probanden basieren.

Da sowohl das Southern British Standard als auch das Network English als theoretische Modelle der englischen Sprache zu betrachten sind und damit bei Verwendung dieser Grundlagen das Kriterium der Akzeptabilität unberücksichtigt bleibt, sollten die Beiträge der Probanden nicht nur von deutschen, sondern auch von britischen und amerikanischen Muttersprachlern korrigiert werden, weil diese den Sprachgebrauch sowohl aus theoretischer als auch aus pragmatischer Sicht beurteilen können. Zur Vermeidung des Problems der interpersonellen Inkonsistenz mußten zudem bei der Korrektur mehrere Muttersprachler miteinbezogen werden. Alle Korrektoren sollten neben theoretischen Kenntnissen und praktischen Erfahrungen in bezug auf die jeweilige Form des Englischen auch über allgemeine linguistische Kenntnisse verfügen, um in Zweifelsfällen die Normgerechtheit sprachlicher Elemente beurteilen zu können. Zusätzlich sollten die Probandenbeiträge jedoch auch von deutschen Muttersprachlern, die Englisch unterrichten, korrigiert werden, weil deutsche Englischlehrer nicht nur die theoretische und partiell auch die praktische Seite der englischen Sprache kennen, sondern darüber hinaus, ebenso wie die Probanden, Deutsch als Erstsprache sprechen. Daher sind sie bei Fehlern, die auf erstsprachlichen Einfluß zurückgehen, in der Regel auch bei sehr subtilem Einfluß der Erstsprache in der Lage, die Fehlerentstehungsursache zu erkennen und angemessene Korrekturbeispiele vorzuschlagen.

\subsection{Methoden der Fehlerklassifizierung}

In der Fehlerlinguistik wurden in den vergangenen Jahren zahlreiche Methoden zur Klassifizierung sprachlicher Abweichungen entwickelt. Die Entscheidung, welches Klassifizierungsverfahren bei einer bestimmten Fehleranalyse zugrunde gelegt werden soll, richtet sich nach der Intention des betreffenden Untersuchungsverfahrens, d. h. nach der sprachlichen Ebene, die im Zentrum der Untersuchung steht. Sprachliche Fehler können nach grammatischen und lexikalischen Kategorien klassifiziert

\footnotetext{
149 vgl. Weiher 1982, $15-16$

150 Wie die Unterrichtserfahrungen des Autors der vorliegenden Arbeit gezeigt haben, beruhen diese Differenzierungsschwierigkeiten häufig darauf, daß die Unterschiede im Englischunterricht deutscher Schulen nur ansatzweise behandelt werden und Britisches und Amerikanisches Englisch in den Lehrwerken zum Teil kombiniert auftreten.
} 
werden, oder auch nach den strukturellen Veränderungen (Morphemvertauschungen etc.), die die sprachlichen Fehler bewirkt haben. ${ }^{151}$ Beide Verfahren zählen zu den sogenannten deskriptiven Taxonomien. ${ }^{152}$ Klassifizierungsverfahren, die auf der Kategorisierung nach grammatischen und lexikalischen Gesichtspunkten basieren, werden als linguistic category classifications bezeichnet. ${ }^{153}$ Klassifizierungsverfahren, bei denen die Struktur einer sprachlichen Äußerung im Vordergrund steht, zählen zu den sogenannten ,, surface structure taxonomies “154.

\subsubsection{Linguistic category classification}

Die Basis der linguistic category classification bilden die unterschiedlichen sprachlichen Ebenen. ${ }^{155}$ Hierzu zählt neben den Bereichen Lexik, Syntax, Morphologie und Orthographie auch die lautliche Ebene. Die Anwendung der linguistic category classification kann sowohl in allgemeiner als auch in spezieller Form erfolgen. Dies bedeutet, Fehlleistungen können entweder ohne weitere Differenzierung lediglich den einzelnen Ebenen zugeordnet werden oder sie werden nach konkreten grammatischen, lexikalischen, orthographischen oder lautlichen Gruppen kategorisiert. ${ }^{156}$ So ist es z. B. möglich, eine Abweichung der Form Yesterday I have seen you ausschließlich als syntaktischen Verstoß zu bezeichnen, zusätzlich kann man sie jedoch der Kategorie ,Vergangenheitstempora‘ zuordnen. Bei Anwendung detaillierter Kategorisierungen sind weitere Abstufungen möglich: Die Fehlleistung könnte darüber hinaus auch als Verstoß beim Gebrauch der Tempora simple past und present perfect bezeichnet werden. ${ }^{157}$

Bei Anwendung einer detaillierten Kategorisierung werden Fehler in der Regel nach dem Prinzip des ranking klassifiziert, d. h., ausgehend von größeren Einheiten werden die Abweichungen in die ihnen zugrundeliegenden kleineren Einheiten gegliedert. ${ }^{158}$ Auf lexikalischer Ebene stellt hier der Gesamttext die größte Einheit dar. Die nächstgrößere Einheit bilden die einzelnen Textabschnitte. Es folgen in der Hierarchie schließlich die einzelnen Sätze, Phrasen, Lexeme und Wortsegmente. ${ }^{159}$ Auf lautlicher Ebene stellt der Satz mit den ihm zugrundeliegenden suprasegmentalen Merkmalen die größte

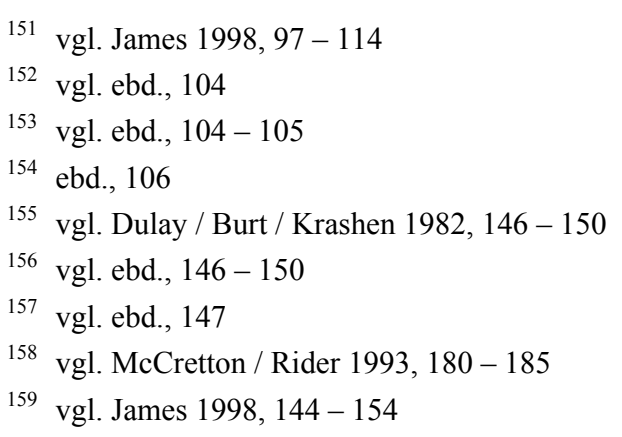


Einheit dar. ${ }^{160}$ Die nächste Stufe bildet die Wortbetonung. Die kleinste Einheit schließlich beinhaltet die Aussprache der einzelnen Phoneme. ${ }^{161}$

Im Bereich Syntax und Morphologie ist die hierarchische Abfolge komplexer und wurde zum Teil linguistisch nicht eindeutig festgelegt. Dies beruht darauf, daß die Trennung zwischen den Bereichen Syntax und Morphologie aufgrund inhaltlicher Überschneidungen beider Bereiche partiell nur undeutlich zu erkennen ist, so daß sie bei Fehleruntersuchungen oftmals zu einer Ebene zusammengefaßt werden. ${ }^{162}$ Auch hier werden Fehler nach dem Prinzip des ranking kategorisiert. ${ }^{163}$ Die größte syntaktische Einheit bildet in der Regel - wie auf lexikalischer Ebene - der Text. Auf der darunterliegenden Stufe stehen auch hier die einzelnen Textabschnitte. Die nachfolgende Einheit besteht aus den verschiedenen Sätzen. Die kleinste Einheit bilden hier die einzelnen Phrasen, Lexeme und Lexemsegmente. ${ }^{164}$

Orthographische Verstöße werden in der Regel in drei Gruppen gegliedert: die Kategorien der Großschreibungsfehler, der Kleinschreibungsfehler sowie der sogenannten Rechtschreibfehler. ${ }^{165}$ Auch hier sind innerhalb der einzelnen Kategorien weitere Unterteilungen möglich. So werden in manchen Untersuchungen Fehler der zuvor genannten Kategorien nach einzelnen Lexemgruppen unterteilt, d. h., es wird unterschieden zwischen Groß- und Kleinschreibungs- bzw. Rechtschreibfehlern beim Gebrauch von Nomina, Adjektiven, Adverbien etc. ${ }^{166}$ Unterteilungen in Unterkategorien bei orthographischen Verstößen sind jedoch wesentlich seltener zu beobachten als in den anderen linguistischen Kategorien.

\subsubsection{Surface structure taxonomy}

Das Prinzip, Fehler nach ihrem strukturellen Erscheinungsbild zu kategorisieren, geht im wesentlichen auf H. C. Dulay, M. K. Burt und S. D. Krashen zurück. ${ }^{167}$ Strukturelle Veränderungen sprachlicher Äußerungen können dadurch hervorgerufen werden, daß sprachliche Elemente in normabweichender Form hinzugefügt, ausgelassen, vertauscht oder fehlerhaft kombiniert werden. Bei Anwendung der surface structure taxonomy werden Fehler daher in die Gruppen omissions, additions, misformations

\footnotetext{
160 vgl. Weiher $1982,69-78$

161 vgl. ebd.

162 vgl. Hecht / Green 1983, 16

163 vgl. McCretton / Rider 1993, 180 - 186

164 vgl. James 1998, 105

165 vgl. James / Klein 1994, 31 - 46

166 vgl. ebd.

167 vgl. Dulay / Burt / Krashen 1982, 150 - 163
} 
und misorderings eingeteilt. ${ }^{168}$ Dabei ist der Unterschied zwischen den Fehlern der einzelnen Kategorien zum Teil nicht immer eindeutig ersichtlich, so daß das wissenschaftliche Fundament dieses Fehlerklassifizierungsverfahrens in den vergangenen Jahren oftmals in Frage gestellt wurde. ${ }^{169}$ Fehler der zuvor genannten Kategorien können sowohl kleinere als auch komplexere Einheiten umfassen.

Abweichungen, die der Kategorie omissions zugeordnet werden, gehen darauf zurück, daß sprachliche Elemente, die in einem bestimmten sprachlichen Zusammenhang zu erwarten gewesen wären, ausgelassen werden. ${ }^{170}$ Omissions unterscheiden sich von den sogenannten ellipsis, die ebenfalls durch das Auslassen sprachlicher Elemente gekennzeichnet sind, dadurch, daß sprachliche Vorgänge, die als ellipsis bezeichnet werden, nur dann als Normverstöße betrachtet werden, wenn ihr Gebrauch gegen die normgerechte Gebrauchsfrequenz verstößt. ${ }^{171}$ Fehler der Kategorie additions entstehen durch das redundante Hinzufügen sprachlicher Elemente. ${ }^{172}$

Zur Kategorie misformations zählen Abweichungen, die dadurch hervorgerufen werden, daß die Struktur einer grammatischen Einheit in einer Form verändert wird, die der Norm der Sprache widerspricht, wie etwa in den Äußerungen he like that book oder I seen her. ${ }^{173}$ Diese Beispiele zeigen auch, daß beim Einordnen der Fehler in diese Kategorie Überschneidungen mit den zuvor genannten Kategorien omissions und additions oft unvermeidbar sind. Fehler der Gruppe misorderings dagegen sind dadurch gekennzeichnet, daß Lexeme oder Lexemgruppen in einer normabweichenden Form angeordnet werden. ${ }^{174}$

Misorderings sind, wie auch die Fehler der zuvor genannten Kategorien, nach Auffassung von Dulay, Burt und Krashen sowohl beim Erst- als auch beim Zweitspracherwerb zu beobachten. ${ }^{175}$ Das Verfahren der surface structure taxonomy wird daher insbesondere bei den Fehleranalysen angewandt, die auf der Theorie der Identitätshypothese basieren. ${ }^{176}$

\footnotetext{
168 vgl. ebd., $154-163$

169 vgl. James 1998, 106

170 vgl. Dulay / Burt / Krashen 1982, $154-156$

171 vgl. James 1998, 106 u. 159

172 vgl. ebd., $156-158$

173 vgl. Dulay / Burt / Krashen 1982, 158 - 162

174 vgl. ebd., $162 \mathrm{f}$

175 vgl. ebd., 150 - 163

176 vgl. Bausch / Kasper 1979, $10 \mathrm{f}$
} 


\subsubsection{Konkretisierung der Klassifizierungsmöglichkeiten}

Der Vergleich der beiden Klassifizierungsverfahren führte zu der Entscheidung, bei der Kategorisierung der Abweichungen in dieser Untersuchung ein Verfahren anzuwenden, das auf einer Kombination der linguistic category classification und der surface structure taxonomy beruht. Die Kombination beider Verfahren ist notwendig, weil linguistic category classifications aufgrund der Abgrenzungsschwierigkeiten zwischen einzelnen linguistischen Ebenen nicht immer eindeutige Klassifizierungen ermöglichen, während das Verfahren der surface structure taxonomy vornehmlich für Fehler entwickelt wurde, die auf biologisch determinierte Faktoren zurückzuführen sind und seine alleinige Anwendung daher die Objektivität des Analyseverfahrens beeinträchtigen könnte.

Konkret werden die Fehlleistungen der vorliegenden Untersuchung nach folgendem Prinzip kategorisiert: Die Abweichungen werden zunächst, soweit eine eindeutige Gruppierung möglich ist, den entsprechenden linguistischen Ebenen zugeordnet. Die Terminologie der einzelnen Elemente richtet sich nach den Bezeichnungen der traditionellen ${ }^{177}$ und der generativen Grammatik ${ }^{178}$.

Bedingt durch die partiell bestehenden Überschneidungen zwischen den Bereichen Syntax und Morphologie werden die beiden Ebenen auch in dieser Arbeit zu einer Gruppe, den syntaktischmorphologischen Abweichungen, zusammengefaßt. Gleiches gilt für die Bereiche Lexik und Pragmatik. Da - wie erwähnt - bei pragmatischen Abweichungen die Entscheidung, ob eine bestimmte Äußerung als normgerecht oder normabweichend zu bezeichnen ist, nicht immer eindeutig getroffen werden kann, werden nur solche pragmatischen Verstöße berücksichtigt, die von der Mehrheit der Korrektoren als normabweichend bezeichnet wurden. Aufgrund der damit verbundenen Entscheidungsschwierigkeiten und der linguistischen Verwandtschaft zwischen den Bereichen Lexik und Pragmatik werden pragmatische Verstöße den lexikalischen Abweichungen zugeordnet.

Wie bereits geschildert, treten Zuordnungsschwierigkeiten jedoch nicht nur bei Elementen linguistischer Bereiche auf, die inhaltlich teilweise übereinstimmen, wie etwa die Bereiche Syntax und Morphologie. Vielmehr konnten solche Zuordnungsschwierigkeiten auch in bezug auf Ebenen beobachtet werden, die inhaltlich größere Unterschiede aufweisen, wie etwa die Bereiche Syntax und Lexik. Betroffen davon sind Elemente, die sowohl syntaktische als auch lexikalische Funktionen erfüllen. Dazu gehören im wesentlichen Präpositionen, Auxiliare und Pronomina.

177 vgl. Cherubim 1976

178 vgl. Radford 1988 u. Gardner 1973 
Präpositionen können zum einen dem lexikalischen Bereich zugeordnet werden, weil sie bedeutungsunterscheidende Funktionen übernehmen. ${ }^{179}$ Zum anderen erfüllen Präpositionen jedoch auch die ursprüngliche Funktion des Kasus im Englischen und übernehmen damit zusätzlich syntaktische Aufgaben.

Hilfsverben sind sowohl lexikalisch als auch syntaktisch von Bedeutung: Einerseits können sie für das semantische Verständnis entscheidend sein. So kann mit Hilfe von Auxiliaren verdeutlicht werden, ob eine Handlung in der Zukunft stattfinden wird oder bereits in der Vergangenheit stattgefunden hat, wie etwa in folgenden Sätzen: Will you go out? oder Did you go out? Auch die unterschiedlichen Stufen der Höflichkeit können durch Hilfsverben zum Ausdruck gebracht werden. Beispiele hierfür bieten die Fragen: Do you want another cup of tea? und Would you like another cup of tea? Andererseits bestimmen Hilfsverben aufgrund ihrer Bedeutung bei der Bildung der Zeitformen und anderer grammatischer Strukturen, wie etwa den question tags, auch die syntaktisch-morphologische Gestaltung sprachlicher Aussagen. So trägt $\mathrm{u}$. a. die Tatsache, daß die Feststellung it's a nice day ein Hilfsverb enthält, dazu bei, daß am Satzende das sogenannte tag isn't it zu erwarten wäre. Ebenso verdeutlicht der Gebrauch des Hilfsverbs am in der Aussage I'm working nicht nur, daß die Handlung, die dem Satz zugrunde liegt, momentan stattfindet. Vielmehr wird der Satz, verglichen mit der Satzstruktur I work, die bei Anwendung der simple form zu gebrauchen wäre, um ein syntaktisches Element erweitert.

Pronomina sind sowohl lexikalisch als auch syntaktisch-morphologisch bedeutsam, weil die Verwechslung unterschiedlicher Pronomina einerseits zu semantischen Veränderungen führen kann und andererseits oftmals auch morphologische Abweichungen zur Folge hat. So führt die Substitution des Pronomens herself durch das Pronomen her in der Aussage she was talking to her dazu, daß der Satz im Deutschen bedeutet, ,sie sprach mit ihr', obwohl der Lernende den deutschen Satz, sie sprach mit sich selbst‘ übersetzen wollte. Außerdem wird jedoch durch das Auslassen der Endsilbe -self die morphologische Struktur des Pronomens verändert.

In der vorliegenden Arbeit wird dem Problem der Zuordnung einzelner Abweichungen durch folgende Vorgehensweise begegnet: Zunächst bilden die zuvor genannten Lexemgruppen aufgrund ihrer semantischen Bedeutung eigene Unterkategorien auf der lexikalischen Ebene. Zusätzlich werden Fehler beim Gebrauch der Präpositionen, Hilfsverben und Pronomina jedoch auch in den Bereich der syntaktisch-morphologischen Abweichungen aufgenommen, wenn die Fehler darauf basieren, daß die

179 vgl. Burgschmidt 1975, $7-10$ 
betreffenden Elemente fälschlicherweise hinzugefügt oder ausgelassen werden und dabei normabweichende Veränderungen entstehen.

Die Kategorisierung und Zuordnung der Abweichungen innerhalb der Hauptkategorien Lexik, SyntaxMorphologie und Orthographie beruht auf dem nachfolgend geschilderten Prinzip.

\subsubsection{Lexikalische Fehler}

Die Zuordnung der lexikalischen Abweichungen basiert auf dem Bestreben, vornehmlich Lexeme zu berücksichtigen, deren semantische und syntaktische Bedeutung entweder gleichgewichtig ist, oder deren semantische Bedeutung überwiegt. Falls die Abweichungen einer bestimmten Lexemgruppe zugeordnet werden können, werden die Fehler der lexikalischen Ebene schließlich nach Wortarten kategorisiert. Beispielsweise werden Fehler, die beim Gebrauch der Auxiliare entstanden sind, der Kategorie ,Hilfsverben` zugeordnet. Aus Gründen der Übersichtlichkeit werden die Kategorien hier nicht weiter unterteilt und enthalten damit auch Lexeme der verschiedenen Unterkategorien. So enthält z. B. die Fehlerkategorie ,Hilfsverben“ sowohl die reinen Hilfsverben als auch die sogenannten Modalverben. Ebenso enthält die Kategorie ,Adverbien` Adverbien des Ortes, der Zeit und der Art und Weise. Elemente, die nicht zu den Lexemen der englischen Sprache gehören, wie etwa Wörter der deutschen Sprache oder Wörter, die von den Lernenden erfunden wurden, werden der Fehlerkategorie ,Gebrauch inexistenter Wörter' zugeordnet.

Aufgrund der Vielzahl der unterschiedlichen Lexemgruppen im Englischen kann in der vorliegenden Arbeit nur eine begrenzte Anzahl von Wortarten aufgenommen und analysiert werden. Dabei werden in erster Linie Wortarten berücksichtigt, die besonders häufig normabweichend verwendet werden und darüber hinaus bei falschem Gebrauch den Kommunikationsverlauf erheblich beeinträchtigen können. Daneben werden auch Lexeme miteinbezogen, die in der Fachdidaktik als typische lexikalische Fehler deutscher Englischlernender bezeichnet werden. Die Berücksichtigung dieser Lexemgruppen erfolgt auch dann, wenn die Anzahl der Verstöße und der Grad der Kommunikationsbeeinträchtigung geringer sind als bei anderen Lexemgruppen.

Bei der Aufnahme der Fehlleistungen in die entsprechenden Kategorien werden darüber hinaus folgende fehlertypologische Kriterien berücksichtigt: Eine Abweichung wird dann einer bestimmten Kategorie zugeordnet, wenn das Lexem, das normabweichend verwendet wurde, zu der Wortart gehört bzw. mit der Wortart verwandt ist, die der Kategorie zugrunde liegt. So wird etwa eine Abweichung der Kategorie ,Adjektiv‘ zugeordnet, wenn statt des Adjektivs, das in dem gegebenen Kontext zu erwarten wäre, ein anderes Adjektiv benutzt wird und diese Verwendung im betreffenden Fall zu einer Normabweichung führt. Ebenso wird eine Abweichung dann in die Kategorie ,Adjektiv“ 
aufgenommen, wenn ein Adjektiv fälschlicherweise durch ein Adverb ersetzt wird, da Adjektive und Adverbien eine ähnliche semantische Funktion übernehmen.

Verstöße, die beim Gebrauch von Nomen und Verben entstehen, werden in der Kategorie ,Wortwahl zusammengefaßt. Diese Kategorisierung beruht darauf, daß sowohl Verben als auch Nomen zu den Wortarten gehören, die im Gegensatz zu Auxiliaren, Adverbien und Präpositionen in erster Linie semantische Funktionen übernehmen. Hinzu kommt, daß Verben und Nomen häufig semantische Einheiten darstellen, und daher nicht - wie etwa viele Kollokationen - getrennt voneinander zu betrachten sind.

\subsubsection{Syntaktisch-morphologische Fehler}

Die Kategorisierung der syntaktisch-morphologischen Fehler beruht auf folgendem Prinzip: Wie auch bei den lexikalischen Fehlern entscheiden die Häufigkeit des Auftretens eines Fehlers und der Grad der Kommunikationsbeeinträchtigung, der von diesem Fehler ausgeht, ob für diesen eine eigene Fehlerkategorie gebildet und nach dem betreffenden Fehlertyp benannt wird. Abweichungen, die weniger häufig auftreten und die Kommunikation weniger beeinflussen, werden in einer Kategorie mit der Bezeichnung ,sonstige Fehler` zusammengefaßt.

Fachdidaktischen Untersuchungen zufolge ${ }^{180}$ führen insbesondere Verstöße gegen das Zeitensystem vor allem innerhalb der Vergangenheitstempora -, das Vertauschen von Wort- und Satzelementen, der normabweichende Gebrauch von infinitiven Strukturen und Partizipialsätzen und die fehlerhafte Verwendung des Gerundiums und des bestimmten bzw. unbestimmten Artikels zu Kommunikationsbeeinträchtigungen bzw. wirken auf den Kommunikationspartner befremdend. Fehler werden daher dann der Unterkategorie ,Wort- bzw. Satzstellungsfehler` zugeordnet, wenn Konstituenten bzw. Sätze vertauscht wurden, ohne daß es dadurch zu einer Substitution von Verbformen kam. Die Substitution einer Verbform durch eine andere, die unter den gegebenen Umständen nicht normgerecht war, wird entsprechend der dadurch bewirkten Veränderung der Oberflächenstruktur der Elemente entweder den Auslassungsfehlern oder den Hinzufügungsfehlern zugeordnet.

Dieses Zuordnungsprinzip soll beispielhaft anhand der progressive-Form verdeutlicht werden. Die Verwendung der simple-Form des Verbs in Sprechsituationen, in denen die progressive-Form zu erwarten gewesen wäre, bewirkt durch Hinzufügung der flektierten Form des Hilfsverbs und des ing-Morphems eine Erweiterung der Verbform. Der Fehler wird daher als Hinzufügungsfehler betrachtet und der als ,Verlaufsform‘ bezeichneten Unterkategorie zugeordnet. Der umgekehrte Fall tritt bei den Auslassungsfehlern ein, wenn die progressive-Form zu erwarten gewesen wäre, der Lernende aber stattdessen die

180 vgl. hierzu Bald 1988, 10 ff 
simple-Form benutzte. Darüber hinaus werden Fehler auch dann als Auslassungs- bzw. Hinzufügungsfehler bezeichnet, wenn die Substitution einer Verbform nicht zum Auslassen oder Hinzufügen von Elementen führte, sondern sich auf den Austausch von Elementen beschränkte, etwa bei der Substitution der Partizipialkonstruktion durch eine nicht partizipial gebrauchte Verbform. Letzteres bewirkt in der Regel vielmehr eine Erweiterung der Verbform. In solchen Fällen werden Fehler dieser Art dennoch der einen oder der anderen Kategorie zugeordnet, weil die geforderte Verbform nicht benutzt wurde. In dieser Hinsicht wurde die in dieser Arbeit partiell angewandte surface structure taxonomy abgewandelt.

Dasselbe Prinzip wird auch bei den in der Kategorie ,syntaktisch-morphologische Fehler eingeordneten Lexemen wie den Adjektiven und Adverbien angewandt. Wird ein Adjektiv statt eines Adverbs benutzt, so führt dies in den meisten Fällen zu einer Elementverkürzung, da eine Vielzahl der Adverbien auf - $l y$ endet, während diese Endung bei Adjektiven in der Regel nicht zu finden ist. Der Fehler wird jedoch der Kategorie ,Auslassung‘ zugeordnet, weil die normgerechte Wortart fehlt. Wie zuvor erwähnt, bilden innerhalb der Kategorien ,Auslassungsfehler` und ,Hinzufügungsfehler` nur solche Abweichungen eigene Unterkategorien, die mit hoher Frequenz auftreten und/oder das Kommunikationsverständnis erheblich beeinträchtigen. Komplexere Satzstrukturen ${ }^{181}$ bilden eigene Hauptkategorien und werden daher nicht als Unterkategorien aufgeführt. Dies gilt auch für Verstöße gegen das Zeitensystem, welches aufgrund seiner Komplexität einer eingehenden Untersuchung und daher einer eigenen Kategorie bedarf.

Innerhalb der Fehlergruppen des Zeitensystems erfolgt eine Einteilung in die drei Zeitformen present tense, past tense und future tense, die wiederum, abgesehen von der jeweiligen progressive-Form, in weitere Unterkategorien aufgeteilt werden. Da die Verlaufsform bereits unter den Auslassungs- und Hinzufügungsfehlern aufgeführt ist, wurde einzig das present tense nicht in weitere Kategorien unterteilt. Für das past tense und das future tense gelten folgende Unterkategorien: Die Vergangenheitstempora werden in drei Gruppen unterteilt, das simple past, das present perfect und das past perfect. $\mathrm{Zu}$ den Untergruppen des future tense zählen das future progressive, das going to-future und das willfuture. Die entsprechenden progressive-Formen werden im Rahmen der Auslassungs- bzw. Hinzufügungsfehler behandelt. Die hierfür vorgesehene Kategorie differenziert dementsprechend zwischen der Verlaufsform in der Gegenwart, der Vergangenheit und der Zukunft, wobei die letzten beiden aus Gründen der Übersichtlichkeit nicht nochmals in die beim Zeitensystem aufgeführten verschiedenen future und past tenses aufgeteilt werden. Die Verlaufsform mit futurischer Funktion bildet mit ihrer present progressive-Entsprechung eine gemeinsame Gruppe. Eine Differenzierung erfolgt jedoch im Verlauf des Analyseverfahrens.

181 vgl. Radford 1988, $194-196$ 


\subsubsection{Orthographische Fehler}

In Anlehnung an das von Hecht/Green ${ }^{182}$ vorgestellte Konzept zur Fehlerkategorisierung und an die in der Sprachdidaktik allgemein vertretene Ansicht, daß die Orthographie eine eigene Kategorie darstelle, bilden die orthographischen Fehler auch in dieser Untersuchung den dritten Bereich innerhalb der Fehlerkategorisierung. Sie werden in die Kategorien ,Großschreibung ‘, ,Kleinschreibung` und ,Rechtschreibung' unterteilt. Eine weitere Gruppe mit der Bezeichnung ,sonstige Fehler ‘ ist für orthographische Fehler vorgesehen, die sich nicht eindeutig den vorgenannten Gruppen zuordnen lassen. Dazu gehören u. a. Fehler, die auf eine veraltete Rechtschreibung zurückzuführen sind oder Abweichungen, die nicht eindeutig als Fehler bezeichnet werden können, jedoch partiell von der orthographischen Norm abweichen, wie die zunehmende Substitution der Segmentabfolge $<$ ght $>$ durch die Grapheme $<t>$ und $<e>$ in Wörtern wie tonite anstelle von tonight. ${ }^{183}$

Zur Vermeidung von Detailauflistungen werden die einzelnen Kategorien innerhalb der Gruppe ,orthographische Fehler` nicht weiter untergliedert. Das Zuordnungsprinzip bei den Gruppen ,Großund Kleinschreibung' ist mit dem der zuvor genannten linguistischen Ebenen identisch. Fehler werden der Kategorie ,Großschreibung' zugeordnet, wenn das Anfangselement des betreffenden Lexems fälschlicherweise klein geschrieben wurde. Analog dazu werden Fehler der Kategorie ,Kleinschreibung' zugeordnet, wenn das Wort entgegen der Schreibnorm groß geschrieben wurde. Die Kategorie ,Rechtschreibung، beinhaltet Lexeme, die durch Abweichungen innerhalb des Wortes oder am Wortende auffallen, wie etwa die Abweichung im Wort ,, interessting “184.

\footnotetext{
182 vgl. Hecht / Green 1983, 30

183 Fehler dieser Art sind oftmals ein Produkt der sogenannten Jugendsprache. Vgl. Bernstein 1971.

184 Hecht / Green 1983, 58
} 


\section{Linguistische Abweichungen deutscher Englischlernender: Gegenwärtiger Kenntnisstand}

Im folgenden wird eine Auswahl typischer Fehler ${ }^{185}$ deutscher Muttersprachler beim Erwerb des Englischen dargestellt. Dabei werden jeweils Beispiele aus den Kategorien Lexik, Syntax/Morphologie und Orthographie benannt und der normgerechten Form gegenübergestellt. Lautliche Abweichungen werden nur dann berücksichtigt, wenn - wie etwa bei manchen orthographischen Fehlern - eine Beziehung zwischen den Abweichungen der zuvor genannten Ebenen und den phonetischen Verstößen besteht. Diese Entscheidung liegt vor allem darin begründet, daß im Mittelpunkt der Arbeit wie zuvor erwähnt - Fehler des schriftlichen Sprachgebrauchs stehen.

Die exemplarische Darstellung der Fehlertypen bezieht sich auf Untersuchungsergebnisse, die seit Beginn der modernen Fremdsprachendidaktik ermittelt wurden. ${ }^{186}$ Aufgrund der Vielzahl der unterschiedlichen Abweichungen werden in den nachfolgenden Ausführungen insbesondere solche Fehler hervorgehoben, die den Kommunikationsverlauf beeinträchtigen, d. h. zu sprachlichen Mißverständnissen führen können, oder die von englischen Muttersprachlern als erheblich normabweichend empfunden werden. Wenngleich die Darstellung auf dem Bestreben beruht, ausschließlich linguistische Fehlleistungen deutscher Muttersprachler zu beschreiben, ist nicht auszuschließen, daß ein Teil der Abweichungen auch bei Englischlernenden beobachtet werden kann, deren Erstsprache nicht Deutsch ist. Da die Fehler deutscher Englischlernender nicht immer auf der Standardform der deutschen Sprache beruhen, sondern manchmal auch auf dialektalen Merkmalen, ${ }^{187}$ werden im folgenden nur solche Fehler dargestellt, die unabhängig von dem Dialekt, den die jeweiligen Lernenden sprechen, bei allen deutschen Muttersprachlern in englischsprachigen Kommunikationssituationen beobachtet werden können.

Der terminologische Hintergrund der einzelnen Fehlertypen wird hier zur Vermeidung von Wiederholungen nur kurz umrissen, da die unterschiedlichen Fehlertypen einschließlich der terminologischen Bezeichnungen bei der Darstellung der Untersuchungsergebnisse detailliert vorgestellt werden. Zudem ist zu berücksichtigen, daß Anzahl und Art der einzelnen Fehlertypen entscheidend vom Kenntnisstand der Lernenden beeinflußt werden. In der Fehlerbeschreibung werden daher in erster Linie solche Abweichungen vorgestellt, die sowohl bei Anfängern als auch bei Lernenden mittleren

185 Falls im folgenden keine Quelle angegeben wird, handelt es sich bei den Fehlerbeispielen um Fehlleistungen, die im Rahmen der unterrichtlichen Tätigkeit des Autors der vorliegenden Arbeit beobachtet wurden.

186 vgl. Gutschow 1967, 77 - 79 u. Melling / Siebenhaar 1956, 10 f u. Morrissey 1981, 173 - 194

187 Diese Aussage betrifft vor allem die phonetische Ebene, spiegelt sich jedoch auch in der Schriftsprache wider. Vgl. hierzu Burgschmidt / Götz 1972, 209 - 225. Die Autoren schildern dabei auch den Einfluß des Dialekts auf die mündliche Sprache. 
Niveaus und fortgeschrittenen Englischlernenden ermittelt wurden. Vorwegnehmend ist jedoch bereits an dieser Stelle zu erwähnen, daß die Fähigkeit, eine bestimmte zielsprachliche Struktur oder ein zielsprachliches Element normgerecht anwenden zu können, im Laufe des Zweitspracherwerbs Veränderungen unterliegt. ${ }^{188}$ Insgesamt konnten im Laufe der Forschungsgeschichte nachfolgend beschriebene Abweichungsformen ermittelt werden, die sowohl in gesteuerten als auch in ungesteuerten Erwerbssituationen zu beobachten waren. ${ }^{189}$

$\mathrm{Zu}$ den bekanntesten Fehlern der lexikalischen Ebene gehören die sogenannten false friends ${ }^{190}$, die im Zusammenhang mit der Darstellung der Untersuchungsergebnisse nochmals Erwähnung finden werden. Ein Lexem wird in der Regel dann als false friend bezeichnet, wenn ein zielsprachliches Wort mit einem Lexem der Erstsprache strukturell nahezu übereinstimmt und dadurch eine Lernerleichterung suggeriert, sich jedoch semantisch von dem erstsprachlichen Wort unterscheidet. False friends sind nicht nur eine Erscheinung der englischen Sprache, sondern sind auch in anderen Sprachen, wie etwa den romanischen, zu finden. Sie sind nicht an eine bestimmte Wortart gebunden, sondern konnten in allen Wortarten nachgewiesen werden. Darüber hinaus kann ein zielsprachliches Lexem, das in einer bestimmten Situation als false friend zu betrachten ist, zu einer anderen Wortart gehören als das erstsprachliche Lexem, dem es strukturell ähnelt. $\mathrm{Zu}$ den klassischen Beispielen dieser Lexemgruppe zählt das englische Wort actually, das an das deutsche Lexem ,aktuell‘ erinnert. Die deutsche Übersetzung von actually lautet jedoch ,eigentlich` bzw. ,tatsächlich', während das Wort ,aktuell‘ je nach sprachlichem Zusammenhang mit den Lexemen current oder topical zu übersetzen wäre. Dieses Beispiel zeigt, daß manche Lexeme bereits dann als false friends zu betrachten sind, wenn eine Ähnlichkeit mit erstsprachlichen Elementen nur partiell besteht, also nur einen Teil des Lexems betrifft. Bei einsilbigen Elementen, wie etwa den Präpositionen on und at kann schon dann von false friends gesprochen werden, wenn die sprachliche Ähnlichkeit jeweils nur ein Segment umfaßt. Diese Präpositionen führen häufig zu Fehlern, weil sie akustisch und optisch partiell mit den deutschen Wörtern ,an` oder ,am‘ übereinstimmen, häufig jedoch auch die deutsche Bedeutung ,auf‘ oder ,um‘ haben.

$\mathrm{Zu}$ den charakteristischsten und häufigsten Fehlerbeispielen der Wortart Verben zählen die Lexeme bring, hear, mean und learn. ${ }^{191}$ Das Wort bring, das eindeutig an das deutsche Wort ,bringen ' erinnert, stimmt nur dann semantisch mit diesem Lexem überein, wenn etwas zu der sich äußernden Person hingebracht wird, wie etwa in der Äußerung „Come over here and bring your book“"192. Nimmt die

\footnotetext{
188 vgl. Vogel 1990, $50-57$

189 s. u. a. Cornell / Schmidt 1980, 94 - 97 u. Drubig 1972, 78 - 91

190 vgl. Breitkreutz 1973, $70-74$

191 vgl. Alfes 1985, 143

192 Swan 1990, Textstelle 117, o. S.
} 
Bewegung jedoch eine andere Richtung ein, d.h., wird etwas von der sich äußernden Person weggebracht, wird das Wort take benutzt, wie in folgendem Beispiel: Take that book to John, please.

Ebenso wird das Lexem hear, das an das deutsche Verb ,hören` erinnert, nur dann verwendet, wenn entweder die anatomische Fähigkeit des Hörens bezeichnet werden soll oder das Hören nicht gezielt erfolgt. Für gezieltes Hören wird das Lexem hear nur in Redesituationen verwendet, in denen sich das Hören auf musikalische Aufführungen, Radio- und Fernsehübertragungen, Reden etc. bezieht. ${ }^{193}$ Ein Beispiel hierfür bietet die Äußerung: „Did you hear Jack's talk on Tuesday? “194 Bei konzentriertem Zuhören hingegen, insbesondere dann, wenn das Gehörte ,nicht sichtbar` ist, wird in der Regel das Lexem listen benutzt, wie etwa in folgender Äußerung: „Listen carefully, please“ ${ }^{195}$. Auch die Lexeme mean und learn unterliegen im Englischen anderen Bedeutungen als die deutschen Lexeme ,meinen‘ und ,lernen‘. Daher kommt es häufig zu fehlerhaften Äußerungen wie:

Fehler: I mean that the left will win the next elections. "196

Korrektur: I think that the left will win the next elections.

Fehler: $\quad$ I was learning for an exam last night.

Korrektur: I was studying for an exam last night.

Wie das erste Korrekturbeispiel zeigt, wird im Englischen zur Verdeutlichung der eigenen Meinung nicht das englische Lexem mean, sondern das Lexem think benutzt. ${ }^{197}$ Im zweiten Beispiel ist der Gebrauch des Verbs learn als Normverstoß zu betrachten, weil der Lernvorgang, der der Vorbereitung einer Prüfung dient, im Englischen mit dem Wort study bezeichnet wird.

Zu den typischen false friends der Wortart Nomen gehört das von dem Wort mean abgeleitete Lexem meaning. Deutsche Englischlernende verwenden das Lexem häufig statt des Wortes opinion. Dabei kommt es beispielsweise zu folgendem Fehler:

Fehler: „What is your meaning about it? “198

Korrektur: What is your opinion about it?

Auch hier beruht die Fehlleistung auf der Ähnlichkeit von zwei Lexemen, dem deutschen Wort ,Meinung' und dem false friend meaning.

\footnotetext{
193 vgl. ebd., Textstelle 287, o. S.

194 ebd.

195 ebd.

196 ebd., Textstelle 385, o. S.

197 vgl. ebd.

198 ebd.
} 
Neben den zuvor genannten Erscheinungsformen von false friends wurde in den Untersuchungen der vergangenen Jahre auch Beispiele für false friends gefunden, die nicht auf einer semantischen Verwandtschaft zwischen den einzelnen Lexemen beruhen. Zu nennen wäre hier das Wort art ${ }^{199}$, das im Deutschen die Bedeutung ,Kunst ' hat und damit in keiner Form semantisch mit dem deutschen Lexem ,Art', das viele Englischlernende als Übersetzung des vorgenannten Lexems benutzen, verwandt ist.

Wenngleich die Gruppe der false friends die meisten Fehler auf lexikalischer Ebene verursacht, wurden innerhalb der Kategorie Lexik weitere fehlerevozierende Lexemgruppen deutlich. Diese finden sich vor allem in der Gruppe der Lexeme, die im Englischen eine andere Numerusform haben als ihre deutschen Entsprechungen. Beispiele bieten die Wörter advice, furniture, information und knowledge. ${ }^{200}$ Obwohl diese Lexeme im Englischen stets im Singular benutzt werden, verwenden deutsche Muttersprachler die betreffenden Wörter oftmals im Plural. Dabei treten u. a. folgende Fehlerformen auf:

Fehler: $\quad$ Your advices were very good.

Korrektur: Your advice was very good.

Fehler: $\quad$ These furnitures are not so good.

Korrektur: This furniture is not so good.

Auch die Gruppe der Hilfsverben bzw. Modalverben gehört zu den Lexemkategorien, die deutsche Muttersprachler häufig normabweichend verwenden. ${ }^{201}$ Dies liegt vor allem darin begründet, daß die Mehrheit der englischen Auxiliare bzw. die unterschiedlichen Formen dieser Lexeme Hilfsverben der deutschen Sprache strukturell ähneln, wie etwa das englische Lexem will dem deutschen Lexem ,wollen', jedoch anderen semantischen Regeln unterliegen als die Lexeme, denen sie scheinbar entsprechen. So wird das englische Lexem will vielfach als Übersetzung des zuvor genannten deutschen Modalverbs benutzt, weil das englische Hilfsverb will der 1. Person Singular Präsens des Verbs ,wollen' optisch und weitgehend auch akustisch entspricht. Es wurden u. a. folgende Abweichungen beobachtet: ${ }^{202}$

Fehler: „I will in the water. “203

Korrektur: I want (I would like) to go into the water.

Fehler: Will you go? (für deutsch: Willst du gehen?)

Korrektur: Would you like to go?

\footnotetext{
199 vgl. Alfes 1985, 143

200 vgl. Hoffmann 1973, 29

201 vgl. Grabowski 1985, 452

202 vgl. Vogel 1989, 140

203 ebd.
} 
Ebenso werden auch die Auxiliare can und must bzw. die von can abgeleitete Verbform could normabweichend verwendet. ${ }^{204}$ Normabweichungen, die beim Gebrauch des Lexems can entstehen, beruhen in der Regel darauf, daß das Lexem im Gegensatz zu dem deutschen Wort ,können“ nicht verwendet wird, um eine Möglichkeit zu verdeutlichen. Während also im Deutschen eine Äußerung wie „Um sechs Uhr kann er schon zu Hause sein“ von deutschen Muttersprachlern nicht als Normabweichung empfunden wird, ist in der englischen Übersetzung des deutschen Satzes das Lexem can unangebracht. ${ }^{205}$ So wurde folgende Abweichung beobachtet:

Fehler: $\quad$ He can be at home. (für deutsch: Vielleicht ist er zu Hause.)

Korrektur: He may be at home.

Auch die Anwendungsschwierigkeiten, die mit der Verbform could verbunden sind, basieren darauf, daß die semantischen Bedeutungen des deutschen Lexems ,konnte', das in bestimmten Zusammenhängen als deutsche Übersetzung benutzt wird, vielfältiger sind als die des betreffenden englischen Verbs. Hier besteht der Unterschied darin, daß das deutsche Lexem ,konnte' als Synonym des deutschen Ausdrucks ,in der Lage sein' betrachtet werden kann, ${ }^{206}$ während das englische Wort could dann nicht als Übersetzung dieses Ausdrucks dienen kann, wenn die Handlung, die der Äußerung zugrunde liegt, tatsächlich stattgefunden hat und abgeschlossen ist. Dies bedeutet, could entspricht nur dem deutschen Lexem ,könnte‘. Zur Verdeutlichung einer Fähigkeit wird im Englischen stattdessen die Lexemkombination was bzw. were able to eingesetzt. Ein Beispiel, das auf diesen Unterschieden beruht, bietet folgende Abweichung:

Fehler: $\quad$ He could help us. (für deutsch: Er konnte uns helfen.)

Korrektur: He was able to help us.

Das zuvor erwähnte Hilfsverb must führt zu Lern- und Anwendungsschwierigkeiten, weil das Lexem strukturell und inhaltlich mit dem deutschen Hilfsverb ,müssen“ verwandt ist, dabei jedoch zum Teil anderen semantischen Bedeutungen unterliegt. ${ }^{207}$ Die Anwendungsschwierigkeiten beruhen auf zwei Faktoren: Zum einen wird das Lexem must im Englischen nur zur Beschreibung dringender Verpflichtungen benutzt, d. h. Verpflichtungen, deren Vermeidung Konsequenzen zur Folge hat. Im Deutschen hingegen kann das Wort ,müssen` auch zur Beschreibung von Handlungen benutzt werden, die nicht als verpflichtend zu betrachten sind, wie etwa in folgenden Äußerungen: „Den Film mußt Du mal sehen“ oder „Ich habe ihn lange nicht gesehen, ich muß ihm mal schreiben.“ Im ersten Beispiel dient das Hilfsverb der Verdeutlichung einer Empfehlung. Im zweiten Beispiel ist das Wort als Synonym für das Lexem ,könnte‘ zu betrachten. Im Englischen hingegen wird bei Handlungen, die

\footnotetext{
204 vgl. Brough / Docherty 1998, 68 - 72

205 vgl. ebd., 68

206 vgl. ebd.

207 vgl. ebd., 70
} 
nicht oder weniger zwingend sind, die Verbform have to benutzt, wie etwa in der Äußerung You have to see this film, it's really great. Der falsche Gebrauch des Hilfsverbs must wird an folgendem Beispiel deutlich:

Fehler: $\quad$ I haven 't seen George for ages. I must drop him a line.

Korrektur: I haven't seen George for ages. I'll have to drop him a line some day.

Der zweite Fehlerentstehungsfaktor, der mit dem Hilfsverb must verbunden ist und dabei weitaus schwerwiegendere Normabweichungen zur Folge hat, beruht darauf, daß das Wort must in Kombination mit dem Lexem not dem deutschen Ausdruck ,mußt nicht“ ähnelt, dabei jedoch im Deutschen als ,darf nicht‘ zu übersetzen wäre. ${ }^{208}$ So könnte ein deutscher Satz wie etwa die Äußerung „Du mußt mir nicht helfen" fälschlicherweise als You must not help me übersetzt werden.

$\mathrm{Zu}$ den charakteristischsten Abweichungen der syntaktisch-morphologischen Ebene gehört vor allem der normabweichende Gebrauch der Infinitiv- und der Gerundstrukturen, der sogenannten that-Sätze und der Konditionalsätze. ${ }^{209}$ Die normabweichende Verwendung von Infinitiv- und Gerundstrukturen äußert sich in der Regel in den beiden nachfolgend beschriebenen Formen. Deutsche Englischlernende, die sich in der Frühphase des Spracherwerbs befinden, verwenden statt des Gerundiums häufig die Infinitivform des Verbs, wie im folgenden Satz: ${ }^{210}$

Fehler: $\quad$ I stopped to smoke last year.

Korrektur: I stopped smoking last year.

Die Fehlleistungen gehen hier in der Regel darauf zurück, daß in der deutschen Vorlage, die die Lernenden ins Englische übertragen, der Infinitiv des Verbs benutzt wird. Bei fortgeschrittenen Lernenden hingegen ist häufig der umgekehrte Fall zu beobachten, d. h., es wird statt des Infinitivs das Gerundium benutzt. Diese Fehlleistungen sind in nahezu allen Fällen als Ergebnis sogenannter Übergeneralisierungen zu betrachten. Dies bedeutet, die Lernenden verwenden das Gerundium auch bei den Verben, die in dem betreffenden sprachlichen Kontext im Infinitiv zu benutzen wären, weil sie davon ausgehen, daß ihre sprachliche Äußerung durch den Gebrauch des Gerundiums authentischer wirkt, d. h. mehr der Sprachform eines englischen Muttersprachlers entspricht. Oftmals sind Fehler dieser Art auch auf Überbetonungen der Gerundstruktur im Englischunterricht zurückzuführen. Ein Beispiel hierfür bietet diese Äußerung:

Fehler: $\quad$ She kissed my hand and made me feeling a fool. ${ }^{211}$

Korrektur: She kissed my hand and made me feel (like) a fool.

\footnotetext{
208 vgl. ebd., 69

209 vgl. Legenhausen 1985, 188 - 196

${ }^{210} \mathrm{Zu}$ ähnlichen Fehlern vgl. ebd., 190.

211 vgl. ebd., 189
} 
Die Schwierigkeiten, die mit dem Gebrauch der that-Sätze verbunden sind, treten insbesondere dann auf, wenn der Satz das Verb want enthält. ${ }^{212}$ Die Normabweichungen beruhen dabei vornehmlich darauf, daß im Deutschen nach dem Lexem ,wollen` unmittelbar das Wort ,daß‘ und ein Objekt folgen können, wie etwa in der Äußerung „Ich will, daß Du mir hilfst.“ Wie das Beispiel zeigt, wird das nachfolgende Verb dabei nicht im Infinitiv benutzt. Im Englischen hingegen folgt dem Lexem want unmittelbar das im Zentrum des Satzes stehende Objekt. Das nachfolgende Verb wird dabei im Infinitiv benutzt. So kommt es u. a. zu folgender Abweichung:

Fehler: He wants that you help him.

Korrektur: He wants you to help him.

Anwendungsschwierigkeiten beim Gebrauch der Konditionalsätze äußern sich insbesondere bei den if-Sätzen des sogenannten hypothetischen und des irrealen Typs. ${ }^{213}$ Diese Abweichungsformen gehen zum einen darauf zurück, daß im Deutschen die ursprünglichen Formen des Konjunktivs II vielfach durch die Umschreibung mit, würde‘ ersetzt werden. Dies bedeutet, statt der veralteten Konjunktivformen wie etwa ,hülfe', ,stürbe‘ oder ,stünde` wird im Deutschen in der Regel eine Umschreibung mit ,würde“ benutzt, wie etwa in der Äußerung „Er würde Dir helfen, wenn er Zeit hätte.“ Hinzu kommt, daß im Deutschen Konditionalformen wie ,würde‘ oder ,könnte‘ auch in dem Satzteil verwendet werden, der die Bedingung zum Ausdruck bringt. Äußerungen wie die folgende werden daher im Deutschen, insbesondere in der gesprochenen Sprache, nicht als Normabweichung betrachtet: „Würdest Du Dich freuen, wenn ich Dir helfen würde?“ Daher kommt es beim Gebrauch des Englischen häufig zu Abweichungen wie:

Fehler: If I would see her I would say hello to her.

Korrektur: If I saw her I would say hello to her.

Fehler: If I would have had time I would have helped you.

Korrektur: If I had had time I would have helped you.

Das erste Beispiel repräsentiert dabei einen Verstoß gegen den sogenannten hypothetischen if-Satz, während das zweite Beispiel als Verstoß gegen den Gebrauch des irrealen if-Satzes zu betrachten ist.

Besonders fehleranfällig ist auf syntaktisch-morphologischer Ebene auch der Bereich des Zeitensystems bzw. der Gebrauch der sogenannten aspects. ${ }^{214}$ Die Verstöße gegen das Zeitensystem beruhen in der Regel auf Vereinfachungen innerhalb der deutschen Sprache, die sowohl bei der Verwendung von

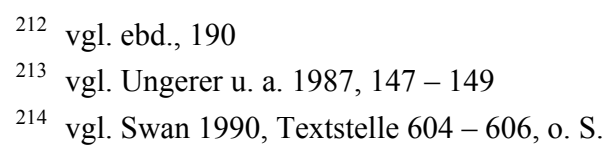


Vergangenheits- als auch von Zukunftstempora zu beobachten sind. Bei den Vergangenheitstempora besteht die Vereinfachung darin, daß das Imperfekt im Deutschen in den meisten Fällen durch das Perfekt ersetzt wird. Eine Äußerung wie „Ich habe gestern gearbeitet“ wird von vielen deutschen Muttersprachlern nicht als normabweichend betrachtet. Im Englischen hingegen besteht eine deutliche Unterscheidung zwischen dem Gebrauch des simple past, das dem deutschen Imperfekt entspricht, und dem present perfect, ${ }^{215}$ das als Entsprechung des deutschen Perfekt zu betrachten ist. So werden im Englischen Ereignisse, die eindeutig in der Vergangenheit liegen, abgeschlossen sind und zeitlich konkretisiert werden, in der Regel im simple past beschrieben. ${ }^{216}$ Bei Handlungen, die noch nicht abgeschlossen sind oder die generell wiederholt werden können und deren Eintreten zeitlich nicht benannt wird, wird gewöhnlich das present perfect benutzt. ${ }^{217} \mathrm{Da}$ im Deutschen - insbesondere in der gesprochenen Sprache - diese Unterscheidung nicht getroffen wird, kommt es u. a. zu folgender Abweichung:

Fehler: $\quad$ I have seen her yesterday.

Korrektur: I saw her yesterday.

Bei den Zukunftstempora bestehen die Vereinfachungen im Deutschen darin, daß zukünftige Handlungen oftmals durch Verwendung des Präsens und einer adverbialen Bestimmung der Zeit verdeutlicht werden, wie etwa in folgendem Satz: „Ich gehe heute abend ins Kino.“ Im Englischen hingegen sind solche syntaktischen Strukturen nur in der Umgangssprache zu finden. In der Standardsprache ist vielmehr, je nachdem, ob das Eintreten der Handlung als definitiv betrachtet wird oder als weniger definitiv, entweder das sogenannte will-future, die progressive form oder das going to-future zu benutzen. ${ }^{218}$ Diese Differenzen werden an folgender Äußerung deutlich:

Fehler: $\quad$ Tonight I go out. ${ }^{219}$

Korrektur: Tonight I'm going out. / Tonight I will go out. / Tonight I'm going to go out.

Fehler beim Gebrauch der sogenannten aspects sind sowohl im Bereich der Gegenwart als auch bei den Tempora der Zukunft und Vergangenheit zu beobachten. Die Schwierigkeit für deutsche Englischlernende besteht dabei in erster Linie beim Gebrauch der sogenannten Verlaufsform, die auf allen Zeitstufen benutzt werden kann, weil im Deutschen im Gegensatz zum Englischen die Verlaufsform in der Regel nicht durch morphologische Veränderungen des Verbs verdeutlicht wird. Im Englischen

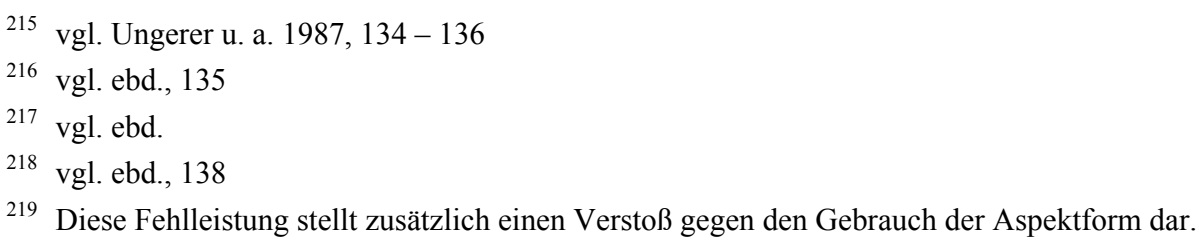


hingegen wird der progressive aspect durch die Verb-Endung -ing angezeigt, während diese VerbEndung bei der simple form nicht benutzt wird. Es kommt daher zu Abweichungen dieser Form:

Fehler: $\quad$ Robert played the piano when I came in.

Korrektur: Robert was playing the piano when I came in.

Die Fehlleistungen der orthographischen Ebene gehen, wie zuvor erwähnt, oft auf phonetische Differenzen zwischen dem Deutschen und dem Englischen zurück. Ein entscheidender Faktor ist dabei die Tatsache, daß der Unterschied zwischen der Schreibweise und der Ausspracheform bestimmter Lexeme im Englischen größer ist als im Deutschen. Zudem verfügt das Englische über Laute, die nicht zum Lautbestand der deutschen Sprache gehören. ${ }^{220}$ Darüber hinaus unterliegt die Groß- und Kleinschreibung im Englischen anderen Regeln als im Deutschen. Die Unterschiede zwischen Schreibweise und Aussprache führen häufig dazu, daß Elemente, die im Englischen nicht gesprochen, jedoch geschrieben werden, beim schriftlichen Gebrauch der englischen Sprache von deutschen Muttersprachlern weggelassen werden, wie etwa in folgendem Satz:

Fehler: $\quad$ He has a lot of dets.

Korrektur: He has a lot of debts.

Ein Beispiel für Fehler, die auf den unterschiedlichen Lautbestand der englischen und deutschen Sprache zurückzuführen sind, bietet folgende Abweichung:

Fehler: $\quad$ Than I went shopping.

Korrektur: Then I went shopping.

Die Abweichung geht darauf zurück, daß das Deutsche nicht über den Laut $/ \mathfrak{a} / 221$ verfügt. Daher substituieren viele deutsche Englischlernende den betreffenden Laut in der gesprochenen Sprache durch das Phonem /e/, weil dieses dem zuvor genannten englischen Phonem maximal ähnelt und die Lernenden das eigentlich zu erwartende Element lautlich nicht produzieren können bzw. den Unterschied zwischen den beiden Lauten nicht realisieren. ${ }^{222}$ Wie das Fehlerbeispiel zeigt, hat dieser phonetische Verstoß schließlich auch Folgen für die Schriftsprache - weil in der Schriftsprache der Laut /æ/ durch den Buchstaben a wiedergegeben wird. Dem Element/e/, das deutsche Englischlernende häufig als Substitution benutzen, entspricht hingegen der Buchstabe e, so daß dieser Buchstabe in vielen Wörtern fälschlicherweise dann benutzt wird, wenn in der gesprochenen Sprache der Laut /æ/ zu erwarten wäre.

\footnotetext{
220 vgl. Weiher 1982, $21-34$

221 vgl. Weiher 1974, 13

222 vgl. ebd. u. Weiher 1982, 164
} 
Fehler, die auf unterschiedliche Regeln der Groß- und Kleinschreibung zurückzuführen sind, äußern sich oftmals bei der Schreibweise von Adjektiven, die der Bezeichnung einer religiösen, politischen oder geographischen Zugehörigkeit dienen. ${ }^{223}$ Die Schwierigkeit entsteht für deutsche Englischlernende dadurch, daß Adjektive, die die zuvor genannten Funktionen erfüllen, im Englischen groß geschrieben werden, wie etwa in dem Satz I am of German origin. Im Deutschen hingegen werden Adjektive dieser Art, vorausgesetzt sie stehen nicht am Satzanfang, klein geschrieben, wie etwa in der Übersetzung des eben genannten Beispiels: ,Ich bin deutscher Abstammung“. Sie werden in der Regel erst dann groß geschrieben, wenn das betreffende Lexem als nominalisiertes Wort zu betrachten ist, wie etwa in dem Ausdruck ,das Deutsche‘. Neben den bereits dargestellten orthographischen Verstößen ist auch eine Vielzahl der Abweichungen in der Groß- und Kleinschreibung regional bedingt und kann daher nicht bei allen deutschen Muttersprachlern beobachtet werden. Hierzu zählen u. a. Abweichungsformen, die auf der sogenannten Auslautverhärtung ${ }^{224}$ beruhen bzw. auf regional bedingten Schwierigkeiten bei der Artikulation stimmhafter und stimmloser Laute. ${ }^{225}$ Wie zuvor erwähnt, sind solche Abweichungsformen aufgrund der Einschränkungen nicht als typische Fehler deutscher Englischlernender zu bezeichnen und gehören damit nicht zu den Fehlleistungen, die im Vordergrund der vorliegenden Arbeit stehen.

\section{Durchführung der Untersuchung}

Das Untersuchungsverfahren wurde - wie eingangs erwähnt - aus organisatorischen Gründen in den Bundesländern Nordrhein-Westfalen und Rheinland-Pfalz durchgeführt. Dreizehn Schulen erklärten sich dazu bereit, an dem Untersuchungsverfahren teilzunehmen: drei Hauptschulen, drei Realschulen, zwei Gymnasien, drei Sprachenschulen sowie zwei Volkshochschulen. Somit war es möglich, alle Schultypen, die für das Untersuchungsverfahren vorgesehen waren, in die Studie miteinzubeziehen. Dabei stimmten die Schulleiter, Fachbereichsleiter und Fachlehrer auch dem Vorhaben zu, die Untersuchung in den unterschiedlichen Jahrgangsstufen, Kursformen bzw. den nach Sprachkenntnisse getrennten Kursen durchführen zu lassen. Da - wie erwähnt - das Testverfahren im Hinblick auf das Ziel, objektive Fehlerergebnisse zu gewinnen, erst in solchen Klassen bzw. Kursen angewandt werden konnte, die bereits drei Jahre Englischunterricht absolviert hatten, sollte die Untersuchung in den traditionellen Schulformen nur die Jahrgangsstufen einbeziehen, die das erste Jahr der Mittelstufe vollendet hatten.

\footnotetext{
223 vgl. Ungerer u. a. 1987, 236

224 vgl. Weiher 1982, 145

225 vgl. ebd.
} 
Ausgehend von dem Bemühen, das Verfahren in einem Zeitraum durchzuführen, in dem die Probanden keine weiteren schulischen Prüfungen zu erwarten hatten, erschien es nach Rücksprache mit den betreffenden Fachlehrern sinnvoll, den Untersuchungszeitraum kurz vor den Abschluß der 7. Klasse zu legen, weil zu dieser Zeit das dritte Lernjahr nahezu beendet war, der Lernprozeß der Teilnehmer jedoch noch nicht durch die Sommerferien unterbrochen worden war. Diese Vorgehensweise war jedoch nur in bezug auf solche Klassen sinnvoll, die seit Beginn der weiterführenden Schule Englisch erlernt hatten, und nicht in bezug auf die Klassen, die mit einer anderen Fremdsprache begonnen hatten. In diesen Klassen wurde die Untersuchung erst ab der Klassenstufe durchgeführt, in der die Klassen bereits drei Jahre im Fach Englisch unterrichtet worden waren.

In den Gymnasien wurde die Untersuchung sowohl in der Mittelstufe als auch in der Oberstufe durchgeführt, um somit - wie zuvor erwähnt - Lernende zu erfassen, die sich in bezug auf die spracherwerbsspezifischen Voraussetzungen zum Teil deutlich voneinander unterscheiden. Die Durchführung in der Sekundarstufe II beschränkte sich jedoch auf die Jahrgangsstufen 11 und 12, weil die Lernenden der Jahrgangsstufe 13 zum Zeitpunkt der Untersuchungsdurchführung entweder das Gymnasium bereits verlassen hatten oder in Abiturprüfungen involviert waren und daher für das Untersuchungsverfahren nicht zur Verfügung standen.

In den Kursen der Sprach- und Volkshochschulen wurde die Untersuchung nur in Kursen durchgeführt, die über einen Kenntnisstand verfügten, der dem eines Teilnehmers einer weiterführenden Schule nach Abschluß des dritten Lernjahrs entspricht.

Konkret wurde die Untersuchung innerhalb der einzelnen Schulformen in folgenden Jahrgangsstufen und Klassen bzw. Kursen durchgeführt: In den Hauptschulen erfolgte die Untersuchung in allen Jahrgangsstufen der Mittelstufe, d. h., es wurden ausgehend von der 7. Klasse alle Jahrgangsstufen dieses Schultyps berücksichtigt. Da Hauptschulen mit Beginn der Jahrgangsstufe 10 differenzieren zwischen Teilnehmern, die die Fachoberschulreife anstreben, und Teilnehmern, die die Schule mit dem Hauptschulabschluß verlassen, wurde die Untersuchung in der Jahrgangsstufe 10 in zwei Klassenformen durchgeführt, den A- und den B-Klassen ${ }^{226}$.

In den Realschulen wurde die Untersuchung nur in den Jahrgangsstufen 7, 8 und 10 durchgeführt, weil Lernende der Schulform Realschule mit Beginn der 9. Klasse die Möglichkeit einer fachlichen Schwerpunktsetzung haben. Dies bedeutet, die Lernenden erhalten neben ihren anderen Fächern in

${ }^{226}$ Klassenbezeichnung A steht für Klassen mit dem Ziel Hauptschulabschluß, B für Klassen mit dem Ziel Fachoberschulreife. A-Klassen werden in der Regel auch als Grundkurs, B-Klassen als Erweiterungskurs bezeichnet. Diese Bezeichnungen werden daher auch bei der Analyse der Fehler in der vorliegenden Arbeit verwendet. 
einem Fach oder einem Fachgebiet, das ihrer Neigung entspricht, zusätzlichen Unterricht. ${ }^{227}$ In bezug auf das Fach Englisch bedeutet dies, daß die Lernbedingungen im regulären Englischunterricht, in dem neben Schülern, die Englisch als Schwerpunktfach gewählt haben, auch Lernende vertreten sind, die sich für einen anderen Schwerpunkt entschieden haben, nicht vergleichbar sind. Die Jahrgangsstufe 9 wurde daher nicht in die Untersuchung einbezogen.

In der Jahrgangsstufe 10 hingegen wird der Unterricht nach Schwerpunktsetzung differenziert. In der Regel wird zwischen sprachlich, naturwissenschaftlich und sozialwissenschaftlich orientierten Lernenden unterschieden. ${ }^{228}$ Lernende, die Englisch als Schwerpunktfach gewählt haben, werden in diesem Fach getrennt von den Lernenden unterrichtet, die sich für einen anderen Schwerpunkt entschieden haben. ${ }^{229}$ Aufgrund dieser Unterscheidung wurde die Untersuchung in den zehnten Klassen der Schulform Realschule jeweils in drei Kurstypen durchgeführt. Aufgrund dieser Unterscheidung wurde die Untersuchung in den zehnten Klassen der Schulform Realschule daher jeweils in drei Kurstypen durchgeführt.

Bei der Durchführung der Untersuchung in den Gymnasien wurde jede Jahrgangsstufe von der 7. bis zur 12. Klasse berücksichtigt. Da Gymnasien in der Oberstufe zwischen Grund- und Leistungskursen unterscheiden, wurden bei der Jahrgangsstufe 12 jeweils zwei Kursformen in die Untersuchung einbezogen, d. h. je zwei Leistungskurse und zwei Grundkurse.

In den Schulen, die dem Bereich Erwachsenenbildung zuzuordnen sind, wurde die Untersuchung sowohl in sogenannten Anfängerkursen als auch in Kursen der sogenannten Mittelstufe und fortgeschrittenen Kursen durchgeführt. Hier konnten jeweils zwei Kurse der zuvor genannten Kategorien untersucht werden. ${ }^{230}$

Die Durchführung der Untersuchung zeigte, daß das Verfassen von Briefen - wie vorausgesetzt allen Probanden vertraut war. Auch waren keine Verständnisschwierigkeiten bei der Bearbeitung der thematischen Vorgaben und beim Ausfüllen der Fragebögen zu erkennen. Insgesamt betrachtet erwies sich die Testgrundlage in der praktischen Anwendung als geeignet.

27 Kultusminister des Landes Nordrhein-Westfalen (Hrsg.) 1994, 29

228 vgl. ebd., 28

229 vgl. ebd., 101

230 Da die Schulen, die dem Bereich Erwachsenenbildung zugeordnet werden können, bei der Einteilung der Lernenden in der Regel keine deutschen Bezeichnungen gebrauchen, sondern die Lernenden den Kategorien beginners, intermediate und advanced learners zuordnen, wurden bei der Einteilung auch in der vorliegenden Arbeit englische Termini benutzt. 


\section{$4 \quad$ Ergebnisse der Untersuchung}

\subsection{Die Probanden}

Quantitativ führte die Untersuchung zu folgenden Ergebnissen (vgl. Tabelle 1 im Anhang): Insgesamt konnten 868 Probanden für die Studie gewonnen werden. 220 Probanden besuchten die Hauptschule und 211 Probanden die Realschule. In den Gymnasien beteiligten sich 288 Probanden an der Untersuchung. Im Bereich der Erwachsenenbildung waren 150 Probanden bereit, an dem Verfahren teilzunehmen. Alle Jahrgangsstufen und Klassen bzw. Kursformen, die für die Untersuchung vorgesehen waren, konnten in das Untersuchungsverfahren einbezogen werden. Wenngleich die Probanden aufgrund ihrer Einteilung nach Schulformen, Jahrgangsstufen und Sprachkenntnissen bereits einzelnen Gruppen zugeordnet waren, erschien es aus Gründen der Übersichtlichkeit sinnvoll, vor der Auswertung der Ergebnisse die Klassen, die der Mittelstufe zuzuordnen sind, in weitere Untergruppen zu untergliedern (vgl. Tabelle 1 im Anhang). Die Teilnehmer der Schulform Hauptschule wurden in drei Gruppen unterteilt. Die erste Gruppe bilden die Schüler der Jahrgangsstufen 7 - 9. Die Zusammenfassung dieser Jahrgangsstufen beruht zum einen darauf, daß die psycho- und soziolinguistischen Aspekte der Teilnehmer dieser Klassen in der Regel nur partiell voneinander abweichen, weil die Untersuchung in den Klassen durchgeführt wurde, die kurz vor Vollendung der Jahrgangsstufe 7 standen, und damit im Vergleich zu den Teilnehmern der 8. und 9. Klasse in bezug auf die Faktoren Alter und Sprachkenntnisse keine gravierenden Unterschiede zu erwarten waren. Zum anderen werden Hauptschüler - wie zuvor erwähnt - in der Jahrgangsstufe 10 nach Teilnehmern getrennt, die den Hauptschulabschluß oder die Fachoberschulreife zum Ziel haben. Diese Unterteilung hat in der Regel auch Auswirkungen auf die psycho- und soziolinguistischen Faktoren, weil Lernende, die die Schule mit der Fachoberschulreife verlassen möchten, oftmals ein höheres Maß an intrinsischer Motivation erkennen lassen, ${ }^{231}$ welche auch Auswirkungen auf die Sprachkenntnisse haben kann. ${ }^{232}$ Hinzu kommt, daß Teilnehmer dieser sogenannten Grund- und Erweiterungskurse in der Regel nicht zusammen unterrichtet werden und der Englischunterricht der beiden Klassenformen thematische Unterschiede erkennen läßt. Die Sprachkenntnisse der Teilnehmer sind daher nicht vergleichbar, so daß die Teilnehmer zwei getrennten Gruppen zugeordnet wurden.

Die Probanden der Schulform Realschule wurden in vier Gruppen unterteilt: Die erste Gruppe bilden die Jahrgangsstufen 7 und 8. Auch hier beruhte die Zusammenfassung darauf, daß sich die betreffenden Jahrgangsstufen in bezug auf die psycho- und soziolinguistischen Aspekte nur marginal unterscheiden.

\footnotetext{
231 Dies wurde in Gesprächen mit den einzelnen Fachlehrern in der Vorbereitungsphase der Studie deutlich.

232 vgl. Norrish 1983, $18-19$
} 
Da aus zuvor erwähnten Gründen die Jahrgangsstufe 9 bei der Untersuchung nicht berücksichtigt wurde, erfolgte die weitere Kategorisierung erst in den Klassen der Jahrgangsstufe 10. Hierbei wurden die Klassen in die zuvor beschriebenen sprachlich, naturwissenschaftlich und sozialwissenschaftlich orientierten Gruppen unterteilt.

Die größte Zusammenfassung wurde bei den Teilnehmern der Schulform Gymnasium vorgenommen. Hierbei wurden die Jahrgangsstufen $7-11$ zu einer Gruppe zusammengefaßt. Wenngleich diese Zusammenfassung zu erheblich größeren psycho- und soziolinguistischen Differenzen innerhalb dieser Gruppe führt, ist sie dennoch vertretbar, weil die Inhalte des Englischunterrichts beim Vergleich der einzelnen Jahrgangsstufen der gymnasialen Mittelstufe weniger Differenzen aufweisen als in Klassen der Haupt- und Realschulen. Dies beruht darauf, daß zum einen die Lerninhalte der gymnasialen Mittelstufe komplexer sind als die Lerninhalte der Mittelstufe der anderen weiterführenden Schulen. Die Vermittlung der Lerninhalte verläuft daher an Gymnasien langsamer. Zum anderen steht den Gymnasien im Gegensatz zu den anderen Schulen zur inhaltlichen Vermittlung auch die Sekundarstufe II zur Verfügung, sie haben dadurch im Vergleich zu anderen Schulen einen zeitlichen Vorteil. Auch die Tatsache, daß die Jahrgangsstufe 11 bereits zur Oberstufe gehört, ist in bezug auf die zuvor genannte Kategorisierung nicht als hinderlich zu betrachten, da eine Unterscheidung in Grund- und Leistungskurse frühestens in der zweiten Hälfte dieser Jahrgangsstufe erfolgt und der Englischunterricht der 11. Klasse in der Regel der Wiederholung und Vertiefung der Aspekte dient, die in den vorhergehenden Jahrgangsstufen vermittelt wurden. Die Probanden der 12. Klasse wurden hingegen wie zuvor erwähnt - nicht zu einer Gruppe zusammengefaßt, sondern nach Teilnehmern der Grundund Leistungskurse unterteilt.

Innerhalb der einzelnen Gruppen der unterschiedlichen Schulformen kam es zu folgender Beteiligung (vgl. Tabelle 1 im Anhang): Bei den Teilnehmern der Schulform Hauptschule umfaßte die Gruppe der Jahrgangsstufen 7 - 9183 Probanden. In der Jahrgangsstufe 10 enthielt die Gruppe der Schüler, die die Schule mit dem Hauptschulabschluß verlassen, 20 Probanden. Die Gruppe der Schüler, die die Fachoberschulreife anstreben, bestand aus 17 Teilnehmern. Die Gruppe der Jahrgangsstufen $7-8$ der Schulform Realschule umfaßte 116 Probanden. In den Klassen der Jahrgangsstufe 10 kam es bei dieser Schulform zu folgender Verteilung: Die Gruppe der sprachlich orientierten Schüler umfaßte 40 Teilnehmer, die Gruppe der sozialwissenschaftlich orientierten Schüler bestand aus 38 Probanden und die Gruppe der naturwissenschaftlich orientierten Schüler aus 17 Probanden.

In der Schulform Gymnasium konnten folgende Teilnehmerzahlen erzielt werden: Die Gruppe der Jahrgangsstufen 7 - 11 umfaßte insgesamt 214 Teilnehmer. Die Gruppe der Oberstufenschüler, die das Fach Englisch als Grundkurs belegt haben, bestand aus 41 Teilnehmern. Die Gruppe des entsprechenden Leistungskurses enthielt 32 Schüler. In bezug auf die Schulformen, die dem Bereich Erwachsenen- 
bildung zuzuordnen sind, kam es zu folgender Verteilung: Die Gruppe der Probanden, die als beginners zu bezeichnen sind, bestand aus 39 Teilnehmern. Die Gruppe der Probanden, die aufgrund der Sprachkenntnisse der Kategorie intermediate learners zugeordnet werden, bestand aus 81 Teilnehmern. Die Gruppe der advanced learners umfaßte 30 Teilnehmer.

Zusammenfassend ist festzustellen, daß die Mehrheit der Probanden der Schulform Gymnasium zuzuordnen ist, während die Gruppe der Teilnehmer des Bereichs Erwachsenenbildung die wenigsten Probanden umfaßt. Dieses Ergebnis ist darauf zurückzuführen, daß die Schulform Gymnasium die größte Anzahl unterschiedlicher Jahrgangsstufen enthält und darüber hinaus in der Oberstufe zwischen zwei Kursformen unterscheidet. Der Bereich Erwachsenenbildung hingegen besteht nur aus drei Gruppen, wenngleich auch hier in den letzten Jahren zunehmend weitere Unterteilungen vorgenommen wurden, die jedoch aus Gründen der Überschaubarkeit in der vorliegenden Studie nicht berücksichtigt wurden.

\subsection{Das Korrektur- und Auswertungsverfahren}

Inhalte des Korrektur- und Auswertungsverfahrens waren die Korrektur der Probandenbriefe sowie die Auswertung der psycho- und soziolinguistischen Informationen über die Teilnehmer, die aus den ausgefüllten Fragebögen hervorgingen. Zur Auswertung der einzelnen Fehlertypen wurde ein Auswertungsbogen erstellt (vgl. Tabelle 31 im Anhang). Im oberen Teil des Bogens wurden die psychound soziolinguistischen Informationen der einzelnen Teilnehmer festgehalten. Im unteren Teil wurden die Art und die Anzahl der einzelnen Fehlleistungen verzeichnet. Die Fehlleistungen wurden dabei nach linguistischen Ebenen geordnet.

Dabei zeigte die Gesamtbetrachtung der Probandenbeiträge, daß die Aufgaben, die den Briefen zugrunde lagen, generell linguistisch bewerkstelligt werden konnten und die psycho- und soziolinguistischen Fragen von den Probanden - wenn auch teilweise unvollständig - ausgefüllt worden waren. Unterschiede wurden in erster Linie in bezug auf die Anzahl der geschriebenen Wörter deutlich. Während die fortgeschrittenen Lernenden durchschnittlich 220 - 250 Wörter schrieben, lag die Anzahl der geschriebenen Wörter bei den weniger fortgeschrittenen Lernenden zum Teil unter 200 Lexemen (vgl. Tabelle 1 im Anhang). Dies betrifft insbesondere die Teilnehmer der Schulform Hauptschule.

Wie zuvor erwähnt, wurden die Probandenbriefe sowohl von deutschen als auch von englischen Muttersprachlern korrigiert. Die erste Korrektur wurde von den deutschen Englischlehrenden durchgeführt, im zweiten Durchgang wurden die Briefe von den britischen und amerikanischen Korrektoren überprüft. Insgesamt betrachtet zeigte der Korrekturverlauf, daß die deutschen Muttersprachler häufig 
zu anderen Korrekturergebnissen kamen als die englischen. Die wesentlichen Unterschiede bestanden darin, daß die deutschen Korrektoren generell mehr sprachliche Elemente als normabweichend bezeichneten als die englischen Muttersprachler, die englischen Muttersprachler hingegen auch Elemente als normabweichend bezeichneten, die von den deutschen Korrektoren als normgerecht betrachtet wurden.

Ein Beispiel für Äußerungen, die nur nach Auffassung der deutschen Korrektoren gegen die Norm des Englischen verstoßen, bietet der Satz The weather is not so bad as you think. ${ }^{233}$ Während die deutschen Korrektoren davon ausgingen, daß das Lexem so durch das Lexem as zu ersetzen sei, wiesen sowohl die britischen als auch amerikanischen Korrektoren darauf hin, daß die Äußerung des Lernenden ebenso korrekt sei wie die doppelte Verwendung des Lexems as, die von den deutschen Linguisten vorgeschlagen wurde.

Ein Beispiel für Äußerungen, die nur von den englischen Muttersprachlern moniert wurden, bietet der Satz Last year I went to England by ferry. ${ }^{234}$ Während alle deutschen Korrektoren das Lexem ferry akzeptierten, stellten die britischen und amerikanischen Korrektoren einstimmig fest, daß das Wort ferry stilistisch inkorrekt sei und stattdessen das Wort boat zu erwarten gewesen wäre.

Abweichungen in bezug auf das Korrekturverhalten waren jedoch auch beim Vergleich der britischen mit den amerikanischen Korrektoren zu erkennen. Dabei zeigte sich generell, daß das Korrekturverhalten der amerikanischen Linguisten toleranter war als das der britischen. Während die amerikanischen Korrektoren nicht nur Elemente akzeptierten, die der Norm des Amerikanischen Englisch entsprechen, sondern auch Elemente, die nur im Britischen Englisch zu erwarten sind, akzeptierten die britischen Korrektoren nur Elemente, die der Norm ihrer eigenen Sprachform entsprachen. Dies betraf nicht nur grammatische, sondern auch lexikalische Elemente. So akzeptierten zum Beispiel die amerikanischen Korrektoren sowohl die Präpositionalphrase at the weekend als auch on the weekend $^{235}$, während die britischen Korrektoren nur die Präposition at als korrekt gelten ließen.

Das Problem der interpersonellen Inkonsistenz äußerte sich jedoch - wie erwartet - auch innerhalb der einzelnen Korrektorengruppen. Betroffen davon waren sowohl die deutschen als auch die englischsprachigen Linguisten. Das Problem war insbesondere in sogenannten borderline cases zu beobachten. Ein Beispiel bietet u. a. die Äußerung Did you see the latest pictures?, die in allen Gruppen in bezug auf die Frage, ob die Äußerung als korrekt oder als falsch zu beurteilen sei, zu

\footnotetext{
${ }^{233}$ Fehlerbeispiel der vorliegenden Studie (im folgenden abgekürzt: FB) der Gruppe advanced learners (adv.) des Bereichs der Erwachsenenbildung (EB) [einzusehen beim Autor]

234 FB der Gruppe Realschule (RS) der Jahrgangsstufe 10, sprachlich orientiert (10spra.)

${ }^{235}$ Beide Präpositionalphrasen wurden in den meisten Lernergruppen wiederholt verwendet.
} 
Unstimmigkeiten führte. Während zum Teil die Meinung vertreten wurde, daß in der betreffenden Äußerung das present perfect zu erwarten gewesen wäre, befürworteten andere Korrektoren den Gebrauch des simple past. Elemente, die zu solchen Unstimmigkeiten führten, wurden nicht in den Fehlerkorpus aufgenommen, weil sie, wie die unterschiedlichen Meinungen verdeutlichen, nicht eindeutig gegen die Kriterien der Grammatikalität und der Akzeptabilität verstoßen.

\subsection{Die Fehler}

Der nach Abschluß des Korrekturverfahrens erstellte Fehlerkorpus zeigt folgendes: Die Probanden produzierten, wie vermutet, Fehler auf allen der zuvor beschriebenen linguistischen Ebenen (vgl. Tabelle 1 im Anhang) und in allen Unterkategorien (vgl. Tabellen 2 bis 11 im Anhang). Insgesamt wurden 12.588 Fehler auf lexikalischer, 21.611 Fehler auf syntaktisch-morphologischer und 4.338 Fehler auf orthographischer Ebene ermittelt. Die meisten Fehler wurden damit auf lexikalischem und auf syntaktischmorphologischem Gebiet produziert, wobei die syntaktisch-morphologischen Fehler gegenüber den lexikalischen überwogen und die orthographischen Fehler den geringsten Teil einnahmen (vgl. Tabelle 1 im Anhang). Insgesamt betrachtet können auf der

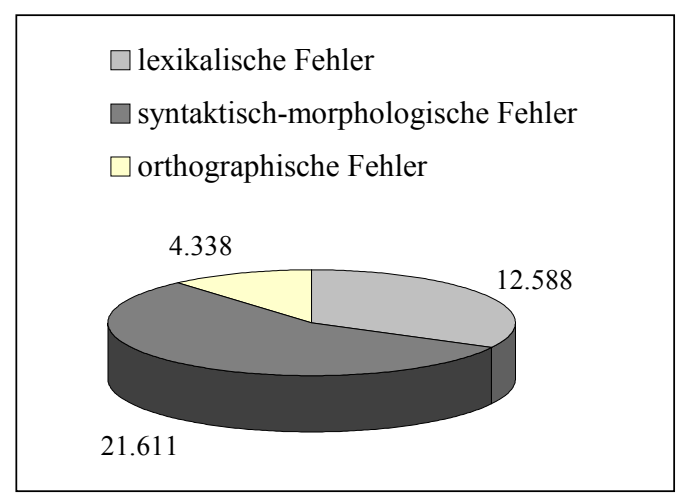

Abbildung 1: Anteil der lexikalischen, syntaktisch-morphologischen und orthographischen Fehler

Grundlage der Fehlertypen, die durch das Korrekturverfahren ermittelt wurden, folgende Aussagen abgeleitet werden:

Die Korrekturergebnisse haben gezeigt, daß die Abweichungen, die in der Fachdidaktik als typische Fehler deutscher Muttersprachler beim Erwerb des Englischen bezeichnet werden, partiell auch in der vorliegenden Studie deutlich wurden. Bei Betrachtung der einzelnen linguistischen Ebenen ist zu erkennen, daß die Lernenden im lexikalischen Bereich sowohl beim Gebrauch von Lexemen mit grammatischer Funktion - wie Hilfsverben und Präpositionen - als auch beim Gebrauch von Lexemen, die in erster Linie semantisch von Bedeutung sind, Fehler verursachten. Dazu gehört der gesamte Bereich der Verbal- und der Nominalebene (vgl. Tabellen 2 und 3 im Anhang). Somit bestätigen die Ergebnisse die in der Fachdidaktik verbreitete Auffassung, daß die lexikalische Sprachverwandtschaft zwischen dem Deutschen und dem Englischen das Auftreten lexikalischer Fehler nicht gänzlich verhindern kann. ${ }^{236}$ Dies wird vor allem bei Betrachtung der Tatsache deutlich, daß sowohl die im Englischen vorhandenen Hilfsverben und Präpositionen als auch der gesamte Bereich der

236 vgl. Gnutzmann 1972, 68 - 72 u. König 1972, 73 - 77 
Pronomina deutsche Entsprechungen kennt und diese Wortarten dennoch zu den größten Fehlerquellen gehören. ${ }^{237}$

Daneben lassen die Fehlerergebnisse erkennen, daß die meisten Abweichungen nicht dadurch entstehen, daß die Lernenden die englische Entsprechung für das deutsche Lexem nicht kennen. Vielmehr basiert der größte Anteil der Abweichungen auf der Tatsache, daß englische Wörter benutzt werden, die isoliert betrachtet tatsächlich der deutschen Vorlage entsprechen, jedoch im betreffenden Kontext unangebracht sind. Dies zeigt, daß die Fehler offensichtlich nicht nur auf linguistischen Differenzen zwischen der Erst- und der Zielsprache beruhen, sondern daß ein erheblicher Anteil der Abweichungen auf die lexikalische Vielseitigkeit der englischen Sprache zurückzuführen ist. Unter Vielseitigkeit ist hier zu verstehen, daß das Englische oft mehrere Lexeme für eine Sache oder einen Zustand kennt, für die oder für den es im Deutschen nur ein Lexem gibt. ${ }^{238}$ Ein Beispiel hierfür bietet das Wort ,klassisch', das im Englischen zwei Entsprechungen kennt, die jedoch nicht austauschbar sind. Dies sind die Lexeme classic und classical. ${ }^{239}$ Das deutsche Wort ,klassisch“ hingegen kann nicht durch ein anderes Lexem ersetzt werden, welches eine ebenso große Ähnlichkeit aufweist wie die zuvor genannten englischen Wörter.

Darüber hinaus verdeutlicht die Tatsache, daß die Fehlertypen, die auf lexikalischer Ebene konstatiert wurden, beinahe bei allen Probanden zu beobachten waren, d. h., das Auftreten dieser Fehlertypen ist nicht an das Erwerbsstadium der Zielsprache gebunden. Vielmehr sind diese Fehler bei Anfängern ebenso zu beobachten wie bei fortgeschrittenen Lernenden (vgl. Tabellen 2 und 3 im Anhang). Eine Abweichung gegenüber den Ergebnissen vorhergehender Fehleruntersuchungen ist in bezug auf die sogenannten false friends zu verzeichnen, die in der Fachliteratur besonders hervorgehoben werden. ${ }^{240}$ Während Lehrbücher immer wieder betonen, daß englische Wörter, die eine starke optische und akustische Ähnlichkeit zu deutschen Wörtern aufweisen und zur selben Wortart gehören, von deutschen Lernenden häufig falsch benutzt werden, ${ }^{241}$ ist die Anzahl solcher Verstöße in der vorliegenden Studie äußerst gering. Offenbar haben die vermehrten Hinweise im Englischunterricht der vergangenen Jahre zu einer Reduktion dieser Fehlertypen geführt.

Konkret wurden dabei innerhalb der einzelnen linguistischen Kategorien die im folgenden aufgelisteten Fehlertypen deutlich (vgl. Tabellen 2 bis 11 im Anhang).

\footnotetext{
237 vgl. Swan 1987, o. S.

238 vgl. Brough / Wittmann 2001, 5

239 vgl. Swan 1990, Textstelle $300-302$, o. S.

240 vgl. Helliwell 1989 u. Brough / Wittmann 2001, 143 - 154

241 vgl. Gnutzmann 1972, $68-72$
} 


\subsubsection{Fehler auf lexikalischer Ebene}

\begin{tabular}{|c|c|c|c|c|c|c|c|}
\hline 1. Fehlergruppe & 2. Fehlergruppe & 3. Fehlergruppe & 4. Fehlergruppe & 5. Fehlergruppe & 6. Fehlergruppe & 7. Fehlergruppe & Sondergruppe \\
\hline Hilfsverben & Wortwahl & $\begin{array}{c}\text { Gebrauch in- } \\
\text { existenter Wörter }\end{array}$ & Präpositionen & Adjektive & Adverbien & Pronomen & Stil \\
\hline
\end{tabular}

\subsubsection{Hilfsverben}

Die erste Fehlergruppe besteht aus Hilfsverben. Diese Fehler bestanden entweder darin, daß ein Hilfsverb fälschlicherweise durch ein anderes Hilfsverb substituiert wurde oder darin, daß nicht die zu erwartende Form des betreffenden Wortes gewählt wurde, wie etwa bei folgendem Fehlertyp: I will a cup of tea ${ }^{242}$ statt I would like a cup of tea. Hierbei wurde das richtige Wort gewählt, jedoch nicht die Konditionalform verwendet, die der Lernende hätte benutzen sollen. Ein Beispiel für eine vollständige Substitution ist in dem Satz Do you do me a favour ${ }^{243} \mathrm{zu}$ erkennen, da statt des Hilfsverbs do korrekterweise das Wort can zu verwenden gewesen wäre.

\subsubsection{Wortwahl}

Die zweite Fehlergruppe, die im lexikalischen Bereich deutlich wurde, bildet der Gebrauch von Wörtern, die für das Verständnis der sprachlichen Äußerung wichtig sind, jedoch im Gegensatz zu Hilfsverben, die etwa die Zeitform verdeutlichen, semantisch von Bedeutung sind. $\mathrm{Zu}$ dieser Wortgruppe gehören in erster Linie Nomen und Verben. Ein Fehlerbeispiel dieser Art bietet der Satz I drive to work by bus every day ${ }^{244}$. Der Fehler liegt hier im Gebrauch des Wortes drive, das statt des zu erwartenden Lexems go gewählt wurde.

\subsubsection{Gebrauch inexistenter Wörter}

Die dritte lexikalische Fehlergruppe stellt der Gebrauch von Wörtern dar, die nicht Bestandteil der englischen Sprache sind und von den Lernenden in Ermangelung des richtigen Wortes erfunden wurden. Dazu zählt u. a. die Wortzusammensetzung ill-house ${ }^{245}$, die statt des Lexems hospital benutzt wurde.

\subsubsection{Präpositionen}

Die vierte Fehlergruppe bilden die Präpositionen. Betroffen waren sowohl Präpositionen, die zeitliche Zusammenhänge verdeutlichen als auch Präpositionen, die im örtlichen Sinne benutzt wurden, wie

\footnotetext{
242 FB der Gruppe RS 10spra.

243 FB der Gruppe EB intermediate (int.)

${ }^{244}$ FB der Gruppe Hauptschule (HS), Erweiterungskurs der Jahrgangsstufe 10 (10E)
} 
etwa in der Äußerung I parked my car before the university ${ }^{246}$. Statt des Lexems before hätte die Präpositionalphrase in front of verwendet werden sollen.

\subsubsection{Adjektive}

Die fünfte Fehlerkategorie, die ermittelt wurde, beinhaltet den falschen Gebrauch der Adjektive. Die Fehler, die dabei auftraten, sind sowohl semantischer als auch grammatischer Art.

Fehler: $\quad$ I will be happy to visit you in September. ${ }^{247}$

Korrektur: I'm looking forward to seeing you in September.

\subsubsection{Adverbien}

Die sechste Fehlerkategorie setzt sich aus Adverbien zusammen. Ähnlich wie bei den Adjektiven beruhen die Fehler in der Regel darauf, daß Adverbien gewählt wurden, die dem eigentlich zu erwartenden Wort in seiner Bedeutung ähneln, jedoch in der betreffenden Äußerung unangebracht sind, wie z. B. bei folgender Abweichung. Der Fehler beruht hier auf dem Gebrauch des Wortes currently, das statt eines Lexems wie etwa presently oder momentarily verwendet wurde.

Fehler: $\quad$ Currently I have to struggle because my work is almost killing me. ${ }^{248}$ Korrektur: Presently/Momentarily I have to struggle because my work is almost killing me.

\subsubsection{Pronomen}

Die meisten Fehler der siebten Fehlergruppe, der Gruppe der Pronomen, gehen darauf zurück, daß ein Pronomen durch ein anderes substituiert wurde, welches in der gegebenen Äußerung unangebracht ist.

Fehler: $\quad$ He is a friend of $m e^{249}$

Korrektur: He is a friend of mine.

\footnotetext{
245 FB der Gruppe HS, Grundkurs der Jahrgangsstufe 10 (10G)

246 FB der Gruppe EB int.

247 FB der Gruppe HS 10G

248 FB der Gruppe EB adv.

249 FB der Gruppe HS 10E
} 


\subsubsection{Sondergruppe: Stil}

Diese Fehlergruppe umfaßt stilistische Äußerungen, d. h. lexikalische Begriffe, die nicht eindeutig als richtig oder falsch zu bezeichnen sind, jedoch in dem Kontext, in dem sie zur Anwendung kamen, unangebracht waren. Daher nehmen sie eine Sonderstellung ein und sind der lexikalischen Ebene nur bedingt zuzuordnen. Ein Beispiel bietet die Formulierung sincerely yours ${ }^{250}$, mit der eine Vielzahl von Briefen endete. Alle Briefe sollten an fiktive oder wirklich existierende Brieffreunde gerichtet sein, zu denen ein vertrautes Verhältnis besteht. Da die Formulierung sincerely yours eher dem formalen Englisch zuzuordnen ist, ist die Äußerung im gegebenen Kontext ungebräuchlich. Der Brief hätte stattdessen mit einem weniger formalen Ausdruck, wie etwa yours oder love enden sollen.

\subsubsection{Fehler auf syntaktisch-morphologischer Ebene}

\begin{tabular}{|c|c|c|c|c|c|}
\hline 1. Fehlergruppe & 2. Fehlergruppe & 3. Fehlergruppe & 4. Fehlergruppe & 5. Fehlergruppe & 6. Fehlergruppe \\
\hline \multirow{2}{*}{ Wort- und Satzstellung } & Auslassungsfehler & Hinzufügungsfehler & Bedingungssätze & Aktiv/Passiv & Zeitensystem \\
\hline & $\begin{array}{l}\text { 1. Verlaufsform } \\
\text { (Gegenwart, } \\
\text { Vergangenheit, } \\
\text { Zukunft) } \\
\text { 2. Infinitiv } \\
\text { 3. Partizipialsätze } \\
\text { 4. Gerundium } \\
\text { 5. Artikel (best. / } \\
\text { unbest.) } \\
\text { 6. Adjektiv } \\
\text { 7. Adverb } \\
\text { 8. sonstige Wortarten } \\
\text { und Bestandteile }\end{array}$ & $\begin{array}{l}\text { 1. Verlaufsform } \\
\text { (Gegenwart, } \\
\text { Vergangenheit, } \\
\text { Zukunft) } \\
\text { 2. Infinitiv } \\
\text { 3. Partizipialsätze } \\
\text { 4. Gerundium } \\
\text { 5. Artikel (best. / } \\
\text { unbest.) } \\
\text { 6. Adjektiv } \\
\text { 7. Adverb } \\
\text { 8. sonstige Wortarten } \\
\text { und Bestandteile }\end{array}$ & & & $\begin{array}{l}\text { 1. Gegenwart } \\
\text { 2. Vergangenheit } \\
\text { a) simple past } \\
\text { b) present perfect } \\
\text { c) past perfect } \\
\text { 3. Zukunft } \\
\text { a) progressive } \\
\text { b) going to } \\
\text { c) will-future }\end{array}$ \\
\hline
\end{tabular}

\subsubsection{Wort- und Satzstellung}

Die erste Fehlergruppe bilden Verstöße gegen die Wort- und Satzstellungsregeln der englischen Sprache. Dazu gehören Fehler, die darauf beruhen, daß die Reihenfolge einzelner Lexeme oder Lexembestandteile innerhalb eines Satzes oder eines Satzteils vertauscht wurden. Die Norm der englischen Sprache bestimmt, daß Temporaladverbialien in der Regel am Anfang oder am Ende eines Satzes plaziert werden.

Fehler: I was yesterday in the cinema. ${ }^{251}$

Korrektur: I was in the cinema yesterday. oder: Yesterday I was in the cinema.

${ }^{250}$ FB der Gruppe EB adv.
251 FB der Gruppe HS 10G 


\subsubsection{Auslassungsfehler}

$\mathrm{Zu}$ der zweiten Gruppe, den Auslassungsfehlern, zählen Abweichungen, die durch das Auslassen syntaktisch-morphologischer Elemente, welche grammatische Funktionen übernommen haben, entstanden sind. Wie oben dargestellt, werden die Auslassungsfehler in acht Unterkategorien unterteilt.

Die erste Unterkategorie besteht aus Fehlern, die auf dem Auslassen der Verlaufsform beruhen. Diese Verstöße sind sowohl im Bereich der Vergangenheitstempora und in der Gegenwartsform als auch bei den Tempora der Zukunft zu beobachten. Aufgrund der zeitlichen Konkretisierung durch die Präpositionalphrase by this time hätte der Lernende das sogenannte future progressive verwenden sollen.

Fehler: $\quad$ On Tuesday by this time I will lie on the beach. ${ }^{252}$

Korrektur: On Tuesday by this time I will be lying on the beach.

Die zweite Unterkategorie setzt sich aus Verstößen zusammen, die auf das Auslassen der Infinitivform to zurückgehen.

Fehler: $\quad$ I want visit my friend. ${ }^{253}$

Korrektur: I want to visit my friend.

Die dritte Unterkategorie besteht aus Abweichungen, die auf den Verzicht auf die Partizipialform zurückgehen.

Fehler: I saw him to repair his car. ${ }^{254}$

Korrektur: I saw him repairing his car.

$\mathrm{Zu}$ der vierten Unterkategorie gehören Gerundialverstöße. Dazu zählt insbesondere das Auslassen des Gerundiums nach Präpositionen.

Fehler: $\quad$ I'm used to drink a cup of tea every morning. ${ }^{255}$

Korrektur: I'm used to drinking a cup of tea every morning.

Die fünfte Unterkategorie bildet die Gruppe der Artikel. Dazu gehören sowohl bestimmte als auch unbestimmte Artikel.

Fehler: We spent our last holidays in Tirol. ${ }^{256}$

Korrektur: We spent our last holiday in the Tyrol.

\footnotetext{
252 FB der Gruppe EB adv.

253 FB der Gruppe HS 10G

${ }^{254}$ FB der Gruppe EB beginners (beg.)

255 FB der Gruppe EB adv.

256 FB der Gruppe EB adv.
} 
Fehler: $\quad$ I have headaches. ${ }^{257}$

Korrektur: I have a headache. ${ }^{258}$

Die sechste Unterkategorie der Auslassungsfehler umfaßt die Gruppe der Adjektive. Fehler, die dieser Kategorie zugeordnet wurden, basieren darauf, daß entweder statt der Adjektive Lexeme benutzt wurden, die zu anderen Wortarten gehören, oder daß anstelle von Prädikativa adverbiale Adjunkte eingesetzt wurden. Zu den häufigsten Fehlern dieses Typs zählt die Verwendung des Adverbs well zur näheren Bestimmung sogenannter verbs of the senses ${ }^{259}$, wie etwa sound, feel oder taste.

Fehler: It tastes very well. ${ }^{260}$

Korrektur: It tastes very good.

Fehler: $\quad$ She is interest. ${ }^{261}$

Korrektur: She is interesting. oder: She is interested.

Die siebte Unterkategorie beinhaltet Fehler beim Gebrauch der Adverbien. Dazu gehören vornehmlich Fehler, die darauf beruhen, daß die eigentlich zu erwartenden Adverbien durch Adjektive ersetzt wurden. Fehler dieser Art werden dem Auslassungsbereich zugeordnet, weil Adverbien im Gegensatz zu Adjektiven auf -ly enden und daher infolge der Verwechslung der Wortarten Segmente ausgelassen werden.

Fehler: You speak English very good. ${ }^{262}$

Korrektur: You speak English very well.

Die achte Unterkategorie besteht aus der Gruppe ,sonstige Wortarten und Bestandteile‘. Dazu gehören alle Fehler, die auf dem Auslassen einzelner Wort- und Satzsegmente beruhen, ohne daß diese Fehler jedoch direkt als Verstoß innerhalb einer bestimmten grammatischen Hauptkategorie oder Wortart bezeichnet werden können. Dazu gehört z. B. das Auslassen des Morphems ,s ' in der 3. Person Singular.

Fehler: $\quad$ The bus leave at 3 o' $^{\prime}$ clock. ${ }^{263}$

Korrektur: The bus leaves at 3 o'clock.

\subsubsection{Hinzufügungsfehler}

Die Korrektur der Probandenbriefe ließ erkennen, da $ß$ die Lernenden nicht nur fälschlicherweise Wort- und Satzelemente auslassen, sondern daß daneben auch Sprachbestandteile redundanterweise

257 FB der Gruppe EB beg.

258 Die Fehlleistung gehört gleichzeitig zu den Hinzufügungsfehlern, weil der Lernende nicht bedacht hat, daß die mit ache verbundenen Wörter zu den Singularwörtern gehören. Das Pluralmorphem ,s‘ ist daher ein Normverstoß.

259 Thomson / Martinet 1987, 156

${ }^{260}$ FB der Gruppe Gymnasium (GY), Grundkurs der Jahrgangsstufe 12 (12G)

261 FB der Gruppe EB beg.

262 FB der Gruppe GY 12G

263 FB der Gruppe HS 10G 
hinzugefügt werden und dadurch Normabweichungen evozieren. Diese Abweichungen bilden die dritte Fehlergruppe. Die Kategorien, die hier gebildet wurden, entsprechen terminologisch den Kategorien des Auslassungsbereichs, sind aber inhaltlich diesen Fehlern diametral entgegengesetzt.

Die erste Unterkategorie der Hinzufügungsfehler, die ,Verlaufsform', besteht aus Abweichungen, die dadurch entstehen, daß die Verlaufsform sowohl in der Gegenwart als auch bei Aussagen über die Vergangenheit und die Zukunft benutzt wurde, obwohl die entsprechenden einfachen Formen zu erwarten gewesen wären. Dabei ist jedoch bei nahezu allen Fehlerbeispielen zu bemerken, daß die Normabweichungen bei den Auslassungsfehlern schwerwiegender sind als bei den Hinzufügungsfehlern. Letzteres ist dadurch begründet, daß das normabweichende Hinzufügen der Verlaufsform auch zu den dialektalen Merkmalen der englischen Sprache gehört, d. h. zum Teil auch bei englischen Muttersprachlern zu beobachten ist. ${ }^{264}$

Fehler: $\quad$ I'm seeing my friend tomorrow. ${ }^{265}$

Korrektur: I'll see my friend tomorrow.

Fehler: $\quad$ I'm living in a little town near Aachen. ${ }^{266}$

Korrektur: I live in a little town near Aachen.

Fehler: $\quad$ I was spending my last holidays with my parents in Spain. ${ }^{267}$

Korrektur: I spent my last holidays with my parents in Spain.

Fehler: $\quad$ We will be remembering our last holiday for a long time. ${ }^{268}$

Korrektur: We will remember our last holiday for a long time.

Die zweite Unterkategorie ,Infinitiv` enthält u. a. folgendes Fehlerbeispiel:

Fehler: $\quad$ I enjoy to go to the beach. ${ }^{269}$

Korrektur: I enjoy going to the beach.

In der dritten Unterkategorie ,Partizipialsätze‘ findet man u. a. folgenden Fehlertyp:

Fehler: $\quad$ We saw a man to walk in the street. ${ }^{270}$

Korrektur: We saw a man walking in the street.

\footnotetext{
${ }^{264}$ Diese Aussage beruht auf Erkenntnissen, die während der Lehrtätigkeit des Autors der vorliegenden Arbeit in Nordostengland gewonnen wurden. Dabei fiel auf, daß z. B. das Lexem want, das regelentsprechend in der einfachen Form verwendet wird, häufig dennoch in der Verlaufsform benutzt wurde, wie etwa in dem Satz I have been wanting to come here for a long time.

265 FB der Gruppe GY 12G

${ }^{266}$ FB der Gruppe HS 10E. Die Äußerung ist dann falsch, wenn eine permanente Handlung vorliegt.

${ }^{267}$ FB der Gruppe HS 10E. Die Äußerung stellt eine Normabweichung dar, weil hier nicht zwei Handlungen miteinander in Verbindung treten.

268 FB der Gruppe HS 10E

269 FB der Gruppe GY, Leistungskurs der Jahrgangsstufe 12 (12L)

${ }^{270}$ FB der Gruppe RS 10spra.
} 
In der vierten Unterkategorie, den gerundialen Hinzufügungsfehlern, bestehen die Abweichungen in der Regel darin, daß das Gerundium nach dem Lexem to benutzt wird, das Wort to dabei jedoch keine Präposition bildet, sondern einen Bestandteil des Infinitivs.

Fehler: $\quad$ I used to working in England. ${ }^{271}$

Korrektur: I used to work in England.

Die fünfte Unterkategorie, die Gruppe des bestimmten und unbestimmten Artikels, betrifft vor allem den redundanten Gebrauch dieser Wortarten bei Eigennamen.

Fehler: $\quad$ I have bought my jacket in the Bond Street. ${ }^{272}$

Korrektur: I have bought my jacket in Bond Street.

Die sechste Unterkategorie, die Gruppe der Adjektive, umfaßt vor allem redundanterweise hinzugefügte Adjektive, die eine besonders positive oder negative Bedeutung vermitteln sollen. Dies ist aufgrund der Tatsache, daß Englisch als eine Sprache der understatements gilt, als normabweichend zu betrachten.

Fehler: $\quad$ Turkey is great and beautiful. ${ }^{273}$

Korrektur: Turkey is great. oder: Turkey is beautiful.

Die siebte Unterkategorie, die Gruppe der Adverbien, enthält hauptsächlich Fehler, die darauf beruhen, daß Adverbien benutzt wurden, obwohl in den betreffenden Äußerungen Adjektive zu erwarten gewesen wären. Normabweichungen dieser Art werden als Hinzufügungsfehler bezeichnet, weil die Verwechslung der Wortarten dazu führt, daß die adverbiale Markierung -ly an das Lexem angefügt wird. Dies zeigt sich etwa im nachfolgenden Beispiel.

Fehler: $\quad$ He looks beautifully. ${ }^{274}$

Korrektur: He looks beautiful.

In der achten Unterkategorie, der Gruppe ,sonstige Wortarten und Bestandteile`, geht das Hinzufügen der Elemente vor allem darauf zurück, daß grammatische Beschränkungen nicht beachtet wurden wie etwa in bezug auf die Anwendungsmöglichkeiten des Pluralmorphems -s.

Fehler: $\quad$ I have three childrens. ${ }^{275}$

Korrektur: I have three children.

\footnotetext{
271 FB der Gruppe EB adv.

272 FB der Gruppe HS 10E

273 FB der Gruppe HS 10G

274 ebd.

275 FB der Gruppe EB beg.
} 


\subsubsection{Bedingungssätze}

Die vierte Fehlergruppe betrifft vor allem die in der Fachdidaktik als if-Sätze bezeichneten Satztypen. Die meisten Fehler dieser Gruppe sind entweder dadurch entstanden, daß eine Konditionalform benutzt wurde, obwohl in der betreffenden Äußerung keine solche zu erwarten gewesen wäre, oder aber dadurch, daß nicht die richtige Konditionalform benutzt wurde.

Fehler: $\quad$ I would come to your party if the weather would be better. ${ }^{276}$

Korrektur: I would come to your party if the weather was better.

\subsubsection{Aktiv/Passiv}

Die Mehrzahl der Abweichungen der fünften Fehlergruppe besteht darin, daß Passiv-Sätze nicht als solche erkannt wurden und stattdessen Aktivformen oder unvollständige Passivformen benutzt wurden.

Fehler: $\quad$ The letter has sent to the wrong address. ${ }^{277}$

Korrektur: The letter has been sent to the wrong address.

Fehler: $\quad$ The product was make in Germany. ${ }^{278}$

Korrektur: The product was made in Germany.

\subsubsection{Zeitensystem}

Im Bereich der 6. Fehlergruppe, den Verstößen beim Gebrauch des Zeitensystems, d. h. den sogenannten tenses and aspects, waren Fehler auf allen Zeitstufen zu erkennen (vgl. Tabellen 6 und $7 \mathrm{im}$ Anhang). Die Abweichungen basieren nahezu alle darauf, daß die Anwendungsbegrenzungen der einzelnen Zeitformen nicht richtig erkannt wurden und daher Zeitformen gewählt wurden, die der eigentlich zu erwartenden Form ähneln, jedoch im betreffenden Kontext unangebracht sind.

In der ersten Unterkategorie - dem Gebrauch der Zeitform Gegenwart - beruhen die Fehler entweder darauf, daß die einfache Gegenwartsform durch die progressive form ersetzt wurde oder darauf, daß anstelle der progressive form die einfache Gegenwartsform benutzt wurde.

Fehler: $\quad$ At the moment we deal with a book about Shakespeare. ${ }^{279}$

Korrektur: At the moment we are dealing with a book about Shakespeare.

Fehler: $\quad$ I am enjoying classical music. ${ }^{280}$

Korrektur: I enjoy classical music.

\footnotetext{
276 FB der Gruppe EB int.

277 FB der Gruppe EB int.

278 FB der Gruppe EB beg.

279 FB der Gruppe GY 12G

280 FB der Gruppe EB adv.
} 
Ähnliche Abweichungen zeigten sich auch bei den Fragesätzen.

Fehler: $\quad$ Are you speaking English well? $?^{281}$

Korrektur: Do you speak English well?

Fehler: What do you do at the moment? ? $^{282}$

Korrektur: What are you doing at the moment?

Auch in der zweiten Unterkategorie - bei den Vergangenheitstempora - sind die Abweichungen darauf zurückzuführen, daß eine Vergangenheitsform durch eine andere substituiert wurde, welche in der betreffenden Aussage von der Norm der englischen Sprache abweicht.

a) simple past

Die Fehler beim Gebrauch des simple past - die erste Gruppe der Unterkategorie ,Vergangenheitstempora' - sind in erster Linie durch Verwechslungen des simple past mit dem present perfect entstanden. Dies bedeutet, es wurde fälschlicherweise vermutet, daß das present perfect anstelle des simple past gewählt werden kann, obwohl im Englischen, im Gegensatz zu den Anwendungsmöglichkeiten der deutschen Sprache, strikt zwischen den Anwendungsmöglichkeiten der beiden Zeitformen unterschieden wird. ${ }^{283}$ Diese Unterscheidung muß insbesondere dann getroffen werden, wenn die betreffende Äußerung eine konkret bestimmbare Zeitangabe enthält. ${ }^{284}$

Fehler: $\quad$ I have worked yesterday. ${ }^{285}$

Korrektur: I worked yesterday.

Auch hier waren Abweichungen ebenso bei den Fragesätzen zu beobachten.

Fehler: $\quad$ Have you worked last week? $?^{286}$

Korrektur: Did you work last week?

b) present perfect

Ähnliche Verwechslungen wurden auch bei der zweiten Gruppe der Vergangenheitstempora konstatiert. Hier wurde in den meisten Fällen das present perfect durch das simple past ersetzt, vermutlich, weil die Lernenden wußten, daß zwischen den Anwendungsmöglichkeiten der beiden Tempora zu unterscheiden ist, diese jedoch in den betreffenden Äußerungen nicht normgerecht realisiert haben.

\footnotetext{
281 FB der Gruppe GY 12G

282 FB der Gruppe EB beg.

283 vgl. Ungerer u. a. 1987, $131-136$

284 vgl. ebd., 135

285 FB der Gruppe RS der Jahrgangsstufe 10, naturwissenschaftlich orientiert (10natw.)

286 ebd.
} 
Fehler: $\quad$ I worked a lot. $^{287}$

Korrektur: I have worked a lot. ${ }^{288}$

Vergleichbare Verstöße sind bei den Fragesätzen zu beobachten.

Fehler: Did you ever go to England? ${ }^{289}$

Korrektur: Have you ever been to England?

c) past perfect

In der dritten Gruppe der Vergangenheitstempora haben Substitutionen des past perfect durch andere Vergangenheitsformen, insbesondere durch das simple past, zur Entstehung der Abweichungen geführt.

Fehler: $\quad$ When I came home I saw immediately that my bike was stolen. ${ }^{290}$

Korrektur: When I came home I saw immediately that my bike had been stolen.

In der dritten Unterkategorie des Bereichs ,Zeitensystem ‘ - den Zukunftstempora - waren, wie auch bei den zuvor genannten Zeitformen, Fehler in allen Typen der betroffenen Zeitform erkennbar. Auch hier gehen die Abweichungen im wesentlichen darauf zurück, daß die Anwendungsmöglichkeiten der einzelnen Zukunftstempora bzw. die damit verbundenen Beschränkungen nicht richtig realisiert wurden.

\section{a) future progressive}

In der ersten Gruppe dieses Fehlerbereichs basieren die Abweichungen vornehmlich darauf, daß statt der progressive form das simple present gewählt wurde.

Fehler: $\quad$ Next year I go to Spain. ${ }^{291}$

Korrektur: Next year I'm going to Spain.

b) going to-future

In der zweiten Gruppe, die als going to-future bezeichnet wurde, sind die Fehler meist dadurch entstanden, daß das going to-future durch das will-future ersetzt wurde.

Fehler: $\quad$ I will definitely visit you next summer. ${ }^{292}$

Korrektur: I'm definitely going to visit you next summer.

287 FB der Gruppe GY 12G

288 Die Aussage steht nicht im Zusammenhang mit einer bestimmten Zeitangabe oder mit einem Ereignis, das in der Vergangenheit stattgefunden hat. Es ist daher das present perfect zu erwarten.

289 FB der Gruppe GY 12G

290 FB der Gruppe GY 12L

291 FB der Gruppe HS 10E

${ }^{292}$ FB der Gruppe EB adv. 
c) will-future

Der umgekehrte Fall ist in der dritten Gruppe des Bereichs Zukunftstempora zu beobachten. Statt des will-future wurde hier meist eine andere Zukunftsform benutzt, insbesondere das going to-future.

Fehler: $\quad$ Perhaps I'm going to come and visit you in my Easter holidays. ${ }^{293}$

Korrektur: Perhaps I will visit you in my Easter holidays.

\subsubsection{Fehler im Bereich Orthographie}

Die erste Fehlergruppe des Bereichs orthographische Abweichungen bildet die Kategorie Großschreibung. Diese Kategorie enthält Abweichungen, die dadurch entstanden sind, daß Lexeme, die normgerecht groß geschrieben werden müßten, klein geschrieben wurden.

Fehler: $\quad$ I speak english and french. ${ }^{294}$

Korrektur: I speak English and French.

Die zweite Fehlergruppe bildet die Kategorie Kleinschreibung. Hier ist der umgekehrte Fall zu beobachten: Statt der zu erwartenden Kleinschreibung wurden Lexeme fälschlicherweise groß geschrieben.

Fehler: $\quad$ Next year I will go to the North of Italy. ${ }^{295}$

Korrektur: Next year I will go to the north of Italy.

Die dritte Kategorie setzt sich aus Rechtschreibfehlern zusammen. Hierzu zählen Abweichungen, bei denen Lexeme entweder innerhalb eines Wortes oder am Wortende falsch geschrieben wurden.

Fehler: $\quad$ I spent my last holidays in Cornwal. ${ }^{296}$

Korrektur: I spent my last holidays in Cornwall.

\subsection{Fehlertypologische Auffälligkeiten in den Probandengruppen}

Der Vergleich der einzelnen Probandengruppen hat in bezug auf die Art und die Anzahl der Fehler (vgl. Tabelle $1 \mathrm{im}$ Anhang) gezeigt, daß die Probanden der Schulform Hauptschule insgesamt die meisten sprachlichen Abweichungen verursachten. Die zweitgrößte Anzahl sprachlicher Verstöße wurde bei den Probanden der Schulform Realschule gefunden. Der drittgrößte Anteil zeigte sich bei den Probanden der Schulform Gymnasium. Die wenigsten Abweichungen waren bei den Teilnehmern

\footnotetext{
293 ebd.

294 FB der Gruppe RS 10spra.

295 ebd.

296 FB der Gruppe EB adv.
} 
des Bereichs Erwachsenenbildung zu erkennen. ${ }^{297}$ Dabei ist jedoch sowohl die Differenz zwischen dem Fehleranteil der Haupt- und der Realschüler als auch die Differenz zwischen den Probanden der Schulform Gymnasium und des Bereichs Erwachsenenbildung als gering zu bezeichnen.

Beim Vergleich der verschiedenen Gruppen der einzelnen Schulformen war zu erkennen, daß die Teilnehmer der Jahrgangsstufen 7 - 9 der Schulform Hauptschule weniger Fehler verursachten als die Teilnehmer der Jahrgangsstufe 10 derselben Schulform. Auch zeigte sich, daß der Fehleranteil der Lernenden, die die Fachoberschulreife anstrebten, größer war als der Fehleranteil der Lernenden, die die Schule mit dem Hauptschulabschluß verlassen würden.

Bei den Teilnehmern der Schulform Realschule war - abgesehen von einer Ausnahme - ein ähnliches Ergebnis zu erkennen. Auch hier zeigte sich, daß der Fehleranteil der Jahrgangsstufen $7-8$ geringer war als der Fehleranteil der Gruppen der Jahrgangsstufe 10. Ausnahmen bildeten hier nur die sprachlich orientierten 10er-Klassen. Die Differenz zwischen dem Fehleranteil der sprachlich orientierten Realschüler und den Teilnehmern der Jahrgangsstufen $7-8$ ist jedoch äußerst gering. Bei Betrachtung der einzelnen 10er-Klassen dieser Schulform war gleichzeitig zu erkennen, daß auch die fachliche Schwerpunktsetzung der Lernenden Auswirkungen auf die Anzahl der Fehler hat. So verursachten die sprachlich orientierten Teilnehmer weniger Fehler als die Teilnehmer, die sich für einen sozialwissenschaftlichen oder naturwissenschaftlichen Schwerpunkt entschieden hatten.

Auch bei den Teilnehmern der Schulform Gymnasium zeigte sich, daß die unteren bis mittleren Jahrgangsstufen zum Teil weniger Fehler verursachten als die Teilnehmer der oberen Jahrgangsstufen. Konkret war hier zu erkennen, daß der Fehleranteil, der in den Jahrgangsstufen 7 -11 ermittelt wurde, geringer war als der Fehleranteil des Grundkurses der Jahrgangsstufe 12.

Beim Vergleich der Ergebnisse, die innerhalb der einzelnen linguistischen Ebenen Lexik, Syntax/ Morphologie und Orthographie gewonnen wurden ${ }^{298}$, war zu erkennen, daß die Teilnehmer der Schulform Hauptschule nicht nur insgesamt, sondern auch innerhalb der einzelnen Sprachbereiche jeweils die meisten Fehler produzierten. Der zweitgrößte Fehleranteil auf lexikalischer, syntaktisch-morphologischer und orthographischer Ebene wurde bei den Teilnehmern der Schulform Realschule ermittelt. Bei Betrachtung der Fehlerergebnisse der Gymnasiasten und der Probanden des Bereichs Erwachsenenbildung zeigte sich, daß der Anteil der lexikalischen Fehler bei den Teilnehmern des Bereichs Erwachsenenbildung geringer war als bei den Gymnasiasten. Auf syntaktisch-morphologischer Ebene

\footnotetext{
297 Das Untersuchungsmaterial kann beim Autor eingesehen werden.

298 vgl. Abb. 2 bis 4
} 
und auf orthographischer Ebene hingegen überwog der Fehleranteil bei den Teilnehmern des Bereichs Erwachsenenbildung.

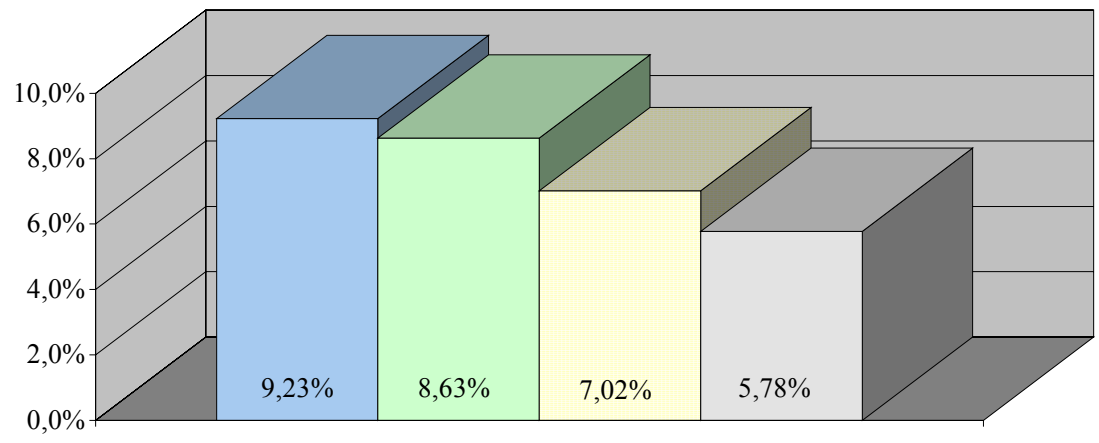

Abbildung 2:

Prozentualer Anteil der lexikalischen Fehler je Schulform
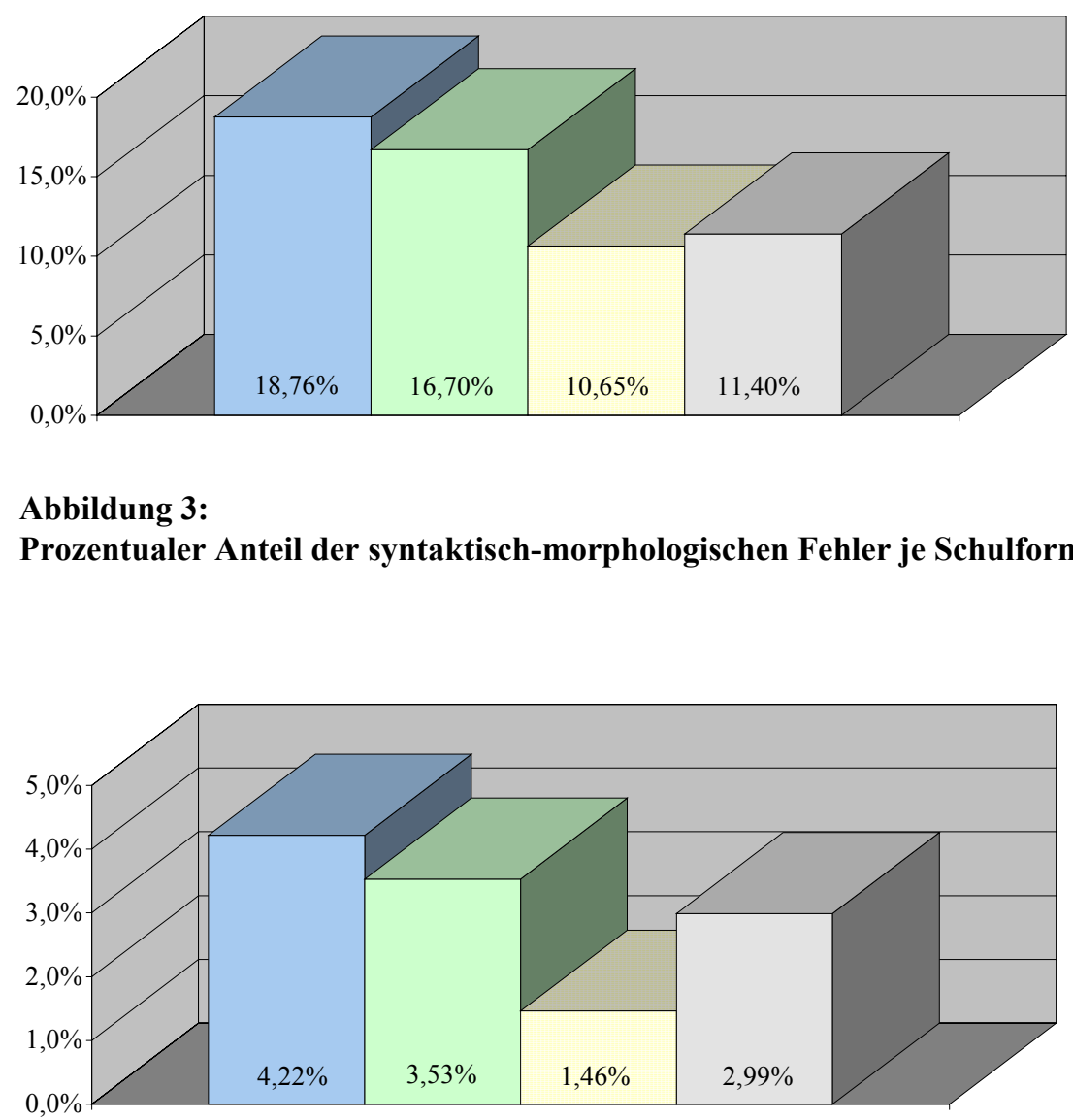
Die Ermittlung der Gruppen, die innerhalb der einzelnen linguistischen Bereiche jeweils die meisten und die wenigsten Abweichungen produzierten und einander im Rahmen des nachfolgenden Analyseverfahrens gegenübergestellt wurden, führte zu folgenden Ergebnissen (vgl. Tabelle $1 \mathrm{im}$ Anhang): Die meisten lexikalischen Fehler wurden von den naturwissenschaftlich orientierten Teilnehmern der Schulform Realschule produziert. Die wenigsten lexikalischen Verstöße waren in der Gruppe der advanced learners des Bereichs Erwachsenenbildung zu erkennen. Auf syntaktisch-morphologischer Ebene wurden die meisten Fehler im Erweiterungskurs der Schulform Hauptschule ermittelt, während die wenigsten syntaktisch-morphologischen Fehler in der Gruppe der advanced learners des Bereichs Erwachsenenbildung konstatiert wurden. Auf orthographischer Ebene waren die meisten Abweichungen in der Gruppe der Jahrgangsstufen 7 - 9 der Schulform Hauptschule zu erkennen. Da die Gruppe jedoch eine Vielzahl von Lernenden enthielt, die nicht deutsch als Erstsprache sprechen, wurde stattdessen der Grundkurs der Jahrgangsstufe 10 dieser Schulform exemplarisch betrachtet, da auch diese Gruppe durch eine besonders hohe Anzahl orthographischer Verstöße auffiel. Die wenigsten orthographischen Verstöße wurden im Leistungskurs der Schulform Gymnasium ermittelt.

Insgesamt betrachtet zeigte der Vergleich der quantitativ jeweils diametral entgegengesetzten Probandengruppen, daß die größte Fehlerdifferenz auf syntaktisch-morphologischer Ebene ermittelt wurde, während die Fehlerunterschiede auf lexikalischer und orthographischer Ebene weniger gravierend waren. Der geringste Unterschied war dabei auf orthographischer Ebene zu erkennen.

\subsection{Der Fragebogen ${ }^{299}$}

Die Auswertung des Fragebogens, der in erster Linie der Ermittlung psycho- und soziolinguistischer Probandeninformationen diente, führte zu folgenden Erkenntnissen (vgl. Tabellen 32 bis 35 im Anhang):

In bezug auf den Faktor Alter zeigte sich, daß die Differenzen zwischen den Probanden, die eine reguläre weiterführende Schule besuchten, in der Regel bei maximal sechs Jahren lagen. Die meisten Probanden dieser Kategorien waren zwischen 13 und 19 Jahren alt. Wenngleich die älteren Schüler Englisch schon länger erlernt hatten als die jüngeren, ist nicht davon auszugehen, daß die Altersunterschiede, die zwischen den Probanden der Unter- und Mittelstufe und den Teilnehmern der gymnasialen Oberstufe bestanden, Auswirkungen auf das Konzentrations- und Lernvermögen der einzelnen

299 vgl. Abb. 5 im Anhang 
Probanden hatten. Die Fehlerunterschiede, die hier deutlich wurden, sind demnach vermutlich nicht auf biologische Aspekte zurückzuführen. ${ }^{300}$

Große Altersunterschiede bestanden hingegen zwischen den Probanden der Unter- und Mittelstufe sowie den Teilnehmern der gymnasialen Oberstufe und den Probanden der Kategorie Erwachsenenbildung. Hier betrug die Altersdifferenz bis zu 40 Jahren. Daher war beim Vergleich der Fehler, die von den Probanden des Bereichs Erwachsenenbildung und von den jugendlichen Probanden produziert wurden, immer darauf zu achten, inwieweit die Abweichungen die Folge einer differierenden Vorgehensweise beim Erlernen der Zweitsprache gewesen sein könnten. Da jedoch die Kategorie Erwachsenenbildung auch Probanden umfaßte, die in bezug auf das Alter mit den Teilnehmern der höheren Klassen der weiterführenden Schulen nahezu übereinstimmten, betrafen die starken Altersdifferenzen nur einen Teil der Lernenden des Bereichs Erwachsenenbildung. Biologische Faktoren bei der Fehlerentstehung kamen hier somit nur partiell zum Tragen.

Die Untersuchung des Faktors Geschlecht zeigte folgendes: Die Auswertung der Teilnehmer, die der Schulform Hauptschule zuzuordnen waren, ergab, daß die Verteilung männlicher und weiblicher Teilnehmer in den jüngeren Klassen gleichgewichtet war. Gleiches gilt auch für die Teilnehmer des sogenannten Erweiterungskurses, d. h. der Kursform, die den Schülern die Möglichkeit des Realschulabschlusses bietet. Innerhalb des Grundkurses der Jahrgangsstufe 10 hingegen war der Anteil männlicher Schüler deutlich größer als der Anteil weiblicher Teilnehmer.

Bei den Teilnehmern der Schulform Realschule waren in allen Jahrgangsstufen deutliche Differenzen zu erkennen. Besonders stark traten die Differenzen in den 10er-Klassen auf, also in der Jahrgangsstufe, in der die Schüler einen fachlichen Schwerpunkt setzen. In der Klasse der sprachlich orientierten Schüler war der Anteil weiblicher Teilnehmer weitaus größer als der männlicher. Dieses Ergebnis ist möglicherweise darauf zurückzuführen, daß weibliche Schüler oft eine größere Neigung zum Erlernen einer Fremdsprache zeigen als männliche. ${ }^{301}$ Bestätigt wird diese Vermutung insbesondere durch die Tatsache, daß die Klasse der naturwissenschaftlich orientieren Teilnehmer der Schulform Realschule ausschließlich aus männlichen Probanden bestand. Auch bei den sozialwissenschaftlich orientierten Schülern überwog der Anteil der männlichen Teilnehmer deutlich. Allerdings ist zu vermuten, daß dieses Ergebnis eher zufällig zustandegekommen ist, da aus keiner der Studien, die zur Vorbereitung des Fragebogens herangezogen wurden, hervorgeht, daß das sozialwissenschaftliche Interesse bei männlichen Teilnehmern stärker ausgeprägt ist als bei weiblichen. In den unteren

\footnotetext{
300 vgl. Vogel 1990, $122-132$

301 Diese Aussage beruht auf Erfahrungen, die der Autor der vorliegenden Arbeit im Rahmen seiner unterrichtlichen Tätigkeit sammeln konnte.
} 
Jahrgangsstufen der Schulform Realschule überwog der Anteil weiblicher Schüler um $14 \%$, wobei auch hier von einer Zufallsverteilung auszugehen ist.

In der Schulform Gymnasium überwog in allen Jahrgangsstufen und allen Kurstypen der Anteil männlicher Schüler deutlich. Es muß jedoch davon ausgegangen werden, daß dieses Ergebnis darauf zurückzuführen ist, daß die Schule, die die Teilnehmer besuchten, ehemals ausschließlich männliche Schüler unterrichtete und die Schulleitung erst in den letzten Jahren vor Durchführung der Untersuchung damit begonnen hatte, auch weibliche Teilnehmer aufzunehmen.

Bei den Teilnehmern des Bereichs Erwachsenenbildung war die geschlechtliche Verteilung wiederum relativ ausgewogen. Nur bei den Probanden, die aufgrund ihrer überdurchschnittlichen Englischkenntnisse als advanced learners bezeichnet wurden, waren Differenzen zu erkennen. In dieser Gruppe überwog der Anteil weiblicher Probanden deutlich. Dieses Ergebnis scheint einerseits die schon bei den Realschülern geäußerte Vermutung zu bestätigen, daß weibliche Schüler stärker am Erlernen von Fremdsprachen interessiert sind als männliche. Andererseits zeigt jedoch die Tatsache, daß die betreffende Gruppe dennoch zu 40 \% aus männlichen Probanden bestand, daß das Geschlecht der Teilnehmer offenbar nur zum Teil für die Entwicklung einer sprachlichen Neigung verantwortlich war.

In bezug auf die Anzahl der Unterrichtsjahre, die die Teilnehmer im Fach Englisch absolviert hatten, wurden, wie bereits das Alter der Probanden nahelegt, deutliche Unterschiede ermittelt. In den verschiedenen Jahrgangsstufen der weiterführenden Schulen lag die Anzahl der Unterrichtsjahre abgesehen von der gymnasialen Oberstufe - zwischen zwei und sieben Jahren. In der gymnasialen Oberstufe war die Anzahl der Unterrichtsjahre höher. Die Angaben, die bei diesen Probanden verzeichnet waren, ließen erkennen, daß die Probanden dieser Gruppen Englisch bereits seit mehr als sieben Jahren, d. h. seit bis zu neun Jahren erlernt hatten. Die höchste Anzahl der Unterrichtsjahre war bei den Probanden des Bereichs Erwachsenenbildung verzeichnet. Dort reichten die Angaben partiell bis zu zehn Unterrichtsjahren. Fehlertypologische Differenzen, die beim Vergleich der einzelnen Probandengruppen und Jahrgangsstufen deutlich wurden, sind demnach auch darauf zurückzuführen, daß die Probanden, die Englisch erst wenige Jahre erlernt hatten, über weniger sprachliche Informationen verfügten als die Probanden, die Englisch seit sechs oder mehr Jahren erlernt hatten.

In bezug auf den Faktor Unterbrechungen, die während des Erwerbs der englischen Sprache auftraten, waren in den Jahrgangsstufen der weiterführenden Schulen keine längeren, d. h. mehr als sechs Monate andauernde Perioden belegt. Langfristige Unterbrechungen von bis zu 16 Jahren waren lediglich bei den Teilnehmern des Bereichs Erwachsenenbildung verzeichnet. Bei der Mehrzahl dieser Probanden war der Zeitraum jedoch kürzer: Die meisten Teilnehmer des Bereichs Erwachsenenbildung gaben einen Unterbrechungszeitraum von bis zu fünf Jahren an. Vermutlich sind die meisten Unter- 
brechungen dadurch begründet, daß viele Teilnehmer nach ihrem Schulabschluß nicht oder nur unregelmäßig in Englisch unterrichtet worden waren und erst zu einem späteren Zeitpunkt wieder Englischunterricht nahmen, etwa weil der Beruf eine Auffrischung oder Fortsetzung der Englischkenntnisse erforderte.

Außerschulische Erfahrungen mit der englischen Sprache waren - abgesehen von den Teilnehmern des Grundkurses der Schulform Hauptschule - in allen Probandengruppen vermerkt. Die geringsten außerschulischen Erfahrungen ließen die Jahrgangsstufen 7 - 9 der Schulform Hauptschule erkennen. Besonders hoch war der Anteil dieser Erfahrungen bei den Gymnasiasten. Vermutlich basiert dieses Ergebnis darauf, daß die Teilnehmer der Schulform Hauptschule in der Regel Kontakte zu Muttersprachlern anderer Zweitsprachen, wie etwa dem Türkischen oder Sprachen slawischer Herkunft hatten. Da das Erlernen der englischen Sprache an dieser Schulform vornehmlich pragmatischen Zwecken, d. h. der Kommunikationsfähigkeit im Berufsleben dient und weniger darauf ausgerichtet ist, im Studium oder in akademischen Berufen zur Anwendung zu kommen, besteht bei Hauptschülern vermutlich weniger Bedarf, außerschulische Englischerfahrungen zu sammeln als bei Schülern anderer Schulformen. Der Englischunterricht an Gymnasien hingegen ist so konzipiert, daß die dort gewonnenen Kenntnisse nach Abschluß der Mittelstufe sowohl in der gymnasialen Oberstufe Verwendung finden können als auch in akademischen Berufen oder in Berufen, die einen Umgang mit geschäftsorientiertem Englisch erfordern. ${ }^{302}$ Aus diesem Grund erhalten die meisten Schüler der Schulform Gymnasium bereits in der Mittelstufe auf Klassenfahrten oder in Arbeitsgruppen Gelegenheit, Kontakte zu englischsprachigen Personen zu knüpfen. ${ }^{303}$

Auffällig ist, daß die sprachlich orientierten Schüler der Schulform Realschule den höchsten Anteil außerschulischer Englischerfahrungen innerhalb der Gruppe der Realschüler verzeichneten. Dies zeigt, daß die Motivation der Schüler, Englisch zu lernen, vermutlich nicht nur auf den schulischen Bereich beschränkt war, sondern daß sich private mit schulischen Interessen verbanden. Bei den Probanden des Bereichs Erwachsenenbildung waren die meisten außerschulischen Erfahrungen mit der englischen Sprache bei den Teilnehmern der Gruppe beginners verzeichnet und nicht in den fortgeschritteneren Kursen. Offenbar führt der außerschulische Kontakt mit der Zielsprache allein nicht immer automatisch zu einer Steigerung der Sprachkenntnisse. Wichtiger scheinen vielmehr die Art der außerschulischen Erfahrungen, die die Lernenden gesammelt haben, und die Intensität dieser Erfahrungen zu sein.

\footnotetext{
302 vgl. Kultusminister des Landes Nordrhein-Westfalen (Hrsg.) 1993

${ }^{303}$ Dies wurde während der Referendariatszeit des Autors dieser Arbeit deutlich.
} 
In den unteren Klassen der weiterführenden Schulen wurde von den meisten Probanden entweder nicht konkret angegeben, in welcher Form außerschulische Erfahrungen mit der englischen Sprache gesammelt worden waren, oder die Erfahrungen beschränkten sich auf Kontakte zu anderen Schülern, die Englisch als Zweitsprache erwarben. Auch bei den Teilnehmern des Bereichs Erwachsenenbildung beschränkten sich die Erfahrungen bei der Mehrheit der Teilnehmer auf Kontakte zu anderen Lernenden, die Englisch als Zweitsprache erwarben, wenngleich hier teilweise angegeben wurde, daß diese Erfahrungen bei längeren Auslandsaufenthalten in englischsprachigen Ländern gesammelt worden waren.

In den höheren Jahrgangsstufen der verschiedenen Schulformen gingen die Erfahrungen - abgesehen von den Teilnehmern der Hauptschule - über Kontakte mit anderen Englischlernenden hinaus. Die meisten Teilnehmer, die außerschulische Erfahrungen gesammelt hatten, gaben an, auch durch Auslandsaufenthalte Kontakte zur englischen Sprache geknüpft zu haben. Dies traf auch auf den Bereich Erwachsenenbildung zu. Besonders hoch war der Anteil der Schüler, die längere Zeit in englischsprachigen Ländern verbracht hatten, im Leistungskurs der Schulform Gymnasium. Auch hier zeigte sich, daß die Motivation, Englisch zu lernen, die bereits durch die Belegung des Leistungskurses im Fach Englisch deutlich wurde, nicht nur schulisch bedingt war, sondern über den Bereich Schule hinausging. Dabei war zu erkennen, daß diese Motivation auch zu einer Fehlerreduzierung beitragen kann, da der Leistungskurs zu den Probandengruppen zählte, die durch eine äußerst geringe Fehleranzahl innerhalb eines linguistischen Bereichs auffielen (vgl. Tabelle 1 im Anhang).

Die Angaben zur Schulform, an der Englisch erworben wurde, gingen weitestgehend mit dem Schultyp einher, an dem die Untersuchung durchgeführt wurde. Alle Probanden der Schulform Hauptschule und Realschule gaben an, Englisch an diesen Schultypen erworben zu haben. Gleiches galt für die Probanden, die der Schulform Gymnasium zuzuordnen waren, wenngleich ein Teil der Teilnehmer, die zur Oberstufe gehörten, zu erkennen gab, daß die Sprache daneben auch an einer Hauptoder Realschule erworben worden war. Dies liegt darin begründet, daß diese Probanden bis zum Abschluß der Fachoberschulreife eine Haupt- oder Realschule besucht hatten und nachfolgend den Entschluß gefaßt hatten, darüber hinaus an einem Gymnasium das Abitur zu absolvieren.

Bei den Probanden des Bereichs Erwachsenenbildung war die Schulform, an der Englisch erworben wurde, sowohl bei den Anfängern als auch bei den fortgeschritteneren Probandengruppen gemischt. In allen Gruppen waren sowohl Haupt- und Realschule als auch die Schulform Gymnasium verzeichnet. Daneben wurden auch Schulformen angegeben, die nicht zu den zuvor genannten gehören, jedoch nicht näher definiert waren. Insgesamt war jedoch zu erkennen, daß die meisten Probanden, die der Gruppe beginners zugeordnet wurden, Englisch an der Schulform Hauptschule erworben hatten. In der Gruppe der Probanden, die als intermediate oder advanced zu bezeichnen waren, war Englisch 
zumeist an Gymnasien erlernt worden. Dabei zeigt der Vergleich der einzelnen Gruppen jedoch, daß bei den Teilnehmern der Gruppe intermediate die Schulform Gymnasium nur etwa von der Hälfte der Lernenden angegeben wurde, während bei den fortgeschrittenen Probanden der prozentuale Anteil derjenigen, die Englisch am Gymnasium erworben hatten, bei 83 \% lag. Da die sprachlichen Anforderungen an Gymnasien aufgrund der akademischen Ausrichtung zum Teil höher sind als an Real- und Hauptschulen, ${ }^{304}$ ist davon auszugehen, daß die Schulform, an der die Zweitsprache erworben wurde, einen entscheidenden Einfluß auf die Sprachkenntnisse der Lernenden hat. Dies wird vor allem durch die Tatsache bestätigt, daß die Gruppe der fortgeschrittenen Lernenden, die - wie erwähnt - angaben, Englisch in erster Linie am Gymnasium erworben zu haben, gleichzeitig die Gruppe der Probanden darstellte, die die wenigsten lexikalischen und syntaktisch-morphologischen Fehler produzierte.

Über einen Schulabschluß bzw. weitere Abschlüsse verfügten nur die Teilnehmer der gymnasialen Oberstufe und die Teilnehmer des Bereichs Erwachsenenbildung. Dabei wurde innerhalb der Gruppen der Gymnasiasten ausschließlich der Abschluß Fachoberschulreife angegeben. In den Gruppen des Bereichs Erwachsenenbildung zählten neben der Fachoberschulreife auch der Haupt- oder Volksschulabschluß und das Abitur bzw. Fachabitur zu den erwähnten Qualifikationen. Auch hier fällt auf, daß innerhalb der Gruppe der beginners der Anteil derjenigen, die über einen Haupt- oder Volksschulabschluß verfügten, überwog und nur ein geringer Teil angab, das Abitur absolviert zu haben. Bei den Teilnehmern hingegen, die als intermediate bezeichnet wurden, hatten 46,9\% das Abitur abgelegt. Bei den fortgeschrittenen Lernenden lag der Anteil der Abiturabsolventen bei 76,7\%. Bei den Probandengruppen intermediate und advanced war der Anteil der Teilnehmer, die über einen Haupt- oder Volksschulabschluß verfügten, gering. Dieses Ergebnis bekräftigt nochmals die bereits zuvor geäußerte Schlußfolgerung, daß die Schulform bzw. die damit verbundenen Abschlüsse die Sprachkenntnisse der Lernenden und die Produktion der Fehler beeinflussen. Je höher die schulischen Anforderungen im Fremdsprachenunterricht sind, desto stärker kommt es zu einer Minderung sprachlicher Abweichungen. ${ }^{305}$

In bezug auf die Einstellung zur englischen Sprache überwogen in nahezu allen Probandengruppen und in allen Jahrgangsstufen positive Aussagen. Ausgenommen von diesem Ergebnis war die Gruppe der naturwissenschaftlich orientierten Realschüler. In dieser Gruppe war deutlich zu erkennen, daß die Mehrheit der Teilnehmer der englischen Sprache gegenüber negativ eingestellt war. Offenbar ging die Tatsache, daß die Schüler dieser Gruppe die Naturwissenschaften bevorzugten, nicht nur mit einer gleichgültigen Einstellung gegenüber Fremdsprachen einher, sondern sogar mit einer ausgesprochenen Abneigung gegen das Erlernen von Fremdsprachen. Dies mag u. a. darauf beruhen, daß die schu-

\footnotetext{
304 vgl. Kultusminister des Landes Nordrhein-Westfalen (Hrsg.) 1989; Kultusminister des Landes Nordrhein-Westfalen (Hrsg.) 1994

305 vgl. Hecht / Green 1983, 49 - 56
} 
lischen Leistungen in den Fremdsprachen nicht den Erwartungen der Schüler und der Lehrenden entsprachen. Auffällig war auch, daß in den Gruppen, in denen die Probanden aus persönlicher Neigung Englisch als Schwerpunktfach gewählt hatten, die Einstellung zur englischen Sprache äußerst positiv war. Zu diesen Gruppen gehörten insbesondere die Gruppe der sprachlich orientierten Realschüler, der Leistungskurs des Schultyps Gymnasium und die Gruppe des Bereichs Erwachsenenbildung, die aufgrund ihrer Leistungen als advanced bezeichnet wurde. Die Tatsache, daß diese Gruppen auch durch geringe Fehlerzahlen auffielen - wenn auch die sprachlich orientierten Realschüler nur bedingt -, weist gleichzeitig darauf hin, daß die positive Einstellung zu einer Sprache offenbar den Lerneffekt erhöhen kann. ${ }^{306}$

Als Grund für das Erlernen der englischen Sprache wurden bei allen Schulformen der weiterführenden Schulen und in allen Jahrgangsstufen zunächst schulische Motive erwähnt. In den höheren Klassen der Schulformen Haupt- und Realschule gab ein Teil der Probanden an, Englisch u. a. auch aus privaten Gründen erlernt zu haben. In der Gruppe der Gymnasiasten wurden private Gründe auch bereits in den unteren Jahrgangsstufen erwähnt. Besonders ausgeprägt war innerhalb der Jahrgangsstufen der weiterführenden Schulen das private Interesse in der Klasse der sprachlich orientierten Realschüler. Auch hier ist zu erkennen, daß die Motivation, eine Fremdsprache zu erlernen, meist aus einer Kombination extrinsischer und intrinsischer Aspekte besteht. In den Gruppen des Bereichs Erwachsenenbildung waren die Gründe für das Erlernen der englischen Sprache vorwiegend extrinsischer Natur. Neben dem schulischen Aspekt wurde hier auch der Faktor Beruf benannt. Daneben wurden jedoch in allen Probandengruppen der Erwachsenenbildung partiell auch private Motive verzeichnet.

Als Herkunftsland des Lehrers wurde in den Jahrgangsstufen der weiterführenden Schulen nahezu ausschließlich Deutschland angegeben. Nur im Leistungskurs der Schulform Gymnasium gab ein geringer Probandenteil zu erkennen, daß die Betreffenden auch von englischen Muttersprachlern unterrichtet worden waren. Auch in den Gruppen des Bereichs Erwachsenenbildung wurden im Englischunterricht vorwiegend deutsche Muttersprachler eingesetzt. Allerdings war hier in allen Gruppen zu erkennen, daß zumindest ein Teil der Schüler auch von englischen Muttersprachlern unterrichtet wurde. Dabei fällt beim Vergleich der Probandengruppen auf, daß die als beginners bezeichneten Gruppen prozentual am wenigsten von englischen Muttersprachlern unterrichtet wurden und die fortgeschrittenen Lernenden den höchsten prozentualen Anteil verzeichneten. Dies legt nahe, daß der Einsatz englischer Muttersprachler im Englischunterricht offenbar lernfördernde Auswirkungen hat und zu einer Verbesserung der Sprachkenntnisse führt. Letzteres beruht vermutlich darauf, daß englische Muttersprachler nicht nur die Sprache als linguistisches Konstrukt, sondern

306 vgl. Vogel 1990, $143-148$ 
gleichzeitig kulturelle Aspekte ihrer Heimat vermitteln. ${ }^{307}$ Hierdurch wirkt der Unterricht für deutsche Muttersprachler authentischer und die zweitsprachlichen Informationen, die an die Lernenden herangetragen werden, können teilweise besser verarbeitet werden als in einem Englischunterricht, dem diese kulturellen Aspekte fehlen. ${ }^{308}$

Die erlernte Form des Englischen war in allen Probandengruppen und Jahrgangsstufen vorwiegend das Britische Englisch. In den Probandengruppen der Hauptschule stellte diese Sprachform die einzige Variante dar, die die Probanden im Unterricht erlernt hatten. Dieses Ergebnis beruht darauf, daß Hauptschüler nahezu ausschließlich Britisches Englisch erlernen, um auf diese Weise Differenzierungsprobleme zwischen den unterschiedlichen Formen der englischen Sprache zu vermeiden. ${ }^{309}$ In den höheren Klassenstufen der Probandengruppen der Realschule gab ein Teil der Schüler an, auch Amerikanisches Englisch erlernt zu haben. Besonders stark vertreten war diese Aussage in der Gruppe der sprachlich orientierten Realschüler. Auch diese Informationen legen nahe, daß das Interesse der Teilnehmer an der englischen Sprache über schulische Motive hinausging, weil an deutschen Realschulen vorwiegend Britisches Englisch vermittelt wird. ${ }^{310}$ Zum Erlernen des Amerikanischen Englisch müssen Schüler der Realschule entweder Zeit in den USA verbringen, private Kontakte zu US-Amerikanern in Deutschland haben oder aber Englisch bei amerikanischen Lehrern an anderen Schulformen erlernt haben. ${ }^{311}$ Die Mehrzahl dieser Faktoren kann nur in der Freizeit der betreffenden Probanden realisiert worden sein und ist daher nicht auf schulische Verpflichtungen zurückzuführen. Das Interesse dieser Teilnehmer, Englisch zu lernen, war somit sowohl linguistisch als auch kulturell begründet, da durch den Umgang mit Muttersprachlern nicht nur die Sprache, sondern auch kulturelle Aspekte des betreffenden Landes vermittelt werden.

Auch in den Gruppen der Schulform Gymnasium stand - wie erwähnt - das Britische Englisch im Vordergrund. Nur ein geringer Anteil der Probanden ließ erkennen, daß daneben auch Amerikanisches Englisch erlernt worden war; am höchsten war der Anteil im Leistungskurs. Dieses Ergebnis beruht darauf, daß ein Teil der Schüler dieses Kurses entweder die USA bereist hatte und dort Kontakte mit US-Amerikanern knüpfen konnte oder als Austauschschüler ein Jahr in den USA verbracht hatte. ${ }^{312}$ Bei den Probanden des Bereichs Erwachsenenbildung waren in allen Gruppen beide Formen der englischen Sprache vertreten. Den höchsten Anteil ließen dabei die als intermediate und advanced bezeichneten Teilnehmer dieser Probandengruppen erkennen. Es ist davon auszugehen, daß dieses

\footnotetext{
307 Dies zeigte sich vor allem in den sprachpraktischen Übungen des Anglistikstudiums des Autors der vorliegenden Arbeit. 308 dto.

309 Dies wurde bei der Durchführung der Untersuchung in den Hauptschulen deutlich.

310 Dies ist insbesondere durch die Lehrwerke belegt, die an Realschulen benutzt werden.

311 Diese Aussage geht auf Erfahrungen während des Referendariats des Autors dieser Arbeit zurück.

312 Dies wurde in den Gesprächen mit den Teilnehmern bei der Vorbereitung des Untersuchungsverfahrens deutlich.
} 
Ergebnis darauf beruht, daß ein großer Anteil der Anfängergruppe Englisch an Volks- oder Hauptschulen erlernt hatte. Wie erwähnt, wurde bzw. wird an diesen Schultypen nahezu ausschließlich Britisches Englisch unterrichtet. ${ }^{313}$ In den anderen Kursen hingegen hatten viele Probanden Englisch an Realschulen oder an Gymnasien erlernt, und dort besteht die Möglichkeit - wie die Ergebnisse zeigten - neben der britischen Form auch das Amerikanische Englisch zu erlernen. Außerdem konnte insbesondere ein Teil der Probanden des fortgeschrittenen Kurses im Rahmen ihres Studiums mit dem sogenannten Business English auch Amerikanisches Englisch erlernen.

Das Erlernen anderer Fremdsprachen neben der englischen Sprache war bei allen Probandengruppen und in allen Jahrgangsstufen verzeichnet. Dabei ließen aus den Probandengruppen der Schulform Hauptschule die wenigsten Teilnehmer Kenntnisse in anderen Fremdsprachen erkennen. Relativ gering war dieser Anteil auch in der Gruppe der sozialwissenschaftlich und naturwissenschaftlich orientierten Realschüler. Hier zeigte sich, daß die Schwerpunktverlagerung auf den Bereich Sozialund Naturwissenschaften nicht nur Auswirkungen auf das Erlernen der englischen Sprache hat, sondern auf den gesamten Bereich der Fremdsprachen. Wenngleich auch an Realschulen das Erlernen einer zweiten Fremdsprache obligatorisch ist, ${ }^{314}$ wird den Schülern die Gelegenheit gegeben, Fremdsprachen mit Beginn der 10. Klasse wieder abzuwählen. ${ }^{315}$ Offenbar gaben nicht alle Teilnehmer an, weitere Fremdsprachen erworben zu haben, weil sie davon ausgingen, daß aufgrund der wenigen Jahre, die sie in einer anderen Fremdsprache unterrichtet worden waren, noch kein Spracherwerb erfolgt war. Den höchsten Anteil weiterer Zweitsprachen zeigte die Gruppe der Gymnasiasten. In dem Leistungskurs dieser Schulform war das Erlernen anderer Fremdsprachen bei jedem Probanden verzeichnet, und auch in den anderen Klassen lag der Anteil bei über $80 \%$. Dieses Ergebnis liegt vor allem darin begründet, daß in Deutschland die Schüler aller Gymnasien mit Beginn der 7. Klasse eine zweite Fremdsprache erlernen müssen. ${ }^{316}$

In der Gruppe der beginners des Bereichs Erwachsenenbildung lag der prozentuale Anteil, der angab, neben Englisch eine andere Fremdsprache erworben zu haben, nur bei $49 \%$. Bei den Probanden der Gruppe intermediate war dieser Anteil nur um 10 \% höher. Dieses Ergebnis basiert darauf, daß viele Probanden dieser Gruppen nicht das Gymnasium besucht hatten, sondern andere Schulformen wie etwa Hauptschulen oder die sogenannte Volksschule. An diesen Schulformen ist bzw. war das Erlernen einer zweiten Fremdsprache nicht obligatorisch, so daß sich zum Teil die Fremdsprachenkenntnisse

313 An den sogenannten Volksschulen, die später durch andere Schultypen ersetzt wurden, war das Erlernen des Britischen Englisch historisch und geographisch bedingt. Aufgrund der Nähe Englands zu Deutschland und der guten politischen Beziehungen, die vor dem 2. Weltkrieg zwischen den beiden Ländern herrschten, bevorzugten deutsche Bildungseinrichtungen bis in die sechziger Jahre des 20. Jahrhunderts traditionell das Britische Englisch. Dies hat bis heute zur Folge, daß das Amerikanische Englisch an den Hauptschulen wenig vertreten ist. Vgl. hierzu Weiher 1982, $15 \mathrm{f}$.

314 vgl. Kultusminister des Landes Nordrhein-Westfalen (Hrsg.) 1994, 28

315 vgl. ebd., $28 \mathrm{f}$

316 vgl. Kultusminister des Landes Nordrhein-Westfalen (Hrsg.) 1993, 36 
der Probanden auf die englische Sprache beschränkten. Bei den fortgeschrittenen Probanden des Bereichs Erwachsenenbildung war - ähnlich wie bei den Gymnasiasten - der prozentuale Anteil derjenigen, die neben Englisch eine weitere Fremdsprache erlernt hatten, wiederum äußerst hoch. Ausschlaggebend für dieses Ergebnis ist vor allem die Tatsache, daß die Mehrheit dieser Lernenden Gymnasien besucht hatte und daher - wie erwähnt - obligatorisch weitere Fremdsprachen zu erlernen hatte.

Die Art der zusätzlichen Fremdsprachen wurde bei den meisten Probanden der Schulform Hauptschule nicht konkret angegeben. Es war jedoch angesichts der Informationen, die aus den Briefen hervorgingen, zu erkennen, daß die Mehrzahl der Teilnehmer eine weitere Fremdsprache - wie Türkisch oder eine slawische Sprache - spricht. Vereinzelt wurden auch romanische Sprachen - wie Spanisch oder Italienisch - angegeben. Bei den Teilnehmern der Schulform Realschule zählte in erster Linie Französisch zu den anderen Fremdsprachen. Auch dies liegt in der Struktur dieser Schulform begründet. Traditionell haben Realschüler die Möglichkeit, neben Englisch auch Französisch zu erwerben. Erst seit wenigen Jahren wird den Schülern die Gelegenheit geboten, auch andere Fremdsprachen - wie etwa Latein oder Spanisch - zu erlernen. ${ }^{317}$ Bei den Gymnasiasten umfaßte die Art der zusätzlichen Fremdsprachen Latein oder Französisch, und bei allen Probanden dieser Schulform wurden auch sonstige Fremdsprachen verzeichnet. Hierzu zählten die unterschiedlichsten romanischen Sprachen, slawische Sprachen und germanische Sprachen wie Holländisch, Dänisch oder Schwedisch. Dies geht auf strukturelle Aspekte der Schulform Gymnasium zurück. An den meisten Gymnasien erhalten die Schüler die Möglichkeit, in Form sogenannter Arbeitsgemeinschaften neben den Standardsprachen Latein und Französisch Sprachen der obengenannten Gruppen zu lernen. ${ }^{318}$

Auch bei den Probanden des Bereichs Erwachsenenbildung wurden in allen Gruppen als zusätzliche Fremdsprachen in erster Linie Latein und Französisch genannt. Dieses Ergebnis beruht - wie bereits erwähnt - darauf, daß die Mehrheit dieser Probanden Realschulen und Gymnasien besucht hatte. Die Tatsache, daß diese Sprachen auch in der Gruppe der Anfänger erwähnt wurden, obwohl diese Probanden vornehmlich Haupt- und Volksschulen besucht hatten, d. h. Schulformen, an denen diese Sprachen nicht oder nur zum Teil unterrichtet werden, beruht vermutlich darauf, daß die Probanden Weiterbildungseinrichtungen wie Volkshochschulen besucht hatten, weil sie diese weiteren Sprachkenntnisse aus beruflichen oder privaten Gründen erwerben wollten.

In bezug auf die Einschätzung der Sprachkenntnisse in dieser/diesen Fremdsprachen fiel auf, daß nur bei den Teilnehmern der Schulform Hauptschule positive Beurteilungen überwogen. In den

\footnotetext{
317 Diese Aussage geht auf Erfahrungen des Autors dieser Arbeit während seiner Lehrtätigkeit zurück. 318 dto.
} 
Gruppen der Jahrgangsstufen 7 - 9 gaben alle Probanden an, die zusätzliche Sprache oder Sprachen, die neben Englisch erworben wurde/n, sehr gut bis gut $(1-2)$ zu sprechen. Auch in dem Kurs der Teilnehmer, die die Fachoberschulreife anstrebten, wurden ihre Sprachkenntnisse von allen Probanden als gut bis befriedigend $(2-3)$ beurteilt. Negative Einschätzungen waren nur bei den Teilnehmern verzeichnet, die die Schule mit dem Hauptschulabschluß verlassen. Jedoch lag auch hier der Anteil der Probanden, die die Sprachkenntnisse als sehr gut - gut $(1-2)$ bezeichneten, bei $44 \%$. Die Teilnehmer der anderen Schulformen hingegen gaben mehrheitlich an, daß ihre Kenntnisse in der oder den betreffenden Sprachen als befriedigend bis ausreichend ( $3-4)$ zu bewerten seien. Besonders gering war der Anteil positiver Einschätzungen bei den Teilnehmern der Schulform Realschule mit sozial- oder naturwissenschaftlichem Schwerpunkt und bei den Teilnehmern des Grundkurses der Schulform Gymnasium.

Auch bei der eigenen Einschätzung der englischen Sprachkenntnisse wurde ermittelt, daß die Probanden mehrheitlich ihre Fähigkeiten als befriedigend bis ausreichend ( $3-4)$ bezeichneten. Eine Ausnahme bildet hierbei lediglich die Gruppe der Jahrgangsstufen 7 - 9 der Schulform Hauptschule. In dieser Gruppe bezeichneten $70 \%$ der Teilnehmer ihre Englischkenntnisse als sehr gut bis gut $(1-2)$. In den anderen Teilnehmergruppen hingegen überwogen eindeutig die negativen Beurteilungen. Besonders hoch war der Anteil äußerst negativer Einschätzungen bei den naturwissenschaftlich orientierten Lernenden der Schulform Realschule und bei den Teilnehmern der Gruppen beginners und intermediate learners des Bereichs Erwachsenenbildung.

Die Auswertung der Ergebnisse bezüglich der Einschätzung des physischen und psychischen Zustandes zeigte, daß die Einschätzung der Probanden der höheren Jahrgangsstufen positiver war als die der jüngeren Lernenden. Nur bei den Teilnehmern des Grundkurses der Schulform Hauptschule der Gruppen der Jahrgangsstufen 7 - 9 dieser Schulform und im Grundkurs der Schulform Gymnasium waren andere Ergebnisse zu beobachten. In diesem Grundkurs gaben 58 \% der Teilnehmer an, sich während des Testverfahrens in einem angespannten und nervösen Zustand befunden zu haben. In der Gruppe der Jahrgangsstufen 7 - 9 war der Anteil der Teilnehmer, die laut eigener Angaben während des Testverfahrens entspannt und ausgeruht waren, ebenso hoch wie der Anteil der Teilnehmer, die während des Testverfahrens angespannt und nervös waren. Im Grundkurs der Schulform Gymnasium lag der Anteil der Teilnehmer, die angaben, bei der Durchführung des Testverfahrens entspannt und ausgeruht gewesen zu sein, bei $25 \%$, während $48 \%$ der Teilnehmer angaben, daß sie während des Testverfahrens angespannt und nervös gewesen waren. Bei den Teilnehmern des Bereichs Erwachsenenbildung zeigte sich, daß die Einschätzung des eigenen physischen und psychischen Zustands in der Gruppe der intermediate learners und bei den fortgeschrittenen Lernenden positiver war als in der Gruppe der Anfänger. 
In bezug auf die Frage, ob vor dem Test Medikamente, Alkohol oder Drogen eingenommen worden waren, wurde in den höheren Jahrgangsstufen die Einnahme der obengenannten Substanzen häufiger angegeben als in den unteren Jahrgangsstufen. Besonders oft wurde die Frage in der Gruppe der naturwissenschaftlich orientierten Realschüler und in den Gruppen beginners und intermediate learners des Bereichs Erwachsenenbildung bejaht.

Bei der Auswertung der Angaben, die in bezug auf körperlich-seelische Beschwerden gemacht wurden, war zu erkennen, daß der Anteil der Probanden, der angab, während des Testverfahrens unter körperlichen und seelischen Beschwerden gelitten zu haben, bei den Teilnehmern der Schulform Hauptschule gering war. In dieser Schulform war der höchste Anteil in den Jahrgangsstufen 7 - 9 zu verzeichnen. In den Gruppen der Schulform Realschule und bei den Teilnehmern des Bereichs Erwachsenenbildung hingegen gab in allen Gruppen mindestens ein Viertel der Teilnehmer an, von körperlich-seelischen Beschwerden betroffen gewesen zu sein. Auch bei den Teilnehmern der Schulform Gymnasium sind die bejahenden Angaben zu diesem Aspekt insgesamt als hoch zu bezeichnen. Auffallend war jedoch, daß der Anteil in den höheren Jahrgangsstufen geringer war als in den unteren. Der geringste Anteil von Probanden mit körperlich-seelischen Beschwerden wurde im Leistungskurs der Jahrgangsstufe 12 verzeichnet.

Die Verwendung von Hilfsmitteln konnte in keiner Schulform bzw. in keiner Probandengruppe nachgewiesen werden. Dieses Ergebnis ist darauf zurückzuführen, daß die Probanden während der Durchführung des Testverfahrens betreut wurden und ihnen vor Beginn des Testverfahrens mitgeteilt wurde, daß die Briefe ohne Hilfsmittel verfaßt werden sollten. 


\section{Die Zweitsprachenerwerbshypothesen und Fehlerentstehungsfaktoren}

Im folgenden wird ein forschungsgeschichtlicher Überblick über die Entwicklung der Zweitspracherwerbshypothesen seit Beginn der modernen Sprachwissenschaft und über die ihnen zugrundeliegenden Aspekte zur Fehlergenese gegeben. Dabei soll insbesondere hervorgehoben werden, welche Positionen die Vertreter der einzelnen Hypothesen gegenüber sprachlichen Fehlern einnahmen.

\section{Die Kontrastivhypothese}

\subsection{Entstehung und Inhalte}

Die Inhalte der Kontrastivhypothese basieren auf dem Grundgedanken der behavioristischen Lerntheorien, die Anfang des 20. Jahrhunderts in den USA entwickelt wurden. Im Zentrum dieser Lerntheorien steht die Annahme, daß Lernen nach dem sogenannten Reiz-Reaktions-Schema verläuft. ${ }^{319}$ Das Grundprinzip dieses Schemas ist die insbesondere von B. F. Skinner geprägte operante Konditionierung, derzufolge menschliches Verhalten steuerbar ist. ${ }^{320}$ Zur Durchführung dieser Steuerung bedarf es eines Verstärkers, der sowohl positiv als auch negativ sein kann. ${ }^{321}$ Positive Verstärker begünstigen das Auftreten einer bestimmten Reaktion, während negative Verstärker Reaktionen unterdrücken. ${ }^{322}$ Nach Skinner erhöhen positive Verstärker die Auftretenswahrscheinlichkeit einer Reaktion dann, wenn sie der Situation zugeführt werden. Negative Verstärker erhöhen die Auftretenswahrscheinlichkeit einer Reaktion, wenn sie der Situation entzogen werden. ${ }^{323}$

In bezug auf das Lernen bedeutet dies, daß alle Faktoren, die zu einer Lernerleichterung beitragen, als positive Verstärker zu betrachten sind. Dazu kann ein Lob des Lehrers gehören, aber auch die Erkenntnis, daß das Erlernen einer bestimmten Struktur zum Erfolg geführt hat, etwa zum Bestehen einer Prüfung beigetragen hat. Zu den negativen Verstärkern gehören alle Faktoren, die das Erlernen bestimmter Strukturen behindern. ${ }^{324}$ Dies könnte beispielsweise ein zu hoher Schwierigkeitsgrad des

\footnotetext{
319 vgl. Zimbardo / Gerrig 1999, $12-13$

320 vgl. Skinner 1966, $213-218$

321 vgl. Zimbardo / Gerrig 1999, $219 \mathrm{f}$

322 vgl. ebd.

323 vgl. Skinner 1966, $213 \mathrm{f}$

324 vgl. Zimbardo / Gerrig 1999, $219-225$
} 
Lernmaterials sein, mangelnde Bestätigung durch den Lehrer oder die Unüberschaubarkeit des Lernmaterials.

Da der Behaviorismus eine naturwissenschaftlich orientierte Richtung der Psychologie darstellt, ${ }^{325}$ beruht die Lernvorstellung der Behavioristen auf Ergebnissen, die empirisch gewonnen wurden. ${ }^{326}$ Die Betrachtungsweise dieser Forschungsrichtung ist dabei objektiv. Kognitive Steuerungen durch den Lernenden wurden bei der Konzeption der behavioristischen Lerntheorie nicht in Betracht gezogen. ${ }^{327}$ In bezug auf das Erlernen einer Zweitsprache wurde aus dieser Betrachtungsweise abgeleitet, daß der Erwerb einer Sprache als reines Input-/Output-Verhalten zu betrachten ist. ${ }^{328}$ Diesem Prinzip zufolge ist das Erlernen einer Sprache ebenso wie andere Lernvorgänge konditionierbar. ${ }^{329}$ Auf der Grundlage der permanenten Vermittlung sprachlicher Strukturen, etwa der Bildung der Fragesätze im Englischen, geht die Anwendung dieser Strukturen in gewohnheitsmäßiges Handeln über. Der Lernende produziert jedoch nur solche sprachlichen Elemente, die zuvor als Input an ihn herangetragen wurden. Sprachliche Elemente, die dem Lernenden bereits bekannt sind, erleichtern dabei den Konditionierungsprozeß, während neue sprachliche Elemente, die der Lernende nicht mit bereits Bekanntem vergleichen kann, den Konditionierungsprozeß behindern. ${ }^{330}$

Der Kerngedanke der Kontrastivhypothese ist daher die Auffassung, daß die Erstsprache des Lernenden den Erwerb einer Zweitsprache determiniert. ${ }^{331}$ Im Vordergrund steht dabei die Theorie, daß Elemente, die in Erst- und Zweitsprache ähnlich sind, leicht und fehlerfrei erlernt werden. Differierende Elemente und Regeln hingegen bereiten Lernschwierigkeiten und führen zu Fehlern. ${ }^{332}$

Konkret bedeutet dies, daß die Erstsprache die Verinnerlichung des zweitsprachlichen Systems beeinflußt, da die Anwendung der Erstsprache bereits als gewohnheitsmäßige Handlung bezeichnet werden kann. ${ }^{333}$ Den Inhalten der Kontrastivhypothese entsprechend überträgt der Lernende ständig seine erstsprachlichen Gewohnheiten in den zweitsprachlichen Erwerbsprozeß. ${ }^{334}$ Dazu gehört etwa bei deutschen Muttersprachlern, die Englisch als Zweitsprache erwerben, die Gewohnheit, Fragesätze nach bereits abgeschlossenen Ereignissen mit dem Hilfsverb have einzuleiten, obwohl die Frage mit dem Lexem did beginnen sollte. Ein Beispiel bietet folgende Äußerung: Have you seen me yester-

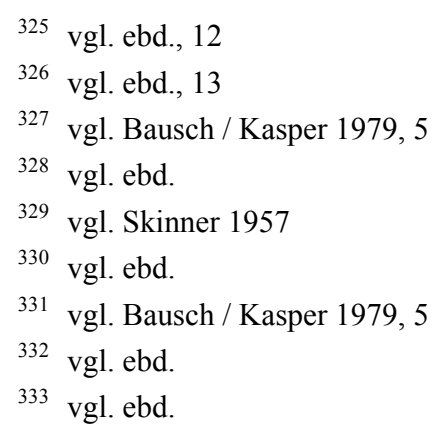


day? ${ }^{335}$ Der Kontrastivhypothese entsprechend sind solche Äußerungen dadurch zu erklären, daß es im Deutschen üblich ist, bei Fragesätzen in bezug auf vergangene Ereignisse das Perfekt zu verwenden, wohingegen im Englischen in solchen Fällen das Imperfekt benutzt wird. ${ }^{336}$ Da der deutsche Englischlernende bei Anwendung seiner Erstsprache erfahren hat, daß der Gebrauch des Imperfekts bei Fragesätzen im Deutschen veraltet ist und möglicherweise als arrogant betrachtet wird, wird er auch im Englischen dazu neigen, aufgrund dieser Konditionierung das Perfekt zu benutzen. Führt der Transferprozeß wie im vorigen Beispiel zu Fehlern, so wird der Vorgang in der Kontrastivhypothese als negativer Transfer bezeichnet. ${ }^{337}$ Die Lernbehinderung, die das erstsprachliche Verhalten dabei bewirkt, wird als proaktive Hemmung bezeichnet, die dabei entstehenden Abweichungen als Interferenzen. ${ }^{338}$ Führt der Transferprozeß hingegen zu einem sprachlichen Resultat, das mit den zweitsprachlichen Regeln und Normen übereinstimmt, so liegt positiver Transfer vor. ${ }^{339}$ Solch ein positiver Transfer liegt dieser Theorie entsprechend etwa dann vor, wenn deutsche Englischlernende den deutschen Satz „Sie wohnt in Köln“ in die sprachlich korrekte englische Form She lives in Cologne übersetzen und dies darauf zurückzuführen ist, daß die englische Zeitform und Satzstellung der deutschen Vorlage exakt entspricht. Der Interferenzbegriff, die Transferformen und die Bezeichnung, proaktive Hemmung' gehen auf Ergebnisse von Experimenten zurück, die Behavioristen mit Tieren durchgeführt haben. Sie sind daher nicht sprachlicher Natur. ${ }^{340}$

Auf der Basis der Annahme, daß Strukturidentität zwischen Erst- und Zweitsprache positiven Transfer bewirkt und eine Lernerleichterung darstellt, Strukturdifferenzen hingegen Fehler verursachen, glaubte man, interferenzfreie und interferenzanfällige Bereiche der Zweitsprache voraussagen zu können. ${ }^{341}$ Mit diesem Mittel versuchte man, das Verhalten der Lernenden vorherzubestimmen. Die Grundlage dieser Voraussagen bildeten sogenannte kontrastive Analysen, ${ }^{342}$ d. h. linguistische Vergleiche der beteiligten Sprachsysteme. Damit wurde die kontrastive Erwerbshypothese zu einer kontrastivanalytischen Hypothese erweitert. ${ }^{343}$ Diese erweiterte Fassung der Hypothese wurde fortan als ,starke Version“ bezeichnet. Kerngedanke ist hier die These, daß linguistische Unterschiede unweigerlich zu Schwierigkeiten beim Erlernen der Zweitsprache führen müßten. Insbesondere in den USA wurden auf der Grundlage dieser These sogenannte hierarchies of difficulty aufgestellt, d. h., es wurde beim Vergleich zweier Sprachsysteme festgelegt, welchen Schwierigkeitsgrad einzelne ziel-

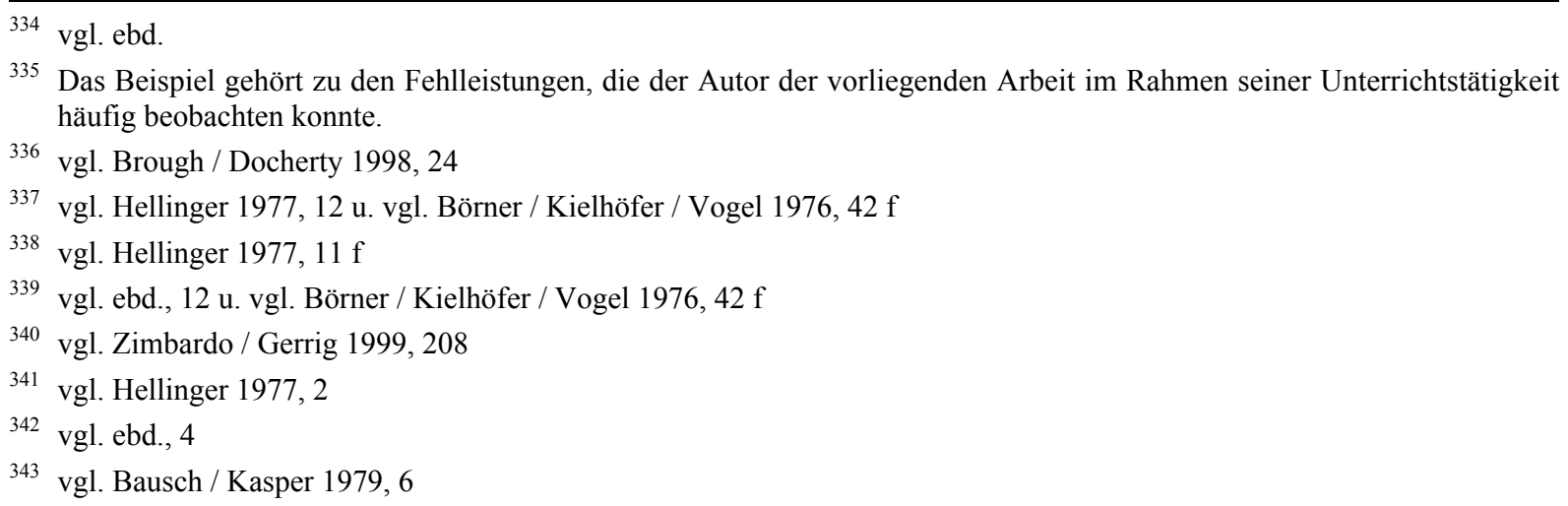


sprachliche Elemente beim Erlernen haben. ${ }^{344}$ Dabei ging man davon aus, daß mit zunehmenden Strukturunterschieden zwischen den Sprachen, die miteinander verglichen wurden, auch die Lernschwierigkeiten zunehmen. ${ }^{345}$

Die Inhalte der Kontrastivhypothese, insbesondere die Hierarchisierung der Schwierigkeitsgrade, hatten seit ihrer Entwicklung in den fünfziger Jahren einen starken Einfluß auf die Erstellung fachdidaktischer Lern- und Lehrwerke. ${ }^{346}$ In Form sogenannter drill-Übungen ${ }^{347}$ wurde insbesondere in den sechziger und siebziger Jahren des 20. Jahrhunderts der Versuch unternommen, die Elemente der Zielsprache zu vermitteln, die als besonders fehleranfällig bezeichnet wurden. Die Vertreter der Kontrastivhypothese fanden ihr Konzept, d. h. die Annahme, daß alle Schwierigkeiten beim Erlernen einer Zweitsprache linguistisch bedingt seien, insbesondere auf lautlicher Ebene bestätigt. ${ }^{348}$ Untersuchungen auf phonetischer Ebene zeigten, daß alle Lernenden einer Erstsprache bei mündlicher Anwendung einer festgelegten Zielsprache - etwa Deutsche beim Erwerb des Englischen - Akzentmerkmale besitzen, die trotz individueller Unterschiede bei allen Betroffenen nachzuweisen waren. ${ }^{349}$

Die Durchführung zahlreicher empirischer Studien führte jedoch bereits in der Frühphase der Kontrastivhypothese zu der Erkenntnis, daß weder die Strukturidentität zwischen Erst- und Zielsprache notwendigerweise zu einer Lernerleichterung noch die Strukturdivergenz zu einer Lernbehinderung führen muß. So wurde insbesondere in den Studien von J. Juhász deutlich, daß nicht nur Differenzen zwischen Erst- und Zielsprache Interferenzen hervorrufen, sondern daß gerade der Kontrastmangel zwischen den beiden Sprachsystemen das Erlernen zweitsprachlichen Materials erschweren kann. ${ }^{350}$ Diese Erscheinung, die als ,homogene Hemmung‘ oder ,Ranschburg'sches Phänomen` bezeichnet wurde, ${ }^{351}$ führte dazu, daß zukünftig auch die Theorie der Schwierigkeitshierarchie in Frage gestellt wurde. ${ }^{352}$ In zahlreichen Untersuchungen wurde deutlich, daß Lernende mitunter gerade solche Begriffe und Strukturen leicht erlernen und memorisieren können, die vollständig von Begriffen und Strukturen der Erstsprache abweichen, obwohl diese Strukturabweichungen der Kontrastivhypothese nach dazu führen müßten, daß die betreffenden Elemente im oberen Bereich der Schwierigkeitshierarchie liegen. ${ }^{353}$

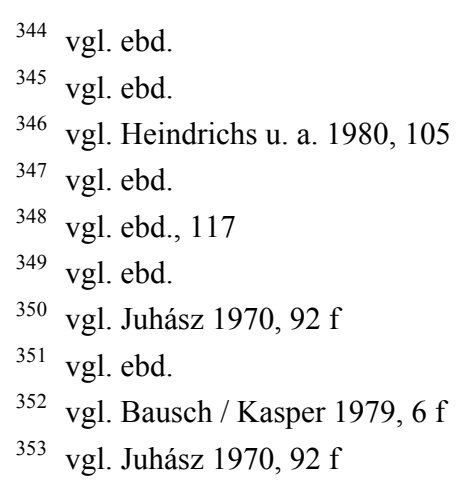


Dabei war zu erkennen, daß gerade die Differenzen zwischen Erst- und Zielsprache die Aufmerksamkeit der Lernenden auf sich ziehen. So weisen Phonetiker immer wieder darauf hin, daß die Aussprache der interdentalen Frikative $/ \theta /$ und $/ \partial /$ bei deutschen Englischlernenden - im Gegensatz zu der weitverbreiteten Meinung - nicht $\mathrm{zu}$ den gravierendsten Ausspracheproblemen deutscher Muttersprachler gehört, weil die für Deutsche fremden Elemente einen starken Aufmerksamkeitseffekt bewirken. ${ }^{354}$ Auch zeigte sich in den Studien, daß Strukturunterschiede - wie etwa die obengenannten Laute - nicht zwingend zu Lernschwierigkeiten führen müssen. ${ }^{355}$ Das Verhalten der Lernenden ließ beispielsweise erkennen, daß die Mehrzahl der deutschen Muttersprachler in der Lage ist, auch solche Laute und grammatikalische Strukturen der englischen Sprache fehlerfrei anzuwenden, für die es im Deutschen keine Entsprechungen gibt. ${ }^{356}$

Gleichzeitig war in diesem Zusammenhang zu erkennen, daß selbst dann, wenn Strukturunterschiede zunächst zu Lernschwierigkeiten führen, aus diesen Schwierigkeiten nicht notwendigerweise Fehler resultieren müssen. ${ }^{357}$ Auch die Lernschwierigkeiten selber können dazu beitragen, daß die Lernenden den Elementen, die anfänglich Probleme verursachen, größere Aufmerksamkeit widmen als den Elementen, die leichter zu erlernen sind. Ein weiterer Kritikpunkt betraf die ausschließlich linguistische Ausrichtung der Kontrastivhypothese. In zahlreichen Untersuchungen konnte nachgewiesen werden, daß der Erwerb einer Zweitsprache nicht nur auf sprachlichen Faktoren beruht, sondern daß affektive Faktoren ebenso berücksichtigt werden müssen wie der soziale Hintergrund der Lernenden und die biologisch-anatomischen Bedingungen, die den Erwerb einer Zweitsprache bestimmen. ${ }^{358}$ Bemängelt wurde in diesem Zusammenhang auch, daß die Kontrastivhypothese nicht unterscheidet zwischen Lernenden, die die Zweitsprache ausschließlich im Fremdsprachenunterricht erworben haben, und Lernenden, bei denen der Zweitspracherwerb unter ungesteuerten Bedingungen verlief. ${ }^{359}$

Einen weiteren Mangel sah man darin, daß die Kontrastivhypothese bei der Annahme eines Rückgriffs auf die Erstsprache nicht berücksichtigt, daß die Lernenden möglicherweise weitere Fremdsprachen erwerben, die ebenfalls Interferenzen verursachen könnten. ${ }^{360}$ Außerdem wird im Rahmen der Hypothese nicht beachtet, daß Lernende häufig auch auf bereits erworbene Regeln und Strukturen der Zielsprache zurückgreifen, wenn sie etwas verdeutlichen möchten und das benötigte Wissen nicht zur

\footnotetext{
354 vgl. Weiher 1982, $162 \mathrm{f}$

355 vgl. Juhász 1970, 92 f

356 Auch im Laufe der unterrichtlichen Tätigkeit des Autors vorliegender Arbeit wurde deutlich, daß Lernende oftmals Wörter besonders gut erlernen, die optisch und akustisch völlig von den entsprechenden deutschen Lexemen abweichen.

357 Während seiner unterrichtlichen Tätigkeit konnte auch der Autor der vorliegenden Arbeit beobachten, daß Lernende, die anfänglich Probleme mit der Anwendung der progressive form haben, diese Probleme in späteren Lernphasen ablegen und diese Form sogar anderen Formen vorziehen.

358 vgl. Vogel 1990, $122-164$

359 Zur Unterscheidung zwischen diesen beiden Formen des Spracherwerbs s. u. a. Bausch / Königs 1983, 316.

360 vgl. Bausch / Kasper 1979, 7
} 
Verfügung steht. ${ }^{361}$ So konnte in vielen Studien nachgewiesen werden, daß deutsche Englischlernende, die bei unregelmäßigen Verben die Vergangenheitsformen nicht kennen, simplifizierend die Endung -ed an das betreffende unregelmäßige Verb anhängen. ${ }^{362}$ Ein Beispiel bietet die Äußerung she byed a book. Demnach kann auch das bereits erworbene Wissen der Zielsprache zu Interferenzen beitragen.

Weiterhin bleibt in der Kontrastivhypothese unbeachtet, daß der Erwerb einer Zweitsprache als Prozeß zu betrachten ist, d. h., die einzelnen Regeln und Elemente werden sukzessiv erworben. Die Dynamik dieses Prozesses verläuft dabei nicht linear: Der Lernende kann eine einmal erworbene Regel nicht notwendigerweise sofort nachhaltig korrekt anwenden. ${ }^{363} \mathrm{Im}$ Fremdsprachenunterricht zeigte sich vielmehr, daß Lernende wiederholt bereits korrekt erworbene Regeln durch eine zweitsprachlich falsche Form ersetzen. ${ }^{364}$ So haben Untersuchungen gezeigt, daß Lernende, obwohl sie sich der Tatsache bewußt waren, daß Fragen mit Modalverben im Englischen nicht mit dem Hilfsverb do eingeleitet werden, diese Regel in einem späteren Erwerbsstadium unerwarteterweise mißachteten. ${ }^{365}$ Es entstanden Fehler der folgenden Form: Do you can come along tomorrow? ${ }^{366}$ Die Kontrastivhypothese hingegen geht von einem statischen Vergleich aus, d. h., es wird erwartet, daß Regeln und Elemente, die als leicht oder als schwer erlernbar eingestuft werden bzw. deren Anwendung zu wenigen oder zu vielen Fehlern führt, permanent zu gleichbleibenden Resultaten führen. ${ }^{367}$

Ein weiterer Kritikpunkt betraf die Vergleichsbasis der kontrastiven Analyse, in der nicht notwendigerweise festgelegt wird, welche Varianten der beiden Sprachen, die miteinander verglichen werden, zugrunde gelegt werden sollen. ${ }^{368}$ Beispielsweise ist es bei einer Sprache wie Englisch, die von 300 - 400 Mio. Muttersprachlern gesprochen wird und über unterschiedliche Normen verfügt, wichtig, daß vor dem Vergleich mit einer anderen Sprache festgelegt wird, welche Norm des Englischen zugrunde gelegt werden soll. Den Annahmen der Kontrastivhypothese entsprechend stellt etwa der Gebrauch des Perfekts in Äußerungen wie „Ich habe dich gerade gesehen“ für deutsche Englischlernende eine Übersetzungshilfe dar, weil der Betreffende der Theorie entsprechend den Satz mit der Äußerung I have just seen you übersetzen wird. Diese Übersetzung wäre jedoch nur dann als uneingeschränkt normgerecht zu bezeichnen, wenn das Britische Englisch als Vergleichsbasis zugrunde

\footnotetext{
361 vgl. Raabe 1980, 79

362 vgl. Drubig 1972, 81

363 vgl. Bausch / Kasper 1979, 7

364 vgl. ebd.

365 vgl. ebd.

366 vgl. ebd.

367 vgl. Rein 1983

368 vgl. Bausch 1973, 159- 182
} 
gelegt würde. Im Amerikanischen Englisch hingegen wäre die deutsche Äußerung mit dem Satz I just saw you zu übersetzen gewesen. ${ }^{369}$

Die zunehmende Kritik an dem ursprünglichen Konzept der Kontrastivhypothese führte schließlich dazu, daß diese in entscheidenden Punkten revidiert wurde. ${ }^{370}$ Die wichtigste Veränderung betrifft den Anspruch, die Entstehung sprachlicher Fehler prognostizieren zu können. Die ,starke Version` der Kontrastivhypothese wurde damit zu einer ,schwachen Version' umgewandelt, ${ }^{371}$ die nun nicht mehr den Anspruch erhob, feststellen zu können, welche Bereiche der Zielsprache Fehler hervorrufen könnten. Vielmehr beschränkt sich die schwache Version auf die Begründung bereits entstandener Fehler und gesteht darüber hinaus ein, daß viele Fehler aufgrund des prozeduralen Verlaufs des Zweitspracherwerbs nicht vorherzusagen sind. ${ }^{372}$

\subsection{Fehlererklärungsfaktoren}

Nach der Umformulierung in eine schwache Version definiert die Kontrastivhypothese fünf zentrale Fehlererklärungsfaktoren ${ }^{373}$, von denen drei Faktoren auf der ursprünglichen Prägung basieren, während die beiden letzteren aus dem neuen Konzept hervorgegangen sind.

\begin{tabular}{|l|l|l|l|}
\hline \multicolumn{3}{|l|}{ Kontrastivhypothese: Fehlererklärungsfaktoren } \\
\hline 1. Starke Version - Interlinguale Interferenz & 2. Schwache Version - Intralinguale Interferenz \\
\hline 1.1 Substitution & $\begin{array}{r}1.2 \text { Über- bzw. Unter- } \\
\text { differenzierung }\end{array}$ & $\begin{array}{l}1.3 \begin{array}{l}\text { Über- bzw. Unter- } \\
\text { repräsentation }\end{array} \\
\text { 1.2 Übergeneralisierung }\end{array}$ & 2.2 Hyperkorrektheit \\
\hline
\end{tabular}

\subsubsection{Interlinguale Interferen $z^{374}$}

\subsubsection{Substitution}

Fehler werden als Substitutionen bezeichnet, wenn der Fremdsprachenlernende zielsprachliche Elemente nicht im Sinne der entsprechenden Norm gebraucht, sondern durch Elemente ersetzt, die den erstsprachlichen in einer entsprechenden erstsprachlichen Kommunikationssituation maximal ähneln.

\footnotetext{
369 vgl. Swan 1990, Textstelle 351, o. S.

370 vgl. Wardhaugh 1970, $123-130$

371 vgl. ebd.

372 vgl. ebd.

373 vgl. Hellinger 1977, 12 - 15

374 Zum Begriff ,Interferenz' s. a. Kaltenbacher 2001b, 29 - 41
} 
Diese Ähnlichkeit kann sich sowohl in der Graphie als auch in der Lautung äußern, ${ }^{375}$ oft handelt es sich auch um eine Kombination beider Faktoren. Mitunter werden fremdsprachliche Elemente bzw. Strukturen vollständig durch erstsprachliche ersetzt, wenn in der Zielsprache keine weiteren, den erstsprachlichen Entsprechungen ähnelnde Elemente und Strukturen vorliegen. Diese Erscheinung ist beispielsweise bei folgender Äußerung zu erkennen:

Fehler: $\quad$ We will meet by Alan. ${ }^{376}$

Korrektur: We will meet at Alan's.

\subsubsection{2 Über- bzw. Unterdifferenzierung}

Überdifferenzierung liegt dann vor, wenn der Lernende fälschlicherweise eine sprachliche Unterscheidung seiner Erstsprache in der Zielsprache an Stellen realisiert, wo eine solche Differenzierung redundant ist. Beispiele für Überdifferenzierungen finden sich u. a. in der Übertragung der im Deutschen normgerechten Unterscheidung der Bewegungsverben in bezug auf öffentliche Transportmittel ins Englische. Deutsche Muttersprachler differenzieren zwischen den Bewegungsverben ,fahren“, ,fliegen', ,schwimmen' etc. stärker als englische native speakers bei den entsprechenden Verben in der englischen Sprache. ${ }^{377}$ Dieser Fehlertyp zeigt sich u. a. in folgendem Beispiel:

Fehler: $\quad$ I will fly to Spain.

Korrektur: I will go to Spain. ${ }^{378}$

Eine Fehlerquelle für Unterdifferenzierungen bietet die im Englischen gängige Differenzierung bei Bewegungsformen im Wasser. Während im Deutschen für alle Arten der Fortbewegung im Wasser das Wort ,schwimmen“ gebraucht wird, wie etwa in der Äußerung „die Kiste schwamm den Fluß hinunter“, unterscheidet man im Englischen zwischen den Verben swim für lebende Objekte und float für tote Materialien bzw. Gegenstände. ${ }^{379}$ So entstehen fehlerhafte Übersetzungen folgenden Typs:

Fehler: $\quad$ The box swam downriver.

Korrektur: The box floated downriver.

375 Dies trifft vor allem bei Homophonie fremd- und erstsprachlicher Elemente innerhalb einzelner Wortarten zu.

376 Soweit nicht anders angegeben, handelt es sich bei den nachfolgenden Fehlerbeispielen um Fehler, die der Autor der vorliegenden Arbeit im Rahmen seiner unterrichtlichen Tätigkeit beobachten konnte.

377 vgl. Brough / Wittmann 2001, 30

378 Diese Äußerung wäre nur dann normgerecht, wenn der Sprecher das Flugzeug selbst fliegen würde.

379 vgl. Hellinger 1977, 14 


\subsubsection{3 Über- bzw. Unterrepräsentation}

Über- bzw. Unterrepräsentationen ${ }^{380}$ sind dadurch gekennzeichnet, daß sprachliche Elemente im Vergleich zur Gebrauchsfrequenz der Zielsprache zu häufig bzw. zu selten benutzt werden, welches in jedem Einzelfall nicht als Fehler zu bezeichnen wäre. Ein Beispiel bietet der verkürzte Relativsatz im Englischen, den die deutsche Sprache nicht kennt, wie etwa bei The man I saw. ${ }^{381}$ Eine Unterrepräsentation liegt dann vor, wenn der deutsche Englischlernende diese Verkürzung niemals benutzt, sondern stattdessen Relativpronomina verwendet, weil er eine solche Struktur aus seiner Erstsprache nicht kennt.

Eine Überrepräsentation liegt vor, wenn der Lernende die verkürzte Form permanent verwendet, weil er glaubt, dadurch einen höheren Kompetenzgrad in der Fremdsprache zu zeigen, oder weil er die Struktur aus persönlichen Neigungen der verlängerten Form vorzieht und daher auf den Gebrauch von Relativpronomina völlig verzichtet.

\subsubsection{Intralinguale Interferenz}

Die Umwandlung der starken Version der Kontrastivhypothese in eine schwache führte dazu, daß neben den interlingualen auch intralinguale Interferenzen berücksichtigt wurden, deren Entstehung durch die Komplexität des zielsprachlichen Sprachsystems evoziert wird. Die schwache Version integrierte in erster Linie zwei intralinguale Interferenzen, die Übergeneralisierung und die Hyperkorrektheit. ${ }^{382}$

\subsubsection{1 Übergeneralisierung}

Sprachliche Abweichungen werden als Übergeneralisierungen bezeichnet, wenn der Lernende bereits erlernte sprachliche Elemente bzw. Strukturen auf Zusammenhänge überträgt, die anderen Regeln unterliegen, ${ }^{383}$ wie etwa der Gebrauch des Hilfsverbs have in Fragesätzen, bei denen have nicht verwendet wird und die Frage stattdessen mit do gebildet wird, beispielsweise bei verbgerichteten Fragen.

Fehler: Have you live in England?

Korrektur: Do you live in England?

\footnotetext{
380 vgl. ebd. und James 1998, 176

381 vgl. Hellinger 1977, 14

382 vgl. ebd., 12

383 vgl. James 1998, 187
} 


\subsubsection{Hyperkorrektheit}

$\mathrm{Zu}$ den Hyperkorrektheiten gehören solche sprachlichen Äußerungen, die, ähnlich wie Über- bzw. Unterrepräsentationen, isoliert betrachtet nicht als Fehler gelten, aber in bestimmten Kommunikationssituationen gegen situative Normen der Zielsprache verstoßen. ${ }^{384}$ Als Beispiel wird in der Fachdidaktik häufig der Gebrauch des sogenannten $\mathrm{RP}^{385}$ angeführt. Hier handelt es sich um eine Ausspracheform der englischen Sprache, die von nur etwa 3\% der englischen Bevölkerung als natürliche Ausspracheform verwendet wird. Von Sprechern, die diesen Akzent aus beruflichen oder gesellschaftlichen Gründen verwenden müssen, wird das RP künstlich in elocution lessons erworben und nahezu ausschließlich in bestimmten Redesituationen benutzt, etwa in Medien, in denen das gesprochene Wort im Vordergrund steht oder bei politischen Anlässen. ${ }^{386}$ Wird diese Sprachform von Englischlernenden, aber auch von native speakers, in allen Konversationssituationen verwendet, kann sie bei bestimmten Gelegenheiten, etwa in informellen Situationen, befremdend und prätentiös klingen. ${ }^{387}$ Auch auf lexikalischer Ebene gibt es ähnliche Erscheinungen. Dies betrifft beispielsweise den Gebrauch von Redewendungen, die normalerweise nur in einem bestimmten Kontext benutzt werden, etwa im Geschäftsleben.

Situation: Zwei Freunde begrüßen sich im $p u b$.

Fehler: How do you do? ${ }^{388}$

Korrektur: How are you?

\section{Die Identitätshypothese}

\subsection{Entstehung und Inhalte}

Die Identitätshypothese beruht auf der Annahme, daß der Erwerb einer Zweitsprache prinzipiell isomorph zum Erwerb der Erstsprache verläuft. ${ }^{389}$ Die Hypothese geht dabei von der Vorstellung aus, daß der Lernende beim Erwerb der Zweitsprache angeborene mentale Prozesse aktiviert. ${ }^{390}$ Diese mentalen Prozesse bewirken, daß die zweitsprachlichen Regeln und Elemente in der gleichen Abfolge erworben werden wie die Regeln und Elemente der Erstsprache. ${ }^{391}$ Aus dieser Annahme leitete man

$384 \mathrm{RP}=$ Received Pronunciation, vgl. ebd., 141.

385 vgl. Weiher 1982, $16-20$

386 vgl. ebd.

387 vgl. ebd.

388 Losgelöst vom sprachlichen Kontext ist diese Äußerung nicht als Fehler zu betrachten. Im gegebenen Zusammenhang verstößt sie jedoch gegen die Norm der englischen Sprache.

389 vgl. Bausch / Kasper 1979, 9

390 vgl. ebd.

391 vgl. ebd. 
ab, daß Entwicklungssequenzen in der zweitsprachlichen Erwerbschronologie durch die Struktur der Zweitsprache gesteuert werden und nicht - wie der Theorie der Kontrastivhypothese entsprechend durch die Struktur der Erstsprache. Fehler beim Zweitspracherwerb werden demnach durch die Struktur der Zweitsprache determiniert, ebenso wie Fehler beim Erstsprachenerwerb auf die Struktur der Erstsprache zurückzuführen sind. ${ }^{392}$

Aufgrund ihrer Entwicklungsbedingtheit werden Fehler beim Zweitspracherwerb von Vertretern der Identitätshypothese in englischsprachigen Ländern als developmental errors ${ }^{393}$ bezeichnet. Die Bezeichnung, interlinguale Interferenzen', die im Rahmen der Kontrastivhypothese geprägt wurde, hat nach Aussage der Identitätshypothese keine Berechtigung. Während die Kontrastivhypothese den Zweitspracherwerb als habit formation versteht, wobei der Lernende die Zweitsprache nach dem Prinzip von trial and error aufnimmt, verläuft der Theorie der Identitätshypothese entsprechend der Erwerb einer Sprache durch kognitive Prozesse. Im Gegensatz zu der These der behavioristisch orientierten Kontrastivhypothese wird der Zweitspracherwerb im Rahmen der Identitätshypothese als aktiver, kreativer Prozeß verstanden. Ein interner, mentaler Mechanismus, der als built-in syllabus bezeichnet wird, filtert aus der Fülle der sprachlichen Daten, die an den Lernenden herangetragen werden, die Information heraus, die der Lernende auf der erreichten Entwicklungsstufe seines Lernprozesses aufnehmen kann. Auf der Grundlage der aufgenommenen Daten bildet der Lernende Hypothesen über das zweitsprachliche Regelsystem, die er während eines kontinuierlichen Überprüfungsprozesses modifiziert, und entwickelt damit seine Übergangskompetenz in Richtung auf die Zweitsprache.

Diese Spracherwerbsmechanismen sind der Identitätshypothese nach universell und können daher sowohl bei Kindern als auch bei Erwachsenen beobachtet werden. Die Spracherwerbsmechanismen werden dabei weder durch das Alter noch durch den Sprachunterricht, das Geschlecht oder die emotionale Einstellung des Lernenden zu der betreffenden Zweitsprache beeinflußt. Diese Theorie basiert insbesondere auf den Ergebnissen empirischer Studien, die mit folgenden Untersuchungsgruppen durchgeführt wurden: ${ }^{394}$

a) Kinder, die ihre Erstsprache erwerben, verglichen mit Kindern, die eine Zweitsprache erwerben

b) Kinder verschiedener Erstsprachen beim Zweitspracherwerb

c) Kinder, die ihre Erstsprache erwerben, verglichen mit Erwachsenen, die eine Zweitsprache erwerben

d) Erwachsene verschiedener Erstsprachen beim Zweitspracherwerb

\footnotetext{
392 vgl. Dulay / Burt / Krashen 1982

393 vgl. Bausch / Kasper 1979, 9
} 
In allen Studien konnte nachgewiesen werden, daß die Lernenden beim Erwerb der syntaktischmorphologischen Regeln vergleichbare Entwicklungssequenzen durchlaufen. Gleichzeitig glaubte man, auf der Basis dieser Untersuchungen beweisen zu können, daß Erst- und Zweitspracherwerb in hohem Maße systematische Prozesse darstellen. Die Art und Weise des Erwerbs variiere nicht beliebig von Individuum zu Individuum und von Sprache zu Sprache. Vielmehr verlaufe der Erwerb einer Sprache nach geordneten Entwicklungssequenzen, in denen die Abfolge der Erwerbsstadien festgelegt sei. Weiterhin geht die Identitätshypothese davon aus, daß diese Entwicklungsprozesse eigene Gesetzmäßigkeiten aufweisen, die nicht durch den sprachlichen Input, der auf die Lernenden einwirkt, beeinflußt werden. Dies bedeutet, daß die Lernenden nicht unbedingt solche Elemente als erste erwerben, die besonders häufig aufgenommen werden.

So wurde deutlich, daß Lernende im Laufe ihrer sprachlichen Entwicklung auf grammmatikspezifische Phänomene und Prozesse reagieren, auf die es im sprachlichen Input keinerlei direkten Hinweis gibt. Außerdem konnte nachgewiesen werden, daß der Erwerb von komplexeren Strukturen zum Teil vor dem Erwerb von weniger komplexen Strukturen erfolgt. Im Rahmen der Studien wurde beispielsweise deutlich, daß Lernende, die Englisch als Zweitsprache erwerben, nicht kontrahierte Formen anwenden, wie etwa will not und are not, auch wenn die Betroffenen bis zu diesem Erwerbsstadium ausschließlich mit kontrahierten Formen konfrontiert wurden. ${ }^{395}$ Die Annahme, daß der sprachliche Input keinen Einfluß auf die Erwerbssequenzen habe, glaubte man auch dadurch bestätigen zu können, daß - wie die Untersuchungen zeigten - der Spracherwerb auch im Falle eines stark reduzierten sprachlichen Inputs Parallelen zu einem normal verlaufenden Spracherwerb aufweist. So konnte gezeigt werden, daß taubgeborene Kinder, die von jedwedem Input - sei er verbal oder in Form von Gesten - ausgeschlossen waren, ein rudimentäres Zeichensystem entwickelten, das alle formalen Eigenschaften der frühen Stadien des normalen Spracherwerbs enthielt. ${ }^{396}$ Außerdem ließen die Studien erkennen, daß Kinder bereits in der Frühphase des Spracherwerbs in der Lage sind, grammatische und lexikalische Regeln abzuleiten, obwohl ihnen das entsprechende sprachliche Vorwissen völlig fehlt. Sie sind dabei auch fähig zu erkennen, welche sprachlichen Bestandteile für die Vermittlung ihrer sprachlichen Botschaft von Bedeutung sind und welche eine untergeordnete Rolle spielen.

Ähnliche Beobachtungen wurden auch im Rahmen der Studien mit Erwachsenen deutlich. Auch hier zeigte sich, daß Lernende einer Zweitsprache durchaus in der Lage sind, über das bereits erworbene Sprachwissen hinaus Strukturen abzuleiten - im Gegensatz zu den früher geltenden Grundsätzen, nach denen die Ableitung dieser Strukturen nur möglich wäre, wenn den Betroffenen ein weit größeres

\footnotetext{
395 vgl. Szagun 2000, 51

396 vgl. Cross 1978, 199216
} 
zweitsprachliches Vorwissen zur Verfügung gestanden hätte. Daneben zeigten die Studien auch, daß Erwachsene durchaus fähig sind, eine Zweitsprache mit erstsprachlicher Kompetenz zu erlernen. Letzteres gilt nicht nur für die syntaktisch-morphologische und die lexikalische Ebene, sondern auch für den gesamten Bereich der Aussprache, der einer besonders ausgeprägten Sprachkompetenz bedarf. In ihrer Gesamtheit bekräftigten die Ergebnisse der Studien die Existenz sogenannter Erwerbsuniversalien und damit die These, daß der Erwerbsprozeß einer Sprache biologisch vorbestimmt sei.

Bereits Mitte der siebziger Jahre jedoch wurde das Konzept der Identitätshypothese heftig kritisiert. Ausgangspunkt dieser Kritik war im wesentlichen die Tatsache, daß trotz aller Hinweise, die für die Existenz einer Universalgrammatik sprechen, keiner Studie der Nachweis gelungen war, daß erstsprachlicher Transfer völlig ausgeschlossen werden kann. Im Gegenteil zeigte sich in nahezu allen Studien, daß der Einfluß der Erstsprache beim Erwerb der Zweitsprache wenigstens partiell zu erkennen war. Die Kritik bezog sich jedoch nicht nur auf die Ergebnisse, die aus den Studien hervorgingen, sondern auch auf das Verfahren, das den einzelnen Studien zugrunde lag. Besonders bemängelt wurde hier die Tatsache, daß die Untersuchungen, die von den Befürwortern der Identitätshypothese zur Unterstützung ihrer Theorie durchgeführt wurden, nahezu ausschließlich syntaktisch-morphologische Aspekte umfaßten. Die lautliche und die lexikalische Ebene wurden im Rahmen dieser Studien kaum berücksichtigt.

Kritisiert wurde darüber hinaus - wenn auch weniger vehement - daß sozio-linguistische Variablen des Lernumfelds, wie etwa die Schulform, an der die Zweitsprache erworben wird, das Lehrmaterial und der Kontakt zu anderen Lernenden, nur ansatzweise in Betracht gezogen wurden. Außerdem wurde in diesem Zusammenhang negativ hervorgehoben, daß die Normenproblematik, die bei der englischen Sprache besonders deutlich zum Tragen kommt, im Rahmen der Identitätshypothese nicht diskutiert wurde.

Angesichts der Kritik ${ }^{397}$ konnte die Identitätshypothese in ihrer ursprünglichen Konzeption nicht aufrechterhalten werden. Einen entscheidenden Beitrag zur partiellen Bewahrung der der Identitätshypothese zugrundeliegenden Theorie leistete die von Wode geprägte Unterscheidung zwischen einer ,starken“ und einer ,schwachen“ Version der Identitätshypothese, ${ }^{398}$ wobei letztere jedoch nur den kindlichen Zweitsprachenlernenden betrifft. Während die ,starke Version' dieselben oberflächenstrukturellen Erwerbssequenzen für Lernende verschiedenen sprachlichen Hintergrunds, Alters und Geschlechts voraussetzt, grenzt Wode dies in seiner, schwachen Version` ein: Unter Beibehaltung der Annahme, daß Erst- und Zweitspracherwerb nach gleichen Prinzipien gesteuert werden, wird die

\footnotetext{
397 Eine Zusammenfassung der wesentlichsten Kritikpunkte aus heutiger Sicht bietet Edmondson 1999, 44 ff.

398 vgl. Wode 1978b, $101-117$
} 
Beschaffenheit der Oberflächenstruktur beim kindlichen Zweitsprachenlernenden von der Gesamtheit der Informationen, über die das Kind verfügt und die in erster Linie auf der Struktur der Erstsprache beruhen, bestimmt. Damit führen die grundsätzlich gleichen Prinzipien bei verschiedenen kindlichen Fremdsprachenlernenden zu unterschiedlichen Oberflächenstrukturen. Da Wode den Begriff ,Identität` vornehmlich auf die Produkt- und weniger auf die Prozeßebene ${ }^{399}$ bezieht, wird die Validität der Identitätshypothese in ihren Prinzipien nur partiell eingeschränkt.

Die Erkenntnis, daß erstsprachlicher Transfer nicht auszuschließen ist und daß dessen Inexistenz empirisch nicht bewiesen werden kann, wurde seit der zweiten Hälfte der siebziger Jahre seltener als Argument zur Widerlegung der Identitätshypothese benutzt. Vielmehr setzte sich die Auffassung durch, daß der Ausschluß erstsprachlichen Transfers von einer kognitiven Zweitspracherwerbstheorie nicht zwingend gefordert werden muß. Stattdessen ermöglichten die Herauslösung des Transferbegriffs aus seinem ursprünglichen behavioristischen Kontext und seine kognitive Uminterpretation und Integration in die modifizierte Fassung der Identitätshypothese eine erweiterte Interpretation des Begriffs ,Zweitspracherwerb‘. Die Verbindung biogenetischer Spracherwerbsprozesse mit der sprachlichen und kommunikativen Vorerfahrung des Lernenden als Grundlage einer Spracherwerbstheorie stellte der Zweitspracherwerbsforschung eine ganzheitliche Sicht des zu untersuchenden Ablaufs zur Verfügung.

Der somit erzielte Konsens wurde nachfolgend jedoch durch die bereits früher diskutierte, von Corder geprägte und später von Krashen aufgegriffene Unterscheidung zwischen second language acquisition und foreign language learning ${ }^{400}$ hinsichtlich der Übertragbarkeit der Identitätshypothese auf alle Erwerbssituationen erneut in Frage gestellt. Corder grenzt den Applikationsbereich der Identitätshypothese explizit auf den ungesteuerten Erwerb (second language acquisition) ein. Das unterrichtsgesteuerte Fremdsprachenlernen (foreign language learning) hingegen sei ein „restructuring continuum, a process of progressively adjusting his (sc. the learner's) mother tongue system to approximate it ever more closely to the target. “ ${ }^{401}$ Krashen unterscheidet nicht nur zwischen den beiden Erwerbsformen, sondern verallgemeinert für den ungesteuerten Zweitspracherwerb - entgegen Wodes Theorie, der ,schwachen Version“ - die Universalität aller Spracherwerbsprozesse bei Kindern und Erwachsenen und schließt für diesen jede Form erstsprachlichen Transfers aus. ${ }^{402}$

\footnotetext{
399 Wenngleich Wode in bezug auf die Abfolge der Erwerbssequenzen zugesteht, daß der Grad der Parallelität durch die Erstsprache determiniert wird, sind Gemeinsamkeiten ihm zufolge unverkennbar. Wodes Ergebnisse beziehen sich jedoch in erster Linie auf den Erwerb der Interrogation.

400 vgl. Corder 1967, 161 - 165. Siehe auch Krashen 1976, 157 - 168.

401 vgl. Corder 1978, $71-93$

402 vgl. Krashen 1976, $157 \mathrm{ff}$
} 
Kennzeichnend für das foreign language learning als einem unter unterrichtsgesteuerten Bedingungen ablaufenden Lernprozeß, ist Krashen zufolge der sogenannte Monitor. ${ }^{403}$ Das danach benannte Monitormodell basiert auf der Annahme, daß Lernende das Regelwissen über die Fremdsprache beim gesteuerten Fremdsprachenerwerb im Gedächtnis speichern. In Form eines Monitors bildet das internalisierte Wissen eine Informations- und Kontrollinstanz. Dieser Monitor kann bei der fremdsprachlichen Produktion und Rezeption konsultiert werden, wenn die dafür notwendigen Performanzbedingungen gewährleistet sind. Von zentraler Bedeutung sind dabei der psycho-physische Zustand des Lernenden und der Zeitfaktor. ${ }^{404}$ Sind die für das Operieren des Monitors notwendigen Performanzbedingungen nicht gegeben, produzieren Lernende unterschiedlicher Erstsprachen ähnliche Fehler und beweisen damit die Validität der universal principles.

Zusammenfassend stellt Krashen die fehlergenetische Bedeutung des Monitor-Modells wie folgt dar: „Errors that result from performance based on the acquired system alone will be consistent across learners/acquirers, regardless of their first language, as acquisition is guided by universal principles. Errors that result from situations in which monitoring is possible will be more idiosyncratic, as they will reflect each learner's conscious mental representation of linguistic regularities in the target language. “ 405 Dieser Auffassung nach ist erstsprachlicher Einfluß somit eng an Lernsituationen im Unterricht geknüpft, während erstsprachlicher Transfer unter ungesteuerten Erwerbsbedingungen nicht zum Tragen kommt. ${ }^{406}$

Die von Krashen u. a. vollzogene Dichotomie fand auch in der Sprachlehrforschung ihren Niederschlag. ${ }^{407}$ Daher konnte sich das Konzept der Identitätshypothese in der - auf einem integrativen Forschungsansatz basierenden - Sprachlehrforschung nicht behaupten. Der Versuch, die Hypothese durch die Einführung einer ,schwachen Version“ und durch die partielle Akzeptanz erstsprachlichen Transfers, die eine ganzheitliche Betrachtungsweise des Lernenden akzentuierten, aufrecht zu erhalten, hatte dennoch nachhaltige Auswirkungen: Auf dieser Basis gründete die sogenannte InterlanguageForschung, die maßgeblich von den Vertretern bzw. partiellen Befürwortern der Identitätshypothese mitgestaltet wurde. ${ }^{408}$

\footnotetext{
403 vgl. Krashen 1977, $152-161$

${ }^{404}$ vgl. Gardner 1985, $125-127$

405 Krashen 1977, 154

$406 \mathrm{Zu}$ Übereinstimmungen mit dieser Annahme siehe insbesondere Corder 1978, 71 - 93 u. Felix 1977, 25 - 34. Felix' Dichotomie von der reproduktiven Kompetenz im Fremdsprachenunterricht und der kreativen Kompetenz im ungesteuerten Fremdsprachenerwerb wurde stets kritisiert. Die Kritik bezieht sich in erster Linie darauf, daß die Bewußtheit von Handlungen kein Indiz für Reproduktivität bzw. Kreativität ist. Kritikern zufolge kann ein Lernender Handlungen bewußt wiederholen oder aber bekannte Operationen bewußt mit neuem sprachlichen Material in neuen Situationen erproben, wobei letzteres eo ipso eine kreative Handlung wäre.

407 vgl. Multhaup 1995, $116-119$

408 vgl. u. a. Dulay / Burt 1974b, 95 - 123 u. Corder 1976, 9 - 34
} 


\subsection{Fehlererklärungsfaktoren}

\subsection{Identitätshypothese: Fehlererklärungsfaktoren}

\begin{tabular}{|l|l|l|l|}
\hline 1. omission errors & 2. addition errors & 3. misformations & 4. misorderings \\
\hline & 2.1 double markings & 3.1 regularization errors & \\
& 2.2 regularization errors & 3.2 archi-forms & \\
& 2.3 simple addition errors & 3.3 alternating forms & \\
\hline
\end{tabular}

Die bei der Bildung der Übergangskompetenz entstehenden zielsprachlichen Abweichungen wurden fortan nicht mehr als ,Fehler' im traditionellen Sinne bezeichnet, sondern als Folge intralingualer Prozesse betrachtet, die als developmental errors ${ }^{409}$ kreative Nebenprodukte des Zweitspracherwerbs darstellen. Dulay/Burt/Krashen ${ }^{410}$ gelang es schließlich in umfassenden Untersuchungen, die den verschiedenen Prozessen zugrundeliegenden Abweichungen näher zu spezifizieren und als surface structure taxonomy darzustellen. Dabei wurden nachfolgend beschriebene Fehlertypen ermittelt und nach ihrem Erscheinungsbild benannt.

\subsection{1 omission errors}

Als omission errors werden Fehlleistungen bezeichnet, die durch das Auslassen eines syntaktischmorphologischen bzw. lexikalischen Elements entstanden sind. Der Fehlertyp umfaßt sowohl das Auslassen größerer syntaktischer Einheiten, wie etwa ganze Satzteile, als auch das Auslassen einzelner Lexeme und Segmente.

Fehler: $\quad$ I working in the garden.

Korrektur: I am working in the garden.

Fehler dieses Typs sind insbesondere in der Frühphase des Erst- und Zweitspracherwerbs zu beobachten. Hierbei beschränken sich die Lernenden oftmals ausschließlich auf die Satzelemente, die für das Verständnis ihrer sprachlichen Aussage von Bedeutung sind: Elemente, die die Bedeutung nicht beeinflussen, werden ausgelassen.

\subsection{2 addition errors}

Addition errors stellen Abweichungen dar, die durch das redundante Hinzufügen sprachlicher Elemente entstanden sind. Man unterscheidet dabei zwischen double markings, regularizations und

409 vgl. Dulay / Burt / Krashen 1982, 165 ff

410 vgl. ebd., 156 
simple addition errors. Welcher Fehlertyp im einzelnen vorliegt, richtet sich nach der sprachlichen Struktur, die durch das Hinzufügen verändert wird.

\subsubsection{1 double markings}

Als double markings werden Fehler bezeichnet, die dadurch entstehen, daß eine grammatische Struktur redundanterweise doppelt verdeutlicht wird. Diese Erscheinung ist sowohl beim Erst- als auch beim Zweitspracherwerb häufig beim Gebrauch der Tempora, der Negationen und bei Anwendung der Pluralformen zu beobachten.

Fehler: $\quad$ He doesn't knows my name.

Korrektur: He doesn't know my name.

Fehler: We didn't went there.

Korrektur: We didn't go there.

Im ersten Beispiel wurde das Morphem $s$, das im simple present der Verdeutlichung der 3. Person Singular dient und bei Negationen durch das Hilfsverb doesn't zum Ausdruck kommt, redundanterweise auch an das Vollverb know angefügt. Im zweiten Beispiel wurde die Zeitform simple past sowohl bei dem Hilfsverb did als auch beim nachfolgenden Vollverb went angewandt, obwohl bei Negationen und Fragen nur das Hilfsverb im simple past verwendet wird.

\subsubsection{2 regularization errors}

Fehlleistungen in der Kategorie der addition errors werden als regularization errors bezeichnet, wenn Markierungselemente regelmäßiger Formen - etwa der Pluralbildung oder der Vergangenheitstempora - bei Lexemen angewandt werden, die zu den unregelmäßigen Formen gehören.

Fehler: $\quad$ sheeps putted

Korrektur: sheep put

Im ersten Beispiel wurde das im Englischen häufig vertretene Pluralallomorph $s$ auf ein Element übertragen, dessen Pluralbildung durch ein sogenanntes Nullallomorph angezeigt wird. Im zweiten Beispiel wurde die Verb-Endung -ed, die bei regelmäßigen Verben zur Bildung des simple past und des Partizips benutzt wird, fälschlicherweise an ein unregelmäßiges Verb angefügt. Bei letzteren werden jedoch diese grammatischen Formen durch andere Elemente gebildet, wie etwa durch die Änderung des Stammvokals. 


\subsubsection{3 simple addition errors}

Die Gruppe der simple addition errors umfaßt Abweichungen, die durch das redundante Hinzufügen sprachlicher Elemente entstehen, ohne daß die Fehler als double markings oder regularizations zu bezeichnen wären. Bei diesem Fehlertyp werden vielmehr zwei oder mehrere entgegengesetzte grammatische Strukturen miteinander kombiniert. Dazu gehört etwa die Verbindung einer Singularform mit einer Pluralform oder die Verbindung einer Zeitform der Vergangenheit mit einer Zeitform, die nur in der Gegenwart anzuwenden ist.

Fehler: $\quad$ The fishes doesn't live in the water.

Korrektur: The fish don't live in the water.

Fehler: Did he goes to the cinema?

Korrektur: Did he go to the cinema?

Im ersten Beispiel wäre nach der Pluralform des Lexems fish die Pluralform des Lexems do zu benutzen gewesen. Im zweiten Beispiel wurde eine Zeitform des simple past mit einer Form des simple present kombiniert. Der Lernende hat dabei nicht beachtet, daß bei Fragen und Negationen im simple past keine Markierung der 3. Person Singular erfolgt.

\subsection{3 misformations}

Fehlleistungen sind als misformations einzuordnen, wenn sie auf einer falsche Struktur oder Morphemform beruhen. Während bei den omission errors notwendige Elemente vollständig ausgelassen werden, geben die misformations zu erkennen, daß die Notwendigkeit des Gebrauchs bestimmter Strukturen oder Formen dem Lernenden grundsätzlich bewußt war. Dabei wurde jedoch eine falsche Form benutzt.

\subsubsection{1 regularization errors}

Regularization errors, die der Kategorie misformations zugeordnet werden, sind dadurch gekennzeichnet, daß die Form eines Wortes, das zu einem bestimmten Erwerbsstadium erlernt wurde, auf Wörter übertragen wird, die von diesem Wort abgeleitet werden, aber strukturell partiell davon abweichen.

Fehler: He was talking to hisself.

Korrektur: He was talking to himself. 
Hier liegt die Fehlleistung darin begründet, daß der Lernende glaubte, das Personalpronomen his auch beim Gebrauch des davon abgeleiteten Reflexivpronomens verwenden zu können. Dies führte innerhalb des Lexems, das er bildete, zu einer Strukturveränderung.

\subsubsection{2 archi-forms}

Eine Abweichung wird als archi-form bezeichnet, wenn ein Lexem einer bestimmten Wortart die Funktion anderer Lexeme dieser Wortart übernimmt.

Fehler: Can you see that dogs?

Korrektur: Can you see those dogs?

Die Fehlleistung liegt darin begründet, daß der Lernende das Demonstrativpronomen that, das nur im Singular anzuwenden ist, nicht durch die Pluralform those ersetzt hat.

\subsubsection{3 alternating forms}

Der Fehlertyp alternating forms liegt dann vor, wenn ein Lexem einer bestimmten Wortart durch ein anderes Lexem dieser Wortart ersetzt wird, das jedoch eine andere grammatische Funktion erfüllt als das substituierte Lexem.

Fehler: $\quad$ The girl asks the boy what he should paint.

Korrektur: The girl asks the boy what she should paint.

Das Pronomen he wurde hier fälschlicherweise zur Verdeutlichung einer femininen Form benutzt.

\subsection{4 misorderings}

Fehler werden als misorderings bezeichnet, wenn lexikalische oder syntaktisch-morphologische Elemente vertauscht werden. Dieser Fehlertyp kann sowohl bei Einzelsegmenten als auch bei ganzen Satzteilen und Sätzen auftreten.

Fehler: I have yesterday seen her.

Korrektur: I saw her yesterday.

Abgesehen von dem Verstoß gegen den Gebrauch der Vergangenheitstempora beruht die Fehlleistung darauf, daß der Lernende die Wortstellungsregeln eines sogenannten adverb of time mißachtet hat. Solche Adverbien stehen gewöhnlich am Satzanfang oder am Satzende. 


\section{Die Interlanguagehypothese}

\subsection{Entstehung und Inhalte}

Die erste Prägung der Interlanguagehypothese entstand bereits Anfang der achtziger Jahre des 20. Jahrhunderts und wurde insbesondere durch L. Selinker in die Zweitspracherwerbsforschung integriert. Die Bezeichnung, Interlanguage` geht auf eine Sprachform zurück, die J. Reinecke 1935 bei Untersuchungen des hawaiianischen Pidgin ermittelte. ${ }^{411}$ Im Rahmen dieser Studien zeigte sich, daß die Sprecher dieser Sprachform ein linguistisches System gebrauchen, das weder mit ihrer Ursprungssprache noch mit der Zielsprache übereinstimmt, sondern Züge beider Sprachformen trägt.

Selinker und andere erkannten, daß eine solche Zwischensprache auch bei Lernenden einer Zweitsprache zu beobachten ist, fügten jedoch erweiternd hinzu, daß Lernende einer Zweitsprache zusätzlich Merkmale entwickeln, die von Erst- und Zweitsprache unabhängig sind. ${ }^{412}$ Diese Merkmale sind sehr individuell und gehen auf die Persönlichkeit des Lernenden zurück. Sie umfassen psychische und physische Faktoren, etwa das Lernverhalten der einzelnen Personen, ihre Fähigkeit, sprachliche Elemente dauerhaft aufzunehmen, die anatomische Beschaffenheit ihrer Artikulationsorgane und die allgemeine körperliche Verfassung der Betreffenden. Teilweise entstehen die Merkmale jedoch auch als Reaktion auf die äußeren Umstände, unter denen die Betreffenden die Zweitsprache erwerben, d. h. durch ihr Lernumfeld.

Die aus diesen Erkenntnissen abgeleitete Interlanguagehypothese beruht daher auf der These, daß Lernende beim Erwerb einer Zweitsprache eine Sprachform entwickeln, die einerseits auf Elementen des zielsprachlichen linguistischen Systems und andererseits auf sozio- und psycholinguistischen Faktoren basiert. Somit wird Zweitspracherwerb nicht nur durch den Kontakt zweier Sprachsysteme gesteuert, sondern auch durch außersprachliche Faktoren. Die Verbindung dieser beiden Komponenten führt schließlich dazu, daß die Lernenden beim Erwerb einer Zweitsprache Prozesse durchlaufen und Strategien anwenden, die schließlich die ,Interlanguageform‘ der Betreffenden prägen. Unter der Bezeichnung ,Prozesse“ sind in der Forschung Abläufe zu verstehen, die den Verlauf des Zweitspracherwerbs bestimmen. Diese sind, wie erwähnt, sprachlichen und außersprachlichen Charakters. Dazu gehören unter anderem die Gestaltung des Unterrichts, in dem die Zweitsprache erworben wird, das Lernumfeld, andere Zweitsprachen, die der Lernende erwirbt, oder die persönliche Einstellung des Lernenden zur Zielsprache.

\footnotetext{
411 vgl. Reinecke 1969 (Erstdruck 1935)

412 vgl. Selinker 1972, $209-231$
} 
Die Bezeichnung ,Strategie‘ umfaßt einerseits die Art und Weise des Erlernens zweitsprachlicher Regeln und Strukturen. Andererseits zählt zu den Strategien auch das Kommunikationsverhalten, das bei Anwendung der Zweitsprache deutlich wird. Hierbei wird in der Regel zwischen offensivem und defensivem Kommunikationsverhalten unterschieden. Eine Strategie wird als offensiv bezeichnet, wenn der Lernende versucht, Kommunikationsbarrieren mit Hilfe seines bereits erworbenen Wissens zu überwinden. Dabei erscheint ihm die Tatsache unbedeutend, daß durch den Rückgriff auf bereits vorhandenes Wissen sprachliche Äußerungen entstehen könnten, die nicht mit der Norm der betreffenden Zielsprache übereinstimmen. Defensives Kommunikationsverhalten liegt in der Regel dann vor, wenn der Lernende ausschließlich auf sprachliche Äußerungen der Zweitsprache zurückgreift, die er in einer normgerechten Form anzuwenden versteht bzw. die ihm als sprachlich korrekt bekannt sind. Dadurch ist das Sprachverhalten des Lernenden sehr beschränkt und wird in der Regel von Kommunikationspartnern als einseitig empfunden.

Fehler, die bei Anwendung einer Zweitsprache entstehen, sind somit das Ergebnis unterschiedlicher Prozesse und Strategien. Da jedoch jeder Lernende von seiner individuellen Biographie geprägt ist, sind auch die Prozesse und Strategien, die im Laufe des Zweitspracherwerbs entwickelt werden, unterschiedlich. Dies wiederum hat nach Aussagen der Interlanguagehypothese zur Folge, daß die Fehler eines Lernenden einer bestimmten Zielsprache nicht unbedingt mit den Fehlern eines anderen Lernenden dieser Sprache übereinstimmen, auch wenn beide Personen die gleiche Erstsprache sprechen.

\subsection{Fehlererklärungsfaktoren}

Nach derzeitigem Forschungsstand werden interlanguages durch sechs psycholinguistische Faktoren ${ }^{413}$ gekennzeichnet, deren Einfluß von Lernenden zu Lernenden variiert.

\begin{tabular}{|c|c|c|c|c|c|}
\hline $\begin{array}{l}\text { 1. language } \\
\text { transfer }\end{array}$ & $\begin{array}{l}\text { 2. transfer of } \\
\text { training }\end{array}$ & $\begin{array}{ll}\text { 3. } & \text { strategies of } \\
\text { second language } \\
\text { learning }\end{array}$ & $\begin{array}{l}\text { 4. } \begin{array}{l}\text { strategies of } \\
\text { second language } \\
\text { communication }\end{array}\end{array}$ & $\begin{array}{l}\text { 5. overgeneralization } \\
\text { of target language } \\
\text { material }\end{array}$ & $\begin{array}{l}\text { 6. lack of } \\
\text { contrast }\end{array}$ \\
\hline & & $\begin{array}{l}3.1 \text { simplification } \\
3.2 \text { complexing }^{414}\end{array}$ & $\begin{array}{l}4.1 \text { formal } \\
\text { reduction strategies } \\
4.2 \begin{array}{l}\text { functional } \\
\text { reduction strategies }\end{array} \\
4.3 \begin{array}{l}\text { productive achieve- } \\
\text { ment strategies }\end{array} \\
4.4 \begin{array}{l}\text { receptive achieve- } \\
\text { ment strategies }\end{array}\end{array}$ & & \\
\hline
\end{tabular}

413 In der ursprünglichen Version der Interlanguagehypothese werden nur fünf Faktoren genannt. Die als lack of contrast bezeichnete Erscheinung wird erst in den späteren Fassungen der Interlanguagehypothese bzw. in den daraus abgeleiteten Hypothesen erwähnt. 


\subsection{1 language transfer}

Der erste Faktor wird als language transfer bezeichnet. Dieser Begriff umfaßt sowohl Übertragungen aus der Erstsprache als auch solche aus anderen Sprachen. Letztere finden vor allem dann statt, wenn der Lernende die Ähnlichkeit zwischen einer ersten und einer zweiten Zweitsprache als stärker wahrnimmt als die Ähnlichkeit zwischen seiner Erstsprache und der zweiten Zweitsprache. So neigen z. B. deutsche Muttersprachler, die Italienisch als zweite romanische Sprache lernen, eher zu Übertragungen aus dem Französischen als zu Übertragungen aus dem Deutschen oder Englischen. Language transfer wird begünstigt, wenn der Lernende in der Erstsprache oder in der ersten Zweitsprache einen sogenannten point of reference findet. Dies sind sprachliche Elemente anderer Sprachen, die der Lernende in der Zielsprache wiederzuerkennen glaubt.

\subsection{2 transfer of training}

Die Bezeichnung transfer of training umfaßt Fehler, die auf die Gestaltung des Fremdsprachenunterrichts zurückzuführen sind. Dazu gehören unter anderem die Wahl der Lehrmaterialien, der Aufbau des Unterrichts, der Einsatz von Medien, die Sprachform des Unterrichtenden und die Auswahl der Übungen. So kann z. B. die Überrepräsentation der progressive form im Englischen deutscher Muttersprachler durch die besonders intensive Vermittlung dieser Form im Englischunterricht hervorgerufen werden.

\subsection{3 strategies of second language learning}

Corder definiert diesen Faktor als "the mental processes whereby a learner creates for himself or discovers a language system underlying the data he is exposed to. “415 Hierzu zählen alle Strategien, die der Lernende zur Aufnahme des zweitsprachlichen Materials verwendet.

\subsubsection{1 simplification}

Fehler der Kategorie simplifications liegen dann vor, wenn der Lernende versucht, sprachliche Elemente zu vereinheitlichen, weil die Vielzahl der unterschiedlichen Informationen nicht verarbeitet werden kann. Dazu gehört u. a. die Fehlleistung, die Vergangenheitsformen unregelmäßiger Verben im Englischen durch regelmäßige Formen zu ersetzen, wie im Beispiel: go - goed.

\footnotetext{
414 In der Fachliteratur werden in diesem Zusammenhang ausschließlich deutsche Begriffe benutzt, das Wort complexing wird in vorliegender Arbeit als Übersetzung des deutschen Wortes ,Komplexierung'verwendet.

${ }^{415}$ Corder 1977, 12
} 


\subsubsection{2 complexing ${ }^{416}$}

Unter complexing ist der Versuch zu verstehen, eine neue zweitsprachliche Struktur dadurch zu erlernen, daß gleichzeitig mit ihr verwandte zweitsprachliche Strukturen erlernt werden. So zeigte sich beispielsweise, daß viele Lernende während der Behandlung des Gerundiums im Unterricht gleichzeitig auch darum bemüht waren, Partizipialformen zu erlernen. Bei der Anwendung dieser Formen kommt es dann jedoch häufig zu Verwechslungen der einzelnen Formen.

Fehler: $\quad$ I used to drinking a cup of tea every morning.

Korrektur: I I used to drink a cup of tea every morning.

Diese Fehlleistung geht offenbar darauf zurück, daß der Lernende bei der Behandlung des Themas ,Gerundium` erfahren hat, daß Präpositionen in Verbindung mit Verben das Gerundium erfordern, wie etwa in dem Satz I'm interested in learning Spanish. Dabei hat der Betreffende bei der Formulierung seiner Äußerung jedoch nicht berücksichtigt, daß die Präposition to Bestandteil des Infinitivs ist und ihr daher nicht das Gerundium folgen kann.

\subsection{4 strategies of second language communication}

\subsubsection{1 formal reduction strategies}

Das Sprachverhalten der Lernenden wird als formal reduction strategy bezeichnet, wenn zweitsprachliche Regeln nicht beachtet oder Elemente, die als kommunikativ unwichtig betrachtet werden bzw. Ausdrucksschwierigkeiten bereiten, von den Lernenden ausgelassen werden. Diese Auslassung einzelner Elemente wird durch den Begriff omission of language items gekennzeichnet.

Auf lautlicher Ebene gehört hierzu u. a. das Auslassen des Pluralmorphems , s nach dem interdentalen Reibelaut $/ \theta /$, das bei deutschen Englischlernenden häufig zu beobachten ist.

Beispiel: I have lived in Berlin for two months.

Fehler: $\quad$ I have lived in Berlin for two $[\ldots . . m \wedge n \theta]$.

Korrektur: I have lived in Berlin for two $[\ldots . . m \wedge n \theta$ s].

416 Dieser Fehlererklärungsfaktor wird hier nur aus Gründen der Vollständigkeit erwähnt. Er wird jedoch im Analysekonzept dieser Arbeit nicht berücksichtigt, weil bis heute in der Zweitspracherwerbsforschung die Auffassung vertreten wird, daß die Simplifizierung als weitaus wichtigere Lernstrategie zu betrachten ist. 
Auf syntaktischer Ebene gehört dazu der bei russischen Englischlernenden oftmals auftretende Verzicht auf den Gebrauch des unbestimmten Artikels.

Fehler: $\quad$ I saw Ø man walking over Ø street.

Korrektur: I saw a man walking over the street.

\subsubsection{2 functional reduction strategies}

Functional reduction strategies bezeichnen Strategien, mit deren Hilfe der Lernende versucht, die kommunikative Aufgabe, die zu bewältigen ist, den Ausdrucksmöglichkeiten seiner interlanguage anzupassen. Sie werden in nachfolgend aufgeführte Unterkategorien unterteilt.

a) topic avoidance

Der Begriff topic avoidance bezeichnet den Versuch, Themen auszuweichen, für die dem Lernenden nicht die nötigen Ausdrucksmittel zur Verfügung stehen. Da die Lernenden in der Regel darum bemüht sind, ihr Vermeidungsverhalten zu verbergen, gehen Themenvermeidungen häufig mit Ablenkungsstrategien einher, mit deren Hilfe der Lernende dem bevorstehenden Thema ausweichen kann. Zu diesen Ablenkungsstrategien gehören unter anderem Redewendungen, die der Lernende sprachlich korrekt beherrscht und deren Wirksamkeit bereits in mehreren Kommunikationssituationen erprobt werden konnte. Dies sind Redewendungen wie beispielsweise by the way, I've been wanting to tell you ...

b) message abandonment

Unter message abandonment ist der Abbruch einer sprachlichen Äußerung zu verstehen, der daraus resultiert, daß dem Lernenden zur Fortsetzung seiner Äußerung die sprachlichen Ausdrucksmittel fehlen. In der Regel wird der Abbruch der Äußerung durch eine idiomatische Redewendung angekündigt, die der Lernende sprachlich korrekt anwenden kann, wie etwa die von Englischlernenden häufig verwendeten Formulierungen that's all oder that's it.

c) meaning replacement

Meaning replacement liegt dann vor, wenn der Lernende nicht über die Ausdrucksmittel verfügt, die er zur Vermittlung seiner Aussage benötigt und diese daher so verändert, daß sie mit Hilfe des bereits erworbenen Wissens verdeutlicht werden kann.

Beispiel: Ein Lernender berichtet über seinen Urlaub in Sydney. Er beabsichtigt zu sagen „Bei meiner Ankunft war die Luft klar und trocken. Am nächsten Tag wurde es jedoch sehr schwül.“ Da dem Lernenden jedoch das englische Wort für ,schwül‘ (humid) nicht bekannt ist, übersetzt er den zweiten 
Satz wie folgt: But on the next day the weather was not so good. Diese Übersetzung resultiert in einer Bedeutungsänderung, da das Wort humid semantisch nicht unbedingt negativ besetzt ist und der konkrete Inhalt des deutschen Wortes ,schwül' durch die Verwendung des Ausdrucks not so good nicht vermittelt wird.

d) modality reduction

Von modality reduction spricht man in der Interlanguage-Forschung, wenn der Lernende es unterläßt, den Beziehungsaspekt in der sprachlichen Interaktion durch die Wahl einer geeigneten Sprechaktmodalität angemessen zum Ausdruck zu bringen.

Fehler: I want to ask you something.

Korrektur: Oh, by the way, Colin, I have been wanting to ask you something. ${ }^{417}$

e) personality reduction

Personality reduction bezeichnet eine Reduktionsstrategie, die bei der Selbstdarstellung der Lernenden auftritt: Viele Lernende beschränken sich bei der Darstellung ihrer eigenen Person aufgrund mangelnden Sprachwissens auf einfache Aussagen, die keiner elaborierten Sprachkenntnisse bedürfen. Dazu gehören u. a. folgende Beispiele: My name is ..., I come from ...

\subsubsection{3 productive achievement strategies}

Unter achievement strategies sind Strategien zu verstehen, mit deren Hilfe der Lernende Kommunikationsprobleme durch Erweiterung seiner kommunikativen Ressourcen löst. Die achievement strategies werden unterteilt in productive und receptive achievement strategies ${ }^{418}$. Erstere sind dadurch gekennzeichnet, daß der Lernende versucht, Ausdrucksdefizite durch Kreativität zu überwinden, das heißt durch den Rückgriff auf Elemente der Erstsprache oder auf bereits erworbene Elemente der Zielsprache bzw. anderer Zweitsprachen.

$\mathrm{Zu}$ den productive achievement strategies gehören folgende Strategietypen, die unterschiedliche Formen der Kompensation darstellen.

417 Wenngleich die Aussage rein linguistisch betrachtet nicht als falsch bezeichnet werden kann, handelt es sich hierbei dennoch um einen Normverstoß, weil das Beispiel einem sehr formalen Gespräch entstammt.

418 Die receptive achievement strategies werden im Analysekonzept der vorliegenden Arbeit nicht berücksichtigt, weil sie in der Regel nicht in Form von normabweichenden Äußerungen auftreten. 
a) language switch

Unter language switch ist der bewußte Rückgriff auf die Erst- oder eine andere Zweitsprache zu verstehen. Dabei weiß der Lernende, daß das Kompensationselement nicht der Zielsprache angehört.

Fehler: Do you want any ... er ... Zinsen.

Korrektur: Will you charge any interest? ${ }^{419}$

b) interlingual transfer (adaption of rules of the mother tongue or another foreign language, also foreignizing)

Bei diesem Strategietyp handelt es sich um Übertragungen aus der Erstsprache oder aus anderen Zweitsprachen. Der Unterschied zu dem zuvor beschriebenen Strategietyp language switch besteht darin, daß die übernommenen Elemente dem Interlanguagesystem adaptiert werden. Ein eindeutiges Beispiel bietet das sogenannte foreignizing, bei dem lexikalische Elemente der Erstsprache phonologisch-morphologisch adaptiert werden, wie in dem folgenden Beispiel eines deutschen Französischlernenden:

Fehler: $\quad$ La société moderne est caractérisée par une escalation dans la brutalité.

Korrektur: La société moderne est caractérisée par une escalade dans la brutalité.

Das Wort escalation existiert im Französischen nicht, es wurde von dem Lernenden aus dem deutschen Lexem ,Eskalation` abgeleitet. Das Lexem, das der Lernende hätte verwenden sollen, lautet escalade.

c) borrowing

Borrowing findet statt, wenn der Lernende Lexeme aus anderen Sprachen übernimmt und dabei davon ausgeht, daß diese Elemente in der Zweitsprache anwendbar sind.

Fehler: Deme una birra, por favor.

Korrektur: Deme una cerveza, por favor. ${ }^{420}$

d) inter-/intralingual transfer (generalization of an interlanguage rule caused by mother tongue or another foreign language)

Im Unterschied $\mathrm{zu}$ den vorgenannten inter- und intralingual bedingten Übertragungen ist dieser Strategietyp dadurch gekennzeichnet, daß der Lernende Elemente aus der Erstsprache oder aus anderen Zweitsprachen übernimmt, die tatsächlich in optisch oder akustisch sehr ähnlicher Form in der Zielsprache existieren. Diese Elemente werden jedoch in der Zielsprache nicht in denselben Zusammen-

\footnotetext{
419 Wenngleich der Kodewechsel nur bei dem Lexem ,Zinsen` deutlich wird, bedarf auch der vorhergehende Satzteil einer Korrektur.

${ }^{420}$ Bei der Entlehnung handelt es sich um das Element birra. Vgl. hierzu Bausch / Kasper 1979, 19.
} 
hängen verwendet wie in den Sprachen, denen sie entnommen wurden. Ein Beispiel bietet die folgende Äußerung eines deutschen Englischlernenden.

Fehler: Have you got a card for a match?

Korrektur: Have you got a game of cards?

Offensichtlich wollte der Lernende fragen: „Haben Sie Karten zum Spielen?“ Der Transfer äußert sich hierbei in dem englischen Wort card, das den Lernenden an das deutsche Wort Karte erinnert. Das Wort ,Spielkarten“ wird im Englischen jedoch nur im Plural verwendet. Das Wort card ohne das Pluralmorphem $-s$ hingegen hat im Englischen die Bedeutung der deutschen Wörter ,Pappe` oder ,Postkarte“.

e) generalization of interlanguage rules/items in new contexts

Dieser Generalisierungstyp unterscheidet sich von den vorgenannten dadurch, daß Regeln und Elemente der Zweitsprache, die in bestimmten Zusammenhängen richtig sind, auf Zusammenhänge übertragen werden, in denen sie unangebracht sind, wie im folgenden Beispiel.

Fehler: $\quad$ I don't know what is it.

Korrektur: I don't know what it is.

Die Wortfolge what is it kann bei Verwendung am Satzanfang durchaus normgerecht sein. Der vorangestellte Satzteil I don't know erfordert jedoch eine Inversion als Komplement.

f) paraphrase (either circumlocution or conversiness)

Von paraphrase spricht man, wenn der Lernende versucht, zweitsprachliche Elemente, die ihm unbekannt sind, durch bekannte Elementen zu umschreiben. Beispiel: a thing you dry your hands with statt a towel.

g) word coinage

Word coinage liegt dann vor, wenn der Lernende ein ihm unbekanntes Element der Zielsprache dadurch kompensiert, daß er Elemente, die er kennt, so zusammensetzt, daß dem Gesprächspartner die Bedeutung verständlich wird. Das Element, das der Lernende geschaffen hat, ist dabei jedoch in der von ihm verwendeten Form nicht Bestandteil der Zielsprache. Beispiel: air thing statt balloon.

h) restructuring

Als restructuring wird der Versuch bezeichnet, Äußerungen, die aufgrund mangelnder Sprachkenntnisse nicht in der geplanten Form zum Ende geführt werden können, so zu verändern, daß der Sinn der geplanten Äußerung dennoch deutlich wird. 
Fehler: $\quad$ My parents has... I have ... er ... four elder sisters.

Korrektur: My parents had had four daughters before they had me.

i) discourse related strategy

Eine Kommunikationsstrategie wird als discourse related strategy bezeichnet, wenn der Lernende nicht versucht, seine Kommunikationsprobleme durch den Rückgriff auf bereits vorhandenes Wissen selbständig zu lösen, sondern seine Sprachprobleme offen zu erkennen gibt. Zwei Erscheinungsformen dieses Strategietyps sind bekannt: der sogenannte appeal to authority und das als admission of ignorance bezeichnete Verhalten. Appeal to authority bedeutet, daß der Lernende seinen Kommunikationspartner direkt um sprachliche Hilfe bittet, etwa weil die zielsprachliche Bedeutung eines bestimmten Lexems vergessen wurde oder weil ein Gespräch oder ein schriftlicher Kommunikationsversuch in der Zielsprache nicht fortgesetzt werden kann.

Beispiel: $\quad$ Hello Sina, how are you? I hope you are ok. I'm ok. Can you help me at English? ${ }^{421}$

Der Begriff admission of ignorance besagt, daß der Lernende mitteilt, daß er die beabsichtigte Äußerung nicht mit den ihm zur Verfügung stehenden sprachlichen Mitteln verdeutlichen kann, ohne dabei um sprachliche Hilfe zu bitten.

Beispiel: $\quad$ Now, I think I have write enough because I don't know what I can write till then.

Wie auch im obigen Beispiel, ist diese Erscheinung häufig dann zu beobachten, wenn die Kommunikationspartner nicht mündlich miteinander in Kontakt treten, sondern in schriftlicher Form, und der Adressat der Äußerung daher nicht unmittelbar sprachliche Hilfe leisten kann.

\subsubsection{4 receptive achievement strategies}

a) inferencing

Die als inferencing bezeichnete Strategie unterscheidet sich von den zuvor genannten productive achievement strategies dadurch, daß sie nicht zur Überwindung von Kommunikationsproblemen während des Kommunikationsverlaufs angewandt wird, sondern zum Verständnis einer mündlichen oder schriftlichen zielsprachlichen Äußerung. Dabei besagt der Begriff inferencing, daß der Lernende versucht, die Bedeutung einer zweitsprachlichen Äußerung durch Rückgriff auf vorhandenes Interlanguage-Wissen bzw. auf erstsprachliches Wissen sowie auf kontextuelle Hinweise zu erschließen. Fehler, die bei der Anwendung dieser Strategie entstehen, beruhen in der Regel darauf, daß die Bedeutung eines Elements falsch interpretiert wird, weil andere Elemente der Zielsprache oder der Erstsprache diese Deutungsweise suggerieren. Ein Beispiel bietet die folgende Äußerung, die auf

421 Das Beispiel stammt aus der Untersuchung, die der vorliegenden Arbeit zugrundeliegt. 
dem Versuch basiert, den deutschen Satz „Mein Bruder ist künstlerisch veranlagt“ ins Englische zu übersetzen.

Fehler: $\quad$ My brother is very artful.

Korrektur: My brother is very artistic.

Hier geht die Abweichung auf die Tatsache zurück, daß der Lernende glaubte, das deutsche Lexem ,künstlerisch` mit dem englischen Wort artful übersetzen zu können, weil bei den meisten englischen Lexemen, die mit der Vorsilbe art- beginnen, dieser Vorsilbe in der deutschen Übersetzung das Lexem ,Kunst' entspricht.

b) monitoring

Der Begriff monitoring, der auf das Monitor-Modell Krashens zurückgeht, beinhaltet folgendes: Lernende, die Zweitsprachen nicht auf natürliche Weise, sondern in gesteuerter Form erwerben, entwickeln eine sprachliche Kontrollinstanz, die als Monitor bezeichnet wird. Diese Kontrollinstanz befähigt den Lernenden dazu, seine zielsprachlichen Äußerungen in bezug auf sprachliche Korrektheit zu überprüfen und ermöglicht es ihm, Fehler nach ihrer Entstehung selbst zu korrigieren. Dieser Monitor wird bei der Produktion zweitsprachlicher Äußerungen jedoch nur dann aktiviert, wenn der Lernende die sprachlichen Regeln oder Elemente, die er zur Verdeutlichung seiner Aussage benötigt, kennt und ihm genügend Zeit zur Anwendung dieser Regeln und Elemente zur Verfügung steht. Außerdem muß dem Lernenden bewußt sein, daß die sprachliche Korrektheit seiner zielsprachlichen Äußerungen Beachtung findet, um ihn für die Notwendigkeit einer korrekten Ausdrucksweise zu sensibilisieren.

Fehler, die darauf beruhen, daß der Monitor nicht aktiviert werden konnte, weil eine oder mehrere dieser Voraussetzungen nicht erfüllt waren, äußern sich in der Regel entweder in Form gravierender Normabweichungen oder in Form sogenannter slips of the pen bzw. slips of the tongue. Zu gravierenden Normabweichungen kommt es, wenn die Monitorsperre auf Unkenntnis einer zielsprachlichen Regel zurückzuführen ist. Ein Beispiel bietet folgende Normabweichung, die daraus entstand, daß der Lernende den deutschen Satz „Das Auto wird gerade repariert“ ins Englische übertragen wollte, ohne über das notwendige Wissen zur Bildung von Passivsätzen im Englischen zu verfügen.

Fehler: $\quad$ The car is repairing.

Korrektur: The car is being repaired.

Slips of the pen bzw. slips of the tongue entstehen häufig dann, wenn Zeitmangel dafür verantwortlich ist, daß der Monitor nicht abgerufen werden konnte. Häufig zu beobachten sind beispielsweise Auslassungen sprachlicher Elemente in zeitlich begrenzten Prüfungen, die bei ausreichend bemessenem Zeitvolumen vermieden worden wären. Fehler dieser Art äußern sich u. a. in folgender Form: 
Fehler: I think the author right.

Korrektur: I think the author is right.

Ähnliche Fehlertypen sind jedoch auch dann zu beobachten, wenn die Zielsprache in einer entspannten Atmosphäre zur Anwendung kommt und der Lernende weiß, daß sprachliche Korrektheit von untergeordneter Bedeutung ist. In solchen Situationen kommt es häufig dazu, daß Lexemendungen, wie etwa die Endsilbe -ly, entfallen, obwohl der Betreffende diese Strukturelemente in formalen Sprachsituationen verwendet hätte.

Fehler: Why are you walking so slow?

Korrektur: Why are you walking so slowly?

\subsection{5 overgeneralization of target language material}

Der Begriff overgeneralization of target language material bezeichnet eine Abweichungsform, die darauf basiert, daß zielsprachliche Regeln oder Elemente auf Strukturen übertragen werden, auf die sie nicht anwendbar sind. Ein typisches Beispiel bietet die Doppelmarkierung des simple past in Fragesätzen, wie etwa in folgender Äußerung.

Fehler: $\quad$ What did he intended to say?

Korrektur: What did he intend to say? ${ }^{422}$

Dabei legt Selinker in der ursprünglichen Version der Interlanguagehypothese nicht eindeutig fest, ob die Entstehung dieser Abweichungsform auf unbewußtes oder auf bewußtes Handeln zurückzuführen ist. Auch bei dem Versuch zu ergründen, inwieweit Prozesse und Strategien der Kontrolle des Lernenden unterliegen, welcher seit der Entwicklung der Interlanguagehypothese einen wichtigen Forschungsaspekt der Zweitspracherwerbsforschung darstellt, konnten zu diesem Punkt keine überzeugenden Ergebnisse erzielt werden. Die Definition des Begriffs overgeneralization ist daher bis heute als ergänzungsbedürftig zu betrachten. Da die Mehrheit der Forschungsergebnisse der vergangenen Jahre jedoch trotz partiell bestehender Zweifel an ihrer Repräsentativität vermuten läßt, daß Prozesse beim Zweitspracherwerb überwiegend unbewußt verlaufen, wird der Fehlertyp auch in der vorliegenden Arbeit als Abweichungsform betrachtet, die sich der Kontrolle des Lernenden entzieht. Dabei unterscheidet sich diese Form der overgeneralization von dem terminologisch identischen Fehlertyp der Kontrastivhypothese dadurch, daß die Entstehung dieser Abweichungsform aus der Sicht der Kontrastivhypothese auf erstsprachlichen Einfluß zurückzuführen ist. Der Interlanguagehypothese nach sind für die Entstehung der overgeneralizations hingegen ausschließlich intralinguale Faktoren verantwortlich.

422 Selinker 1972, 218 


\subsection{6 lack of contrast}

Die als lack of contrast bzw. im deutschen Sprachraum als Kontrastmangel bezeichnete Erscheinung wurde erst in späteren Entwicklungsphasen in das Konzept der Interlanguagehypothese aufgenommen. Der Kontrastmangel basiert auf interlingualen und/oder intralingualen Faktoren. Diese Erscheinung wird auch als Ranschburg'sches Phänomen bezeichnet. Juhász definiert den Fehlerentstehungsfaktor wie folgt: „Bei gleicher Intensität und gleichem Gefühlswert werden aus einer gleichzeitig (oder nahezu gleichzeitig) einwirkenden Menge von Reizen die einander unähnlichen bevorzugt, während die einander ähnlichen bzw. identischen aufeinander hemmend einwirken.“ ${ }^{423}$ Kontrastmangel kann „optisch, akustisch oder auch begrifflich begründet““424 sein.

Fehler: $\quad$ You will become a letter tomorrow.

Korrektur: You will get a letter tomorrow.

Im vorliegenden Beispiel, das bei einem deutschen Englischlernenden beobachtet wurde, beruht die Fehlleistung auf der Ähnlichkeit zwischen dem deutschen Wort ,bekommen' und dem englischen Verb to become.

\subsection{Exkurs: Differenzierung der Begriffe ,Prozesse' und ,Strategien'}

Nach Loslösung des Begriffs ,Strategie“ von der traditionellen Fehlerlinguistik Mitte der siebziger Jahre wurde der Begriff in der Zweitspracherwerbsforschung definitorisch ausgedehnt. Dies führte zu wachsender Unklarheit darüber, inwiefern Strategien sich von anderen Mitteln der Sprachproduktion unterscheiden bzw. welche Bedeutung dem Begriff letztendlich zugrunde liegt. ${ }^{425}$ Die angesichts der vielfältigen Beiträge deutlich werdende Tendenz, den Strategiebegriff neu zu definieren bzw. vorhergehende Definitionen zu verbessern, erschwerte die Unterscheidung zwischen Strategien und Prozessen zunehmend. Vereinzelt führte sie gar zu einer synonymen Verwendung beider Begriffe. ${ }^{426}$ Während Selinker ${ }^{427}$ davon ausgeht, daß Strategien sowohl bewußt als auch unbewußt verwendet werden können, definieren A. Knapp-Potthoff / K. Knapp Strategien als „,... kognitive Operationen, die vom Lerner zielgerichtet und intentional angewendet werden. Strategien sind demzufolge“ vom Lernenden wählbar, d. h., die Anwendung von Strategien ist vom Lerner manipulierbar, sie sind ,lernund lehrbar.“428 Prozesse hingegen definieren Knapp-Potthoff/Knapp als „mentale Operationen, die

\footnotetext{
423 Juhász 1983, 44

424 Hecht / Green 1983, 44

${ }^{425} \mathrm{Zu}$ einer ausführlichen Diskussion der mit der Strategiedefinition verbundenen Probleme siehe Zimmermann 1997, 95 113.

426 vgl. u. a. Hecht / Green 1983, 44 ff

427 vgl. Selinker 1972, $209 \mathrm{ff}$ u. Selinker / Swain / Dumas 1975, $139-152$

428 Knapp-Potthoff / Knapp 1982, 133 ff
} 
sich auf das automatisch ablaufende Erkennen und Speichern von sprachlichen Elementen und Regelmäßigkeiten und auf deren automatische Aktualisierung bei Produktion und Rezeption beziehen. Der Ablauf von Prozessen vollzieht sich unbewußt und vom Lerner unbeeinflußbar.“‘429

Diese Definition schließt nicht aus, daß Strategien auch routiniert verwendet werden können. KnappPotthoff/Knapp nehmen in ihrer definitorischen Abgrenzung die Sicht des Lernenden ein, wohingegen in der früheren Forschung Strategien zumeist an fehlerhaften Äußerungen der Lernenden festgemacht und beinahe ausschließlich aus der Sicht des Lehrers bzw. Analysators untersucht wurden. ${ }^{430}$ Ein Versuch zur Lösung des definitorischen Problems, der in jüngerer Zeit im Rahmen der deutschen Strategieforschung ${ }^{431}$ unternommen wird, ist die Aufspaltung des Strategiebegriffs in a) die Ebene des beobachtbaren Verhaltens und Handelns, b) die Ebene des nicht unmittelbar beobachtbaren kognitiven Prozesses und c) die Ebene der mentalen (und damit nicht unmittelbar beobachtbaren) Repräsentationen im Gedächtnis. Dabei werden die Strategien der ersten Ebene als ,manifeste Strategien“ oder ,Techniken', die der zweiten Ebene als ,mentale Strategien“ und die der dritten Ebene als ,Strategiewissen` bezeichnet. ${ }^{432}$

Wenngleich diese Differenzierung Überlappungen zwischen den einzelnen Ebenen nicht ausschließen kann, verdeutlicht die Unterscheidung das Bemühen, die unterschiedlichen Aspekte des Spracherwerbs differenziert und umfassend zugleich zu erfassen. Somit wird die Tendenz erkennbar, pauschale Definitionen der Begriffe ,Strategie‘ und ,Proze $\beta^{\star}$ zu vermeiden und stattdessen Definitionen aus dem Blickpunkt des Spracherwerbs zu entwickeln. Der Interlanguagehypothese in ihrer gegenwärtigen Form liegt ein Strategie-Prozeß-Verständnis zugrunde, das der von KnappPotthoff/Knapp vorgeschlagenen Differenzierung entspricht und damit eine Erweiterung der ursprünglichen Verwendung darstellt. Betont wird in der aktuellen Form der Interlanguagehypothese insbesondere, daß strategische Prozesse durch Übung automatisiert werden können und in der Folge auch unbewußt ablaufen. ${ }^{433}$ Die Interlanguage-Forschung unterscheidet, wie oben erwähnt, zwischen Lern- und Kommunikationsstrategien. Lernstrategien ,sind konstitutive Elemente des zweitsprachlichen bzw. Interlanguage-Regelbildungsprozesses, die der Lerner systematisch zur Bildung, Überprüfung und Revision von Hypothesen über die Zweitsprache einsetzt.“434 In der ursprünglichen, von Selinker begründeten Form der Interlanguagehypothese konzentrierte sich die Diskussion der Frage,

\footnotetext{
429 ebd.

430 vgl. Selinker 1969, 67 ff

431 vgl. Rampillon / Zimmermann 1997, 100 - 113

432 vgl. ebd.

433 vgl. Pauels 1995, 628

434 vgl. Bausch / Kasper 1979, 17
} 
welche Faktoren Lernstrategien determinieren, im wesentlichen auf Simplifizierungen und Komplexierungen. $^{435}$

Die heutige Sichtweise ist wesentlich differenzierter. Lernstrategien können nach derzeitigem Forschungsstand in kognitive, metakognitive und soziale ${ }^{436}$ sowie rezeptive Strategien unterteilt werden. Letztere sind passiv bzw. inferentiell und erfordern keine aktive Gesprächsteilnahme. Hierbei „sucht der Lerner vielmehr in seinem Input (d. h. in den Äußerungen von Muttersprachlern) nach verifizierenden Daten und zieht Schlüsse für die Angemessenheit oder Unangemessenheit seiner Hypothesen. ${ }^{“ 437}$ Die ersten drei Strategien ${ }^{438}$ erfordern Lerneraktivität. Kognitive Strategien bestimmen die Vorgehensweise bei der Erarbeitung von Lernmaterial und Lernaufgaben. Dies kann mental durch die Bildung von Schemata geschehen oder auch dadurch, daß das zu erlernende Material in sinnvolle Kategorien geordnet wird. ${ }^{439}$ Die sozialen Strategien kommen in der Interaktion mit anderen Personen zum Tragen oder „wirken auf die Kontrolle affektiver Faktoren ein.“440

Metakognitive Lernstrategien bilden nach heutigem Wissensstand einen integralen Bestandteil des Sprachverarbeitungsmechanismus. Sie sind an der erfolgreichen Bewältigung aller kognitiven Lernaufgaben beteiligt und können daher als exekutive Fertigkeiten verstanden werden, die der Analyse, Reflexion, Planung und Kontrolle dienen. Damit wirken sie direkt auf die Informationsverarbeitung ein. ${ }^{441}$ Kommunikationsstrategien werden im Gegensatz zu Lernstrategien zur Bewältigung aktueller Kommunikationsschwierigkeiten bzw. zur Überbrückung von Wissenslücken eingesetzt, wenn der Lernende das Kommunikationsproblem als solches erkannt hat. Sie werden in der Regel in funktionale und formale Reduktionsstrategien unterteilt. ${ }^{442}$ Formale Reduktionsstrategien werden eingesetzt, wenn der Lernende auf den Gebrauch von Interlanguage-Elementen verzichtet, die er „für kommunikativ unwichtig hält und/oder die ihm Ausdrucksschwierigkeiten bereiten. ${ }^{“ 443}$ Der Lernende versucht somit, „ein ausreichendes Maß an fluency sicherzustellen. ““444 Als Beispiel hierfür nennen Bausch/Kasper den

\footnotetext{
435 vgl. Selinker 1972, $219 \mathrm{ff}$

436 vgl. Chamot / O’Malley 1990, $197 \mathrm{ff}$

437 Desgranges 1990, 28

438 Knapp-Potthoff / Knapp nehmen eine andere Kategorisierung vor. Ihnen zufolge können die aktiven Lernstrategien in produktiv-interaktive, metasprachlich-interaktive und diskurs-interaktive Lernstrategien unterteilt werden. Allerdings bezieht sich die von den Autoren vorgeschlagene Kategorisierung auf den Einsatz von Strategien beim ungesteuerten Zweitspracherwerb. Vgl. Knapp-Potthoff / Knapp 1982, 135.

439 vgl. Pauels 1995, 628

440 ebd.

441 vgl. Dechert 1997, 13. Zusammenfassungen der wichtigsten metakognitiven Strategien finden sich bei Chamot / O'Malley 1990, 17 u. Dechert 1997, 13 - 14.

442 In der heutigen Interlanguage-Forschung werden Reduktionsstrategien nicht nur in formale und funktionale Strategien unterteilt. Sie werden darüber hinaus von den achievement strategies (Elaborationsstrategien) unterschieden. In der ursprünglichen Version der Interlanguagehypothese hingegen bildeten die achievement strategies eine Untergruppe der funktionalen Reduktionstrategien. Vgl. Bausch / Kasper 1979, 17 ff.

443 ebd., 17

444 Kostrzewa / Cheon-Kostrzewa 1996, 89
} 
Verzicht auf den Gebrauch des Stoßtons im Dänischen, bzw. auf syntaktischer Ebene den Gebrauch des unbestimmten und bestimmten Artikels. ${ }^{445}$

Funktionale Reduktionsstrategien stellen eine Anpassung der zu bewältigenden kommunikativen Aufgabe an die Ausdrucksmöglichkeiten der interlanguage des Lernenden dar. Bis heute konnte in der Interlanguage-Forschung die Frage nicht befriedigend beantwortet werden, inwieweit aus der Verwendung von Kommunikationsstrategien, die ursprünglich nicht dem Erlernen von Regeln dienten, dennoch ein Lernprozeß resultieren kann. Trotz unzureichender empirischer Daten neigt die Forschung verstärkt dazu, letzteres zu bestätigen. Knapp-Potthoff/Knapp gehen davon aus, daß „die Wahrnehmung von Diskrepanzen zwischen Kommunikationszielen und sprachlichen Möglichkeiten des Lerners vor allem dann, wenn es sich um wiederkehrende gleiche Diskrepanzen handelt ${ }^{\star 446}$, eine Motivation schaffen kann, „nicht ständig nach neuen ad-hoc Lösungen zu suchen, sondern seine Lernersprache langfristig zu verändern, also weiterzulernen.““447 Demnach ermöglichen Kommunikationsstrategien bewußtes Lernen, wenn mit ihrer Hilfe längerfristige Kommunikationskrisen überwunden werden können.

\subsubsection{Fossilisierung}

Der Begriff ,Fossilisierung` bezeichnet eine Erscheinung, die nach Selinker neben den zuvor dargestellten Prozessen und Strategien ein wesentliches Merkmal von interlanguages darstellt. ${ }^{448}$ Unter Fossilisierungen sind Sprachformen zu verstehen, ,die weder der Norm der Zielsprache, noch dem aktuellen Lernstand der Lernenden entsprechen und sich darüber hinaus noch als äußerst resistent gegenüber Lernbemühungen erweisen. “449 Sie repräsentieren den Rückfall des Lernenden auf eine frühere, für überwunden gehaltene Lernstufe. Dieser Rückfall wird in der Regel als back-sliding bezeichnet und kann sowohl einzelne Strukturen und Elemente aller linguistischer Ebenen betreffen als auch „den gesamten Bereich der Lernersprache.“450 Als Beispiele nennt Selinker u. a. den Gebrauch des uvularen Lauts /r/ französischer Englischlernender und die Anwendung der Zeit-OrtReihenfolge deutscher Muttersprachler beim Gebrauch der englischen Sprache. ${ }^{451}$ Das Auftreten fossilisierter lernersprachlicher Strukturen ist insbesondere dann zu beobachten, wenn die Kommunikationsgegenstände, mit denen der Lerner konfrontiert wird, von ihm als schwierig eingestuft wird bzw. er sich

\footnotetext{
445 vgl. Bausch / Kasper 1979, 17

446 Knapp-Potthoff / Knapp 1982, 135

447 ebd.

448 vgl. Selinker 1972, $215 \mathrm{ff}$

449 Vogel 1990, 29

450 ebd., 31

451 vgl. Selinker 1972, 215. Der Begriff der Fossilisierung hat bis heute nicht an Aktualität verloren. Dies belegen u. a. Untersuchungen von Towell und Hawkins. Vgl. Towell / Hawkins 1994.
} 
„in einem Zustand der Angst befindet oder extrem gespannt ist.“452 Kommt es zu einer Stagnierung der Lernersprache, d. h., entwickelt sich die Lernersprache nicht mehr auf die Norm der Zielsprache hin, wird aber dennoch als „Kommunikationsmittel wie eine natürliche Sprache verwendet ${ }^{\star 453}$, so spricht man von einer „Pidginisierung ${ }^{\star 454}$ der Lernersprache.

Der Begriff der Fossilisierung in seiner gegenwärtigen Form ${ }^{455}$ erscheint erst in der präzisierten Fassung der Interlanguagehypothese und beruht auf den Ergebnissen, die beim Vergleich vorpubertärer kindlicher Sprachäußerungen mit der Lernersprache von Erwachsenen ermittelt wurden. In seinem ursprünglichen Interlanguage-Modell, das sich nur auf das Fremdsprachenlernen Erwachsener bezog, ging Selinker davon aus, daß Fossilisierungen nur bei postpubertären Lernenden zu beobachten sind und es nur einer geringen Minderheit von Zweitsprachenlernenden (etwa 5\%) gelingt, eine Zweitsprache bis zur vollständigen Beherrschung zu erlernen. ${ }^{456}$ Selinker, der aufgrund dieses Befunds die Existenz einer sogenannten latenten Psychostruktur bestätigt sah, die „als Analogiekonstrukt zum vorpubertären language aquisition device ${ }^{\text {، } 457} \mathrm{zu}$ betrachten ist, berief sich dabei insbesondere auf Forschungsergebnisse der Neurologen W. Penfield / E. Roberts ${ }^{458}$ und E. H. Lenneberg ${ }^{459}$ und übertrug diese auf den Zweitspracherwerb.

Penfield/Roberts hatten bei der Untersuchung von Aphasieerscheinungen festgestellt, „daß bis zum Abschluß der zerebralen Reifung im Pubertätsalter Aphasien behoben werden können, während dies im postpubertären Alter nicht mehr der Fall ist.“460 Diese Erscheinung wird von Lenneberg mit der Elastizität des kindlichen Gehirns erklärt, „,die bis zur Pubertät beiden Gehirnhälften die Steuerung der Sprachfunktionen überläßt. “461 Er führt das Nichtvorhandensein dieser Flexibilität des Gehirns nach Abschluß der Pubertät darauf zurück, daß ,der Lateralisierungsvorgang bzw. Spezialisierungsvorgang des Gehirns zu diesem Zeitpunkt abgeschlossen ist und also nunmehr die wichtigen Sprachfunktionen normalerweise linksseitig im Gehirn fixiert sind, und die rechte Gehirnhälfte diese Funktionen nicht mehr übernehmen kann. “462 Lenneberg überträgt diese Erkenntnis auf den gesamten Erstspracherwerb. Demzufolge gibt es auch beim Erstspracherwerb eine sogenannte sensible Phase bzw. kritische Periode, „die sich vom zweiten Lebensjahr, ab dem die Sprechmotorik und die Funktionsbereitschaft des

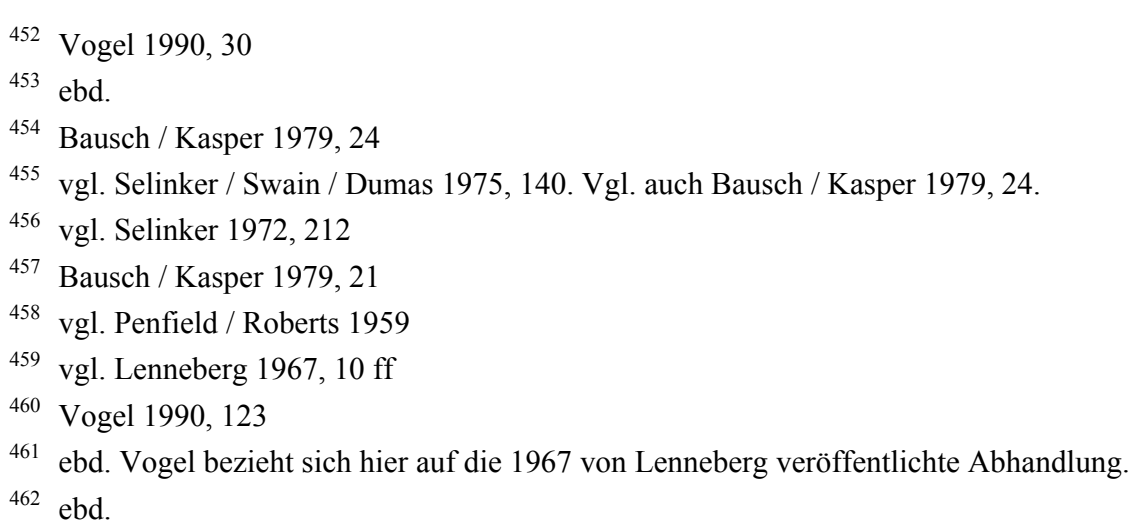


Zentralnervensystems verfügbar sind, bis zur Pubertät, dem Zeitpunkt an dem die endgültige Spezialisierung der Hemisphären abgeschlossen ist, erstreckt.“463

Nach Lenneberg ist es dem Menschen nur in dieser Phase möglich, eine Sprache in der Form zu erlernen, wie sie von einem Muttersprachler gesprochen wird. Nach Abschluß der Pubertät jedoch ,und dem Abschluß der zerebralen Dominanz ist der Mensch als sprachlich erwachsen anzusehen, und es ist ihm nicht mehr möglich, eine Sprache akzentfrei und ohne Mühe und Unterweisung auf natürlichem Wege lernen zu können. ““64 Das Auftreten von Fossilisierungen, das auch im vorpubertären kindlichen Zweitspracherwerb beobachtet werden konnte, zeigte jedoch, daß der Begriff der Fossilisierung und damit die gesamte Interlanguagehypothese auch auf den kindlichen Fremdsprachenlernenden übertragen werden mußte. Hierbei bezogen sich Kritiker der latenten Psychostruktur vor allem auf Forschungsergebnisse zur kortikalen Lateralisation. Diese zeigten, daß die Entstehung eines foreign accent aphatischen Befunden zufolge - auf die sich Selinker stützte - in der Pubertät im Alter zwischen 11 und 13 Jahren anzusetzen ist und in manchen Befunden bereits im Alter von 5 Jahren belegt ist. ${ }^{465}$ Diese Ergebnisse forderten eine Erweiterung des Interlanguage-Modells auf den präpubertären Lernenden.

Anhand von Untersuchungen wurde in den nachfolgenden Jahren schließlich u. a. auch von Selinker ermittelt, daß neben den neurophysiologischen Faktoren sozialpsychologische Aspekte maßgeblich an der Entstehung von Fossilisierungen beteiligt sind. ${ }^{466}$ Dem nachfolgend entwickelten Konzept lag folglich nicht nur der Faktor ,Alter' als maßgebliches Kriterium zur Entstehung von Fossilisierungen zugrunde. Einbezogen wurden nun auch sozialpsychologische Faktoren wie „die Kommunikationsbedürfnisse des Lerners, seine Identifizierung mit der und Akkulturation in die zweitsprachliche Kommunikationsgemeinschaft“ sowie die „Rückmeldung, die der Lerner in seiner Interaktion mit native speakers über sein Sprachverhalten erhält.“467 Wie später ermittelt werden konnte, führt „,das Zusammenwirken von vorwiegend positiver Rückmeldung auf der kognitiven und affektiven Ebene zur Stabilisierung und Fossilisierung von Interlanguage-Elementen“468, während negative Rückmeldungen eine Destabilisierung bewirken können.

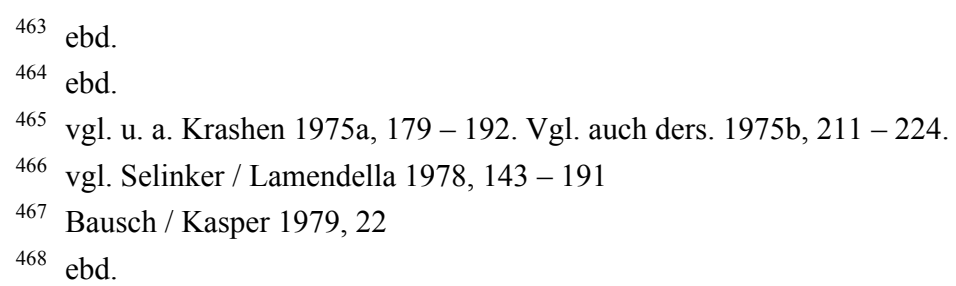




\subsection{2 ,Systematizität' und ,Variabilität'}

Die Strukturen und Regeln einer Sprache unterliegen stets einer gewissen Systematik, die die Anwendung der einzelnen Strukturen und Elemente der betreffenden Sprache bestimmt. Interlanguages stellen diesbezüglich eine Sonderform dar. Einerseits variieren Lernersprachen je nach Erwerbsbedingungen erheblich, andererseits verfügt jede Lernersprache über systematische Erscheinungen, die sie mit anderen Lernersprachen teilt und die daher für diese Sprachform kennzeichnend sind. ${ }^{469}$ Das Auftreten dieses sprachlichen Verhaltens erfolgt nicht zufällig und willkürlich, sondern unterliegt

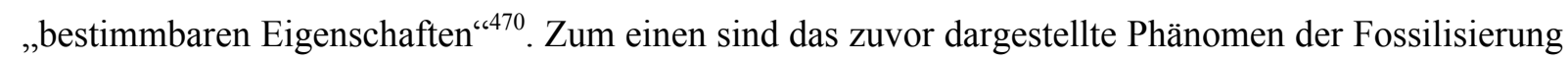
und die im Rahmen der Interlanguagehypothese dargestellten Fehlleistungskategorien sowohl in fossilisierter als auch in sporadisch auftretender Form in allen Lernersprachen - wenn auch in unterschiedlich ausgeprägter Weise - zu finden. Zum anderen erfolgt das Auftreten der Fehler nicht zufällig, sondern wird von einer gewissen Systematik geleitet, die sowohl die Fehlerart als auch den Fehlerort und die Oberflächenstruktur des Fehlers determiniert. ${ }^{471}$ So konnte am Beispiel der Regularisierungsfehler gezeigt werden, daß die Abweichungen nicht grundsätzlich gegen Morphemkombinationsmöglichkeiten des Englischen verstoßen, sondern daß sie nur nicht der im Sinne der Norm zu erwartenden Form entsprechen. ${ }^{472}$

Kombinationen wie die eines auf $-t$ auslautendes Wortes mit dem nachfolgenden Suffix -ted - wie im Fehlerbeispiel putted $^{473}$ - sind im Englischen durchaus vertreten, beispielsweise in der Vergangenheitsform des Verbs omit. Mißachtet wurde hier lediglich, daß ein unregelmäßiges Verb anderen past tense-Bildungen unterliegt. Es wurden bislang keine Fehlerbeispiele ermittelt, bei denen Regularisierungsfehler dieser Art durch grundsätzlich inexistente past tense-Markierungen gebildet wurden. Ebenso wurden bei allen ermittelten Fehlern fundamentale Prinzipien der im Englischen möglichen Lautkombinationen beachtet, d. h., es wurden keine Abweichungen beobachtet, bei denen zum Beispiel ausschließlich Konsonanten mit Konsonanten kombiniert wurden. Regelmäßigkeiten wurden darüber hinaus nicht nur auf der morphologischen Ebene, sondern auch auf der lexikalisch-morphologischen Ebene beobachtet. So konnte empirisch ermittelt werden, daß das fälschliche Hinzufügen der progressive-Form wie auch die fehlerhafte Wahl des past tense marker auf bestimmte Verbgruppen beschränkt sind. ${ }^{474}$

\footnotetext{
469 vgl. Gass / Selinker 1994, $165 \mathrm{ff}$

470 Vogel 1990, 47

471 vgl. ebd., $46-60$

472 vgl. Clark / Clark 1977, 276 ff

473 vgl. James 1998, 107

474 vgl. Clark / Clark 1977, 284 f
} 
Diese Systematizität gilt insbesondere für Sprachformen, die auf identischen Erst- und Zielsprachen basieren, aber auch für Lernersprachen, denen unterschiedliche Erst- und Zielsprachen zugrunde liegen. Dies bedeutet, daß in allen Lernersprachen Fehlleistungen wie overgeneralizations oder Formen des interlingual transfer beobachtet werden können, selbst wenn Erst- und Zielsprache der miteinander verglichenen Lernenden völlig divergieren. Sind hingegen beim Vergleich verschiedener Lernender Erst- und Zielsprache identisch, so kann nicht nur von partiellen Ähnlichkeiten in bezug auf die im Laufe des Zweitspracherwerbs zu beobachtenden Fehler gesprochen werden. Die Parallelen hinsichtlich bestimmter Lernvorgänge und Lernschwierigkeiten sind in der Regel vielmehr so zahlreich, daß für diese Lernenden Lehrbücher und Grammatiken erstellt werden können, die für die meisten Lernenden des betreffenden Sprachraums geeignet sind.

So haben die mit Beginn der Sprachlehrforschung einsetzenden Untersuchungen am Beispiel von Englisch als Zielsprache gezeigt, daß bei Englischlernenden unterschiedlicher Nationen overgeneralizations, addition errors, misformations und Fehler der Gruppe interlingual transfer zu beobachten waren. Darüber hinaus konnten trotz individueller Unterschiede zwischen einzelnen Lernenden Fehler ermittelt werden, die für die Sprecher einer bestimmten Erstsprache charakteristisch sind und hinsichtlich des strukturellen und erwerbssequentiellen Auftretens Gemeinsamkeiten aufweisen. ${ }^{475}$ Die Mehrzahl der Abweichungen, die in dem im Verlauf dieser Arbeit ermittelten Fehlerkorpus enthalten sind, können partiell auch bei anderen Englischlernenden - etwa bei Lernenden, die Französisch oder eine skandinavische Sprache als Erstsprache sprechen - beobachtet werden. Dazu zählen unter anderem das Auslassen der progressive form oder double markings beim Gebrauch des past tense. ${ }^{476}$ Andererseits gibt es Fehlleistungen, anhand derer englische Muttersprachler eindeutig deutsche Englischlernende erkennen können. Ein Beispiel hierfür auf der phonologischen Ebene bietet der typische Akzent deutscher Muttersprachler beim Erwerb der englischen Sprache. ${ }^{477}$

Variabilität ${ }^{478}$ als zweites charakteristisches Merkmal von Lernersprachen wird im wesentlichen von drei Faktoren ${ }^{479}$ bestimmt: der Zeit, den Vorkommensbedingungen und dem Lernenden. Die zeitliche Variabilität von Lernersprachen äußert sich in erster Linie darin, daß jede Lernersprache in bezug auf den Gebrauch sprachlicher Elemente und Strukturen im Laufe des Erwerbsprozesses einer Zielsprache ständigen Veränderungen unterliegt. Die Variabilität kann dabei sowohl korrekte als auch inkorrekte Elemente umfassen: Interlanguage-Elemente, die im Sinne der Zielsprache des Lernenden als korrekt zu bezeichnen sind und in dieser Form über einen gewissen Erwerbszeitraum hin benutzt worden sind,

\footnotetext{
475 vgl. dazu exemplarisch Granger 1993, 57- 71

476 vgl. ebd.

477 vgl. hierzu insbesondere Weiher 1974, 161 - 163

478 vgl. Gass / Selinker 1994, 165 ff u. vgl. Vogel 1990, $51-54$

479 vgl. Vogel 1990, $51-54$
} 
können in einer bestimmten Erwerbsphase in fehlerhafter Form angewandt werden. Auf der anderen Seite ist auch der umgekehrte Fall zu beobachten, wenn bislang fehlerhafte benutzte Strukturen oder Elemente ab einem bestimmten Zeitpunkt fehlerfrei verwendet werden. In beiden Fällen ist dieser Prozeß umkehrbar. Im Idealfall ist es jedoch so, daß sich Lernersprachen kontinuierlich auf die Norm der Zielsprache hin entwickeln. Die zeitliche Variabilität von Lernersprachen unterscheidet sich in diesem Zusammenhang von der Variabilität natürlicher Sprachen dadurch, daß die Veränderungen von interlanguages in der Regel nicht von den einzelnen Sprechern beeinflußt werden können und nicht intentional ablaufen. Außerdem verläuft der Wandel einer natürlichen Sprache langsamer und ist im Normalfall nicht vorhersagbar.

Beim gesteuerten Zweitspracherwerb hingegen realisieren die einzelnen Sprecher die Veränderung ihrer Lernersprache. Sie können sowohl ihren Lernfortschritt als auch dessen Rückgang messen, beispielsweise anhand schulischer Leistungsbeurteilungen oder in Kommunikationssituationen mit anderen Englischlernenden oder englischen Muttersprachlern. ${ }^{480}$ Dabei sind sie sich der Tatsache bewußt, daß sie ihren Lernprozeß beeinflussen können. Das Erlernen von Sprachen verläuft dabei zumeist nach unterrichtlichen Zwängen und ist hinsichtlich des zeitlichen Verlaufs an institutionelle Vorschriften gebunden.

Die Variabilität der Lernersprache in bezug auf die Vorkommensbedingungen äußert sich darin, daß die Art der lernersprachlichen Äußerungen - d. h. die Kommunikationsgeschwindigkeit, die Anzahl der Fehler und die stilistische Form beim Vergleich der verschiedensten Ausprägungen und Situationen zweitsprachlicher Produktion - Unterschiede aufweist. Untersuchungen haben zum Beispiel gezeigt, daß die Anzahl der Fehler bei Übersetzungen deutlich höher liegt als bei Nacherzählungen. Ebenso überwiegt die Anzahl der Fehler in formalen Testsituationen im Vergleich zur Fehleranzahl in lernersprachlichen Äußerungen, denen kein Testverfahren zugrunde liegt. ${ }^{481}$

Gleiches gilt für informelle Kommunikationszusammenhänge: Hier produzieren Lernende in der Regel weniger Fehler als in formellen Kommunikationszusammenhängen. Unterschiede wurden auch beim Vergleich von Kommunikationssituationen mit native speakers der betreffenden Zielsprache und mit anderen Zweitsprachenlernenden der angestrebten Sprachform erkennbar. Anhand von Untersuchungen konnte gezeigt werden, daß Zweitsprachenlernende beim Kontakt mit Muttersprachlern der Zielsprache auf ein größeres Maß an Sprachkorrektheit bedacht sind und daher Strategien anwenden, mit deren Hilfe Fehler nach Möglichkeit vermieden werden können. ${ }^{482}$ Beim Kontakt mit Nicht-

\footnotetext{
480 vgl. Timm 1996b, $174-190$

481 vgl. Doyé 1996, 192 - 208

482 vgl. Schuhmann / Schuhmann 1977, 241 - 249
} 
Muttersprachlern der Zielsprache hingegen wurde der verstärkte Gebrauch von Produktionsstrategien deutlich, d. h., das Bedürfnis nach kommunikativem Austausch überwog gegenüber dem Bestreben, möglichst korrekt zu sprechen.

So ließen Kommunikationssituationen mit anderen Zweitsprachenlernenden der betreffenden Sprachform erkennen, daß die Bereitschaft, Strukturen und Elemente einzusetzen, die erst vor kurzer Zeit erlernt wurden und zu deren Anwendung der Lernende wenige oder keine Gelegenheiten hatte, weitaus größer ist als beim Kontakt mit native speakers dieser Sprachform. ${ }^{483}$ Weitere Unterschiede wurden beim Vergleich von freier bzw. spontaner Rede, zu deren Vorbereitung der Lernende keine Zeit hatte, und geplanter und vorbereiteter Rede deutlich. ${ }^{484}$ Hier zeigte sich, daß Qualität und Quantität des Gebrauchs sprachlicher Strukturen und Elemente in der Regel bei spontaner Kommunikation wesentlich begrenzter sind als bei vorbereiteten Formen der Rede.

Die Tatsache, daß die Variabilität von Lernersprachen neben diesen Faktoren und dem zeitlichen Aspekt auch auf die unterschiedlichen Persönlichkeitsmerkmale bzw. persönlichkeitsbezogenen Eigenschaften der einzelnen Lernenden zurückzuführen ist, ${ }^{485}$ wurde bereits durch die im Zusammenhang mit der Systematizität erwähnten "individuelle[n] Unterschiede" ${ }^{486}$ thematisiert. Wie zuvor erwähnt, sind Muttersprachler bzw. fortgeschrittene Lernende einer bestimmten Sprache in der Regel in der Lage, die nationale bzw. sprachgemeinschaftliche Zugehörigkeit von Fremdsprachenlernenden zu erkennen. Dennoch sind die auf eine bestimmte Zielform gerichteten Lernersprachen von native speakers derselben Sprache beim Vergleich meist nicht identisch, wie die unterrichtliche Praxis zeigt. ${ }^{487}$ Individuelle Unterschiede basieren im wesentlichen auf „Persönlichkeitsfaktoren wie Empathiefähigkeit, Extro- bzw. Introvertiertheit (...) Alter, Motiviertheit und Lernstil““488, den sprachlichen und kommunikativen Vorerfahrungen, wie auch auf der Art des Fremdsprachenunterrichts, durch den die Lernenden die betreffende Sprachform erworben haben. ${ }^{489}$

So fällt es Sprechern, die in der Lage sind, sich in die Kultur bzw. Redeweise der Sprecher einer bestimmten Zielsprache hineinzuversetzen, leichter, fehlerfreie Äußerungen zu produzieren als Lernenden, die sich von der erstsprachlichen Denkweise nicht lösen können und fremde Lautung und Struktur als fremd und unnatürlich empfinden. Ebenso konnte mittels empirischer Studien gezeigt werden, daß die Lernersprache extrovertierter Lernertypen auf einer verstärkten Anwendung von

\footnotetext{
483 vgl. Widdowson 1989, 128 - 137

484 vgl. Vogel 1990, 53

485 vgl. ebd.

486 ebd., S. 54.

487 vgl. Tarone 1983, $141-163$

488 Vogel 1990, 54
} 
Elaborationsstrategien basiert und somit in Kommunikationssituationen mehr Elemente und Strukturen zur Anwendung kommen als bei introvertierten Lernertypen. ${ }^{490}$ Andererseits führt das Sprachverhalten extrovertierter Lernender oftmals zu einer höheren Fehlerquote als die Kommunikationsweise der diametral entgegengesetzten Lernertypen, die in der Regel um Sprachkorrektheit bemüht sind. ${ }^{491}$

Die Auswirkungen des Faktors ,Alter ‘ hinsichtlich der Variabilität von Lernersprachen konnten bislang nur partiell geklärt werden. Es konnte jedoch gezeigt werden, daß sich die Erwerbsgeschwindigkeit der Zielsprache von Lernenden mit identischen Erstsprachen und nahezu identischen psychosozialen Voraussetzungen unterscheidet, wenn eine Lernergruppe das Alter der Maturitas erreicht hat und die andere sich in der präpubertären Phase befindet. Hier verläuft der Erwerb der erstgenannten Gruppe langsamer als bei letzterer. ${ }^{492}$ Es gilt als langjährig erwiesen, daß der von Lernendem zu Lernendem unterschiedlich ausgeprägte Faktor ,Motivation` zur Variabilität von Lernersprachen beiträgt und nach wie vor ein entscheidendes Problem im schulischen Unterricht darstellt. ${ }^{493}$ Lernende, bei denen ein hoher Motivationsgrad zu verzeichnen ist, erwerben die angestrebte Zielsprache nicht nur schneller, sondern unterscheiden sich auch hinsichtlich der Qualität der zweitsprachlichen Produkte von Lernenden, die dem Spracherwerb unmotiviert entgegentreten.

Der Faktor ,Lernstil‘ führt zur Variabilität von Lernersprachen, weil er sowohl die Qualität als auch die Quantität des Spracherwerbs beeinflußt. In zahlreichen Untersuchungen konnte ermittelt werden, daß Lernende, welche Methoden der drei wesentlichen Lernkategorien ,Problemfindung', ,Problemanalyse` und ,Problemlösung ‘ anwenden, Fehler erfolgreicher vermeiden als Lernende, die ein einseitiges Lernkonzept verfolgen. ${ }^{494}$ Auch die sprachliche und kommunikative Vorerfahrung der Lernenden, zu der insbesondere Kenntnisse anderer Fremdsprachen bzw. die Beherrschung der Erstsprache gehören, führt zur Variabilität von Lernersprachen: Lernende, die bereits andere Zielsprachen erworben und/oder die Regularitäten ihrer Erstsprache und deren Ausnahmen erkannt haben, wissen in der Regel, welche Aspekte der Sprache beim Spracherwerb von besonderer Bedeutung sind und welche Lernformen zu größtmöglichem Erfolg beim Zweitspracherwerb führen.

Darüber hinaus ist die Form des Fremdsprachenunterrichts ${ }^{495}$ ein entscheidender, zur Variabilität von Lernersprachen beitragender Faktor, weil der Fremdsprachenunterricht bei den meisten Lernenden den einzigen Kontakt zur Fremdsprache darstellt. Einseitige Lehrmethoden wie etwa Grammatik-

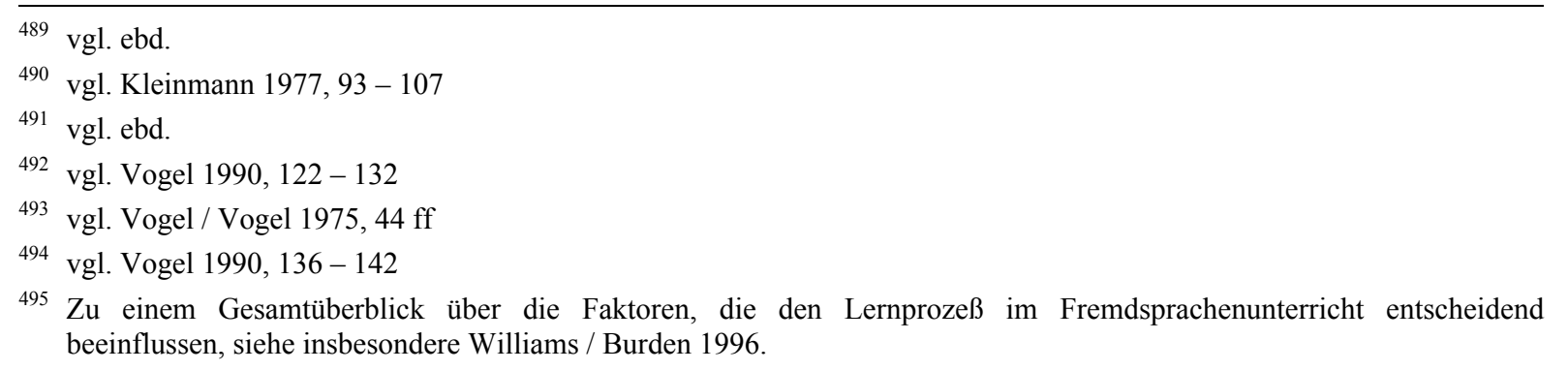


zentriertheit, sowie Faktoren wie fehlende Authentizität, nicht lernergemäße Segmentierung des Lernstoffs, fehlende Übung in der Sprachanwendung und die Nichtberücksichtigung individueller Lernstile können zu den sogenannten induced errors ${ }^{496}$ führen. Diese sind bei Lernenden, die einen Fremdsprachenunterricht erfahren haben, der diese Merkmale nicht aufweist, nur selten bzw. gar nicht zu finden.

496 vgl. Stenson 1983, $256-271$ 


\section{Die Fehleranalyse}

Die Analyse sprachlicher Fehler bedarf einer Vielzahl sprachlicher und außersprachlicher Vorinformationen, die bei der Untersuchung, die der vorliegenden Arbeit zugrunde liegt, mit Hilfe des zuvor beschriebenen Fragenkatalogs ermittelt wurden. Diese Vorinformationen betreffen sowohl die Persönlichkeit des Fehlerproduzenten als auch die äußeren Faktoren, die den Zweitspracherwerb des Lernenden begleitet haben, wie etwa die Schulausbildung oder Kontakte zu Muttersprachlern der Zielsprache.

Bei der Analyse der sprachlichen Fehler werden - wie eingangs erwähnt - die Fehlleistungen herangezogen, die jeweils in den Probandengruppen mit den meisten und mit den wenigsten Fehlern der einzelnen linguistischen Kategorien ermittelt wurden. Auf diesem Wege soll zurückverfolgt werden, welche Faktoren dazu beigetragen haben, daß eine bestimmte Probandengruppe besonders viele bzw. wenige Abweichungen verursachte. Fehlleistungen der anderen Probandengruppen werden dann berücksichtigt, wenn sie zur Unterstützung der Fehleranalyse beitragen.

Wie die Darstellung der einzelnen Zweitspracherwerbshypothesen verdeutlichte, ist die Entstehung sprachlicher Fehler als äußerst subtiler Vorgang zu betrachten. Selbst bei Berücksichtigung möglichst vieler Vorinformationen und bei Einbeziehung aller in Frage kommenden Fehlererklärungsfaktoren ist nicht auszuschließen, daß bei manchen Fehlern Faktoren mitgewirkt haben, die weder aus dem Fragebogen noch aus den Erklärungsansätzen der einzelnen Erwerbshypothesen hervorgehen.

Damit jedoch auch solche Faktoren berücksichtigt oder wenigstens partiell berücksichtigt werden können, wird bei der Analyse der einzelnen Fehler von folgenden Voraussetzungen ausgegangen: Das Analyseverfahren stützt sich sowohl auf die Informationen, die aus dem Fragebogen hervorgehen als auch auf die Erklärungsfaktoren der Hypothesen und auf das gesamte sprachliche Umfeld, das den jeweiligen Fehler umgibt. Letzteres bedeutet, daß nicht nur der Satz oder das Element herangezogen wird, in dem ein Fehler auftritt, sondern der gesamte schriftliche Beitrag des Lernenden.

Da jedoch sowohl die Beiträge der Probanden als auch die Erklärungsfaktoren der Hypothesen nur ein begrenztes Erklärungspotential zur Verfügung stellen und als theoretische Hilfsmittel betrachtet werden müssen, werden auch Erfahrungen aus der täglichen Unterrichtspraxis zugrunde gelegt. Die Analyse sprachlicher Fehler ist nicht mit naturwissenschaftlichen Vorgängen zu vergleichen. Es ist zu erwarten, daß die in Frage kommenden Faktoren nicht in allen Fällen als zutreffend oder unzutreffend bezeichnet werden können. Vielmehr ist in manchen Fällen einzuräumen, daß sowohl der eine als auch der andere Fehlerentstehungsfaktor oder auch beide gleichzeitig zutreffen können. 
Voranzustellen ist auch, daß Vermutungen, die im Laufe des Analyseverfahrens unweigerlich angestellt werden, keineswegs als wissenschaftlich unzulässig zu betrachten sind. Sie verdeutlichen vielmehr, daß sprachliche Fehler linguistische Erscheinungen darstellen, deren Analyse über den Bereich Sprache hinaus weitere Faktoren einzubeziehen hat. Diese Grundprinzipien vorausgesetzt, basiert die Analyse der Fehler auf folgender Vorgehensweise: Da das Ziel vorliegender Arbeit darin besteht, sowohl die Erklärungsfaktoren der Kontrastivhypothese als auch die der Identitäts- und der Interlanguagehypothese anzuwenden, werden die Erklärungsfaktoren dieser Hypothesen zu einem Gesamtmodell zusammengeführt. Das bedeutet, die Fehlererklärungsfaktoren der einzelnen Erwerbshypothesen werden nicht separat betrachtet, sondern immer im Zusammenhang mit den Faktoren der jeweiligen anderen Hypothesen. Diese Vorgehensweise soll gewährleisten, daß die Erklärungsfaktoren nicht miteinander konkurrieren, sondern einander ergänzen.

Das Analysemodell, das für die vorliegende Untersuchung entwickelt wurde, enthält daher drei Untersuchungsaspekte: einen sprachlichen Untersuchungsaspekt, einen entwicklungsspezifischen Untersuchungsaspekt und einen Untersuchungsaspekt, der die Lernstrategien und Lernprozesse als mögliche Fehlerquelle in Betracht zieht. Damit jedoch trotz der Verbindung der einzelnen Theorien nachvollzogen werden kann, aus welcher Hypothese die Erklärungsfaktoren hervorgegangen sind, wurde die Bezeichnung der Fehlertypen in ihrer ursprünglichen Terminologie beibehalten. Somit ist bei Betrachtung des Gesamtmodells deutlich zu erkennen, welcher Erklärungsfaktor welcher Hypothese zuzuordnen ist. Für die praktische Durchführung der Analyse wurden Analyseordner entworfen, die alle verfügbaren Fehlererklärungsfaktoren enthalten. ${ }^{497}$ Diese Ordner wurden so angelegt, daß die Chronologie der einzelnen Erklärungsfaktoren der zeitlichen Entwicklung der einzelnen Erwerbshypothesen entspricht. So besteht der erste Teil aus sprachlichen Erklärungsfaktoren, d. h. den Faktoren, die auf dem Prinzip der Kontrastivhypothese in ihrer starken und ihrer schwachen Form beruhen. Der zweite Teil enthält die Faktoren der Identitätshypothese, die sprachentwicklungsspezifische Aspekte als Fehlerquelle in den Vordergrund stellen. Im dritten Teil schließlich werden die Fehler der Interlanguagehypothese entsprechend aus lernpsychologischer Sicht betrachtet.

Der Aufbau der Analyseordner gleicht einem Buch, das in unterschiedliche Rubriken unterteilt ist, wobei die einzelnen Rubriken nach den jeweiligen Fehlererklärungsfaktoren benannt sind. Die Ordner sind dabei nach den einzelnen linguistischen Ebenen getrennt, d.h., es wurde ein Ordner für lexikalische, einer für syntaktisch-morphologische und einer für orthographische Fehler erstellt. Die linguistischen Ebenen wiederum sind in einzelne Kategorien unterteilt. Beispielsweise ist im Ordner für lexikalische Verstöße eine Rubrik vorgesehen, die ausschließlich Verstöße beim Gebrauch der Präpositionen behandelt. Ebenso enthält der Ordner für syntaktisch-morphologische Verstöße eine

497 Die Ordner können beim Autor der vorliegenden Arbeit eingesehen werden. 
Rubrik, die nur Fehler beim Gebrauch des Gerundiums behandelt. In gleicher Weise beinhaltet der Ordner für den Bereich Orthographie z. B. eine Rubrik, die sich ausschließlich mit Rechtschreibfehlern befaßt.

Der Aufbau der Analyseordner bzw. die in ihnen enthaltenen Fehlererklärungsrubriken entsprechen dem Prinzip eines Siebs. Alle Fehler, die in das Analyseverfahren einbezogen werden, werden somit sowohl an dem linguistischen System als auch an entwicklungsspezifischen und lernpsychologischen Erklärungsfaktoren gemessen. Dies bedeutet, bei jeder Abweichung wird überprüft, inwieweit die Entstehung des Fehlers mit den Erklärungsfaktoren der Kontrastiv-, der Identitäts- und der Interlanguagehypothese begründet werden kann.

Nachdem alle Fehler der betreffenden Kategorie - z. B. die Gruppe der Hilfsverben - den Analysekatalog durchlaufen haben, wird schließlich ermittelt, wie hoch der proportionale Anteil der einzelnen Erklärungsfaktoren ist. Damit die Erklärungsfaktoren jedoch nicht voneinander getrennt betrachtet werden, ist gleichzeitig zu untersuchen, welcher Zusammenhang zwischen den in Frage kommenden Erklärungsfaktoren bestehen könnte. Das Analyseprinzip soll an folgendem Fehlerbeispiel eines deutschen Englischlernenden dargestellt werden.

Fehler: $\quad$ At the moment I'm hearing a lot of music. ${ }^{498}$

Korrektur: At the moment I'm listening to music a lot.

Schon bei erster Betrachtung ist zu erkennen, daß der Fehler auf unterschiedliche Quellen zurückzuführen ist, die nicht nur sprachlicher Natur sind, sondern auch entwicklungsspezifischen und lernpsychologischen Einfluß vermuten lassen. Der sprachliche Aspekt wird durch das Lexem hear deutlich. Es ist davon auszugehen, daß der Lernende den deutschen Satz „zur Zeit höre ich viel Musik“ ins Englische übertragen wollte. Das Lexem hear, das den ersten Fehler darstellt, wurde offensichtlich benutzt, weil das Lexem dem deutschen Wort ,hören“ optisch und akustisch ähnelt. Dabei hat der Lernende zwei Aspekte nicht bedacht: Zum einen gehört das Wort hear zur Wortgruppe der sogenannten statischen Verben. ${ }^{499}$ Die Besonderheit dieser Verbgruppe liegt darin, daß statische Verben in der Regel nicht in der progressive form benutzt werden. Dazu gehören nahezu alle Verben, die mit den Sinnesorganen in Zusammenhang stehen. Statt der progressive form wäre in der oben dargestellten Äußerung die simple form zu erwarten gewesen, da Verben dieser Gruppe in der Regel ausschließlich in dieser Grundform benutzt werden.

\footnotetext{
498 FB der Gruppe HS 10E

499 vgl. Swan 1990, Textstellen 502 f, o. S.
} 
Zum anderen ist das Wort hear in dem angegebenen Zusammenhang unangebracht. Das Lexem hear wird im Englischen entweder benutzt, um die anatomische Fähigkeit, etwas hören zu können, wie in dem Satz I can hear well auszudrücken, oder aber zur Schilderung akustischer Eindrücke, die der Betreffende nicht steuern kann. Dazu zählt etwa folgende Äußerung: When I open the window I can hear the traffic. Hören im Sinne von Zuhören wird im Englischen jedoch mit dem Lexem listen to übersetzt. Aus dem Gesprächszusammenhang geht aber hervor, daß es sich in der betreffenden Situation nicht um Musik handelt, die sich der Steuerung des Lernenden entzieht, sondern um Musik, die der Lernende intentional hört. Somit wäre der Satz unter Verwendung des Wortes listen zu übersetzen gewesen.

Terminologisch betrachtet ist dieser Fehler bei Anwendung der Kontrastivhypothese als Substitution, gegebenenfalls auch als Übergeneralisierung zu bezeichnen. ${ }^{500}$ Eine Substitution liegt hierbei vor, weil ein englisches Lexem durch ein Wort ersetzt wird, das einer deutschen Vorlage optisch und akustisch ähnelt. Daneben ist der Fehler auch als Übergeneralisierung zu betrachten, weil das Wort hear im Englischen existiert, jedoch hier in einem falschen Zusammenhang benutzt wird. Die Verallgemeinerung wurde in diesem Fall durch die sprachliche Verwandtschaft zu dem Wort ,hören` ausgelöst.

Nach Anwendung der Fehlererklärungsfaktoren der Kontrastivhypothese wird überprüft, inwieweit der Fehler als Folge eines entwicklungsbedingten Vorgangs betrachtet werden kann. Dabei ist zunächst zu untersuchen, ob die Kategorien, die im Rahmen der Identitätshypothese erstellt wurden, Fehler dieses Typs enthalten.

Dies bedeutet, es muß zuerst untersucht werden, ob Fälle verzeichnet sind, in denen Lernende, die Englisch unter natürlichen Bedingungen erlernten, Wörter durch Lexeme ersetzten, die dem eigentlich zu erwartenden Begriff semantisch ähneln, jedoch im betreffenden Kontext ungeeignet waren. Zweitens ist zu überprüfen, ob die Aufzeichnungen Fälle enthalten, in denen Lernende unter natürlichen Erwerbsbedingungen die progressive form benutzten, obwohl die simple form zu erwarten gewesen wäre. Schließlich muß entschieden werden, welcher Fehlertyp hier vorliegt. Da es sich um das Hinzufügen redundanter grammatischer Elemente handelt, fällt die Abweichung in den Bereich der addition errors. ${ }^{501}$ Im vorliegenden Beispiel zeigt eine Überprüfung der empirischen Ergebnisse, daß der Fehler in der Tat zu den typischen Abweichungen englischer Kinder beim Erwerb ihrer eigenen Erstsprache gehört. Untersuchungen haben gezeigt, daß Lernende, die Englisch in dem Land erwerben, in dem die englische Sprache Verkehrssprache ist, in fortgeschrittenen Erwerbsstadien oftmals nicht zwischen

\footnotetext{
500 vgl. Kap. II 1.1.2 der vorliegenden Arbeit: Fehlererklärungsfaktoren der Kontrastivhypothese

501 vgl. Kap. II 1.2.2 der vorliegenden Arbeit: Fehlererklärungsfaktoren der Identitätshypothese
} 
dem Gebrauch der simple form und der progressive form differenzieren können. ${ }^{502}$ Somit ist der Fehler auch in die Kategorien der Identitätshypothese einzuordnen.

Fehlertypologisch ist die Abweichung als simple addition error ${ }^{503}$ zu betrachten, weil keine Elemente vertauscht, ausgelassen oder in einer Form kombiniert werden, die gänzlich der Norm des Englischen widerspricht. Vielmehr wurde eine grammatische Konstruktion in strukturell richtiger Form benutzt, es wurde lediglich nicht bedacht, daß die Form in dem gegebenen Zusammenhang unangebracht ist.

In einem dritten Schritt ist schließlich zu überprüfen, inwieweit es sich hier um eine Fehlleistung handelt, die partiell auch auf bewußt und unbewußt ablaufende Prozesse während des Spracherlernens und der Sprachanwendung zurückgeführt werden kann, d. h. inwieweit die Erklärungsfaktoren der Interlanguagehypothese ${ }^{504}$ bei der Analyse der Abweichung behilflich sein können. Die Betrachtung der Abweichung zeigt dabei, daß der Fehler in der Tat auf unbewußt und bewußt ablaufenden Faktoren basieren könnte. Zum einen kann der Fehler dadurch entstanden sein, daß das Thema progressive form im Englischunterricht überbetont wurde. Eine Überbetonung entsteht insbesondere dann, wenn eine bestimmte grammatische Form ohne thematischen und methodischen Wechsel über einen längeren Zeitraum hinweg behandelt wird. Der Überbetonungseffekt wird noch verstärkt, wenn der Lehrende permanent sogenannte Signalwörter erwähnt, die den Lernenden darauf aufmerksam machen sollen, daß eine bestimmte grammatische Konstruktion zu verwenden ist. Die Präpositionalphrase at the moment, die den Anfang des genannten Fehlerbeispiels bildet, zählt zu den Signalwörtern, die in den Lehrbüchern des Englischunterrichts an deutschen Schulen immer wieder hervorgehoben werden. Es ist also denkbar, daß die Anwendung dieser Präpositionalphrase den Lernenden unweigerlich dazu veranlaßte, die progressive form zu benutzen.

Auch die Verwendung des Wortes hear kann unbewußt verlaufen sein und basiert möglicherweise auf Faktoren des Unterrichts. Letzteres könnte z. B. zutreffen, wenn die Unterscheidung zwischen den Anwendungsmöglichkeiten der Lexeme listen und hear im Unterricht nicht deutlich genug aufgezeigt wurde und der Schwerpunkt auf den Anwendungsmöglichkeiten des Lexems hear lag. Da alle Fehler, die möglicherweise auf Faktoren des Unterrichts basieren, im Rahmen der Interlanguagehypothese unter dem Begriff transfer of training zusammengefaßt werden, ${ }^{505}$ wird die Fehlleistung in die dafür vorgesehene Kategorie des Analysekatalogs aufgenommen.

\footnotetext{
502 vgl. Vogel 1989, $134-136$

503 vgl. Kap. II 1.2.2 der vorliegenden Arbeit: Fehlererklärungsfaktoren der Identitätshypothese

504 vgl. Kap. II 3 der vorliegenden Arbeit: Die Interlanguagehypothese

505 vgl. ebd.
} 
Neben den unbewußt ablaufenden Entstehungsursachen kann die Abweichung jedoch auch als Ergebnis eines Sprach- und Lernverhaltens gedeutet werden, das bewußt verlaufen ist, d. h. vom Lernenden gesteuert wurde: Zum einen könnte sie als Resultat einer Simplifizierung betrachtet werden, die während des Lernprozesses stattgefunden hat. Möglicherweise hat der Lernende beim Erlernen der progressive form bewußt darauf verzichtet, zwischen der Anwendung sogenannter statischer und dynamischer Verben ${ }^{506} \mathrm{zu}$ unterscheiden, weil er auf diese Weise versuchte, den Lernprozeß übersichtlicher zu gestalten. In diesem Fall hätte sich der Lernende bewußt dafür entschieden, in Redesituationen, die seiner Meinung nach die progressive form erwarten lassen, grundsätzlich auf diese Form zurückzugreifen, ohne Berücksichtigung etwaiger Ausnahmen. Somit könnte die Abweichung auch das Ergebnis einer Lernstrategie darstellen und wäre damit der Rubrik strategies of second language communication - simplification ${ }^{507}$ des Analysekatalogs zuzuordnen.

Daneben ist der Fehler auch als Ergebnis einer bewußt ablaufenden Kommunikationsstrategie zu betrachten, weil es möglich ist, daß der Lernende eine zweitsprachliche Regel bewußt generalisiert hat. Dies bedeutet, es ist nicht auszuschließen, daß der Lernende aufgrund der Tatsache, daß die Mehrzahl der englischen Verben in der progressive form steht, wenn eine momentan oder vorübergehend stattfindende Handlung zum Ausdruck gebracht werden soll, glaubte, diese Struktur auch im vorliegenden Fall anwenden zu müssen. Zudem ist es möglich, daß der Lernende die mit statischen Verben verbundenen Regeln in der Situation, in der das Fehlerbeispiel entstanden ist - etwa aufgrund der zeitlichen Beschränkung des Testverfahrens - nicht abrufen konnte. Möglich ist auch, daß diese Regel aufgrund neu erworbenen Sprachwissens vorübergehend verdrängt wurde. Auch die Verwendung des Lexems hear kann das Ergebnis einer Handlung sein, die der Lernende bewußt vollzogen hat. Deutsche Englischlernende erwerben das Wort hear in der Regel früher als das Lexem listen. So ist es möglich, daß das Element hear im Sprachbewußtsein des Lernenden stärker verankert ist als das zuletzt genannte Lexem und der Lernende daher zunächst auf das ihm vertrautere Element zurückgreift. Die Assoziation zu dem deutschen Lexem ,hören' mag dabei die Verwendung des Wortes hear unterstützt haben, so daß hierbei interlingual transfer zusätzlich mitgewirkt haben könnte. Das Fehlerbeispiel wird somit darüber hinaus in den Kategorien productive achievement strategies - b) interlingual transfer (adaption of rules of the mothertongue or another foreign language, also foreignizing) - e) generalization of interlanguage rules/items in new contexts und in die Kategorie overgeneralization, die für unbewußte Übertragungen vorgesehen ist, aufgenommen. Aufgrund der zuvor erwähnten optischen und akustischen Ähnlichkeit zum deutschen Wort ,hören“ wird die Abweichung abschließend auch in der Kategorie lack of contrast festgehalten. ${ }^{508}$

\footnotetext{
506 vgl. Swan 1990, Textstellen 502 f., o. S.

507 vgl. Kap. II 3 der vorliegenden Arbeit: Die Interlanguagehypothese

508 vgl. ebd.
} 
Da sowohl die Kontrastiv- als auch die Identitäts- und die Interlanguagehypothese Möglichkeiten zur Analyse der Entstehung dieses Fehlers bieten, ist abschließend zu untersuchen, welche Folgen daraus in bezug auf die Bedeutung der einzelnen Hypothesen resultieren. Als Ergebnis ist in diesem Fall festzuhalten, daß die Interlanguagehypothese quantitativ betrachtet die meisten Fehlererklärungsmöglichkeiten bietet. In diesem Zusammenhang ist jedoch zu betonen, daß diese Feststellung nicht bedeutet, daß die Interlanguagehypothese auch das größte fehleranalytische Potential besitzt. Der Schwerpunkt der Interlanguagehypothese liegt auf dem Verhalten des Lernenden während des Erlernens der Zielsprache und während ihrer Anwendung. Im Vordergrund steht dabei das individuelle Verhalten des jeweiligen Lernenden und der aktive Umgang mit der betreffenden Zielsprache. Wenngleich auch diese Aspekte eine entscheidende Rolle bei der Analyse der Fehler spielen, zeigt jedoch die Analyse des zuvor behandelten Fehlerbeispiels, daß auch linguistische Entstehungsfaktoren und biologische Aspekte zum Tragen kommen. Dies bedeutet, auch wenn nur wenige Faktoren der Kontrastiv- und der Identitätshypothese nachgewiesen werden konnten, ist das Erklärungspotential dieser Hypothesen dennoch ebenso wichtig wie das der Interlanguagehypothese, da diese Faktoren nicht auf einen Lernenden begrenzt sind, sondern auf andere deutsche Muttersprachler beim Erwerb des Englischen übertragen werden können. Insbesondere in Hinblick auf die Erarbeitung von Fehlervermeidungsmodellen ist es daher wichtig, nicht nur die Fehler einzelner Individuen zu betrachten, sondern festzustellen, welche Gemeinsamkeiten innerhalb der Sprechergruppe einer bestimmten Erstsprache in bezug auf das Erlernen einer bestimmten Zielsprache festzustellen sind.

In Hinblick auf das Beispiel at the moment I'm hearing a lot of music läßt sich die Analyse der Abweichung in folgender Form zusammenfassen: Die Entstehung der Abweichung beruht auf einer Kombination sprachsystembezogener, entwicklungsspezifisch determinierter und interlanguagespezifischer Faktoren. Der Einfluß des erstsprachlichen Systems äußert sich vornehmlich in der Wahl des Lexems hear. Entwicklungsspezifisch determinierte Aspekte und der Einfluß strategisch-prozeduralen Verhaltens zeigen sich vor allem in der Anwendung der progressive form. Der Gebrauch dieser Struktur resultiert zum einen daraus, daß die normabweichende Anwendung der progressive form eine natürliche Begleiterscheinung des Erwerbs der englischen Sprache darstellt. ${ }^{509}$ Dieser Vorgang ist entwicklungsspezifisch vorprogrammiert und kann von den Lernenden nur bedingt beeinflußt werden. Zum anderen kann die Anwendung dieser Struktur darauf zurückzuführen sein, daß der Lernende sprachliche Regeln sowohl bewußt als auch unbewußt generalisiert, weil er aufgrund der Vielzahl der sprachlichen Informationen, die an ihn herangetragen wurden, nicht immer in der Lage ist, normgerechte Strukturen zu verwenden. Schließlich kann auch die Ähnlichkeit zwischen dem deutschen Wort ,hören' und dem englischen Lexem to hear zur Entstehung der Abweichung beigetragen haben.

509 vgl. Vogel 1989, $134-136$ 


\section{Lexikalische Abweichungen}

Bei Gegenüberstellung der Probandengruppen, die insgesamt die meisten und wenigsten lexikalischen Abweichungen verzeichnen (vgl. Tabelle 1 im Anhang), wurde folgendes deutlich: Bei Betrachtung der Fehlertypen, die in den Gruppen ermittelt wurden, war zu erkennen, daß die Art der Fehler bzw. die Lexemtypen, die zu den jeweiligen Abweichungen führten, in beiden Gruppen identisch sind (vgl. Tabellen 2 und 3 im Anhang). Dies bedeutet, daß die Anwendungsschwierigkeiten, die bei Lernenden mit geringen lexikalischen Kenntnissen auftreten, auch bei Lernenden mit fortgeschrittenen Kenntnissen zu beobachten sind. Unterschiede bestehen nur in bezug auf das quantitative Auftreten der einzelnen Fehlertypen, wenngleich auch hier nur partiell Differenzen festzustellen waren.

Damit wird deutlich, daß der Kenntnisstand der Probanden keinen fehlertypologischen Einfluß hatte. Die Anwendung der drei Erwerbshypothesen hat gezeigt, daß in beiden Probandengruppen sowohl die Fehlererklärungsfaktoren der Kontrastivhypothese als auch die der Interlanguagehypothese zum Tragen kommen (vgl. Tabellen 12 und 13 im Anhang). Ein Einfluß der Faktoren, die der Identitätshypothese zugrunde liegen, konnte in keiner der beiden Gruppen nachgewiesen werden. Diesen Ergebnissen zufolge gehen lexikalische Fehler bei beiden Lernertypen vornehmlich auf Faktoren des linguistischen Systems und auf Faktoren, die aus dem strategischen Verhalten der Lernenden resultieren, zurück. Dabei zeigen die Ergebnisse, daß der strategisch-prozedurale Einfluß in beiden Gruppen geringfügig größer ist als die rein linguistischen Komponenten. Gleichzeitig wurde deutlich, daß der Einfluß der Erstsprache auch in fortgeschrittenen Erwerbsstadien das Kommunikationsverhalten der Lernenden maßgeblich bestimmt. Die Erkenntnisse aus der Analyse der verschiedenen Fehlertypen ${ }^{510}$ werden nachfolgend im einzelnen beschrieben.

\subsection{Hilfsverben}

Bei Gegenüberstellung der Fehlereintragungen in der Lexemgruppe Hilfsverben zeigt der Vergleich der Fehlertypen, daß in beiden Probandengruppen sowohl bei den einfachen Hilfsverben als auch bei den modalen Hilfsverben Fehler zu verzeichnen sind. Der quantitative Fehleranteil, der auf den Gebrauch dieser Lexemgruppe zurückgeht, ist nahezu deckungsgleich. In beiden Probandengruppen ist die Anzahl der Eintragungen relativ gering (vgl. Tabellen 2 und 3 im Anhang). Dieses Ergebnis verdeutlicht, daß die Anwendung der Hilfsverben selbst bei Lernenden mit ausgeprägten lexikalischen Lernschwierigkeiten nicht zu den wichtigsten Sprachproblemen deutscher Englischlernender zählt. Andererseits zeigt jedoch die Tatsache, daß dieser Fehlertyp auch bei Lernenden mit fortgeschrittenem

510 Die Fehlertypen und -beispiele, die im folgenden erwähnt werden, können beim Autor der vorliegenden Arbeit eingesehen werden. 
lexikalischen Wissen auftritt, daß Hilfsverben zu den Lexemgruppen gehören, die auch in fortgeschrittenen Stadien des Zweitspracherwerbs Anwendungsschwierigkeiten bereiten können.

Diese Fehler treten selbst dann auf, wenn - wie im Fall der Probanden mit den wenigsten lexikalischen Abweichungen (PwenlAb) - die Betreffenden außerschulische Kontakte zur englischen Sprache hatten und über einen längeren Zeitraum sprachliche Erfahrungen in englischsprachigen Ländern sammeln konnten. Auch das Erlernen anderer Fremdsprachen - etwa Latein oder Französisch -, das bei nahezu allen PwenlAb vermerkt wurde, führt nicht zu einer völligen Fehlervermeidung, obwohl Lernende insbesondere durch den Erwerb romanischer Sprachen erfahren, daß Hilfsverben besonderer Beachtung bedürfen, weil sie wichtige grammatische und semantische Funktionen übernehmen. ${ }^{511}$ Somit haben die linguistischen Kenntnisse der Lernenden keinen nachweisbaren Einfluß auf die Typologie der Hilfsverbfehler und auf die Häufigkeit ihres Auftretens. Beim Vergleich der beiden Probandengruppen wird jedoch deutlich, daß die Fehler, die die Probanden mit den meisten lexikalischen Abweichungen (PmeilAb) produzieren, elementarer sind als die Fehler, die in der entgegengesetzten Gruppe ermittelt wurden. Unter ,elementar' ist hierbei zu verstehen, daß die Lernenden beim Gebrauch der Hilfsverben zum Teil grundsätzlich von der Norm der englischen Sprache abweichen. Ein Beispiel bietet folgende Äußerung:

Fehler: $\quad$ Last week my friends and I haven't seen us. ${ }^{512}$

Korrektur: Last week my friends and I didn't see each other.

In diesem Beispiel liegen zwei grundsätzliche Normabweichungen vor. Zum einen wäre statt des Hilfsverbs have das Lexem do zu erwarten gewesen. Zum anderen ist die Zeitform, die der Lernende benutzt, normabweichend, da statt des verwendeten simple present die simple past form des Hilfsverbs korrekt gewesen wäre. Bei den PwenlAb hingegen basiert die Mehrzahl der Fehler darauf, daß lediglich unterschiedliche Formen eines bestimmten Lexems miteinander verwechselt wurden, ohne dabei jedoch Lexeme grundsätzlich zu vertauschen. Auch sind die benutzten Formen in der Regel nicht als grundsätzlich falsch zu bezeichnen. Sie sind vielmehr in dem Kontext, in dem sie benutzt werden, unangebracht, weil durch die Zeitform, die die Lernenden benutzten, semantische Aussagen ungewollt verändert wurden, wie in folgendem Beispiel:

Fehler: $\quad$ Do you think I will forget your birthday? ${ }^{513}$

Korrektur: Did you think I would forget your birthday?

\footnotetext{
511 vgl. Thomson / Martinet 1987, $109-115$

512 Das Beispiel stammt aus der Probandengruppe mit den meisten lexikalischen Abweichungen - PmeilAb (FB der Gruppe RS 10natw.).

513 Das Beispiel stammt aus der Gruppe mit den wenigsten lexikalischen Abweichungen - PwenlAb (FB der Gruppe EB adv.).
} 
Das Beispiel läßt erkennen, daß der Lernende Fragesätze normgerecht bilden kann und Satzelemente syntaktisch korrekt anzuwenden versteht. Auch die zugrundeliegenden Lexeme sind grundsätzlich nicht als falsch zu bewerten. Der Satz stellt damit keine generelle Normabweichung dar und zeigt, daß der Lernende über fortgeschrittene Englischkenntnisse verfügt. Dennoch sind die Verbformen in der betreffenden Aussage ungeeignet, weil der Textzusammenhang erkennen läßt, daß die deutsche Aussage, die der Betreffende ins Englische übertragen wollte, lautete: „Hast du geglaubt, ich würde deinen Geburtstag vergessen?“ Es wäre demnach die simple past form der Lexeme will und do zu erwarten gewesen.

Bei Anwendung der Fehlererklärungsfaktoren der Kontrastivhypothese ist zu erkennen, daß der Einfluß der Erstsprache Deutsch bei den PmeilAb in Form von Substitutionen sowie Über- bzw. Unterdifferenzierungen deutlich wird (vgl. Tabelle 14 im Anhang), d. h. Faktoren, die der starken Version der Kontrastivhypothese zuzuordnen sind. ${ }^{514}$ Ein eindeutiges Beispiel bietet die zuvor genannte Äußerung Last week my friends and I haven't seen us. Die Substitution besteht darin, daß der Lernende das Element didn't, das in der von ihm geplanten Äußerung korrekterweise zu benutzen gewesen wäre, durch das Element haven't ersetzt hat, da dieses dem erstsprachlichen Lexem, das der Lernende zu übersetzen versucht hat, maximal ähnelt: In diesem Fall ist davon auszugehen, daß das deutsche Hilfsverb ,haben' als Vorlage gedient hat. ${ }^{515}$ Gleichzeitig handelt es sich um einen erstsprachlich bedingten Differenzierungsfehler, weil der Lernende nicht bedacht hat, daß der Gebrauch des Hilfsverbs have anderen Beschränkungen unterliegt als der des deutschen Wortes ,haben“. Das bedeutet, der Lernende war sich der Tatsache nicht bewußt, daß im Englischen zur Bildung der Vergangenheitsform Negationen unter Verwendung der Hilfsverben did oder had zu bilden sind, wenn die sprachliche Aussage auf ein abgeschlossenes Ereignis verweist.

Ähnliches ist bei Anwendung der Kontrastivhypothese auch in der entgegengesetzten Probandengruppe zu beobachten. Auch hier kommen nur die Fehlererklärungsfaktoren ,Substitution “ und ,Über-“ bzw. ,Unterdifferenzierung‘ zum Tragen (vgl. Tabelle 15 im Anhang). Der Unterschied besteht allein darin, daß das reine Ersetzen sprachlicher Elemente wesentlich öfter auftrat als bei den PmeilAb. Offensichtlich sind fortgeschrittene Lernende aufgrund ihres größeren Wortschatzes besser in der Lage, zwischen den Gebrauchsnormen englischer und deutscher Lexeme zu unterscheiden und konzentrieren sich bei Anwendung der Sprache mehr auf sprachliche Unterschiede als Lernende mit einem geringeren Wortschatz. Gleichzeitig führt diese Aufmerksamkeit jedoch dazu, daß elementare Fehler wie Substitutionen verstärkt auftreten. Ein Beispiel hierfür bietet folgende Fehlleistung:

514 vgl. Kap. II 1.1.2 der vorliegenden Arbeit: Fehlererklärungsfaktoren der Kontrastivhypothese

515 Auch der normabweichende Gebrauch des Lexems us stellt eine Substitution dar, die jedoch nicht den Bereich der Hilfsverben betrifft und an dieser Stelle daher nicht behandelt wird. 
Fehler: $\quad$ The police wasn 't of much help. ${ }^{516}$

Korrektur: The police weren't of much help.

Hierbei ist zu vermuten, daß der Lernende das Wort were, das zu erwarten gewesen wäre, durch das Lexem was ersetzt hat, weil der Begriff ,Polizei` im Deutschen im Singular benutzt wird. Da der Lernende damit gleichzeitig eine grammatische Sonderregel mißachtet hat, die sich auf Begriffe bezieht, die nur im Plural verwendet werden, kann hier auch von einer Unterdifferenzierung gesprochen werden.

Beim Versuch, die Entstehung dieser Abweichung mit Hilfe der Erklärungsfaktoren der Interlanguagehypothese zu begründen, wurde deutlich, daß von den zur Verfügung stehenden Erklärungsfaktoren nur der Einfluß der sogenannten strategies of second language communication ${ }^{517}$ nachzuweisen ist (vgl. Tabellen 16 und 17 im Anhang). Zu den Hauptfehlerquellen gehört in beiden Probandengruppen das Generalisieren von Interlanguageelementen und -regeln. Dies bedeutet, die Fehler resultieren aus der Annahme, daß ein Element oder eine Regel, die in einem bestimmten Kommunikationszusammenhang richtig war, ohne Einschränkung auch in anderen Kommunikationszusammenhängen angewendet werden kann. So ist die oben erwähnte Fehlleistung Last week my friends and I haven't seen us aus der Gruppe der PmeilAb der Interlanguagehypothese zufolge als bewußte generalization zu betrachten. Der Lernende ging davon aus, daß die Negation have not, die etwa beim present perfect vorzufinden ist, auch in anderen Zeitformen benutzt werden kann. Das Verhalten ist dabei als strategisch einzustufen, weil der Lernende versuchte, mit den lexikalischen Möglichkeiten, die ihm zur Verfügung standen, einen vollständigen englischen Satz zu bilden, um auf diese Weise seine sprachliche Aussage zu verdeutlichen. Gleiches gilt für das zuvor genannte Fehlerbeispiel Do you think I will forget your birthday? Auch hier wurden sprachlich bekannte Mittel so eingesetzt, daß der Kommunikationspartner die beabsichtigte Aussage verstehen kann. Die Strategie besteht in diesem Fall darin, daß der Lernende sich seiner Fähigkeiten bewußt war, sowohl Fragesätze zu bilden als auch zukünftige Ereignisse grammatisch anzudeuten und er davon ausging, mit Hilfe dieses Wissens neue sprachliche Zusammenhänge bewältigen zu können.

Bei Anwendung der Interlanguagehypothese ist zu erkennen, daß die Anzahl der bewußten generalizations bei den PmeilAb größer ist als in der entgegengesetzten Gruppe. Andererseits traten Generalisierungen bei den PwenlAb auch auf prozeduraler Ebene auf. Dies bedeutet, die Lernenden wandten overgeneralizations zum Teil auch unbewußt an, während diese Erscheinung bei den PmeilAb nicht nachgewiesen werden konnte. Dies läßt darauf schließen, daß die Verallgemeinerung sprachlicher Regeln zum Zwecke des Ausgleichs von Kommunikationsdefiziten in fortgeschrittenen Phasen des

\footnotetext{
516 PwenlAb

517 vgl. Kap. II 3 der vorliegenden Arbeit: Die Interlanguagehypothese
} 
Spracherwerbs zum Teil nicht mehr der Kontrolle der Lernenden unterliegt. Darüber hinaus zeigt der Vergleich beider Gruppen, daß weder inter-/intralingual transfer noch lack of contrast bei den PwenlAb an der Fehlerentstehung beteiligt waren. Der fehlerfördernde Einfluß der Erstsprache, der sowohl in Form von intralingual transfer als auch zum Teil als lack of contrast auftritt, nimmt demnach in bezug auf die Gruppe Hilfsverben mit zunehmenden Sprachkenntnissen ab.

Bei den PwenlAb sind - wenn auch nur in wenigen Fällen - daneben manche Abweichungen auf paraphrase zurückzuführen. Allgemein ist damit nach Ermittlung der Unterschiede und Gemeinsamkeiten zwischen beiden Gruppen festzuhalten, daß die Fehlerquellen bei den Anfängern und den Lernenden mit ausgeprägten lexikalischen Kenntnissen im wesentlichen deckungsgleich sind. Bei beiden Lernertypen entstehen die Fehler nicht während des passiven Erlernens der Zielsprache, sondern bei ihrer aktiven Anwendung. Ein Einfluß anderer Sprachen als der Erstsprache Deutsch ist in keiner der beiden Gruppen nachzuweisen. Ebensowenig kann ein Einfluß der Gestaltung des Unterrichts oder der Konzeption der Lehrmaterialien nachgewiesen werden. Die Hauptfehlerquelle besteht in beiden Lernergruppen in dem Versuch, Kommunikationsdefizite durch Rückgriff auf bereits vorhandenes Wissen auszugleichen, wobei die PmeilAb zum größeren Teil auf ihr erstsprachliches Wissen zurückgriffen. Insgesamt läßt das strategische Verhalten der PwenlAb eine größere Risikobereitschaft erkennen als das sprachliche Verhalten der entgegengesetzten Gruppe. Gleichzeitig fällt die Begründung der Fehlerentstehung bei den fortgeschrittenen Lernenden schwerer als bei den Anfängern, weil die Fehlerentstehung bei fortgeschrittenen Lernenden teilweise einer Automatisierung unterliegt und sich daher der bewußten Sprachsteuerung entzieht. 


\subsection{Wortwahl}

Der Vergleich der beiden Probandengruppen zeigt, daß die Kategorie der Wortwahlfehler bei allen Probanden insgesamt die meisten Fehler enthält (vgl. Tabellen 2 und 3 im Anhang). Bei genauerer Betrachtung der Fehler ist festzustellen, daß in beiden Gruppen nahezu die Hälfte aller Wortwahlfehler zur Unterkategorie der sogenannten false friends ${ }^{518}$ gehört. Dies sind Lexeme, die den Lernenden optisch und akustisch an Wörter erinnern, die er aus seiner Erstsprache oder anderen Zweitsprachen kennt und die er in dieser Sprache bzw. diesen Sprachen zur Verdeutlichung seiner Aussage benutzen könnte. Dabei wurde jedoch von beiden Probandentypen nicht bedacht, daß die englischen Lexeme, die aufgrund dieser scheinbaren Verwandtschaft benutzt wurden, im Englischen eine andere Bedeutung haben als in den Sprachen, denen die Lexeme entlehnt wurden. Ein typisches Beispiel bietet die folgende Äußerung:

Fehler: $\quad$ I was quite impressed by the nature. ${ }^{519}$

Korrektur: I was quite impressed by the countryside.

Das Wort nature, das in der vorliegenden Äußerung den false friend-Effekt bildet, ist offensichtlich darauf zurückzuführen, daß der Lernende versuchte, den deutschen Satz „Ich war von der Natur ziemlich beeindruckt“" ins Englische zu übertragen. Der Lernende berichtete an dieser Stelle von seinem Urlaub. Das deutsche Wort ,Natur' ist in diesem Zusammenhang also als Synonym für das deutsche Lexem ,Landschaft‘ zu betrachten. Wie das Korrekturbeispiel zeigt, wäre dieser Begriff mit dem Wort countryside, gegebenenfalls auch wilderness, zu übersetzen gewesen. Das Wort nature hingegen entspricht nur in sogenannten Komposita, wie etwa dem Begriff nature lover, dem deutschen Wort ,Natur‘. Ansonsten entspricht das englische Lexem nature semantisch den deutschen Lexemen ,Ursprung', ,Wesen' oder ,Beschaffenheit‘. ${ }^{520}$

Ein Beispiel für den Gebrauch eines false friends in der Gruppe der PmeilAb bietet folgende Äußerung:

Fehler: $\quad$ She is very small but that isn't so wild. ${ }^{521}$

Korrektur: She is very small but that is ok/that doesn't matter very much.

Das Wort wild, das hierbei als false friend zu betrachten ist, entstammt offenbar dem deutschen umgangssprachlichen Ausdruck, das ist nicht so wild‘. Während in der deutschen Umgangssprache die Wortkombination ,nicht so wild‘ als Ersatzform für den Ausdruck ,nicht so schlimm ‘ betrachtet werden kann, ist das englische Lexem wild mit den deutschen Wörtern ,animalisch`, ,ungebändigt‘

\footnotetext{
518 vgl. Helliwell 1989

519 PwenlAb

520 vgl. Brough / Wittmann 2001, 85

521 PmeilAb
} 
oder ,unberührt` zu übersetzen. Daher besitzt das Wort im Englischen keine der Bedeutungen, die mit dem deutschen Wort ,wild‘ verbunden werden. Aus diesen beiden Fehlerbeispielen wird ersichtlich, daß false friends sowohl in frühen als auch in fortgeschrittenen Phasen des Zweitspracherwerbs auftreten können. Gleichzeitig wird deutlich, daß auch die Art der einzelnen Fehler in beiden Gruppen übereinstimmt. Während die Untersuchung der Hilfsverbfehler zeigte, daß die Verstöße der PmeilAb elementarer waren als die der entgegengesetzten Gruppe, sind beim Vergleich der false friends-Fehler keine qualitativen Unterschiede zu erkennen. Die Fehlergruppe zählt damit zu den Abweichungen, die offensichtlich eine weitgehende Resistenz gegenüber den Fehlervermeidungsversuchen zeigen, die im Englischunterricht oder im Kontakt mit Muttersprachlern unternommen werden. Fehler dieser Art gehören daher zu den gravierendsten Lernproblemen der lexikalischen Ebene.

Wortwahlfehler, die nicht zur Unterkategorie der false friends gehören, bestehen in der Verwechslung von Wörtern, die sich semantisch ähneln, jedoch nicht als Synonyme betrachtet werden dürfen. Anders als die false friends, die in der Regel eine gänzlich andere Bedeutung haben als das Wort, an das sie erinnern, haben die Lexeme dieser Kategorie isoliert betrachtet oftmals tatsächlich die Bedeutung des Wortes, das zu erwarten gewesen wäre. Dies zeigt sich deutlich in folgendem Beispiel:

Fehler: $\quad$ I hope our next common holiday will still be exciting. ${ }^{522}$

Korrektur: I hope our next shared holiday will be exciting again.

Die Grundlage des Fehlers bildet - neben dem syntaktischen Verstoß - das Wort common. Offensichtlich ist die Fehlleistung bei dem Versuch, das deutsche Wort, gemeinsam“ zu übersetzen, entstanden. Wenngleich das Lexem common in der Tat als Übersetzungsmöglichkeit für das deutsche Adjektiv ,gemeinsam‘ betrachtet werden kann, kommt diese Bedeutung nur in Verbindung mit dem Hilfsverb have zum Tragen, wie beispielsweise in dem englischen Ausdruck to have something in common. Wird das Wort common jedoch einem Hauptwort vorangestellt, so lautet die deutsche Übersetzung dieses Lexems ,gewöhnlich', ,ordinär', ,gemeinschaftlich`oder, weitverbreitet‘.

Wenngleich diese Wortverwechslungen in beiden Probandengruppen zu beobachten sind, sind beim Vergleich der Lernergruppen bei diesem Fehlertyp - anders als bei den false friends - qualitative Unterschiede festzustellen. Die Verwechslungen, die bei den PwenlAb auftreten, sind in der Regel subtiler als die Fehler, die in der entgegengesetzten Gruppe konstatiert wurden. Die Wortverwechslungen der PwenlAb lassen erkennen, daß die Lernenden über sehr gute Wortschatzkenntnisse verfügen. Dies wird auch im zuletzt genannten Fehlerbeispiel deutlich: Das Lexem common, das der Lernende fälschlicherweise verwendete, gehört normalerweise nicht zum Wortschatz eines Lernenden, der über geringe oder mittelmäßige Wortschatzkenntnisse verfügt. In 
den Lehrbüchern deutscher Schulen wird das Wort frühestens zum Ende der Mittelstufe eingeführt, d. h., nachdem die Schüler Englisch seit mindestens drei Jahren erlernt haben. ${ }^{523}$ Außerdem weist der Satz, in dem das Wort benutzt wurde, keine grundsätzlichen Strukturverstöße auf. Während des Korrekturverfahrens zeigte sich auch, daß - obgleich alle Korrektoren das Wort im gegebenen Kontext als unangebracht bezeichneten - die Entscheidung, ob es sich bei dessen Gebrauch um einen eindeutigen Fehler handelt, einiger Überlegung bedurfte. Bei den Fehlern der PmeilAb hingegen traten solche Zweifel nicht auf. Die Fehler konnten immer bereits bei erster Betrachtung als Normverstöße identifiziert werden, wie etwa in folgendem Beispiel:

Fehler: $\quad$ We will drive at the bike. ${ }^{524}$

Korrektur: We are going to ride our bikes.

Neben der falschen Futurform liegt der Verstoß darin, daß das Wort drive in der betreffenden Äußerung unangebracht ist. Die Fehlleistung ist dabei als elementar zu bezeichnen, weil Englischlernende bereits in der Frühphase des Zweitspracherwerbs erfahren, daß die Kollokation to ride a bike lautet. ${ }^{525}$ Das Wort drive wird hingegen in der Regel nur dann benutzt, wenn von einem vierrädrigen Fahrzeug die Rede ist und die Person, auf die sich das Wort drive bezieht, das Fahrzeug selber steuert. ${ }^{526}$

Insgesamt ist jedoch - ähnlich wie bei den Hilfsverben - festzustellen, daß die sprachlichen und außersprachlichen Differenzen, die beim Vergleich der beiden Probandengruppen zu erkennen sind, nur geringe Auswirkungen auf das quantitative Auftreten der Wortwahlfehler haben. Trotz der Tatsache, daß die PwenlAb sprachliche Erfahrungen in englischsprachigen Ländern sammeln konnten und darüber hinaus oft auch andere Fremdsprachen erlernt hatten, gehören die Wortwahlfehler zu den umfangreichsten Fehlerkategorien des lexikalischen Bereichs. Auch die allgemeine Schulbildung und die Schulform, an der Englisch erlernt wurde, haben keinen Einfluß auf die Anzahl der Fehler. Vielmehr ist anzunehmen, daß die Vielzahl sprachlicher und außersprachlicher Informationen, mit denen die fortgeschrittenen Lernenden im Laufe der Jahre konfrontiert wurden, ihr Differenzierungsvermögen im Bereich Wortschatz beeinträchtigt haben.

Beim Versuch, die Entstehung der Wortwahlfehler zu begründen, wurde bei Anwendung der Fehlererklärungsfaktoren der Kontrastiv- und der Interlanguagehypothese folgendes deutlich (vgl. Tabellen 12 und 13 im Anhang): Anwendungsmöglichkeiten der Interlanguagehypothese sind in der Gruppe der PmeilAb deutlich öfter gegeben als solche der Kontrastivhypothese. Dennoch kommen bei weit

523 Diese Aussage geht auf Erfahrungen zurück, die der Autor der vorliegenden Arbeit bei der Anwendung unterschiedlicher Schulbücher während seiner Referendariatszeit sammeln konnte.

524 PmeilAb

525 Diese Aussage geht auf Erfahrungen zurück, die der Autor der vorliegenden Arbeit bei der Anwendung unterschiedlicher Schulbücher während seiner Referendariatszeit sammeln konnte.

526 vgl. Brough / Wittmann 2001, $30 \mathrm{f}$ 
mehr als der Hälfte aller Fehlleistungen auch die Fehlererklärungsfaktoren der Kontrastivhypothese zum Tragen. Allgemein betrachtet ist somit die Entstehung der Fehler durch eine Kombination linguistischer und strategisch-prozeduraler Faktoren zu begründen. Die Zuordnungsergebnisse im Bereich der Kontrastivhypothese zeigen, daß rein linguistische Fehler ausschließlich in Form von Substitutionen und Über- bzw. Unterdifferenzierungen auftreten (vgl. Tabellen 14 und 15 im Anhang). Dabei kommen - wie bei den Hilfsverben - auch hier nur Faktoren der starken Version der Kontrastivhypothese zur Anwendung. Da diese Version ausschließlich erstsprachlichen Einfluß als Fehlerquelle anerkennt, ist also davon auszugehen, daß die linguistischen Differenzen zwischen deutschen und englischen Lexemen maßgeblich an der Entstehung der Wortwahlfehler beteiligt sind. Von den Fehlertypen der starken Version ist dabei besonders häufig das Substituieren englischsprachiger Elemente, die den Lernenden an deutsche Lexeme erinnern, zu beobachten, wie in folgendem Beispiel:

Fehler: $\quad$ OK, that's it. Make it good. ${ }^{527}$

Korrektur: That's all for now. Be good and take care.

Es ist eindeutig zu erkennen, daß es sich hier um einen Übersetzungsversuch des deutschen Satzes „Mach es gut“ handelt. Das Wort make wird jedoch als Abschiedsgruß im Englischen nicht verwendet. Das Auftreten erstsprachlich bedingter Über- bzw. Unterdifferenzierungen ist in folgendem Beispiel zu erkennen:

Fehler: In my holidays my moneybag is stolen by a man. ${ }^{528}$

Korrektur: During my vacation my wallet was stolen by a man.

Offensichtlich wußte der Lernende, daß im Englischen - ähnlich wie im Deutschen - Lexeme oftmals durch Wortzusammensetzungen gebildet werden, wie etwa in dem Lexem bottle opener. Er hat jedoch nicht berücksichtigt, daß diese Wortbildungsregel im Englischen in manchen Fällen - zu denen auch das Wort wallet gehört - nicht zutrifft. Der Einfluß der Erstsprache Deutsch äußert sich dabei zum einen darin, daß der Lernende eine Wortbildungsregel, die im Deutschen nahezu immer zutrifft, ohne weitere Differenzierung ins Englische übertragen hat. Zum anderen wird der Einfluß auch in der Wahl der Lexeme money und bag deutlich. Vermutlich hat hier das deutsche Wort ,Geldbörse‘ als Vorlage gedient. - Beide Fehlleistungen klassifizieren die Abweichung als Unterdifferenzierung.

Die Ergebnisse, die bei Anwendung der Interlanguagehypothese ermittelt wurden (vgl. Tabellen 16 und 17 im Anhang), entsprechen nahezu den Resultaten, die bei der Analyse der Hilfsverbfehler erzielt wurden. Die meisten Fehler gehen auf strategisches Verhalten der Lernenden während der Sprachanwendung zurück. Reduction strategies, interlingual transfer und insbesondere bewußte generalizations stellen auch hier die größten Fehlerquellen dar. Im Gegensatz zu den Hilfsverben ist

527 PmeilAb 
aber hier bei einem Teil der Fehler zu vermuten, daß sie auf unbewußtes Verhalten zurückzuführen sind. Daneben hat partiell auch lack of contrast zur Entstehung der Abweichungen beigetragen. Das Zusammenwirken der einzelnen Faktoren zeigt sich z. B. in folgender Äußerung:

Fehler: $\quad$ Please write me back and told me if I can come next spring. ${ }^{529}$ Best greetings ${ }^{530}$ Korrektur: Please write back to me and let me know if I can come next spring. Best wishes

Neben den syntaktisch-morphologischen Abweichungen ist die Äußerung aufgrund des Gebrauchs der Lexeme told und greetings als Normverstoß zu bezeichnen. Der Mangel an Ausdrucksformen, der sich hierbei in erster Linie in Form der sogenannten modality and personality reduction äußert, wird sowohl beim Lexem told als auch beim Lexem greetings deutlich. Es ist davon auszugehen, daß dem Lernenden aufgrund seiner geringen Wortschatzkenntnisse nur wenige englische Wörter zur Übersetzung der deutschen Lexeme ,erzählen` oder ,mitteilen` zur Verfügung standen. Gleiches gilt für das deutsche Wort ,Grüße'. Da er jedoch darum bemüht war, seine sprachliche Aussage zu verdeutlichen, hat er auf die Elemente zurückgegriffen, die ihm zur Verfügung standen, obwohl er damit Gefahr lief, von der Norm der englischen Sprache abzuweichen.

Interlingual transfer als weitere Kommunikationsstrategie, die in diesem Beispiel deutlich wird, ist vor allem bei dem Wort greetings zu erkennen. Offensichtlich ist diese Abweichung - wie erwähnt auf das deutsche Lexem ,Grüße' zurückzuführen, da das Wort dem englischen Lexem greetings strukturell ähnelt. Dies spricht gleichzeitig auch dafür, daß lack of contrast an der Fehlerentstehung beteiligt gewesen sein könnte. Bei den Fehlern handelt es sich zusätzlich auch um bewußte generalizations, weil der Lernende nicht berücksichtigt hat, daß die Anwendungsmöglichkeiten der Lexeme tell und greetings beschränkt sind. Das Wort tell wird in der Regel in Aufforderungen nur dann benutzt, wenn der Kommunikationspartner eine sofortige Antwort erwartet. ${ }^{531}$ Das Lexem greetings ist nur als Eingangsformel, etwa auf Postkarten, üblich und wird normalerweise in Briefen gar nicht benutzt. ${ }^{532}$ Die Möglichkeit, daß die Generalisierungen auch unbewußt verlaufen sein könnten, induziert insbesondere das Lexem told. Das Wort wurde im schriftlichen Beitrag des Lernenden mehrfach verwendet, so daß vermutet werden kann, daß der Teilnehmer aufgrund des Zeitfaktors und der testbedingten Anspannung nicht die Möglichkeit hatte, auf ein anderes Lexem zurückzugreifen.

Bei den Verstößen der PwenlAb sind Anwendungsmöglichkeiten der Kontrastiv- und der Interlanguagehypothese etwas seltener gegeben als in der zuvor behandelten Probandengruppe, wobei hier die Interlanguagehypothese öfter zur Anwendung kommt als die Kontrastivhypothese (vgl. Tabellen 12

\footnotetext{
528 PmeilAb

529 Der Ausdruck next spring wurde bereits im vorhergehenden Text des Probanden mehrfach erwähnt und ist daher in diesem Zusammenhang überflüssig.

530 PmeilAb

531 vgl. Brough / Wittman 2001, 100
} 
bis $17 \mathrm{im}$ Anhang). Dennoch ist auch in dieser Gruppe die Fehlerentstehung auf eine Mischung linguistischer und strategisch-prozeduraler Faktoren zurückzuführen. Bei Anwendung der Faktoren der Kontrastivhypothese stimmen die Kategorien, die nachzuweisen sind, nahezu mit den Kategorien überein, die in der entgegengesetzten Probandengruppe zum Tragen kommen (vgl. Tabellen 14 und 15 im Anhang). Auch hier äußert sich der Einfluß der Erstsprache in erster Linie in Form von Substitutionen und Unter- bzw. Überdifferenzierungen. Nur in wenigen Fällen ist eine Übergeneralisierung im Sinne der schwachen Version der Kontrastivhypothese zu erkennen. In allen anderen Fällen kommen - wie bei der entgegengesetzten Probandengruppe - nur Faktoren der starken Version dieser Erwerbshypothese zur Anwendung. Deckungsgleich ist auch, daß das Substituieren sprachlicher Elemente stärker hervortritt als Über- bzw. Unterdifferenzierungen. Der Einfluß der Faktoren der Kontrastivhypothese ist $u$. a. an folgendem Beispiel zu erkennen:

Fehler: $\quad$ Then we can speak about our next journey in spring and can also exchange views about personal things. ${ }^{533}$

Korrektur: Then we can talk about our next journey in spring and can also discuss personal matters.

Der Einfluß der deutschen Sprache äußert sich vor allem in der Verwendung der Lexeme speak und things, ist aber vermutlich auch bei dem Ausdruck exchange views vorhanden. Die Wahl der Lexeme speak und things läßt eindeutig darauf schließen, daß es sich hier um Übersetzungen der deutschen Wörter ,sprechen' und ,Dinge' handelt. Die Fehlleistungen wurden dabei sowohl durch die lautliche als auch durch die optische Ähnlichkeit zwischen den deutschen und den englischen Lexemen verursacht. Somit sind die Fehler als Substitutionen zu bezeichnen. Das Wort exchange, das nur von einem Teil der Korrektoren als normabweichend betrachtet wurde, stellt eine Unterdifferenzierung dar, die auf das deutsche Wort ,austauschen` zurückzuführen ist.

\subsection{Inexistente Wörter}

Der Vergleich der entgegengesetzten Probandengruppen zeigt, daß der Gebrauch inexistenter Wörter als ein Lernproblem betrachtet werden muß, das nicht nur bei Lernenden mit extremen lexikalischen Schwierigkeiten zu beobachten ist, sondern auch bei Lernenden, die über fortgeschrittene Wortschatzkenntnisse verfügen (vgl. Tabellen 2 und 3 im Anhang). Schon vor Anwendung der Erwerbshypothesen ist an dieser Stelle festzuhalten, daß beide Gruppen deutscher Englischlernender ihre Kommunikationslücken u. a. durch das Erfinden vermeintlich englischer Lexeme auszugleichen versuchten. Somit ist festzustellen, daß beide Probandentypen die zweitsprachlichen Elemente, die sie im Laufe des Spracherwerbs verinnerlicht hatten, nicht nur in der Form wiedergaben, in der sie erworben worden waren, sondern daß sie mit diesen Elementen auch kreativ umgingen. Der quantitative Ver-

\footnotetext{
532 vgl. Swan 1990, Textstelle 274, o. S.

533 PwenlAb
} 
gleich der Fehler zeigt allerdings, daß dieses Verhalten bei Lernenden mit ausgeprägten lexikalischen Defiziten weitaus stärker zur Anwendung kam als bei Lernenden mit überdurchschnittlich guten Wortschatzkenntnissen. Während der Gebrauch inexistenter Wörter bei den PmeilAb quantitativ die zweitgrößte Fehlerkategorie bildet, stellt dieser Fehlertyp in der entgegengesetzten Probandengruppe die Fehlerkategorie mit den wenigsten Eintragungen dar (vgl. Tabellen 2 und 3 im Anhang). Demnach sind fortgeschrittene Lernende weitaus besser in der Lage zu erkennen, ob bestimmte lexikalische Elemente, die die Lernenden selber prägen, tatsächlich zum Bestand der englischen Sprache gehören könnten oder nicht. Auch die Art der Wortprägungen unterscheidet sich in beiden Probandengruppen.

Bei den PmeilAb wurde die Mehrzahl der inexistenten Wörter nach dem Vorbild der deutschen Sprache gebildet. Dies bedeutet, die Betreffenden wandten entweder deutsche Wortbildungsregeln an und erfanden auf diese Weise ein Lexem, das im Wortschatz der englischen Sprache nicht existiert oder sie benutzten ein deutsches Lexem und veränderten dieses morphologisch so, daß die Struktur des Wortes der eines englischen Lexems entsprach. In vielen Fällen wurden auch englische Wörter gänzlich durch deutsche Wörter ersetzt, teils durch Anpassung des deutschen Lexems an die englische Rechtschreibung, teils jedoch auch ohne jegliche Anpassung an die englische Sprache. Die Anwendung deutscher Wortbildungsregeln äußerte sich meist in Form der sogenannten Kompositabbildung, die im Deutschen zur Entstehung von Wörtern wie beispielsweise ,Solidaritätsbeitrag' oder ,Scheinselbständigkeit‘ führte. Analog zu dieser deutschen Regel substituierten die Probanden ein unbekanntes englisches Lexem dadurch, daß sie zwei ihnen bekannte Wörter miteinander kombinierten, um daraus ein Wort zu bilden, das eine ähnliche Aussage vermittelt wie das eigentlich zu erwartende Lexem. Beispiele hierfür bieten u.a. Wörter wie, dive-glasses ' für goggles oder ,coolcupboard ' für refrigerator. Im Englischen sind Komposita jedoch weniger häufig zu beobachten. Dies beruht vor allem darauf, daß die Kargheit morphologischer Markierung eine große Freiheit insbesondere in der Verwendung substantivischer Modifikatoren erlaubt, aber auch darauf, daß eine große Anzahl der englischen Lexeme lateinischen Ursprungs ist $^{534}$ und im Lateinischen Komposita seltener benutzt werden als in den germanischen Sprachen. ${ }^{535}$ Die Anpassung deutscher Wörter an die morphologische Struktur englischer Lexeme äußert sich in der Regel durch Veränderungen der Endsilbe oder des letzten Phonems, wie etwa in folgendem Beispiel:

Fehler: $\quad$ a Packing cigarettes ${ }^{536}$

Korrektur: a pack of cigarettes

Hier ist der Fehler auf die lautliche Ähnlichkeit zwischen der Endsilbe, -ung' im deutschen Wort ,Packung' und der englischen Endsilbe ,-ing', die bei englischen Hauptwörtern häufig zu finden ist,

\footnotetext{
534 vgl. Baugh / Cable 1978, 46 - 52

535 vgl. Ungerer u. a. $1987,239 \mathrm{f}$

536 PmeilAb
} 
zurückzuführen. Offensichtlich hat der Lernende zunächst erkannt, daß das Morphem ,-ung‘ eindeutig der deutschen Sprache zuzuordnen ist und daher nicht benutzt werden kann. Die oben erwähnte Ähnlichkeit hat ihn jedoch dazu bewogen, auf die Verwendung einer Endsilbe nicht ganz zu verzichten, sondern auf die Lautkombination, ,-ing' zurückzugreifen, zumal das Wort packing im Englischen tatsächlich existiert - wenn auch nicht mit der Bedeutung, Packung‘. Bestätigt wird die Annahme, daß es sich hier um die Übernahme eines deutschen Wortes handelt, auch durch die Großschreibung des fehlerhaften Lexems. Eine direkte Übernahme deutscher Wörter mit bzw. ohne Anpassung an die Rechtschreibung der englischen Sprache fand in folgenden Beispielen statt:

Fehler: $\quad$ The Markt was very crowd. ${ }^{537}$

Korrektur: The market place was very crowded.

Fehler: $\quad$ I have a friend in Russland. ${ }^{538}$

Korrektur: I have a friend in Russia.

Im ersten Fehlerbeispiel wurde das Wort ,Markt“ ohne morphologische oder orthographische Anlehnung an die englische Sprache übernommen. Im zweiten Beispiel hat der Lernende lediglich das Graphem $<\beta>$, das nicht Bestandteil der englischen Sprache ist, dem Englischen angepaßt. ${ }^{539}$

Bei den PwenlAb spiegeln die inexistenten Wörter, die benutzt wurden, wider, daß die Lernenden über ein umfangreicheres linguistisches Wissen verfügten als die entgegengesetzte Probandengruppe. Dies ist zum einen daran zu erkennen, daß die Wortprägungen stärker an die englische Sprache angepaßt wurden. Zum anderen äußert sich das umfassendere linguistische Wissen auch darin, daß die Wortprägungen zum Teil nicht nur auf das Englische oder das Deutsche zurückgingen, sondern auch auf andere Zweitsprachen. Dies verdeutlicht folgendes Beispiel:

Fehler: $\quad$ Retour to the hotel my bike have had a puncture.

Korrektur: Returning to the hotel I discovered that my bike had had a puncture.

Die Fehlleistung, die auf lexikalischer Ebene in der Anwendung des Wortes retour besteht, zeigt, daß der Lernende neben Englisch auch eine romanische Sprache erlernt hat. ${ }^{540}$

Die Anwendung der Erwerbshypothesen führte zu folgenden Ergebnissen: Der Vergleich der Probandengruppen zeigt, daß die Kontrastivhypothese in der Gruppe der PmeilAb häufiger zur Anwendung kommt als die Interlanguagehypothese (vgl. Tabellen 12 und 13 im Anhang). In der Gruppe der PwenlAb hingegen überwiegen die Anwendungsmöglichkeiten der Interlanguagehypothese. Demnach ist der Fehleranteil, der auf erstsprachlichen Einfluß

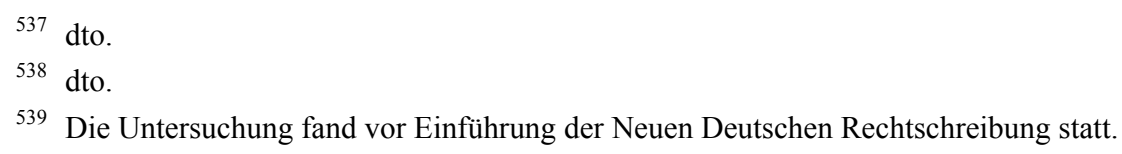


zurückzuführen ist, bei den PmeilAb größer als in der entgegengesetzten Gruppe. Bei deutschen Englischlernenden mit überdurchschnittlichen lexikalischen Kenntnissen basiert die Entstehung inexistenter Wörter diesem Ergebnis entsprechend stärker auf strategischem Verhalten, d. h. auf dem Versuch, durch Rückgriff auf bekannte Lexeme Sprachlücken zu überbrücken. Dennoch beweist die Tatsache, daß auch in dieser Teilnehmergruppe ein erheblicher Anteil der Fehler auf die Entstehungsfaktoren der Kontrastivhypothese zurückgeführt werden kann, daß der Einfluß der deutschen Sprache als wichtige Fehlerentstehungsquelle zu betrachten ist.

Die genauere Betrachtung der einzelnen Fehlererklärungsfaktoren zeigt, daß in beiden Probandengruppen bei Anwendung der Kontrastivhypothese nur der Fehlererklärungsfaktor ,Substitution“ nachzuweisen ist (vgl. Tabellen 14 und 15 im Anhang). Demnach besteht die Differenz, die hinsichtlich der Anwendungsmöglichkeit der Kontrastivhypothese zu beobachten ist, lediglich auf quantitativer Ebene. Qualitativ ist vielmehr festzustellen, daß sowohl bei den PwenlAb als auch bei den PmeilAb inexistente Wörter dadurch entstanden sind, daß englische Wörter durch Lexeme ersetzt wurden, die der Lernende aus seiner Erstsprache abgeleitet hat. Diese Ableitungen basieren in der Regel darauf, $\mathrm{da} ß$ die Lernenden aufgrund der Tatsache, daß mitunter lautliche und optische Parallelen zwischen deutschen Wörtern und ihren englischen Entsprechungen bestehen, glaubten, lexikalische Lücken durch Rückgriff auf ein deutsches Lexem kompensieren zu können.

Auch bei Anwendung der Erklärungsfaktoren der Interlanguagehypothese wird deutlich, daß der Einfluß der Erstsprache Deutsch bei den PmeilAb stärker zum Tragen kommt als in der entgegengesetzten Probandengruppe (vgl. Tabellen 16 und 17 im Anhang). Dies verdeutlicht insbesondere die hohe Anzahl der Eintragungen in der Kategorie language switch. Bei den PwenlAb hingegen enthält diese Kategorie keine Eintragungen. In Hinblick auf die Frage, welche Erklärungsfaktoren der Interlanguagehypothese in beiden Gruppen nachgewiesen werden konnten, ist weiterhin festzuhalten, daß der Erklärungsfaktor lack of contrast bei den PwenlAb kaum ins Gewicht fällt, in der entgegengesetzten Probandengruppe jedoch einen entscheidenden Fehlerentstehungsfaktor darstellt. Die Untersuchung der hier einzuordnenden Fehlertypen zeigt darüber hinaus, daß die meisten dieser Abweichungen auf Kontrastmangel zwischen den einzelnen englischen Lexemen basieren und nicht auf Kontrastmangel zwischen deutschen und englischen Wörtern. Dieses Ergebnis bestätigt die von Gegnern der Kontrastivhypothese aufgestellte These, daß nicht nur Differenzen zwischen zielsprachlichen und erstsprachlichen Strukturen Fehler verursachen können, sondern auch strukturelle Ähnlichkeiten zwischen den verschiedenen Elementen der Zielsprache. ${ }^{541}$ Zudem bestätigt es auch die bereits weiter oben erwähnte Theorie, daß mit der Erweiterung des Wortschatzes nicht unbedingt auch eine Lernerleichterung einhergeht. Vielmehr kann ein Wissenszuwachs auf lexikalischer Ebene

\footnotetext{
540 In diesem Fall handelt es sich um eine Entlehnung aus dem Französischen.

541 vgl. Juhász 1970
} 
durchaus dazu führen, daß die betreffenden Lernenden aufgrund struktureller Ähnlichkeiten partiell nicht mehr dazu imstande sind, korrekt zwischen den erworbenen Lexemen zu unterscheiden.

Der Vergleich der beiden Probandengruppen zeigt darüber hinaus, daß die Fehler in beiden Gruppen auf kreativem Sprachverhalten basieren und nicht auf sprachlichen Einschränkungen. Dies wird durch die Tatsache bestätigt, daß weder bei den PmeilAb noch bei den PwenlAb Eintragungen in den Kategorien modality und personality reduction enthalten sind (vgl. Tabellen 16 und 17 im Anhang). Auffällig ist auch, daß die als meaning replacement bezeichnete Fehlerkategorie nur bei den PwenlAb Eintragungen enthält. Nur diese Lernenden erfinden also Ausdrücke, durch die die eigentlich geplante sprachliche Aussage vollständig verändert wird. Bei den PmeilAb hingegen lassen die Wortprägungen eine stärkere semantische Ähnlichkeit zu den Lexemen, die eigentlich zu erwarten gewesen wären, erkennen. Demnach sind das kreative Sprachverhalten und damit verbunden die Bereitschaft, Fehler zu produzieren, bei den PwenlAb stärker ausgeprägt als in der entgegengesetzten Probandengruppe.

\subsection{Präpositionen}

Im Bereich der Präpositionalfehler konnten alle Abweichungsformen, die während des Korrekturverfahrens ermittelt wurden, sowohl bei den PmeilAb als auch bei den PwenlAb beobachtet werden. In beiden Probandengruppen zeigten sich Differenzierungsschwierigkeiten bei der Anwendung von Präpositionen, die der Verdeutlichung zeitlicher Zusammenhänge und räumlicher Verhältnisse dienen. Dazu zählen vor allem die Präpositionen in, on und at sowohl in ihrer zeitlichen als auch in ihrer räumlichen Bedeutung. Anwendungsprobleme waren auch - obgleich weniger häufig - beim Gebrauch der Präpositionen for und since zu erkennen, insbesondere im Zusammenhang mit den Zeitformen simple past und present perfect. Auch die Präposition by führte insbesondere bei den PmeilAb zu Anwendungsschwierigkeiten. In dieser Gruppe diente sie häufig im Zusammenhang mit Ortsverhältnissen als Substitutionselement für die Präposition at, die in den gegebenen Situationen korrekterweise zu verwenden gewesen wäre.

Fehler wurden darüber hinaus - wenn auch in geringerem Umfang und nahezu ausschließlich bei den PwenlAb - beim Gebrauch der Lexeme over/above, under/below, before/in front of beobachtet. Dabei zeigte sich, daß die Anwendungsschwierigkeiten, die dabei zu erkennen waren, darauf beruhen, daß die Präpositionen over und above, under und below sowie in front of und before sich ähnelnde semantische Funktionen und teilweise auch dieselbe deutsche Übersetzung haben, im Englischen jedoch nicht austauschbar sind. Seltener war in beiden Probandengruppen zu beobachten, daß auch solche Präpositionen falsch verwendet wurden, die im direkten Zusammenhang mit einem Verb stehen, 
wie etwa in dem Fehlerbeispiel talk with, das darauf beruht, daß statt des Lexems with die Präposition to hätte verwendet werden müssen.

Zusammengefaßt zeigt der Vergleich der entgegengesetzten Probandengruppen neben der Tatsache, daß typologisch betrachtet insgesamt nur wenige Abweichungen zu beobachten sind, daß der Präpositionsgebrauch der PwenlAb auf fortgeschrittenene Sprachkenntnisse zurückzuführen ist, während die Fehler in der entgegengesetzten Probandengruppe elementarer sind. Dies bedeutet, die PmeilAb verursachten Fehler, die aufgrund der dort umfassenderen Spracherfahrungen in der entgegengesetzten Gruppe nur selten auftraten. So sind Äußerungen wie etwa das Fehlerbeispiel I will send you the picture I'm on (richtig: I'll send you the picture I'm in) nahezu ausschließlich bei den PmeilAb zu beobachten. Andererseits zeigt jedoch das Korrekturverfahren - wenn auch in anderen Zusammenhängen -, daß die Präpositionen, die bei den PmeilAb auf elementarer Ebene normabweichend verwendet werden, auf fortgeschrittenerer Ebene auch bei den PwenlAb zu Anwendungsschwierigkeiten führen können. So zeigte sich, daß auch die PwenlAb oftmals nicht in der Lage waren zu unterscheiden, ob eine bestimmte Präpositionalphrase mit der Präposition on oder in zu übersetzen ist. Dies trat insbesondere dann auf, wenn der räumliche Bezug nicht eindeutig zu erkennen war. Ein Beispiel für dieses Problem bietet die deutsche Präpositionalphrase ,auf der Straße', die, je nach beabsichtigter Aussage, entweder mit dem Ausdruck in the street oder mit on the street übersetzt werden muß. ${ }^{542}$

Die Anwendung der Erwerbshypothesen zeigt, daß - ähnlich wie bei der Fehlerkategorie ,Inexistente Wörter ${ }^{\star}$ - die Anwendungsmöglichkeiten der Kontrastivhypothese bei den PmeilAb überwiegen (vgl. Tabelle 12 im Anhang). In der entgegengesetzten Probandengruppe hingegen kommen eindeutig die Erklärungsfaktoren der Interlanguagehypothese häufiger zur Anwendung (vgl. Tabelle 13 im Anhang). Dies bestätigt die zuvor getroffene Feststellung, daß die Fehler der PwenlAb subtiler sind, d. h. mehr auf dem strategischen Verhalten der Probanden basieren als in der entgegengesetzten Teilnehmergruppe. Bei den PmeilAb hingegen ist eine stärkere Anlehnung an die deutsche Sprache zu erkennen. Hier zeigt sich bei der Mehrzahl der Präpositionsfehler, daß die Abweichungen auf dem Versuch basieren, englische Lexeme durch Elemente zu ersetzen, die den Lernenden lautlich und optisch an die Präposition erinnerten, die im betreffenden Zusammenhang in der deutschen Sprache zu verwenden gewesen wäre. Ein Beispiel bietet folgende Aussage:

Fehler: $\quad$ Yesterday I was by my friend. ${ }^{543}$

Korrektur: Yesterday I was at my friend's.

\footnotetext{
542 Die Präposition in wird verwendet, um zu verdeutlichen, daß sich eine Person oder ein Gegenstand in dieser Straße befindet, während die Präposition on nur benutzt wird, um zu verdeutlichen, daß eine Person oder ein Gegenstand auf der Straße liegt.

543 PmeilAb
} 
Hierbei handelt es sich eindeutig um eine nahezu wörtliche Übersetzung des deutschen Satzes „Gestern war ich bei meinem Freund.“ Die Wahl der Präposition by basiert dabei auf der Tatsache, daß das Deutsche über das Lexem ,bei‘ verfügt, welches dem englischen Element by optisch und akustisch nahezu entspricht. Die Fehler der PwenlAb hingegen zeigen, daß sich die Teilnehmer geradezu gegensätzlich verhielten, d. h., sie wählten Präpositionen, die den deutschen Präpositionen möglichst unähnlich sind, wie etwa in folgendem Beispiel:

Fehler: $\quad$ I saw a picture of a king who ruled above England. ${ }^{544}$

Korrektur: I saw a picture of a king who ruled over England.

Die Präposition over, die in dem betreffenden Beispiel zu benutzen gewesen wäre, wurde vermutlich daher nicht gebraucht, weil der Lernende es vermeiden wollte, eine Präposition zu benutzen, die dem deutschen Lexem ,über` optisch und akustisch zu sehr entspricht. Somit wählte er aus kommunikationsstrategischen Gründen ein Lexem, das der Präposition over semantisch ähnelt, strukturell jedoch vollkommen von der deutschen Präposition, über ‘ abweicht. Da jedoch auch bei den PwenlAb die Faktoren der Kontrastivhypothese häufig zur Anwendung kommen, ist auch in dieser Probandengruppe der Einfluß der deutschen Sprache als wichtiger Fehlerentstehungsfaktor hervorzuheben.

Bei genauerer Betrachtung der Fehlererklärungsfaktoren der einzelnen Erwerbshypothesen wird jedoch - wie auch in der zuletzt beschriebenen Fehlerkategorie - deutlich, daß von den Faktoren der Kontrastivhypothese in beiden Probandengruppen nur die Kategorie ,Substitution" nachzuweisen ist (vgl. Tabelle 14 und 15 im Anhang). Damit zeigt sich, daß nicht nur der Einfluß der deutschen Sprache in beiden Gruppen zu erkennen ist, sondern daß selbst fortgeschrittene Lernende englische Elemente durch Lexeme ersetzen, die an deutsche Worte erinnern.

Bei Anwendung der Erklärungsfaktoren der Interlanguagehypothese wird deutlich, daß in beiden Probandengruppen die Mehrzahl der Präpositionalfehler auf das Generalisieren von Interlanguageregeln und -elementen in neuen Sprachzusammenhängen zurückgeführt werden kann (vgl. Tabellen 16 und 17 im Anhang). Demnach basieren die Fehler der Interlanguagehypothese zufolge in erster Linie auf dem strategischen Verhalten, das die Lernenden während des Kommunikationsverlaufs anwandten. Dieses Sprachverhalten kam dabei in beiden Probandengruppen bewußt zur Anwendung, wenngleich die Lernenden teilweise auch unbewußtes Sprachverhalten erkennen ließen. Darüber hinaus zeigen die Ergebnisse, daß sowohl bei den PmeilAb als auch bei den PwenlAb partiell auch lack of contrast an der Fehlerentstehung beteiligt war.

544 PwenlAb 
Das Zusammenwirken der einzelnen Faktoren der Kontrastiv- und Interlanguagehypothese wird an folgenden Beispielen deutlich:

Fehler: I'll go with some friends in a disco. ${ }^{545}$

Korrektur: I'm going to a discotheque with some friends.

Fehler: We're often go into a pub. ${ }^{546}$

Korrektur: We often go to a pub.

Fehler: We went there by my car. ${ }^{547}$

Korrektur: We went there in my car.

Fehler: I'm looking forward for seeing you. ${ }^{548}$

Korrektur: I'm looking forward to seeing you.

Das erste Fehlerbeispiel, in dem der Lernende statt der zu erwartenden Präposition to das Lexem in verwendete, läßt eindeutig darauf schließen, daß er von der deutschen Präposition ,in` fehlgeleitet wurde, d. h., es wurde hier ein englischsprachiges Lexem durch ein anderes englisches Wort ersetzt, das einem deutschen Element strukturell nahezu entspricht. In bezug auf das zweite Beispiel kann die Abweichung, die auf der Anwendung der Präposition into basiert, sowohl als Substitution als auch als bewußte generalization gedeutet werden. Als Substitution kann sie eingestuft werden, weil davon auszugehen ist, daß, ähnlich wie im vorhergehenden Beispiel, die deutsche Präposition ,in‘, an der sich der Lernende bei seiner Übersetzung orientierte, zur Entstehung der Abweichung beigetragen hat. Als Generalisierung eines Elements, das der Lernende zu einem früheren Erwerbsstadium internalisiert hatte, ist der Fehler einzuordnen, weil die Präposition into im Englischen in der Tat dazu benutzt wird zu verdeutlichen, daß eine Person oder Sache in einen geschlossenen Raum gelangt, wie etwa in der Phrase go into a room. Im obigen Beispiel ist die Einrichtung pub aber nicht als Raum zu betrachten, sondern als öffentliche Institution, wie etwa eine Schule oder eine Kirche. Es wäre daher das Wort to zu verwenden gewesen.

Generalisierungen, sowohl auf bewußter als auch auf unbewußter Ebene, zeigen sich insbesondere in den letzten beiden Fehlerbeispielen, die aus der Gruppe der PwenlAb stammen. Das Fehlerbeispiel We went there by my car ist als Generalisierung einzustufen, weil der Lernende offensichtlich in der Vergangenheit verinnerlicht hat, daß bei Transportmitteln in der Regel die Präposition by zu benutzen ist. Das Hinzufügen des Personalpronomens $m y$ führt jedoch in dieser Äußerung dazu, daß das Wort car nicht mehr ein allgemeines Transportmittel bezeichnet, sondern das Eigentum des betreffenden

\footnotetext{
545 PmeilAb

546 dto.

547 PwenlAb

548 dto.
} 
Sprechers. Der Norm der englischen Sprache entsprechend wäre in einem solchen Fall die Präposition in zu verwenden gewesen. ${ }^{549}$

Da der Lernende - wie der Text insgesamt zeigte - Präpositionen in der Regel normgerecht verwendet, ist zu vermuten, daß es sich in diesem Fall auch um eine Fehlleistung handelt, die unbewußt entstanden ist und damit als overgeneralization zu betrachten ist. Die Tatsache, daß die deutsche Sprache über das Wort ,bei' verfügt, das dem englischen Lexem by optisch und akustisch ähnelt, mag - obwohl das deutsche Wort ,bei` zur Verdeutlichung anderer Zusammenhänge dient - die unbewußt verlaufende Handlung zusätzlich motiviert haben. Somit ist nicht auszuschließen, daß es sich bei der vorliegenden Generalisierung marginal auch um eine Substitution im Sinne der Kontrastivhypothese handelt.

Das unbewußte wie auch das bewußte Generalisieren von Interlanguageelementen wird besonders im letzten Beispiel deutlich. Diese Fehlleistung ist möglicherweise unbewußt verlaufen, weil der Lernende durch Anwendung der Präposition forward wahrscheinlich ungewollt an das Lexem for erinnert wurde, das häufig im Zusammenhang mit Gerundialkonstruktionen verwendet wird. Andererseits ist es jedoch auch möglich, daß der Proband die Präposition for absichtlich benutzte, weil das Element for nicht nur in der Redewendung to look forward to enthalten ist, sondern auch in einer englischen Übersetzung des deutschen Verbs ,suchen', das u. a. mit dem Ausdruck to look for übersetzt werden kann. So könnte es sein, daß der Lernende aufgrund der ihm bekannten Kombinationen der Elemente look und for glaubte, bei Verwendung des Verbs look bei allen nachfolgenden Präpositionen die Präposition for benutzen zu müssen.

Zusammenfassend betrachtet zeigen die Analyse der Fehlleistungen sowie die Art und die Anzahl der Verstöße, die während des Korrekturverfahrens ermittelt wurden, daß Präpositionen zu den fehleranfälligsten Elementen der englischen Sprache gehören. Ihre Entstehung läßt sich auf eine Verbindung linguistischer und außersprachlicher Faktoren zurückführen, d. h. auf erstsprachlichen Einfluß und auf das strategisch-prozedurale Verhalten der Lernenden während der Sprachanwendung. Die Betrachtung der psycho- und soziolinguistischen Faktoren zeigt dabei, daß Präpositionsfehler selbst dann auftreten können, wenn die Lernenden intensiven Kontakt zu englischsprachigen Muttersprachlern hatten bzw. mehrere Monate in englischsprachigen Ländern verbracht hatten. Auch linguistische Erfahrungen, die über das Erlernen der englischen Sprache hinausgehen, wie etwa Kenntnisse in anderen Sprachen oder intensiver Grammatikunterricht in der Erstsprache, tragen nur ansatzweise zu einer Reduktion der Anwendungsprobleme bei. Die Komplexität englischer Präpositionen, d. h. die subtilen semantischen Unterschiede von Elementen wie etwa under und below, und die unterschiedlichen Übersetzungs- und Anwendungsmöglichkeiten scheinbar einfacher Elemente - wie etwa der Präposition in - bedürfen

549 vgl. Ungerer u. a. 1987, 113 
sowohl in der Frühphase als auch in den fortgeschrittenen Phasen des Zweitspracherwerbs einer besonderen Hervorhebung im Englischunterricht deutscher Muttersprachler.

\subsection{Adjektiv}

Die Anzahl der Abweichungen, die beim Gebrauch der Lexemgruppe Adjektiv bei den PmeilAb und den PwenlAb ermittelt wurde, zeigt, daß quantitativ betrachtet die Anwendungsschwierigkeiten beim Vergleich beider Probandengruppen nahezu identisch sind. In beiden Gruppen ist die Anzahl der Verstöße im Vergleich zu anderen Fehlerkategorien niedrig (vgl. Tabellen 2 und 3 im Anhang): Damit ist zu erkennen, daß Lern- und Gebrauchsschwierigkeiten im Zusammenhang mit dieser Wortgruppe in beiden Probandengruppen relativ selten auftreten, wobei die Anwendungsschwierigkeiten bei den PmeilAb stärker ausgeprägt sind als bei den PwenlAb. Dennoch sei bereits an dieser Stelle vermerkt, daß dieses Ergebnis nicht zu der Annahme führen sollte, daß Adjektive zu den Elementen der englischen Sprache gehören, die von Englischlernenden ohne Schwierigkeiten internalisiert werden. Vielmehr zeigt die Tatsache, daß Adjektiv-Fehler auch in fortgeschrittenen Erwerbsphasen auftreten können, daß diese im Englischunterricht einer besonderen Hervorhebung bedürfen.

Im Verlauf des Korrekturverfahrens fiel zudem auf, daß die Schwierigkeiten bei der Bewertung von Adjektiv-Fehlern bei den englischen Muttersprachlern größer waren als bei den deutschen Korrektoren. Die englischen Muttersprachler konnten während des Korrekturverfahrens oftmals nicht entscheiden bzw. waren sich nicht einig, ob ein bestimmtes Element, das von einem Probanden benutzt wurde, letztlich gegen die semantischen oder grammatischen Gebrauchsregeln englischer Adjektive verstößt oder nicht. ${ }^{550}$ In solchen Fällen wurde die Entscheidung in der Regel von den deutschen Korrektoren getroffen, da sie Englisch als Zweitsprache sprechen und daher ein neutraleres Korrekturverhalten zeigen als englische Muttersprachler. Dies liegt vermutlich darin begründet, daß sich deutsche Korrektoren oftmals stärker an dem grammatischen Modell einer Sprache orientieren als an deren praktischen Anwendungsmöglichkeiten.

In bezug auf die Adjektivfehler, die innerhalb der Probandengruppen ermittelt wurden, ist festzustellen, daß die Fehler der PmeilAb einerseits darin bestanden, daß statt der Adjektivform eines bestimmten Lexems dessen Partizipialform benutzt wurde, wie etwa im folgenden Beispiel:

550 Dies liegt vor allem darin begründet, daß sich der schriftliche Adjektivgebrauch deutlich von dem mündlichen unterscheidet. In der gesprochenen Sprache neigen auch englische Muttersprachler dazu, gegen die Grammatikregeln bezüglich des Gebrauchs von Adjektiven zu verstoßen. So ist es häufig zu beobachten, daß Adjektive benutzt werden, obwohl in dem betreffenden Kontext ein Adverb zu erwarten wäre, wie etwa in der Aussage She runs quick, die während eines Gesprächs mit einem Briten vom Autor der vorliegenden Arbeit aufgezeichnet wurde. Zu Adjektivverstößen bei englischen Muttersprachlern siehe u. a. Swan 1990, Textstelle 12, o. S. 
Fehler: It's an interested film. ${ }^{551}$

Korrektur: It's an interesting film.

Andererseits waren jedoch auch Adjektivfehler zu beobachten, die aus semantischen Gründen als Normverstöße zu bewerten waren. Ein Beispiel bietet folgende Äußerung:

Fehler: $\quad$ The weather was easy. ${ }^{552}$

Korrektur: The weather was pleasant.

Bei den PwenlAb hingegen war die überwiegende Mehrzahl der Adjektivfehler semantischen Ursprungs. Nur vereinzelt war daneben zu beobachten, daß die Probanden grammatisch begründete Fehler produzierten. Hierzu zählen Fehler, die darauf beruhen, daß statt des Prädikativums, das nach intransitiven Verben wie sound zu erwarten wäre, ein adverbiales Adjunkt selegiert wird. Betroffen davon ist insbesondere das Lexem good, das in einigen Fällen durch das adverbiale Adjunkt well ersetzt wurde, wie etwa in folgendem Beispiel:

Fehler: $\quad$ It sounds well. ${ }^{553}$

Korrektur: It sounds good.

Zu den semantisch begründeten Verstößen zählen vor allem Fehler, die auf mangelndes semantisches Differenzierungsvermögen zurückzuführen sind, d. h., die Probanden substituierten ein bestimmtes englisches Wort durch ein Lexem, das dem eigentlich zu erwartenden Lexem semantisch ähnelt. Dabei wurde jedoch in der Regel nicht berücksichtigt, daß zwischen dem Substitutionselement und dem eigentlich zu erwartenden Lexem subtile semantische Unterschiede bestehen, wie etwa in folgendem Beispiel:

Fehler: $\quad$ We were looking for a cheap restaurant. ${ }^{554}$

Korrektur: We were looking for an inexpensive restaurant.

Der Fehler entstand hier dadurch, daß der Lernende offensichtlich eine Übersetzung für das deutsche Lexem ,preiswert‘ suchte. Er hat dabei jedoch nicht in Betracht gezogen, daß das Wort cheap im Zusammenhang mit öffentlich zugänglichen Einrichtungen wie Gaststätten sowie auch im Zusammenhang mit Gegenständen oft benutzt wird, um zu verdeutlichen, daß die betreffenden Einrichtungen oder Gegenstände von minderwertiger Qualität sind. ${ }^{555}$ Das Wort inexpensive hingegen, das in dem Korrekturbeispiel vorgeschlagen wurde, impliziert, daß sich die Minderung nur auf den Preis und nicht auf die Qualität bezieht. Wie bei den vorhergehenden Fehlergruppen besteht der Unterschied

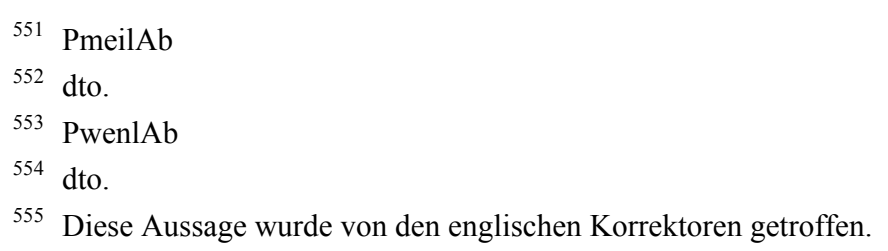


zwischen den semantisch bedingten Adjektivfehlern beider Probandengruppen darin, daß die Verwechslungen bei den PwenlAb subtileren Ursprungs sind als in der entgegengesetzten Probandengruppe. Trotz der Fehler lassen die PwenlAb erkennen, daß sie über ein anspruchsvolles Vokabular der englischen Sprache verfügen, während sich die Fehler der PmeilAb auf elementarer Ebene befinden. Dies ist u.a. an den zuvor dargestellten Beispielen zu erkennen: Das verwendete Wort easy im Beispiel der PmeilAb ist in keiner Weise mit den eigentlich zu erwartenden Lexemen pleasant oder nice verwandt. Die Gemeinsamkeit zwischen den Lexemen besteht lediglich darin, daß sowohl das Wort easy als auch die Wörter pleasant und nice positiv belegt sind. Hingegen läßt der Gebrauch des Wortes cheap in der entgegengesetzten Probandengruppe erkennen, daß die Lernenden ein Lexem gewählt haben, das mit dem Element inexpensive, das korrekterweise zu benutzen gewesen wäre, semantisch verwandt ist.

Die Analyse der Fehler auf der Grundlage der Kontrastiv- und der Interlanguagehypothese zeigte folgendes: In beiden Probandengruppen überwiegen eindeutig die Anwendungsmöglichkeiten der Interlanguagehypothese, dabei kommt diese Hypothese bei den PwenlAb öfter zur Anwendung als in der entgegengesetzten Probandengruppe (vgl. Tabellen 12 und 13 im Anhang). Auch die Zahl der Fehler, die auf Faktoren der Kontrastivhypothese zurückgeführt werden können, ist bei den PwenlAb höher. Somit sind der Einfluß der Erstsprache und der Einfluß strategischer und prozeduraler Faktoren bei den PwenlAb ausgeprägter und der Anteil der Fehler, der mit Hilfe der Erwerbshypothesen analysiert werden kann, größer als bei den PmeilAb.

Bei genauerer Betrachtung der einzelnen Kategorien der Kontrastiv- und der Interlanguagehypothese zeigt sich, daß bei Anwendung der Kontrastivhypothese in der Gruppe der PmeilAb nur die Fehlerkategorie ,Substitution“ nachgewiesen werden konnte (vgl. Tabelle 14 im Anhang). In der entgegengesetzten Probandengruppe wurden zusätzlich Über- bzw. Unterdifferenzierungen konstatiert, wenngleich auch hier zu beobachten war, daß die Mehrzahl der Fehler auf dem Ersetzen sprachlicher Elemente basierte (vgl. Tabelle 15 im Anhang). Zusammenfassend ist festzustellen, daß in beiden Probandengruppen erstsprachlicher Einfluß vorwiegend in seiner eindeutigsten Form deutlich wird, d. h., die Lernenden haben zweitsprachliche Elemente durch andere Elemente ersetzt, die in struktureller und/oder lautlicher Hinsicht Assoziationen zu erstsprachlichen Lexemen hervorrufen.

In bezug auf die Anwendbarkeit der Faktoren der Interlanguagehypothese fällt auf, daß die Ergebnisse, die bei den Adjektivverstößen ermittelt wurden, nahezu mit den Ergebnissen, die bei der Untersuchung der stilistischen Fehler gewonnen wurden, übereinstimmen (vgl. Tabellen 16 und $17 \mathrm{im}$ Anhang). In beiden Probandengruppen überwiegt insgesamt der Einfluß funktionaler Reduktionsstrategien. Bei den PmeilAb jedoch ist die Mehrzahl der Fehler bei Anwendung der Interlanguagehypothese auf modality and personality reduction zurückzuführen, während in der entgegengesetzten 
Probandengruppe im wesentlichen bewußte generalizations für die Entstehung der Abweichungen verantwortlich sind. Daneben zeigte sich, wie bei den stilistischen Fehlern, daß bei den PmeilAb partiell auch der Einfluß unbewußter overgeneralizations zu konstatieren ist. Dieses trifft, anders als bei den stilistischen Verstößen, hier auch auf die Gruppe der PwenlAb zu. Wie bei den stilistischen Verstößen basiert auch bei den Verstößen im Bereich der Adjektive ein, wenngleich geringer, Teil der Abweichungen auf dem Fehlertyp paraphrase. Darüber hinaus ist bei den PwenlAb lack of contrast als weitere mögliche Fehlerentstehungsursache in Betracht zu ziehen. Der Einfluß der obengenannten Faktoren zeigt sich u. a. an folgenden Beispielen:

Fehler: $\quad$ How do you found our last holiday in Mallorca? I hope good. ${ }^{556}$

Korrektur: What did you think about our last holiday in Mallorca? I hope it was also enjoyable for you.

Fehler: $\quad$ Inspite of everything the work is very interesting. ${ }^{557}$

Korrektur: Inspite of all the work is very challenging.

Fehler: $\quad$ It was not very funny. ${ }^{558}$

Korrektur: It was awful.

Die Fehlleistung des ersten Beispiels beruht - in bezug auf die Kategorie ,Adjektiv“ - auf dem Gebrauch des Lexems good. ${ }^{559}$ Wenngleich die Bemerkung nicht unmittelbar als Normverstoß zu bezeichnen ist, neigen englische Muttersprachler bei Fragen nach Empfindungen dazu, den Kernpunkt der Frage zu umschreiben, um dadurch die Privatsphäre des Kommunikationspartners nicht zu verletzen. ${ }^{560}$ Dabei liegt bei Gebrauch des Wortes good eindeutig eine Substitution vor, da sich der Lernende an dem deutschen Lexem ,gut' orientiert hat, das dem englischen Lexem good lautlich und optisch ähnelt. Zusätzlich zum erstsprachlichen Einfluß kann die Wahl dieses Wortes jedoch auch als Ergebnis einer Reduktionsstrategie interpretiert werden, weil der Lernende vermutlich das Wort auch in der Absicht benutzte, sprachliche Ausschweifungen zu vermeiden und es dem Kommunikationspartner dennoch zu ermöglichen, die Frageabsicht zu verstehen. So war es ihm möglich, die Anwendung von Lexemen zu umgehen, die ihm aufgrund seltenen Gebrauchs weniger bekannt sind als das Wort good und deren Anwendung möglicherweise eine umfangreichere syntaktische Konstruktion erfordert hätte.

Neben diesem bewußten Reduktionsverhalten kann das Lexem good jedoch auch als Beispiel einer unbewußten Fehlerentstehung betrachtet werden, da es durchaus möglich ist, daß der Lernende das

\footnotetext{
556 PmeilAb

557 PwenlAb

558 dto.

559 Die Verbform found stellt ebenfalls eine Normabweichung dar. Fehler dieser Art werden im Rahmen der syntaktischmorphologischen Abweichungen analysiert.

560 vgl. Lojewski 1991, 175
} 
Lexem bereits so häufig benutzt hatte, daß die Anwendung ohne weitere Überlegung automatisch erfolgte.

Im zweiten Beispiel kommen die Fehlerentstehungsfaktoren der Erwerbshypothesen an folgenden Punkten zum Tragen: Die Tatsache, daß es sich hierbei um eine Substitution handelt, die auf den Einfluß der Erstsprache Deutsch zurückgeführt werden kann, wird darin deutlich, daß bei der Wahl des Lexems interesting eindeutig das deutsche Adjektiv ,interessant‘ als Vorlage gedient hat. Gleichzeitig ist die Fehlleistung als eine durch die Erstsprache verursachte Unterdifferenzierung zu betrachten, weil der Lernende offensichtlich davon ausgegangen ist, daß das Wort interesting im Englischen in gleichen Zusammenhängen benutzt werden kann wie das deutsche Wort ,interessant‘. Im Deutschen kann dieses Wort in bezug auf eine berufliche Tätigkeit, aber auch in bezug auf Dinge oder Personen als Synonym für Adjektive wie ,herausfordernd', ,spannend‘ oder , abwechslungsreich` verwendet werden. Im Englischen dagegen ist der Bedeutungsumfang des Wortes interesting limitierter. Wird das Wort interesting in Verbindung mit einer Tätigkeit oder einem Beruf benutzt, so bedeutet dies für englische Muttersprachler, daß die Tätigkeit als nicht monoton empfunden wird. ${ }^{561}$ Aus dem Gesamtzusammenhang des Briefes, den der Proband verfaßt hat, geht jedoch hervor, daß der Betreffende seine Tätigkeit als interessant im deutschen Sinne des Wortes empfand. Im Englischen wäre zur Verdeutlichung einer solchen Aussage entweder, wie im Korrekturbeispiel angegeben, das Wort challenging zu verwenden oder aber Lexeme wie versatile oder many-sided.

Die Anwendung der Faktoren der Interlanguagehypothese ergab, daß der Gebrauch des Lexems interesting auch als eine Generalisierung eines Interlanguageelements einzuordnen ist, die bewußt oder auch unbewußt verlaufen sein könnte. Eine bewußte generalization wäre gegeben, wenn der Lernende - ähnlich wie im Beispiel, das der entgegengesetzten Gruppe entnommen wurde - versuchte, durch die Wahl des Wortes interesting ein Lexem zu benutzen, mit dessen Hilfe er seine sprachliche Aussage verdeutlichen könnte, ohne dabei Wörter zu verwenden, die ihm weniger vertraut sind. Der Fehler könnte jedoch auch als Ergebnis einer unbewußten overgeneralization entstanden sein, weil das Wort interesting zu den Elementen des Englischen gehört, die häufig benutzt werden. Verstärkt durch die Tatsache, daß gleiches auch für das deutsche Lexem ,interessant ‘ gilt sowie durch den Kontrastmangel zwischen interesting und, interessant', ist es durchaus möglich, daß der Lernende die Anwendungsbeschränkungen des Lexems interesting nicht weiter hinterfragt hat und das Wort automatisch benutzte.

Das letzte Beispiel verdeutlicht, daß zum Teil auch der Fehlertyp paraphrase für die Entstehung der Abweichungen verantwortlich ist. Die Fehlleistung basiert hier auf dem Ausdruck not very funny, der

561 Diese Aussage wurde von den englischen Korrektoren getroffen. 
als Normabweichung zu bezeichnen ist, weil er nicht die Redeabsicht des Lernenden verdeutlicht. Die Situation, die der Lernende zu beschreiben versuchte, wurde als äußerst unangenehm empfunden. Selbst im Englischen, als der Sprache der understatements, wäre an dieser Stelle ein ausdrucksstärkeres Lexem, wie etwa das Wort awful zu erwarten. Offenbar hat der Lernende das Wort zu paraphrasieren versucht. Dies kann dadurch begründet sein, daß er davon ausging, daß Untertreibungen im Englischen immer angebracht sind. In diesem Fall läge eine bewußte generalization vor. Denkbar ist jedoch auch, daß dem Lernenden zum Zeitpunkt der Anwendung kein anderes Lexem zur Verfügung stand und er daher das eigentlich zu erwartende Wort zu umschreiben versuchte.

Insgesamt betrachtet zeigt die Analyse der Fehler der Kategorie ,Adjektiv“, daß sowohl die Kontrastivals auch die Interlanguagehypothese wichtige Faktoren zur Untersuchung der Fehlergenese zur Verfügung stellen, wenn auch die Interlanguagehypothese öfter zur Anwendung kam. Dabei ergänzen sich die beiden Hypothesen bei der Analyse der Fehler. So verdeutlicht u. a. das letzte Beispiel, daß, selbst wenn die meisten Fehler als Ergebnis bewußter oder unbewußter overgeneralization zu betrachten sind und teilweise auf lack of contrast basieren, diese Strategien und Prozesse durch Einflüsse im Bereich des linguistischen Systems verstärkt wurden.

\subsection{Adverb}

Die Ergebnisse, die in bezug auf den Fehlertyp Adverb ermittelt wurden, zeigen, daß die Anwendungsschwierigkeiten, die mit dieser Lexemgruppe verbunden sind, sowohl bei den PmeilAb als auch bei den PwenlAb quantitativ mit den Kategorien ,Hilfsverben“ und ,Adjektive“ vergleichbar sind (vgl. Tabellen 12 und $13 \mathrm{im}$ Anhang). Sie befinden sich damit auf der unteren bis mittleren Ebene der Schwierigkeitshierarchie. Dabei sind die Anwendungsprobleme bei den PwenlAb geringfügig schwächer ausgeprägt als bei der entgegengesetzten Probandengruppe. Bei den PmeilAb beruhen die Verstöße in der Regel darauf, daß statt eines zu erwartenden Adverbs ein Adjektiv verwendet wurde, wie etwa in folgendem Beispiel:

Fehler: $\quad$ Today I don't feel good. ${ }^{562}$

Korrektur: Today I don't feel well.

Bei den PwenlAb liegen die Verstöße mehrheitlich auf semantischer Ebene, d. h., die Probanden verwendeten statt des Adverbs, das eigentlich zu erwarten gewesen wäre, ein Wort, das diesem Element semantisch ähnelt, jedoch aufgrund subtiler Bedeutungsunterschiede nicht als Ersatzform benutzt werden kann. Zum anderen beruhen die Fehler, die in dieser Gruppe zu beobachten waren, auch darauf, daß ein Teil der englischen Lexeme nicht zwischen einer Adjektiv- und einer Adverbialform unter- 
scheidet und die Probanden fälschlicherweise das für Adverbien charakteristische Suffix -ly an das Lexem anfügten, wie das nachstehende Beispiel zeigt:

Fehler: $\quad$ He drives fastly. ${ }^{563}$

Korrektur: He drives fast.

Bei Anwendung der Erwerbshypothesen fällt auf, daß die Erklärungsfaktoren der Kontrastivhypothese in der Gruppe der PmeilAb stärker zum Tragen kommen als die der Interlanguagehypothese (vgl. Tabelle 12 im Anhang). Demnach ist die Hauptentstehungsursache der Fehler in dieser Gruppe in dem Einfluß erstsprachlicher Faktoren zu sehen. In der entgegengesetzten Probandengruppe überwiegt das Erklärungspotential der Interlanguagehypothese (vgl. Tabelle 13 im Anhang), so daß hier die Mehrzahl der Fehler auf das strategisch-prozedurale Verhalten der Lernenden beim Erlernen und beim Gebrauch der Zielsprache zurückzuführen ist. In beiden Lernergruppen ist jedoch die Differenz in bezug auf die Anwendbarkeit der beiden Hypothesen gering. Erstsprachliche und strategisch-prozedurale Faktoren sind folglich etwa zu gleichen Teilen für die Entstehung der Abweichungen verantwortlich.

Die genauere Betrachtung der einzelnen Faktoren zeigt, daß die Form erstsprachlichen Einflusses in beiden Gruppen weitgehend identisch ist. Die Auswirkungen der Erstsprache Deutsch äußern sich in erster Linie darin, daß die Probanden englische Adverbien durch andere Adverbien des Englischen ersetzten, die sie an Lexeme der deutschen Sprache erinnerten (vgl. Tabellen 14 und 15 im Anhang). Letzteres verdeutlicht u. a. das zuvor genannte Fehlerbeispiel Today I don't feel good. Hier ist eindeutig zu erkennen, daß der Gebrauch des Lexems good auf das deutsche Lexem ,gut ${ }^{\star}$ zurückzuführen ist. Ähnliche Substitutionsformen sind - wie das folgende Beispiel zeigt - auch bei den PwenlAb zu beobachten:

Fehler: $\quad$ My boys were lightly disappointed. ${ }^{564}$

Korrektur: My boys were slightly disappointed.

Auch hier ist davon auszugehen, daß der Gebrauch des normabweichenden Elements - in diesem Fall des Lexems lightly - auf einer deutschen Vorlage beruht. Vermutlich beruht die Anwendung dieses Wortes auf dem Versuch, die deutsche Lexemkombination, leicht enttäuscht‘ ins Englische zu übertragen.

Der Einfluß der Erstsprache zeigt sich bei den PmeilAb nicht nur in der Fehlerkategorie ,Substitution', sondern partiell auch in Form von Unter- bzw. Überdifferenzierungen (vgl. Tabelle 14 im Anhang), die ebenfalls anhand des zuvor erwähnten Lexems good verdeutlicht werden können. Die Wahl dieses

\footnotetext{
562 PmeilAb

563 PwenlAb

564 PwenlAb
} 
Lexems ist als Unterdifferenzierung zu bewerten, weil der Lernende nicht berücksichtigte, daß - im Gegensatz zum deutschen Lexem , gut ${ }^{\star}$ - Adjektiv- und Adverbform des Lexems good strukturell keinerlei Entsprechungen aufweisen. Die Übereinstimmungen zwischen beiden Probandengruppen, die in bezug auf die Form erstsprachlichen Einflusses deutlich wurden, zeigen zudem, daß ein höherer lexikalischer Wissensstand der Lernenden den Einfluß der Erstsprache in seiner elementarsten Form, d. h. das Ersetzen zielsprachlicher Strukturen durch erstsprachliche, nicht verhindert. Somit sind die fehlergenetischen Ursachen adverbialer Verstöße bei den PmeilAb und den PwenlAb aus der Sicht der Kontrastivhypothese betrachtet nahezu deckungsgleich.

Bei Anwendung der Fehlererklärungsfaktoren der Interlanguagehypothese zeigt der Vergleich der Ergebnisse, daß bei den PmeilAb mehr Fehlererklärungsfaktoren zur Anwendung kommen als in der entgegengesetzten Probandengruppe (vgl. Tabellen 16 und 17 im Anhang). Die Mehrzahl der Abweichungen geht hier auf den Gebrauch von Reduktionsstrategien, insbesondere den sogenannten achievement strategies zurück. Die meisten Abweichungen verzeichnen die Kategorien interlingual transfer und generalization of interlanguage rules/items in new contexts. Daneben kommt auch lack of contrast als wichtige Fehlerentstehungsursache zum Tragen. Somit ist die Entstehung der adverbialen Verstöße bei den PmeilAb zum einen dadurch begründet, daß die Lernenden versuchten, sich auf eine einzige lexikalische Form zu beschränken - meist auf die Form, die das betreffende Lexem als Adjektiv besitzt - und dabei formal nicht zwischen dem Gebrauch von Adjektiven und Adverbien unterschieden. Zum anderen basieren die Fehler darauf, daß sich die Lernenden an Strukturen ihrer Erstsprache orientierten und dabei mißachteten, daß sich Adjektive und Adverbien im Englischen in der Regel morphologisch, entweder durch Veränderung bzw. durch Substitution des gesamten Wortes oder durch Hinzufügen der Endung -ly, unterscheiden. Die Differenzierungsschwierigkeiten sind jedoch nicht nur auf den Einfluß der Erstsprache zurückzuführen, sondern wurden zusätzlich dadurch verstärkt, daß auch im Englischen Adjektiv- und Adverbform manchmal identisch sind. Dies führte dazu, daß die Lernenden schließlich diese strukturelle Identität auch auf solche Elemente übertrugen, die zwischen der Form eines Adjektivs und der Form eines Adverbs unterscheiden.

Auch die Anwendbarkeit der Fehlererklärungsmöglichkeiten der Interlanguagehypothese läßt sich anhand des zuvor dargestellten Fehlerbeispiels Today I don't feel good verdeutlichen: Der Einfluß von Reduktionsstrategien, interlingual transfer und bewußter generalization wird hier in folgenden Aspekten sichtbar: Die Anwendung einer Reduktionsstrategie zeigt sich darin, daß der Lernende nur das Lexem good verwendete und damit die Anwendung des Lexems well und die Regeln, die diesem Lexem zugrunde liegen, vermied. Dieses Verhalten kann darauf beruhen, daß der Lernende nicht mit dem Lexem well vertraut war und er daher versuchte, sich auf den Gebrauch eines Lexems zu beschränken, dessen Anwendungsmöglichkeiten er zu kennen glaubte. Dabei ist davon auszugehen, daß die Ähnlichkeit zwischen dem deutschen Lexem, gut` und dessen englischer Entsprechung dieses 
Verhalten begünstigt hat, so daß damit auch interlingual transfer als Fehlerentstehungsursache zum Tragen kommt. Gleichzeitig kann der Gebrauch des Wortes auch als Ergebnis einer bewußten generalization interpretiert werden, weil in der Tat im Englischen in manchen Zusammenhängen nach dem Verb feel das Wort good benutzt wird. In dem gegebenen Beispiel erhält der Satz durch die Verwendung dieses Lexems jedoch eine Bedeutung, die vom Lernenden nicht beabsichtigt war. Bezieht sich das Wort good auf das Lexem feel, so bedeutet dies, daß sich der Betreffende in einer guten Stimmung befindet. Folgt dem Verb feel jedoch das Adverb well, so wird das körperliche Befinden des Betreffenden näher definiert. Da jedoch - wie der sprachliche Zusammenhang zeigt - der Lernende nicht über seinen psychischen Zustand, sondern über seine körperliche Verfassung berichtete, ist der Gebrauch des Lexems good als Normabweichung zu bezeichnen. Die zuvor erwähnte Ähnlichkeit zwischen dem deutschen Wort ,gut ${ }^{\star}$ und dem englischen Adjektiv good spricht schließlich auch dafür, daß die Fehlleistung zusätzlich auch auf lack of contrast zurückgeführt werden kann.

Bei den PwenlAb basiert die Entstehung adverbialer Verstöße interlanguagespezifisch betrachtet im wesentlichen darauf, daß die semantischen Anwendungsmöglichkeiten zuvor erworbener Adverbien verallgemeinert wurden und die Elemente in sprachlichen Situationen zur Anwendung kamen, in denen andere Lexeme zu erwarten gewesen wären (vgl. Tabelle 17 im Anhang). Wie das sprachliche Umfeld der betreffenden Fehler zeigt, entstehen diese Generalisierungen meist bewußt. Nur bei einer Minderheit der Verstöße gibt es Anhaltspunkte dafür, daß die Abweichungen u. a. als Ergebnis prozeduralen, d. h. unbewußten Verhaltens betrachtet werden können. Darüber hinaus sind in wenigen Fällen die Fehler auch auf paraphrase zurückzuführen.

Sowohl das bewußte als auch das unbewußte Generalisieren zielsprachlicher Regeln zeigt sich im oben erwähnten Fehlerbeispiel He drives fastly: Die Endung -ly ist in der Regel tatsächlich als morphologisches Erkennungsmerkmal englischer Adverbien zu betrachten. Der Lernende hat jedoch nicht bedacht, daß das Wort fast zu den Lexemen des Englischen gehört, die morphologisch nicht zwischen einer Adjektiv- und einer Adverbialform unterscheiden. ${ }^{565}$ Die Einstufung des Fehlers als unbewußte overgeneralization beruht vor allem darauf, daß das Wort fast im schriftlichen Beitrag des Lernenden teils normgerecht und teils normabweichend verwendet wurde.

Der Fehlertyp paraphrase als Ursache adverbialer Verstöße ist in folgendem Beispiel zu erkennen:

Fehler: $\quad$ Please give me a ring on one of the next evenings. ${ }^{566}$

Korrektur: Please give me a ring some evening soon.

565 vgl. Swan 1990, Textstelle 12, o. S.
566 PwenlAb 
Hier ist davon auszugehen, daß der Fehler darauf basiert, daß der Lernende die Formulierung some evening soon nicht in seinem Sprachspeicher abrufen konnte und daher seine Aussage durch eine Umschreibung verdeutlichen mußte.

Zusammenfassend betrachtet ist damit die Entstehung der adverbialen Verstöße in beiden Probandengruppen auf eine Kombination erstsprachlich bedingter und strategischer Faktoren zurückzuführen. Die Ursachen der Fehlerentstehung unterscheiden sich dabei zum einen darin, daß der Einfluß der Erstsprache bei den PmeilAb stärker zum Tragen kommt als bei den PwenlAb. Zum anderen beruhen die Fehler bei den PmeilAb auf defensivem Sprachverhalten, während bei den PwenlAb Generalisierungen bewußter und unbewußter Art als Hauptentstehungsfaktoren zu betrachten sind.

\subsection{Pronomen}

Die Fehlleistungen, die beim Gebrauch der Pronomen ermittelt wurden, zeigen, daß die Anwendungsschwierigkeiten im Bereich dieser Lexemgruppe bei den PmeilAb weitaus größer sind als in der entgegengesetzten Probandengruppe (vgl. Tabellen 2 und 3 im Anhang). Mit zunehmenden lexikalischen Kenntnissen geht also - im Gegensatz zu vielen anderen Wortarten - auch die Anzahl pronominaler Verstöße zurück.

Bei Anwendung der Fehlererklärungsfaktoren der Erwerbshypothesen wurde ermittelt, daß die Fehlererklärungsfaktoren der Interlanguagehypothese bei den PmeilAb deutlich überwiegen (vgl. Tabelle 12 im Anhang). Nur $8 \%$ der pronominalen Verstöße lassen erkennen, daß erstsprachlicher Einfluß an der Fehlerentstehung beteiligt war. Bei den PwenlAb ist das Gegenteil zu beobachten: In dieser Probandengruppe überwiegen eindeutig die Fehlererklärungsfaktoren der Kontrastivhypothese (vgl. Tabelle 13 im Anhang). Insgesamt wird deutlich, daß beide Erwerbshypothesen bei den PwenlAb ein größeres Fehlererklärungspotential bieten als in der entgegengesetzten Gruppe. Somit ist festzustellen, daß die Entstehung von pronominalen Verstößen bei fortgeschrittenen Lernenden eindeutiger begründet werden kann als bei Lernenden mit erheblichen lexikalischen Anwendungsschwierigkeiten.

Die Betrachtung der einzelnen Fehlererklärungsfaktoren zeigt, daß bei den PmeilAb bei Anwendung der Kontrastivhypothese nur der Fehlerentstehungsfaktor Substitution nachgewiesen werden konnte, während in der entgegengesetzten Probandengruppe neben Substitutionen auch erstsprachlich bedingte Über- und Unterdifferenzierungen zum Tragen kommen (vgl. Tabellen 14 und 15 im Anhang). Dies bedeutet, daß der Einfluß der Erstsprache bei den PwenlAb nicht nur stärker an der Fehlerentstehung beteiligt war als bei den PmeilAb, sondern daß bei ersteren die Ausprägungsformen erstsprachlichen Einflusses darüber hinaus vielseitiger sind als in der entgegengesetzten Probandengruppe. 
In bezug auf die Fehlerentstehungsfaktoren der Interlanguagehypothese ist zu erkennen, daß die Abweichungen bei den PmeilAb sowohl auf inter-/intralingual transfer als auch auf bewußte generalizations und auf lack of contrast zurückzuführen sind (vgl. Tabelle 16 im Anhang). Bei den PwenlAb basieren die Fehlerentstehungsursachen auf reduction strategies, bewußten generalizations, paraphrase und lack of contrast (vgl. Tabelle $17 \mathrm{im}$ Anhang). Inter-/intralingual transfer konnte in dieser Gruppe nicht nachgewiesen werden. Demnach ist interlanguagespezifisch betrachtet die Fehlerentstehung bei den PmeilAb auf eine Kombination erstsprachlich bedingter Faktoren und zielsprachlicher Generalisierungen zurückzuführen, während die Hauptentstehungsursachen bei den PwenlAb in der Generalisierung bereits erworbener Elemente der Zielsprache und im Versuch, durch Umschreibungen Sprachdefizite auszugleichen, zu sehen sind.

Der Einfluß der unterschiedlichen Fehlerentstehungsursachen kann an folgenden Beispielen verdeutlicht werden:

Fehler: $\quad$ At the moment my situation is good. ${ }^{567}$

Korrektur: At the moment I'm very well.

Fehler: $\quad$ It was a great time for me together with you. ${ }^{568}$

Korrektur: I had a great time with you.

Im ersten Beispiel ist das Substituieren zielsprachlicher Elemente durch erstsprachliche daran zu erkennen, daß die Äußerung my situation is good eindeutig als Übersetzung der deutschen Vorlage ,meine Situation ist gut ${ }^{\star}$ zu betrachten ist. Daher erscheint es aus Sicht der Interlanguagehypothese gerechtfertigt, diese Abweichung der Kategorie interlingual transfer zuzuordnen.

Die oben erwähnte optische und akustische Übereinstimmung zwischen den Lexemen, gut ${ }^{\star}$ und good lassen darüber hinaus darauf schließen, daß der Fehler außerdem auf lack of contrast zurückzuführen ist. Da das Lexem good grammatisch durchaus gemeinsam mit dem Lexem situation benutzt werden kann, seine Verwendung im vorliegenden Fall jedoch aus stilistischen Gründen als Normabweichung zu bezeichnen ist, ist die Fehlleistung zudem als Ergebnis einer Generalisierung zweitsprachlicher Elemente zu betrachten.

Auch im zweiten Fehlerbeispiel ist die Fehlleistung u. a. der Kategorie ,Substitution` zuzuordnen, da eindeutig ein deutscher Ausdruck als Übersetzungsvorlage gedient hat. Die Präpositionalphrase together with you ist zweifellos durch die Übersetzung des deutschen Ausdrucks ,zusammen mit Dir entstanden. Jedoch hat der Lernende nicht bedacht, daß die syntaktischen Strukturen der englischen

$\begin{array}{ll}567 & \text { PmeilAb } \\ & \\ 568 & \text { PwenlAb }\end{array}$ 
Sprache in der Regel durch syntaktisch-morphologische Knappheit geprägt sind und Lexeme ausgelassen werden, die nicht zum Verständnis der Aussage benötigt werden. Da die Formulierung ,zusammen mit Dir` im Deutschen generell zulässig ist, bei der Übersetzung jedoch semantische Regeln des Englischen nicht berücksichtigt wurden, ist die Abweichung gleichzeitig auch als erstsprachlich bedingte Unterdifferenzierung zu betrachten. Aus der Sicht der Interlanguagehypothese kann der Fehler außerdem als bewußte generalization gedeutet werden, da die Wortkombination together with you losgelöst vom sprachlichen Kontext im Englischen normgerecht wäre. Die Ähnlichkeit zum oben erwähnten deutschen Ausdruck läßt vermuten, daß darüber hinaus lack of contrast an der Fehlerentstehung beteiligt war.

Paraphrase als weitere Fehlerentstehungsursache äußert sich darin, daß der Lernende den eigentlich zu erwartenden Ausdruck nicht verwendet hat, sondern stattdessen versuchte, mit Hilfe einer Umschreibung die in diesem Kontext nicht abrufbare Formulierung zu kompensieren.

\subsection{Sondergruppe: Stil}

Fehler, die der Sondergruppe ,Stil` zuzuordnen sind, treten in beiden Probandengruppen mit nahezu gleicher Häufigkeit auf (vgl. Tabellen 2 und 3 im Anhang). Verglichen mit den zuvor behandelten Fehlerkategorien der lexikalischen Ebene ähneln die Anwendungsschwierigkeiten, die mit dem normgerechten Gebrauch stilistischer Formulierungen verbunden sind, denen der Fehlerkategorie ,Hilfsverben“. Die hier beobachteten Anwendungsprobleme befinden sich dabei auf mittlerer Stufe der Schwierigkeitshierarchie. Wie eingangs erwähnt, nehmen die Fehler der stilistischen Ebene eine Sonderposition ein, da stilistische Abweichungen oft nicht als eindeutig falsch zu bezeichnen sind, sondern die Formulierungen in der Regel nur in dem Kontext, in dem sie verwendet werden, von der Norm der englischen Sprache abweichen. In anderen Redesituationen hingegen kann die betreffende stilistische Formulierung möglicherweise durchaus der Norm des Englischen entsprechen. Wie bei den zuvor behandelten Fehlerkategorien unterscheiden sich auch die stilistischen Verstöße, die in der Gruppe der PmeilAb beobachtet wurden, in ihrer sprachlichen Qualität von den stilistischen Abweichungen der entgegengesetzten Probandengruppe. Unter sprachlicher Qualität ist hier zu verstehen, daß die Formulierungen, die bei den PmeilAb als fehlerhaft eingestuft wurden, stärker von der Norm der englischen Sprache abweichen als die Formulierungen, die bei den PwenlAb als fehlerhaft konstatiert wurden.

Wenngleich - wie erwähnt - stilistische Formulierungen in anderen sprachlichen Situationen als der vorgegebenen nicht notwendigerweise als fehlerhaft zu bezeichnen sind, konnten in der Gruppe der PmeilAb auch Ausdrücke ermittelt werden, die in nahezu jeder Redesituation als normabweichend zu 
bezeichnen sind, wohingegen dies in der entgegengesetzten Probandengruppe nicht zu beobachten war. Beispiele hierfür bieten folgende Äußerungen:

Fehler: $\quad$ Please write me back and told me if I can come next spring. Best greeting ${ }^{569}$

Korrektur: Please drop me a line and let me know if next spring is convenient for you.

Fehler: $\quad$ I wish you a very good life. ${ }^{570}$

Korrektur: I hope that everything in your life will turn out well for you.

Fehler: $\quad$ That's it for now. ${ }^{571}$

Korrektur: That's all I can tell you at the moment.

Fehler: $\quad$ I will ring you up in the next days to arrange a date. Sincerely yours - Wolfgang ${ }^{572}$ Korrektur: I will ring you up and then we can think about a date. Love-Wolfgang ${ }^{573}$

Der entscheidende stilistische Verstoß des ersten Beispiels liegt in dem Gebrauch der Formulierung Best greetings. ${ }^{574}$ Das Lexem greetings wird im schriftlichen Sprachgebrauch - wie oben erwähnt - in der Regel nur als Eingangsformel sehr informeller Kommunikationsmittel wie etwa Postkarten benutzt. $^{575}$ Als Abschluß einer förmlicheren schriftlichen Mitteilung kommt der Ausdruck im Englischen jedoch nicht vor. Stattdessen wären Ausdrücke wie Yours oder Love zu erwarten, vorausgesetzt, die Kommunikationspartner sind miteinander vertraut. ${ }^{576}$ Das zweite Fehlerbeispiel, das ebenfalls am Ende eines Probandenbriefes ermittelt wurde, weicht vollständig von der Norm der englischen Sprache ab, weil die Formulierung I wish you a very good life impliziert, daß der Verfasser des Briefes nicht wieder mit dem Kommunikationspartner in Kontakt treten wird. Außerdem wirkt der Satz aufgrund der Tatsache, daß sich keine weiteren Sätze anschließen, ironisch. Die Betrachtung des Gesamtbriefes zeigt jedoch deutlich, daß der Proband keinen ironischen Eindruck hinterlassen wollte. Eine weitere Abweichung von der Norm der englischen Sprache besteht darin, daß im Englischen, als einer Sprache der understatements, Gefühle, ob positiver oder negativer Art, auf sprachlicher Ebene in der Regel weniger deutlich zum Ausdruck kommen. ${ }^{577}$

In den beiden zuletzt genannten Fehlerbeispielen hingegen könnten beide Formulierungen grundsätzlich am Ende einer schriftlichen Konversation benutzt werden. Die Beispiele wurden dennoch als Normabweichungen bezeichnet, weil die von den Probanden benutzten Ausdrücke, d. h. sowohl die

\footnotetext{
569 PmeilAb

570 dto

571 PwenlAb

572 dto.

573 Die Satzerweiterung and then we can think about ... wurde nicht von allen Korrektoren als unbedingt notwendig erachtet. Es wurde jedoch seitens der englischen Korrektoren betont, daß die Formulierung I will ring you up ohne weiteren Einschub sehr fordernd klingt und damit von den Höflichkeitsvorstellungen englischer Muttersprachler abweicht.

574 Die Fehlleistung ,told' stellt einen Verstoß im Bereich des Zeitensystems dar. Sie wird in diesem Textteil nicht analysiert, da hier stilistische Verstöße im Vordergrund stehen.

575 vgl. Swan 1990, Textstelle 274, o. S.

576 vgl. ebd., Textstelle 361, o. S.

577 Diese Aussage beruht auf persönlichen Erfahrungen des Autors der vorliegenden Arbeit.
} 
Formulierung That's it for now als auch die Redewendung Sincerely yours, nicht in den entsprechenden Gesprächskontext passen. Der Ausdruck That's it for now wäre eher im Bereich der Medien zu erwarten, etwa wenn ein Reporter seinen Zuhörern $\mathrm{zu}$ erkennen gibt, daß eine Informationsübertragung vorerst beendet ist. Gebräuchlich ist der Ausdruck auch in geschäftlichen Situationen, etwa bei Bestellungen. Die Formulierung That's it for now könnte dazu dienen, dem Geschäftspartner mitzuteilen, daß eine Bestellung oder ein geschäftlicher Auftrag vorerst abgeschlossen ist - aber auch in einer solchen Situation wird der Ausdruck That's it for now nur mündlich benutzt, außer im besonders vertraulichen Kontext. Am Ende eines Briefes bedarf es einer weniger abrupten Formulierung, die den Kommunikationspartner in persönlicher Form erkennen läßt, daß der andere Kommunikationspartner den Text nun beenden wird. ${ }^{578}$

Der Ausdruck sincerely yours, der dem letzten Fehlerbeispiel zugrunde liegt, ist nicht nur ein Ausdruck, der am Ende eines Gesprächs benutzt werden kann, sondern darüber hinaus auch eine Formulierung, die für die Kommunikationsform Brief bezeichnend ist. ${ }^{579}$ Allerdings hat der Lernende hier nicht beachtet, daß dieser Ausdruck in der Regel nur beim Gebrauch des sogenannten Business English bzw. formalen Englisch benutzt wird. ${ }^{580}$ Bei privaten Briefkontakten - wie in dem Brief, dem das Beispiel entnommen wurde - sind jedoch eher Formulierungen zu erwarten, die Vertrautheit zwischen den Kommunikationspartnern implizieren. Diese könnte durch Lexeme wie love oder yours zum Ausdruck gebracht werden. ${ }^{581}$

Die Anwendung der Fehlererklärungsfaktoren der Erwerbshypothesen führte zu folgenden Ergebnissen: Zunächst fällt auf, daß im Gegensatz zu den vorhergehenden Fehlerkategorien in beiden Probandengruppen die Erklärungsfaktoren der Kontrastivhypothese stärker zur Anwendung kommen als die der Interlanguagehypothese (vgl. Tabellen 12 und 13 im Anhang). Besonders deutlich zeigt sich dies in der Gruppe der PwenlAb. So ist bereits an dieser Stelle festzuhalten, daß stilistische Fehler beim Erwerb der englischen Sprache in erster Linie auf den Einfluß der Erstsprache Deutsch zurückzuführen sind. Dies betrifft Lernende, die sich in der Frühphase des Zweitspracherwerbs befinden, vor allem aber Lernende in fortgeschrittenen Erwerbsstadien. Letzteres ist vermutlich darauf zurückzuführen, daß die PwenlAb im Gegensatz zu den PmeilAb - von wenigen Ausnahmen abgesehen - in Berufen tätig waren, in deren Rahmen sie sich intensiv mit stilistischen Aspekten der deutschen Sprache auseinandersetzen müssen. Diese sprachlichen Auseinandersetzungen könnten dazu beigetragen haben, daß deutsche Formulierungen auch bei Anwendung der englischen Sprache zum Tragen kamen.

\footnotetext{
578 vgl. Swan 1990, Textstelle 361, o. S.

579 vgl. ebd.

580 vgl. ebd.

581 vgl. ebd.
} 
Bei den PmeilAb hingegen ist der Einfluß der Erstsprache vermutlich in erster Linie darauf zurückzuführen, daß die Lernenden - abgesehen vom zweitsprachlichen Unterricht an der Schule selten Gelegenheit hatten, bereits erworbene stilistische Formulierungen der englischen Sprache in realen Gesprächssituationen einzuüben.

Die Anwendung der Erklärungsfaktoren der einzelnen Hypothesen ergab, daß von den Faktoren, die aus der Kontrastivhypothese abgeleitet wurden, in beiden Probandengruppen die Fehlerkategorien over-/underdifferentiation und overindulgence/underrepresentation nachgewiesen werden konnten (vgl. Tabellen 14 und 15 im Anhang). Damit kommen sowohl bei den PmeilAb als auch bei den PwenlAb ausschließlich Fehlerkategorien der starken Version der Kontrastivhypothese zur Anwendung. In bezug auf beide Probandengruppen ist somit festzustellen, daß ein Teil der entstandenen Fehler ausschließlich auf die linguistische Struktur der deutschen Sprache zurückgeführt werden kann, ohne daß dabei ein Einfluß anderer Zweitsprachen oder strategisches Verhalten zum Tragen kommt. Dies verdeutlichen folgende Beispiele:

Fehler: $\quad$ Goodbye til the 7. september. ${ }^{582}$

Korrektur: Bye, see you on the $7^{\text {th }}$ of September.

Fehler: $\quad$ Please tell me on which day we could meet. ${ }^{583}$

Korrektur: Please let me know which day is convenient for you so that we can meet and see each other.

Das erste Beispiel ist zweifelsfrei darauf zurückzuführen, daß die Fehlleistung auf der Übersetzung des deutschen Satzes ,bis zum 7. September` beruht. Obgleich der Lernende bei seiner Übersetzung Lexeme benutzte, die im Deutschen akustisch nicht in ähnlicher Form vorhanden sind, hat er, wie in der deutschen Vorlage, bei der Übersetzung einsilbige Lexeme verwendet und sie syntaktisch der deutschen Struktur entsprechend eingesetzt. Somit entspricht die Struktur des englischen Satzes eindeutig der deutschen Vorlage. Als stilistischer Verstoß und nicht als Abweichung vom linguistischen System ist die Fehlleistung zu werten, weil in mündlichen Gesprächssituationen Äußerungen wie till the 7 th of september von englischen Muttersprachlern zum Teil durchaus verwendet werden. ${ }^{584}$

Auch das zweite Beispiel läßt erkennen, daß es sich hier um eine direkte Übersetzung vom Deutschen ins Englische handelt. Als Vorlage diente hierbei eindeutig der deutsche Satz „Bitte sag mir, an welchem Tag wir uns treffen können.“ Isoliert betrachtet ist diese Äußerung nicht als Normabweichung zu bezeichnen. Nach Aussagen der englischen Korrektoren widerspricht die Äußerung jedoch den Kommunikationsprinzipien der englischen Sprache. Die Aufforderung please tell me wirkt

\footnotetext{
582 PmeilAb

583 PwenlAb

584 Dies wurde in Gesprächen, die der Autor der vorliegenden Arbeit mit englischen Muttersprachlern führte, deutlich.
} 
in einem Brief zu direkt. Alle Korrektoren urteilten, daß der Verfasser des Briefes sprachlich zurückhaltender wirken und dem Kommunikationspartner damit mehr Entscheidungsfreiheit vermitteln solle. In einem formelleren Gesprächszusammenhang hingegen, etwa zwischen zwei Geschäftspartnern, die darum bemüht sind, eine wichtige Entscheidung möglichst kurzfristig zu fällen, wäre der Satz Please tell me on which day we could meet durchaus normentsprechend. Letzteres beruht vor allem darauf, daß der Satz keinerlei Abweichungen vom linguistischen System enthält. Sowohl syntaktisch als auch lexikalisch läßt der Satz keine Normverstöße erkennen, sondern verdeutlicht im Gegenteil, etwa aufgrund der normgerechten Anwendung der Präposition on und des Hilfsverbs could, daß der Lernende über sehr fortgeschrittene Englischkenntnisse verfügt.

In bezug auf die Erklärungsfaktoren der Interlanguagehypothese bestehen zwischen den beiden Probandengruppen deutliche Unterschiede (vgl. Tabellen 16 und 17 im Anhang). Bei den PmeilAb ist die Mehrzahl der Fehler, interlanguagespezifisch betrachtet, auf reduction strategies zurückzuführen. Dies bedeutet, die Fehler basieren meist darauf, daß die Lernenden versuchten, bei Anwendung der Zielsprache Englisch möglichst wenige Ausdrucksformen zu verwenden - teils um auf diese Weise die Entstehung sprachlicher Fehler zu vermeiden, teils weil den Lernenden die sprachlichen Ausdrucksmittel, mit deren Hilfe sie den Sprachverlauf hätten fortsetzen können, nicht zur Verfügung standen. Dieses sprachliche Verhalten kam in den meisten Fällen bewußt zur Anwendung. Nur bei wenigen Abweichungen ist anzunehmen, daß sie möglicherweise auch unbewußt entstanden sind. Darüber hinaus ist bei einem kleinen Teil der Fehlleistungen davon auszugehen, daß sie partiell auch auf Faktoren des Fremdsprachenunterrichts und auf interlingualen Einfluß zurückgeführt werden können. Der Einfluß der unterschiedlichen Faktoren kann an folgenden Beispielen verdeutlicht werden:

Fehler: How do you do? I'm very happy, and you? ${ }^{585}$

Korrektur: How are you? I hope you are well. At the moment I'm enjoying myself and I hope that everything is ok with you, too.

Fehler: I have no ideas. Ciao. ${ }^{586}$

Korrektur: I don't know what else to tell you. See you soon-Yours

Das erste Beispiel zeigt sowohl den Einfluß von Faktoren des Unterrichts und von Reduktionsstrategien als auch die Tatsache, daß die Fehlleistungen möglicherweise partiell auch auf unbewußtes Handeln zurückzuführen sind. Stilistisch ist in diesem Beispiel vor allem der Ausdruck how do you do zu bemängeln. Die Benutzung dieser Redewendung wurde zum einen durch Faktoren des Unterrichts verursacht, weil die Redewendung veraltet ist und im Englischen in der Regel - wenn überhaupt - nur noch im Bereich des Business English bzw. des formalen Englisch verwendet wird. Es ist unwahrscheinlich, daß der Lernende, aus dessen Fragebogen hervorgeht, daß nahezu keine außerschulischen

\footnotetext{
585 PmeilAb

586 dto.
} 
Erfahrungen mit der englischen Sprache gesammelt wurden, diese Formulierung in einem Sprachkontext erworben hat, der außerhalb des schulischen Bereichs liegt. Der Erwerb und die Anwendung der Formulierung basieren vermutlich entweder darauf, daß der Lehrer eine veraltete Form des Englischen benutzte und der Lernende diese Form aufgrund häufiger Anwendung verinnerlicht hat oder darauf, daß im Englischunterricht Schulbücher benutzt worden waren, deren Inhalte nicht an den heutigen Stand der englischen Sprache angepaßt waren. Möglich ist jedoch auch, daß die Schüler im Unterricht das Verfassen einfacher englischer Geschäftsbriefe erlernten und der Lernende auf diese Weise auf die Redewendung aufmerksam wurde, weil die Formulierung how do you do im Business English auch heutzutage, wie erwähnt, durchaus üblich ist. ${ }^{587}$ Unbewußtes Handeln ist in diesem Beispiel deshalb nicht auszuschließen, weil es möglich ist, daß der Unterrichtende die Formulierung so häufig benutzt hat oder aber sie durch das Verfassen geschäftlicher Briefe so intensiv eingeübt wurde, daß der Lernende die deutsche Frage „Wie geht es dir?“ automatisch mit der Redewendung how do you do übersetzte.

Die Anwendung von Reduktionsstrategien zeigt sich in dem sich anschließenden Satz: I'm very happy and you? Hier war der Lernende darum bemüht, mit möglichst wenigen sprachlichen Elementen eine kommunikative Aussage zu vermitteln. Damit vermied er zum einen den normgerechten Gebrauch der Verlaufsform, weil der Satz nicht auf einen momentan andauernden Zustand hinweist, zum anderen aber auch den Gebrauch der Präpositionalphrase at the moment, die aufgrund der Präposition at von Lernenden in frühen Erwerbsstadien als schwierig empfunden wird. Gerade aufgrund der sprachlichen Zurückhaltung des Lernenden sind jedoch stilistische Abweichungen entstanden, weil im Englischen die Darstellung von Gefühlszuständen in der Regel durch sprachliche Umschreibungen oder Einleitungen erfolgt, die nicht sofort den Kern der Sache erfassen. ${ }^{588}$ Dabei ist es im Englischen tatsächlich üblich, daß der Darstellung eigener Gemütszustände die Frage nach der Situation des Kommunikationspartners folgt. Die verkürzte Frage and you? allerdings wird von englischen Muttersprachlern als kühl und zurückhaltend empfunden. ${ }^{589}$

Das zweite Beispiel verdeutlicht neben Reduktionsverhalten auch den Einfluß interlingualen Transfers. Der abrupte Kommunikationsabbruch, der nach dem Satz I have no ideas erfolgte, ist ein Beispiel für das Reduktionsverhalten in Form des sogenannten message abandonment (vgl. Tabelle $16 \mathrm{im}$ Anhang): Der Lernende hat bewußt darauf verzichtet, den Kommunikationsverlauf fortzusetzen. Dieses Verhalten beruhte vermutlich auf einer Kombination motivationsbedingter und sprachlicher Faktoren. Einerseits ist davon auszugehen, daß der Lernende mangels extrinsischer und intrinsischer

\footnotetext{
587 vgl. Pons Englisch Aktiv 2002, 32

588 vgl. Lojewski 1991, 175

589 Dies bestätigten die englischen Korrektoren, die sich an der Auswertung der Studie, die der vorliegenden Arbeit zugrundeliegt, beteiligten.
} 
Motivation darauf verzichtete, den Brief fortzusetzen. Letzteres bestätigt vor allem die Tatsache, daß die Einstellung des Lernenden gegenüber der englischen Sprache auf dem Fragebogen als negativ bezeichnet wurde. Andererseits kann auch vermutet werden, daß sich der Lernende außerstande sah, seine weiteren Gedanken sprachlich vermitteln zu können und den Kommunikationsverlauf daher beendete.

Interlingual transfer als weitere Fehlerursache wird vor allem in der Wahl des italienischen Lexems ciao sichtbar. Offenbar ist der Lernende davon ausgegangen, daß das Wort ciao wie im Deutschen auch im Englischen als Modewort benutzt wird. Dabei wurde jedoch nicht beachtet, daß die englische Sprache für die Übernahme sogenannter Lehnwörter weniger offen ist als das Deutsche. Auch hier ist nicht auszuschließen, daß das Lexem aufgrund seiner häufigen Anwendung bei jüngeren deutschen Muttersprachlern einem Automatismus unterliegt und daher unbewußt benutzt wurde.

Bei den PwenlAb geht die Mehrzahl der Abweichungen bei Anwendung der Fehlererklärungsfaktoren der Interlanguagehypothese auf das Generalisieren von Interlanguageregeln und -elementen in neuen sprachlichen Zusammenhängen zurück (vgl. Tabelle 17 im Anhang). Zum Teil, wenn auch seltener, entstanden die Fehler auch durch das Paraphrasieren sprachlicher Elemente. Die Anwendung von Reduktionsstrategien konnte nur in wenigen Fällen nachgewiesen werden. Aus der Perspektive der Interlanguagehypothese betrachtet unterscheidet sich damit die Genese der stilistischen Fehler der PwenlAb deutlich von der Genese der entgegengesetzten Probandengruppe. Während der größte Teil der Fehler der PmeilAb durch das Bemühen entstand, möglichst wenige sprachliche Mittel zu benutzen, liegen die Ursachen der Fehlerentstehung bei den PwenlAb in der Verallgemeinerung bereits erlernter Ausdrücke und in dem Versuch, nicht abrufbare stilistische Wendungen durch Umschreibungen zu verdeutlichen (vgl. Tabelle 17 im Anhang). Letztere zeigen sich z. B. an der folgenden, schon in anderem Zusammenhang aufgeführten Äußerung:

Fehler: $\quad$ Then we can speak about our next journey in spring and can also exchange views about personal things. ${ }^{590}$

Korrektur: Then we can plan our next journey in spring and can talk about personal matters.

Das Verallgemeinern sprachlicher Elemente wird vor allem am Gebrauch des Verbs speak about deutlich. Wenngleich der Ausdruck an dieser Stelle nicht als völlig normabweichend zu bezeichnen ist, handelt es sich hier dennoch um eine stilistisch unpassende Ausdrucksform, weil die Formulierung speak about zu allgemein ist und nicht wirklich das Ziel des Briefverfassers erfaßte. Der Ausdruck wäre dann zu verwenden gewesen, wenn der gemeinsame Urlaub in der Vergangenheit stattgefunden und somit der Austausch von Erinnerungen im Vordergrund gestanden hätte. Da hier jedoch der

590 PwenlAb 
Wunsch geäußert werden sollte, den gemeinsamen Urlaub zu organisieren, wäre an dieser Stelle ein Lexem zu erwarten gewesen, das auf die Durchführung einer in der Zukunft liegenden Handlung schließen läßt. Die Verwendung des Wortes plan hätte die beabsichtigte Bedeutung zum Ausdruck gebracht.

Paraphrase als zweite Fehlerentstehungsursache äußert sich in der Umschreibung exchange views about personal things. Statt der Verwendung der einfachen Formulierung talk about personal matters versuchte der Lernende, der offenbar in der gegebenen Situation nicht auf eine einfachere Formulierung zurückgreifen konnte, seine sprachliche Aussage durch eine komplexe Aneinanderreihung formaler Lexeme zu verdeutlichen. Dabei wurde jedoch nicht beachtet, daß in der englischen Sprache, insbesondere in Gesprächssituationen zwischen einander vertrauten Personen, einfache, kurze Darstellungen bevorzugt werden. Darüber hinaus ist der Ausdruck exchange views in der Regel keine Formulierung, die zwischen zwei Brieffreunden verwendet wird, sondern wäre entweder in geschäftlichen Situationen einzusetzen oder in Situationen, in denen Fachleute eines bestimmten Bereichs eine Angelegenheit des betreffenden Fachbereichs diskutieren. Auch der Ausdruck personal things weicht in der betreffenden Situation von der Norm der englischen Sprache ab, weil er nicht besagt, daß man sich - wie beabsichtigt - über persönliche Angelegenheiten unterhalten möchte. Mit personal things bezeichnet man im Englischen persönliche Gegenstände, d. h. Gegenstände, die zu einer bestimmten Person gehören, wie etwa Wertgegenstände, Möbel, Kleidung etc. Der Ausdruck personal matters hingegen, der in dem Korrekturbeispiel verwendet wurde, besagt eindeutig, daß der Briefverfasser vertraute Gespräche über Freunde, Beziehungen, berufliche Dinge, private Probleme etc. führen möchte.

Insgesamt betrachtet zeigt die Analyse der stilistischen Fehler, daß - anders als bei den zuvor behandelten lexikalischen Fehlerkategorien - die Unterschiede zwischen den Fehlerentstehungsursachen beider Probandengruppen größer sind als die Gemeinsamkeiten. Die PwenlAb produzierten Fehler dadurch, daß sie ihre bereits erworbenen Kenntnisse anwandten, um auf diese Weise bewußt Kommunikationslücken zu schließen. Die PmeilAb verhielten sich defensiv, d. h. sprachlich zurückhaltend, und ließen dadurch Fehler entstehen, die teilweise auch unbewußt verursacht wurden.

Bei Betrachtung der psycho- und soziolinguistischen Voraussetzungen der Lernenden wird zudem deutlich, daß die Unterschiede, die in den Probandengruppen festzustellen waren, auf stilistischer Ebene stärkere Auswirkungen haben als beim Gebrauch der zuvor behandelten Lexemgruppen. Offenbar haben die ausgiebigen Erfahrungen in englischsprachigen Ländern und mit englischen Muttersprachlern, die bei den PwenlAb weit öfter vorhanden waren als bei der entgegengesetzten Probandengruppe, bei ersteren zu der Erkenntnis geführt, daß Wissenslücken durch Umschreibungen und Verallgemeinerungen geschlossen werden können und der Kommunikationspartner auf diese 
Weise die sprachliche Aussage versteht, auch wenn dieses Sprachverhalten zu Fehlern führt. Bei den PmeilAb hingegen führte die Tatsache, daß nur wenige von ihnen Erfahrungen dieser Art sammeln konnten, dazu, daß sie Kommunikationseinschränkungen oder sogar einen vollständigen Kommunikationsabbruch einer Fortsetzung des Gesprächs vorzogen, um auf diese Weise der Entstehung sprachlicher Fehler vorzubeugen. Somit zeigt sich auf stilistischer Ebene deutlicher als bei den anderen Lexembereichen, daß Auslandsaufenthalte, Erfahrungen mit englischen Muttersprachlern sowie ein breites Spektrum allgemeiner linguistischer Kenntnisse positiv auf den Kommunikationsverlauf wirken. Zudem sind - wie erwähnt - stilistische Abweichungen nicht als Verstöße gegen das linguistische System zu betrachten.

\section{Syntaktisch-morphologische Abweichungen}

Wie bei den lexikalischen Abweichungen zeigte die Gegenüberstellung (vgl. Tabelle 1 im Anhang) der Gruppe der Probanden mit den meisten syntaktisch-morphologischen Abweichungen (PmeismAb) und der Gruppe der Probanden mit den wenigsten syntaktisch-morphologischen Abweichungen (PwensmAb), daß die Fehler, die in den beiden Probandengruppen ermittelt wurden, typologisch übereinstimmen (vgl. Tabellen 4 bis 9 im Anhang). In beiden Gruppen wurden Fehler sowohl auf der Ebene ganzer Sätze als auch bei einzelnen Satzelementen ermittelt. Unter ,Ebene ganzer Sätze` ist zu verstehen, daß die Fehlleistungen nicht in der Verschiebung oder morphologischen Veränderung einzelner Lexeme oder Konstituenten bestehen, sondern eine Umstrukturierung des gesamten Satzes zur Folge haben. Fehler, die einzelne Satzelemente betreffen, äußern sich in beiden Gruppen darin, daß die Struktur eines bestimmten Lexems bzw. bestimmter Lexeme verändert wurde oder aber darin, daß die syntaktische Stellung einzelner Satzteile oder -elemente von der Norm der englischen Sprache abweicht.

$\mathrm{Zu}$ den Fehlern, die Auswirkungen auf die gesamte Satzebene haben, zählen das Vertauschen von Elementen zusammenhängender Lexemgruppen, beispielsweise einer komplexen Aneinanderreihung verschiedener Adverbien des Ortes und der Zeit sowie die normabweichende Bildung von Fragesätzen. Auch der falsche Gebrauch der Zustandsformen Aktiv und Passiv ist diesem Fehlerbereich zuzuordnen. Ein Beispiel für Fehler, die einzelne Satzelemente betreffen, bietet der falsche Gebrauch des Gerundiums in folgender Äußerung:

Fehler: I'm used to drink tea every morning. ${ }^{591}$

Korrektur: I'm used to drinking tea every morning.

591 Wie auch im vorhergehenden Kapitel handelt es sich bei den nachfolgenden Fehlerbeispielen - falls nicht anders angegeben - um Abweichungen, die der Autor der vorliegenden Arbeit im Rahmen seiner unterrichtlichen Tätigkeit ermitteln konnte. 
$\mathrm{Zu}$ den Abweichungen, die die gesamte Satzstruktur betreffen, gehört u. a. folgende Form der Fragesatzbildung:

Fehler: Lives your brother in Berlin?

Korrektur: Does your brother live in Berlin?

Neben diesen Abweichungen stellt der fehlerhafte Gebrauch des Zeitensystems in beiden Probandengruppen einen wichtigen Fehlerkomplex dar, wobei sowohl bei den PmeismAb als auch bei den PwensmAb die Fehlerkategorie simple past durch eine besonders hohe Anzahl von Verstößen auffällt (vgl. Tabellen 6 und 7 im Anhang).

Bei genauerer Betrachtung der einzelnen Fehlertypen wird - wie bei den Abweichungen auf lexikalischer Ebene - deutlich, daß der Unterschied zwischen den Abweichungsformen der PmeismAb und der PwensmAb darin besteht, daß die Fehler der PmeismAb elementarer sind bzw. einen stärkeren Abweichungscharakter haben als die Fehler, die in der entgegengesetzten Probandengruppe ermittelt wurden. Konkret bedeutet dies, daß die Fehler der PmeismAb oft eine größere Orientierung am Deutschen erkennen lassen oder aber schwerwiegender sind als bei den PwensmAb. Letzteres äußert sich darin, daß die beabsichtigte sprachliche Aussage aufgrund des Fehlers schlechter zu verstehen ist. Ein Beispiel für eine direkte Anlehnung an das Deutsche bietet folgender Satz:

Fehler: I have you yesterday in the bus seen. ${ }^{592}$

Korrektur: I saw you on the bus yesterday.

Eine starke Beeinträchtigung der beabsichtigten Aussage wird in folgender Äußerung deutlich:

Fehler: $\quad$ I hope were arrived to Spain with my family. ${ }^{593}$

Korrektur: I hope to go to Spain with my family.

Das Korrekturbeispiel basiert auf der Vermutung, daß der Lernende den deutschen Satz „Ich hoffe, daß ich nächstes Jahr mit meiner Familie nach Spanien fahren kann“ zu vermitteln suchte. Ohne Betrachtung des Gesamttextes, in dem der Satz enthalten ist, wäre die Bedeutung der Äußerung kaum zu verstehen gewesen, da sie verschiedene Interpretationsmöglichkeiten zugelassen hätte. So ist dieser Aussage bei isolierter Betrachtung weder eindeutig zu entnehmen, ob der Lernende über ein vergangenes Ereignis oder über ein zukünftiges zu sprechen beabsichtigt, noch, welche semantische Verbindung zwischen dem Verb hope und der Ankunft in Spanien besteht. Die Unsicherheit entsteht dabei in erster Linie durch die Passivkonstruktion were arrived, wird jedoch durch den Gebrauch des

\footnotetext{
592 Das Beispiel stammt aus der Probandengruppe mit den meisten syntaktisch-morphologischen Abweichungen PmeismAb (FB der Gruppe HS 10E).

593 dto.
} 
Lexems hope und der Präposition to noch verstärkt. Eine Interpretationsmöglichkeit wäre somit, daß die Ankunft in Spanien bereits stattgefunden hat, eine andere, daß die Reise noch bevorsteht.

Bei den Probanden, die sich durch eine äußerst geringe Anzahl syntaktisch-morphologischer Verstöße von den anderen unterscheiden, ist zum einen der Einfluß der deutschen Sprache bei ähnlichen semantischen Aussagen weniger auffällig und zum anderen das Verständnis der beabsichtigten Aussagen trotz der enthaltenen Abweichung leichter, wenngleich die Erstsprache Deutsch auch hier eine wichtige Fehlerentstehungsquelle darstellt. Der Unterschied zu den Fehlertypen der PmeismAb wird u. a. in folgendem Beispiel deutlich:

Fehler: $\quad$ I would be pleased if you spend some days of our summer holidays with us in our new home. ${ }^{594}$

Korrektur: I would be delighted if you were to spend a few days of our summer holidays in our new home with us.

Neben dem semantischen Aspekt, der sich insbesondere in der Wahl des Lexems some und ansatzweise auch in der Wahl des Wortes pleased zeigt, ist die Äußerung aufgrund der Verwendung der Verbform spend als Abweichung zu betrachten. Bedingt durch die Konditionalkonstruktion I would be pleased und das sich anschließende Lexem if ist die Botschaft der Äußerung jedoch ohne Schwierigkeiten zu verstehen. Auch die Wortwahl läßt erkennen, daß der Lernende mit dem Englischen bereits vertraut ist.

Auf quantitativer Ebene zeigt der Vergleich der beiden Probandengruppen, daß die Kategorien der Auslassungs- und Hinzufügungsfehler jeweils besonders hohe Fehlerergebnisse verzeichnen (vgl. Tabellen 4 und 5 im Anhang). Dabei ist bei den PmeismAb der Anteil der Hinzufügungsfehler größer als der der Auslassungsfehler, während bei den PwensmAb der umgekehrte Fall zu beobachten ist. Auffällig hoch ist daneben, wie bereits erwähnt, in beiden Probandengruppen der Anteil der Fehler, die beim Gebrauch des simple past entstanden sind. Auch die Anzahl der Wort- und Satzstellungsfehler ist signifikant (vgl. Tabellen 8 und 9 im Anhang). Dabei ist der Fehleranteil der Wort- und Satzstellungsfehler bei den PmeismAb höher als in der entgegengesetzten Probandengruppe.

Bei den PmeismAb fällt darüber hinaus auf, daß die Probanden deutliche Schwierigkeiten bei der Anwendung des Infinitivs (vgl. Tabelle 4 im Anhang) und der Zeitform will-future (vgl. Tabelle 6 im Anhang) haben. Diese Schwierigkeiten sind bei den PwensmAb wesentlich geringer, wenngleich die Fehlerkategorie will-future auch in dieser Probandengruppe einen höheren Fehleranteil verzeichnet als die anderen Zeitformen der Zukunft (vgl. Tabelle 7 im Anhang). Weiterhin ergibt der Vergleich der beiden Gruppen - wie zu erwarten -, daß der Fehleranteil in den fortgeschrittenen Grammatik-

594 Das Beispiel stammt aus der Probandengruppe mit den wenigsten syntaktisch-morphologischen Abweichungen PwensmAb (FB der Gruppe EB adv.). 
bereichen, die erst in höheren Stufen des Spracherwerbs erlernt werden, bei den PwensmAb größer ist als in der entgegengesetzten Probandengruppe. Dies betrifft die Fehlerkategorien past perfect, present perfect sowie die Gruppe der Bedingungssätze (vgl. Tabellen 6 bis 9 im Anhang). In diesem Zusammenhang ist festzustellen, daß der Gebrauch des present perfect insbesondere fortgeschrittenen Englischlernenden Probleme bereitet, weil diese die Anwendung des present perfect vom Gebrauch des simple past abgrenzen müssen. Englischlernende in frühen Erwerbsphasen hingegen konzentrieren sich in der Regel auf eine Zeitform und verzichten auf jegliche Differenzierung. ${ }^{595}$ Der ausschließliche Gebrauch des present perfect kann daher gerade von Lernenden, die sich in der Frühphase des Spracherwerbs befinden, normgerecht bewältigt werden.

Insgesamt betrachtet lassen die quantitativen Unterschiede und Übereinstimmungen, die beim Vergleich der beiden Probandengruppen ermittelt wurden, erkennen, daß die größten syntaktisch-morphologischen Probleme deutscher Englischlernender in der Anwendung syntaktisch-morphologischer Einheiten liegen, die nicht die gesamte Struktur des Satzes betreffen, sondern einzelne Lexeme oder Lexemendungen (vgl. Tabellen 4 und 5 im Anhang). Dies wird insbesondere durch die hohe Anzahl der Abweichungen in den Bereichen der Auslassungs- und Hinzufügungsfehler deutlich. Aufgrund der Tatsache, daß die Fehlerzahlen in beiden Probandengruppen äußerst hoch sind, ist zu erkennen, daß Schwierigkeiten hier auch in fortgeschrittenen Erwerbsphasen auftreten und im Englischunterricht deutscher Muttersprachler einer besonderen Aufmerksamkeit bedürfen. Eine genauere Untersuchung dieser Fehlertypen zeigte, daß die Abweichungen in der Regel darauf basierten, daß die betreffenden Lexeme entweder redundanterweise hinzugefügt wurden oder an Stellen, an denen sie zu erwarten gewesen wären, ausgelassen wurden. Ein typisches Beispiel für das redundante Hinzufügen bietet die Anfügung des 3. Person Singular-Morphems , s ' in folgender Äußerung:

Fehler: $\quad$ I works in the garden. ${ }^{596}$

Korrektur: I work in the garden.

Das Auslassen wichtiger Elemente wird an folgendem Beispiel deutlich:

Fehler: $\quad$ At the moment I sitting in the office. ${ }^{597}$

Korrektur: At the moment I'm sitting in the office.

Neben diesen Formen der Auslassungs- und Hinzufügungsfehler gehören darüber hinaus das normabweichende Auslassen oder Hinzufügen von Artikeln und Adverbien zu den meistvertretenen Fehler-

\footnotetext{
595 vgl. u. a. Vogel 1989, $125 \mathrm{ff}$

596 PmeismAb

597 PwensmAb
} 
formen dieser Kategorien, ${ }^{598}$ wobei beide Lexemgruppen bei den PwensmAb seltener normabweichend verwendet wurden als bei den PmeismAb (vgl. Tabellen 4 und 5 im Anhang). Beispiele für das redundante Hinzufügen oder normabweichende Auslassen dieser Lexemgruppen bieten u.a. folgende Äußerungen:

Fehler: I live in the Goethestraße. ${ }^{599}$

Korrektur: I live in Goethestraße.

Fehler: $\quad$ At beginning I liked my job very much. ${ }^{600}$

Korrektur: At the beginning I liked my job very much.

Fehler: We really had a terribly time. ${ }^{601}$

Korrektur: We really had a terrible time.

Fehler: $\quad$ At the moment I work real hard. ${ }^{602}$

Korrektur: At the moment I'm working really hard.

Im ersten Beispiel ist die Einfügung des bestimmten Artikels als normabweichend zu bezeichnen, weil im Englischen - im Gegensatz zum Deutschen - der bestimmte Artikel bei der Benennung von Straßen, Parks und Plätzen, die einen Namen tragen, nicht verwendet wird. ${ }^{603}$ Im Zusammenhang mit diesen Orten wird der Artikel nur benutzt, wenn auf eine bestimmte Straße oder auf einen bestimmten Park oder Platz verwiesen wird, ${ }^{604}$ wie etwa in folgender Äußerung: I don't live in the Goethestraße you mean, I live in a different one.

Im zweiten Fehlerbeispiel wäre der Artikel hingegen zu erwarten gewesen, weil das Wort beginning nicht zu der Gruppe der zuvor genannten Lexeme gehört und bei nicht näher spezifizierten Nomen, die in Verbindung mit der Präposition at benutzt werden, ein Artikel zu verwenden ist.

Das dritte Beispiel dokumentiert das oben erwähnte Ersetzen eines Adjektivs durch ein Adverb. Der Fehler, der durch den Gebrauch des Adverbs terribly entstand, ist als grammatischer Verstoß zu betrachten, weil sich das Wort terrible auf das nachfolgende Nomen time bezieht und daher das Lexem als Adjektiv und nicht als Adverb zu benutzen ist. Das vierte Fehlerbeispiel verdeutlicht den umgekehrten Fall, das Ersetzen eines Adverbs durch ein Adjektiv. Das Wort really dient hier zur näheren Beschreibung des Lexems hard und gehört damit zur Gruppe der Adverbien.

\footnotetext{
598 Wie bereits bei der Darstellung des Fehlerkategoriesierungsverfahrens erwähnt, ist unter den Bezeichnungen ,Auslassung“ oder ,Hinzufügung' jedoch nicht zu verstehen, daß ganze Lexeme ausgelassen oder hinzugefügt wurden, sondern vielmehr, daß statt des zu erwartenden Artikels oder Adverbs Lexeme benutzt wurden, die zu anderen Wortarten gehören. Im Falle der Adverbien bestanden die Fehler in der Regel darin, daß die Adverbien durch Adjektive ersetzt wurden.

599 PmeismAb

${ }^{600}$ PwensmAb

601 dto.

602 dto.

603 vgl. Ungerer u. a. 1987, 73 - 76
} 
Neben diesen Fehlertypen gehört darüber hinaus in der Gruppe der PmeismAb auch das Auslassen der Infinitiv-Form zu den regelmäßig zu beobachtenden Fehlerformen, wenngleich weniger häufig als das Auslassen der vorgenannten Lexemgruppen. Ein Beispiel bietet folgende Äußerung:

Fehler: $\quad$ I want go swimming. ${ }^{605}$

Korrektur: I want to go swimming.

Aufgrund der Komplexität der syntaktisch-morphologischen Ebene und der Vielzahl der Fehlergruppen, die hier zu beobachten sind, beschränkt sich nachfolgende Darstellung der Fehleranalyse auf solche Kategorien, die durch besonders hohe Fehlerzahlen hervortreten und in beiden Probandengruppen belegt sind. Auch hat die Analyse der Auslassungs- und Hinzufügungsfehler gezeigt, daß die Faktoren, die zur Entstehung der Abweichung beigetragen haben, in allen Unterkategorien dieses Bereichs nahezu übereinstimmen. Die Beschreibung der Fehlerentstehung dieser Kategorie wird sich daher auf die Unterkategorie ,sonstige Wortarten und Bestandteile' beschränken, weil diese die meisten Abweichungen enthält. Hinzu kommt, daß Fehler beim Gebrauch von Adverbien, wenngleich sie - wie zuvor beschrieben - zu den häufigsten Erscheinungen des Hinzufügungs- und Auslassungsbereichs gehören, bereits im Rahmen der lexikalischen Abweichungen dargestellt wurden. Aus diesen Gründen werden bei der nachfolgenden Beschreibung der Fehleranalyse folgende Kategorien berücksichtigt:

1. Wort- und Satzstellungsfehler

2. Der Gebrauch des simple past

3. Hinzufügen und Auslassen sonstiger Wortarten und Bestandteile

Bei Anwendung der Fehlererklärungsfaktoren der drei Erwerbshypothesen wurden folgende Ergebnisse ermittelt: Im Gegensatz zu den lexikalischen Abweichungen konnten auf syntaktischmorphologischer Ebene sowohl die Erklärungsfaktoren der Kontrastiv- und der Interlanguagehypothese als auch die der Identitätshypothese jeweils in beiden Lernergruppen nachgewiesen werden (vgl. Tabellen 18 und 19 im Anhang). Dabei überwiegt in beiden Lernergruppen das Erklärungspotential der Interlanguagehypothese, wenngleich die Differenz zur Anwendbarkeit der Erklärungsfaktoren der Identitätshypothese gering ist. Weniger Erklärungsmöglichkeiten bieten die Faktoren der Kontrastivhypothese. Da jedoch in beiden Lernergruppen die Erklärungsfaktoren dieser Hypothese bei insgesamt mehr als $50 \%$ der Fehler angewendet werden konnten, sind auch erstsprachlich bedingte Faktoren als wichtige Fehlerentstehungsursache syntaktisch-morphologischer Abweichungen zu bezeichnen.

\footnotetext{
604 vgl. ebd., $73 \mathrm{f}$

605 PmeismAb
} 
Prinzipiell zeigen die Ergebnisse, die beim Vergleich deutlich wurden, daß die Ursachen syntaktischmorphologischer Fehler bei deutschen Englischlernenden mit äußerst geringen Grammatikkenntnissen und Lernenden mit umfassenden Grammatikkenntnissen weitgehend übereinstimmen. In beiden Gruppen ist die Fehlerentstehung sowohl auf den Einfluß der Erstsprache und auf biologisch determinierte Faktoren als auch auf die strategische Vorgehensweise beim Erlernen und bei der Anwendung der Zielsprache zurückzuführen (vgl. Tabellen 20 bis 25 im Anhang). Im Vergleich zu den lexikalischen Abweichungen, die ausschließlich mit Hilfe der Faktoren der Kontrastiv- und der Interlanguagehypothese nachvollzogen werden können, ist die Entstehung syntaktisch-morphologischer $\mathrm{Ab}$ weichungen komplexer, da hier auch solche Faktoren zum Tragen kommen, die Bestandteil des Erwerbs natürlicher Sprachen sind. Die Analyse zeigt dabei, daß - ähnlich wie bei den lexikalischen Abweichungen - die Differenzen zwischen den Erwerbsbedingungen der PmeismAb und der PwensmAb in bezug auf das Gesamtergebnis von untergeordneter Bedeutung sind: Trotz der Tatsache, daß die PwensmAb Englisch nicht ausschließlich unter schulischen Bedingungen erworben hatten, sondern darüber hinaus Kontakte $\mathrm{zu}$ englischen Muttersprachlern pflegten und teils über Auslandserfahrungen in englischsprachigen Ländern verfügten, stimmen die Faktoren, die zur Entstehung der Abweichungen beigetragen haben, mit den Faktoren überein, die bei den PmeismAb zum Tragen kommen. Auch die grundsätzlichen linguistischen Differenzen - wie etwa Kenntnisse in anderen Zweitsprachen, die bei den PmeismAb nur ansatzweise vorhanden sind - und die Unterschiede zwischen den Schulformen, die die Probanden besucht haben, beeinflussen die Fehlerentstehung nur marginal.

Bei der Analyse der Fehler, die aus obengenannten Gründen im weiteren Verlauf exemplarisch dargestellt werden, wurden nachfolgend beschriebene Ergebnisse ermittelt.

\subsection{Wort- und Satzstellungsfehler}

Bei den Wort- und Satzstellungsfehlern kommen - wie oben erwähnt - die Erklärungsfaktoren der Interlanguagehypothese am häufigsten zur Anwendung (vgl. Tabellen 18 und 19 im Anhang). In der Gruppe der PmeismAb konnten Fehlerentstehungsursachen dieser Hypothese bei allen Wort- und Satzstellungsfehlern nachgewiesen werden. Der nächstgrößte Fehlerentstehungsanteil ist auf die Faktoren der Identitätshypothese zurückzuführen. Die Faktoren der Kontrastivhypothese kommen in dieser Kategorie am wenigsten zum Tragen. Angesichts der Tatsache, daß die Differenzen zwischen der Anwendbarkeit der einzelnen Hypothesen jedoch nicht als gravierend zu bezeichnen sind, stellen sowohl interlanguagespezifische als auch entwicklungsbedingte und erstsprachliche Faktoren wichtige Ursachen der Fehlerproduktion dar. 
Bei den PwensmAb zeigt sich hier erstmals, daß zwei Hypothesen, die Identitäts- und die Interlanguagehypothese, im gleichen Umfang zum Tragen kommen (vgl. Tabelle 19 im Anhang). Faktoren dieser Hypothesen konnten bei allen Fehlern nachgewiesen werden. Auch hier bietet die Kontrastivhypothese das geringste Fehlererklärungspotential. Dennoch ist auch bei den Ergebnissen dieser Probandengruppe zu erkennen, daß erstsprachliche Faktoren eine wichtige Fehlerentstehungsquelle darstellen, da erstsprachlicher Einfluß bei weit mehr als der Hälfte aller Abweichungen nachgewiesen werden konnte. Somit sind die Faktoren, die die Wort- und Satzstellungsfehler verursacht haben, in beiden Probandengruppen identisch.

Bei genauerer Betrachtung der Fehlerentstehungsursachen der Hypothesen zeigt sich, daß erstsprachlicher Einfluß bei den PmeismAb in erster Linie in Form von Substitutionen deutlich wird (vgl. Tabelle 20 im Anhang). Das heißt, die Probanden ersetzten englische Satzbaumuster durch Satzkonstruktionen der deutschen Sprache. Daneben äußert sich der Einfluß der Erstsprache auch in Form sogenannter Hyperkorrektheiten. In sehr seltenen Fällen ist die Anlehnung an das Deutsche auch an erstsprachlich bedingten Generalisierungen zu erkennen. Die verschiedenen Auswirkungen der Erstsprache in dieser Lernergruppe lassen sich an folgendem Beispiel verdeutlichen:

Fehler: $\quad$ Did have you seen me yesterday? ${ }^{606}$

Korrektur: Did you see me yesterday? ${ }^{607}$

Die Fehlleistung stellt zunächst eine Substitution dar, weil der Lernende nach Gebrauch des Lexems did auf eine syntaktische Struktur der deutschen Sprache zurückgriff und die eigentlich zu erwartende englische Struktur durch diese ersetzte. Es ist davon auszugehen, daß der Teilsatz have you seen me yesterday auf dem deutschen Fragesatz „Hast du mich gestern gesehen?“ basiert. Dies äußert sich sowohl in der Stellung des Hilfsverbs have als auch im Gebrauch der Verbform seen. Daneben verdeutlicht dieser Satzteil, daß die Abweichung partiell auch als Übergeneralisierung interpretiert werden kann, weil der Lernende mit dem Gebrauch der Konstruktion have you seen eine syntaktische Form verwendete, die im Englischen tatsächlich existiert, in der betreffenden Situation jedoch als Normabweichung zu betrachten ist, weil aufgrund der Zeitangabe ausschließlich die Verwendung des Hilfsverbs did korrekt gewesen wäre. Der Fehler wurde durch die lautliche und optische Ähnlichkeit zwischen der deutschen Frage „Hast du mich gesehen?“ und der present perfect-Form have seen ausgelöst.

Darüber hinaus kann die Fehlleistung jedoch auch als Ergebnis einer hyperkorrekten Sprachanwendung betrachtet werden, weil der Lernende in dem Fehlerbeispiel zwei unterschiedliche Zeit-

\footnotetext{
606 PmeismAb

${ }^{607}$ Da der Wortstellungsfehler auf dem falschen Gebrauch der Zeitformen simple past und present perfect beruht, ist die Abweichung auch als Beispiel für den normabweichenden Gebrauch des englischen Zeitensystems zu betrachten.
} 
formen miteinander kombinierte, das simple past und das present perfect. Die Hyperkorrektheit in dieser Kombination basiert vermutlich darauf, daß der Lernende zu einem früheren Zeitpunkt internalisiert hatte, daß Fragesätze, die sich auf ein vergangenes Ereignis beziehen, mit dem Hilfsverb did einzuleiten sind. Der Betreffende hatte jedoch auch verinnerlicht, daß in bestimmten Fällen - etwa bei Handlungen, die noch nicht abgeschlossen sind - das present perfect zu verwenden ist und die Frage demnach mit dem Hilfsverb have eingeleitet wird. Aus Unsicherheit und um den Verstoß gegen eine der beiden Möglichkeiten der Fragesatzbildung zu vermeiden, kam es schließlich zu der oben dargestellten syntaktischen Konstruktion. Die Unsicherheit des Lernenden wurde dabei vermutlich durch die strukturellen Gemeinsamkeiten zwischen der Verbform have seen und der Perfektform des deutschen Verbs ,sehen“ noch verstärkt.

Bei den PwensmAb äußert sich der Einfluß des Deutschen sowohl in Form von Substitutionen und Über- und Unterdifferenzierungen als auch in Form von Übergeneralisierungen (vgl. Tabelle $21 \mathrm{im}$ Anhang). Dies wird an folgendem Beispiel deutlich:

Fehler: $\quad$ Do you have already plans for your holiday next year? ${ }^{608}$

Korrektur: Have you already made plans for your holiday next year? ${ }^{609}$

Die Äußerung ist zunächst als Substitution zu betrachten, weil die syntaktische Struktur des Satzes auf dem Gebrauch des Präsens beruht, das in der deutschen Vorlage, die der Lernende ins Englische zu übertragen versuchte, als normgerecht zu bezeichnen ist. Der deutsche Satz, an dem sich der Lernende orientierte, lautete dabei vermutlich: „Hast Du schon Pläne für Deinen Urlaub im nächsten Jahr?“ Damit hat der Lernende die syntaktische Struktur des zielsprachlichen Satzes durch eine Struktur des Deutschen ersetzt.

Daneben kann die Abweichung jedoch auch der Fehlerkategorie ,Unter- bzw. Überdifferenzierung“ zugeordnet werden, weil der Lernende die flexiblen Möglichkeiten, die er im Deutschen zur Verdeutlichung seiner semantischen Aussage hat, auf die englische Sprache übertrug. Im Deutschen kann die obengenannte Frage in grammatisch unterschiedlichen Formen gestellt werden. Zum einen könnte der Fragesatz der oben dargestellten Form entsprechen. Zum anderen hätte der Lernende jedoch auch das Perfekt benutzen können, wie es im Englischen korrekt wäre. In diesem Fall würde der Fragesatz im Deutschen lauten: „Hast du schon Pläne für deinen Urlaub im nächsten Jahr gemacht?“ Im Englischen hingegen wird das simple present zur Verdeutlichung der beabsichtigten Aussage nur in der Umgangssprache verwendet, während sich in der normgerechten Sprachform Fragesätze, die mit der

608 PwensmAb

609 Wie beim zuvorgenannten Beispiel beruht auch hier das Vertauschen der Lexeme auf einem Verstoß gegen das englische Zeitensystem, so daß die Äußerung auch ein Beispiel für den normabweichenden Gebrauch der Zeitform simple present darstellt. 
Verbform $d o$ eingeleitet werden, auf permanent andauernde Handlungen beziehen. Weiß der Fragende jedoch nicht, ob die Handlung, auf die sich die Frage bezieht, schon eingetreten ist oder nicht, so wird im Englischen in der Regel das present perfect verwendet, so daß der Fragesatz mit dem Hilfsverb have beginnt. Da der Lernende die Differenzierungsmöglichkeiten des Deutschen auch im Englischen einzusetzen versuchte, ist die Abweichung als Überdifferenzierung zu betrachten.

Schließlich ist der Fehler auch als erstsprachlich bedingte Übergeneralisierung zu werten, weil hier eine Regel der englischen Sprache, d. h. die Bildung des Fragesatzes mit dem Hilfsverb do, die unter bestimmten Bedingungen als normgerecht zu bezeichnen ist, auf sprachliche Kontexte übertragen wurde, die andere Satzkonstruktionen erfordern. Ausgelöst wurde die Fehlleistung dabei durch die zuvor erwähnte Tatsache, daß die Aussage im Deutschen auf unterschiedliche Art und Weise verdeutlicht werden kann, während das Englische über diese Flexibilität nicht verfügt.

Insgesamt betrachtet zeigen die Ergebnisse, die bei Anwendung der Kontrastivhypothese ermittelt wurden, daß die Art und Weise, in der erstsprachlicher Einfluß an der Fehlerentstehung beteiligt war, bei den PmeismAb und den PwensmAb übereinstimmt. In beiden Gruppen dominiert das Ersetzen zielsprachlicher Strukturen durch Strukturen der Erstsprache. Die große Anzahl der Hyperkorrektheiten in der Gruppe der PmeismAb und die große Anzahl der Über- und Unterdifferenzierungen sowie Übergeneralisierungen in der entgegengesetzten Probandengruppe zeigen in diesem Zusammenhang, daß die PmeismAb den Einfluß der Erstsprache bewußter wahrnehmen als die PwensmAb. Während erstere darum bemüht sind, möglichst viele Strukturen miteinander zu kombinieren und zu diesem Zweck sowohl erstsprachliche als auch zielsprachliche Elemente verwenden, ist das Bewußtsein für sprachliche Differenzen bei den PwensmAb weit schwächer ausgeprägt. Vielmehr führen die Ergebnisse zur Annahme, daß mit zunehmenden syntaktisch-morphologischen Kenntnissen das Vermögen, zwischen erst- und zielsprachlichen Strukturen sowie den Strukturen innerhalb der Zielsprache zu unterscheiden, abnimmt bzw. das Bemühen, zwischen den einzelnen Strukturen zu differenzieren, schwächer ist als in früheren Erwerbsphasen.

Auch beim Versuch, die Entstehung der Wort- und Satzstellungsfehler mit Hilfe der Faktoren der Identitätshypothese zu begründen, sind im wesentlichen zunächst Übereinstimmungen zu erkennen. Entwicklungsspezifisch betrachtet sind in beiden Probandengruppen die meisten Abweichungen der Kategorie misorderings zuzuordnen (vgl. Tabellen 22 und 23 im Anhang), weil die Mehrzahl der Abweichungen nicht auf die strukturelle Veränderung einzelner Lexeme, sondern auf grundsätzliche Verstöße gegen die Wortstellungsregeln der englischen Sprache zurückzuführen ist. Die Verstöße hatten dabei nicht immer eine morphologische Veränderung der Lexeme zur Folge, wenngleich bei den meisten Abweichungen das Vertauschen einzelner Lexeme auch strukturelle Auswirkungen auf die Elemente hat, die durch das Vertauschen unmittelbar betroffen waren. Den Thesen der Identitäts- 
hypothese zufolge gehört die normabweichende Anordnung der einzelnen Satzelemente zu den häufigsten Erscheinungen des Erwerbs natürlicher Sprachen und ist sowohl in natürlichen als auch in unterrichtlich gesteuerten Erwerbssituationen zu beobachten. Ein typisches Beispiel für das Vertauschen von Satzelementen, das sowohl bei Kindern, die Englisch als Erstsprache erwerben, ${ }^{610}$ als auch bei Englischlernenden, die bereits über eine andere Erstsprache verfügen, beobachtet werden kann, bietet folgende Abweichung:

Fehler: $\quad$ Last year I go with my letter-friend on holiday in Turkey. ${ }^{611}$

Korrektur: Last year I went on holiday with my pen-friend to Turkey.

Bei diesem Fehlerbeispiel handelt es sich um eine Abweichung der Kategorie misorderings, die mit einer strukturellen Veränderung der vertauschten Lexeme einhergeht. Die Redewendung go on holiday bildet eine zusammenhängende syntaktische Einheit, die der Norm des Englischen entsprechend nicht durch andere Lexeme unterbrochen wird. Der Einschub with my letter-friend widerspricht daher den Regeln der syntaktischen Anordnung dieser Verbalphrase. Der Gebrauch der Verbform go stellt dabei zusätzlich einen Verstoß gegen das englische Zeitensystem dar. Die Gemeinsamkeit zwischen obengenannter Äußerung und den Fehlern englischsprachiger Kinder besteht darin, daß die Fehlleistung neben dem Einfluß erstsprachlicher Faktoren auch dadurch entstanden sein könnte, daß der Lernende die Anordnung der Lexeme nach semantischen Schwerpunkten festgelegt hat. Die Abweichung könnte demnach u. a. darauf basieren, daß der Lernende die Lexeme nach der Wichtigkeit ihrer semantischen Informationen geordnet hat.

Ein ähnliches Verhalten ist auch bei Kindern in frühen Erwerbsstadien zu beobachten. Studien zum Erstspracherwerb haben gezeigt, daß Kinder zum einen in der Regel nur die Elemente benutzen, die sie zur Vermittlung ihrer sprachlichen Botschaft benötigen, und zum anderen bei der syntaktischen Anordnung die Lexeme zuerst verwenden, die ihnen semantisch wichtiger erscheinen als andere. ${ }^{612}$ Dieses Verhalten gehört zu den Theoriebildungen, die einen entscheidenden Faktor des Erstspracherwerbs darstellen: Kinder überprüfen während des Erwerbs ihrer Erstsprache, ähnlich wie Lernende beim Zweitspracherwerb, ständig die semantische und grammatische Bedeutung der einzelnen Satzelemente und selektieren die sprachlichen Informationen, die an sie herangetragen werden. ${ }^{613}$ Alle semantischen und grammatischen Regeln, die das Kind innerhalb des Entwicklungsstadiums, in dem es sich befindet, nicht zwingend zur sprachlichen Verständigung benötigt, gelangen erst in einem späteren Entwicklungsstadium in den Sprachspeicher. ${ }^{614}$ Die sprachlichen Elemente, die für das Kind

\footnotetext{
610 vgl. Szagun 2000, $25-28$

611 PmeismAb

${ }^{612}$ vgl. Dulay / Burt / Krashen 1982, 162

613 vgl. Szagun 2000, $99-129$

614 vgl. ebd.
} 
von kommunikativer Bedeutung sind und daher verinnerlicht werden, unterliegen bei ihrer Anwendung einer hierarchischen Struktur ${ }^{615}$ : Bei der syntaktischen Anordnung werden - wie zuvor erwähnt - zuerst die Elemente verwendet, die die Hauptinformationen der beabsichtigten sprachlichen Botschaft enthalten.

Die Vermutung, daß der Wortstellungsfehler des Lernenden unter anderem auf entwicklungsbedingtes Verhalten zurückzuführen ist, wird durch die Verwendung der Verbform go noch verstärkt, da diese als ein typisches Merkmal kindlichen Reduktionsverhaltens betrachtet werden kann. Auch bei Kindern ist häufig zu beobachten, daß statt einer Vergangenheitsform die Grundform des Verbs benutzt wird, weil die Kinder erfahren haben, daß ihre sprachliche Aussage trotz dieser grammatischen Einschränkung in der Regel verstanden wird. ${ }^{616}$

Auch bei den PwensmAb lassen die Abweichungen, die der Kategorie misorderings zuzuordnen sind, Parallelen zu Abweichungen erkennen, die beim Erstspracherwerb von Kindern zu beobachten sind. Dies verdeutlicht folgendes Beispiel:

Fehler: $\quad$ So, I often can be relaxed. ${ }^{617}$

Korrektur: So, I can often be relaxed.

Linguistisch betrachtet besteht die Fehlleistung darin, daß sogenannte adverbs of frequency ${ }^{618}$ der Norm entsprechend den Hilfsverben nachgestellt und nicht vor diesen eingesetzt werden. Wie die Untersuchungen von Dulay/Burt/Krashen ${ }^{619}$ gezeigt haben, sind Abweichungen dieser oder ähnlicher Art nicht nur bei Kindern während des Erstspracherwerbs zu beobachten, sondern auch bei Erwachsenen, die Englisch unter natürlichen Bedingungen in einem englischsprachigen Land erwerben. In diesem Zusammenhang wurde auch deutlich, daß das Vertauschen von adverbialen Bestimmungen der Zeit bei Englischlernenden, die unterschiedliche Erstsprachen erworben haben, nachgewiesen werden konnte. ${ }^{620}$ Diese Feststellung unterstützt die Theorie der Identitätshypothese, daß der Erwerb der syntaktischen Struktur einer Sprache an biologisch festgelegte Prozesse gebunden ist, die bei allen Lernenden - einschließlich der dabei auftretenden Fehler - identisch verlaufen.

In bezug auf die Erklärungsfaktoren der Identitätshypothese fällt darüber hinaus auf, daß die Entstehung der Wort- und Satzstellungsfehler entwicklungsspezifisch betrachtet auch auf den Gebrauch

\footnotetext{
615 vgl. ebd.

${ }^{616}$ vgl. Dulay / Burt / Krashen 1982, $165-170$

617 PwensmAb

618 vgl. Thomson / Martinet 1987, $56-58$

619 vgl. Dulay / Burt / Krashen 1982, 162

620 vgl. ebd.
} 
sogenannter archi-forms und auf den als alternating forms beschriebenen Vorgang zurückgeführt werden kann (vgl. Tabellen 22 und 23 im Anhang). Der Anteil dieser Entstehungsursachen ist im Vergleich zu der zuvor behandelten Fehlerkategorie misorderings jedoch in beiden Gruppen gering, wobei sie bei den PmeismAb etwas deutlicher zum Tragen kommen als in der entgegengesetzten Probandengruppe. Ein typisches Beispiel für den Gebrauch von archi-forms bei ersteren stellt folgender Normverstoß dar:

Fehler: In the summer holiday I have time then I came to you. ${ }^{621}$

Korrektur: If I have time during the summer holiday I hope I can come and visit you.

In der entgegengesetzten Gruppe äußerten sich archi-forms etwa in folgender Form:

Fehler: $\quad$ At the office I prepare the yearly calculation of the financial contribution we have to pay this year to Brussels. ${ }^{622}$

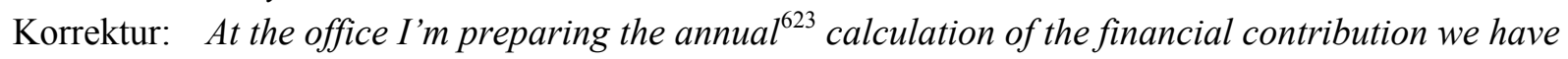
to pay to Brussels this year.

Die Gegenüberstellung der Fehlleistungen zeigt, daß die Fehler der Kategorie archi-forms bei den PmeismAb wesentlich schwerwiegender sind als in der entgegengesetzten Gruppe. Dennoch sind beide Abweichungen als archi-forms zu bezeichnen, weil in beiden Fehlerbeispielen die Elementvertauschungen darauf zurückzuführen sind, daß die Probanden elementare syntaktische Strukturen verwendeten, im jeweiligen Zusammenhang jedoch komplexere Formen erforderlich gewesen wären. Im ersten Beispiel resultiert die Verwendung der archi-form in einem Satzstellungsfehler, weil der Lernende zur Beschreibung eines möglicherweise eintretenden Ereignisses die syntaktische Struktur eines einfachen Aussagesatzes benutzt hat. Da das Eintreten des Ereignisses, das der Lernende in seiner Äußerung ankündigt, an eine Bedingung gebunden ist, wurde von allen Korrektoren einstimmig bestätigt, daß ein Konditionalsatz zu erwarten gewesen wäre. Wie die Struktur des Fehlerbeispiels zeigt, stimmt die Lexemanordnung jedoch nicht mit der eines Bedingungssatzes überein, so daß der Satz einer Korrektur der Wortstellung bedarf. Auch als Aussagesatz enthält die Äußerung zwei Fehler: Zum ersten müßte die Aussage korrekterweise in zwei Sätze unterteilt werden, d. h., nach dem Lexem time wäre ein neuer Satzanfang zu erwarten. Hinzu kommt, daß die Verbform came im gegebenen Kontext eine Normabweichung bildet. Der Lernende hätte statt des simple past das will-future benutzen sollen bzw. das simple present, wenn der Satz als umgangssprachliche Äußerung zu interpretieren ist.

${ }^{621}$ PmeismAb

${ }^{622}$ PwensmAb

${ }^{623}$ Wenngleich ein Teil der Korrektoren das Wort yearly nicht als unpassend bezeichnet hat, stellte die Mehrheit der Korrektoren fest, daß das Wort annual im gegebenen Kontext angemessener erscheint, da das Wort sich auf einen bürokratischen Vorgang bezieht. 
Im zweiten Beispiel äußert sich die Anwendung der archi-form darin, daß das Lexem prepare nicht in der Verlaufsform benutzt wird, sondern in der simple form. Da der Lernende jedoch ein momentan stattfindendes Ereignis beschreibt und nicht eine gewohnheitsmäßige Handlung, ist die simple form im gegebenen Kontext als normabweichend zu betrachten.

Der als alternating forms bezeichnete Fehlertyp äußert sich bei den PmeismAb u. a. in folgender Form:

Fehler: $\quad$ A man had took my bag.

Korrektur: A man had taken my bag.

Bei den PwensmAb war u. a. folgender Fehler, der auf dieser Abweichungsform basiert, zu beobachten:

Fehler: $\quad$ The police hasn't find my car.

Korrektur: The police haven't found my car.

Im ersten Beispiel ist der Normverstoß linguistisch betrachtet auf den falschen Gebrauch der Partizipform des Verbs take zurückzuführen. Gleiches trifft auf die Verbform find im zweiten Fehlerbeispiel zu. Dieses enthält jedoch darüber hinaus eine zweite Abweichung, die darauf basiert, daß das Wort police im Englischen als Plurallexem zu betrachten ist. Verben, die sich auf dieses Lexem beziehen, werden daher in der pluralen Konjugationsform benutzt. Dieser Verstoß ist als Fehler der Kategorie archi-forms zu betrachten, während der falsche Gebrauch der Partizipform ein typisches Beispiel der Fehlerkategorie alternating forms darstellt. Der Vergleich der Fehlerbeispiele zeigt, daß die Fehler dieser Kategorie in beiden Probandengruppen nahezu identisch sind. Während die Fehler der Gruppe archi-forms bei den PmeismAb schwerwiegender waren als in der entgegengesetzten Probandengruppe, sind diese Unterschiede bei den alternating forms kaum zu erkennen. Die einzige Auffälligkeit besteht darin, daß sich die Fehler dieser Kategorie bei den PmeismAb auf den Gebrauch der reinen Vergangenheitstempora konzentrieren, während sie bei den Probanden der entgegengesetzten Gruppe häufig beim Gebrauch des present perfect zu beobachten waren. Zahlreiche Studien zum Erstund zum Zweitspracherwerb haben ergeben, daß sowohl Fehler der Kategorie archi-forms als auch sogenannte alternating forms in nahezu identischer Form bei Kindern, die Englisch als Erstsprache erwerben und bei Lernenden, die Englisch unter unterrichtlich gesteuerten Bedingungen erwerben, zu beobachten sind. ${ }^{624}$ Diese Übereinstimmungen waren auch dann zu erkennen, wenn letztere unterschiedliche Erstsprachen besaßen.

Weitere Parallelen zwischen den Fehlern der zuvor genannten Kategorien, die bei deutschen Englischlernenden in unterrichtlichen Erwerbssituationen zu erkennen sind, und den Fehlern von Kindern oder

${ }^{624}$ vgl. Dulay / Burt / Krashen 1982, 165 ff 
Erwachsenen, die Englisch in natürlichen Situationen erwerben, äußern sich auch beim Gebrauch der Verneinungen, wie etwa in folgendem Beispiel:

Fehler: I no have it. ${ }^{625}$

Korrektur: I don't have it.

In zahlreichen Untersuchungen wurde deutlich, daß die genannten Personengruppen häufig Negierungen bilden, ohne das Lexem do zu verwenden und stattdessen ausschließlich die Lexeme no oder not benutzen. ${ }^{626}$ Dabei kann die Position dieser Lexeme variieren, d. h., die Wörter werden zum Teil auch am Satzanfang oder am Satzende benutzt. ${ }^{627}$

Ein weiterer Fehlertyp der Identitätshypothese, Abweichungen der Kategorie omissions, kommt in bezug auf die Wortstellungsfehler bei den PmeismAb zum Tragen. Ein Beispiel für diesen Fehlertyp bietet folgende Äußerung:

Fehler: In two weeks I finished school.

Korrektur: In two weeks school will have finished.

Die Fehlleistung ist darauf zurückzuführen, daß der Lernende auf ein zukünftiges Ereignis verweist, jedoch das dem Satz zugrundeliegende Verb im simple past benutzt. Auch hier hat der Vergleich mit Studien, die zur Überprüfung entwicklungsbedingter Fehler durchgeführt wurden, gezeigt, daß Fehler dieser Kategorie ebenfalls in frühen Stadien des natürlichen Spracherwerbs zu beobachten sind. ${ }^{628}$ Der Identitätshypothese zufolge basieren auch diese Fehlleistungen auf entwicklungsbedingten Selektionsprozessen. Diese dienen den Lernenden dazu, in den Anfangsstadien des Erst- und Zweitspracherwerbs - wie erwähnt - die Vielzahl der Informationen, die an sie herangetragen werden, beim aktiven Sprachgebrauch auf solche Elemente zu beschränken, die besonders wichtig für die Vermittlung der beabsichtigten Aussage sind.

Zusammenfassend betrachtet zeigen die Ergebnisse, die bei Anwendung der Fehlerentstehungsfaktoren der Identitätshypothese ermittelt wurden, daß die Art und Weise, in der entwicklungsbedingte Faktoren an der Entstehung von Wort- und Satzstellungsfehlern beteiligt sind, nur partiell an den syntaktischmorphologischen Wissensstand der Lernenden gebunden ist. Übereinstimmend ist sowohl bei den PmeismAb als auch bei den PwensmAb zu erkennen, daß die Wort- und Satzstellungsfehler unter anderem auf das entwicklungsbedingte Vertauschen von Satzelementen und/oder auf morphologische Veränderungen von einzelnen Lexemen zurückzuführen sind. Bei den PmeismAb kommt bei der Ent-

\footnotetext{
${ }^{625}$ PmeismAb

${ }^{626}$ vgl. Dulay / Burt / Krashen 1982, 179

627 vgl. ebd.

${ }^{628}$ Siehe dazu auch Dulay / Burt / Krashen 1982, 165 ff.
} 
stehung der Wort- und Satzstellungsfehler darüber hinaus als weiterer entwicklungsbedingter Vorgang die Fehlerkategorie omissions zum Tragen: das Auslassen ganzer syntaktischer Einheiten. Die Tatsache, daß es sich hierbei um eine Erscheinung handelt, die beim natürlichen Spracherwerb in der Regel nur in sehr frühen Erwerbsphasen zu beobachten ist, während Fehler der Gruppen archi-forms und alternating forms auch in späteren Erwerbsphasen auftreten können, läßt darauf schließen, daß das Spektrum entwicklungsbedingter Faktoren bei Anfängern größer ist als bei fortgeschrittenen Lernenden.

Neben diesen Ergebnissen hat die Anwendung der Erklärungsfaktoren der Interlanguagehypothese auf die Entstehung der Wort- und Satzstellungsfehler zu folgenden Erkenntnissen geführt: Bereits bei erster Betrachtung der Zuordnungsergebnisse ist festzustellen, daß sich die Anwendungsmöglichkeiten dieser Hypothese im Gegensatz zu den Abweichungen auf lexikalischer Ebene nicht auf wenige Faktoren konzentrieren, sondern eine größere Verteilung erkennen lassen (vgl. Tabellen 24 und 25 im Anhang). Dies trifft insbesondere auf die Gruppe der PmeismAb zu. Dabei zeigen die Zuordnungsergebnisse, daß die Entstehung der Wort- und Satzstellungsfehler aus der Sicht der Interlanguagehypothese sowohl auf unterrichtlich bedingte Faktoren zurückzuführen ist als auch auf Lern- und Kommunikationsstrategien und unbewußt ablaufende overgeneralizations bei der Sprachanwendung. Darüber hinaus ist bei den PmeismAb auch lack of contrast zwischen erst- und zielsprachlichen Elementen bzw. zwischen einzelnen zielsprachlichen Strukturen für das Zustandekommen der Abweichungen verantwortlich. Unterrichtlich bedingte Faktoren kommen in dieser Probandengruppe stärker zum Tragen als bei den PwensmAb, während der Einfluß von Lern- und Reduktionsstrategien und productive achievement strategies bei letzteren deutlicher zu erkennen ist. Auch der Fehlerentstehungsanteil, der auf unbewußt ablaufende Prozesse zurückzuführen ist, ist bei den PwensmAb wesentlich größer als in der entgegengesetzten Probandengruppe.

Somit ist festzustellen, daß die Entstehung der Wort- und Satzstellungsfehler bei den PmeismAb öfter als in der entgegengesetzten Probandengruppe durch äußere Einflüsse hervorgerufen wurde, d. h. durch Faktoren, die nicht aus dem sprachlichen Verhalten der Probanden selber resultierten. Die Lernenden können diese Aspekte - wie etwa die Gestaltung des Unterrichts - nur partiell beeinflussen. Die PwensmAb waren gegen den Einfluß äußerlicher Faktoren resistenter. In dieser Gruppe stehen Fehlerentstehungsfaktoren, die aus dem sprachlichen Lern- und Anwendungsverhalten der Probanden resultierten, stärker im Vordergrund. Hingegen ist in beiden Probandengruppen übereinstimmend zu erkennen, daß aus der Reihe der Reduktionsstrategien besonders häufig die als modality reduction bezeichnete Strategie zur Anwendung kam (vgl. Tabellen 24 und 25 im Anhang). Gleiches gilt für die Kategorien interlingual transfer, inter-/intralingual transfer und paraphrase. Zudem fällt in beiden Gruppen auf, daß das Erklärungspotential der Kommunikationsstrategien größer ist als das der anderen Fehlerentstehungsfaktoren, wenngleich bei den PwensmAb die Differenz zwischen dem 
Fehlerentstehungsanteil der reduction strategies und productive achievement strategies und den unbewußten overgeneralizations als gering zu bezeichnen ist.

Aufgrund der zuvor genannten fehlergenetischen Gemeinsamkeiten ist zu konstatieren, daß in beiden Probandengruppen die Mehrzahl der Fehler auf dem Versuch basiert, Kommunikationsdefizite durch den Rückgriff auf bekannte Strukturen der Zielsprache, anderer Zweitsprachen oder der Erstsprache auszugleichen. Darüber hinaus ist in beiden Gruppen auch die Reduzierung sprachlicher Elemente beim Erlernen und bei der Anwendung der Zielsprache für die Entstehung der Abweichungen verantwortlich (vgl. Tabellen 24 und 25 im Anhang). Der Einfluß beider Faktoren zeigt sich in folgenden Beispielen:

Fehler: I was last summer together with you in Italy. ${ }^{629}$

Korrektur: Last summer we went to Italy together.

Fehler: $\quad$ I believe a visit to us is a good opportunity to shorten the way to Italy where your brother is living. ${ }^{630}$

Korrektur: I believe a visit to us would be a good way of shortening the trip to Italy where your brother lives.

Linguistisch betrachtet beruht die Abweichung im ersten Beispiel darauf, daß konkrete Zeitangaben

- wie etwa Uhrzeiten oder Jahresangaben - im Englischen entweder am Satzanfang oder am Satzende stehen. Im Fehlerbeispiel befindet sich die Zeitangabe jedoch in der Mitte des Satzes. Zusätzlich basiert der Fehler auch auf einer stilistischen Abweichung, die durch den Gebrauch des Hilfsverbs was verursacht wurde. Da die Reise nach Italien, die als Kernaussage des Satzes zu betrachten ist, einen aktiven Vorgang bezeichnet, wäre statt einer Form des statischen Verbs to be ein Verb der Bewegung - wie etwa travel oder go - zu erwarten gewesen. Dieses Verb ist dabei syntaktisch unmittelbar mit seinem Bezugswort verknüpft, so daß die Verbalphrase im Englischen go to Italy lauten müßte.

Die obengenannten Faktoren der Interlanguagehypothese werden im ersten Fehlerbeispiel an folgenden Punkten deutlich: Der Einfluß unterrichtlich bedingter Faktoren, die zur Entstehung der Wortwahlfehler beigetragen haben könnten, äußert sich im ersten Beispiel im Gebrauch des Hilfsverbs have. Möglicherweise wurden die Anwendung des Hilfsverbs be und die simple past form dieses Lexems im Englischunterricht überbetont und dadurch andere Lexeme vorübergehend verdrängt. Die syntaktische Stellung des Ausdrucks last summer und des Adverbs together läßt darüber hinaus vermuten, daß die Differenzen zwischen der Position adverbialer Bestimmungen in den Sprachen Deutsch und Englisch im Unterricht nicht deutlich genug hervorgehoben wurden. Dies könnte dazu geführt haben, daß der Lernende in seiner englischen Äußerung Satzbauformen seiner Erstsprache oder anderer Zweit- 
sprachen verwendete. Auch die Annahme, daß die Wortstellungsfehler auf einer Lernstrategie, der sogenannten simplification, basieren, ${ }^{631}$ läßt sich anhand der Verbform was verdeutlichen. Es ist möglich, daß sich der Lernende bei dem Versuch, die Strukturen der englischen Sprache zu verinnerlichen, zunächst auf bestimmte Lexeme und deren Verbformen konzentriert hat, um dadurch den Lernprozeß übersichtlicher zu gestalten. In diesem Fall hätte er sich während des Lernvorgangs zunächst auf elementare Worte wie das Lexem be beschränkt, so daß ihm zur Verdeutlichung seiner sprachlichen Aussage keine anderen Lexeme zur Verfügung standen und er zwangsläufig auf die Verbform was zurückgreifen mußte. Der Gebrauch dieser Verbform spricht jedoch auch dafür, daß die Wortstellungsfehler zusätzlich auf einer Reduktionsstrategie basieren, weil - wie zuvor erwähnt sowohl das Wort be als auch die Verbform was zu den elementarsten Lexemen der englischen Sprache gehören. Mittels ihrer Verwendung vermied der Lernende somit den Gebrauch stilistisch anspruchsvollerer Verben.

Der Einfluß sogenannter productive achievement strategies, der sich in Form von inter-/intralingual transfer äußert, zeigt sich im ersten Fehlerbeispiel zum einen darin, daß der Lernende bei der Anordnung der Satzelemente Strukturen der deutschen Sprache zugrunde gelegt hat. Dies wird daran deutlich, daß die syntaktische Struktur des englischen Satzes Wort für Wort der deutschen Vorlage „Ich war letztes Jahr zusammen mit dir in Italien“ entspricht. Auch die Tatsache, daß der Lernende das Hilfsverb to be und die entsprechende Verbform was benutzte, ist auf den Einfluß der Erstsprache Deutsch zurückzuführen. Die Verbform basiert, wie erwähnt, eindeutig auf der 3. Person Singular Imperfektform des deutschen Hilfsverbs ,sein‘. Intralingual transfer, d. h. Interferenzen, die auf innersprachliche Strukturen des Englischen zurückzuführen sind, werden vor allem an der Position des Wortes together deutlich. Im Englischen ist es durchaus möglich, das Lexem together vor der Phrase with you zu benutzen. Allerdings entspricht diese Lexemstellung der Norm der englischen Sprache nur dann, wenn dem Personalpronomen you ein weiterer Nebensatz folgt und nicht - wie im Fehlerbeispiel - ein Objekt mit vorangestellter Präposition. Es ist somit möglich, daß der Lernende glaubte, die prinzipiell korrekte Lexemkombination together with you auch in der Satzkonstruktion benutzen zu können, mit der er seine Aussage verdeutlichen wollte. Da dieser Vorgang gleichzeitig als eine Verallgemeinerung zielsprachlicher Elemente interpretiert werden kann, ist die Fehlleistung auch als Generalisierung einzustufen. Diese kann auch unbewußt verlaufen sein, weil der Lernende das Lexem together in seinen Ausführungen nicht nur normabweichend, sondern an anderer Stelle auch normgerecht verwendet hat.

Darüber hinaus ist der Fehler vermutlich auch auf lack of contrast zurückzuführen, denn die Phrase together with you ähnelt dem deutschen Satzteil ,zusammen mit Dir', der dem Lernenden wahr-

${ }^{631}$ vgl. Kap. II 3.3.2 der vorliegenden Arbeit: Fehlererklärungsfaktoren der Interlanguagehypothese 
scheinlich als Vorlage gedient hat. Diese Ähnlichkeit kann dazu beigetragen haben, daß der Lernende die zuvor genannten syntaktischen Beschränkungen, die der englischen Phrase zugrunde liegen, nicht beachtet hat.

Auch die Wortstellungsfehler, die in der Äußerung I believe a visit to us is a good opportunity to shorten the way to Italy where your brother is living enthalten sind, basieren auf mehreren Faktoren. Zum einen hätte der Lernende statt des Hilfsverbs is das Lexem would benutzen sollen, da er in seiner Äußerung einen Vorschlag unterbreitet und daher eine Konditionalform zu verwenden gewesen wäre. Zum anderen ist das Wort opportunity im gegebenen Kontext stilistisch unpassend, da der Lernende offensichtlich das deutsche Wort ,Möglichkeit‘ ins Englische zu übertragen suchte. Bei der Übersetzung des Satzes ins Englische ist jedoch - wie im Korrekturbeispiel - das Wort way zu benutzen. Letzteres wird in der Regel in Verbindung mit der Präposition of verwendet, so daß das unmittelbar folgende Verb im Gerundium stehen muß. Der Lernende benutzte jedoch den Infinitiv. Die Wortstellungsfehler sind somit auf eine Kombination syntaktischer und semantischer Abweichungen zurückzuführen.

Der Einfluß unterrichtlich bedingter Faktoren äußert sich hier vor allem im Gebrauch des progressive aspects in der Lexemkombination is living. Die progressive form ist hier offensichtlich darauf zurückzuführen, daß diese Form im Englischunterricht des Lernenden überbetont wurde und er daher nicht mehr zwischen dem Gebrauch der simple und der progressive form unterscheiden konnte. Außerdem ist die Abweichung möglicherweise als Folge einer Lernstrategie zu interpretieren, weil der Lernende eventuell bewußt darauf verzichtete, zwischen dem Gebrauch der simple und der progressive form zu unterscheiden. Dabei könnte er den Schwerpunkt auf den Gebrauch der progressive form gelegt haben, weil diese Form von Englischlernenden oft als charakteristisches Merkmal der englischen Sprache betrachtet wird.

Der Einfluß von Reduktionsstrategien äußert sich vor allem darin, daß der Lernende im ersten Teil seiner Aussage statt des Gerundiums die Infinitivform to shorten benutzte. Damit griff er auf eine elementare Struktur des Englischen zurück und vermied somit den Gebrauch einer komplexeren Struktur. Der Gebrauch des Infinitivs weist gleichzeitig darauf hin, daß die Fehlleistung auch auf interlingualem Transfer basiert, weil im Deutschen im gegebenen sprachlichen Kontext der Infinitiv des Verbs ,kürzen` durchaus als normgerecht zu bezeichnen ist. Intralingual transfer zeigt sich in der Tatsache, daß im Englischen Infinitivformen generell häufiger zur Anwendung kommen als Gerundialkonstruktionen. In der Äußerung des Lernenden wäre jedoch aufgrund der Präposition of, die dem zu verwendenden Lexem way hätte folgen sollen, das Gerundium zu benutzen gewesen. Die häufigen Anwendungsmöglichkeiten des Infinitivs im Englischen sprechen darüber hinaus dafür, den Fehler zusätzlich als Ergebnis einer unbewußten overgeneralization einzustufen, weil der Lernende in seinen 
Ausführungen Gerundial- und Infinitivstrukturen teils normabweichend und teils normgerecht verwendete. Die Umschreibung des eigentlich zu erwartenden Ausdrucks a good way of shortening zeigt schließlich, daß der Fehler auch der Kategorie paraphrase zugeordnet werden kann.

\subsection{Simple Past}

In der Fehlerkategorie simple past ist in bezug auf die Anwendbarkeit der Erwerbshypothesen festzustellen, daß in beiden Probandengruppen ähnliche Faktoren zur Entstehung der Abweichungen beigetragen haben (vgl. Tabellen 18 und 19 im Anhang), wenngleich sich die Art der Fehler - wie erwähnt - fehlertypologisch unterscheidet. Während bei den PmeismAb nahezu ausschließlich morphologische Verbveränderungen zu den Abweichungen führten, entstanden die Verstöße in der Gruppe der PwensmAb dadurch, daß das simple past mit dem present perfect verwechselt wurde.

Bei Betrachtung der einzelnen Erwerbshypothesen ist die Anzahl der Einträge im Bereich der Identitätsund der Interlanguagehypothese in beiden Probandengruppen identisch (vgl. Tabellen 18 und 19 im Anhang). Somit ist das Erklärungspotential beider Hypothesen gleichbedeutend. Der Einfluß der Faktoren der Kontrastivhypothese kommt in der Gruppe der PmeismAb weniger zum Tragen als der Einfluß entwicklungs- und interlanguagespezifischer Faktoren, während erstsprachlich bedingte Faktoren bei den PwensmAb als wichtigste Fehlerentstehungsursachen zu betrachten sind. Dabei ist jedoch in dieser Gruppe die Differenz zwischen den Anwendungsmöglichkeiten der Kontrastivhypothese und der Identitäts- und der Interlanguagehypothese gering.

Bei Betrachtung der Unterkategorien der einzelnen Erwerbshypothesen fällt auf, daß die Fehler sowohl bei den PmeismAb als auch bei den PwensmAb den Erklärungsfaktoren der Kontrastivhypothese entsprechend auf Substitutionen, Über- bzw. Unterdifferenzierungen und Übergeneralisierungen zurückgehen (vgl. Tabellen 20 und 21 im Anhang). Auch bei Anwendung der Identitätshypothese sind Übereinstimmungen zu erkennen: In beiden Probandengruppen äußert sich der Einfluß entwicklungsbedingter Faktoren darin, daß die Teilnehmer sogenannte archi-forms benutzten oder auf Sprachformen zurückgriffen, die der Kategorie alternating forms zuzuordnen sind (vgl. Tabellen 22 und 23 im Anhang). Auch sind in beiden Gruppen Abweichungen auf das redundante Hinzufügen sprachlicher Elemente - addition errors - und auf den als regularization bezeichneten Vorgang zurückzuführen.

Nahezu deckungsgleiche Ergebnisse zeigte auch der Versuch, die Fehlleistungen mit Hilfe der Interlanguagehypothese zu begründen. In beiden Probandengruppen sind die Fehler durch unterrichtliche Faktoren, Lernstrategien, bewußt eingesetzte Kommunikationsstrategien und unbewußte overgenera- 
lizations hervorgerufen worden (vgl. Tabellen 24 und 25 im Anhang). Darüber hinaus war in beiden Gruppen auch lack of contrast an der Fehlerentstehung beteiligt.

Das Zusammenwirken der unterschiedlichen Fehlerentstehungsfaktoren ist an folgenden Fehlerbeispielen zu belegen:

Fehler: $\quad$ A young man had stolen my bike last week. ${ }^{632}$

Korrektur: A young man stole my bike last week.

Fehler: $\quad$ Last Monday I was at the travel agency and the travel agent gived me some information about France. ${ }^{63}$

Korrektur: Last Monday I was at the travel agency and the travel agent gave me some information about France.

Fehler: $\quad$ Last weekend someone stole my bike. We have had a wonderful ride along the river Rhine. ${ }^{634}$

Korrektur: Last weekend someone stole my bike. We had a wonderful ride along the river Rhine.

Das Substituieren zielsprachlicher Elemente durch erstsprachliche äußert sich deutlich im ersten und im dritten Fehlerbeispiel, stellt vermutlich jedoch auch im zweiten Beispiel eine Fehlerursache dar. Im ersten Beispiel ist dieser Fehlertyp eindeutig am Gebrauch der Verbform had stolen zu erkennen, wobei davon auszugehen ist, daß der Fehler auf der deutschen Verbform , hat gestohlen` basiert. Diese Übertragung ist darauf zurückzuführen, daß, wie bereits zuvor erwähnt, das Perfekt im gegebenen sprachlichen Kontext im Deutschen, insbesondere in der Umgangssprache, nicht als normabweichende Zeitform gilt, während im Englischen im Zusammenhang mit der Zeitangabe last week das Imperfekt, d. h. das simple past zu verwenden gewesen wäre. Ähnliches wird im dritten Fehlerbeispiel deutlich. Hier ist die Fehlerkategorie ,Substitution“ an der gewählten Formulierung have had zu erkennen, deren Zeitform offenbar auf die deutsche Perfektform ,habe gemacht“ zurückzuführen ist: Vermutlich hat hier die deutsche Phrase ,haben eine Fahrradtour gemacht' als Vorlage gedient. Auch in diesem Satz wäre aufgrund der Zeitangabe last weekend korrekterweise das simple past zu benutzen gewesen. Im zweiten Beispiel ist das Substituieren zielsprachlicher Strukturen nur ansatzweise zu erkennen. Es ist zu vermuten, daß der Lernende statt der Verbform gave die normabweichende Form give deshalb benutzte, weil die Übersetzung auf dem deutschen Lexem, gab' basierte und das Fehlerelement gived aufgrund des Endkonsonanten - $d$ eine größere akustische Ähnlichkeit zur deutschen Vorlage aufweist als das Lexem gave.

Auch Über- bzw. Unterdifferenzierungen und erstsprachlich bedingte Übergeneralisierungen sind in allen Beispielen zu belegen. Im ersten Fehlerbeispiel hat der Lernende zum einen nicht zwischen den

\footnotetext{
${ }^{632}$ PmeismAb

${ }^{633}$ PwensmAb

634 dto.
} 
Anwendungsmöglichkeiten des Perfekts im Englischen und Deutschen unterschieden. Daher ist der Fehler als Unterdifferenzierung zu bezeichnen. Zum anderen wurde hier bedingt durch erstsprachliche Strukturen eine Zeitform des Englischen in einem Zusammenhang benutzt, in dem eine andere Zeitform zu verwenden gewesen wäre, so daß die Fehlleistung auch eine Übergeneralisierung darstellt.

Ähnliches gilt für das zweite Fehlerbeispiel. Auch hier ist die Fehlerbezeichnung ,Unterdifferenzierung“ zutreffend, weil der Lernende nicht zwischen dem Gebrauch starker und schwacher Verben unterschieden hat und daher die simple past-Form des starken Verbs give durch Anfügen der Endung -ed markierte. Da hiermit eine Regel des Englischen, die nur auf schwache Verben zutrifft, auf andere Zusammenhänge übertragen wurde, ist die Abweichung zusätzlich als Übergeneralisierung einzustufen. Ebenso ist das dritte Fehlerbeispiel als Differenzierungsfehler und Übergeneralisierung zu betrachten, weil der Lernende nicht zwischen dem normgerechten Gebrauch des Perfekts in der Erst- und in der Zielsprache unterschieden hat und gleichzeitig der Gebrauch des Perfekts auf sprachliche Zusammenhänge übertragen wurde, in denen die Anwendungsmöglichkeiten des present perfect nicht zum Tragen kommen.

Die Anwendbarkeit von Fehlererklärungsfaktoren der Identitätshypothese wird in den drei Beispielen an folgenden Aspekten deutlich: Das entwicklungsbedingte Hinzufügen sprachlicher Elemente ist in allen Fehlerbeispielen nachzuweisen. Im ersten Beispiel äußert sich der Fehlertyp darin, daß durch die Verwendung des past perfect redundanterweise ein Hilfsverb und das Wortelement $-n$ hinzugefügt wurden. Im zweiten Beispiel bildet der Endkonsonant $-d$ das redundante Element, im dritten Beispiel äußert sich das normabweichende Hinzufügen im Gebrauch des Hilfsverbs have. Der als regularization bezeichnete Fehlertyp tritt im zweiten Beispiel auf, in dem der Fehler - wie erwähnt - auf der normabweichenden Verwendung der simple past-Form des Lexems give beruht. Sowohl dieses Beispiel als auch der Gebrauch des present perfect in der Abweichung des dritten Fehlerbeispiels zeigen außerdem, daß die Fehler zusätzlich auch auf die Verwendung sogenannter archi-forms bzw. alternating forms zurückzuführen sind.

Die Annahme, daß es sich bei diesen Abweichungsformen um entwicklungsbedingte Fehler handelt, beruht dabei auf der Tatsache, daß ähnliche Fehlertypen auch bei Kindern beobachtet werden konnten, die Englisch als Erstsprache erwerben. ${ }^{635}$ Dies gilt vor allem für den normabweichenden Gebrauch der simple past-Form, der dem zweiten Fehlerbeispiel zugrunde liegt. ${ }^{636}$ Die vorgenannten Fehlertypen treten beim Erstspracherwerb insbesondere dann auf, wenn die Kinder in einem bestimmten Erwerbsstadium mehrere, sich strukturell ähnelnde Zeitformen erwerben und aufgrund

\footnotetext{
635 vgl. Szagun 2000, $66-70$

636 vgl. ebd.
} 
der Informationsfülle nur partiell in der Lage sind, zwischen den unterschiedlichen Zeitformen zu unterscheiden. ${ }^{637}$

Auch der Einfluß von Fehlererklärungsfaktoren der Interlanguagehypothese ist in allen drei Fehlerbeispielen zu erkennen. Die Vermutung, daß unterrichtlich bedingte Faktoren an der Fehlerentstehung beteiligt gewesen sein könnten, beruht vor allem darauf, daß die Lernenden das simple past in ihren schriftlichen Beiträgen nicht konsequent durch eine andere Zeitform bzw. eine normabweichende simple past-Form ersetzten, sondern das simple past zum Teil auch normgerecht verwendet haben. Es ist also möglich, daß die Lernenden aufgrund einer Überbetonung der Zeitformen simple past und present perfect im Englischunterricht und der damit verbundenen Informationsdichte zeitweise nicht mehr in der Lage waren, zwischen den Anwendungsmöglichkeiten der beiden Zeitformen zu unterscheiden. Unterrichtserfahrungen haben gezeigt, daß die Vermittlung sprachlicher Regeln und die Gegenüberstellung sprachlicher Formen auch kontraproduktive Ergebnisse zur Folge haben können. ${ }^{638}$ So kann die Vermittlung sprachlicher Strukturen statt der erhofften Verbesserung bei den Lernenden auch Unsicherheiten in bezug auf die Anwendung der betreffenden Elemente hervorrufen. ${ }^{639}$ Bestärkt wird der Eindruck, daß Faktoren des Unterrichts an der Fehlerentstehung beteiligt gewesen sein könnten, vor allem durch die Tatsache, daß, nach Aussagen der Unterrichtenden, die beiden Zeitformen kurz vor Durchführung der Studie im Englischunterricht der PwensmAb behandelt worden waren.

Im zweiten Fehlerbeispiel wird auch der Einfluß von Lernstrategien deutlich: Die Fehlleistung gived ist vermutlich teilweise darauf zurückzuführen, daß sich der Verfasser zur Erleichterung des Lernprozesses zunächst auf die Vergangenheitsbildung schwacher Verben konzentrierte und diese Struktur auch auf starke Verben übertrug. Diese Annahme wird dadurch bekräftigt, daß der Lernende in seinem gesamten Beitrag ausschließlich diese Form der Vergangenheitsbildung benutzt hat und in keinem Fall Strukturen anwandte, die bei starken Verben zu erwarten gewesen wären.

Kommunikationsstrategien, insbesondere bewußt angewandte overgeneralizations, sind in den genannten Beispielen vor allem daran zu erkennen, daß die Lernenden versuchten, durch Verwendung bereits erworbener Strukturen sprachliche Zusammenhänge zu bewältigen, für die diese Strukturen nicht geeignet waren. Dies zeigt im ersten Beispiel die Verwendung des past perfect, im zweiten Beispiel die simple past-Markierung durch die Endung -ed und im dritten Beispiel die Anwendung des present perfect. Da in allen drei Fällen Strukturen verwendet wurden, die tatsächlich Bestandteil der englischen Sprache sind, war zusätzlich auch intralingual transfer an der Fehlerentstehung beteiligt.

\footnotetext{
637 vgl. ebd.

638 Diese Aussage beruht auf Erfahrungen des Autors der vorliegenden Arbeit, die während des Referendariats im Englischunterricht gewonnen wurden.

639 vgl. Juhász 1970, o. S.
} 
Interlingual transfer zeigt sich insbesondere darin, daß die fehlerhaft verwendeten Strukturen Ähnlichkeiten zu deutschen Strukturen aufweisen. Dies läßt gleichzeitig auf den Einfluß der Fehlerentstehungsursache lack of contrast schließen. Der Wechsel zwischen normgerechter und normabweichender Anwendung des simple past führt schließlich zu der Annahme, daß ein Teil der overgeneralizations auch auf unbewußtes Verhalten zurückzuführen ist.

Zusammengefaßt zeigt die Analyse des Fehlertyps simple past, daß die Ursachen der Fehlerentstehung bei den PmeismAb und den PwensmAb nahezu identisch sind (vgl. Tabellen 18 und 19 im Anhang) und bei beiden Lernertypen als äußerst komplex zu bezeichnen sind. Das simple past zählt damit zu den Zeitformen, die in allen Phasen des Zweitspracherwerbs als fehleranfällig zu betrachten sind und aufgrund der mit ihnen verbundenen Lernschwierigkeiten auch in fortgeschrittenen Erwerbsphasen einer besonderen Hervorhebung im Englischunterricht deutscher Englischlernender bedürfen. Dabei ist jedoch besonderer Wert darauf zu legen, daß das simple past immer im Zusammenhang mit anderen Zeitformen der Vergangenheit $\mathrm{zu}$ behandeln ist und Differenzen zwischen den Anwendungsmöglichkeiten des Perfekts im Deutschen und im Englischen besonders betont werden müssen.

\subsection{Hinzufügen und Auslassen sonstiger Wortarten und Bestandteile}

Der Schwerpunkt der folgenden Ausführungen liegt auf der Beschreibung der sogenannten Auslassungsfehler, da die Genese der Hinzufügungsfehler zum Teil bereits durch die vorhergehende Analyse des Fehlerbeispiels gived verdeutlicht wurde. Dabei wurde festgestellt, daß die Entstehung von Hinzufügungsfehlern auf einer Kombination erstsprachlicher entwicklungs- und interlanguagespezifischer Faktoren beruht (vgl. Tabellen 18 und 19 im Anhang).

In bezug auf die Auslassungsfehler der Kategorie ,sonstige Wortarten und Bestandteile‘ ist bei Anwendung der Erwerbshypothesen zu erkennen, daß die Ergebnisse, die in den beiden Gruppen gewonnen wurden, partiell übereinstimmen (vgl. Tabellen 18 und 19 im Anhang). In beiden Probandengruppen ist das Erklärungspotential der Identitäts- und der Interlanguagehypothese nahezu gleichbedeutend. Ein Unterschied besteht lediglich darin, daß die Häufigkeit der Anwendbarkeit beider Hypothesen bei den PmeismAb übereinstimmt, während in der entgegengesetzten Probandengruppe das Erklärungspotential der Identitätshypothese geringfügig größer ist.

In bezug auf die Kontrastivhypothese ist zu konstatieren, daß die Häufigkeit der Anwendbarkeit ihrer Fehlererklärungsfaktoren in der Gruppe der PwensmAb mit der Häufigkeit der Anwendungsmöglichkeiten der Interlanguagehypothese übereinstimmt. In der entgegengesetzten Probandengruppe kommen die Erklärungsmöglichkeiten der Kontrastivhypothese weniger häufig zur Anwendung als 
die der anderen Hypothesen (vgl. Tabelle 18 im Anhang). Die Differenz ist jedoch als unbedeutend zu bezeichnen.

Insgesamt ist also festzustellen, daß die Entstehung von Auslassungsfehlern bei den Lernenden beider Gruppen auf eine Kombination linguistischer, entwicklungsbedingter und interlanguagespezifischer Faktoren in nahezu gleicher Verteilung zurückgeführt werden kann. Dabei zeigt sich auch, daß der Einfluß der Erstsprache auf die Fehlerentstehung bei zunehmenden Sprachkenntnissen nicht abnimmt, sondern ebenso deutlich zum Tragen kommt wie der Einfluß biologisch vorbestimmter Faktoren und strategisch-prozeduraler Aspekte. Die Anzahl der Auslassungsfehler, die in beiden Probandengruppen ermittelt wurde, zeigt, daß das Auslassen syntaktischer Elemente nicht nur bei Anfängern, sondern auch bei fortgeschrittenen Lernenden als bedeutendes Sprachproblem zu betrachten ist, wenngleich der Fehleranteil bei den PmeismAb größer ist als in der entgegengesetzten Gruppe (vgl. Tabellen 4 und 5 im Anhang).

Innerhalb der Unterkategorien der einzelnen Hypothesen wurden nachfolgend dargestellte Ergebnisse deutlich. Aus der Sicht der Kontrastivhypothese ist die Fehlerentstehung in beiden Probandengruppen auf Substitutionen, Über- bzw. Unterdifferenzierungen und Übergeneralisierungen zurückzuführen (vgl. Tabellen 20 und 21 im Anhang). Dies bedeutet, beide Lernertypen produzierten Auslassungsfehler dadurch, daß zielsprachliche Elemente durch erstsprachliche ersetzt wurden bzw. dadurch, daß die Lernenden bei Anwendung der Zielsprache sprachliche Differenzierungen übernahmen, die im gegebenen Kontext im Deutschen vorzunehmen wären oder auf Differenzierungen verzichteten, weil sie im Deutschen nicht erforderlich sind. Zusätzlich entstanden Auslassungsfehler dadurch, daß erstsprachliche Regeln bei zielsprachlichen Strukturen angewandt wurden, die anderen Regeln unterliegen. Quantitativ steht das Substituieren englischer Elemente in beiden Probandengruppen an erster Stelle. An zweiter Stelle steht in beiden Gruppen der Fehlertyp Übergeneralisierung, während Über- und Unterdifferenzierungen in geringerer Anzahl auftraten. Darüber hinaus ist festzustellen, daß Differenzen in bezug auf die Anwendbarkeit der einzelnen Unterkategorien bei den PmeismAb deutlicher zu erkennen sind als in der entgegengesetzten Probandengruppe. So ist die Differenz zwischen dem Anteil der Substitutionen und der anderen Fehlerentstehungsursachen bei den PwensmAb gering, während bei den Probanden der entgegengesetzten Gruppe eine deutliche Differenz festzustellen ist. Das heißt, die Verteilung der Fehlerentstehungsfaktoren ist bei den PwensmAb gleichmäßig, bei den PmeismAb hingegen konzentriert sich die Fehlerentstehung auf den Faktor Substitution.

Auch in bezug auf die Anwendbarkeit der Identitätshypothese stimmen die Faktoren, die zur Fehlerentstehung beigetragen haben, in beiden Probandengruppen überein (vgl. Tabellen 22 und $23 \mathrm{im}$ Anhang). Sowohl bei den PmeismAb als auch bei den PwensmAb ist die Entstehung der meisten Fehler auf das Auslassen syntaktischer Elemente und auf den Gebrauch von archi-forms bzw. alter- 
nating forms zurückzuführen. Darüber hinaus treten in beiden Probandengruppen auch Abweichungen auf, die das Ergebnis entwicklungsbedingter Elementvertauschungen sind, also in der Kategorie misorderings zusammengefaßt werden. Dies Ergebnis läßt darauf schließen, daß der sprachliche Kenntnisstand der Lernenden nur geringen Einfluß auf die Typologie entwicklungsbedingter Fehler hat. Vielmehr weist dieses Ergebnis darauf hin, daß die entwicklungsbedingten Lernschwierigkeiten, die bei den PmeismAb zu konstatieren sind, auch den Lernprozeß der PwensmAb beeinflussen.

Auch die Überprüfung der Anwendungsmöglichkeiten der Interlanguagehypothese ergab in bezug auf beide Probandengruppen ähnliche Ergebnisse. In beiden Gruppen ist die Fehlerentstehung interlanguagespezifisch betrachtet auf Faktoren des Unterrichts, Lern- und Kommunikationsstrategien, unbewußte overgeneralizations und lack of contrast zurückzuführen (vgl. Tabellen 24 und $25 \mathrm{im}$ Anhang). Die einzelnen Kommunikationsstrategien, die in den Probandengruppen jeweils zum Tragen kommen, sind nahezu identisch. In beiden Gruppen basieren die Fehlleistungen im wesentlichen auf Faktoren der Kategorien modality und personality reduction, inter-/intralingual transfer, paraphrase und word coinage. Der Einfluß der Fehlerentstehungsfaktoren, der aus den drei Hypothesen abzuleiten ist, zeigt sich beispielsweise in folgenden Äußerungen:

Fehler: $\quad$ I know not what to write. ${ }^{640}$

Korrektur: I don't know what to write.

Fehler: $\quad$ I work in the office for ten years. ${ }^{641}$

Korrektur: I have worked in the office for ten years.

Folgende Faktoren der Kontrastivhypothese kommen hier zum Tragen: Das Substitutieren zielsprachlicher Elemente äußert sich im ersten Beispiel darin, daß der Lernende die deutsche Verneinung ,ich weiß nicht` Wort für Wort ins Englische übertragen hat und dabei die englischen Verneinungsregeln nicht berücksichtigte. Im zweiten Beispiel ist der Fehlertyp Substitution an der Verwendung des einfachen Präsens zu erkennen. Hier mißachtete der Lernende, daß Handlungen, die in der Vergangenheit begonnen haben und immer noch andauern, wie im vorliegenden Fall, im Englischen nicht wie im Deutschen durch das simple present, sondern durch das present perfect beschrieben werden. Zusätzlich sind die Abweichungen auch als Unterdifferenzierungen einzustufen, da in beiden Fehlerbeispielen nicht zwischen deutschen und englischen Strukturen unterschieden wurde. Der Fehlertyp ,Übergeneralisierung ' ist im zweiten Fehlerbeispiel zu konstatieren, da der Lernende eine Struktur, die im gegebenen Kontext im Deutschen durchaus zulässig wäre, in einem englischen Ausdruck benutzte, der eine andere Struktur erfordert hätte.

\footnotetext{
640 PmeismAb

641 PwensmAb
} 
Der Einfluß entwicklungsbedingter Faktoren wird an folgenden Aspekten deutlich: Zunächst gehören beide Fehlerbeispiele zu den Abweichungsformen, die beim gesteuerten wie beim ungesteuerten Zweitspracherwerb zu beobachten sind ${ }^{642}$ und auch in unterschiedlichen Phasen des Erstspracherwerbs auftreten. ${ }^{643}$ Die Einstufung als omission error ist dabei in bezug auf beide Beispiele möglich. Im ersten Beispiel ist das Auslassen des Hilfsverbs do als omission error zu betrachten, im zweiten das Auslassen des Lexems have.

Da in beiden Fehlertypen grammatische Strukturen der englischen Sprache dazu benutzt wurden, andere grammatische Strukturen zu ersetzen, die in den gegebenen sprachlichen Situationen eigentlich zu verwenden gewesen wären, können beide Fehler darüber hinaus auch der Kategorie archi-forms zugeordnet werden. Im ersten Beispiel ist die Verneinung know not als archi-form zu betrachten, weil der Lernende hier eine archaische Form benutzte, die im Englischen nur noch poetisch oder in der Umgangssprache - meist humorvoll gemeint - verwendet wird, bzw. in Texten des 16. und 17. Jahrhunderts erscheint ${ }^{644}$ und er mit dieser Struktur die normgerechte Verneinung don't know ersetzte. Im zweiten Beispiel diente der Gebrauch des simple present als Ersatzform für das present perfect, das zur Bildung einer grammatisch korrekten Äußerung zu verwenden gewesen wäre. Die Tatsache, daß in beiden Sätzen Sprachformen auftreten, die tatsächlich Bestandteile der englischen Sprache sind oder waren bzw. in besonderen Formen des Englischen, hier der Umgangssprache, benutzt werden, zeigt gleichzeitig, daß beide Abweichungen auch auf dem Fehlertyp alternating forms beruhen.

Der Fehlerentstehungsfaktor misorderings zeigt sich vor allem im ersten Beispiel. Diese Äußerung ist nicht nur aufgrund der Auslassung des Elementes do als Fehlleistung zu bezeichnen, sondern auch, weil das Adverb not eine falsche syntaktische Position einnimmt. Da das Auslassen des Hilfsverbs have im zweiten Beispiel zum einen die Zeitform verändert und zum anderen zu einer Verschiebung der Lexemstruktur führt, ist der Fehlertyp misordering jedoch ansatzweise auch in diesem Beispiel zu erkennen.

Auch die Auswirkungen interlanguagespezifischer Faktoren sind in beiden Fehlerbeispielen festzustellen. Die Annahme, daß bei beiden Abweichungen u. a. Faktoren des Unterrichts zur Fehlerentstehung geführt haben, basiert darauf, daß die normabweichende Bildung der Verneinung und die Verwechslung der Zeit- und Verlaufsformen nicht nur in diesen beiden Beispielen ermittelt wurden, sondern auch bei den anderen Teilnehmern des Kurses, den die Verfasser der beiden Äußerungen

\footnotetext{
${ }^{642}$ vgl. Dulay / Burt / Krashen 1982, 165 ff

643 vgl. ebd.

${ }^{644}$ vgl. Baugh / Cable 1978, 253 - 295
} 
besuchten. Es ist daher in bezug auf die Äußerung I know not what to write denkbar, daß entweder die Verneinung mit to do im Unterricht nicht deutlich genug hervorgehoben wurde oder daß in den Unterrichtsmaterialien, die den Lernenden zur Verfügung standen, Verneinungsformen überwogen, die nicht mit dem Hilfsverb do gebildet werden. In der Formulierung I work in the office for ten years besteht der Einfluß unterrichtlicher Faktoren möglicherweise darin, daß die Unterschiede, die zwischen den Anwendungsmöglichkeiten des Perfekts im Deutschen und im Englischen bestehen, noch nicht genügend thematisiert worden waren. Auch die Tatsache, daß die Präposition for den Gebrauch des present perfect bedingt, wurde im Unterricht vermutlich nicht eingehend hervorgehoben.

Daneben ist aufgrund der Beobachtung, daß in beiden Fehlerbeispielen statt einer komplexen grammatischen Struktur sprachliche Elemente verwendet wurden, die zu den Lerninhalten des Anfangsunterrichts gehören, auch ein Einfluß der Lernstrategie simplification zu vermuten.

Die Auswirkungen formaler und funktionaler Reduktionsstrategien, d. h. Fehler der Kategorien omission of language items und modality reduction (vgl. Tabelle 24 und 25 im Anhang) zeigen sich darin, daß in beiden Beispielen Elemente ausgelassen wurden, die für das Verständnis der Aussage von Bedeutung sind. Der Fehlertyp meaning replacement ist besonders im zweiten Fehlerbeispiel zu erkennen. Hier wurde, bedingt durch das Auslassen des present perfect, nicht zum Ausdruck gebracht, daß die Handlung in der Vergangenheit begonnen hat und immer noch andauert. Vielmehr entsteht aufgrund der Abweichung der Eindruck, daß die Handlung in zehn Jahren enden und danach nicht weiter fortgesetzt wird. Wie der sprachliche Kontext, in dem der Fehler ermittelt wurde, zeigt, widerspricht diese Aussage jedoch dem Inhalt, den der Verfasser vermitteln wollte.

Interlingual transfer zeigt sich in beiden Fehlerbeispielen darin, daß Strukturen der deutschen Sprache übernommen wurden. Dabei hat im ersten Beispiel die deutsche Äußerung ,Ich weiß nicht‘ als Vorlage gedient und im zweiten der deutsche Satz ,Ich arbeite seit zehn Jahren in diesem Büro. Zusätzlich sind die Abweichungen auch den Fehlerkategorien intralingual transfer und generalization of interlanguage rules zuzuordnen, da - wie bereits erwähnt - in beiden Fehlerbeispielen Elemente verwendet werden, die in der Tat als Bestandteile der englischen Sprache zu betrachten sind.

Der Fehlertyp paraphrase ist, wenn auch nur ansatzweise, im ersten Beispiel zu konstatieren. Hier wurde die Verneinung do not, die in dem gegebenen Zusammenhang zu erwarten gewesen wäre, durch die veraltete Struktur know not umschrieben bzw. ersetzt. 
Da die grammatischen Strukturen, die jeweils normabweichend verwendet werden, in den Beiträgen der Probanden zum Teil auch normgerecht zur Anwendung kamen, ist in bezug auf beide Beispiele zu vermuten, daß die Abweichungen partiell auch unbewußt entstanden sind. Sie können daher auch der Kategorie overgeneralization zugeordnet werden.

Auch der Einfluß des Fehlerentstehungsfaktors lack of contrast zeigt sich in beiden Abweichungen. Im ersten Beispiel hat u. a. die Ähnlichkeit zwischen dem englischen Adverb not und dem deutschen Lexem ,nicht` zur Entstehung der Abweichung beigetragen. Im zweiten Beispiel ist ebenfalls lack of contrast als Fehlerentstehungsursache zu vermuten, weil die normgerechte Äußerung I have worked in the office for ten years strukturell dem deutschen Satz „Ich habe in diesem Büro zehn Jahre gearbeitet“ ähnelt. Da die Handlung dem deutschen Satz zufolge jedoch beendet ist und diese Aussage daher nicht der Intention des Lernenden entspräche, benutzte er Lernende statt des Perfekts das simple present.

Wenngleich die quantitative Verteilung der Fehlerentstehungsfaktoren beim Vergleich der beiden Probandengruppen zum Teil unterschiedlich ist, verdeutlichen die Ergebnisse dennoch, daß die fehlergenetischen Faktoren keine gravierenden Unterschiede aufweisen. Bei der Erarbeitung fehlerprophylaktischer Konzepte sind folglich in bezug auf die Auslassungsfehler zwischen Anfängern und fortgeschrittenen Lernenden keine erheblichen Differenzen zu berücksichtigen.

\section{Orthographische Abweichungen}

Wie bei den linguistischen Ebenen Lexik und Syntax-Morphologie ist auch auf orthographischer Ebene zu erkennen, daß beim Vergleich der Gruppe der Probanden mit den meisten orthographischen Abweichungen (PmeioAb) mit der Gruppe der Probanden mit den wenigsten orthographischen Abweichungen (PwenoAb) fehlertypologische Übereinstimmungen bestehen (vgl. Tabellen 10 und $11 \mathrm{im}$ Anhang). Dies bedeutet, die Anwendungsschwierigkeiten beider Probandengruppen ähneln sich. Wie eingangs erwähnt, wurden in beiden Probandengruppen drei Fehlertypen ermittelt: Fehler, die bei normabweichender Anwendung der Groß- und der Kleinschreibung entstanden sowie Fehler, die innerhalb oder am Ende einzelner Lexeme auftraten und der Kategorie ,Rechtschreibung ' zugeordnet wurden. Dabei zeigt die Gegenüberstellung der einzelnen Fehlerkategorien, daß die Anzahl der Fehler der Kategorie ,Großschreibung ‘ bei den PwenoAb höher ist als in der entgegengesetzten Probandengruppe. Diesem Ergebnis zufolge neigen Lernende mit fortgeschrittenen orthographischen Kenntnissen trotz ihres Wissens häufiger als Lernanfänger dazu, Lexeme klein zu schreiben, obwohl die betreffenden Wörter den orthographischen Regeln des Englischen entsprechend groß zu schreiben wären. 
Die Lernprobleme der anderen Kategorien hingegen überwiegen in der Gruppe der PmeioAb deutlich. Dies betrifft vor allem die Kategorie ,Kleinschreibung' (vgl. Tabellen 10 und 11 im Anhang). Während dieser Fehlertyp bei den PwenoAb kaum beobachtet werden konnte, bildet er in der entgegengesetzten Probandengruppe die zweitgrößte Fehlerkategorie. Eine quantitative Übereinstimmung zwischen beiden Probandengruppen besteht nur in bezug auf die Fehlerkategorie, Rechtschreibung'. Dieser Fehlertyp ist in beiden Gruppen als größtes Lernproblem im Bereich der Orthographie zu bezeichnen.

Diese Beobachtungen lassen bereits vor Anwendung der Erwerbshypothesen die Vermutung zu, daß die Differenzen, die beim Vergleich der Kategorien ,Großschreibung‘ und ,Kleinschreibung` deutlich wurden, darauf basieren, daß die Großschreibungsregeln von den PwenoAb partiell verdrängt wurden. Diese Verdrängung ist wahrscheinlich darauf zurückzuführen, daß die Lernenden durch den zunehmenden schriftlichen Umgang mit der englischen Sprache immer deutlicher erfahren haben, daß die Kleinschreibung im Englischen - im Gegensatz zum Deutschen - überwiegt. Aus dieser Erkenntnis resultierte vermutlich ein hyperkorrektes orthographisches Verhalten, welches sich darin äußerte, daß die Kleinschreibung häufiger zur Anwendung kam als die Großschreibung, weil die Lernenden darum bemüht waren, sprachliche Fehler zu vermeiden. Die Anzahl der Fehler in der Kategorie ,Kleinschreibung' ist in dieser Gruppe dementsprechend gering. Jedoch mißachteten sie häufiger als die Probanden der entgegengesetzten Gruppe, daß englische Lexeme in bestimmten Fällen - wie etwa Adjektive, die von Ländern abgeleitet werden - mit einem Großbuchstaben beginnen.

Lernende mit erheblichen orthographischen Lernschwierigkeiten hingegen orientieren sich vermutlich stärker an den Regeln der Erstsprache Deutsch, in der die Großschreibung - wie erwähnt - eine größere Bedeutung hat als im Englischen. Die Anzahl der Verstöße ist in dieser Kategorie daher geringer.

Beim Vergleich der Anwendungsmöglichkeiten der Erwerbshypothesen fällt auf, daß die Identitätshypothese in beiden Probandengruppen weit seltener zur Anwendung kommt als die Kontrastiv- und die Interlanguagehypothese. Das Erklärungspotential der Identitätshypothese ist in beiden Probandengruppen äußerst gering (vgl. Tabellen 26 und 27 im Anhang). Die Anwendbarkeit von Erklärungsfaktoren der Identitätshypothese konnte in beiden Probandengruppen ausschließlich in der Fehlerkategorie ,Rechtschreibung' nachgewiesen werden, wobei die Faktoren bei den PmeioAb stärker stärker hervortraten. Darüber hinaus zeigt der Vergleich, daß sowohl die Kontrastiv- als auch die Interlanguagehypothese bei den PmeioAb häufiger zum Tragen kommt als bei den PwenoAb. Dabei ist jedoch die Differenz zwischen den Anwendungsmöglichkeiten der Interlanguagehypothese in beiden Gruppen relativ gering, während der Unterschied in bezug auf die Anwendungsmöglichkeiten der Kontrastivhypothese deutlich erkennbar ist. 
Insgesamt betrachtet zeigt die Untersuchung der Entstehung der orthographischen Abweichungen mit Hilfe der drei Erwerbshypothesen, daß, trotz der im einzelnen unterschiedlichen Gewichtung, der Einfluß der Erstsprache und der Einfluß strategisch-prozeduraler Faktoren in beiden Probandengruppen als wichtigste Fehlerentstehungsursachen zu betrachten sind. Dabei kommen beide Faktoren bei den PmeioAb stärker zum Tragen als in der entgegengesetzten Gruppe. Dies gilt vor allem für die entwicklungsbedingten Faktoren. Demnach läßt der Einfluß entwicklungsbedingter Faktoren mit zunehmenden orthographischen Kenntnissen nach. Innerhalb der einzelnen Fehlerkategorien wurden nachfolgend beschriebene Faktoren deutlich.

\subsection{Großschreibung}

Der Vergleich der Ergebnisse im Bereich der Fehlerkategorie ,Großschreibung` zeigt, daß der Einfluß der Erstsprache bei den PmeioAb ebenso deutlich nachgewiesen werden konnte wie der Einfluß strategisch-prozeduraler Faktoren. Entwicklungsbedingte Aspekte kommen hier - wie erwähnt - nicht zum Tragen (vgl. Tabelle 26 im Anhang). Demnach beruht die Genese der Großschreibungsfehler in dieser Probandengruppe auf linguistischen und interlanguagespezifischen Entstehungsursachen in gleichmäßiger Verteilung.

Bei Betrachtung der Unterkategorien der Kontrastivhypothese ist zu erkennen, daß die Fehler dieser Probandengruppe auf Substitutionen sowie Über- bzw. Unterdifferenzierungen zurückzuführen sind (vgl. Tabelle 28 im Anhang). Somit entstanden die Fehler aus linguistischer Sicht zum einen dadurch, $\mathrm{da}$ zielsprachliche Elemente durch Elemente ersetzt wurden, die im gegebenen Kontext in der Erstsprache zur Anwendung kämen. Zum anderen basierte die Fehlerentstehung darauf, daß linguistische Unterscheidungen zwischen Erst- und Zielsprache entweder überbetont oder aber nicht beachtet wurden.

Bei Anwendung der Fehlererklärungsfaktoren der Interlanguagehypothese zeigt sich, daß nur die Kategorien interlingual transfer und generalization of interlanguage rules zum Tragen kommen (vgl. Tabelle 30 im Anhang). Lernerspezifisch betrachtet ist die Entstehung der Großschreibungsfehler somit ausschließlich auf Strategien zurückzuführen, die bewußt angewandt wurden. Dabei versuchten die Lernenden zum einen, orthographische Anwendungsschwierigkeiten durch Übertragung sprachlicher Regeln der Erstsprache oder anderer Zweitsprachen zu lösen. Zum anderen basierte die Fehlerentstehung darauf, daß bereits erworbene Regeln der Zielsprache in sprachlichen Situationen verwendet wurden, die anderen Regeln unterliegen.

Auch in der Gruppe der PwenoAb ist - wie erwähnt - die Fehlerentstehung ausschließlich durch linguistische und strategisch-prozedurale Faktoren zu begründen. Dabei zeigt sich, daß erstsprachlich 
bedingte Faktoren wesentlich seltener zur Anwendung kommen als in der entgegengesetzten Probandengruppe, während der Einfluß interlanguagespezifischer Aspekte größer ist (vgl. Tabellen 26 und $27 \mathrm{im}$ Anhang). Somit entstanden die meisten Fehler in dieser Gruppe eindeutig durch die Anwendung von Lern- bzw. Kommunikationsstrategien sowie durch unbewußt ablaufende Prozesse bei der Sprachanwendung. Da jedoch auch erstsprachlich bedingte Faktoren zu $24 \%$ nachgewiesen werden konnten, dürfen diese Faktoren bei der Erarbeitung von Fehlervermeidungskonzepten nicht außer acht gelassen werden, sondern sind als wichtige Fehlerentstehungsursache zu betrachten.

Bei Anwendung der Fehlerentstehungsfaktoren der Kontrastivhypothese ist zu erkennen, daß in dieser Probandengruppe nur die Kategorie ,Substitution` zu beobachten ist (vgl. Tabelle 28 im Anhang): Linguistisch betrachtet beruhen die Fehler ausschließlich auf dem Ersetzen zielsprachlicher Elemente durch erstsprachliche. Auch bei Anwendung der Interlanguagehypothese zeigt sich, daß - wie in der entgegengesetzten Probandengruppe - nur zwei Kategorien nachzuweisen sind (vgl. Tabelle 30 im Anhang). Übereinstimmung besteht in der Anwendbarkeit der Kategorie generalization of interlanguage rules. Im Gegensatz zu den Fehlern der PmeioAb basierten in dieser Probandengruppe jedoch auch Abweichungen auf unbewußten overgeneralizations. Der Einfluß der verschiedenen Faktoren der beiden Hypothesen wird in folgenden Äußerungen deutlich:

Fehler: $\quad$ The british food was not so good. ${ }^{645}$

Korrektur: The British food was not so good.

Fehler: $\quad$ Do you remember our last holidays we spent together in South africa? ${ }^{646}$

Korrektur: Do you remember our last holidays we spent together in South Africa?

Fehler: $\quad$ I'm going to travel to Hamburg next tuesday. ${ }^{647}$

Korrektur: I'm going to travel to Hamburg next Tuesday.

Im ersten Beispiel wird der Fehlerentstehungsfaktor Substitution daran deutlich, daß der Lernende bei der Schreibweise des Lexems British eine deutsche Regel zugrunde gelegt hat. Der Großbuchstabe wurde also durch den entsprechenden Kleinbuchstaben ersetzt, weil Adjektive - auch wenn sie eine Nationalität bezeichnen - im Deutschen klein geschrieben werden, wenn das betreffende Wort nicht als Bestandteil eines Eigennamens zu betrachten ist. Im Englischen werden Adjektive jedoch groß geschrieben, wenn das Lexem eine Nationalität oder eine ethnische oder religiöse Gruppe beschreibt. Da der Lernende diese Differenzierung nicht beachtete, ist die Abweichung gleichzeitig als Unterdifferenzierung zu bezeichnen.

645 Das Beispiel stammt aus der Probandengruppe mit den meisten orthographischen Abweichungen - PmeioAb (FB der Gruppe HS 10G). Zur Ausnahmeregelung bezüglich dieser Probandengruppe siehe Kap. I 4.4 der vorliegenden Arbeit: Fehlertypologische Auffälligkeiten in den Probandengruppen.

${ }^{646}$ Das Beispiel stammt aus der Probandengruppe mit den wenigsten orthographischen Abweichungen - PwenoAb (FB der Gruppe GY 12L).

647 PmeioAb 
Die Auswirkungen der Erstsprache in der Gruppe der PwenoAb zeigen sich im zweiten Fehlerbeispiel. Hier äußert sich der Fehlerentstehungsfaktor Substitution in der Schreibweise des Lexems South africa. Dabei ist die normabweichende Schreibweise offensichtlich dadurch entstanden, daß das Wort ,Südafrika‘, im Gegensatz zu seiner englischen Entsprechung, zusammen geschrieben wird und der Wortteil ,africa‘ daher mit einem Kleinbuchstaben beginnt.

Die Fehlerentstehungsfaktoren der Interlanguagehypothese werden an folgenden Aspekten deutlich: Interlingual transfer äußert sich im ersten Beispiel darin, daß der Lernende - wie erwähnt - bei der Schreibweise des Adjektivs British eine deutsche Regel zugrunde gelegt hat. Da die Mehrzahl der englischen Adjektive in der Tat klein geschrieben wird und der Lernende demnach eine Schreibweise benutzte, die im Englischen durchaus existiert, jedoch beim betreffenden Lexem nicht zur Anwendung kommt, ist der Fehler auch der Kategorie generalization of interlanguage rules zuzuordnen. Die Vermutung, daß es sich bei der Abweichung um eine bewußte generalization handelt, wird dadurch verstärkt, daß der Lernende in seinem schriftlichen Beitrag alle Adjektive klein geschrieben hat und damit offensichtlich eine zuvor erworbene Sprachregel konsequent befolgte.

In der Gruppe der PwenoAb ist ein Einfluß interlanguagespezifischer Faktoren in Form einer bewußten generalization zu vermuten: Der Ausdruck South africa könnte u. a. deshalb falsch geschrieben worden sein, weil das zweite Element einer Wortzusammensetzung bei den meisten englischen Komposita klein geschrieben wird, auch wenn das erste Lexem dieser Zusammensetzung mit einem Großbuchstaben beginnt - ein Beispiel hierfür bietet der zuvor genannte Ausdruck British food. Es ist daher vorstellbar, daß diese Schreibweise dem Lernenden möglicherweise vertrauter war als die Schreibweise, die bei Ausnahmen wie dem Lexem South Africa anzuwenden ist.

Der Einfluß einer unbewußten overgeneralization zeigt sich im dritten Beispiel: Die Abweichung Tuesday ist darauf zurückzuführen, daß - wie zuvor erwähnt - die Mehrzahl der englischen Lexeme innerhalb eines Satzes klein geschrieben wird. Hier hat der Lernende nicht beachtet, daß es von dieser Regel jedoch eine Reihe von Ausnahmen gibt, zu denen auch die Wochentage zählen. Dabei ist auszuschließen, daß die Fehlerentstehung auf bewußtes Handeln zurückzuführen ist, weil im Beitrag des Probanden die Schreibweise der Ausnahmen bei allen anderen Lexemen beachtet wurde, jedoch bei dem Wort Tuesday der Aufmerksamkeit des Lernenden entgangen war.

\subsection{Kleinschreibung}

Bei Gegenüberstellung der Fehleranzahl ist in bezug auf die Fehlerkategorie ,Kleinschreibung - wie erwähnt - zu erkennen, daß die Anwendungsschwierigkeiten, die mit den Regeln der Kleinschreibung 
verbunden sind, bei den PmeioAb deutlich stärker zum Tragen kommen als in der entgegengesetzten Probandengruppe (vgl. Tabellen 10 und 11 im Anhang). Die Untersuchung dieser Fehler mit Hilfe der Erwerbshypothesen zeigt, daß in der Gruppe der PmeioAb sowohl die Kontrastiv- als auch die Interlanguagehypothese stärker zur Anwendung kommen als in der entgegengesetzten Probandengruppe (vgl. Tabellen 26 und 27 im Anhang). Bei ersteren konnten erstsprachliche Fehlerentstehungsfaktoren häufiger nachgewiesen werden als strategisch-prozedurale Aspekte. Die Differenz zwischen den Anwendungsmöglichkeiten der beiden Hypothesen ist dabei jedoch relativ gering. Diesem Ergebnis zufolge ist der Einfluß der Erstsprache bei Lernanfängern stärker an der Fehlerentstehung im Bereich der Orthographie beteiligt als das strategisch-prozedurale Verhalten.

Auch in der Gruppe der PwenoAb überwiegt das Erklärungspotential der Kontrastivhypothese. Allerdings ist hier ein erheblicher Unterschied zwischen den Anwendungsmöglichkeiten der beiden Hypothesen zu verzeichnen (vgl. Tabelle 27 im Anhang). Demnach waren in dieser Lernergruppe erstsprachliche Faktoren weitaus stärker an der Fehlerentstehung beteiligt als interlanguagespezifische Aspekte.

Die Anwendung der Unterkategorien der einzelnen Erwerbshypothesen führte $\mathrm{zu}$ folgenden Ergebnissen: Übereinstimmend mit den Erkenntnissen, die in der Kategorie ,Großschreibung“ gewonnen wurden, sind auch in der vorliegenden Fehlerkategorie die Abweichungen aus Sicht der Kontrastivhypothese in der Gruppe der PmeioAb sowohl auf Substitutionen als auch auf Über- bzw. Unterdifferenzierungen zurückzuführen (vgl. Tabelle 28 im Anhang). In der entgegengesetzten Probandengruppe hingegen konnte nur der Fehlererklärungsfaktor Substitution nachgewiesen werden. Interlanguagespezifisch betrachtet basiert die Fehlerentstehung bei den PmeioAb ausschließlich auf productive achievement strategies. Hier wurden sowohl inter-/intralingual transfer als auch die Fehlerkategorie generalization of interlanguage rules als Fehlerursachen ermittelt (vgl. Tabelle $30 \mathrm{im}$ Anhang). In der entgegengesetzten Probandengruppe hingegen wurden die Fehler ausschließlich durch unbewußte overgeneralizations verursacht. Folgende Beispiele verdeutlichen die Auswirkungen der genannten Faktoren:

Fehler: $\quad$ I drive next year in the Winter holiday to München. ${ }^{648}$

Korrektur: For my winter holidays next year I'm going to Munich.

Fehler: $\quad$ In my last Holidays I stayed with my class in Italy. ${ }^{649}$

Korrektur: During my last holidays I was in Italy with my class.

Im ersten Beispiel zeigt sich der Fehlerentstehungsfaktor ,Substitution“ darin, daß der Lernende eine Schreibweise des Englischen durch eine Schreibweise des Deutschen ersetzt hat: Er hat das Wort

648 PmeioAb 
,Winter ${ }^{6}$ groß geschrieben, weil dies der deutschen Schreibweise von Jahreszeiten entspricht. Im Englischen hingegen werden Jahreszeiten - im Gegensatz zu Wochen- und Monatsnamen - klein geschrieben. Da der Lernende diese Differenzierung nicht beachtet hat, kann die Abweichung auch der Kategorie ,Unterdifferenzierung` zugeordnet werden. Auch im zweiten Beispiel ist der Fehlerentstehungsfaktor Substitution an dem Ersetzen englischsprachiger Elemente zu erkennen. In diesem Fall ist die fehlerhafte Schreibweise des Lexems holidays darauf zurückzuführen, daß das Wort ,Ferien“ im Deutschen groß geschrieben wird, während das Lexem holiday im Englischen bekanntlich nicht zu den Wörtern gehört, die mit einem Großbuchstaben beginnen.

Die Auswirkungen von Faktoren, die der Interlanguagehypothese zugrunde liegen, zeigen sich an folgenden Punkten: Der Fehlerentstehungsfaktor interlingual transfer ist im ersten Beispiel daran zu erkennen, daß Schreibweisen des Deutschen - wie zuvor erwähnt - ins Englische übertragen wurden. Die Abweichung des zweiten Beispiels wurde jedoch dieser Kategorie nicht zugeordnet, weil der Fehlertyp im Beitrag des Probanden nur einmal auftrat und demnach, interlanguagespezifisch betrachtet, andere Faktoren überwiegen. Die Annahme, daß im ersten Beispiel auch intralingual transfer an der Fehlerentstehung beteiligt war, ist dadurch begründet, daß andere Lexeme, die eine ähnlich semantische Funktion übernehmen wie die Jahreszeiten, im Englischen tatsächlich groß geschrieben werden. Es ist also möglich, daß der Lernende glaubte, diese Schreibweise auch bei dem Lexem winter anwenden zu müssen. Da es sich hier gleichzeitig um eine Übertragung englischer Schreibweisen auf Strukturen handelt, die anderen Regeln unterliegen, ist die Abweichung zusätzlich als generalization of interlanguage rules zu bezeichnen.

In der Gruppe der PwenoAb kommt bei Anwendung der Interlanguagehypothese ausschließlich der Fehlerentstehungsfaktor overgeneralization zum Tragen. Im dargestellten Beispiel ist davon auszugehen, daß der Fehler ausschließlich auf einer unbewußten Übertragung einer Interlanguageregel basiert. Fehlergenetisch betrachtet unterscheidet sich diesem Ergebnis zufolge die Entstehung der Fehler der Kategorie ,Kleinschreibung ' in den beiden Probandengruppen dadurch, daß in der Gruppe der PmeioAb sowohl linguistische als auch interlanguagespezifische Faktoren stärker an der Fehlerentstehung beteiligt waren als in der entgegengesetzten Gruppe. Dabei kommen in ersterer auch die unterschiedlichen Formen erstsprachlichen und interlanguagespezifischen Einflusses stärker zum Tragen. Demnach sind die Faktoren, die zur Fehlerentstehung beigetragen haben, in dieser Gruppe vielseitiger als bei den PwenoAb. Darüber hinaus zeigt der Vergleich der Ergebnisse, daß bei den PmeioAb offensives Sprachverhalten überwiegt, während in der entgegengesetzten Gruppe unbewußtes Verhalten als wichtigster Fehlerentstehungsfaktor zu betrachten ist. In diesem Zusammenhang verdeutlichen die Ergebnisse also auch, daß der Einfluß der Erstsprache mit zunehmenden orthographischen Kenntnissen keineswegs nachläßt. 


\subsection{Rechtschreibung}

In der Kategorie ,Rechtschreibung' konnte - wie erwähnt - in beiden Probandengruppen auch entwicklungsspezifischer Einfluß nachgewiesen werden (vgl. Tabellen 26 und 27 im Anhang). Dieser Fehlerentstehungsfaktor kommt bei den PmeioAb jedoch wesentlich öfter zur Anwendung als in der entgegengesetzten Probandengruppe - bei den PwenoAb sind die Faktoren der Identitätshypothese von nahezu untergeordneter Bedeutung. Weiterhin zeigt der Vergleich, daß das Erklärungspotential der Interlanguagehypothese in beiden Probandengruppen größer ist als das der Kontrastivhypothese, dabei jedoch bei den PwenoAb deutlicher zum Tragen kommt als in der entgegengesetzten Gruppe. Schließlich ist bei Gegenüberstellung der Ergebnisse zu erkennen, daß der Einfluß der Erstsprache bei den PmeioAb größer ist als bei den PwenoAb, wenngleich die Differenz hier gering ist.

Innerhalb der Unterkategorien der einzelnen Hypothesen kommt es zu folgenden Ergebnissen: Bei Anwendung der Kontrastivhypothese konnte in beiden Probandengruppen nur der Fehlerentstehungsfaktor Substitution nachgewiesen werden (vgl. Tabelle 28 im Anhang). Aus der Reihe der Erklärungsfaktoren der Identitätshypothese kommt bei den PmeioAb sowohl die Kategorie omission errors als auch die Kategorie simple addition errors zum Tragen, während bei der entgegengesetzten Gruppe der zuletzt genannte Fehlerentstehungsfaktor als einzige Auswirkung entwicklungsbedingter Aspekte zu betrachten ist (vgl. Tabelle 29 im Anhang).

Bei Anwendung der Interlanguagehypothese stimmen die Ergebnisse beider Probandengruppen nahezu überein (vgl. Tabelle $30 \mathrm{im}$ Anhang). In beiden Gruppen ist die Fehlerentstehung auf productive achievement strategies, auf unbewußte overgeneralizations und auf lack of contrast zurückzuführen. Productive achievement strategies äußern sich bei den PmeioAb sowohl in Form von inter/intralingual transfer als auch in Form des Fehlertyps generalization of interlanguage rules. In der entgegengesetzten Probandengruppe kommen dieselben Faktoren mit Ausnahme des intralingual transfer zum Tragen.

Zusammenfassend ist also festzustellen, daß die Ursachen der Fehlerentstehung in beiden Gruppen bis hin zu den Unterkategorien nahezu übereinstimmen. Unterschiede bestehen - wie erwähnt - lediglich in der Tatsache, daß entwicklungsbedingte Faktoren bei Lernenden mit erheblichen Anwendungsschwierigkeiten stärker zum Tragen kommen und auch die Formen entwicklungsbedingten Einflusses vielseitiger sind. Auch der Einfluß interlanguagespezifischer Faktoren ist in dieser Probandengruppe differenzierter, wenngleich, quantitativ betrachtet, der Einfluß strategisch-prozeduraler Aspekte bei den PwenoAb deutlicher zum Tragen kommt. Die Auswirkungen der verschiedenen Faktoren werden an folgenden Beispielen deutlich: 
Fehler: $\quad$ Our last holiday was very beutifull. ${ }^{650}$

Korrektur: Our last holiday was very beautiful.

Fehler: $\quad$ But know I have to do some importent things. ${ }^{651}$

Korrektur: But now I have to do some important things.

Im ersten Beispiel äußert sich der Fehlerentstehungsfaktor Substitution in der Schreibweise der Endsilbe -ful des Lexems beautiful. Hierbei ist davon auszugehen, daß die Ähnlichkeit zwischen der Lautung der betreffenden Endsilbe und dem deutschen Lexem, voll', das mit einem Doppelkonsonanten endet und häufig auch als Suffix verwendet wird, zu der normabweichenden Schreibweise geführt hat.

Im zweiten Beispiel zeigt sich derselbe Fehlerentstehungsfaktor in der Schreibweise des Adjektivs important. Der Fehler basiert hier auf der bereits zuvor erwähnten Tatsache, daß viele deutsche Englischlernende nicht zwischen der Aussprache der Laute /e/ und /æ/ differenzieren können und oft den zuletzt genannten Laut durch den ersten ersetzen, weil sie entweder die akustischen Unterschiede nicht wahrnehmen oder artikulatorische Schwierigkeiten bei der Bildung des Lautes /æ/ haben. Diese Substitution führt schließlich - wie in obigem Beispiel - dazu, daß der betreffende Laut im schriftlichen Englisch durch den Buchstaben e zum Ausdruck gebracht wird, und nicht - wie eigentlich zu erwarten - durch den Buchstaben $a$, weil das $e$ das Phonem /e/ repräsentiert.

Die Fehlerentstehungsfaktoren, die bei Anwendung der Identitätshypothese zum Tragen kommen, werden an folgenden Punkten deutlich: Der Fehlerentstehungsfaktor omission errors, der in der Gruppe der PmeioAb nachgewiesen werden konnte, äußert sich im ersten Beispiel darin, daß bei der Schreibweise des Lexems beautiful u. a. der Buchstabe $a$ ausgelassen wurde. Die Einstufung dieses Fehlers als entwicklungsbedingter Verstoß beruht darauf, daß Abweichungen dieser Art auch beim natürlichen Zweitspracherwerb und beim Erstspracherwerb beobachtet werden können. ${ }^{652}$ Das Auslassen solcher Elemente beruht entweder darauf, daß die betreffenden Buchstaben als redundant betrachtet werden, weil sie zur Verdeutlichung der beabsichtigten Aussage irrelevant erscheinen oder darauf, daß das ausgelassene Element einen Laut darstellt, den die Lernenden als schwierig empfinden.

Im zweiten Fehlerbeispiel ist der Einfluß entwicklungsbedingter Faktoren, der sich hier - wie erwähnt - nur in Form des simple addition errors äußert, an der Schreibweise des Lexems know zu erkennen. Auch dieser Fehlertyp gehört zu den Begleiterscheinungen des natürlichen Zweitspracherwerbs und

\footnotetext{
650 PmeioAb

651 PwenoAb

${ }^{652}$ vgl. Dulay / Burt / Krashen 1982
} 
des Erstspracherwerbs. ${ }^{653}$ Dabei haben Studien zu diesen Erwerbsformen gezeigt, daß Lernende während der Verinnerlichung sogenannter homophoner Ausdrücke oder Wörter, die sich wie die Lexeme know und now optisch ähneln, vorübergehend oftmals nicht zwischen der Schreibweise der einzelnen Wörter unterscheiden können. ${ }^{654}$ In diesem Zusammenhang wurde deutlich, daß diese Abweichungsform auch in äußerst fortgeschrittenen Phasen der zuvor genannten Erwerbsformen auftritt. ${ }^{655}$

Die Auswirkungen der Fehlerentstehungsfaktoren der Interlanguagehypothese zeigen sich im ersten Beispiel vor allem in der Schreibweise der bereits zuvor hervorgehobenen Endsilbe -ful. Wie erwähnt, ist diese Schreibweise zum einen darauf zurückzuführen, daß die Endsilbe -ful optisch und akustisch an das deutsche Suffix ,-voll‘ erinnert. Daher ist der Fehler der Kategorie interlingual transfer zuzuordnen. Gleichzeitig ähnelt die Endsilbe jedoch auch dem englischen Adjektiv full, so daß die Abweichung darüber hinaus auch als Ergebnis intralingual transfer und einer generalization of interlanguage rules betrachtet werden kann, weil der Lernende hier eine zielsprachliche Struktur auf ein Element der Zielsprache übertragen hat, das auf einer anderen Struktur basiert. Dabei zeigt die Tatsache, daß die Fehlleistung auf intra- und interlingualen Übereinstimmungen basiert, daß lack of contrast ebenfalls als Entstehungsursache zu berücksichtigen ist.

Der Einfluß einer unbewußter overgeneralization äußert sich in der Schreibweise der ersten Silbe des Lexems beautiful. Hier ist von einer unbewußten und nicht von einer bewußten Generalisierung auszugehen, weil die fehlerhafte Schreibweise nur einmal auftrat, wohingegen der Teilnehmer das Wort beautiful in seinem Beitrag mehrfach korrekt benutzt hat. Im zweiten Fehlerbeispiel wird interlanguagespezifischer Einfluß sowohl in der Schreibweise des Lexems know als auch in der Schreibweise des Lexems important deutlich. Dabei basiert die Schreibweise des Lexems know auf einer unbewußten overgeneralization, kann aber zusätzlich auch auf lack of contrast zurückzuführen sein. Die Einstufung als unbewußte overgeneralization ist berechtigt, weil die Fehlleistung im Probandenbeitrag nur in diesem Satz beobachtet werden konnte, während das Lexem ansonsten richtig geschrieben wurde. Lack of contrast ist als Fehlerentstehungsursache in Betracht zu ziehen, weil die Lexeme know und now sich optisch ähneln.

Das Fehlerbeispiel important, das auf eine Vokalverwechslung zurückzuführen ist, verdeutlicht sowohl den Einfluß von interlingual transfer als auch den Einfluß der Fehlerkategorien generalization und lack of contrast. Interlingual transfer zeigt sich - wie erwähnt - darin, daß deutsche Muttersprachler das Phonem /æ/ häufig durch das Phonem /e/ ersetzen, da sie den Vokal/æ/ nicht

\footnotetext{
653 vgl. ebd.

${ }^{654}$ vgl. Juhász 1970

655 vgl. ebd.
} 
immer normgerecht artikulieren können und daher auf einen Laut zurückgreifen, der sowohl im Deutschen als auch im Englischen zu finden ist und dabei dem zielsprachlichen Element ähnelt. Aufgrund dieser Ähnlichkeit beruht die Abweichung gleichzeitig auch auf lack of contrast. Schließlich ist die Abweichung auch der Kategorie generalization zuzuordnen, da hier ein zielsprachliches Phonem auf ein Lexem der Zielsprache übertragen wurde, das diesen Laut nicht enthalten sollte. 


\section{Zusammenfassung der Untersuchungsergebnisse}

\section{Vergleich der Untersuchungsergebnisse mit den Ergebnissen früherer Studien zur Fehlertypologie deutscher Englischlernender}

Der erste Untersuchungsaspekt der vorliegenden Arbeit, die fehlertypologische Analyse der Abweichungen deutscher Englischlernender, hat zu folgenden Ergebnissen geführt: Der Vergleich der einzelnen Fehlertypen hat gezeigt, daß die Art der Abweichungen, die deutsche Englischlernende bei Anwendung der englischen Sprache produzieren, trotz unterschiedlicher Voraussetzungen der Lernenden tendenziell übereinstimmen. Dies bedeutet, Englischlernende, die Deutsch als Erstsprache erworben haben, erzeugen Fehlertypen, die - ungeachtet partieller Übereinstimmungen mit Lernenden anderer Erstsprachen - für deutsche Muttersprachler bezeichnend sind. Diese tendenziellen Übereinstimmungen sind auch dann zu beobachten, wenn sich die einzelnen deutschen Englischlernenden in bezug auf die Faktoren Ausbildung, Auslands- und Anwendungserfahrungen, emotionale Einstellung zur Zielsprache, Alter und Geschlecht voneinander unterscheiden. Zusammenfassend ist also auf der Grundlage der hier gewonnenen Erkenntnisse zu bestätigen, daß deutsche Englischlernende über eine charakteristische Fehlertypologie verfügen.

Im Hinblick auf die Art der ermittelten Abweichungen stimmen die Resultate partiell mit den zum Vergleich herangezogenen Studien ${ }^{656}$ überein, lassen dabei jedoch auch erkennen, daß die Ergebnisse vorhergehender Studien in bezug auf das quantitative Auftreten der einzelnen Fehlertypen einer entscheidenden Ergänzung bedürfen. Zunächst bestätigen die Ergebnisse das Vorkommen einheitlicher Merkmale im Verlauf des Spracherwerbs deutscher Englischlernender. Die Beiträge der Teilnehmer zeigen, daß deutsche Englischlernende bereits in der Frühphase des Zielspracherwerbs in der Lage sind, elementare Strukturen der englischen Sprache in einer Form anzuwenden, die von englischen Muttersprachlern oder Englischlernenden anderer Erstsprachen verstanden wird. Dabei zeigt die Art und Anzahl der ermittelten Verstöße jedoch, daß diese Kommunikationsfähigkeit nicht notwendigerweise mit einer geringen Fehlerproduktion einhergeht. Vielmehr ist den hier gewonnenen Fehlerergebnissen zu entnehmen, daß die bei deutschen Muttersprachlern häufig vertretene Meinung, der Erwerb der englischen Sprache erfolge in der Regel, etwa im Vergleich zur französischen, problemlos und die Anzahl der Normabweichungen sei - anders als beim Erwerb romanischer Sprachen - unbedeutend, nicht bestätigt werden kann.

${ }^{656}$ Siehe hierzu exemplarisch: Grabowski 1985; Hecht / Green 1983; Legenhausen 1985. 
Ganz im Gegensatz zu dieser Meinung lassen die Fehlerergebnisse erkennen, daß selbst in äußerst fortgeschrittenen Erwerbsstadien die Anwendung von Strukturen der englischen Sprache, die in der Frühphase des Englischunterrichts vermittelt wurden, Abweichungen verursachen kann. Die weitverbreitete Auffassung, die Sprachverwandtschaft zwischen dem Deutschen und dem Englischen erleichtere deutschen Muttersprachlern das Erlernen der englischen Sprache, kann daher nur bedingt akzeptiert werden. Dabei zeigen die Ergebnisse dieser Arbeit, daß die Fehler alle linguistischen Ebenen umfassen. Wenngleich diese Erkenntnis auch aus anderen Fehleruntersuchungen hervorgeht, zeigt sich in der vorliegenden Arbeit deutlicher als in vorhergehenden Studien, daß die Fehlerverteilung in den Bereichen Lexik, Syntax/Morphologie und Orthographie ungleichmäßig ist.

Im Gegensatz zu der ausgewogeneren Verteilung, die in anderen Studien konstatiert wird, wurden die meisten Anwendungsschwierigkeiten der Probanden in vorliegender Untersuchung auf syntaktischmorphologischer Ebene ermittelt. Die Fehler, die auf dieser Ebene auftraten, betrafen alle Elemente, deren Anwendung die Struktur eines Satzes oder einzelner Satzelemente beeinflußt. Die Abweichungen umfaßten dabei sowohl größere syntaktische Einheiten als auch kleinere Strukturelemente. Zu den Fehlern, die Auswirkungen auf größere syntaktische Einheiten hatten, zählen vor allem die Wort- und die Satzstellungsfehler. Die Fehler dieser Kategorie basierten in der Regel darauf, daß Satzteile oder Lexeme nicht der syntaktischen Struktur der englischen Sprache angepaßt wurden, sondern der Struktur der Erstsprache, anderer Zweitsprachen oder einer syntaktischen Struktur des Englischen, die in dem Kontext, in dem sie zur Anwendung kam, nicht als normgerecht zu bezeichnen ist. Zu den Verstößen der syntaktischen Ebene gehören auch Fehler in den Bereichen des Zeitensystems und der Zustandsformen Aktiv/Passiv.

In bezug auf das Zeitensystem wurde deutlich, daß hier insbesondere die Unterscheidung zwischen dem Gebrauch des simple past und des present perfect zu Normabweichungen führte. Dabei zeigte sich, daß diese Zeitformen im Englischunterricht einer noch deutlicheren Hervorhebung bedürfen, als es die Ergebnisse vorhergehender Studien bereits implizieren. Anwendungsschwierigkeiten zeigten sich jedoch auch bei anderen Zeitformen der Vergangenheit und der Gegenwart sowie bei den verschiedenen Futurtempora. Hierzu zählen die Zeit- und Verlaufsformen past perfect, present simple und present progressive, future progressive, going to-future und will-future. Besonders hervorzuheben ist hierbei das will-future, wobei zu erkennen war, daß diese Zeitform in erster Linie von den Probanden mit geringen syntaktisch-morphologischen Kenntnissen falsch verwendet wurde.

$\mathrm{Zu}$ den Fehlern, die im Bereich der kleineren syntaktischen Einheiten zu beobachten waren, gehört insbesondere der Gebrauch von Satzformen, die sich durch das Hinzufügen oder Auslassen einzelner Morpheme oder Lexeme von anderen Satzformen unterscheiden, jedoch nicht notwendigerweise 
- wie bei der Wort- und Satzstellung - die gesamte Satzstruktur betreffen. Hierzu zählen zum einen der nicht normgerechte Gebrauch der sogenannten aspects - wie etwa der progressive form -, der Artikel, der Adjektiv- und Adverbialformen sowie der fehlerhafte Gebrauch der grammatischen Strukturen Gerundium, Partizip, Infinitiv und der sogenannten if-clauses. Zum anderen umfaßt diese Gruppe die Anwendung der unterschiedlichen Pluralmorpheme und des Elements $s$ in der 3. Person Singular sowie der Verb-Endung -ed bei regelmäßigen Verben. Dabei wurden diese Elemente teils redundanterweise hinzugefügt und teils ausgelassen, obwohl ihre Verwendung im gegebenen Kontext notwendig gewesen wäre.

Der nächstgrößte Fehlerkomplex trat auf lexikalischer Ebene auf. Die Fehler, die hier zu beobachten waren, betrafen sowohl Lexeme, die grammatische Funktionen übernehmen, wie etwa Auxiliare, als auch Lexeme, die in erster Linie semantisch von Bedeutung sind, beispielsweise Substantive und Verben. Diese wurden durch andere Elemente der betreffenden Wortgruppe ersetzt, wodurch die beabsichtigte Aussage des Lernenden semantisch verändert wurde. Weiterhin umfaßt der Komplex der lexikalischen Ebene auch sogenannte inexistente Wörter, d.h. Wörter, die von den Lernenden erfunden wurden und weder in der Erst- noch in der Zielsprache des Lernenden existieren.

Der drittgrößte Fehlerkomplex entstammte dem Bereich der Orthographie. Die Fehler, die hier ermittelt wurden, umfassen sowohl den Themenbereich Groß-/Kleinschreibung als auch die fehlerhafte Schreibweise innerhalb einzelner Lexeme, die in der vorliegenden Arbeit der Kategorie ,Rechtschreibung' zugeordnet wurde.

Neben den allgemeinen Ergebnissen, die bei Gegenüberstellung der linguistischen Ebenen deutlich wurden, zeigte sich bei genauerer Betrachtung der einzelnen Fehlertypen, daß im Vergleich zu den Ergebnissen vorhergehender Studien auf lexikalischer Ebene eine Verlagerung der Fehlerhäufigkeit zu erkennen war. Die Schwierigkeiten beim Gebrauch der Lexemgruppen ,Hilfsverben“ und ,Adjektive/Adverbien', die in der fachdidaktischen Literatur besonders betont werden, kamen in der Studie, die der vorliegenden Arbeit zugrunde liegt, weniger deutlich zum Tragen. Vielmehr ist anhand der Anzahl der Verstöße in diesem Bereich festzustellen, daß sich diese Lexemgruppen auf der unteren bis mittleren Stufe der Schwierigkeitshierarchie befinden. Dies gilt gleichermaßen für Lernende mit erheblichen lexikalischen Schwierigkeiten wie für Lernende mit überdurchschnittlich hohen lexikalischen Kenntnissen.

Insbesondere im Falle der Hilfsverben wurde deutlich, daß die Lernenden beider Gruppen durchaus in der Lage waren, zwischen dem Gebrauch der einzelnen englischen Auxiliare zu differenzieren. Die 
diesbezüglichen Schwierigkeiten, die fachdidaktischen Aussagen nach bei deutschen Englischlernenden bestehen, äußern sich erst dann, wenn die Lernenden entscheiden müssen, welche Form eines bestimmten Hilfsverbs im gegebenen Kontext zu verwenden ist. Auch beim Gebrauch der Lexemgruppen Adjektiv und Adverb entstanden Anwendungsschwierigkeiten nur bei den sogenannten Ausnahmen. Dies betrifft vor allem Lexeme, die in bestimmten Zusammenhängen strukturell keinen Unterschied zwischen der Adjektiv- und der Adverbialform erkennen lassen, wie etwa das Lexem fast. Gleichzeitig ist in diesem Rahmen zu konstatieren, daß Verstöße beim Gebrauch der Adverbien, die darauf basieren, daß statt der Adverbialform, insbesondere prädikativ, die Form eines Adjektivs benutzt wird - wie etwa in der Äußerung she works quick - häufig auch bei englischen Muttersprachlern zu beobachten sind. Somit sind sie nicht als typisch deutsche Verstöße zu bezeichnen, sondern lediglich als Fehler, die u. a. bei deutschen Englischlernenden auftreten.

Weiterhin zeigte sich in der Untersuchung, daß Wortwahlfehler, d. h. die abweichende Verwendung von Lexemen, die ausschließlich semantische Funktionen übernehmen, entgegen ihrer Einordnung in der fachdidaktischen Literatur einer stärkeren Hervorhebung bedürfen. Dieser Fehlertyp stand den Ergebnissen vorliegender Arbeit zufolge sowohl bei den PmeilAb als auch bei den PwenlAb auf der obersten Stufe der Schwierigkeitshierarchie. Dabei hat die genauere Analyse dieser Fehler gezeigt, daß der Bereich Wortwahl nicht nur im gleichen Ausmaß zu behandeln ist wie etwa grammatisch bedeutende Lexemgruppen, sondern sogar zu den Schwerpunktbereichen der lexikalischen Ebene gehören sollte, weil die Entstehung von Abweichungen in diesem Bereich nicht als ausschließlich linguistisches Problem zu betrachten ist, sondern oftmals auch auf interkulturellen Interferenzen beruht. Dies bedeutet, Wortwahlfehler entstehen häufig dadurch, daß die Lernenden aufgrund mangelnder Kenntnisse der Kultur englischsprachiger Länder, wie etwa deren Humor, Höflichkeitsnormen etc., nicht in der Lage sind, zwischen dem Gebrauch einzelner englischer Lexeme zu differenzieren.

Zudem wurde im Verlauf des Analyseverfahrens deutlich, daß die beständige Vermittlung neuer Wortschatzelemente nicht unbedingt zu einer Wissensbereicherung der Lernenden führen muß, sondern auch kontraproduktiv sein kann, weil die Differenzierungsschwierigkeiten durch die Vermittlung neuer Lexeme zunehmen können. Vielmehr erscheint es im Hinblick auf eine Fehlerreduktion gerade bei fortgeschrittenen Englischlernenden wichtig, zu einem gegebenen Zeitpunkt die Vermittlung neuer Lexeme zu reduzieren und stattdessen $\mathrm{zu}$ verdeutlichen, inwieweit sich die bereits erworbenen Lexeme semantisch voneinander unterscheiden.

Differenzen zu früheren Studien wurden auch bezüglich der Art der einzelnen Wortwahlfehler deutlich. So zeigte sich im Rahmen des Korrekturverfahrens, daß innerhalb der Fehlerkategorie, die in der 
Fremdsprachendidaktik als false friends bezeichnet wird, ${ }^{657}$ Veränderungen stattgefunden haben. In diesem Bereich traten Elemente, die in der Vergangenheit häufig beobachtet worden waren, in der vorliegenden Studie nur vereinzelt auf. Selbst bei den PmeilAb konnte nur in wenigen Fällen nachgewiesen werden, daß false friends, die traditionell als typische Fehlerelemente deutscher Englischlernender bezeichnet werden ${ }^{658}$ - wie etwa die Elemente become oder actually - immer noch als fehlerevozierend zu betrachten wären. Vielmehr ist zu erkennen, daß deutsche Muttersprachler, die heutzutage Englisch lernen, die Probleme, die mit den zuvor genannten oder ähnlichen Lexemen verbunden sind, weitgehend überwunden haben. Stattdessen zählen gegenwärtig vornehmlich solche Lexeme zur Gruppe der false friends, die nicht nur optisch und akustisch mit deutschen Lexemen zu vergleichen sind, sondern teils sogar semantisch dem deutschen Element entsprechen, dem sie ähneln. Dies sind Lexeme wie beispielsweise die englischen Wörter learn oder bring, die in manchen Zusammenhängen tatsächlich als ,lernen‘ bzw. ,bringen‘ übersetzt werden können, in anderen Fällen jedoch abweichende Bedeutungen besitzen.

Eine weitere Differenz zu früheren Studien war in bezug auf die Fehlerkategorie ,inexistente Wörter‘ zu erkennen. Während in diesen Studien inexistente Wörter als Fehlertyp nur als Randerscheinung betrachtet wurden, zeigte sich in der Untersuchung, die der vorliegenden Arbeit zugrunde liegt, daß gerade bei Lernenden, die sich in der Frühphase des Erwerbs der englischen Sprache befinden, der Gebrauch inexistenter Wörter zu einem der häufigsten Fehlertypen gehört. Dies ist zum einen dadurch begründet, daß Englischlernende heutzutage häufig Wörter erlernen, die akustisch an ein englisches Lexem erinnern bzw. auch zum Lexembestand der englischen Sprache gehören, jedoch in der Form, in der sie vom Lernenden benutzt werden, als inexistente Wörter zu betrachten sind. Zu diesen gehört beispielsweise das Wort handy, das eine Übersetzung des deutschen Adjektivs , bequem‘ bildet. Seine Verwendung als Synonym für das englische Kompositon mobile phone stellt eine typische Fehlleistung deutscher Englischlernender dar.

In anderen Fällen ist der Gebrauch inexistenter Wörter vermutlich darauf zurückzuführen, daß sich das sprachliche Verhalten der Lehrenden verändert hat, d. h., das Korrekturverhalten heutiger Fremdsprachenlehrer und ihre Einstellung gegenüber sprachlichen Fehlern sind in der Regel entspannter als in der Vergangenheit. So ist es häufig zu beobachten, daß Fremdsprachenlehrer im Bemühen, den Redefluß der Lernenden nicht zu hemmen, sprachliche Fehler nicht oder nur ansatzweise korrigieren. Dadurch entsteht bei vielen Englischlernenden das Bewußtsein, daß es das wichtigste Ziel sei, den Kommunikationsverlauf fortzusetzen, auch wenn dies nur durch das Erfinden von Ersatzwörtern für nicht abrufbare Elemente oder durch die direkte Übernahme erstsprachlicher Elemente möglich ist.

\footnotetext{
657 vgl. Helliwell 1989

658 vgl. Brough / Wittmann 2001, 143
} 
Darüber hinaus sind die linguistischen Möglichkeiten, d. h. die Möglichkeiten neben den Standardsprachen Englisch, Französisch und Latein auch andere Sprachen zu erlernen, heutzutage vielfältiger als in der Vergangenheit, weil viele Schulen weitere Sprachen anbieten. Dies erhöht das Risiko, daß Englischlernende, die parallel auch andere Zweitsprachen erwerben, linguistische Elemente dieser Sprachen beim Gebrauch des Englischen miteinbeziehen. Hinzu kommt, daß der Einfluß unterschiedlicher Kulturen und damit auch der Sprachen dieser Kulturen in Deutschland zugenommen hat, so daß ein Teil der deutschen Englischlernenden nicht nur Deutsch als Erstsprache spricht, sondern zusätzlich auch andere europäische oder außereuropäische Sprachen. Dies kann dazu führen, daß die Lernenden bei Anwendung des Englischen auch auf andere Erstsprachen zurückgreifen und dadurch Wörter prägen, die nicht zum Lexembestand des Englischen gehören.

Neben diesen Differenzen zu vorangegangenen Studien wurde im Rahmen der Untersuchung deutlich, daß die Hervorhebung der Probleme, die in der Fachdidaktik in bezug auf den Gebrauch der Lexemgruppe ,Präposition' beschrieben werden, ${ }^{659}$ nicht nur gerechtfertigt ist, sondern daß die Beschäftigung mit diesen sogar noch einer Intensivierung bedarf. Diese Erkenntnis beruht darauf, daß die Anzahl der Präpositionsfehler sowohl bei den PmeilAb als auch bei den PwenlAb größer war als bei anderen Lexemen, die grammatische Funktionen übernehmen sowie darauf, daß auch die Genese der Präpositionsfehler als äußerst komplex zu betrachten ist. Diese Komplexität entsteht zum einen dadurch, daß die Anwendungsregeln der englischen Präpositionen nicht immer deutlich definiert sind ${ }^{660}$ - daher können Präpositionsfehler auch bei englischen Muttersprachlern beobachtet werden. ${ }^{661}$ Zum anderen bestehen zwischen vielen englischen und deutschen Präpositionen optische und akustische Gemeinsamkeiten. Diese Übereinstimmungen führen oft zu Fehlern, weil die sich ähnelnden Elemente in der Regel in den beiden Sprachen nicht denselben Anwendungsbeschränkungen unterliegen.

\section{Die Anwendbarkeit der Fehlererklärungsfaktoren der Kontrastiv-, der Identitäts- und der Interlanguagehypothese}

Bei der Überprüfung der Anwendbarkeit der einzelnen Fehlererklärungsfaktoren konnten in bezug auf die linguistischen Ebenen Lexik, Syntax-Morphologie und Orthographie nachfolgend dargestellte Ergebnisse ermittelt werden.

\footnotetext{
659 vgl. Burgschmidt 1975

${ }^{660}$ vgl. Swan 1990, Textstelle 483, o. S.

661 Dies zeigte sich wiederholt in Gesprächen mit englischen Muttersprachlern. Vgl. hierzu auch Crystal, 65 - 67.
} 


\subsection{Lexikalische Ebene}

Auf lexikalischer Ebene zeigte sich, daß die Faktoren der Identitätshypothese nur ansatzweise nachgewiesen werden konnten und selbst in den wenigen Fällen, in denen eine Beteiligung von biologisch determinierten Faktoren an der Fehlerentstehung nicht auszuschließen war, kein wissenschaftlich nachvollziehbarer Beweis für diese Beteiligung erbracht werden konnte. Diese Beobachtung muß nicht zwingend bedeuten, daß lexikalische Fehler deutscher Englischlernender in keiner Form auf natürliche Sequenzen des Spracherwerbs zurückzuführen sind. Die Spekulationen, die beim Versuch, diese Sequenzen für die Fehlerentstehung verantwortlich zu machen, angestellt werden müssen, sind jedoch zu vage, als daß die Identitätshypothese in bezug auf die lexikalischen Abweichungen als geeignetes Fehleranalysemodell betrachtet werden könnte.

Bei Anwendung der Fehlererklärungsfaktoren der Kontrastiv- und der Interlanguagehypothese zeigte sich hingegen in allen Fehlerkategorien der lexikalischen Ebene, daß sowohl erstsprachliche als auch strategisch-prozedurale Faktoren an der Fehlerentstehung beteiligt waren, deren Einfluß jedoch bei jedem Fehlertyp in unterschiedlichem Maße zum Tragen kam. Der Vergleich der Anwendbarkeit dieser Faktoren zeigte bei Gegenüberstellung der beiden entgegengesetzten Probandengruppen, d. h. den PmeilAb und den PwenlAb, daß die Nachweisbarkeit der Faktoren der Interlanguagehypothese in beiden Gruppen überwog. Somit konnte der Einfluß erstsprachlicher Faktoren weniger häufig nachgewiesen werden als strategisch-prozedurale Fehlerentstehungsgründe.

Wenngleich dieses Ergebnis zunächst zu der Annahme führt, daß folglich die Bedeutung der Interlanguagehypothese größer ist als die der Kontrastivhypothese, ist einer genaueren Betrachtung des Analyseverfahrens zu entnehmen, daß dieser Annahme nur mit größter Einschränkung zuzustimmen ist. Zum einen ist die Differenz zwischen der Anwendbarkeit der beiden Hypothesen in beiden Probandengruppen quantitativ als gering zu bezeichnen. Zum anderen resultiert die Differenz in der Anwendbarkeit beider Hypothesen vor allem daraus, daß die Fehlererklärungsfaktoren der Interlanguagehypothese flexibler sind als die der Kontrastivhypothese. Erstere verfügen über einen größeren Zuordnungsspielraum und bieten damit mehr Interpretationsmöglichkeiten, weil die Bezeichnungen der einzelnen Fehlerentstehungsfaktoren oftmals weniger spezifisch sind als die der Kontrastivhypothese.

Somit wird in der Interlanguagehypothese weniger eindeutig festgelegt, welche Fehlertypen den einzelnen Kategorien zuzuordnen sind. So ist zum Beispiel der Bezeichnung Substitution, die im Rahmen der Kontrastivhypothese geprägt wurde, deutlich zu entnehmen, daß diese Kategorie Fehlertypen umfaßt, die auf dem Ersetzen lexikalischer Elemente basieren. Da die ursprüngliche Version der Kontrastivhypothese, zu der auch die Kategorie ,Substitution` gehört, ausschließlich auf einem Ver- 
gleich der Erst- mit der Zielsprache beruht, besteht kein Zweifel daran, daß es sich hier um das Ersetzen zielsprachlicher Elemente durch erstsprachliche handelt. In der Interlanguagehypothese hingegen wird beispielsweise für eine Kategorie wie die Fehlergruppe interlingual transfer nicht eindeutig festgelegt, welche Form des interlingualen Transfers diese Kategorie letztendlich umfaßt - d. h., es wird hier nicht klar definiert, ob die Übertragungen aus der Erstsprache oder aus anderen Zweitsprachen stammen. Falls andere Zweitsprachen miteinbezogen werden, ist ebenfalls nicht zu erkennen, welche Sprachen jeweils zum Tragen kommen.

Dieses Zuordnungsproblem wird nicht nur im Bereich der Kommunikationsstrategien deutlich, sondern auch in bezug auf die Lernstrategien. So definiert zum Beispiel die als simplification ${ }^{662}$ bezeichnete Lernstrategie nicht, welche Vereinfachungsformen der Begriff umfaßt. Während des Analyseverfahrens stellte sich etwa die Frage, ob der Verzicht auf den Gebrauch der Adverbialendung -ly als Ergebnis einer Vereinfachungsstrategie zu betrachten ist, die der Lernende bereits während des Erlernens englischer Elemente anwandte, um den Überblick über die unterschiedlichen Lexemformen zu behalten oder ob dieser Fehler darauf beruhte, daß der Lernende glaubte, er müsse auf die Endung -ly verzichten, weil hier eine der sogenannten Ausnahmen vorliegt, wie etwa bei den Lexemen hard und fast.

Diese Zuordnungsschwierigkeiten bzw. Interpretationsmöglichkeiten führen schließlich dazu, daß der Fehlerlinguist während der Analyse sprachlicher Fehler mehr Möglichkeiten hat, Abweichungen der Interlanguagehypothese zuzuordnen als anderen Hypothesen und in der Regel von diesen Möglichkeiten Gebrauch macht, um dadurch zu verhindern, daß etwa ein wichtiger Fehlerentstehungsaspekt übersehen wird. Da solche Schwierigkeiten - wie erwähnt - bei der Kontrastivhypothese kaum auftreten, ist diese für die Erklärung lexikalischer Abweichungen nahezu ebenso bedeutend wie die Interlanguagehypothese.

In diesem Zusammenhang ist darauf hinzuweisen, daß die Tatsache, daß eine Erwerbshypothese mehr Zuordnungsergebnisse verzeichnet als eine andere, nicht notwendigerweise bedeutet, daß die Hypothese mit geringeren Zuordnungszahlen als weniger hilfreiches Fehleranalyseinstrumentarium zu betrachten ist, oder daß der Fehlerentstehungsaspekt der betreffenden Hypothese eine untergeordnete Bedeutung hat. Vielmehr sind häufig die Fehlerentstehungsaspekte der Hypothese, die weniger Eintragungen enthält, im Hinblick auf ein zukünftiges Vermeiden des Fehlers entscheidender als die Fehlerentstehungsfaktoren der These, die mehr Zuordnungsmöglichkeiten bietet. So kann zum Beispiel eine Äußerung wie he is a friend of me, die aufgrund der Verwendung des Pronomens me als Abweichung zu bezeichnen ist, in der Tat als Ergebnis einer Vereinfachungsstrategie und einer Reduktionsstrategie

${ }^{662}$ vgl. hierzu Richards 1975, $115-126$ 
bezeichnet werden: Indem der Lernende die Anwendungsbeschränkungen des Wortes me mißachtet, vermeidet er, zwischen den unterschiedlichen Übersetzungsformen des deutschen Lexems ,mir ${ }^{`} \mathrm{zu}$ unterscheiden und benutzt me auch dann, wenn eigentlich das Pronomen mine zu verwenden wäre. Darüber hinaus kann der Fehler auch als Ergebnis einer unbewußten Übergeneralisierung gedeutet werden, weil der Lernende eventuell so häufig mit dem Wort me konfrontiert wurde, daß dadurch andere Pronomina, die in seinem Wissensspeicher festgehalten sind, verdrängt wurden. Somit bietet die Interlanguagehypothese gleich drei Interpretationsmöglichkeiten für eine einzige Abweichung.

Aus der Perspektive der Kontrastivhypothese hingegen läßt die Abweichung maximal zwei Zuordnungsmöglichkeiten zu. Dies ist zum einen die Einordnung in die Kategorie ,Substitution', weil der Fehler auch darauf zurückzuführen ist, daß der Lernende nach einer Übersetzung des deutschen Lexems ,mir' suchte und das englische Lexem me diesem akustisch und optisch ähnelt. Zum anderen kann es sich hier auch um eine durch die Erstsprache Deutsch verursachte Unterdifferenzierung handeln, wenn der Lernende zu einem früheren Zeitpunkt verinnerlicht hat, daß das englische Lexem me unter bestimmten Umständen zur Übersetzung des deutschen Lexems ,mir` benutzt wird und er, obwohl er weitere Übersetzungsmöglichkeiten kennt, aufgrund der Ähnlichkeit zwischen me und ,mir‘ das Wort mine außer acht läßt.

Wenngleich somit mehr Zuordnungsmöglichkeiten zu den Erklärungsfaktoren der Interlanguagehypothese bestehen, ist die Fehlerentstehungsursache, die aus der Kontrastivhypothese abzuleiten ist, in Hinblick auf eine zukünftige Fehlervermeidung bedeutender, d. h. weniger leicht zu beseitigen. Als Ergebnis einer Vereinfachungs- oder Reduktionsstrategie wie auch einer unbewußten Übergeneralisierung könnte dem Fehler möglicherweise durch eine Veränderung des Lernkonzepts und durch fortdauernde Korrektur begegnet werden. Erstsprachlicher Einfluß als Fehlerentstehungsursache ist hingegen weitaus resistenter gegen Lern- und Lehrmethoden, weil das erstsprachliche Wissen in der Regel mental so tief verankert ist, daß Versuche der Einflußnahme von außen oftmals erfolglos sind. Unter der Bezeichnung ,tief verankert' ist hier zu verstehen, daß Wissenselemente, die in früher Kindheit erworben wurden, fester gespeichert sind als Wissenselemente, die der Lernende in späteren Lebensphasen aufgenommen hat.

Während also die Gestaltung des zweitsprachlichen Lernkonzepts und die Aneignung einer Reduktionsstrategie sowie die bewußte und die unbewußte Anwendung zweitsprachlichen Wissens erst in späteren Lebensphasen erfolgen, sind erstsprachliche Aspekte mit den Ursprungserfahrungen des Lernenden verknüpft. Sie sind damit ein festerer Bestandteil der Persönlichkeit des Lernenden als solche Aspekte, die in späteren Lebensphasen erworben wurden. Darüber hinaus sind die Komponenten, die den Erwerb der Erstsprache bestimmen, nicht nur psychologischer, sondern oft auch bio- 
logischer Natur. ${ }^{63}$ Daher sind Fehlerentstehungsfaktoren, die auf erstsprachlichem Einfluß basieren, komplexer als strategisch-prozedurale Entstehungsfaktoren.

Neben diesen allgemeinen Ergebnissen und Aussagen zur Anwendbarkeit der Erklärungsfaktoren der einzelnen Hypothesen wurden detailliertere Erkenntnisse in bezug auf die verschiedenen lexikalischen Fehlerkategorien gewonnen, die im folgenden erläutert werden. Im Falle der Lexemgruppe ,Hilfsverben' war der Unterschied zwischen der Anwendbarkeit der beiden Hypothesen in der Gruppe der PmeilAb gering. Diesem Ergebnis ist zu entnehmen, daß die Entstehung von Hilfsverbfehlern bei Lernenden mit großen lexikalischen Lernschwierigkeiten auf erstsprachlichen und strategisch-prozeduralen Faktoren in gleicher Verteilung beruht. Konkret basieren die Hilfsverbfehler bei diesen Lernenden darauf, daß sich die deutschen und englischen Hilfsverben partiell ähneln, die englischen Hilfsverben jedoch anderen Anwendungsbeschränkungen unterliegen als die deutschen.

Bei deutschen Englischlernenden mit überdurchschnittlich hohen lexikalischen Kenntnissen hingegen sind strategisch-prozedurale Faktoren stärker an der Entstehung der Hilfsverbfehler beteiligt als der Einfluß der Erstsprache Deutsch. Diese Erkenntnis läßt den Schluß zu, daß in bezug auf die Lexemgruppe ,Hilfsverben` mit zunehmenden lexikalischen Kenntnissen eine Reduktion erstsprachlichen Einflusses bei der Fehlerentstehung einhergeht. Die Art des erstsprachlichen Einflusses ist jedoch bei Lernenden mit extremen lexikalischen Schwierigkeiten und Lernenden mit fortgeschrittenen lexikalischen Kenntnissen identisch. Bei beiden Lernertypen zeigt sich der Einfluß der Erstsprache Deutsch daran, daß zielsprachliche Elemente durch andere Elemente der Zielsprache ersetzt werden, die erstsprachlichen strukturell ähneln, und daran, daß die Lernenden die Anwendungsbeschränkungen englischer Hilfsverben mißachten, weil sie sich aufgrund optischer und akustischer Übereinstimmungen mit deutschen Hilfsverben an Regeln orientieren, die nur für die deutschen Lexeme gelten.

In bezug auf den Einfluß strategisch-prozeduraler Faktoren sind die Fehlerentstehungsursachen bei Lernenden mit großen lexikalischen Defiziten komplexer. Während die Fehlerentstehung bei diesen Lernenden sowohl auf Reduktionen als auch auf Generalisierungen und Kontrastmangel zurückzuführen ist, basiert die Entstehung der Abweichungen bei fortgeschrittenen Lernenden im wesentlichen auf bewußt und unbewußt stattfindenden Generalisierungen. Reduktionsstrategien sind hier nur von untergeordneter Bedeutung.

Bei der Analyse der Fehlerkategorie ,Wortwahl' wurde deutlich, daß - wie zuvor - sowohl bei Lernenden mit großen lexikalischen Anwendungsschwierigkeiten als auch bei fortgeschrittenen Lernenden die strategisch-prozeduralen Fehlerentstehungsfaktoren überwiegen. Die Differenz zwischen

663 vgl. Szagun 2000, $185-187$ 
dem Einfluß der Erstsprache und den strategisch-prozeduralen Faktoren ist in dieser Kategorie bei Lernenden mit geringen lexikalischen Kenntnissen größer als bei den Hilfsverben. Bei Lernenden mit überdurchschnittlichen lexikalischen Fähigkeiten hingegen ist die Differenz zwischen dem Einfluß der Faktoren der Kontrastiv- und der Interlanguagehypothese hier geringer, wenngleich auch hier der Einfluß strategisch-prozeduraler Faktoren überwiegt. Die genauere Analyse zeigte dabei, daß - ähnlich wie bei den Hilfsverben - in bezug auf die Art und Weise erstsprachlichen Einflusses beim Vergleich der beiden Probandengruppen keine Differenzen bestanden. Die Ergebnisse zeigen, daß die Wortwahlfehler sowohl bei deutschen Englischlernenden mit erheblichen lexikalischen Schwierigkeiten als auch bei Englischlernenden, die über ein großes lexikalisches Wissen verfügen, auf Substitutionen und Unterdifferenzierungen basieren. Der Einfluß strategisch-prozeduraler Faktoren ist bei Lernenden mit erheblichen lexikalischen Anwendungsschwierigkeiten komplexer. Während bei diesen Lernenden sowohl Reduktionsstrategien als auch interlingualer Einfluß, Generalisierungen und Kontrastmangel zum Tragen kommen, konzentriert sich der strategisch-prozedurale Einfluß bei fortgeschrittenen Lernenden auf das Generalisieren von Interlanguageelementen. Reduktionsstrategien sind nur sehr geringfügig an der Fehlerentstehung beteiligt.

Der Gebrauch inexistenter Wörter ist bei deutschen Englischlernenden mit erheblichen Lexikdefiziten hauptsächlich auf den Einfluß der deutschen Sprache zurückzuführen, wenngleich die Auswirkungen strategisch-prozeduraler Faktoren auch maßgeblich zur Fehlerentstehung beitragen. Auch bei fortgeschrittenen Lernenden trägt der Einfluß der Erstsprache erheblich zur Entstehung der Fehler bei, hier überwiegt jedoch der Einfluß von Strategien und Prozessen während der Sprachanwendung. Übereinstimmend ist jedoch wie zuvor festzustellen, daß die Art und Weise des Einflusses der Erstsprache Deutsch in beiden Gruppen identisch ist und sich darin äußert, daß zielsprachliche Elemente durch andere Elemente ersetzt werden, die strukturell deutschen Lexemen ähneln.

Interlanguagespezifisch betrachtet ist die Entstehung der inexistenten Wörter bei Lernenden mit erheblichen lexikalischen Schwierigkeiten darauf zurückzuführen, daß sie zum Ausgleich von Kommunikationslücken gezielt entweder auf die Erstsprache oder auf andere Zweitsprachen zurückgreifen. Bei Lernenden mit überdurchschnittlichen lexikalischen Kenntnissen hingegen ist der Kontrastmangel zwischen deutschen und englischen Elementen bzw. zwischen einzelnen englischen Lexemen für die Fehlerentstehung verantwortlich.

Bei den Präpositionsfehlern zeigte die Anwendung der Kontrastivhypothese, daß die Abweichungen in beiden Probandengruppen im wesentlichen dadurch entstanden, daß Lexeme, die eigentlich zu erwarten gewesen wären, durch Präpositionen ersetzt wurden, die Ähnlichkeiten zu deutschen Präpositionen aufweisen. Hierbei zeigte sich, daß die Theorie, auf der die Kontrastivhypothese basiert, nur partiell befürwortet werden kann und daß einem entscheidenden Kritikpunkt, der sich gegen die 
Validität der Kontrastivhypothese richtet, zuzustimmen ist: Gegner der Kontrastivhypothese haben immer wieder bemängelt, daß die Hypothese ihren Schwerpunkt auf den Vergleich struktureller Unterschiede zwischen Erst- und Zielsprache legt und dabei außer acht läßt, daß gerade auch strukturelle Übereinstimmungen zur Entstehung von Fehlern beitragen können. ${ }^{664}$ Die in vorliegender Arbeit durchgeführte Analyse der Präpositionsfehler zeigte dieser Kritik entsprechend, daß die meisten Präpositionsfehler darauf zurückzuführen sind, daß eine Reihe der englischen Präpositionen deutschen Präpositionen aufgrund der gemeinsamen sprachgeschichtlichen Herkunft beider Sprachen optisch und akustisch ähnelt. Die Ähnlichkeit von englischen und deutschen Präpositionen als Fehlerentstehungsursache wurde sowohl bei den PmeilAb als bei den PwenlAb beobachtet.

Auch aus strategisch-prozeduraler Sicht wurden beim Vergleich der Probandengruppen Übereinstimmungen ermittelt. Sowohl bei den PmeilAb als auch bei den PwenlAb waren die meisten Fehler darauf zurückzuführen, daß bereits erlernte Elemente bzw. die mit ihnen verbundenen Anwendungsregeln in Zusammenhängen benutzt wurden, in denen diese Regeln nicht gelten. So kann das deutsche Lexem ,auf` häufig mit dem englischen Lexem on übersetzt werden, in bezug auf bestimmte Gegenstände, wie beispielsweise Bilder, ist das deutsche Wort , auf ' jedoch mit dem Lexem in zu übersetzen. ${ }^{665}$ Diese Übertragungen verlaufen meist bewußt - in beiden Probandengruppen traten allerdings auch Fälle auf, die auf unbewußte Übertragungen schließen lassen. Daneben zeigte sich auf interlanguagespezifischer Ebene, daß auch Kontrastmangel zwischen deutschen und englischen Präpositionen in beiden Gruppen zur Entstehung der Fehler beigetragen hat.

Auf stilistischer Ebene konnten in beiden Probandengruppen die Faktoren, die der Kontrastivhypothese zugrunde liegen, öfter nachgewiesen werden als die der Interlanguagehypothese. Besonders wenig kamen die interlanguagespezifischen Fehlererklärungsfaktoren bei den PwenlAb zum Tragen. Die Hauptentstehungsursache stilistischer Fehler stellt somit sowohl bei deutschen Englischlernenden, die über ein geringes lexikalisches Wissen verfügen als auch bei solchen mit überdurchschnittlichen Lexikkenntnissen die Erstsprache Deutsch dar. Der Einfluß der deutschen Sprache äußert sich jeweils darin, daß sich die Lernenden bei Anwendung stilistischer Formulierungen an Ausdrücken ihrer Erstsprache orientieren. Dabei werden zum einen Elemente der Zielsprache durch andere Elemente ersetzt, die in bezug auf ihre Struktur Assoziationen zur Erstsprache hervorrufen. In anderen Fällen äußert sich der Einfluß der Erstsprache darin, daß die Lernenden nicht zwischen den unterschiedlichen englischen Formulierungen differenzieren, weil diese Differenzierungen im Deutschen redundant wären. Im englischen Sprachraum hingegen wird zwischen den unterschiedlichen codes und registers

\footnotetext{
${ }^{664}$ vgl. Bausch / Kasper 1979, 6 - 7

665 vgl. Swan 1990, Textstelle 88, o. S.
} 
unterschieden, welches dazu führt, daß die Umgangssprache stärker von der formalen Sprache abweicht als im Deutschen (abgesehen von Besonderheiten der regionalen Dialekte im deutschen Sprachraum).

Aus der Sicht der Interlanguagehypothese basierte die Fehlerentstehung bei den PmeilAb vor allem darauf, daß sich die Lernenden auf eine geringe Anzahl lexikalischer Elemente beschränkten, obwohl in den gegebenen Situationen differenziertere Formulierungen notwendig gewesen wären. Bei den PwenlAb hingegen waren die meisten stilistischen Verstöße auf bewußt stattfindende Generalisierungen bereits erworbener Sprachregeln zurückzuführen. Es wurden also stilistische Formulierungen, die während des Spracherwerbs verinnerlicht worden waren, in sprachlichen Zusammenhängen benutzt, in denen sie als Normabweichungen einzustufen sind.

In bezug auf die Verstöße, die im Rahmen der Fehlerkategorie ,Adjektiv' konstatiert wurden, waren sowohl bei den PmeilAb als auch bei den PwenlAb die Faktoren der Interlanguagehypothese häufiger anwendbar als die der Kontrastivhypothese, wobei die Differenz in der Anwendbarkeit der Hypothesen bei den PwenlAb größer war.

Kontrastivanalytisch betrachtet zeigte sich der Einfluß der Erstsprache in beiden Probandengruppen daran, daß Adjektive, die im betreffenden Kontext zur Verdeutlichung der Aussage notwendig gewesen wären, durch Adjektive ersetzt wurden, deren Bedeutung die Lernenden aus dem Deutschen zu kennen glaubten, weil die betreffenden Lexeme deutschen Adjektiven strukturell ähneln. Bei den PwenlAb wurde der Einfluß der deutschen Sprache in einigen Fällen auch daran deutlich, daß sie beim Gebrauch der englischen Adjektive zu wenig differenzierten, weil Differenzierungen in bezug auf die entsprechenden Lexeme des Deutschen nicht erforderlich sind.

Die Analyse der Abweichungen auf der Grundlage der Interlanguagehypothese zeigte, daß bei den PmeilAb die Fehler Resultate sprachlicher Reduktionen und unbewußt stattfindender Übergeneralisierungen waren. Dies bedeutet, die Probanden verwendeten ein Adjektiv, das sie in einer sehr frühen Phase des Zweitspracherwerbs internalisiert hatten und dessen Anwendungsmöglichkeiten sie zu kennen glaubten, weil ihnen das betreffende Lexem vertraut und einfach erschien. Dabei erkannten sie nicht, daß die Aussage, die sie vermitteln wollten, mit diesem Lexem nicht zum Ausdruck gebracht werden kann. Aufgrund der häufigen Anwendungen der betreffenden Lexeme entstanden solche Fehler unbewußt, d. h., ohne daß die Lernenden die Gebrauchsmöglichkeiten des jeweiligen Elements überprüften.

Bei den PwenlAb war vor allem der Versuch, die Bedeutung bestimmter Adjektive zu verallgemeinern, ohne dabei semantische Beschränkungen zu beachten, für die Entstehung der Fehler verantwortlich. 
Zum Teil wurden diese Verallgemeinerungen durch die strukturellen Entsprechungen zwischen deutschen und englischen Adjektiven - wie etwa bei den Lexemen ,interessant ${ }^{\star}$ und interesting hervorgerufen.

Bei der Analyse der Fehler der Kategorie ,Adverb` war die Häufigkeit der Anwendungsmöglichkeiten der verschiedenen Fehlererklärungsfaktoren bei beiden Lernertypen nahezu identisch. Bei den PmeilAb überwog der Anteil der erstsprachlich bedingten Fehler leicht, während bei den PwenlAb geringfügig mehr Fehler auf strategisch-prozedurale Faktoren zurückzuführen waren. Wie bei den vorhergehenden Lexemgruppen äußerte sich der Einfluß der Erstsprache meist in Form von Substitutionen, d. h., die Lernenden benutzten zum Teil Adverbien, die an deutsche Elemente erinnern. Diese Form des erstsprachlichen Einflusses dominierte in allen Probandengruppen.

Der Versuch, die Entstehung der adverbialen Verstöße durch die Fehlerentstehungsfaktoren der Interlanguagehypothese zu erklären, führte zu dem Ergebnis, daß die Fehlergenese bei den PmeilAb sowohl auf Reduktionsstrategien und interlingualen Transfer als auch auf Verallgemeinerungen semantischer Anwendungsmöglichkeiten zurückzuführen war. Die Fehlerentstehung basierte aus der Sicht der Interlanguagehypothese also zum einen darauf, daß die Lernenden bestimmte Adverbien nicht verwendeten, weil ihnen die Bedeutung der Lexeme nicht vertraut war. In anderen Fällen verzichteten sie auf Adverbien bzw. Adverbialformen, weil sie davon ausgingen, daß ihre sprachliche Aussage auch ohne das Anhängen des Suffixes -ly oder sogar eine vollständige Lexemänderung - wie etwa den Wechsel von good zu well - verständlich sein würde. Weiterhin waren die Fehler diesem Ergebnis zufolge auch darauf zurückzuführen, daß sich die Lernenden bei der Anwendung englischer Adverbien an Adverbien anderer Sprachen orientierten bzw. Regeln, die für bestimmte englische Adverbien gelten, auf Elemente übertrugen, die anderen Regeln unterliegen. Darüber hinaus basierten die adverbialen Verstöße in dieser Gruppe auch auf Kontrastmangel zwischen deutschen und englischen Strukturen und auf Kontrastmangel innerhalb der englischen Sprache. Letzterer führte dazu, daß oftmals anstelle von Adverbien Adjektive verwendet wurden, da sich im Englischen die Mehrzahl der Adverbien nur durch die Endsilbe - $l y$ von den entsprechenden Adjektiven unterscheidet. Bei den PwenlAb hingegen konzentrierte sich die Fehlerentstehung interlanguagespezifisch betrachtet auf die bewußte Generalisierung von Interlanguageelementen sowie auf Paraphrasierungen und unbewußte Übergeneralisierungen.

Zusammenfassend betrachtet waren damit die Entstehungsursachen der adverbialen Verstöße im Bereich des Einflusses der Erstsprache nahezu identisch, d. h., in beiden Probandengruppen äußerte sich der Einfluß der Erstsprache in erster Linie darin, daß englische Adverbien durch Lexeme ersetzt wurden, die semantisch oder strukturell deutschen Lexemen ähneln. Die Betrachtung aus interlanguagespezifischer Sicht hingegen zeigte Unterschiede. Während die Fehlerentstehung der 
Interlanguagehypothese zufolge bei den PmeilAb sowohl auf Reduktionsstrategien als auch auf Erweiterungsstrategien und Kontrastmangel zurückzuführen war, basierte die Fehlerentstehung in der entgegengesetzten Gruppe zum einen auf unbewußten Generalisierungen und zum anderen auf dem Versuch, durch Erweiterung des eigenen sprachlichen Potentials Kommunikationsprobleme zu überwinden. Demnach ist das sprachliche Verhalten von Lernenden mit erheblichen lexikalischen Schwierigkeiten vielseitiger und die sprachlichen Handlungen finden ausschließlich bewußt statt. Bei fortgeschrittenen Lernenden konzentriert sich das sprachliche Verhalten auf Generalisierungen, die zum Teil auch unbewußt verlaufen.

Der Vergleich der Ergebnisse, die bei der Analyse der pronominalen Verstöße deutlich wurden, zeigte, daß zwischen beiden Probandengruppen deutliche Unterschiede bestanden. Bei den PmeilAb dominierte eindeutig der Einfluß interlanguagespezifischer Faktoren, der fehlergenetische Einfluß der Erstsprache war von untergeordneter Bedeutung. Bei den PwenlAb hingegen überwog der Einfluß erstsprachlicher Faktoren. Hier kamen interlanguagespezifische Aspekte weniger zum Tragen. Der Unterschied zwischen den Anwendungsmöglichkeiten beider Faktoren war in dieser Gruppe jedoch gering.

Innerhalb der Unterkategorien der Kontrastiv- und der Interlanguagehypothese zeigte sich, daß erstsprachlicher Einfluß bei den PmeilAb nur in Form von Substitution auftrat. In der entgegengesetzten Probandengruppe äußerte sich der Einfluß der Erstsprache zusätzlich darin, daß die Lernenden nicht zwischen dem Gebrauch deutscher und englischer Pronomen differenzierten und erstsprachliche Regeln verallgemeinerten. Auch in bezug auf den Einfluß interlanguagespezifischer Faktoren waren deutliche Unterschiede zu erkennen. Bei den PmeilAb äußerten sich interlanguagespezifische Faktoren in Form von inter- und intralingualem Transfer, bewußten Generalisierungen und Kontrastmangel. In der entgegengesetzten Probandengruppe wurde der Einfluß derselben Faktoren nachgewiesen, hinzu kamen jedoch auch Reduktionsstrategien und Paraphrasierungen. Inter- oder intralingualer Transfer konnte nicht nachgewiesen werden.

Zusammenfassend ist festzustellen, daß erstsprachlicher Einfluß in bezug auf die Gruppe der Pronomen bei den PwenlAb einen höheren Stellenwert einnahm als in der entgegengesetzten Probandengruppe. Außerdem waren bei den PwenlAb auch die Ausprägungsformen erstsprachlichen Einflusses vielseitiger. In bezug auf den Einfluß interlanguagespezifischer Faktoren waren sowohl Übereinstimmungen als auch Unterschiede zu erkennen. In beiden Probandengruppen zeigte sich, daß die Fehlerentstehung teilweise auf bewußten Generalisierungen von Interlanguageelementen und Kontrastmangel basierte. Der interlanguagespezifische Einfluß unterschied sich jedoch dadurch, daß bei den PmeilAb die Fehler weiterhin auf inter- und intralingualem Transfer basierten, während in der 
entgegengesetzten Probandengruppe zusätzlich Reduktionsstrategien und Paraphrasierungen nachgewiesen werden konnten.

\subsection{Syntaktisch-morphologische Ebene}

Bei der Analyse der syntaktisch-morphologischen Abweichungen wurde deutlich, daß die Fehlerentstehung sowohl bei den PmeismAb als auch bei den PwensmAb auf den Einfluß der Erstsprache, auf entwicklungsbedingten Einfluß und auf das strategisch-prozedurale Verhalten der Lernenden zurückzuführen war. Im Gegensatz zu den lexikalischen Abweichungen kamen damit auf dieser linguistischen Ebene auch die Fehlerentstehungsfaktoren der Identitätshypothese zum Tragen. Die Ursachen, die syntaktisch-morphologische Abweichungen hervorrufen, sind demnach komplexer als die Faktoren, die zur Entstehung lexikalischer Abweichungen beitragen. Hier zeigte sich, daß in beiden Probandengruppen strategisch-prozedurale Faktoren, d. h. Aspekte der Interlanguagehypothese, den größten Einfluß auf die Fehlerentstehung hatten. Der zweitgrößte Fehleranteil war auf die Auswirkungen entwicklungsbedingter Faktoren zurückzuführen. Somit bildete der Einfluß der Erstsprache die geringste Fehlerentstehungsursache. Da er jedoch in beiden Probandengruppen bei mehr als der Hälfte der Abweichungen an der Fehlerentstehung beteiligt war, müssen die Erklärungsfaktoren aller drei Hypothesen als wichtige Fehlerquellen betrachtet werden. Dabei wurde jedoch auch deutlich, daß alle Hypothesen bei den PwensmAb öfter zur Anwendung kamen als in der entgegengesetzten Probandengruppe. Die Fehlerentstehung war bei diesen Lernenden demnach komplexer.

Die fehlergenetischen Übereinstimmungen, die trotz der Differenzen insgesamt deutlich wurden, zeigten, daß die Ursachen für die normabweichende Verwendung syntaktisch-morphologischer Elemente prinzipiell bei allen Lernertypen beobachtet werden können und demnach nur partiell an das syntaktisch-morphologische Wissen der Lernenden gebunden sind. Die genauere Betrachtung der einzelnen Fehlerkategorien bestätigte, daß die Fehlerentstehungsfaktoren in beiden Probandengruppen übereinstimmten. Unterschiede waren lediglich bei der Gewichtung der Entstehungsursachen zu erkennen. So zeigte die Analyse der Wort- und Satzstellungsfehler, daß diese in beiden Probandengruppen auf erstsprachlichen, entwicklungsbedingten und strategisch-prozeduralen Faktoren basierten. Gleichzeitig wurde beim Vergleich der Ergebnisse deutlich, daß in beiden Probandengruppen das Ausmaß des Einflusses entwicklungsbedingter und strategisch-prozeduraler Faktoren nahezu identisch war. In beiden Gruppen konnten die Auswirkungen strategisch-prozeduraler Faktoren bei allen Abweichungen nachgewiesen werden. Gleiches gilt für die Auswirkungen entwicklungsbedingter Aspekte, jedoch nur in bezug auf die PwensmAb. In der entgegengesetzten Probandengruppe kam entwicklungsbedingter Einfluß etwas seltener zum Tragen, stellte jedoch auch hier die zweitwichtigste Fehlerquelle dar. Auch in bezug auf den Einfluß der Erstsprache waren die Ergebnisse nahezu 
deckungsgleich. Wenngleich dieser Faktor in beiden Probandengruppen die geringste Entstehungsursache bildete, beruhten die Fehler beider Gruppen doch zu mehr als $80 \%$ u. a. auf Interferenzen der Erstsprache.

In bezug auf die Unterkategorien der einzelnen Entstehungsfaktoren äußerten sich die Auswirkungen der Erstsprache bei den PmeismAb in Form von Substitutionen, Übergeneralisierungen und Hyperkorrektheiten, wobei der Faktor ,Substitution' quantitativ an erster Stelle stand. In der entgegengesetzten Probandengruppe entfielen die Hyperkorrektheiten, jedoch kamen zusätzlich Über- bzw. Unterdifferenzierungen zum Tragen. Auch hier bildeten die Substitutionen die umfangreichste Unterkategorie, die Verteilung der Faktoren war jedoch insgesamt gleichmäßiger, d. h., der erstsprachliche Einfluß äußerte sich ebenso in Form von Differenzierungsschwierigkeiten und Verallgemeinerungen erstsprachlicher Regeln.

Die Erscheinungsformen entwicklungsbedingten Einflusses hingegen waren in beiden Probandengruppen nahezu identisch. Die Wort- und Satzstellungsfehler basierten aus der Sicht der Identitätshypothese schwerpunktmäßig darauf, daß Satzelemente in der Reihenfolge der Bedeutung, die die Elemente für die Lernenden hatten, angeordnet wurden. Hier handelt es sich um einen Vorgang, der auch beim Erlernen anderer Sprachen beobachtet werden kann und im Laufe fortgeschrittener Erwerbsphasen nachläßt. Ansatzweise wurde entwicklungsbedingter Einfluß in beiden Lernergruppen jedoch auch darin deutlich, daß oftmals syntaktische Strukturen durch andere Strukturen ersetzt wurden. Beispielsweise wurden Fragesätze nach dem Prinzip von Aussagesätzen gebildet oder Verneinungen nicht mit Hilfe des Verbs to do verdeutlicht, obwohl diese Verneinungsform in der entsprechenden Äußerung zu erwarten gewesen wäre.

Auch in bezug auf den Einfluß interlanguagespezifischer Faktoren waren beim Vergleich der beiden Probandengruppen Übereinstimmungen zu erkennen. In beiden Gruppen waren die Fehler u. a. auf Faktoren des Unterrichts, Lernstrategien, funktionale Reduktionsstrategien und Erweiterungsstrategien zurückzuführen. Teilweise basierten die Abweichungen in beiden Gruppen auch auf unbewußten Übergeneralisierungen, jedoch war dieser Fehlerentstehungsfaktor bei den PwensmAb stärker ausgeprägt als in der entgegengesetzten Probandengruppe. Bei den PmeismAb war zusätzlich auch Kontrastmangel an der Fehlerentstehung beteiligt. Somit unterschied sich die Fehlerentstehung interlanguagespezifisch betrachtet lediglich dadurch, daß die Wort- und Satzstellungsfehler bei den PmeismAb neben strategisch-prozeduralen Faktoren auch auf strukturellen Entsprechungen zwischen dem Deutschen und dem Englischen basierten, während in der entgegengesetzten Gruppe die Fehler partiell auch durch unbewußte Übergeneralisierungen entstanden waren. 
Bei der Analyse der Auslassungsfehler war in bezug auf die Kategorie ,sonstige Wortarten und -bestandteile' bei den PmeismAb der Einfluß entwicklungsbedingter und interlanguagespezifischer Faktoren quantitativ identisch. Die Faktoren der Identitäts- und der Interlanguagehypothese stellten die Ursachen für die Entstehung der meisten Abweichungen dar. Die Differenz zur Anwendbarkeit von Faktoren der Kontrastivhypothese war jedoch gering. Somit waren die Auswirkungen der Erstsprache auch erheblich an der Fehlerentstehung beteiligt. In der Gruppe der PwensmAb war der Einfluß der Faktoren aller drei Hypothesen quantitativ nahezu identisch.

Die Betrachtung der Unterkategorien der einzelnen Erwerbshypothesen zeigte, daß in beiden Probandengruppen erstsprachlicher Einfluß vornehmlich in Form von Substitutionen, Über- und Unterdifferenzierungen und Übergeneralisierungen auftrat. Somit war die Fehlerentstehung aus Sicht der Kontrastivhypothese in beiden Probandengruppen auf das Ersetzen zielsprachlicher Elemente, Differenzierungsschwierigkeiten zwischen deutschen und englischen Strukturen und sprachlichen Generalisierungen zurückzuführen. Auch in bezug auf den Einfluß entwicklungsbedingter Faktoren gab es in beiden Probandengruppen Übereinstimmungen. Hier basierte die Fehlerentstehung zum einen darauf, daß Elemente, die als redundant betrachtet wurden, nicht zur Anwendung kamen und zum anderen darauf, daß verschiedene grammatische Strukturen durch andere Strukturen ersetzt wurden. Zusätzlich entstanden Abweichungen auch durch das entwicklungsbedingte Vertauschen sprachlicher Strukturen.

Auch die Anwendung der Interlanguagehypothese führte $\mathrm{zu}$ ähnlichen Ergebnissen. In beiden Probandengruppen basierten die Auslassungsfehler auf Faktoren des Unterrichts, Lernstrategien und Kommunikationsstrategien, inter- und intralingualem Transfer sowie bewußten Generalisierungen und Paraphrasierungen. Darüber hinaus traten in beiden Probandengruppen auch Fehler als Ergebnis von unbewußten Übergeneralisierungen und Konstrastmangel auf. Zusammenfassend ist festzustellen, daß Auslassungsfehler sowohl bei deutschen Englischlernenden mit geringen syntaktischmorphologischen Kenntnissen als auch bei Lernenden mit überdurchschnittlichen Kenntnissen interlanguagespezifisch darauf basieren, daß grammatische Unterschiede zwischen Erst- und Zielsprache nicht beachtet werden und Elemente aus entwicklungsbedingten Gründen ausgelassen, vertauscht oder ersetzt werden. Gleichzeitig sind die Fehler jedoch auch auf Faktoren des Unterrichts, Lern- und Kommunikationsstrategien, prozedurale Faktoren sowie inter- und intralinguale Strukturentsprechungen zurückzuführen.

Auch in der Fehlerkategorie simple past war die Fehlerentstehung in beiden Probandengruppen auf erstsprachliche, entwicklungsbedingte und interlanguagespezifische Faktoren zurückzuführen. Die nähere Betrachtung der Ergebnisse zeigte, daß in beiden Probandengruppen der Fehlerentstehungseinfluß entwicklungsbedingter und interlanguagespezifischer Faktoren quantitativ übereinstimmte. 
Während diese Faktoren jedoch in der Gruppe der PmeismAb die wichtigste Fehlerentstehungsursachen bildeten, überwog in der entgegengesetzten Probandengruppe der Einfluß der Erstsprache. In dieser Gruppe war jedoch die Differenz zu den anderen Fehlerentstehungsfaktoren gering, während bei den PmeismAb erstsprachliche Faktoren deutlich seltener nachgewiesen werden konnten als die übrigen Faktoren. Demnach rekurrieren fortgeschrittene Lernende bei Anwendung des simple past stärker auf die Erstsprache als Lernende mit erheblichen syntaktisch-morphologischen Lern- und Anwendungsschwierigkeiten.

Bei Betrachtung der Unterkategorien wurde deutlich, daß der Einfluß der Erstsprache in beiden Probandengruppen im Form von Substitutionen, Über- und Unterdifferenzierungen und Übergeneralisierungen auftrat. Bei den PmeismAb führte der Einfluß der Erstsprache darüber hinaus auch zu Hyperkorrektheiten. Typologisch stimmten die Ergebnisse somit nahezu überein. Die genauere Analyse zeigte jedoch, daß alle Erscheinungsformen erstsprachlichen Einflusses bei den PwensmAb deutlich stärker zum Tragen kamen als in der entgegengesetzten Probandengruppe. Daraus ist zu schließen, daß der Einfluß der Erstsprache mit zunehmenden syntaktisch-morphologischen Kenntnissen beim Gebrauch der Zeitform simple past nicht nachläßt, sondern im Laufe des Zweitspracherwerbs sogar deutlicher hervortritt.

Bei Anwendung der Erklärungsfaktoren der Identitätshypothese wurde in beiden Lernergruppen entwicklungsbedingter Einfluß in Form von Auslassungs- und Hinzufügungsfehlern deutlich. Dieser äußerte sich darüber hinaus auch darin, daß die Lernenden das simple past durch andere Zeitformen ersetzten - zu denen neben dem simple present, das insbesondere in der Gruppe der PmeismAb häufig als Ersatzform zur Anwendung kam, das present perfect zählte. Im wesentlichen stimmte die Form entwicklungsbedingten Einflusses in beiden Probandengruppen typologisch überein, wenngleich bei den PmeismAb darüber hinaus auch Fehler darauf zurückzuführen waren, daß sie Satzelemente vertauschten oder grammatische Markierungen redundanterweise hinzufügten.

Auch die Analyse der Fehlerentstehung mit Hilfe der Erklärungsfaktoren der Interlanguagehypothese zeigte, daß die Fehlerentstehungsfaktoren in beiden Probandengruppen größtenteils übereinstimmten. In beiden Gruppen waren die Fehler sowohl auf unterrichtlich bedingte Faktoren als auch auf Lernstrategien, formale und funktionale Reduktionsstrategien sowie die sogenannten productive achievement strategies zurückzuführen. Aus der Reihe dieser Strategien kamen in beiden Probandengruppen die Unterkategorien meaning replacement, modality reduction, inter-/intralingual transfer, omission of language items, generalizations of interlanguage rules/items in new contexts, paraphrase sowie word coinage zur Anwendung. Darüber hinaus resultierten in beiden Gruppen die Fehler teilweise auch aus unbewußten Übergeneralisierungen und Kontrastmangel. 
Zusammenfassend ist festzustellen, daß die Entstehung der simple past-Verstöße in beiden Probandengruppen oftmals darauf basierte, daß die Probanden versuchten, das simple past durch Zeitformen zu ersetzen, die in der betreffenden Situation in der Erstsprache zu verwenden gewesen wären. In anderen Fällen entstanden Abweichungen dadurch, daß Elemente ersetzt oder ausgelassen und vertauscht wurden, weil der Gebrauch der Zeitform bzw. Verlaufsform - entwicklungsspezifisch betrachtet - nicht in Einklang mit den natürlichen Erwerbssequenzen stand. Darüber hinaus waren die Verstöße in beiden Gruppen auch dadurch begründet, daß die Zeitform simple past im Unterricht unzureichend vermittelt worden war und die Lernenden versuchten, den Lernvorgang zu vereinfachen. Weiterhin waren Abweichungen in beiden Gruppen das Ergebnis sprachlicher Reduktionen und des Versuchs, durch Erweiterung des bereits verinnerlichten Wissens Kommunikationsdefizite auszugleichen. $\mathrm{Zu}$ den wichtigsten Fehlerentstehungsfaktoren gehörten dabei sprachliche Generalisierungen, die sowohl bewußt als auch unbewußt verliefen und zum Teil auch auf inter- und intralingualen Differenzierungsschwierigkeiten basierten.

\subsection{Orthographische Ebene}

Die Analyse der Abweichungen, die auf orthographischer Ebene ermittelt wurden, zeigte, daß in beiden Probandengruppen entwicklungsspezifischer Einfluß bei der Fehlerentstehung kaum von Bedeutung war. In der Gruppe der PmeioAb war dieser jedoch deutlicher zu erkennen als in der entgegengesetzten Probandengruppe. Der Einfluß interlanguagespezifischer Faktoren kam bei den PwenoAb deutlich stärker zum Tragen als erstsprachlich bedingte Fehlerentstehungsfaktoren. Bei den PmeioAb hingegen war die Differenz zwischen diesen beiden Fehlerentstehungsfaktoren wesentlich geringer. Strategisch-prozedurales Verhalten war bei den PwenoAb also eindeutig stärker an der Fehlerentstehung beteiligt als der Einfluß der Erstsprache.

Die Untersuchung der einzelnen Fehlerkategorien erbrachte, daß erstsprachliche und entwicklungsbedingte Faktoren in bezug auf die Fehlerkategorie ,Großschreibung' in der Gruppe der PmeioAb gleichbedeutend waren. Aus der Sicht der Kontrastivhypothese konnte die Fehlerentstehung in dieser Gruppe sowohl darauf zurückgeführt werden, daß die Probanden zielsprachliche Schreibweisen durch erstsprachliche ersetzten als auch darauf, daß die Lernenden nicht zwischen den unterschiedlichen Schreibweisen im Englischen differenzierten, weil diese Differenzierungen im betreffenden Kontext im Deutschen redundant wären. Ein Beispiel bietet etwa die Schreibweise von Adjektiven, die sich auf ethnische Gruppen oder Nationalitäten beziehen. Diese werden - wie bereits zuvor erwähnt - im Englischen groß geschrieben, während eine solche Unterscheidung zu anderen Adjektiven im Deutschen nicht vorgenommen wird. In der entgegengesetzten Probandengruppe hingegen äußerte sich der Einfluß der Erstsprache ausschließlich darin, daß englische Schreibweisen durch deutsche substituiert 
wurden. Somit war der Einfluß der Erstsprache bei den PmeioAb differenzierter als in der entgegengesetzten Probandengruppe. Entwicklungsbedingter Einfluß hingegen konnte in keiner der Probandengruppen nachgewiesen werden, die Fehlerentstehung in der Kategorie ,Großschreibung ‘ war also in keinem Fall auf biologisch determinierte Faktoren zurückzuführen.

Bei Betrachtung der Faktoren der Interlanguagehypothese zeigte sich in beiden Probandengruppen, daß die Fehlerentstehung vornehmlich darauf basierte, daß orthographische Regeln des Englischen generalisiert wurden. Diese Generalisierungen verliefen bei den PwenoAb teilweise unbewußt. Bei den PmeioAb traten auch Großschreibungsfehler aufgrund von interlingualem Transfer auf. Da jedoch in beiden Probandengruppen die bewußten Generalisierungen den Hauptentstehungsfaktor bildeten, wurde bei der Untersuchung der Fehlergenese in bezug auf die Interlanguagehypothese keine wesentlichen Unterschiede zwischen beiden Gruppen festgestellt.

Die Analyse der Fehlerkategorie ,Kleinschreibung' zeigte, daß - wie bei der vorhergehenden Fehlerkategorie - entwicklungsspezifischer Einfluß in keiner Probandengruppe an der Fehlerentstehung beteiligt war. In beiden Gruppen bildete der Einfluß der Erstsprache die wichtigste Fehlerentstehungsursache. Gleichzeitig ließ der Vergleich der beiden Probandengruppen erkennen, daß die Differenz zwischen dem Einfluß erstsprachlicher und interlanguagespezifischer Faktoren bei den PmeioAb geringer war als in der entgegengesetzten Gruppe. Während bei den PmeioAb die zuvor genannten Fehlerentstehungsfaktoren in nahezu gleicher Quantität auftraten, lag der Schwerpunkt der Fehlerentstehung in der entgegengesetzten Probandengruppe eindeutig auf dem Einfluß der Erstsprache.

Der Vergleich der Ergebnisse, die bei Betrachtung der einzelnen Kategorien deutlich wurden, ergab in Hinblick auf die Kontrastivhypothese, daß die Fehlerentstehung in beiden Probandengruppen darauf zurückzuführen war, daß die Lernenden zielsprachliche Schreibweisen durch erstsprachliche ersetzten. In der Gruppe der PmeioAb basierten zusätzlich auch Fehler darauf, daß die Lernenden nicht zwischen den einzelnen zielsprachlichen Schreibweisen differenzierten, weil solche Differenzierungen im Deutschen redundant wären.

Die Anwendung der Fehlererklärungsfaktoren der Interlanguagehypothese zeigte, daß interlanguagespezifischer Einfluß bei den PmeioAb vielseitiger ist war in der entgegengesetzten Probandengruppe. Bei ersteren äußerten sich interlanguagespezifische Faktoren sowohl in Form von inter- und intralingualem Transfer als auch in Form von bewußten Generalisierungen. Bei den PwenoAb hingegen war interlanguagespezifischer Einfluß nur daran zu erkennen, daß die Probanden Regeln der Zielsprache unbewußt generalisierten. Zusammenfassend ist hinsichtlich der Entstehung der Kleinschreibungs- 
fehler festzustellen, daß die Fehlerentstehung bei den PmeioAb in bezug auf die Faktoren aller Hypothesen komplexer war als in der entgegengesetzten Probandengruppe.

Bei der Analyse der Fehler der Kategorie ,Rechtschreibung ${ }^{6}$ wurde ermittelt, daß in beiden Probandengruppen, im Gegensatz zu den anderen Fehlern der orthographischen Ebene, sowohl erstsprachlicher als auch entwicklungs- und interlanguagespezifischer Einfluß nachzuweisen war. Dabei stellte in beiden Gruppen übereinstimmend der interlanguagespezifische Einfluß die wichtigste Fehlerentstehungsursache dar. Der zweitgrößte Anteil der Fehler war in beiden Probandengruppen auf den Einfluß der Erstsprache zurückzuführen, während entwicklungsbedingte Faktoren in den wenigsten Fällen die Ursache der Abweichungen darstellten. Außerdem zeigten die Ergebnisse, daß sowohl der Einfluß der Erstsprache als auch der Einfluß entwicklungsspezifischer Faktoren bei den PmeioAb stärker zum Tragen kamen als in der entgegengesetzten Probandengruppe. Interlanguagespezifischer Einfluß hingegen war bei den PwenoAb öfter festzustellen.

Die Anwendung der einzelnen Fehlererklärungskategorien der drei Hypothesen zeigte, daß der Einfluß der Erstsprache in beiden Probandengruppen ausschließlich in Form von Substitutionen zum Tragen kam. Die Anwendung der Faktoren der Identitätshypothese hingegen ließ Unterschiede erkennen. Während die Rechtschreibfehler bei den PmeioAb aus entwicklungsspezifischer Sicht dadurch entstanden sind, daß die Probanden zum einen Elemente ausgelassen und zum anderen Elemente redundanterweise hinzugefügt haben, konzentrierte sich die Fehlerentstehung bei den Probanden der entgegengesetzten Gruppe auf das redundante Hinzufügen sprachlicher Elemente.

Bei Anwendung der Erklärungsfaktoren der Interlanguagehypothese wiederum waren Übereinstimmungen zu erkennen. In beiden Probandengruppen basierte die Fehlerentstehung im wesentlichen auf interlingualem Transfer, bewußten und unbewußten Generalisierungen und auf Kontrastmangel. Der einzige fehlergenetische Unterschied bestand darin, daß bei den PmeioAb neben interlingualem Transfer auch intralingualer Transfer deutlich wurde, während diese Fehlerentstehungsursache in der entgegengesetzten Probandengruppe nicht nachgewiesen werden konnte.

Zusammenfassend ist in bezug auf die Analyse der Rechtschreibfehler zu konstatieren, daß die Fehlergenese beim Vergleich der beiden Probandengruppen insgesamt keine erheblichen Abweichungen zeigte. Es wurde jedoch deutlich, daß - wie auch in den vorhergehenden Fehlerkategorien - die Fehlerentstehung der PmeioAb komplexer war als in der entgegengesetzten Probandengruppe. In letzterer konzentrierte sich die Entstehung der Rechtschreibfehler sowohl in bezug auf den entwicklungsbedingten als auch auf den interlanguagesspezifischen Anteil auf wenige Faktoren, wenngleich diese einseitige Verteilung bei Anwendung der Interlanguagehypothese weniger deutlich zu erkennen war. 


\section{Schlußbetrachtung}

Die Auswertung der Untersuchungsergebnisse vorliegender Arbeit bestätigt die zu Beginn der Arbeit aufgestellte Behauptung, daß die Genese sprachlicher Fehler nur dann nachvollzogen werden kann, wenn die Abweichungen aus unterschiedlichen Perspektiven betrachtet werden. $\mathrm{Zu}$ einer umfassenden Erklärung der Entstehung linguistischer Abweichungen deutscher Englischlernender bedarf es sowohl der Anwendung der Kontrastiv-, als auch der Identitäts- und der Interlanguagehypothese. Die Einbeziehung aller drei Hypothesen gewährleistet, daß die Fehlerentstehung einerseits unter strukturellen Aspekten, andererseits jedoch auch unter sprachentwicklungsspezifischen und strategisch-prozeduralen Gesichtspunkten untersucht wird.

Dabei zeigte sich, daß die Faktoren der einzelnen Hypothesen - obwohl sie unterschiedlichen linguistischen Ansätzen zuzuordnen sind - ineinander übergreifen und nicht voneinander getrennt, d. h. als unabhängige Variablen zu betrachten sind. Dies gilt insbesondere für Fehlleistungen auf syntaktischmorphologischer Ebene, trifft jedoch auch auf die lexikalischen und orthographischen Abweichungen zu, wenngleich die Faktoren der Identitätshypothese bei lexikalischen Abweichungen nicht zum Tragen kommen und auch bei orthographischen Abweichungen von untergeordneter Bedeutung sind. In bezug auf die syntaktisch-morphologische Ebene und mit Einschränkung auch auf die lexikalische und die orthographische Ebene wurde im Rahmen der Analyse deutlich, daß die Inhalte einer bestimmten Hypothese erst dann einen fehleranalytischen Beitrag leisten können, wenn zuvor die Faktoren einer anderen Hypothese angewandt wurden.

So werden etwa bestimmte strategische Verhaltensweisen, die auf erstsprachlichem Einfluß basieren, erst dann verständlich, wenn die Differenzen zwischen Erst- und Zielsprache zuvor in Form eines kontrastiven Vergleichs der beiden Sprachsysteme, d. h. unter Anwendung der Faktoren der Kontrastivhypothese erarbeitet wurden. Ebenso kann die Frage, warum bestimmte erstsprachlich und strategisch bedingte Fehler in bestimmten Phasen des Spracherwerbs auftreten und in anderen Erwerbsphasen nicht bzw. weniger häufig beobachtet werden können, nur dann plausibel beantwortet werden, wenn die sprachlichen Besonderheiten, die innerhalb der einzelnen Sequenzen natürlichen Spracherwerbs sowohl bei Lernenden einer Erst- als auch einer Zweitsprache auftreten, miteinbezogen werden. Konzentriert sich also die Fehleranalyse auf ein bzw. zwei Verfahren, dann bleiben solche Fehlerentstehungsfaktoren offen, die nur unter Anwendung aller verfügbaren Methoden begründet werden können. 
In Hinblick auf die fehlergenetischen Erklärungen, die aus den einzelnen Hypothesen abgeleitet werden können, kann der vehementen Kritik ${ }^{666}$ an den Erklärungsfaktoren der Kontrastivhypothese und der ihr zugrundeliegenden Theorie auf der Grundlage der Ergebnisse vorliegender Arbeit nur partiell zugestimmt werden. Der Einfluß der Erstsprache ist sowohl bei Anfängern als auch bei fortgeschrittenen Lernenden unverändert als eine der wichtigsten Fehlerentstehungsursachen zu betrachten. Dabei zeigte sich, daß erstsprachliche Auswirkungen oftmals nicht bei erster Betrachtung der betreffenden Fehlleistung deutlich werden, sondern erst dann als solche zu erkennen sind, wenn der gesamte sprachliche Kontext, in dem der Fehler ermittelt wurde, fehlergenetisch analysiert wird.

Auch der von Gegnern der Kontrastivhypothese aufgestellten Behauptung, Fehlervorhersagen und das Erstellen von Schwierigkeitshierarchien seien wissenschaftlich nicht vertretbar, ${ }^{667}$ kann nur partiell zugestimmt werden: Wie die Fehlerbeispiele gezeigt haben, ist unverändert davon auszugehen, daß strukturelle und semantische Unterschiede zwischen der Erstsprache Deutsch und der Zielsprache Englisch zu Lern- und Anwendungsschwierigkeiten führen. Mit Zunahme der Differenzen zwischen Erst- und Zielsprache steigt auch die Zahl der Fehler, was die Aufstellung von Hierarchien ermöglichen würde. Die Auswirkungen dieser strukturellen und semantischen Differenzen sind sowohl bei Anfängern als auch bei fortgeschrittenen Lernenden zu beobachten. Es muß jedoch in diesem Zusammenhang hervorgehoben werden, daß diese Schwierigkeiten Schwankungen unterliegen können. Es ist also möglich, daß ein Englischlernender ein bestimmtes Element oder eine bestimmte Struktur des Englischen teils normgerecht und teils normabweichend verwendet.

In bezug auf eklatante Strukturdifferenzen ist der Kritik im wesentlichen zuzustimmen. In vorliegender Studie war zu erkennen, daß zielsprachliche Elemente, die sich inhaltlich oder strukturell gravierend von Elementen des Deutschen unterscheiden, häufiger normgerecht als normabweichend verwendet werden, weil sie gerade aufgrund ihrer Unterschiedlichkeit die Aufmerksamkeit der Lernenden wecken. Beispielsweise war die Anzahl der Fehler beim Gebrauch des going to-future oder der sogenannten question tags ${ }^{668}$ äußerst gering, weil diese Strukturen im Deutschen nicht bzw. nur ansatzweise zu finden sind und von den Lernenden offensichtlich als charakteristische Merkmale der englischen Sprache betrachtet werden, die eine besondere Beachtung verdienen.

Auch in einem weiteren Punkt, der Behauptung, daß strukturelle Gemeinsamkeiten zwischen Erst- und Zielsprache zu Lernerleichterungen führten, ${ }^{669}$ ist der Kontrastivhypothese partiell zu widersprechen. Wie von Gegnern der Kontrastivhypothese bereits konstatiert wurde, konnte auch in der vorliegenden

\footnotetext{
666 vgl. Bausch / Kasper 1979, 6-7

667 vgl. ebd.

668 vgl. Swan 1990, Textstelle 515, o. S.
} 
Arbeit ermittelt werden, daß - im Gegensatz zur Annahme der Hypothese - insbesondere solche Elemente Fehler verursachen können, die sowohl in der Erst- als auch in der Zielsprache zu finden sind. So zeigte sich zum Beispiel, daß der Gebrauch der Präposition in oftmals zu Abweichungen führt, eben weil das Element nicht nur Bestandteil der englischen, sondern auch der deutschen Sprache ist.

Trotz der Tatsache, daß der Kritik an der Kontrastivhypothese partiell zuzustimmen ist, kann diese Hypothese dennoch einen wichtigen Beitrag zur Analyse sprachlicher Fehler leisten. Dies betrifft nicht nur die allgemeine Theorie der Hypothese, sondern auch die einzelnen Fehlererklärungskategorien, $d . h$. die Formen des erstsprachlichen Einflusses, die in der Kontrastivhypothese beschrieben werden. So konnte im Rahmen der Fehleranalyse gezeigt werden, daß erstsprachlicher Einfluß in der Tat meist in Form von Substitutionen und Über- bzw. Unterdifferenzierungen auftritt. In diesem Zusammenhang wurde auch deutlich, daß der Versuch, durch eine Ergänzung der Fehlererklärungsfaktoren das Erklärungspotential der ersten Version der Kontrastivhypothese zu erweitern, redundant ist, weil die meisten dieser hinzugefügten Faktoren bei Anwendung der Theorie der Kontrastivhypothese nicht nachgewiesen werden konnten und zudem in der Interlanguagehypothese enthalten sind.

Auch bei der Analyse der Abweichungen mit Hilfe der Identitätshypothese wurde deutlich, daß die Kritik, die an dieser Hypothese geäußert wurde, in bezug auf die syntaktisch-morphologische Ebene zurückgewiesen werden muß. Die Ergebnisse der vorliegenden Arbeit haben gezeigt, daß entwicklungsspezifische Faktoren bei grammatischen Verstößen nicht vernachlässigt werden dürfen, sondern als wichtige Fehlerentstehungsquelle zu betrachten sind. Die Anwendung dieser Faktoren kann Einblicke in die Vorgänge des Zweitspracherwerbs bieten. Gleichzeitig verdeutlicht sie, daß sprachliche Fehler nicht nur auf linguistischen und strategisch-prozeduralen Ursachen basieren, sondern teilweise auch als natürliche Erscheinungen des Spracherwerbs zu betrachten sind. Der Lehrende sollte also sein Korrekturverhalten den entwicklungsbedingten Fehlern anpassen - d. h., er sollte den Lernenden beim Auftreten entwicklungsbedingter Fehler vermehrt Möglichkeiten zur Selbstkorrektur bieten. Dadurch kann verhindert werden, daß durch die Korrekturen des Lehrers möglicherweise Strukturen verfrüht eingeführt werden, die aufgrund der biologisch festgelegten Erwerbssequenzen erst zu einem späteren Erwerbsstadium internalisiert werden können.

Der kontraproduktive Effekt der vorzeitigen Vermittlung zweitsprachlicher Strukturen kann dabei darin bestehen, daß das Element, das der Unterrichtende im Rahmen der Korrektur vermittelt, im Sprachspeicher des Lernenden nicht als Bestandteil des zweitsprachlichen Repertoires akzeptiert wird und zukünftig entweder gar nicht oder nur normabweichend zur Anwendung kommt. Es erscheint daher in bezug auf entwicklungsbedingte Fehler sinnvoll, dem Lernenden beim Auftreten dieser

669 vgl. Bausch / Kasper 1979, 5 - 7 
Fehlertypen möglichst viel Zeit zur Überprüfung des Repertoires der von ihm bereits internalisierten Elemente zu geben, um ihm auf diesem Wege zu ermöglichen, das Element, das in dem betreffenden Kontext korrekterweise zu verwenden wäre, selbst zu erkennen.

In bezug auf die Identitätshypothese konnte damit im Rahmen vorliegender Arbeit bestätigt werden, daß auch biologisch determinierte Faktoren an der Entstehung linguistischer Abweichungen deutscher Englischlernender beteiligt sind. Ihre Auswirkungen beschränken sich jedoch nahezu ausschließlich auf die syntaktisch-morphologische Ebene und bedürfen zu ihrer Korrektur einer weniger lehrergesteuerten Herangehensweise als lexikalische oder orthographische Abweichungen. Bei der Analyse der Fehlleistungen zeigte sich allerdings, daß das Auftreten entwicklungsbedingter Fehler bei deutschen Muttersprachlern, die Englisch unter unterrichtlich gesteuerten Erwerbsbedingungen internalisieren, weitaus länger zu beobachten ist als bei Lernenden, die die Sprache auf natürlichem Wege verinnerlichen. Der von Kritikern geäußerten Behauptung, daß unterrichtlich gesteuerter und natürlicher Zweitspracherwerb unterschiedlich verlaufen, ${ }^{670}$ ist also partiell zuzustimmen, wenngleich diese Erkenntnis nicht die Validität der Identitätshypothese in Frage stellt.

Bei Anwendung der Interlanguagehypothese zeigte sich, daß diese Hypothese einerseits über das größte Erklärungspotential verfügt, andererseits jedoch die einzelnen Fehlererklärungsfaktoren konkretisierungsbedürftig sind. Dies führt dazu, daß die fehleranalytischen Aussagen, die im Zusammenhang mit der Interlanguagehypothese getroffen werden können, weniger wissenschaftlich fundiert sind als die Ergebnisse, die etwa bei Anwendung der Kontrastivhypothese erzielt werden. Dies beruht zum einen darauf, daß - wie bereits bei der Darstellung der Gesamtergebnisse erwähnt - die Definitionen der einzelnen Erklärungsfaktoren als vage zu betrachten sind. Zum anderen basiert die Unklarheit der Ergebnisse auch auf der Tatsache, daß die Interlanguagehypothese durch die Berücksichtigung unbewußt ablaufender Prozesse bei der Fehlerentstehung fehlergenetische Faktoren einbezieht, die sich jeglicher Überprüfung entziehen. Prozesse dieser Art entstehen im Unterbewußtsein und werden daher von den Betroffenen nicht wahrgenommen. Sie können daher weder von den Lernenden noch von Fehlerlinguisten nachvollzogen werden. Darüber hinaus zeigte sich, daß ein Teil der Fehlererklärungsfaktoren der Interlanguagehypothese redundant ist, d. h., Entstehungsursachen, die einer Kategorie zugrunde liegen, sind zum Teil gleichzeitig auch Bestandteil einer anderen Kategorie. Dies betrifft vor allem den als language transfer ${ }^{671}$ bezeichneten Fehlerentstehungsfaktor.

Trotz dieser Schwächen der Interlanguagehypothese wurde dennoch im Rahmen der Analyse deutlich, daß der Grundgedanke dieser Theorie, d. h. der Versuch, die Persönlichkeit des Lernenden und seine

\footnotetext{
${ }^{670}$ vgl. Apeltauer 2003, $13-15$

${ }^{671}$ vgl. Kaltenbacher 2001b, $29 \mathrm{f}$
} 
individuellen Verhaltensweisen bei der Fehleranalyse miteinzubeziehen, einen wichtigen fehlerlinguistischen Ansatz bildet. Wie die Analyse zeigte, kann das Auftreten von Fehlergruppen innerhalb einer bestimmten Sprachgemeinschaft teilweise verallgemeinert werden, läßt jedoch teils auch individuelle Unterschiede erkennen.

Darüber hinaus bietet die Interlanguagehypothese Ansätze, die in den anderen Erwerbshypothesen nicht berücksichtigt werden. Dies betrifft insbesondere die Aspekte Fossilisierung, Systematizität und Variabilität. Hier bietet ausschließlich die Interlanguagehypothese eine Erklärung für das Auftreten von Abweichungsformen, die in allen Erwerbsphasen zu beobachten sind bzw. von Abweichungsformen, die der Lernende bereits überwunden hatte und die unerwarteterweise erneut auftreten.

Neben den oben dargestellten Ergebnissen hat die vorliegende Arbeit gezeigt, daß sprachliche Fehler zu den Erscheinungen der Linguistik gehören, die wissenschaftlich nur partiell begründet werden können und auch in Zukunft intensiver Untersuchungen bedürfen. Die Aussagen, die in bezug auf die Entstehung linguistischer Abweichung getroffen werden können, sind in den wenigsten Fällen als eindeutige Ergebnisse zu bezeichnen. Sie beruhen vielmehr auf einer Kombination wissenschaftlich nachweisbarer Ergebnisse und didaktischer Vermutungen, die aus der unterrichtlichen Erfahrung resultieren. 



\section{Literaturverzeichnis}

\section{Primärliteratur}

Alfes, L. 1985: „,Falsche‘ und ,halbehrliche‘ Freunde - Ein Problem der Wortschatzdidaktik“, in: Englisch, $20 / 1985$, S. 143

Andreasson, A.-M. 1994: „Norm as a pedagogical paradigm“, in: World Englishes 13 / 1994, S. 395 - 409 , zit. nach: James, C. 1998: Errors in Language Learning and Use: Exploring Error Analysis, London / New York

Apeltauer, E. 1987: „Einführung in den gesteuerten Zweitspracherwerb“, in: E. Apeltauer (Hrsg.): Gesteuerter Zweitspracherwerb. Voraussetzungen und Konsequenzen für den Unterricht, München, S. 11 - 43

Apeltauer, E. 2003: Grundlagen des Erst- und Fremdsprachenerwerbs - Eine Einführung. Fernstudieneinheit 15. Fernstudienprojekt zur Fort- und Weiterbildung im Bereich Germanistik und Deutsch als Fremdsprache Teilbereich Deutsch als Fremdsprache, Kassel u. a.

Barnickel, K.-D. 1992: Falsche Freunde. Ein vergleichendes Wörterbuch Deutsch-Englisch, Heidelberg

Baugh, A. C. / Cable, T. 1978: A History of the English Language, London u. a.

Bausch, K.-R. 1973: „Kontrastive Linguistik“, in: W. A. Koch (Hrsg.): Perspektiven der Linguistik, Bd. 1, Stuttgart, S. $159-182$

Bausch, K.-R. / Kasper, G. 1979: „Der Zweitsprachenerwerb: Möglichkeiten und Grenzen der ,großen“ Hypothesen“, in: Linguistische Berichte, 64 / 1979, S. 5

Bernstein, B. 1971: Class, Codes and Control, London

Bianchi, M. / Busch, B. / Sommet, P. 1987: Partnerschaftliches Lernen im Fremdsprachenunterricht, Bonn / Frankfurt a. M.

Börner, W. / Kielhöfer, B. / Vogel, K. 1976: Französisch lehren und lernen. Aspekte der Sprachlehrforschung, Kronberg

Breitkreutz, H. 1973: „,False Friends“ und ihre unterrichtliche Behandlung“, in: Die Neueren Sprachen, $72 / 1973$, S. $70-74$

Brenni, J. 1964: American English - A bibliography, Philadelphia

Brough, S. / Docherty, V. J. 1998: Praktische Grammatik Englisch, Berlin / München u. a.

Brough, S. / Wittmann, C. K. 2001: Englisch - Typische Fehler vermeiden - Ein unterhaltsamer Ratgeber zur richtigen Wortwahl, Berlin / München

Burgschmidt, E. 1975: Die englischen Präpositionen, Dortmund

Burgschmidt E. / Götz, D. 1972: „Kontrastive Phonologie Deutsch-Englisch und Mundartinterferenz“, in: Linguistik und Didaktik, 11 / 1972, S. $209-225$

Burgschmidt, E. / Götz, D. 1974: Kontrastive Linguistik Deutsch/Englisch, München

Cherubim, D. 1976: Grammatische Kategorien. Das Verhältnis von traditioneller und moderner Sprachwissenschaft, Tübingen

Chomsky, N. 1959: „Rezension von Skinner, B. (1957): Verbal Behavior“, New York, in: Language, 35 / 1959 , S. $26-58$

Chomsky, N. 1965: Aspects of the Theory of Syntax, Cambridge M. A.

Christ, H. / Hüllen, W. 1995: „Fremdsprachenunterricht“, in: K.-R. Bausch, / H. Christ / H.-J. Krumm (Hrsg.): Handbuch des Fremdsprachenunterrichts, Tübingen, S. $1-7$

Clark, H. H. / Clark, E. V. 1977: Psychology and Language, New York

Cook, V. J. 1973: „The Comparison of Language Development in Native Children and Foreign Adults“, in: IRAL, XI / 1973, S. $13-28$

Corder, S. P. 1967: „The Significance of Learners’ Errors“, in: IRAL, V / 1967, S. 161 - 170 
Corder, S. P. 1976: „The Study of Interlanguage“, in: G. Nickel (Hrsg.): Proceedings of the $4^{\text {th }}$ International Congress of Applied Linguistics, Bd.2, Stuttgart, S. 9 - 34

Corder, S. P. 1978: „Language-Learner Language“, in: J. C. Richards (Hrsg.): Understanding Second and Foreign Language Learning. Issues and Approaches, Rowley, Mass., S. $71-93$

Cross, T. G. 1978: „Mother's speech and it's acquisition with rate of linguistic development in young children“, in: W. Waterson / C. E. Snow (Hrsg.), The development of communication, Chichester / New York

Crystal, D. 1984: Who Cares About English Usage?, Suffolk

Davies, A. 1991: The Native Speaker in Applied Linguistics, Edinburgh

Dechert, H. W. 1997: „Metakognition und Zweitsprachenerwerb“, in: U. Rampillon / G. Zimmermann (Hrsg.) 1997: Strategien und Techniken beim Erwerb fremder Sprachen, Ismaning, S. 13

Desgranges, I. 1990: Korrektur und Spracherwerb: Selbst- und Fremdkorrekturen in Gesprächen zwischen deutschen und ausländischen Kindern, Frankfurt a. M.

Doyé, P. 1996: „Prüfung der Handlungskompetenz durch pragmatische Tests“, in: G. Bach / J.-P. Timm: Englischunterricht, Tübingen, S. $192-208$

Drubig, B. 1972: „Zur Analyse syntaktischer Fehlleistungen“, in: G. Nickel: Fehleranalyse, Berlin, S. 78 - 91

Dulay, H. C. / Burt, M. K. 1974a: „You Can’t Learn Without Goofing. An Analysis of Children's Second Language Errors“, in: J. C. Richards, (Hrsg.): Error Analysis. Perspectives on Second Language Acquisition, London, S. $95-123$

Dulay, H. C. / Burt, M. K. 1974b: „Natural Sequences in Child Second Language Acquisition“, in: Language Learning, 24 / 1974, S. $37-53$

Dulay, H. C. / Burt, M. K. 1977: „Remarks on Creativity in Language Acquisition“, in: M. K. Burt / H. C. Dulay / M. Finocchiaro (Hrsg.): Viewpoints on English as a Second Language, New York, S. 95 - 126

Dulay, H. C. / Burt, M. K. / Krashen, S. D. 1982: Language Two, New York

Edge, J. 1989: Mistakes and Correction, London

Faerch, C. / Kasper, G. (Hrsg.) 1987: Introspection in Second Language Research, Clevedon

Fanselow, G. / Felix, S. W. 1993: Sprachtheorie - Eine Einführung in die Generative Grammatik. Band 1: Grundlagen und Zielsetzungen, Tübingen / Basel

Gardner, T. 1973: Hauptströmungen der modernen Linguistik, Göttingen

Gardner, R. C. 1985: Social Psychology and Language Learning. The Role of Attitudes and Motivation, London

Gass, S. M. / Selinker, L. 1994: Second Language Acquisition, New Jersey

Giera, F. 1980: „Fehler - ein ungelöstes Problem?“, in: Praxis des neusprachlichen Unterrichts, 27 / 1980 , S. 356

Gnutzmann, C. 1972: „Zur Analyse lexikalischer Fehler“, in: G. Nickel: Fehleranalyse, Berlin, S. 68 - 72

Grabowski, E. 1985: „Besonders fehleranfällige sprachliche Mittel des engeren Verbverbands im Englischen“, in: Fremdsprachenunterricht, 29 / 1985, S. $452-456$

Gutschow, H. 1967: „Zur Analyse von Schülerfehlern“, in: Englisch, 3 / 1967, S. 77 - 79

Havranek, G. 2001: „Zur Effizienz der mündlichen Korrektur“, in: M. B. Sellner (Hrsg.): Fremdsprachendidaktik und Zweitspracherwerb im Kontext, Frankfurt a. M., S. 10

Hecht, K. / Green, P. S. 1983: Fehleranalyse und Leistungsbewertung im Englischunterricht der Sekundarstufe I. Linguistische, lernpsychologische und didaktische Analyse von Englischfehlern in Briefen von deutschen und englischen Lernern, Donauwörth

Heindrichs, W. / Gester, F. W. / Kelz, H. P. 1980: Sprachlehrforschung. Angewandte Linguistik und Fremdsprachendidaktik, Stuttgart

Hellinger, M. 1977: Kontrastive Grammatik Deutsch/Englisch, Tübingen

Helliwell, M. 1989: Can I Become a Beefsteak?, Oxford

Herrmann, J. 1994: „Datengewinnung in der Zweitsprachenforschung. Einige empirische Befunde zur Effizienz verschiedener Verfahren“, in: Die Neueren Sprachen, 93 / 1994, S. 566 - 584

Hoffmann, H. G. 1973: Englisch compact - Ein Begleiter für alle, die Englisch lernen, München 
James, C. 1998: Errors in Language Learning and Use: Exploring Error Analysis, London / New York, S. 5 ff

James, C. / Klein, K. 1994: „Foreign language learners’ spelling and proof-reading strategies“, in: Papers and Studies in Contrastive Linguistics, Vol. 29, S. $31-46$

Juhász, J. 1970: Probleme der Interferenz, München

Juhász, J. 1983: „Probleme der Interferenz“, in: K. Hecht / P. S. Green: Fehleranalyse und Leistungsbewertung im Englischunterricht der Sekundarstufe I. Linguistische, lernpsychologische und didaktische Analyse von Englischfehlern in Briefen von deutschen und englischen Lernern, Donauwörth, S. 44

Kachru, B. 1985: „Standards, codification and sociolinguistic realism: the English language in the Outer Circle“, in: R. Quirk / H. G. Widdowson (Hrsg.): English in the World - Teaching and Learning the Language and Literatures, Cambridge, S. $11-30$

Kaltenbacher, M. 2001a: Universal Grammar and Parameter Resetting in Second Language Acquisition, Frankfurt a. M.

Kaltenbacher, M. 2001b: „Zweitspracherwerb und Erstsprachverlust: Zur fremdsprachlichen Interferenz im Übersetzungsprozeß“, in: M. B. Sellner (Hrsg.): Fremdsprachendidaktik und Zweitspracherwerb im Kontext, Frankfurt a. M., S. $29-41$

Kleinmann, H. H. 1977: „Avoidance Behavior in Adult Second Language Acquisition“, in: Language Learning, 27 / 1977, S. $93-107$

Knapp-Potthoff, A. / Knapp, K. 1982: Fremdsprachenlernen und -lehren. Eine Einführung in die Didaktik der Fremdsprachen vom Standpunkt der Zweitspracherwerbsforschung, Stuttgart

König, E. 1972: „Fehleranalyse und Fehlertherapie im lexikalischen Bereich“, in: G. Nickel: Fehleranalyse, Berlin, S. $73-77$

Kostrzewa, F. / Cheon-Kostrzewa, B. J. 1996: „Strategien in zielsprachlicher Kommunikation“, in: Neusprachl. Mitteilungen, 49 / 1996, S. 88 - 93

Krashen, S. D. 1975a: „The Development of Cerebral Dominance and Language learning: More Evidence“, in: D. P. Dato (Hrsg.): Developmental Psycholinguistics: Theory and Application, Washington D.C., S. 179 192

Krashen, S. D. 1975b: „The Critical Period for Language Acquisition and Its Possible Bases“, in: D. Aaronson / R.W. Rieber (Hrsg.): Developmental Psycholinguistics and Communication Disorders, New York, S. 211 224

Krashen, S. D. 1976: „Formal and Informal Linguistic Environments in Language Acquisition and Language Learning“, in: TESOL Quarterly, 10 / 1976, S. 157 - 168

Krashen, S. D. 1977: „The Monitor Model for Adult Second Language Performance“, in: M. K. Burt / H. C. Dulay / M. Finocchiaro (Hrsg.): Viewpoints on English as a Second Language, New York, S. $152-161$

Kultusminister des Landes Nordrhein-Westfalen (Hrsg.) 1989: Richtlinien Englisch - Hauptschule, Heft 3205 , Frechen

Kultusminister des Landes Nordrhein-Westfalen (Hrsg.) 1993: Richtlinien und Lehrpläne Englisch Gymnasium - Sekundarstufe I, Heft 3417, Frechen

Kultusminister des Landes Nordrhein-Westfalen (Hrsg.) 1994: Richtlinien und Lehrpläne Englisch - Realschule, Heft 3303, Frechen

Lado, R. 1965: Language testing, Oxford

Legenhausen, L. 1975: Fehleranalyse und Fehlerbewertung. Untersuchungen an englischen Reifeprüfungsnacherzählungen, Berlin

Legenhausen, L. 1985: „Typische Fehler im Bereich der englischen Satzkomplemente“, in: Der Fremdsprachliche Unterricht, 19 / 1985, S. 188 - 196

Lenneberg, E. H. 1967: Biological Foundations of Language, New York

Libben, G. 2000: „Representation and Processing in the Second Language Lexicon“, in: J. Archibald (Hrsg.): Second Language Acquisition and Linguistic Theory, Malden, Mass. / Oxford, S. 228 - 249

Lightbrown, P. M. 1990: „Process-product research on second language learning in classrooms“, in: B. Harley et. al. (Hrsg.): The Development of Second Language Proficiency, Cambridge, S. 82 - 92 
Lojewski, W. von 1991: Die Briten sind anders - Erfahrungen rund um Big Ben, München

McCretton, E. / Rider, N. 1993: „Error Gravity and Error Hierarchies“, in: IRAL, XXXI / 1993, S. 180 - 185

Melling, J. / Siebenhaar, R. H. 1956: Hauptschwierigkeiten der englischen Sprache, Heidelberg

Morrissey, M. D. 1981: „Error Grammar“, in: Die Neuere Sprachen, 80 / 1981, S. 173 - 194

Multhaup, U. / Wolff, D. 1992: „Prozeßorientierung in der Fremdsprachendidaktik. Statt einer Einleitung“, in: U. Multhaup / D. Wolff: Prozeßorientierung in der Fremdsprachendidaktik, Frankfurt a. M., S. 7 - 13

Multhaup, U. 1995: Psycholinguistik und fremdsprachliches Lernen, Ismaning

Norrish, J. 1983: Language Learners and their errors, London

O’Malley, J. M. / Chamot, A. U. 1990: Learning Strategies in Second Language Acquisition, Cambridge

Palmer, H. E. 1921: The Principles of Language Study, London

Pauels, W. 1995: „Sprachbegabung im Lichte kognitiver Zweitsprachenerwerbsmodelle“, in: Die Neueren Sprachen, 94 / 1995, S. 628

Penfield, W. / Roberts, L. 1959: Speech and Brain Mechanisms, Princeton

Pons Englisch Aktiv 2002, Stuttgart

Pons Großwörterbuch 1988, Collins deutsch-englisch, englisch-deutsch, Stuttgart

Poulisse, N. 1997: „Slips of the tongue and their correction in L2 learner speech - metalinguistic awareness and second language acquisition“, in: H. W. Dechert (Hrsg.): Metacognitions and Second Language Acquisition Multilingual Matters, Clevedon

Quirk, R. / Widdowson, H. G. 1985: „The English language in a global context“, in: R. Quirk / H. G. Widdowson (Hrsg.): English in the World - Teaching and Learning the Language and Literatures, Cambridge, S. 16

Raabe, H. 1980: „Der Fehler beim Fremdsprachenerwerb und Fremdsprachengebrauch“, in: D. Cherubim, (Hrsg.): Fehlerlinguistik, Tübingen, S. $61-68$

Radford, A. 1988: Transformational Grammar, Cambridge

Rampillon, U. / Zimmermann, G. (Hrsg.) 1997: Strategien und Techniken beim Erwerb fremder Sprachen, Ismaning

Rein, K. 1983: Einführung in die kontrastive Linguistik, Darmstadt

Reinecke, J. 1969: Language and Dialect in Hawaii, Honolulu (Erstdruck 1935)

Samarin, W. J. 1962: „Lingua francas of the world“, in: F. A. Rice (Hrsg.): Studies of the role of second languages, Washington

Schneider, K. P. 1992: „Prozesse und Strategien der Verbalisierung visueller Stimuli in der Fremdsprache“, in: U. Multhaup / D. Wolff: Prozeßorientierung in der Fremdsprachendidaktik, Frankfurt a. M., S. $34-40$

Schuhmann, F. M. / Schuhmann, J. H. 1977: „Diary of a language learner. An introspective study of second language learning“, in: D. Brown / C. A. Yorio / R. Crymes, S. $241-249$

Selinker, L. 1969: „Language transfer“, in: General Linguistics, 9 / 1969, S. 67 - 92

Selinker, L. 1972: „Interlanguage“, in: IRAL, X / 1972, S. 209 - 231

Selinker, L. / Lamendella, J. T. 1978: „Two Perspectives on Fossilization in Interlanguage Learning“, in: Interlanguage Studies Bulletin, 3 / 1978, S. 143 - 191

Selinker, L. / Swain, M. / Dumas, G. 1975: „The Interlanguage Hypothesis Extended to Children“, in: Language Learning, 25 / 1975, S. $139-152$

Skinner, B. F. 1957: Verbal behavior, New York

Skinner, B. F. 1966: „What is the experimental analysis of behavior?“, in: Journal of the Experimental Analysis of Behavior, 9 / 1966, S. $213-218$

Stenson, N. 1983: „Induced errors“, in: B. W. Robinett / J. Schachter (Hrsg.): Second Language Learning: Contrastive Analysis, Error Analysis and Related Aspects, Ann Arbor, S. 256 - 271

Strang, B. M. H. 1970: A History of English, London 
Swan, M. 1987: Learner English - A teacher's guide to interference and other problems, Cambridge

Swan, M. 1990: Practical English Usage, Oxford

Szagun, G. 2000: Sprachentwicklung beim Kind, Weinheim

Tarone, E. 1983: „On the Variability of Interlanguage Systems“, in: Applied Linguistics, 4 / 1983, S. 141 - 163

Thomson, A. J. / Martinet, A. V. 1987: A Practical English Grammar, Oxford

Timm, J.-P. 1996a: „Neue Perspektiven: Konsequente Schülerorientierung“, in: G. Bach, / J.-P. Timm (Hrsg.): Englischunterricht, Tübingen, S. 271

Timm, J.-P. 1996b: „Fehlerkorrektur zwischen Handlungsorientierung und didaktischer Steuerung“, in: G. Bach / J.-P. Timm (Hrsg.): Englischunterricht, Tübingen, S. 174 - 191

Towell, R. / Hawkins, R. 1994: Approaches to Second Language Acquisition, Clevedon

Trudgill, P. / Hannah, J. 1982: International English. A Guide to Varieties of Standard English, London

Ungerer, F. u. a. 1987: A Grammar of Present-Day English, Stuttgart

Vogel, K. 1990: Lernersprache - Linguistische und psycholinguistische Grundfragen zu ihrer Erforschung, Tübingen

Vogel, K. / Vogel, S. 1975: Lernpsychologie und Fremdsprachenerwerb, Tübingen

Vogel, T. 1989: „Tempus und Aspekt im Natürlichen Zweitsprachenerwerb“, in: B. Kettemann (Hrsg.): Englisch als Zweitsprache, Tübingen, S. $123-147$

Wardhaugh, R. 1970: „The contrastive analysis hypothesis“, in: TESOL Quarterly, 4 / 1970, S. 123 - 130

Weiher, E. 1974: Lautwahrnehmung und Lautproduktion im Englischunterricht für Deutsche, Diss., Kiel

Weiher, E. 1982: Praktische englische Phonetik, Bonn 1982

Weller, F. R. 1991: „Auswahlbibliographie zur Fehlerkunde (Veröffentlichungen 1977 - 1990)“, in: Die Neueren Sprachen, 90 / 1991, S. $710-732$

Widdowson, H. G. 1989: „Knowledge of Language and Ability for Use“, in: Applied Linguistics, 10 / 1989 , S. $128-137$

Wode, H. 1978a: „L1 Erwerb, L2 Erwerb und Fremdsprachenunterricht“, in: Die Neueren Sprachen, 77 / 1978 , S. $452-465$

Wode, H. 1978b: „Developmental Sequences in Naturalistic L2 Acquisition“, in: E. Marcussen-Hatch (Hrsg.): Second Language Acquisition. A Book of Readings, Rowley, Mass., S. $101-117$

Wode, H. 1988: Einführung in die Psycholinguistik, Ismaning

Young-Scholten, M. / Archibald, J. 2000: „Second Language Syllable Structure“, in: J. Archibald (Hrsg.): Second Language Acquisition and Linguistic Theory, Malden, Mass. / Oxford, S. 64 - 101

Zimbardo, Ph. G. / Gerrig, R. J. 1999: Psychologie, Berlin / Heidelberg

Zimmermann, G. 1997: „Anmerkungen zum Strategienkonzept“, in: U. Rampillon / G. Zimmermann (Hrsg.): Strategien und Techniken beim Erwerb fremder Sprachen, Ismaning, S. 95 - 113

\section{Sekundärliteratur}

Bald, W.-D. 1988: Kernprobleme der englischen Grammatik. Sprachliche Fakten und ihre Vermittlung, Berlin

Baur, R. S. / Rehbein, J. 1979: „Lerntheorie und Lernwirklichkeit. Zur Aneignung des deutschen Artikels bei türkischen Schülern. Ein Versuch mit der Galperinschen Konzeption“, in: Osnabrücker Beiträge zur Sprachtheorie, $10 / 1979$, S. $70-104$

Bausch, K.-R. / Königs, F. G. 1983: „,Lernt‘ oder ,erwirbt‘ man Fremdsprachen im Unterricht“, in: Die Neueren Sprachen, 82 / 1983, S. 316

Cornell A. / Schmidt, H. 1980: „Zur Analyse von deutsch-englischen Interferenzerscheinungen“, in: Praxis des neusprachlichen Unterrichts, 27 / 1980, S. $94-97$ 
Edmondson, W. 1999: Twelve Lectures on Second Language Acquisition. Foreign Language Teaching and Learning Perspectives, Tübingen

Felix, S. W. 1977: „Kreative und reproduktive Kompetenz im Zweitsprachenerwerb“, in: H. Hunfeld (Hrsg.): Neue Perspektiven der Fremdsprachendidaktik, Kronberg, S. 25 - 34

Granger, S. 1993: „International Corpus of Learner English“, in: J. Aarts / P. de Haan / N. Oostdijk (Hrsg.): English Language Corpora: Design, Analysis and Exploitation, Amsterdam, S. 57 - 71

Kaspari, D. 1995: „Kreativität im fremdsprachlichen Unterricht“, in: Praxis des neusprachlichen Unterrichts, 4 / 1995, S. 345 - 353

Richards, J. C. 1975: „Simplification - A Strategy in the Adult Acquisition of a Foreign Language - An Example from Indonesian/Malay“, in: Language Learning, 25 / 1975, S. 115 - 126

Selinker, L. 1992: Rediscovering Interlanguage, London

Williams, M. / Burden, B. 1996: Psychology for Language Teachers, Cambridge

Wolff, D. 1994: „Sprachpsychologie, Psycholinguistik und Fremdsprachenunterricht. Zur Anbahnung einer Beziehung. Dokumentiert in Aufsätzen aus den Neueren Sprachen von 1970 - 1992“, in: Die Neueren Sprachen, 93 / 1994, S. 103 - 1237 


\section{Tabellen}

Tabelle 1: Quantitatives Fehlerverhältnis innerhalb der Probandengruppen.

Tabelle 2: Anzahl der lexikalischen Fehler in der Probandengruppe mit den meisten

lexikalischen Abweichungen ....

Tabelle 3: Anzahl der lexikalischen Fehler in der Probandengruppe mit den wenigsten lexikalischen Abweichungen ...

Tabelle 4: Anzahl der syntaktisch-morphologischen Fehler in der Probandengruppe mit den meisten syntaktisch-morphologischen Abweichungen - Auslassungs- und Hinzufügungsfehler

Tabelle 5: Anzahl der syntaktisch-morphologischen Fehler in der Probandengruppe mit den wenigsten syntaktisch-morphologischen Abweichungen - Auslassungs- und Hinzufügungsfehler

Tabelle 6: Anzahl der syntaktisch-morphologischen Fehler in der Probandengruppe mit den meisten syntaktisch-morphologischen Abweichungen - Zeitensystem

Tabelle 7: Anzahl der syntaktisch-morphologischen Fehler in der Probandengruppe mit den wenigsten syntaktisch-morphologischen Abweichungen - Zeitensystem ....

Tabelle 8: Anzahl der syntaktisch-morphologischen Fehler in der Probandengruppe mit den meisten syntaktisch-morphologischen Abweichungen - Fehler auf Satzebene

Tabelle 9: Anzahl der syntaktisch-morphologischen Fehler in der Probandengruppe mit den wenigsten syntaktisch-morphologischen Abweichungen - Fehler auf Satzebene .....

Tabelle 10: Anzahl der orthographischen Fehler in der Probandengruppe mit den meisten orthographischen Abweichungen.

Tabelle 11: Anzahl der orthographischen Fehler in der Probandengruppe mit den wenigsten orthographischen Abweichungen.

Tabelle 12: Anwendbarkeit der Erwerbshypothesen in der Probandengruppe mit den meisten lexikalischen Abweichungen .

Tabelle 13: Anwendbarkeit der Erwerbshypothesen in der Probandengruppe mit den wenigsten lexikalischen Abweichungen .....

Tabelle 14: Kontrastivhypothese: Anwendbarkeit der Fehlererklärungsfaktoren in der Probandengruppe mit den meisten lexikalischen Abweichungen .....

Tabelle 15: Kontrastivhypothese: Anwendbarkeit der Fehlererklärungsfaktoren in der Probandengruppe mit den wenigsten lexikalischen Abweichungen

Tabelle 16: Interlanguagehypothese: Anwendbarkeit der Fehlererklärungsfaktoren in der Probandengruppe mit den meisten lexikalischen Abweichungen .....

Tabelle 17: Interlanguagehypothese: Anwendbarkeit der Fehlererklärungsfaktoren in der Probandengruppe mit den wenigsten lexikalischen Abweichungen

Tabelle 18: Anwendbarkeit der Erwerbshypothesen in der Probandengruppe mit den meisten syntaktisch-morphologischen Abweichungen

Tabelle 19: Anwendbarkeit der Erwerbshypothesen in der Probandengruppe mit den wenigsten syntaktisch-morphologischen Abweichungen

Tabelle 20: Kontrastivhypothese: Anwendbarkeit der Fehlererklärungsfaktoren in der Probandengruppe mit den meisten syntaktisch-morphologischen Abweichungen

Tabelle 21: Kontrastivhypothese: Anwendbarkeit der Fehlererklärungsfaktoren in der Probandengruppe mit den wenigsten syntaktisch-morphologischen Abweichungen .....

Tabelle 22: Identitätshypothese: Anwendbarkeit der Fehlererklärungsfaktoren in der Probandengruppe mit den meisten syntaktisch-morphologischen Abweichungen

Tabelle 23: Identitätshypothese: Anwendbarkeit der Fehlererklärungsfaktoren in der Probandengruppe mit den wenigsten syntaktisch-morphologischen Abweichungen ... 
Tabelle 24: Interlanguagehypothese: Anwendbarkeit der Fehlererklärungsfaktoren in der Probandengruppe mit den meisten syntaktisch-morphologischen Abweichungen.....

Tabelle 25: Interlanguagehypothese: Anwendbarkeit der Fehlererklärungsfaktoren in der Probandengruppe mit den wenigsten syntaktisch-morphologischen Abweichungen

Tabelle 26: Anwendbarkeit der Erwerbshypothesen in der Probandengruppe mit den meisten orthographischen Abweichungen

Tabelle 27: Anwendbarkeit der Erwerbshypothesen in der Probandengruppe mit den wenigsten orthographischen Abweichungen....

Tabelle 28: Kontrastivhypothese: Anwendbarkeit der Fehlererklärungsfaktoren in den Probandengruppen mit den meisten und mit den wenigsten orthographischen Abweichungen.....

Tabelle 29: Identitätshypothese: Anwendbarkeit der Fehlererklärungsfaktoren in den Probandengruppen mit den meisten und mit den wenigsten orthographischen Abweichungen.....

Tabelle 30: Interlanguagehypothese: Anwendbarkeit der Fehlererklärungsfaktoren in den Probandengruppen mit den meisten und mit den wenigsten orthographischen Abweichungen.....

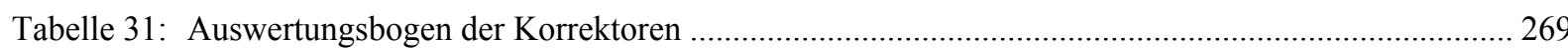

Tabelle 32: Auswertungsbogen der psycho- und soziolinguistischen Fragen / Hauptschule ............................ 270

Tabelle 33: Auswertungsbogen der psycho- und soziolinguistischen Fragen / Realschule............................ 271

Tabelle 34: Auswertungsbogen der psycho- und soziolinguistischen Fragen / Gymnasium.......................... 272

Tabelle 35: Auswertungsbogen der psycho- und soziolinguistischen Fragen / Erwachsenenbildung .............. 273

Abb. 5: $\quad$ Fragebogen und Aufgabenstellung des Briefes...................................................................... 275 


\begin{tabular}{|c|c|c|c|c|c|c|c|c|c|c|c|c|c|c|}
\hline 邑 & वे & 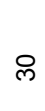 & $\begin{array}{l}\text { q } \\
\stackrel{N}{N}\end{array}$ & 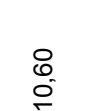 & $\begin{array}{l}\circ \\
\circ \\
\stackrel{\circ}{0} \\
\text { o }\end{array}$ & 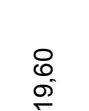 & $\begin{array}{l}\stackrel{\circ}{N} \\
\stackrel{N}{N}\end{array}$ & $\begin{array}{l}\mathscr{\infty} \\
\stackrel{\infty}{\infty}\end{array}$ & $\begin{array}{l}\stackrel{\circ}{\sim} \\
\stackrel{\sim}{\sim}\end{array}$ & \multirow[b]{2}{*}{$\begin{array}{l}\infty \\
\infty \\
\stackrel{+}{ \pm}\end{array}$} & \multirow[b]{2}{*}{$\begin{array}{l}\stackrel{\circ}{\Lambda} \\
\stackrel{0}{\infty} \\
\stackrel{0}{\infty}\end{array}$} & \multirow[b]{2}{*}{$\begin{array}{l}\stackrel{0}{2} \\
\stackrel{0}{\circ} \\
\omega^{\circ}\end{array}$} & \multirow[b]{2}{*}{$\begin{array}{l}\stackrel{\circ}{\circ} \\
\stackrel{9}{+}\end{array}$} & \multirow[b]{2}{*}{ 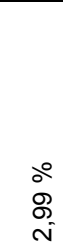 } \\
\hline 古 & $\stackrel{ \pm}{\Xi}$ & $\bar{\infty}$ & $\begin{array}{l}\infty \\
\infty \\
\infty \\
\stackrel{\leftrightarrow}{0}\end{array}$ & 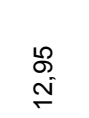 & $\begin{array}{l}\circ \\
\stackrel{0}{\circ} \\
\dot{\omega}\end{array}$ & $\begin{array}{l}\text { o } \\
\stackrel{\sim}{\sim} \\
\text { N. }\end{array}$ & $\begin{array}{l}\circ \\
\text { oे } \\
\text { ळे }\end{array}$ & 足 & $\underset{\stackrel{\aleph}{N}}{\stackrel{\sim}{N}}$ & & & & & \\
\hline 足 & षं & 足 & \begin{tabular}{l}
$\stackrel{N}{N}$ \\
\multirow{N}{\sim}{}
\end{tabular} & $\begin{array}{l}\bar{\Xi} \\
\stackrel{\sim}{\sim}\end{array}$ & $\begin{array}{l}\stackrel{0}{0} \\
\infty \\
\infty \\
\infty\end{array}$ & $\begin{array}{l}\stackrel{R}{2} \\
0 \\
N\end{array}$ & 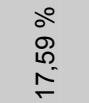 & $\underset{\infty}{\stackrel{N}{c}}$ & 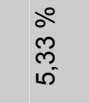 & \multirow{4}{*}{ 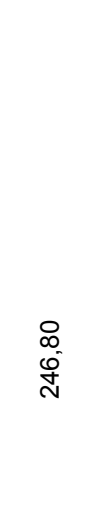 } & \multirow{4}{*}{$\begin{array}{l}\stackrel{\circ}{\circ} \\
\hat{N} \\
\stackrel{\sigma}{\sigma}\end{array}$} & \multirow{4}{*}{$\begin{array}{l}\stackrel{\circ}{\circ} \\
\stackrel{0}{\sim}\end{array}$} & \multirow{4}{*}{$\begin{array}{l}\circ \\
\tilde{0} \\
0 \\
0\end{array}$} & \multirow{4}{*}{$\begin{array}{l}\stackrel{\circ}{\circ} \\
\text { o } \\
\text { o }\end{array}$} \\
\hline ১) & $\stackrel{\vec{N}}{\sim}$ & ల్ల & $\begin{array}{l}\stackrel{q}{+} \\
\stackrel{+}{N}\end{array}$ & 茴 & $\begin{array}{l}\circ \\
8 \\
8 \\
i\end{array}$ & $\begin{array}{l}\hat{m} \\
\text { îे }\end{array}$ & $\begin{array}{l}\stackrel{\circ}{\circ} \\
\stackrel{o}{+} \\
\infty\end{array}$ & $\stackrel{\text { g }}{\text { N }}$ & $\begin{array}{l}\circ \\
8 \\
\circ\end{array}$ & & & & & \\
\hline ১) & $\stackrel{\text { I }}{\underset{N}{N}}$ & $\bar{\gamma}$ & $\begin{array}{l}\stackrel{\sim}{\infty} \\
\stackrel{5}{N}\end{array}$ & $\begin{array}{l}\text { ơ } \\
\text { ì }\end{array}$ & 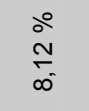 & $\frac{q}{-\infty}$ & $\begin{array}{l}\stackrel{\circ}{\circ} \\
\stackrel{9}{+} \\
\stackrel{2}{ }\end{array}$ & $\begin{array}{l}\stackrel{R}{\sim} \\
\leftarrow\end{array}$ & $\begin{array}{l}\stackrel{0}{\circ} \\
\infty \\
\infty \\
\leftarrow\end{array}$ & & & & & \\
\hline ১ঠ & $\begin{array}{l}\frac{5}{1} \\
i\end{array}$ & $\stackrel{+}{\sim}$ & $\begin{array}{l}\stackrel{\sim}{N} \\
\stackrel{\sim}{\sim}\end{array}$ & 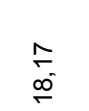 & 号 & $\stackrel{\sim}{\sim}$ & 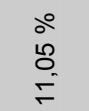 & $\stackrel{+}{\stackrel{N}{m}}$ & 号 & & & & & \\
\hline œ & 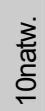 & 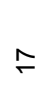 & 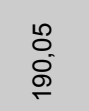 & $\begin{array}{l}\tilde{\sigma} \\
\text { ì }\end{array}$ & 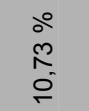 & $\begin{array}{l}8 \\
\frac{-}{a}\end{array}$ & $\begin{array}{l}\stackrel{0}{\circ} \\
\hat{n} \\
\stackrel{N}{N}\end{array}$ & 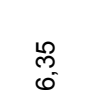 & $\begin{array}{c}\stackrel{\circ}{\circ} \\
\stackrel{\mathrm{m}}{\infty}\end{array}$ & \multirow{4}{*}{$\begin{array}{l}\text { N } \\
\text { N } \\
\text { N }\end{array}$} & \multirow{4}{*}{$\begin{array}{l}\circ \\
\text { ¿̊ } \\
10 \\
0 \\
0\end{array}$} & \multirow{4}{*}{$\begin{array}{l}\stackrel{0}{0} \\
0 \\
0 \\
\infty\end{array}$} & \multirow{4}{*}{ ○ } & \multirow{4}{*}{ 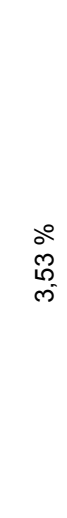 } \\
\hline 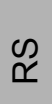 & 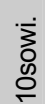 & $\stackrel{\infty}{\infty}$ & ্ֻ] & $\begin{array}{l}\stackrel{\infty}{N} \\
\stackrel{\sigma}{\sigma}\end{array}$ & $\begin{array}{l}\stackrel{\circ}{0} \\
\stackrel{0}{\infty}\end{array}$ & $\underset{\text { ల్ల }}{\tilde{ల}}$ & $\begin{array}{l}\stackrel{0}{0} \\
0 \\
\underline{5}\end{array}$ & 志 & 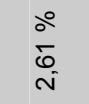 & & & & & \\
\hline 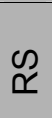 & $\begin{array}{l}\frac{\pi}{0} \\
\frac{0}{0}\end{array}$ & q & $\begin{array}{l}\mathcal{N} \\
\text { ș } \\
\text { Ni }\end{array}$ & 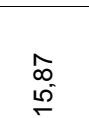 & $\begin{array}{l}\stackrel{\circ}{ } \\
\stackrel{8}{\circ}\end{array}$ & $\begin{array}{l}\hat{f} \\
\text { fi }\end{array}$ & $\begin{array}{l}\stackrel{0}{N} \\
\stackrel{N}{\infty}\end{array}$ & $\frac{}{65}$ & 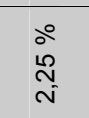 & & & & & \\
\hline D) & $\begin{array}{l}\infty \\
1 \\
i\end{array}$ & $\stackrel{\circ}{r}$ & 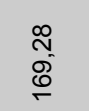 & $\begin{array}{l}\widehat{\infty} \\
\stackrel{-}{\Gamma} \\
\stackrel{5}{\sigma}\end{array}$ & $\begin{array}{l}\stackrel{0}{\circ} \\
\frac{0}{\infty}\end{array}$ & $\underset{\text { s. }}{\stackrel{5}{*}}$ & $\begin{array}{l}\stackrel{\circ}{ } \\
\infty \\
\infty \\
\underset{\leftarrow}{\leftarrow}\end{array}$ & $\begin{array}{l}8 \\
\text { is }\end{array}$ & $\begin{array}{l}\stackrel{\circ}{\circ} \\
\stackrel{\leftrightarrow}{\sim} \\
\stackrel{N}{N}\end{array}$ & & & & & \\
\hline 年 & 빰 & 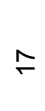 & $\begin{array}{l}\underset{\sigma}{\sigma} \\
\stackrel{\sigma}{\sigma}\end{array}$ & 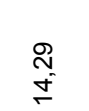 & 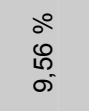 & 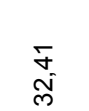 & 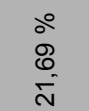 & స్ & 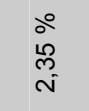 & \multirow{3}{*}{$\begin{array}{l}\hat{\kappa} \\
\stackrel{\infty^{-}}{\leftarrow}\end{array}$} & \multirow{3}{*}{$\begin{array}{l}\text { ॰ } \\
\text { ले } \\
\text { oे }\end{array}$} & \multirow{3}{*}{$\begin{array}{l}\stackrel{\circ}{0} \\
\stackrel{N}{\sigma} \\
\sigma\end{array}$} & \multirow{3}{*}{$\begin{array}{l}\stackrel{0}{0} \\
\stackrel{\infty}{\infty}\end{array}$} & \multirow{3}{*}{ 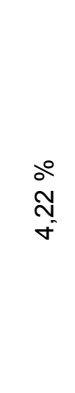 } \\
\hline OS & 巳 & ని & $\frac{n}{\pi}$ & $\begin{array}{l}8 \\
\infty \\
\infty\end{array}$ & $\begin{array}{l}\stackrel{0}{0} \\
\vdots \\
0\end{array}$ & $\begin{array}{l}\stackrel{8}{\infty} \\
\stackrel{\infty}{c}\end{array}$ & $\begin{array}{l}\stackrel{0}{ } \\
\text { ఫे } \\
0 \\
0\end{array}$ & $\begin{array}{l}\stackrel{R}{\sim} \\
\stackrel{\sigma}{*}\end{array}$ & $\begin{array}{l}\stackrel{0}{\infty} \\
\infty \\
\infty\end{array}$ & & & & & \\
\hline \multirow[t]{2}{*}{$\frac{N}{I}$} & $\begin{array}{l}9 \\
1 \\
i\end{array}$ & $\stackrel{\infty}{\sim}$ & $\begin{array}{l}\stackrel{2}{2} \\
\text { ơ } \\
\stackrel{2}{2}\end{array}$ & $\begin{array}{l}\infty \\
\infty \\
\sigma^{-1}\end{array}$ & $\begin{array}{c}\circ \\
\circ \\
\infty \\
\infty \\
\infty\end{array}$ & $\begin{array}{l}\stackrel{t}{0} \\
\stackrel{0}{0}\end{array}$ & $\begin{array}{l}\stackrel{0}{0} \\
\stackrel{0}{0} \\
\underline{b}\end{array}$ & $\begin{array}{l}8 \\
5 \\
15\end{array}$ & $\begin{array}{l}\text { oे } \\
\text { bे } \\
\text { in }\end{array}$ & & & & & \\
\hline & & 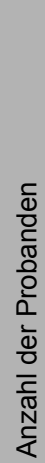 & 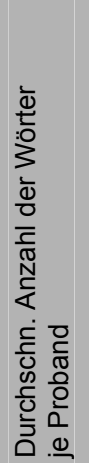 & 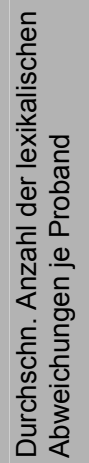 & 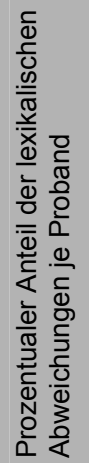 & 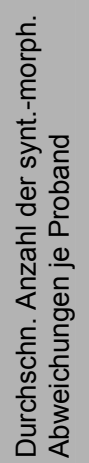 & 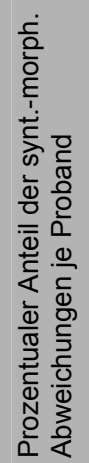 & 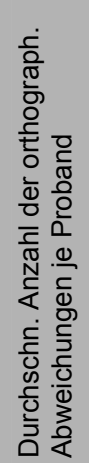 & 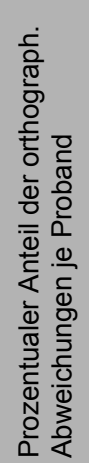 & 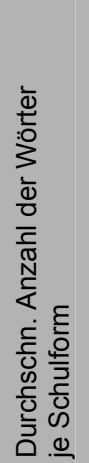 & 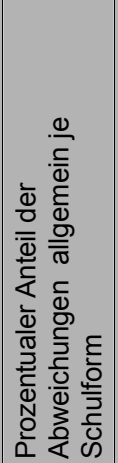 & 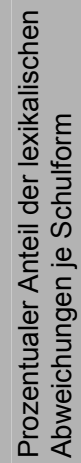 & 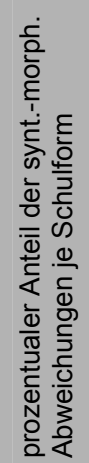 & 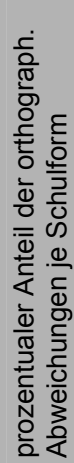 \\
\hline
\end{tabular}

Tabelle 1: Quantitatives Fehlerverhältnis innerhalb der Probandengruppen (die stark umrandeten Gruppen repräsentieren die Teilnehmer mit den jeweils meisten und wenigsten Fehlern in der betreffenden linguistischen Kategorie) 


\begin{tabular}{|l|r|}
\hline Lexemgruppen & Fehleranzahl \\
\hline Hilfsverben & $26(15)$ \\
Wortwahl & $168(95)$ \\
\hline inex. Wörter & $125(71)$ \\
\hline Präposition & $101(57)$ \\
\hline Adjektiv & $23(13)$ \\
\hline Adverb & $25(14)$ \\
\hline Pronomen & $109(62)$ \\
\hline Sondergruppe: Stil & $35(20)$ \\
\hline
\end{tabular}

Tabelle 2:

Anzahl der lexikalischen Fehler in der Probandengruppe mit den meisten lexikalischen Abweichungen ${ }^{672}$

\begin{tabular}{|l|rrrr|}
\hline & $\begin{array}{c}\text { Auslassungs- } \\
\text { fehler }\end{array}$ & \multicolumn{2}{|c|}{$\begin{array}{c}\text { Hinzufügungs- } \\
\text { fehler }\end{array}$} \\
\hline $\begin{array}{l}\text { a) Verlaufsform } \\
\text { G/V/F }\end{array}$ & 9 & $(5)$ & 5 & $(3)$ \\
\hline b) Infinitiv & 25 & $(14)$ & 5 & $(3)$ \\
\hline c) Partizipialsätze & 4 & $(2)$ & 2 & $(1)$ \\
\hline d) Gerundium & 12 & $(7)$ & 0 & $(0)$ \\
\hline $\begin{array}{l}\text { e) Artikel } \\
\text { (best./unbest.) }\end{array}$ & 35 & $(20)$ & 37 & $(21)$ \\
\hline f)Adjektiv & 5 & $(3)$ & 19 & $(11)$ \\
\hline g)Adverb & 72 & $(41)$ & 32 & $(18)$ \\
\hline $\begin{array}{l}\text { h) sonst. Wortarten / } \\
\text { Bestandteile }\end{array}$ & $224(127)$ & $279(158)$ \\
\hline
\end{tabular}

Tabelle 4:

Anzahl der syntaktisch-morphologischen Fehler in der Probandengruppe mit den meisten syntaktischmorphologischen Abweichungen ${ }^{673}$ - Auslassungsund Hinzufügungsfehler

\begin{tabular}{|c|c|}
\hline & Zeitensystem \\
\hline a) Gegenwart & $12(7)$ \\
\hline b) Vergangenheit & \\
\hline simple past & $86(49)$ \\
\hline present perfect & $2(1)$ \\
\hline past perfect & $2(1)$ \\
\hline c) Zukunft & $7(4)$ \\
\hline progressive & $2(1)$ \\
\hline going to-future & $26(15)$ \\
\hline will-future & \\
\hline
\end{tabular}

Tabelle 6:

Anzahl der syntaktisch-morphologischen Fehler in der Probandengruppe mit den meisten syntaktischmorphologischen Abweichungen ${ }^{674}$ - Zeitensystem

\begin{tabular}{|l|c|}
\hline Lexemgruppen & Fehleranzahl \\
\hline Hilfsverben & 25 \\
Wortwahl & 139 \\
\hline inex. Wörter & 10 \\
\hline Präposition & 51 \\
\hline Adjektiv & 14 \\
\hline Adverb & 20 \\
\hline Pronomen & 30 \\
\hline Sondergruppe: Stil & 29 \\
\hline
\end{tabular}

Tabelle 3:

Anzahl der lexikalischen Fehler in der Probandengruppe mit den wenigsten lexikalischen Abweichungen

\begin{tabular}{|l|c|c|}
\hline & $\begin{array}{c}\text { Auslassungs- } \\
\text { fehler }\end{array}$ & $\begin{array}{c}\text { Hinzufügungs- } \\
\text { fehler }\end{array}$ \\
\hline $\begin{array}{l}\text { a) Verlaufsform } \\
\text { G/V/F }\end{array}$ & 9 & 10 \\
\hline b) Infinitiv & 9 & 3 \\
\hline c) Partizipialsätze & 3 & 0 \\
\hline d) Gerundium & 12 & 5 \\
\hline $\begin{array}{c}\text { e) Artikel } \\
\text { (best./unbest.) }\end{array}$ & 21 & 17 \\
\hline f)Adjektiv & 10 & 13 \\
\hline g) Adverb & 35 & 31 \\
\hline $\begin{array}{c}\text { h) sonst. Wortarten / } \\
\text { Bestandteile }\end{array}$ & 182 & 120 \\
\hline
\end{tabular}

Tabelle 5:

Anzahl der syntaktisch-morphologischen Fehler in der Probandengruppe mit den wenigsten syntaktischmorphologischen Abweichungen - Auslassungsund Hinzufügungsfehler

\begin{tabular}{|c|c|}
\hline & Zeitensystem \\
\hline a) Gegenwart & 7 \\
\hline b) Vergangenheit & \\
\hline simple past & 34 \\
\hline present perfect & 10 \\
\hline past perfect & 12 \\
\hline c) Zukunft & \\
\hline progressive & 2 \\
\hline going to-future & 0 \\
\hline will-future & 12 \\
\hline
\end{tabular}

Tabelle 7:

Anzahl der syntaktisch-morphologischen Fehler in der Probandengruppe mit den wenigsten syntaktischmorphologischen Abweichungen - Zeitensystem

\footnotetext{
${ }^{672}$ Die Zahlen in Klammern geben die tatsächlich gemachten Fehler an, um sie jedoch mit der zweiten Probandengruppe vergleichen zu können, wurden sie entsprechend der Personenanzahl angepaßt.

673 dto.

674 dto.
} 


\begin{tabular}{|l|c|}
\hline & Fehler auf Satzebene \\
\hline Wort- / Satzstellung & $99(56)$ \\
\hline Bedingungssätze & $0(0)$ \\
\hline Aktiv / Passiv & $2(1)$ \\
\hline
\end{tabular}

Tabelle 8:

Anzahl der syntaktisch-morphologischen Fehler in der Probandengruppe mit den meisten syntaktisch-morphologischen Abweichungen ${ }^{675}$ - Fehler auf Satzebene

\begin{tabular}{|l|c|}
\hline & Fehlerhäufigkeit \\
\hline Großschreibung & $13(8)$ \\
\hline Kleinschreibung & $45(28)$ \\
\hline Rechtschreibung & $94(59)$ \\
\hline sonstige & $(0)$ \\
\hline
\end{tabular}

Tabelle 10:

Anzahl der orthographischen Fehler in der Probandengruppe mit den meisten orthographischen Abweichungen ${ }^{676}$

\begin{tabular}{|c|c|c|c|}
\hline & 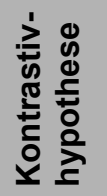 & 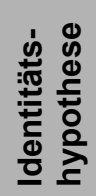 & 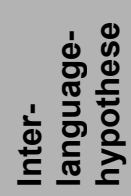 \\
\hline Hilfsverben & 93 & 0 & 100 \\
\hline Wortwahl & 65 & 0 & 96 \\
\hline inex. Wörter & 66 & 0 & 59 \\
\hline Präposition & 51 & 0 & 47 \\
\hline Adjektiv & 23 & 0 & 38 \\
\hline Adverb & 71 & 0 & 64 \\
\hline Pronomen & 8 & 0 & 32 \\
\hline Sondergruppe: Stil & 85 & 0 & 75 \\
\hline Durchschnitt & $58 \%$ & 0 & $64 \%$ \\
\hline
\end{tabular}

Tabelle 12:

Anwendbarkeit der Erwerbshypothesen in der Probandengruppe mit den meisten lexikalischen Abweichungen - Zuordnung zu den Hypothesen in \% ${ }^{677}$

\begin{tabular}{|l|c|}
\hline & Fehler auf Satzebene \\
\hline Wort- / Satzstellung & 35 \\
\hline Bedingungssätze & 5 \\
\hline Aktiv / Passiv & 1 \\
\hline
\end{tabular}

Tabelle 9:

Anzahl der syntaktisch-morphologischen Fehler in der Probandengruppe mit den wenigsten syntaktisch-morphologischen Abweichungen - Fehler auf Satzebene

\begin{tabular}{|l|c|}
\hline & Fehlerhäufigkeit \\
\hline Großschreibung & 29 \\
\hline Kleinschreibung & 6 \\
\hline Rechtschreibung & 53 \\
\hline sonstige & 0 \\
\hline
\end{tabular}

Tabelle 11:

Anzahl der orthographischen Fehler in der Probandengruppe mit den wenigsten orthographischen Abweichungen

\begin{tabular}{|c|c|c|c|}
\hline & 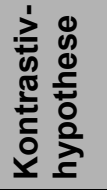 & 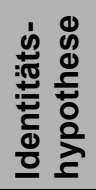 & 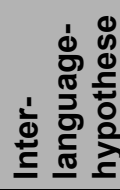 \\
\hline Hilfsverben & 32 & 0 & 56 \\
\hline Wortwahl & 52 & 0 & 62 \\
\hline inex. Wörter & 50 & 0 & 80 \\
\hline Präposition & 41 & 0 & 57 \\
\hline Adjektiv & 36 & 0 & 71 \\
\hline Adverb & 60 & 0 & 65 \\
\hline Pronomen & 60 & 0 & 57 \\
\hline Sondergruppe: Stil & 93 & 0 & 21 \\
\hline Durchschnitt & $53 \%$ & 0 & $59 \%$ \\
\hline
\end{tabular}

Tabelle 13:

Anwendbarkeit der Erwerbshypothesen in der Probandengruppe mit den wenigsten lexikalischen Abweichungen - Zuordnung zu Hypothesen in \% ${ }^{678}$

\footnotetext{
675 dto.

676 dto

${ }^{677}$ Die Fehlererklärungsfaktoren der Identitätshypothese kommen bei den Probanden mit den meisten lexikalischen Abweichungen nicht zum Tragen.

${ }^{678}$ dto.
} 


\begin{tabular}{|c|c|c|c|c|c|}
\hline & 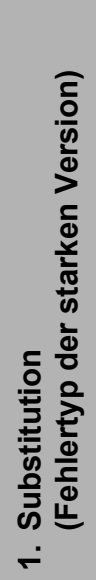 & 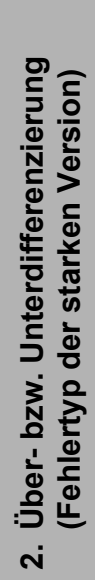 & 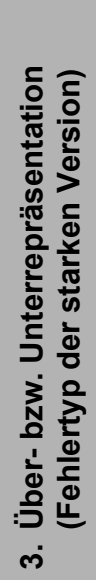 & 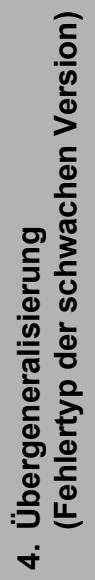 & 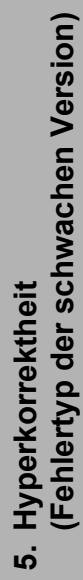 \\
\hline Hilfsverben & 47 & 47 & 0 & 0 & 0 \\
\hline Wortwahl & 42 & 25 & 0 & 0 & 0 \\
\hline Gebrauch inex. Wörter & 66 & 0 & 0 & 0 & 0 \\
\hline Präposition & 51 & 0 & 0 & 0 & 0 \\
\hline Adjektiv & 23 & 0 & 0 & 0 & 0 \\
\hline Adverb & 64 & 7 & 0 & 0 & 0 \\
\hline Pronomen & 8 & 0 & 0 & 0 & 0 \\
\hline Sondergruppe: Stil & 35 & 45 & 5 & 0 & 0 \\
\hline
\end{tabular}

Tabelle 14:

Kontrastivhypothese: Anwendbarkeit der Fehlererklärungsfaktoren in der Probandengruppe mit den meisten lexikalischen Abweichungen - Zuordnung in \% 


\begin{tabular}{|c|c|c|c|c|c|}
\hline & 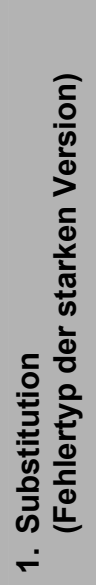 & 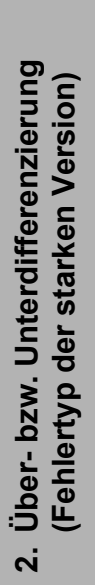 & 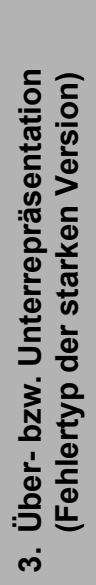 & 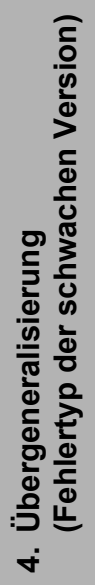 & 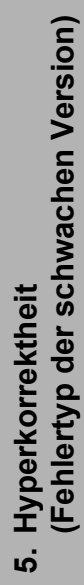 \\
\hline Hilfsverben & 28 & 4 & 0 & 0 & 0 \\
\hline Wortwahl & 40 & 12 & 0 & 1 & 0 \\
\hline Gebrauch inex. Wörter & 50 & 0 & 0 & 0 & 0 \\
\hline Präposition & 41 & 0 & 0 & 0 & 0 \\
\hline Adjektiv & 21 & 14 & 0 & 0 & 0 \\
\hline Adverb & 60 & 0 & 0 & 0 & 0 \\
\hline Pronomen & 47 & 10 & 0 & 3 & 0 \\
\hline Sondergruppe: Stil & 83 & 7 & 3 & 0 & 0 \\
\hline
\end{tabular}

Tabelle 15:

Kontrastivhypothese: Anwendbarkeit der Fehlererklärungsfaktoren in der Probandengruppe mit den wenigsten lexikalischen Abweichungen - Zuordnung in \% 


\begin{tabular}{|c|c|c|c|c|c|c|c|c|c|c|c|}
\hline & 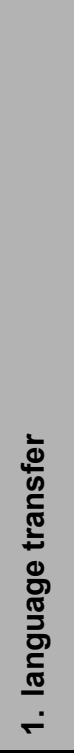 & 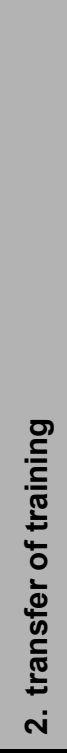 & 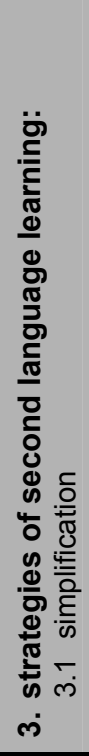 & 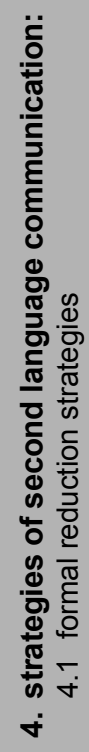 & 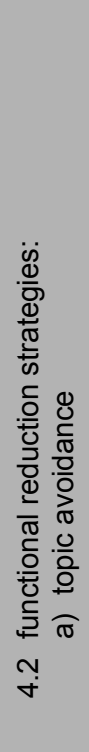 & 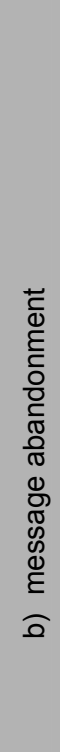 & 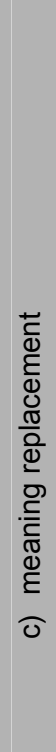 & 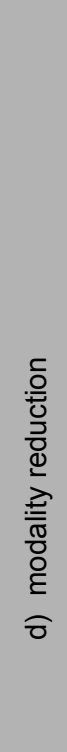 & 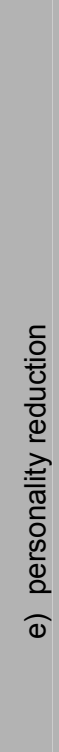 & 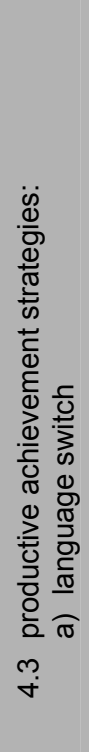 & 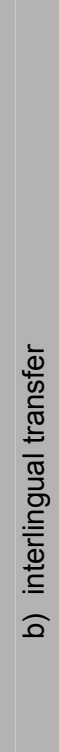 \\
\hline Hilfsverben & 0 & 0 & 0 & 0 & 0 & 0 & 0 & 20 & 0 & 0 & 13 \\
\hline Wortwahl & 0 & 0 & 1 & 0 & 0 & 0 & 0 & 15 & 6 & 0 & 20 \\
\hline Gebrauch inex. Wörter & 0 & 0 & 0 & 0 & 0 & 0 & 0 & 0 & 0 & 39 & 4 \\
\hline Präposition & 0 & 0 & 0 & 0 & 0 & 0 & 0 & 0 & 2 & 0 & 7 \\
\hline Adjektiv & 0 & 0 & 0 & 0 & 0 & 0 & 0 & 23 & 8 & 0 & 0 \\
\hline Adverb & 0 & 0 & 0 & 0 & 0 & 0 & 0 & 7 & 7 & 0 & 21 \\
\hline Pronomen & 0 & 0 & 0 & 0 & 0 & 0 & 0 & 0 & 0 & 0 & 8 \\
\hline Sondergruppe: Stil & 0 & 5 & 0 & 0 & 0 & 5 & 0 & 35 & 25 & 0 & 5 \\
\hline
\end{tabular}

\begin{tabular}{|c|c|c|c|c|c|c|c|c|c|c|c|}
\hline & 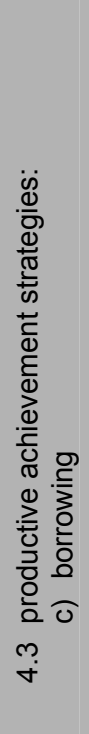 & 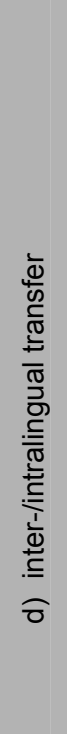 & 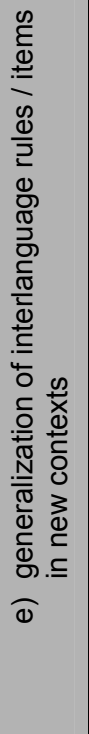 & 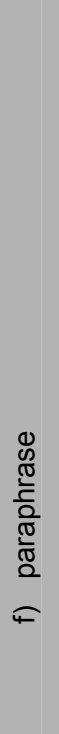 & 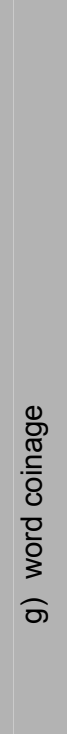 & 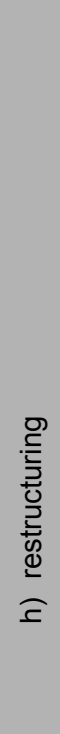 & 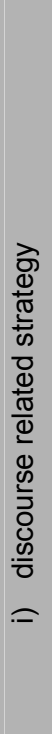 & 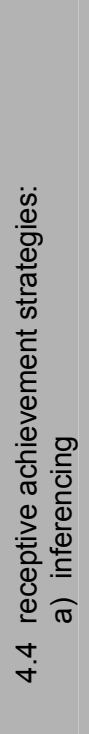 & 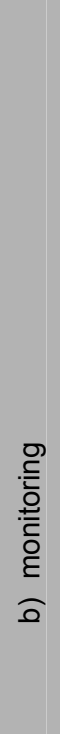 & 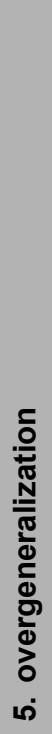 & 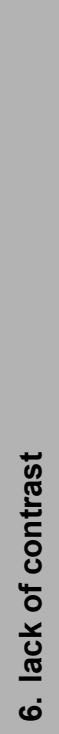 \\
\hline Hilfsverben & 0 & 7 & 73 & 0 & 0 & 0 & 0 & 0 & 0 & 0 & 7 \\
\hline Wortwahl & 0 & 11 & 47 & 0 & 0 & 0 & 0 & 0 & 0 & 4 & 17 \\
\hline Gebrauch inex. Wörter & 3 & 0 & 0 & 0 & 14 & 0 & 0 & 0 & 0 & 1 & 4 \\
\hline Präposition & 0 & 0 & 32 & 0 & 0 & 0 & 0 & 0 & 0 & 5 & 11 \\
\hline Adjektiv & 0 & 0 & 0 & 0 & 0 & 0 & 0 & 0 & 0 & 8 & 0 \\
\hline Adverb & 0 & 7 & 14 & 0 & 0 & 0 & 0 & 0 & 0 & 0 & 14 \\
\hline Pronomen & 0 & 2 & 27 & 0 & 0 & 0 & 0 & 0 & 0 & 0 & 10 \\
\hline Sondergruppe: Stil & 0 & 0 & 0 & 0 & 0 & 0 & 0 & 0 & 0 & 5 & 0 \\
\hline
\end{tabular}

Tabelle16:

Interlanguagehypothese: Anwendbarkeit der Fehlererklärungsfaktoren in der Probandengruppe mit den meisten lexikalischen Abweichungen - Zuordnung in \% 


\begin{tabular}{|c|c|c|c|c|c|c|c|c|c|c|c|}
\hline & 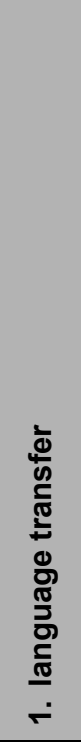 & 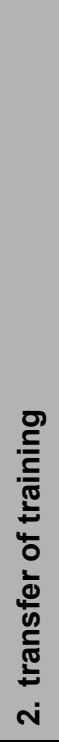 & 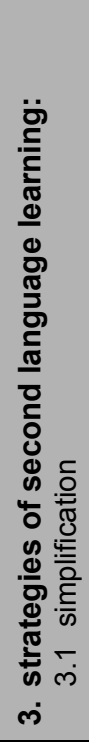 & 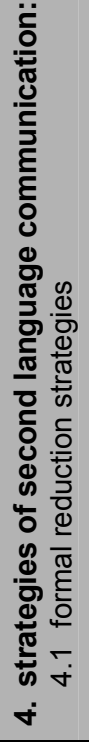 & 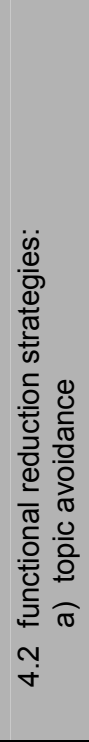 & 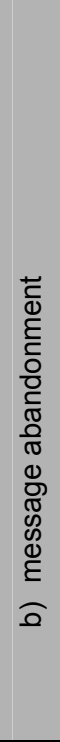 & 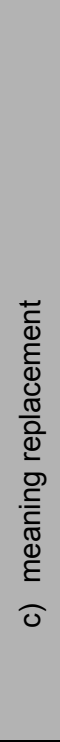 & 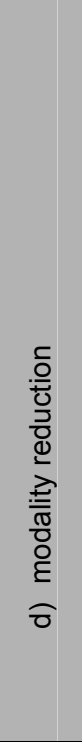 & 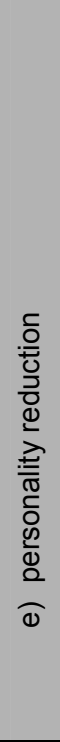 & 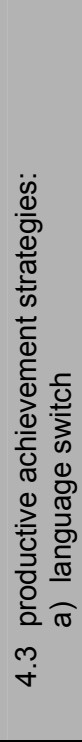 & 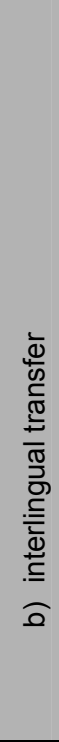 \\
\hline Hilfsverben & 0 & 0 & 0 & 0 & 0 & 0 & 0 & 4 & 0 & 0 & 0 \\
\hline Wortwahl & 0 & 0 & 0 & 0 & 0 & 0 & 1 & 4 & 0 & 0 & 0 \\
\hline Gebrauch inex. Wörter & 0 & 0 & 0 & 0 & 0 & 0 & 30 & 0 & 0 & 0 & 20 \\
\hline Präposition & 0 & 0 & 0 & 0 & 0 & 0 & 0 & 0 & 0 & 0 & 0 \\
\hline Adjektiv & 0 & 0 & 0 & 0 & 0 & 0 & 0 & 0 & 0 & 0 & 0 \\
\hline Adverb & 0 & 0 & 0 & 0 & 0 & 0 & 0 & 0 & 0 & 0 & 0 \\
\hline Pronomen & 0 & 0 & 0 & 0 & 0 & 0 & 0 & 3 & 0 & 0 & 0 \\
\hline Sondergruppe: Stil & 0 & 0 & 0 & 0 & 0 & 0 & 0 & 3 & 0 & 0 & 0 \\
\hline & 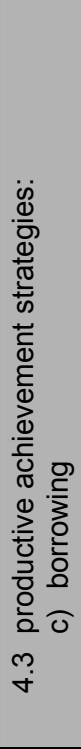 & 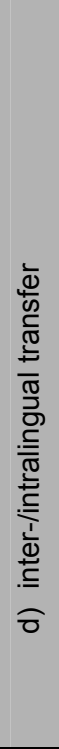 & 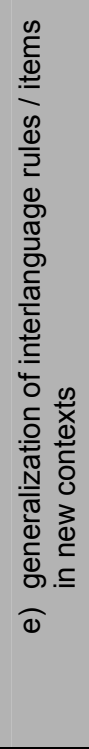 & 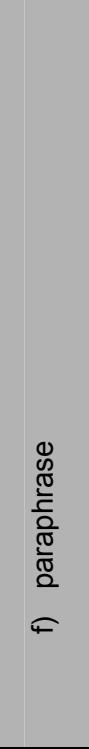 & 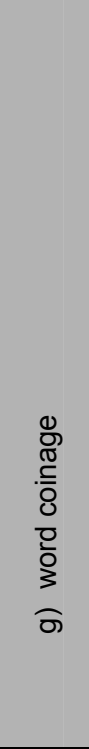 & 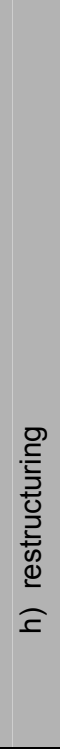 & 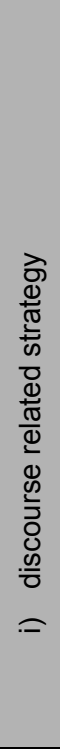 & 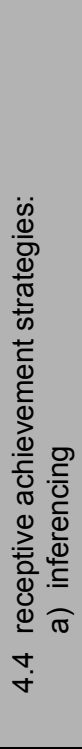 & 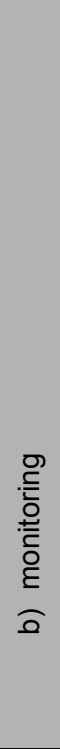 & 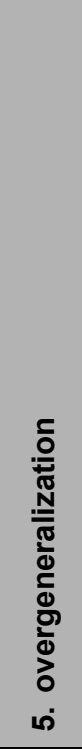 & 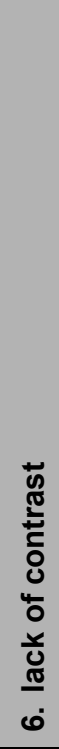 \\
\hline Hilfsverben & 0 & 0 & 40 & 4 & 0 & 0 & 0 & 0 & 0 & 8 & 0 \\
\hline Wortwahl & 0 & 0 & 43 & 4 & 1 & 0 & 0 & 0 & 0 & 5 & 5 \\
\hline Gebrauch inex. Wörter & 10 & 0 & 0 & 0 & 0 & 0 & 0 & 0 & 0 & 0 & 50 \\
\hline Präposition & 0 & 0 & 41 & 0 & 0 & 0 & 0 & 0 & 0 & 12 & 10 \\
\hline Adjektiv & 0 & 0 & 57 & 7 & 0 & 0 & 0 & 0 & 0 & 7 & 14 \\
\hline Adverb & 0 & 0 & 40 & 15 & 0 & 0 & 0 & 0 & 0 & 10 & 0 \\
\hline Pronomen & 0 & 0 & 30 & 20 & 0 & 0 & 0 & 0 & 0 & 0 & 7 \\
\hline Sondergruppe: Stil & 0 & 0 & 10 & 7 & 0 & 0 & 0 & 0 & 0 & 0 & 0 \\
\hline
\end{tabular}

Tabelle17:

Interlanguagehypothese: Anwendbarkeit der Fehlererklärungsfaktoren in der Probandengruppe mit den wenigsten lexikalischen Abweichungen - Zuordnung in \% 


\begin{tabular}{|c|c|c|c|}
\hline & Kontrastivhypothese & Identitätshypothese & Interlanguagehypothese \\
\hline 1. Wort / Satzstellung & 84 & 98 & 100 \\
\hline 2. Auslassungsfehler: & 52 & 82 & 91 \\
\hline a) Verlaufsform: G/V/F & 4 & 3 & 3 \\
\hline b) Infinitiv & 9 & 7 & 7 \\
\hline c) Partizipialsätze & 2 & 1 & 1 \\
\hline d) Gerundium & 4 & 3 & 4 \\
\hline e) Artikel (best./unbest.) & 10 & 11 & 10 \\
\hline f) Adjektiv & 2 & 2 & 2 \\
\hline g) Adverb & 13 & 11 & 12 \\
\hline h) sonst. Wortarten / Bestandteile & 56 & 63 & 63 \\
\hline 3. Hinzufügungsfehler: & 68 & 87 & 97 \\
\hline a) Verlaufsform: G/V/F & 1 & 2 & 1 \\
\hline b) Infinitiv & 1 & 2 & 1 \\
\hline c) Partizipialsätze & 1 & 1 & 0,5 \\
\hline d) Gerundium & 0 & 0 & 0 \\
\hline e) Artikel (best./unbest.) & 12 & 10 & 10 \\
\hline f) Adjektiv & 5 & 4 & 5 \\
\hline g) Adverb & 11 & 8 & 9 \\
\hline h) sonst. Wortarten / Bestandteile & 68 & 74 & 73 \\
\hline 4. Bedingungssätze & 0 & 0 & 0 \\
\hline 5. Aktiv / Passiv & 100 & 1 & 100 \\
\hline 6. Zeitensystem: & 40 & 71 & 100 \\
\hline a) Gegenwart & 3 & 5 & 9 \\
\hline \multicolumn{4}{|l|}{ b) Vergangenheit: } \\
\hline - simple past & 48 & 63 & 63 \\
\hline - present perfect & 3 & 1 & 1 \\
\hline - past perfect & 3 & 1 & 1 \\
\hline \multicolumn{4}{|l|}{ c) Zukunft: } \\
\hline - progressive & 13 & 4 & 5 \\
\hline - going to-future & 3 & 1 & 1 \\
\hline - will-future & 26 & 21 & 19 \\
\hline Durchschnitt & $57 \%$ & $76 \%$ & $81 \%$ \\
\hline
\end{tabular}

Tabelle 18:

Anwendbarkeit der Erwerbshypothesen in der Probandengruppe mit den meisten syntaktisch-morphologischen Abweichungen - Zuordnung zu den Hypothesen in \% 


\begin{tabular}{|c|c|c|c|}
\hline & Kontrastivhypothese & Identitätshypothese & Interlanguagehypothese \\
\hline 1. Wort / Satzstellung & 86 & 100 & 100 \\
\hline 2. Auslassungsfehler: & 86 & 96 & 98 \\
\hline a) Verlaufsform: G/V/F & 3 & 3 & 3 \\
\hline b) Infinitiv & 4 & 3 & 3 \\
\hline c) Partizipialsätze & 1 & 1 & 1 \\
\hline d) Gerundium & 5 & 4 & 4 \\
\hline e) Artikel (best./unbest.) & 7 & 7 & 8 \\
\hline f) Adjektiv & 4 & 4 & 4 \\
\hline g) Adverb & 13 & 12 & 13 \\
\hline h) sonst. Wortarten / Bestandteile & 64 & 65 & 64 \\
\hline 3. Hinzufügungsfehler: & 72 & 92 & 94 \\
\hline a) Verlaufsform: G/V/F & 1 & 5 & 5 \\
\hline b) Infinitiv & 2 & 2 & 2 \\
\hline c) Partizipialsätze & 0 & 0 & 0 \\
\hline d) Gerundium & 0 & 3 & 3 \\
\hline e) Artikel (best./unbest.) & 11 & 9 & 9 \\
\hline f) Adjektiv & 7 & 7 & 7 \\
\hline g) Adverb & 8 & 9 & 10 \\
\hline h) sonst. Wortarten / Bestandteile & 71 & 65 & 64 \\
\hline 4. Bedingungssätze & 100 & 80 & 100 \\
\hline 5. Aktiv / Passiv & 0 & 100 & 100 \\
\hline 6. Zeitensystem: & 70 & 96 & 99 \\
\hline a) Gegenwart & 6 & 8 & 9 \\
\hline b) Vergangenheit: & 76 & 74 & 74 \\
\hline - simple past & 48 & 45 & 45 \\
\hline - present perfect & 7 & 14 & 13 \\
\hline - past perfect & 20 & 16 & 16 \\
\hline c) Zukunft: & 19 & 18 & 17 \\
\hline - progressive & 2 & 3 & 3 \\
\hline - going to-future & 0 & 0 & 0 \\
\hline - will-future & 17 & 15 & 14 \\
\hline Durchschnitt & $69 \%$ & $94 \%$ & $98,5 \%$ \\
\hline
\end{tabular}

Tabelle 19:

Anwendbarkeit der Erwerbshypothesen in der Probandengruppe mit den wenigsten syntaktischmorphologischen Abweichungen - Zuordnung zu den Hypothesen in \% 


\begin{tabular}{|c|c|c|c|c|c|}
\hline & 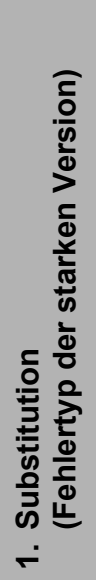 & 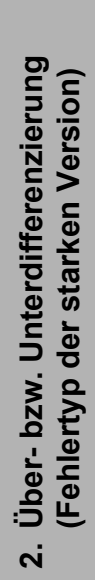 & 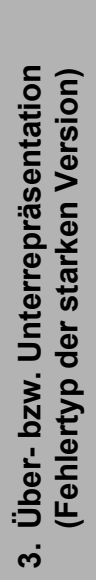 & 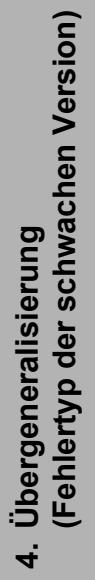 & 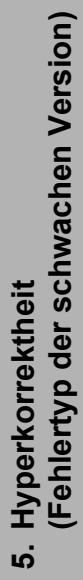 \\
\hline 1. Wort / Satzstellung & 66 & 0 & 0 & 4 & 20 \\
\hline \multicolumn{6}{|l|}{ 2. Auslassungsfehler: } \\
\hline a) Verlaufsform: G/V/F & 100 & 100 & 0 & 100 & 0 \\
\hline b) Infinitiv & 64 & 36 & 0 & 29 & 7 \\
\hline c) Partizipialsätze & 50 & 50 & 0 & 100 & 0 \\
\hline d) Gerundium & 71 & 14 & 0 & 43 & 0 \\
\hline e) Artikel (best./unbest.) & 45 & 40 & 0 & 35 & 5 \\
\hline f) Adjektiv & 33 & 0 & 33 & 33 & 0 \\
\hline g) Adverb & 22 & 27 & 0 & 24 & 0 \\
\hline h) sonst. Wortarten / Bestandteile & 43 & 19 & 2 & 28 & 0 \\
\hline \multicolumn{6}{|l|}{ 3. Hinzufügungsfehler: } \\
\hline a) Verlaufsform: G/V/F & 0 & 33 & 0 & 33 & 0 \\
\hline b) Infinitiv & 33 & 0 & 0 & 33 & 0 \\
\hline c) Partizipialsätze & 0 & 0 & 0 & 100 & 0 \\
\hline d) Gerundium & 0 & 0 & 0 & 0 & 0 \\
\hline e) Artikel (best./unbest.) & 86 & 19 & 0 & 10 & 0 \\
\hline f) Adjektiv & 64 & 27 & 36 & 45 & 0 \\
\hline g) Adverb & 67 & 33 & 28 & 50 & 0 \\
\hline h) sonst. Wortarten / Bestandteile & 50 & 21 & 8 & 33 & 4 \\
\hline 4. Bedingungssätze & 0 & 0 & 0 & 0 & 0 \\
\hline 5. Aktiv / Passiv & 100 & 0 & 0 & 0 & 0 \\
\hline \multicolumn{6}{|l|}{ 6. Zeitensystem: } \\
\hline a) Gegenwart & 14 & 0 & 0 & 14 & 0 \\
\hline \multicolumn{6}{|l|}{ b) Vergangenheit: } \\
\hline - simple past & 29 & 8 & 0 & 12 & 4 \\
\hline - present perfect & 0 & 100 & 0 & 100 & 0 \\
\hline - past perfect & 0 & 100 & 0 & 100 & 0 \\
\hline \multicolumn{6}{|l|}{ c) Zukunft: } \\
\hline - progressive & 100 & 75 & 0 & 100 & 25 \\
\hline - going to-future & 100 & 100 & 0 & 100 & 0 \\
\hline - will-future & 47 & 20 & 0 & 40 & 0 \\
\hline
\end{tabular}

Tabelle 20:

Kontrastivhypothese: Anwendbarkeit der Fehlererklärungsfaktoren in der Probandengruppe mit den meisten syntaktisch-morphologischen Abweichungen - Zuordnung in \% 


\begin{tabular}{|c|c|c|c|c|c|}
\hline & 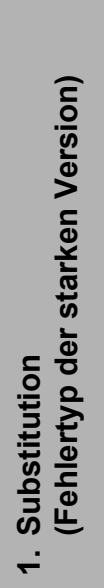 & 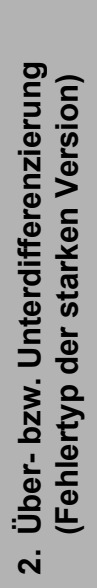 & 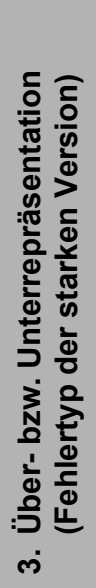 & 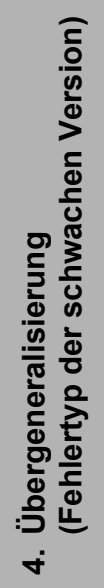 & 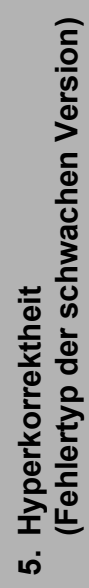 \\
\hline 1. Wort / Satzstellung & 86 & 66 & 0 & 69 & 0 \\
\hline \multicolumn{6}{|l|}{ 2. Auslassungsfehler: } \\
\hline a) Verlaufsform: G/V/F & 89 & 56 & 0 & 89 & 0 \\
\hline b) Infinitiv & 100 & 89 & 0 & 100 & 0 \\
\hline c) Partizipialsätze & 67 & 67 & 0 & 67 & 0 \\
\hline d) Gerundium & 92 & 92 & 0 & 92 & 0 \\
\hline e) Artikel (best./unbest.) & 67 & 67 & 0 & 71 & 10 \\
\hline f) Adjektiv & 90 & 90 & 0 & 50 & 0 \\
\hline g) Adverb & 91 & 91 & 0 & 77 & 0 \\
\hline h) sonst. Wortarten / Bestandteile & 83 & 78 & 0 & 82 & 0,5 \\
\hline \multicolumn{6}{|l|}{ 3. Hinzufügungsfehler: } \\
\hline a) Verlaufsform: G/V/F & 0 & 10 & 0 & 10 & 10 \\
\hline b) Infinitiv & 100 & 100 & 0 & 100 & 0 \\
\hline c) Partizipialsätze & 0 & 0 & 0 & 0 & 0 \\
\hline d) Gerundium & 0 & 0 & 0 & 0 & 0 \\
\hline e) Artikel (best./unbest.) & 94 & 94 & 0 & 94 & 0 \\
\hline f) Adjektiv & 77 & 46 & 0 & 77 & 0 \\
\hline g) Adverb & 39 & 29 & 0 & 35 & 0 \\
\hline h) sonst. Wortarten / Bestandteile & 84 & 75 & 0 & 83 & 0 \\
\hline 4. Bedingungssätze & 100 & 100 & 0 & 100 & 0 \\
\hline 5. Aktiv / Passiv & 0 & 0 & 0 & 0 & 0 \\
\hline \multicolumn{6}{|l|}{ 6. Zeitensystem: } \\
\hline a) Gegenwart & 43 & 29 & 0 & 43 & 43 \\
\hline \multicolumn{6}{|l|}{ b) Vergangenheit: } \\
\hline - simple past & 76 & 62 & 0 & 96 & 0 \\
\hline - present perfect & 40 & 10 & 0 & 40 & 0 \\
\hline - past perfect & 92 & 67 & 0 & 83 & 0 \\
\hline \multicolumn{6}{|l|}{ c) Zukunft: } \\
\hline - progressive & 50 & 50 & 0 & 50 & 0 \\
\hline - going to-future & 0 & 0 & 0 & 0 & 0 \\
\hline - will-future & 75 & 75 & 0 & 75 & 0 \\
\hline
\end{tabular}

Tabelle 21:

Kontrastivhypothese: Anwendbarkeit der Fehlererklärungsfaktoren in der Probandengruppe mit den wenigsten syntaktisch-morphologischen Abweichungen - Zuordnung in \% 


\begin{tabular}{|c|c|c|c|c|c|c|c|c|}
\hline & $\begin{array}{l}\text { 음 } \\
\text { 은 } \\
0 \\
\frac{0}{0} \\
\frac{0}{0} \\
\frac{0}{E} \\
0 \\
-\end{array}$ & 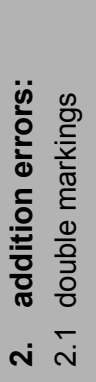 & 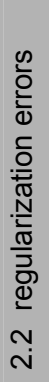 & 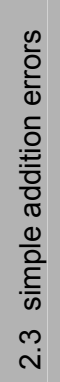 & 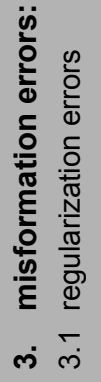 & 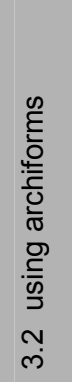 & 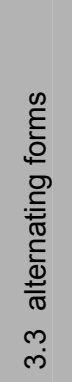 & 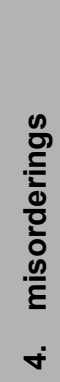 \\
\hline 1. Wort / Satzstellung & 7 & 0 & 0 & 0 & 0 & 5 & 7 & 95 \\
\hline \multicolumn{9}{|l|}{ 2. Auslassungsfehler: } \\
\hline a) Verlaufsform: G/V/F & 100 & 0 & 0 & 0 & 0 & 20 & 40 & 0 \\
\hline b) Infinitiv & 11 & 0 & 0 & 14 & 0 & 7 & 21 & 21 \\
\hline c) Partizipialsätze & 50 & 0 & 0 & 50 & 0 & 50 & 0 & 0 \\
\hline d) Gerundium & 57 & 0 & 0 & 0 & 0 & 71 & 86 & 29 \\
\hline e) Artikel (best./unbest.) & 95 & 0 & 0 & 0 & 0 & 15 & 40 & 15 \\
\hline f) Adjektiv & 33 & 0 & 0 & 0 & 0 & 0 & 33 & 33 \\
\hline g) Adverb & 39 & 0 & 0 & 0 & 0 & 2 & 2 & 22 \\
\hline h) sonst. Wortarten / Bestandteile & 78 & 0 & 0 & 0 & 0 & 11 & 24 & 32 \\
\hline \multicolumn{9}{|l|}{ 3. Hinzufügungsfehler: } \\
\hline a) Verlaufsform: G/V/F & 0 & 0 & 0 & 33 & 0 & 0 & 67 & 0 \\
\hline b) Infinitiv & 33 & 0 & 0 & 33 & 0 & 67 & 0 & 0 \\
\hline c) Partizipialsätze & 0 & 0 & 0 & 0 & 0 & 100 & 0 & 0 \\
\hline d) Gerundium & 0 & 0 & 0 & 0 & 0 & 0 & 0 & 0 \\
\hline e) Artikel (best./unbest.) & 0 & 0 & 0 & 81 & 0 & 0 & 33 & 14 \\
\hline f) Adjektiv & 9 & 0 & 0 & 36 & 0 & 18 & 9 & 36 \\
\hline g) Adverb & 0 & 0 & 0 & 72 & 0 & 0 & 0 & 17 \\
\hline h) sonst. Wortarten / Bestandteile & 0 & 4 & 1 & 65 & 1 & 8 & 21 & 28 \\
\hline 4. Bedingungssätze & 0 & 0 & 0 & 0 & 0 & 0 & 0 & 0 \\
\hline 5. Aktiv / Passiv & 0 & 0 & 0 & 0 & 0 & 0 & 100 & 100 \\
\hline \multicolumn{9}{|l|}{ 6. Zeitensystem: } \\
\hline a) Gegenwart & 0 & 0 & 0 & 14 & 0 & 71 & 29 & 14 \\
\hline \multicolumn{9}{|l|}{ b) Vergangenheit: } \\
\hline - simple past & 10 & 2 & 2 & 10 & 8 & 49 & 49 & 2 \\
\hline - present perfect & 100 & 0 & 0 & 0 & 0 & 0 & 100 & 0 \\
\hline - past perfect & 100 & 0 & 0 & 0 & 0 & 0 & 0 & 0 \\
\hline \multicolumn{9}{|l|}{ c) Zukunft: } \\
\hline - progressive & 75 & 0 & 0 & 0 & 0 & 25 & 75 & 0 \\
\hline - going to-future & 100 & 0 & 0 & 0 & 0 & 0 & 100 & 0 \\
\hline - will-future & 67 & 0 & 0 & 0 & 0 & 20 & 93 & 7 \\
\hline
\end{tabular}

Tabelle 22:

Identitätshypothese: Anwendbarkeit der Fehlererklärungsfaktoren in der Probandengruppe mit den $\underline{\text { meisten }}$ syntaktisch-morphologischen Abweichungen - Zuordnung in \% 


\begin{tabular}{|c|c|c|c|c|c|c|c|c|}
\hline & 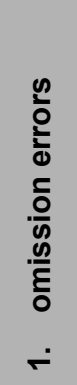 & 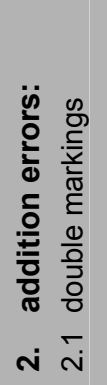 & 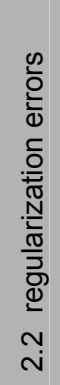 & 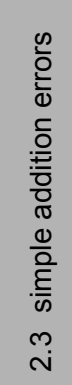 & 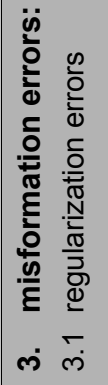 & 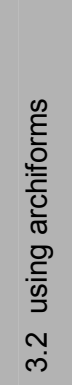 & 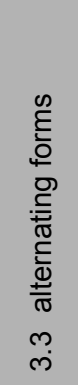 & 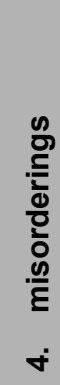 \\
\hline 1. Wort / Satzstellung & 0 & 0 & 0 & 0 & 0 & 3 & 3 & 100 \\
\hline \multicolumn{9}{|l|}{ 2. Auslassungsfehler: } \\
\hline a) Verlaufsform: G/V/F & 78 & 0 & 0 & 0 & 0 & 78 & 67 & 0 \\
\hline b) Infinitiv & 100 & 0 & 0 & 0 & 0 & 78 & 78 & 33 \\
\hline c) Partizipialsätze & 67 & 0 & 0 & 0 & 0 & 100 & 100 & 0 \\
\hline d) Gerundium & 83 & 0 & 0 & 0 & 0 & 67 & 92 & 8 \\
\hline e) Artikel (best./unbest.) & 95 & 0 & 0 & 0 & 0 & 10 & 67 & 10 \\
\hline f) Adjektiv & 100 & 0 & 0 & 0 & 0 & 0 & 30 & 0 \\
\hline g) Adverb & 89 & 0 & 0 & 3 & 0 & 3 & 6 & 6 \\
\hline h) sonst. Wortarten / Bestandteile & 97 & 0 & 0 & 0 & 0 & 28 & 42 & 31 \\
\hline \multicolumn{9}{|l|}{ 3. Hinzufügungsfehler: } \\
\hline a) Verlaufsform: G/V/F & 0 & 0 & 0 & 100 & 0 & 80 & 80 & 0 \\
\hline b) Infinitiv & 0 & 0 & 0 & 100 & 0 & 100 & 100 & 0 \\
\hline c) Partizipialsätze & 0 & 0 & 0 & 0 & 0 & 0 & 0 & 0 \\
\hline d) Gerundium & 0 & 0 & 0 & 100 & 0 & 100 & 100 & 0 \\
\hline e) Artikel (best./unbest.) & 0 & 0 & 0 & 100 & 0 & 47 & 47 & 6 \\
\hline f) Adjektiv & 0 & 0 & 0 & 85 & 0 & 0 & 0 & 15 \\
\hline g) Adverb & 0 & 0 & 0 & 52 & 0 & 10 & 20 & 16 \\
\hline h) sonst. Wortarten / Bestandteile & 1 & 1 & 0 & 98 & 0 & 27 & 26 & 26 \\
\hline 4. Bedingungssätze & 20 & 0 & 0 & 0 & 0 & 80 & 80 & 0 \\
\hline 5. Aktiv / Passiv & 100 & 0 & 0 & 0 & 0 & 100 & 100 & 0 \\
\hline \multicolumn{9}{|l|}{ 6. Zeitensystem: } \\
\hline a) Gegenwart & 0 & 0 & 0 & 43 & 0 & 71 & 71 & 0 \\
\hline \multicolumn{9}{|l|}{ b) Vergangenheit: } \\
\hline - simple past & 6 & 0 & 0 & 59 & 3 & 91 & 88 & 0 \\
\hline - present perfect & 70 & 0 & 10 & 0 & 10 & 80 & 70 & 0 \\
\hline - past perfect & 42 & 0 & 0 & 8 & 0 & 75 & 83 & 17 \\
\hline \multicolumn{9}{|l|}{ c) Zukunft: } \\
\hline - progressive & 50 & 0 & 0 & 50 & 0 & 100 & 100 & 0 \\
\hline - going to-future & 0 & 0 & 0 & 0 & 0 & 0 & 0 & 0 \\
\hline - will-future & 58 & 0 & 0 & 0 & 0 & 50 & 83 & 25 \\
\hline
\end{tabular}

Tabelle 23:

Identitätshypothese: Anwendbarkeit der Fehlererklärungsfaktoren in der Probandengruppe mit den wenigsten syntaktisch-morphologischen Abweichungen - Zuordnung in \% 


\begin{tabular}{|c|c|c|c|c|c|c|c|c|c|c|c|}
\hline & 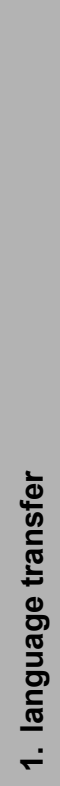 & 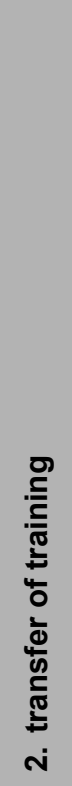 & 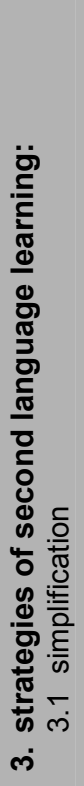 & 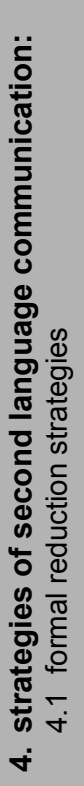 & 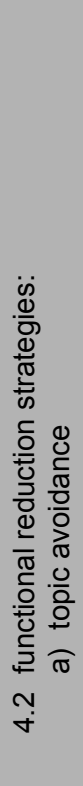 & 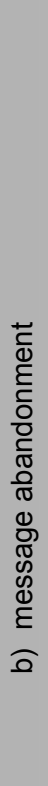 & 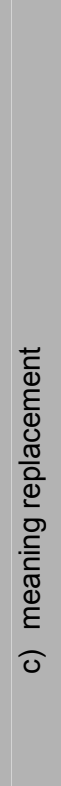 & 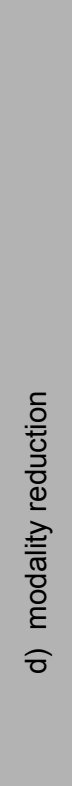 & 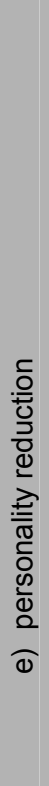 & 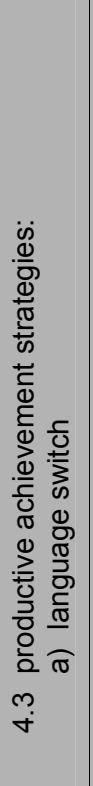 & 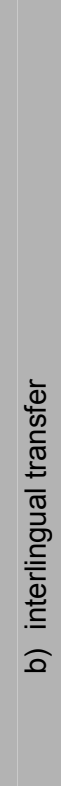 \\
\hline 1. Wort / Satzstellung & 18 & 27 & 64 & 9 & 0 & 2 & 2 & 61 & 0 & 0 & 63 \\
\hline \multicolumn{12}{|l|}{ 2. Auslassungsfehler: } \\
\hline a) Verlaufsform: G/V/F & 20 & 60 & 100 & 100 & 0 & 0 & 20 & 80 & 0 & 0 & 100 \\
\hline b) Infinitiv & 0 & 57 & 79 & 79 & 0 & 0 & 43 & 93 & 0 & 0 & 57 \\
\hline c) Partizipialsätze & 0 & 100 & 50 & 100 & 0 & 0 & 0 & 100 & 0 & 0 & 50 \\
\hline d) Gerundium & 0 & 29 & 100 & 57 & 0 & 0 & 43 & 100 & 0 & 0 & 57 \\
\hline e) Artikel (best./unbest.) & 25 & 50 & 75 & 95 & 0 & 0 & 25 & 85 & 0 & 10 & 50 \\
\hline f) Adjektiv & 0 & 33 & 100 & 67 & 0 & 0 & 67 & 100 & 0 & 0 & 33 \\
\hline g) Adverb & 0 & 7 & 54 & 41 & 0 & 2 & 15 & 56 & 0 & 0 & 24 \\
\hline h) sonst. Wortarten / Bestandteile & 0 & 37 & 90 & 88 & 0 & 5 & 45 & 86 & 0 & 0 & 34 \\
\hline \multicolumn{12}{|l|}{ 3. Hinzufügungsfehler: } \\
\hline a) Verlaufsform: G/V/F & 0 & 67 & 0 & 0 & 0 & 0 & 33 & 33 & 0 & 0 & 0 \\
\hline b) Infinitiv & 0 & 67 & 67 & 33 & 0 & 0 & 67 & 67 & 0 & 0 & 0 \\
\hline c) Partizipialsätze & 0 & 100 & 0 & 0 & 0 & 0 & 0 & 0 & 0 & 0 & 0 \\
\hline d) Gerundium & 0 & 0 & 0 & 0 & 0 & 0 & 0 & 0 & 0 & 0 & 0 \\
\hline e) Artikel (best./unbest.) & 0 & 19 & 62 & 0 & 0 & 0 & 48 & 71 & 0 & 0 & 76 \\
\hline f) Adjektiv & 0 & 35 & 55 & 9 & 0 & 0 & 45 & 36 & 0 & 0 & 55 \\
\hline g) Adverb & 0 & 67 & 17 & 0 & 0 & 0 & 22 & 11 & 0 & 0 & 61 \\
\hline h) sonst. Wortarten / Bestandteile & 0 & 45 & 35 & 1 & 0 & 0 & 33 & 46 & 0 & 0 & 39 \\
\hline 4. Bedingungssätze & 0 & 0 & 0 & 0 & 0 & 0 & 0 & 0 & 0 & 0 & 0 \\
\hline 5. Aktiv / Passiv & 0 & 0 & 100 & 0 & 0 & 0 & 0 & 100 & 0 & 0 & 100 \\
\hline \multicolumn{12}{|l|}{ 6. Zeitensystem: } \\
\hline a) Gegenwart & 0 & 86 & 0 & 0 & 0 & 0 & 86 & 14 & 0 & 0 & 14 \\
\hline \multicolumn{12}{|l|}{ b) Vergangenheit: } \\
\hline - simple past & 16 & 45 & 71 & 22 & 0 & 0 & 35 & 22 & 0 & 8 & 22 \\
\hline - present perfect & 0 & 100 & 0 & 100 & 0 & 0 & 0 & 100 & 0 & 0 & 0 \\
\hline - past perfect & 0 & 100 & 100 & 100 & 0 & 0 & 0 & 100 & 0 & 0 & 0 \\
\hline \multicolumn{12}{|l|}{ c) Zukunft: } \\
\hline - progressive & 0 & 50 & 75 & 75 & 0 & 0 & 0 & 75 & 0 & 0 & 100 \\
\hline - going to-future & 0 & 0 & 100 & 100 & 0 & 0 & 0 & 100 & 0 & 0 & 100 \\
\hline - will-future & 0 & 53 & 73 & 67 & 0 & 0 & 27 & 100 & 0 & 0 & 47 \\
\hline
\end{tabular}

Tabelle 24:

Interlanguagehypothese: Anwendbarkeit der Fehlererklärungsfaktoren in der Probandengruppe mit den meisten syntaktisch-morphologischen Abweichungen - Zuordnung in \% (Fortsetzung nächste Seite) 


\begin{tabular}{|c|c|c|c|c|c|c|c|c|c|c|c|}
\hline & 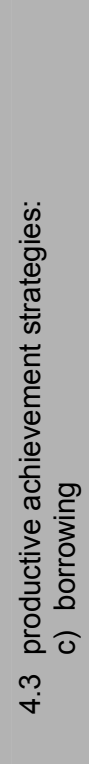 & 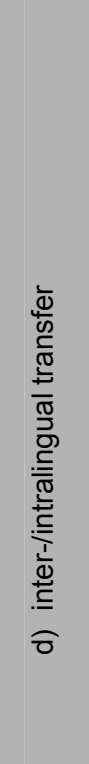 & 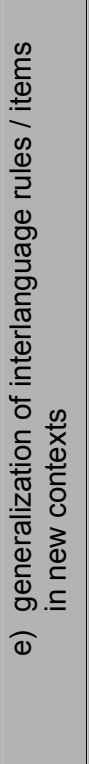 & 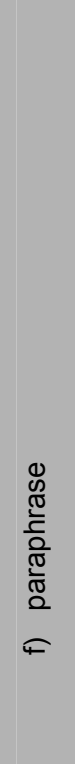 & 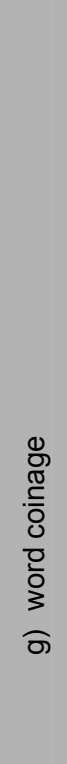 & 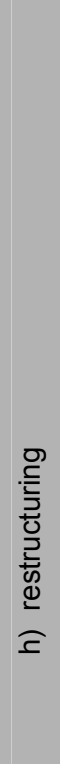 & 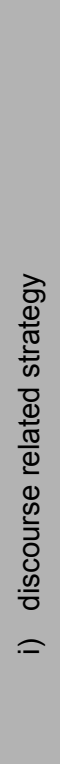 & 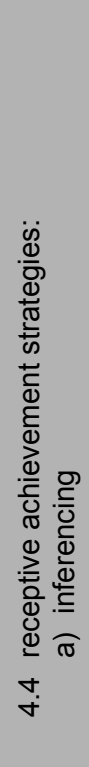 & 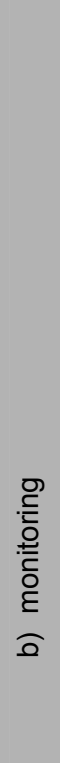 & 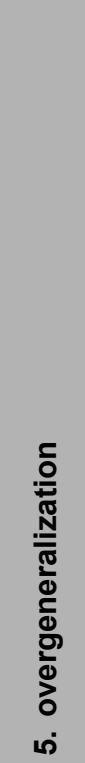 & 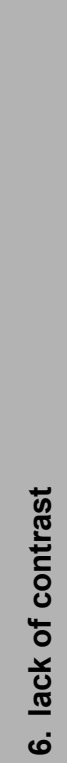 \\
\hline 1. Wort / Satzstellung & 0 & 89 & 16 & 34 & 5 & 2 & 0 & 0 & 0 & 16 & 27 \\
\hline \multicolumn{12}{|l|}{ 2. Auslassungsfehler: } \\
\hline a) Verlaufsform: G/V/F & 0 & 100 & 100 & 20 & 0 & 0 & 0 & 0 & 0 & 100 & 0 \\
\hline b) Infinitiv & 0 & 71 & 71 & 29 & 0 & 0 & 0 & 0 & 0 & 71 & 7 \\
\hline c) Partizipialsätze & 0 & 100 & 100 & 50 & 0 & 0 & 0 & 0 & 0 & 100 & 50 \\
\hline d) Gerundium & 0 & 86 & 43 & 57 & 43 & 0 & 0 & 0 & 0 & 57 & 0 \\
\hline e) Artikel (best./unbest.) & 15 & 95 & 95 & 35 & 0 & 0 & 0 & 0 & 0 & 65 & 20 \\
\hline f) Adjektiv & 0 & 67 & 33 & 33 & 33 & 0 & 0 & 0 & 0 & 33 & 33 \\
\hline g) Adverb & 7 & 37 & 39 & 17 & 2 & 0 & 0 & 0 & 0 & 41 & 7 \\
\hline h) sonst. Wortarten / Bestandteile & 0 & 77 & 70 & 49 & 14 & 0 & 2 & 0 & 1 & 72 & 20 \\
\hline \multicolumn{12}{|l|}{ 3. Hinzufügungsfehler: } \\
\hline a) Verlaufsform: G/V/F & 0 & 0 & 100 & 67 & 33 & 0 & 0 & 0 & 0 & 67 & 0 \\
\hline b) Infinitiv & 0 & 67 & 100 & 33 & 0 & 0 & 0 & 0 & 0 & 67 & 0 \\
\hline c) Partizipialsätze & 0 & 0 & 100 & 0 & 0 & 0 & 0 & 0 & 0 & 100 & 0 \\
\hline d) Gerundium & 0 & 0 & 0 & 0 & 0 & 0 & 0 & 0 & 0 & 0 & 0 \\
\hline e) Artikel (best./unbest.) & 5 & 95 & 90 & 29 & 52 & 0 & 0 & 0 & 0 & 95 & 5 \\
\hline f) Adjektiv & 0 & 91 & 100 & 27 & 36 & 0 & 0 & 0 & 0 & 91 & 27 \\
\hline g) Adverb & 0 & 94 & 94 & 22 & 0 & 0 & 0 & 0 & 0 & 83 & 22 \\
\hline h) sonst. Wortarten / Bestandteile & 1 & 68 & 69 & 45 & 16 & 0 & 0 & 0 & 0 & 63 & 18 \\
\hline 4. Bedingungssätze & 0 & 0 & 0 & 0 & 0 & 0 & 0 & 0 & 0 & 0 & 0 \\
\hline 5. Aktiv / Passiv & 0 & 100 & 0 & 100 & 0 & 0 & 0 & 0 & 0 & 0 & 0 \\
\hline \multicolumn{12}{|l|}{ 6. Zeitensystem: } \\
\hline a) Gegenwart & 0 & 29 & 100 & 29 & 0 & 0 & 0 & 0 & 0 & 100 & 14 \\
\hline \multicolumn{12}{|l|}{ b) Vergangenheit: } \\
\hline - simple past & 2 & 69 & 92 & 12 & 37 & 0 & 0 & 0 & 0 & 87 & 39 \\
\hline - present perfect & 0 & 100 & 100 & 0 & 0 & 0 & 0 & 0 & 0 & 100 & 0 \\
\hline - past perfect & 0 & 100 & 100 & 0 & 0 & 0 & 0 & 0 & 0 & 100 & 0 \\
\hline \multicolumn{12}{|l|}{ c) Zukunft: } \\
\hline - progressive & 0 & 100 & 100 & 0 & 0 & 0 & 0 & 0 & 0 & 100 & 100 \\
\hline - going to-future & 0 & 100 & 100 & 100 & 0 & 0 & 0 & 0 & 0 & 100 & 0 \\
\hline - will-future & 0 & 73 & 93 & 47 & 40 & 0 & 0 & 0 & 0 & 87 & 33 \\
\hline
\end{tabular}

Fortsetzung Tabelle 24:

Interlanguagehypothese: Anwendbarkeit der Fehlererklärungsfaktoren in der Probandengruppe mit den meisten syntaktisch-morphologischen Abweichungen - Zuordnung in \% 


\begin{tabular}{|c|c|c|c|c|c|c|c|c|c|c|c|}
\hline & 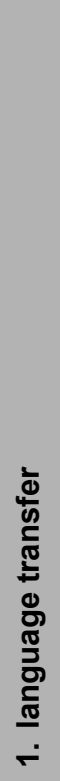 & 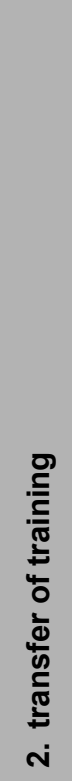 & 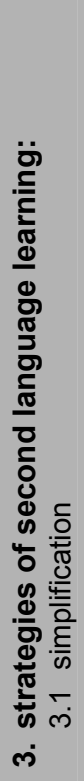 & 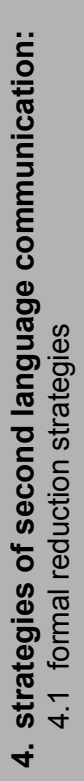 & 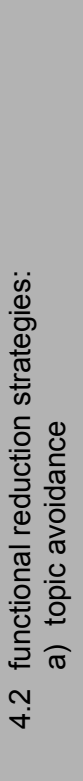 & 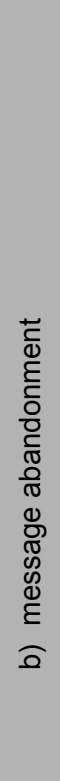 & 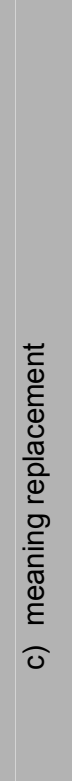 & 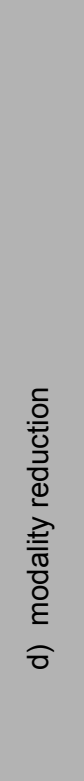 & 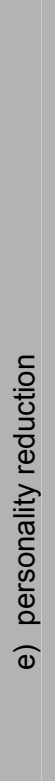 & 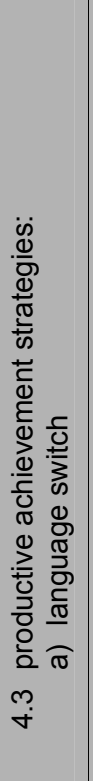 & 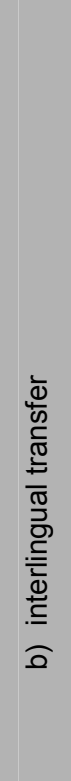 \\
\hline 1. Wort / Satzstellung & 3 & 11 & 89 & 0 & 0 & 0 & 20 & 94 & 0 & 3 & 86 \\
\hline \multicolumn{12}{|l|}{ 2. Auslassungsfehler: } \\
\hline a) Verlaufsform: G/V/F & 0 & 44 & 78 & 78 & 0 & 0 & 67 & 78 & 0 & 0 & 89 \\
\hline b) Infinitiv & 0 & 67 & 100 & 78 & 0 & 0 & 78 & 100 & 0 & 0 & 100 \\
\hline c) Partizipialsätze & 0 & 33 & 100 & 67 & 0 & 0 & 100 & 100 & 0 & 0 & 67 \\
\hline d) Gerundium & 8 & 75 & 100 & 86 & 0 & 0 & 50 & 100 & 0 & 0 & 92 \\
\hline e) Artikel (best./unbest.) & 5 & 48 & 81 & 95 & 0 & 0 & 24 & 81 & 0 & 5 & 67 \\
\hline f) Adjektiv & 0 & 0 & 100 & 100 & 0 & 0 & 50 & 100 & 0 & 0 & 90 \\
\hline g) Adverb & 9 & 97 & 89 & 0 & 0 & 60 & 97 & 0 & 0 & 0 & 91 \\
\hline h) sonst. Wortarten / Bestandteile & 2 & 14 & 96 & 97 & 0 & 0 & 73 & 92 & 0 & 1 & 80 \\
\hline \multicolumn{12}{|l|}{ 3. Hinzufügungsfehler: } \\
\hline a) Verlaufsform: G/V/F & 0 & 100 & 0 & 0 & 0 & 0 & 100 & 30 & 0 & 0 & 0 \\
\hline b) Infinitiv & 0 & 33 & 100 & 0 & 0 & 0 & 0 & 100 & 0 & 0 & 100 \\
\hline c) Partizipialsätze & 0 & 0 & 0 & 0 & 0 & 0 & 0 & 0 & 0 & 0 & 0 \\
\hline d) Gerundium & 0 & 100 & 0 & 0 & 0 & 0 & 80 & 20 & 0 & 0 & 0 \\
\hline e) Artikel (best./unbest.) & 0 & 35 & 94 & 0 & 0 & 0 & 41 & 82 & 0 & 0 & 94 \\
\hline f) Adjektiv & 0 & 77 & 69 & 0 & 0 & 0 & 69 & 77 & 0 & 0 & 77 \\
\hline g) Adverb & 0 & 45 & 23 & 0 & 0 & 0 & 45 & 32 & 0 & 0 & 39 \\
\hline h) sonst. Wortarten / Bestandteile & 0 & 0 & 76 & 0 & 0 & 0 & 53 & 70 & 0 & 0 & 85 \\
\hline 4. Bedingungssätze & 0 & 100 & 100 & 20 & 0 & 0 & 100 & 100 & 0 & 0 & 0 \\
\hline 5. Aktiv / Passiv & 0 & 0 & 100 & 100 & 0 & 0 & 0 & 100 & 0 & 0 & 0 \\
\hline \multicolumn{12}{|l|}{ 6. Zeitensystem: } \\
\hline a) Gegenwart & 0 & 100 & 0 & 0 & 0 & 0 & 86 & 0 & 0 & 0 & 29 \\
\hline \multicolumn{12}{|l|}{ b) Vergangenheit: } \\
\hline - simple past & 0 & 79 & 88 & 9 & 0 & 0 & 91 & 88 & 0 & 0 & 76 \\
\hline - present perfect & 0 & 90 & 60 & 70 & 0 & 0 & 70 & 70 & 0 & 0 & 40 \\
\hline - past perfect & 0 & 83 & 100 & 42 & 0 & 0 & 92 & 100 & 0 & 0 & 92 \\
\hline \multicolumn{12}{|l|}{ c) Zukunft: } \\
\hline - progressive & 0 & 100 & 50 & 50 & 0 & 0 & 100 & 50 & 0 & 0 & 50 \\
\hline - going to-future & 0 & 0 & 0 & 0 & 0 & 0 & 0 & 0 & 0 & 0 & 0 \\
\hline - will-future & 0 & 42 & 75 & 58 & 0 & 0 & 92 & 83 & 0 & 0 & 67 \\
\hline
\end{tabular}

Tabelle 25:

Interlanguagehypothese: Anwendbarkeit der Fehlererklärungsfaktoren in der Probandengruppe mit den wenigsten syntaktisch-morphologischen Abweichungen - Zuordnung in \% (Fortsetzung nächste Seite) 


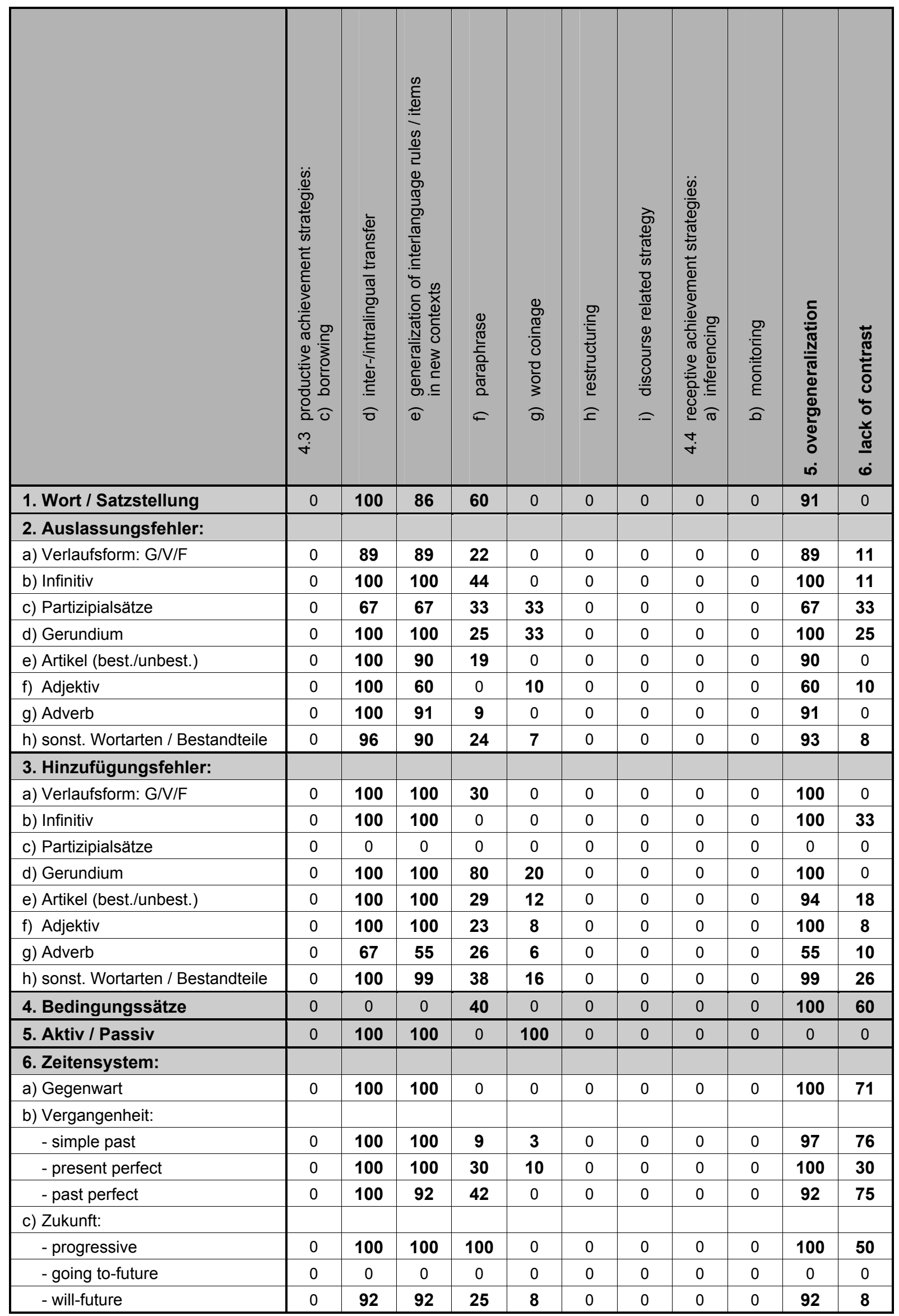

Fortsetzung Tabelle 25:

Interlanguagehypothese: Anwendbarkeit der Fehlererklärungsfaktoren in der Probandengruppe mit den wenigsten syntaktisch-morphologischen Abweichungen - Zuordnung in \% 


\begin{tabular}{|c|c|c|c|}
\hline $\begin{array}{l}\text { orthographische } \\
\text { Kategorien }\end{array}$ & 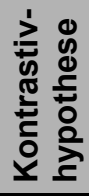 & 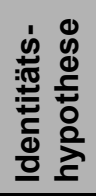 & 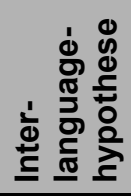 \\
\hline Großschreibung & 50 & 0 & 50 \\
\hline Kleinschreibung & 96 & 0 & 89 \\
\hline Rechtschreibung & 29 & 15 & 54 \\
\hline
\end{tabular}

Tabelle 26:

Anwendbarkeit der Erwerbshypothesen in der Probandengruppe mit den meisten orthographischen $\mathrm{Ab}$ weichungen - Zuordnung zu den Hypothesen in \% ${ }^{679}$

\begin{tabular}{|c|c|c|c|}
\hline & 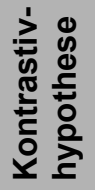 & 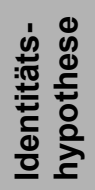 & 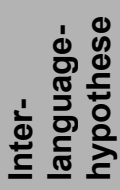 \\
\hline Großschreibung & 24 & 0 & 79 \\
\hline Kleinschreibung & 67 & 0 & 33 \\
\hline Rechtschreibung & 25 & 2 & 72 \\
\hline
\end{tabular}

Tabelle 27:

Anwendbarkeit der Erwerbshypothesen in der Probandengruppe mit den wenigsten orthographischen $\mathrm{Ab}$ weichungen - Zuordnung zu Hypothesen in $\%{ }^{680}$

\begin{tabular}{|c|c|c|c|c|c|c|}
\hline & & 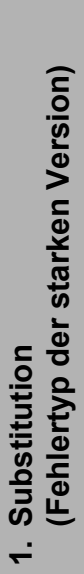 & 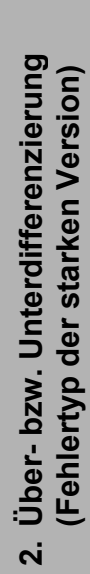 & 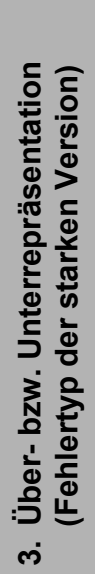 & 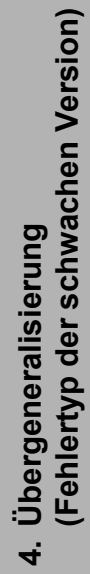 & 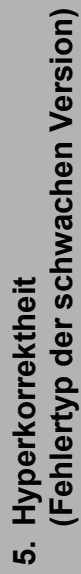 \\
\hline \multirow{3}{*}{ 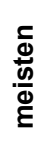 } & Großschreibung & 50 & 50 & 0 & 0 & 0 \\
\hline & Kleinschreibung & 61 & 89 & 0 & 0 & 0 \\
\hline & Rechtschreibung & 29 & 0 & 0 & 0 & 0 \\
\hline \multirow{3}{*}{ 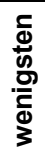 } & Großschreibung & 24 & 0 & 0 & 0 & 0 \\
\hline & Kleinschreibung & 67 & 0 & 0 & 0 & 0 \\
\hline & Rechtschreibung & 25 & 0 & 0 & 0 & 0 \\
\hline
\end{tabular}

Tabelle 28:

Kontrastivhypothese: Anwendbarkeit der Fehlererklärungsfaktoren in den Probandengruppen mit den meisten und mit den wenigsten orthographischen Abweichungen - Zuordnung in \%

\footnotetext{
${ }^{679}$ Die Fehlererklärungsfaktoren der Identitätshypothese kommen bei den Probanden mit den meisten lexikalischen Abweichungen nicht zum Tragen.

${ }^{680}$ dto.
} 


\begin{tabular}{|c|c|c|c|c|c|c|c|c|c|}
\hline & & 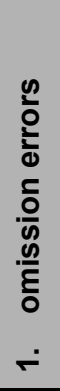 & 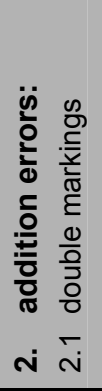 & 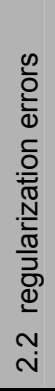 & 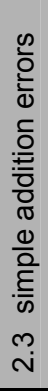 & 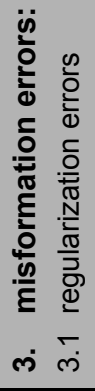 & 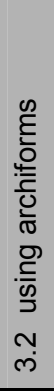 & 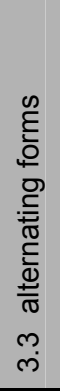 & 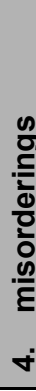 \\
\hline \multirow{3}{*}{ 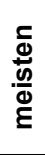 } & Großschreibung & 0 & 0 & 0 & 0 & 0 & 0 & 0 & 0 \\
\hline & Kleinschreibung & 0 & 0 & 0 & 0 & 0 & 0 & 0 & 0 \\
\hline & Rechtschreibung & 10 & 0 & 0 & 7 & 0 & 0 & 0 & 0 \\
\hline \multirow{3}{*}{ 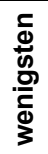 } & Großschreibung & 0 & 0 & 0 & 0 & 0 & 0 & 0 & 0 \\
\hline & Kleinschreibung & 0 & 0 & 0 & 0 & 0 & 0 & 0 & 0 \\
\hline & Rechtschreibung & 0 & 0 & 0 & 2 & 0 & 0 & 0 & 0 \\
\hline
\end{tabular}

Tabelle 29:

Identitätshypothese: Anwendbarkeit der Fehlererklärungsfaktoren in den Probandengruppen mit den meisten und mit den wenigsten orthographischen Abweichungen - Zuordnung in \% 


\begin{tabular}{|c|c|c|c|c|c|c|c|c|c|c|c|c|}
\hline & & 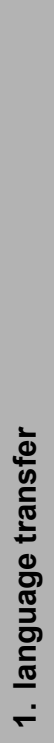 & 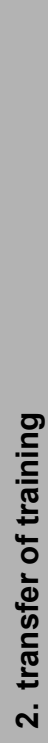 & 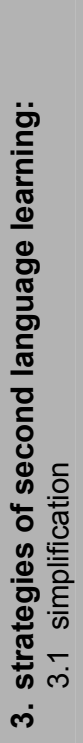 & 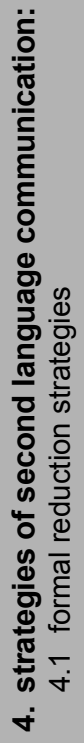 & 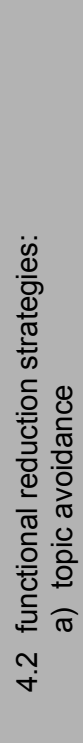 & 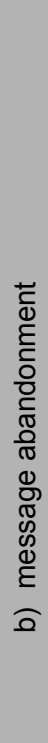 & 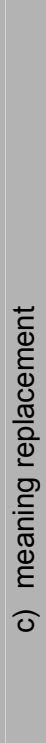 & 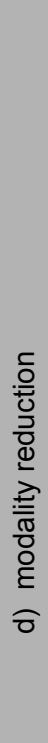 & 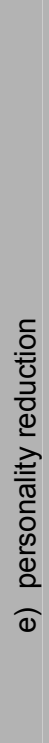 & 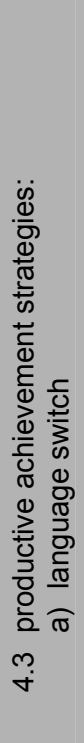 & 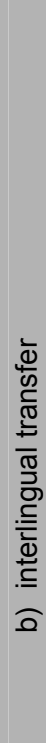 \\
\hline \multirow{3}{*}{ 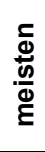 } & Großschreibung & 0 & 0 & 0 & 0 & 0 & 0 & 0 & 0 & 0 & 0 & 13 \\
\hline & Kleinschreibung & 0 & 0 & 0 & 0 & 0 & 0 & 0 & 0 & 0 & 0 & 25 \\
\hline & Rechtschreibung & 0 & 0 & 0 & 0 & 0 & 0 & 0 & 0 & 0 & 0 & 10 \\
\hline \multirow{3}{*}{ 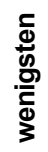 } & Großschreibung & 0 & 0 & 0 & 0 & 0 & 0 & 0 & 0 & 0 & 0 & 0 \\
\hline & Kleinschreibung & 0 & 0 & 0 & 0 & 0 & 0 & 0 & 0 & 0 & 0 & 0 \\
\hline & Rechtschreibung & 0 & 0 & 0 & 0 & 0 & 0 & 0 & 0 & 0 & 0 & 2 \\
\hline
\end{tabular}

\begin{tabular}{|c|c|c|c|c|c|c|c|c|c|c|c|c|}
\hline & & 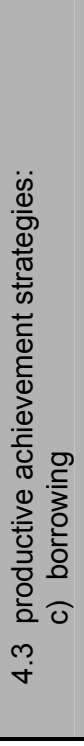 & 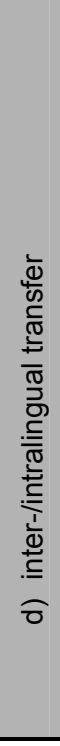 & 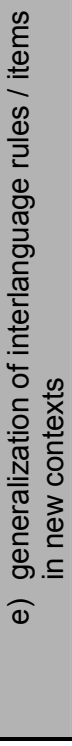 & 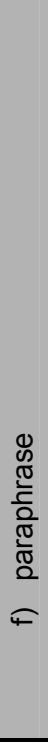 & 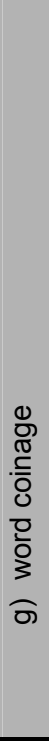 & 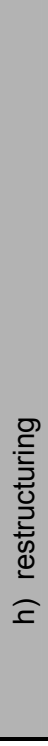 & 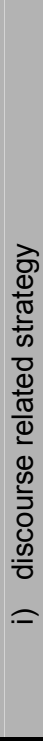 & 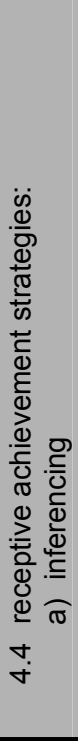 & 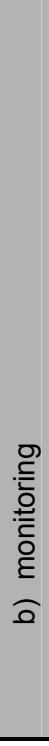 & 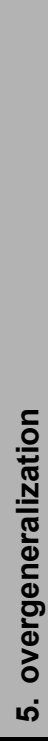 & 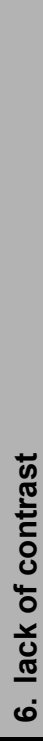 \\
\hline \multirow{3}{*}{ 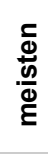 } & Großschreibung & 0 & 0 & 50 & 0 & 0 & 0 & 0 & 0 & 0 & 0 & 0 \\
\hline & Kleinschreibung & 0 & 25 & 75 & 0 & 0 & 0 & 0 & 0 & 0 & 0 & 0 \\
\hline & Rechtschreibung & 0 & 7 & 14 & 0 & 0 & 0 & 0 & 0 & 0 & 3 & 41 \\
\hline \multirow{3}{*}{ 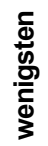 } & Großschreibung & 0 & 0 & 59 & 0 & 0 & 0 & 0 & 0 & 0 & 21 & 0 \\
\hline & Kleinschreibung & 0 & 0 & 0 & 0 & 0 & 0 & 0 & 0 & 0 & 33 & 0 \\
\hline & Rechtschreibung & 0 & 0 & 17 & 0 & 0 & 0 & 0 & 0 & 0 & 8 & 60 \\
\hline
\end{tabular}

Tabelle 30:

Interlanguagehypothese: Anwendbarkeit der Fehlererklärungsfaktoren in den Probandengruppen mit den meisten und mit den wenigsten orthographischen Abweichungen - Zuordnung in \% 
Jahrgangsstufe und Schulform: HS (A/B, RS (A/B oder sprachl./sowi.), Gymn.

(GK/LK), Erwachsenenbildung

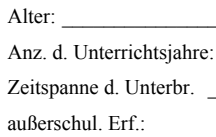

Pronomen:

\section{Bemerkungen:}

$\mathrm{m} / \mathrm{w}$

Unterbrechungen: /nd

Auslandsaufenth.: USA/GB/Austr./nd/sonst.:

Beruf/Stud./Au Pair/Urlaub/nd/sonst.:

Arb. m. engl.-spr. Kollegen: ja/nein/nd

Bez. z. engl.-spr. Personen: ja/nein/nd

Bez. z. Kindern, d. Engl. a. d. Schule lernen:

HS/RS/Gymn./komm.Sprachenschule/VHS/nd/

Abschlüsse: Volksschule/Hauptschule/

mittl. Reife/Abitur/Fachabitur/nd/sonst.:

Berufsausbildung/Studium/nd/sonst:

gut $/ \mathrm{mittel} / \mathrm{schlecht} / \mathrm{nd}$

private/berufliche/schulische/nd/sonst. Gründe $\mathrm{D} / \mathrm{US} / \mathrm{GB} / \mathrm{nd} /$ sonst.

Latein (1,2,3,4,5, nd), Franz. (1,2,3,4,5, nd),

$1,2,3,4,5$, nd

ausgeruht/nd/sonst.:

Nikotin/Medik./Alkohol/nd/sonst.

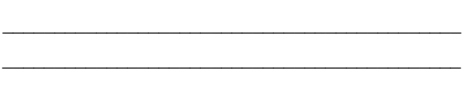

Anz. d. Fehler insges.

synt./morphol. Fehler:

1. Wort/Satzstellung:

2. Auslassungsfehler:

a) Verlaufsform: G/V/F

b) Infinitiv:

c) Patizipialsätze:

d) Gerundium:

e) Artikel (best./unbest.):

f) Adjektiv:

g) Adverb:

h) sonst. Wortarten/Bestandteile:

3. Hinzufügungsfehler:

a) Verlaufsform: G/V/F

b) Infinitiv:

c) Patizipialsätze:

d) Gerundium:

e) Artikel (best./unbest.):

f) Adjektiv:

g) Adverb:

h) sonst. Wortarten/Bestandteile:

4. Bedingungssätze:

5. Aktiv/Passiv:

6. Zeitensystem:

a)Gegenwart:

b)Vergangenheit:

- s.p.:

p.p.:

- pa.p.

c) Futur:

- progr.:

- to be going to:

- will-Future:
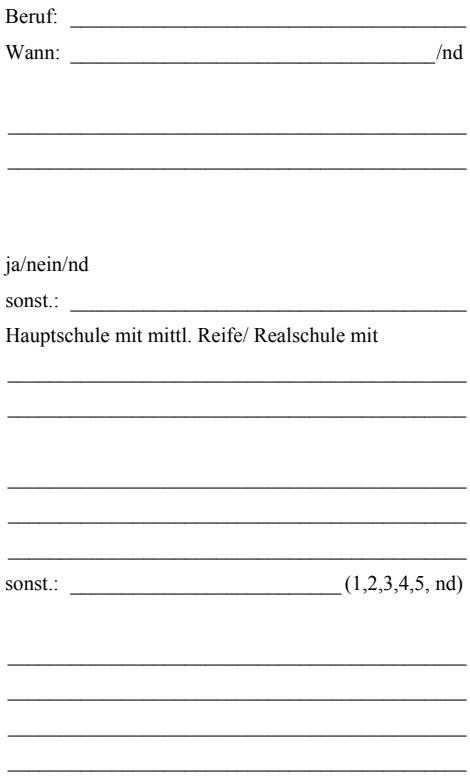

Orthogr. Fehler

Großschreibung:

Kleinschreibung:

Rechtschreibung:

sonst::

Sonst. Fehler:

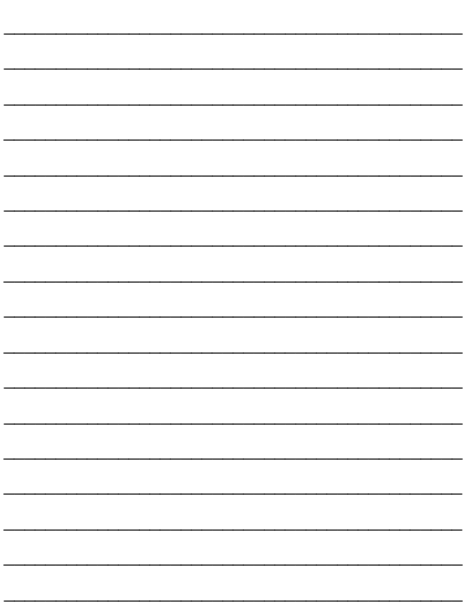

Tabelle 31: Auswertungsbogen der Korrektoren 


\begin{tabular}{|c|c|c|c|}
\hline & HS $7-9$ & HS $10 \mathrm{G}$ & HS $10 \mathrm{E}$ \\
\hline Alter & $13-17$ & $16-18$ & $16-18$ \\
\hline Geschlecht & 50 \% männl. / 50 \% weibl. & 70 \% männl. / 30 \% weibl. & 50 \% männl. / 50 \% weibl. \\
\hline Unterrichtsjahre Englisch & $3-6$ & $6-7$ & $5-7$ \\
\hline Unterbrechungen & $5 \%$ & - & - \\
\hline $\begin{array}{l}\text { Zeitraum der Unter- } \\
\text { brechung }\end{array}$ & weniger als 6 Monate & - & - \\
\hline $\begin{array}{l}\text { außerschulische } \\
\text { Erfahrungen mit der } \\
\text { englischen Sprache }\end{array}$ & $15 \%$ & - & $50 \%$ \\
\hline $\begin{array}{l}\text { Art der außerschulischen } \\
\text { Erfahrungen mit der } \\
\text { englischen Sprache }\end{array}$ & $\begin{array}{l}5 \% \text { Kontakt zu anderen } \\
\text { Schülern, die Englisch als } \\
\text { Zweitsprache erwerben }\end{array}$ & - & $\begin{array}{l}25 \% \text { Kontakt mit englischen } \\
\text { Muttersprachlern } \\
25 \% \text { nicht konkretisiert }\end{array}$ \\
\hline $\begin{array}{l}\text { Schulform, an der Eng- } \\
\text { lisch erworben wurde }\end{array}$ & Hauptschule & Hauptschule & Hauptschule \\
\hline Schulabschluß & - & - & - \\
\hline weitere Abschlüsse & - & - & - \\
\hline $\begin{array}{l}\text { Einstellung zur eng- } \\
\text { lischen Sprache }\end{array}$ & $\begin{array}{ll}\text { sehr gut / gut } & 50 \% \\
\text { mittelmäßig } & 25 \% \\
\text { negativ } & 16 \% \\
\text { keine } & 9 \%\end{array}$ & $\begin{array}{ll}\text { sehr gut / gut } & 50 \% \\
\text { mittelmäßig } & 25 \% \\
\text { negativ } & - \\
\text { keine } & 25 \%\end{array}$ & $\begin{array}{ll}\text { sehr gut / gut } & 95 \% \\
\text { mittelmäßig } & 5 \% \\
\text { negativ } & - \\
\text { keine } & -\end{array}$ \\
\hline \multirow{2}{*}{$\begin{array}{l}\text { Grund für das Erlernen } \\
\text { der englischen Sprache }\end{array}$} & \multirow{2}{*}{$100 \%$ schulisch } & $100 \%$ schulisch & $100 \%$ schulisch \\
\hline & & daneben $35 \%$ privates Interesse & daneben $25 \%$ privates Interesse \\
\hline $\begin{array}{l}\text { Herkunftsland des } \\
\text { Lehrers (deutsch- oder } \\
\text { englischsprachig) }\end{array}$ & deutschsprachig & deutschsprachig & deutschsprachig \\
\hline $\begin{array}{l}\text { erlernte Form des Eng- } \\
\text { lischen (BrE oder AE) }\end{array}$ & $100 \% \mathrm{BrE}$ & $100 \% \mathrm{BrE}$ & $100 \% \mathrm{BrE}$ \\
\hline $\begin{array}{l}\text { andere Fremdsprachen } \\
\text { neben Englisch }\end{array}$ & $30 \%$ & $34 \%$ & $25 \%$ \\
\hline $\begin{array}{l}\text { Art der zusätzlichen } \\
\text { Fremdsprache (Latein, } \\
\text { Französisch, sonstige) }\end{array}$ & sonstige & sonstige & sonstige \\
\hline $\begin{array}{l}\text { Einschätzung der } \\
\text { Sprachkenntnisse in } \\
\text { dieser/diesen } \\
\text { Fremdsprache/n }\end{array}$ & Note $1-2-100 \%$ & $\begin{array}{l}\text { Note } 1-2 \backslash 44 \% \\
\text { Note } 3-4 \backslash 44 \% \\
\text { Note } 5 \longrightarrow 12 \%\end{array}$ & Note $2-3>100 \%$ \\
\hline $\begin{array}{l}\text { eigene Einschätzung der } \\
\text { engl. Sprachkenntnisse }\end{array}$ & $\begin{array}{l}\text { Note } 1-2 \backslash 70 \% \\
\text { Note } 3-4 \longrightarrow 27 \% \\
\text { Note } 5 \longrightarrow 3 \%\end{array}$ & 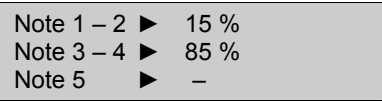 & $\begin{array}{l}\text { Note } 1-2 \backslash \begin{array}{l}12 \% \\
\text { Note } 3-4 \longrightarrow 88 \% \\
\text { Note } 5\end{array}-\quad- \\
\end{array}$ \\
\hline $\begin{array}{l}\text { Einschätzung des } \\
\text { physischen und } \\
\text { psychischen Zustandes }\end{array}$ & $\begin{array}{ll}\text { entspannt / ausgeruht } & 30 \% \\
\text { angespannt / nervös } & 30 \% \\
\text { müde } & 15 \%\end{array}$ & $\begin{array}{l}\text { entspannt / ausgeruht } 42 \% \\
\text { angespannt / nervös } \\
\text { müde }\end{array}$ & 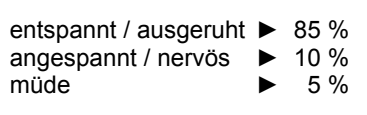 \\
\hline $\begin{array}{l}\text { vor dem Test wurden } \\
\text { eingenommen }\end{array}$ & $\begin{array}{ll}\text { Medikamente } & 26 \% \\
\text { Alkohol } & - \\
\text { Drogen } & \\
\text { (Nikotin/Koffein) } & 12 \% \\
\end{array}$ & $\begin{array}{ll}\text { Medikamente } & 10 \% \\
\text { Alkohol } & - \\
\text { Drogen } & \\
\text { (Nikotin/Koffein) } & -\end{array}$ & $\begin{array}{ll}\text { Medikamente } & - \\
\text { Alkohol } & 0,5 \% \\
\text { Drogen } & \\
\text { (Nikotin/Koffein) } & -\end{array}$ \\
\hline $\begin{array}{l}\text { körperlich-seelische } \\
\text { Beschwerden }\end{array}$ & $6 \%$ & $0 \%$ & $18 \%$ \\
\hline Hilfsmittel & keine & keine & keine \\
\hline
\end{tabular}

Tabelle 32:

Auswertungsbogen der psycho- und soziolinguistischen Fragen / Hauptschule 


\begin{tabular}{|c|c|c|c|c|}
\hline & RS $7-8$ & RS 10 spra. & RS 10 sowi. & RS 10 natw. \\
\hline Alter & $12-15$ & $15-18$ & $14-17$ & $15-18$ \\
\hline Geschlecht & $43 \%$ männl. / 57 \% weibl. & $26 \%$ männl. / $74 \%$ weibl. & $65 \%$ männl. / $35 \%$ weibl. & 100 \% männl. \\
\hline $\begin{array}{l}\text { Unterrichtsjahre } \\
\text { Englisch }\end{array}$ & $3-5$ & $5-7$ & $4-7$ & $5-7$ \\
\hline Unterbrechungen & $12 \%$ & - & - & $25 \%$ \\
\hline $\begin{array}{l}\text { Zeitraum der Unter- } \\
\text { brechung }\end{array}$ & bis zu 6 Monate & & & bis zu 2 Monate \\
\hline $\begin{array}{l}\text { außerschulische } \\
\text { Erfahrungen mit der } \\
\text { englischen Sprache }\end{array}$ & $40 \%$ & $70 \%$ & $46,2 \%$ & $35 \%$ \\
\hline $\begin{array}{l}\text { Art der außerschu- } \\
\text { lischen Erfahrungen } \\
\text { mit der engl. Sprache }\end{array}$ & nicht definiert & $\begin{array}{l}40 \% \text { Kontakt mit engl. } \\
\text { Muttersprachlern } \\
30 \% \text { Auslandsaufenthalt } \\
20 \% \text { nicht konkretisiert }\end{array}$ & $\begin{array}{l}\text { 7,7 \% Kontakt mit engl. } \\
\text { Muttersprachlern } \\
\text { 38,5\% Auslandsaufenthalt }\end{array}$ & $35 \%$ Auslandsaufenthalt \\
\hline $\begin{array}{l}\text { Schulform, an der Eng- } \\
\text { lisch erworben wurde }\end{array}$ & Realschule & Realschule & Realschule & Realschule \\
\hline Schulabschluß & - & - & - & - \\
\hline weitere Abschlüsse & - & - & - & - \\
\hline $\begin{array}{l}\text { Einstellung zur eng- } \\
\text { lischen Sprache }\end{array}$ & $\begin{array}{ll}\text { sehr gut / gut } & 60 \% \\
\text { mittelmäßig } & 20 \% \\
\text { negativ } & 10 \% \\
\text { keine } & 10 \%\end{array}$ & $\begin{array}{ll}\text { sehr gut / gut } & 90 \% \\
\text { mittelmäßig } & 10 \% \\
\text { negativ } & - \\
\text { keine } & -\end{array}$ & \begin{tabular}{|l} 
sehr gut / gut \\
mittelmäßig
\end{tabular} & $\begin{array}{ll}\text { sehr gut / gut } & 30 \% \\
\text { mittelmäßig } & - \\
\text { negativ } & 45 \% \\
\text { keine } & 25 \%\end{array}$ \\
\hline \multirow{2}{*}{$\begin{array}{l}\text { Grund für das Erlernen } \\
\text { der engl. Sprache }\end{array}$} & \multirow[b]{2}{*}{$100 \%$ schulisch } & $100 \%$ schulisch & $100 \%$ schulisch & $100 \%$ schulisch \\
\hline & & $\begin{array}{l}\text { daneben } 50 \% \text { privates } \\
\text { Interesse }\end{array}$ & $\begin{array}{l}\text { daneben } 14 \% \text { privates } \\
\text { Interesse }\end{array}$ & $\begin{array}{l}\text { daneben } 10 \% \text { privates } \\
\text { Interesse }\end{array}$ \\
\hline $\begin{array}{l}\text { Herkunftsland des } \\
\text { Lehrers (deutsch- oder } \\
\text { englischsprachig) }\end{array}$ & deutschsprachig & deutschsprachig & deutschsprachig & deutschsprachig \\
\hline $\begin{array}{l}\text { erlernte Form des Eng- } \\
\text { lischen (BE oder AE) }\end{array}$ & $100 \% \mathrm{BrE}$ & $\begin{array}{l}100 \% \mathrm{BrE} \\
50 \% \text { daneben } \mathrm{AE}\end{array}$ & $\begin{array}{l}100 \% \text { BrE } \\
11 \% \text { daneben } \mathrm{AE}\end{array}$ & $\begin{array}{l}100 \% \mathrm{BrE} \\
12,5 \% \text { daneben } \mathrm{AE}\end{array}$ \\
\hline $\begin{array}{l}\text { andere Fremdsprachen } \\
\text { neben Englisch }\end{array}$ & $85 \%$ & $100 \%$ & $20 \%$ & $45 \%$ \\
\hline $\begin{array}{l}\text { Art der zusätzlichen } \\
\text { Fremdsprache (Latein, } \\
\text { Französisch, sonstige) }\end{array}$ & Französisch & Französisch & Französisch & $\begin{array}{l}\text { Französisch } \\
\text { sonstige }\end{array}$ \\
\hline $\begin{array}{l}\text { Einschätzung der } \\
\text { Sprachkenntnisse in } \\
\text { dieser/diesen } \\
\text { Fremdsprache/n }\end{array}$ & $\begin{array}{l}\text { Note } 1-2>15 \% \\
\text { Note } 3-4>85 \%\end{array}$ & $\begin{array}{l}\text { Note } 1-2 \longrightarrow 25 \% \\
\text { Note } 3-4>75 \%\end{array}$ & $\begin{array}{l}\text { Note } 1-2 \longrightarrow 2,6 \% \\
\text { Note } 3-4 \longrightarrow 97,4 \%\end{array}$ & $\begin{array}{l}\text { Note } 1-2 \longrightarrow 6 \% \\
\text { Note } 3-4 \longrightarrow 94 \%\end{array}$ \\
\hline $\begin{array}{l}\text { eigene Einschätzung } \\
\text { der englischen } \\
\text { Sprachkenntnisse }\end{array}$ & $\begin{array}{l}\text { Note } 1-2 \longrightarrow 22 \% \\
\text { Note } 3-4>75 \% \\
\text { Note } 5 \longrightarrow 3 \%\end{array}$ & $\begin{array}{l}\text { Note } 1-2>40,5 \% \\
\text { Note } 3-4 \quad 57,0 \% \\
\text { keine Ang. } 2,5 \%\end{array}$ & $\begin{array}{l}\text { Note } 1-2 \longrightarrow 10 \% \\
\text { Note } 3-4 \longrightarrow 78 \% \\
\text { Note } 5 \longrightarrow 12 \%\end{array}$ & $\begin{array}{l}\text { Note } 1-2>2 \% \\
\text { Note } 3-4 \quad 65 \% \\
\text { Note } 5>33 \%\end{array}$ \\
\hline $\begin{array}{l}\text { Einschätzung des } \\
\text { physischen und } \\
\text { psychischen Zustandes }\end{array}$ & 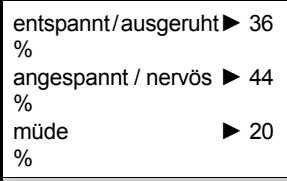 & $\begin{array}{l}\text { entspannt/ausgeruht } \longrightarrow 48 \\
\% \\
\text { angespannt / nervös } \longrightarrow 26 \\
\% \\
\begin{array}{l}\text { müde } \\
\%\end{array} \\
\end{array}$ & 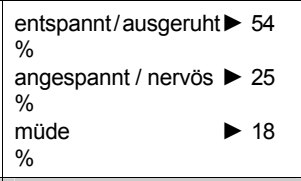 & 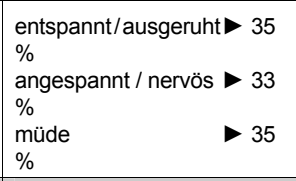 \\
\hline $\begin{array}{l}\text { vor dem Test wurden } \\
\text { eingenommen }\end{array}$ & $\begin{array}{l}\text { Medikamente - } \\
\text { Alkohol } \\
\text { Drogen } \\
\text { (Nikotin/Koffein) - }\end{array}$ & $\begin{array}{ll}\text { Medikamente } & 11,0 \% \\
\text { Alkohol } & - \\
\text { Drogen } & \\
\text { (Nikotin/Koffein) } & 18,3 \% \\
\end{array}$ & \begin{tabular}{|ll} 
Medikamente & $1,3 \%$ \\
Alkohol & $2,6 \%$ \\
Drogen & \\
(Nikotin/Koffein) & $>19,2 \%$ \\
\end{tabular} & \begin{tabular}{|ll} 
Medikamente & $>5,9 \%$ \\
Alkohol & $14,7 \%$ \\
Drogen & \\
(Nikotin/Koffein) & $>32,4 \%$ \\
\end{tabular} \\
\hline $\begin{array}{l}\text { körperlich-seelische } \\
\text { Beschwerden }\end{array}$ & $33 \%$ & $35 \%$ & $25 \%$ & $25 \%$ \\
\hline Hilfsmittel & keine & keine & keine & keine \\
\hline
\end{tabular}

Tabelle 33:

Auswertungsbogen der psycho- und soziolinguistischen Fragen / Realschule 


\begin{tabular}{|c|c|c|c|}
\hline & GY $7-11$ & GY 12 G & GY 12 L \\
\hline Alter & $13-18$ & $18-19$ & $18-19$ \\
\hline Geschlecht & 60 \% männl. / 40 \% weibl. & 55 \% männl. / 45 \% weibl. & 77 \% männl. / 23 \% weibl. \\
\hline Unterrichtsjahre Englisch & $2-6$ & $6-8$ & $6-9$ \\
\hline Unterbrechungen & $3 \%$ & - & - \\
\hline $\begin{array}{l}\text { Zeitraum der Unter- } \\
\text { brechung }\end{array}$ & nicht näher definiert & - & - \\
\hline $\begin{array}{l}\text { außerschulische } \\
\text { Erfahrungen mit der } \\
\text { englischen Sprache }\end{array}$ & $67 \%$ & $100 \%$ & $100 \%$ \\
\hline $\begin{array}{l}\text { Art der außerschulischen } \\
\text { Erfahrungen mit der } \\
\text { englischen Sprache }\end{array}$ & $\begin{array}{l}55 \% \text { Kontakt zu anderen } \\
\text { Schülern, die Englisch als } \\
\text { Zweitsprache erwerben, } \\
\text { Urlaubsreisen und } \\
\text { Schüleraustausch }\end{array}$ & $\begin{array}{l}50 \% \text { Auslandsaufenthalte } \\
55 \% \text { Kontakt zu anderen } \\
\text { Schülern, die Englisch als } \\
\text { Zweitsprache erwerben, } \\
\text { Urlaubsreisen und } \\
\text { Schüleraustausch }\end{array}$ & $\begin{array}{l}90 \% \text { Auslandsaufenthalte und } \\
\text { Kommunikationserfahrung mit } \\
\text { Muttersprachlern im eigenen Land } \\
10 \% \text { Kontakt zu anderen } \\
\text { Schülern, die Englisch als } \\
\text { Zweitsprache erwerben }\end{array}$ \\
\hline $\begin{array}{l}\text { Schulform, an der Eng- } \\
\text { lisch erworben wurde }\end{array}$ & $100 \%$ Gymnasium & $\begin{array}{l}77 \% \text { Gymnasium } \\
23 \% \text { Hauptschule / Realschule }\end{array}$ & $\begin{array}{l}67 \% \text { Gymnasium } \\
33 \% \text { Realschule }\end{array}$ \\
\hline Schulabschluß & - & - & - \\
\hline weitere Abschlüsse & $20 \%$ Mittlere Reife & $100 \%$ Mittlere Reife & $100 \%$ Mittlere Reife \\
\hline $\begin{array}{l}\text { Einstellung zur eng- } \\
\text { lischen Sprache }\end{array}$ & $\begin{array}{ll}\text { sehr gut / gut } & 50 \% \\
\text { mittelmäßig } & 30 \% \\
\text { negativ } & 8 \% \\
\text { keine } & 12 \%\end{array}$ & $\begin{array}{ll}\text { sehr gut / gut } & 68 \% \\
\text { mittelmäßig } & 28 \% \\
\text { negativ } & 4 \% \\
\text { keine } & -\end{array}$ & $\begin{array}{ll}\text { sehr gut / gut } & 87 \% \\
\text { mittelmäßig } & 2 \% \\
\text { negativ } & - \\
\text { keine } & 11 \%\end{array}$ \\
\hline \multirow{2}{*}{$\begin{array}{l}\text { Grund für das Erlernen } \\
\text { der englischen Sprache }\end{array}$} & $100 \%$ schulisch & $100 \%$ schulisch & $100 \%$ schulisch \\
\hline & daneben $40 \%$ privates Interesse & daneben $28 \%$ privates Interesse & daneben $32 \%$ privates Interesse \\
\hline $\begin{array}{l}\text { Herkunftsland des } \\
\text { Lehrers (deutsch- oder } \\
\text { englischsprachig) }\end{array}$ & deutschsprachig & deutschsprachig & $\begin{array}{r}92 \% \text { deutschsprachig } \\
8 \% \text { englischsprachig }\end{array}$ \\
\hline $\begin{array}{l}\text { erlernte Form des Eng- } \\
\text { lischen (BrE oder AE) }\end{array}$ & $\begin{array}{l}100 \% \mathrm{BrE} \\
15 \% \text { daneben } \mathrm{AE}\end{array}$ & $\begin{array}{l}82 \% \mathrm{BrE} \\
16 \% \mathrm{AE} \\
2 \% \text { keine Angabe }\end{array}$ & $\begin{array}{l}100 \% \mathrm{BrE} \\
20 \% \text { daneben } \mathrm{AE}\end{array}$ \\
\hline $\begin{array}{l}\text { andere Fremdsprachen } \\
\text { neben Englisch }\end{array}$ & $85 \%$ & $83 \%$ & $100 \%$ \\
\hline $\begin{array}{l}\text { Art der zusätzlichen } \\
\text { Fremdsprache (Latein, } \\
\text { Französisch, sonstige) }\end{array}$ & $\begin{array}{l}\text { Latein } \\
\text { Französisch } \\
\text { sonstige }\end{array}$ & $\begin{array}{l}\text { Latein } \\
\text { Französisch } \\
\text { sonstige }\end{array}$ & $\begin{array}{l}\text { Latein } \\
\text { Französisch } \\
\text { sonstige }\end{array}$ \\
\hline $\begin{array}{l}\text { Einschätzung der } \\
\text { Sprachkenntnisse in } \\
\text { dieser/diesen } \\
\text { Fremdsprache/n }\end{array}$ & $\begin{array}{l}\text { Note } 1-2 \longrightarrow 15 \% \\
\text { Note } 3-4 \longrightarrow 80 \% \\
\text { Note } 5 \longrightarrow 5 \%\end{array}$ & $\begin{array}{l}\text { Note } 1-2 \longrightarrow 7 \% \\
\text { Note } 3-4 \longrightarrow 80 \% \\
\text { Note } 5 \longrightarrow 13 \%\end{array}$ & $\begin{array}{l}\text { Note } 1-2 \longrightarrow 10 \% \\
\text { Note } 3-4 \longrightarrow 85 \% \\
\text { Note } 5 \longrightarrow 5 \%\end{array}$ \\
\hline $\begin{array}{l}\text { eigene Einschätzung der } \\
\text { engl. Sprachkenntnisse }\end{array}$ & $\begin{array}{l}\text { Note } 1-2 \longrightarrow 27 \% \\
\text { Note } 3-4 \longrightarrow 71 \% \\
\text { Note } 5 \longrightarrow 2 \%\end{array}$ & $\begin{array}{l}\text { Note } 1-2 \longrightarrow 17 \% \\
\text { Note } 3-4 \longrightarrow 78 \% \\
\text { Note } 5 \longrightarrow 5 \%\end{array}$ & $\begin{array}{l}\text { Note } 1-2 \longrightarrow 39 \% \\
\text { Note } 3-4=61 \% \\
\text { Note } 5\end{array}$ \\
\hline $\begin{array}{l}\text { Einschätzung des } \\
\text { physischen und } \\
\text { psychischen Zustandes }\end{array}$ & $\begin{array}{ll}\text { entspannt / ausgeruht } & 41 \% \\
\text { angespannt / nervös } & 31 \% \\
\text { müde } & 28 \% \\
\text { keine Angabe } & -\end{array}$ & $\begin{array}{ll}\text { entspannt / ausgeruht } & 25 \% \\
\text { angespannt / nervös } & 48 \% \\
\text { müde } & 17 \% \\
\text { keine Angabe } & 10 \%\end{array}$ & $\begin{array}{ll}\text { entspannt / ausgeruht } & 53 \% \\
\text { angespannt / nervös } & 20 \% \\
\text { müde } & 27 \% \\
\text { keine Angabe } & -\end{array}$ \\
\hline $\begin{array}{l}\text { vor dem Test wurden } \\
\text { eingenommen }\end{array}$ & $\begin{array}{l}\text { Medikamente }>2 \% \\
\text { Alkohol } \\
\text { Drogen } \\
\text { (Nikotin/Koffein) }\end{array}$ & $\begin{array}{l}\text { Medikamente }>2 \% \\
\text { Alkohol } \\
\begin{array}{l}\text { Drogen } \\
\text { (Nikotin/Koffein) }\end{array}-\text { - }\end{array}$ & $\begin{array}{ll}\text { Medikamente } & - \\
\text { Alkohol } & - \\
\text { Drogen } & \\
\text { (Nikotin/Koffein) } & -22 \%\end{array}$ \\
\hline $\begin{array}{l}\text { körperlich-seelische } \\
\text { Beschwerden }\end{array}$ & $30 \%$ & $23 \%$ & $14 \%$ \\
\hline Hilfsmittel & keine & keine & keine \\
\hline
\end{tabular}

Tabelle 34:

Auswertungsbogen der psycho- und soziolinguistischen Fragen / Gymnasium 


\begin{tabular}{|c|c|c|c|}
\hline & EB - beginners & EB - intermediate & EB - advanced \\
\hline Alter & $\begin{array}{l}16-19 \text { Jahre } 21 \% \\
20-25 \text { Jahre } 44 \% \\
26-30 \text { Jahre } 20 \% \\
31-35 \text { Jahre } 8 \% \\
36-40 \text { Jahre } 5 \% \\
41-45 \text { Jahre }- \\
46-50 \text { Jahre }- \\
51-55 \text { Jahre }- \\
56-60 \text { Jahre }- \\
\text { keine Angabe }- \\
\end{array}$ & $\begin{array}{l}16-19 \text { Jahre } 6 \% \\
20-25 \text { Jahre } 23 \% \\
26-30 \text { Jahre } 28 \% \\
31-35 \text { Jahre } 19 \% \\
36-40 \text { Jahre } 14 \% \\
41-45 \text { Jahre } 3 \% \\
46-50 \text { Jahre } 6 \% \\
51-55 \text { Jahre } 1 \% \\
56-60 \text { Jahre } 1 \% \\
\text { keine Angabe } 1 \%\end{array}$ & $\begin{array}{l}16-19 \text { Jahre } 3 \% \\
20-25 \text { Jahre } 10 \% \\
26-30 \text { Jahre } 17 \% \\
31-35 \text { Jahre } 30 \% \\
36-40 \text { Jahre } 17 \% \\
41-45 \text { Jahre } 3 \% \\
46-50 \text { Jahre } 7 \% \\
51-55 \text { Jahre } 6 \% \\
56-60 \text { Jahre } 3 \% \\
\text { keine Angabe } 3 \%\end{array}$ \\
\hline Geschlecht & 55 \% männl. / 45 \% weibl. & 50 \% männl. / 50 \% weibl. & 40 \% männl. / 60 \% weibl. \\
\hline Unterrichtsjahre Englisch & \begin{aligned}$<1$ Jahr & \multicolumn{2}{c}{$2 \%$} \\
$1-2$ Jahre & - \\
$3-4$ Jahre & $25 \% \\
5-6$ Jahre & $55 \% \\
7-10$ Jahre & $>18 \%\end{aligned}$ & $\begin{aligned}<1 \text { Jahr } & 1 \% \\
1-2 \text { Jahre } & 6 \% \\
3-4 \text { Jahre } & 2 \% \\
5-6 \text { Jahre } & 30 \% \\
7-10 \text { Jahre } & 56 \% \\
>10 \text { Jahre } & 4 \% \\
\text { keine Angabe } & 1 \%\end{aligned}$ & $\begin{aligned}<1 \text { Jahr } & - \\
1-2 \text { Jahre } & 3 \% \\
3-4 \text { Jahre } & 3 \% \\
5-6 \text { Jahre } & 20 \% \\
7-10 \text { Jahre } & 67 \% \\
>10 \text { Jahre } & 7 \% \\
\text { keine Angabe } & -\end{aligned}$ \\
\hline Unterbrechungen & $50 \%$ & $53 \%$ & - \\
\hline $\begin{array}{l}\text { Zeitraum der Unter- } \\
\text { brechung }\end{array}$ & $\begin{aligned}<5 \text { Jahre } & 25,5 \% \\
6-10 \text { Jahre } & 12,8 \% \\
11-15 \text { Jahre } & 7,7 \% \\
>16 \text { Jahre } & 5,0 \% \\
\text { nicht näher def. } & -\end{aligned}$ & $\begin{array}{r}<5 \text { Jahre } 26 \% \\
6 \text { - } 10 \text { Jahre } 17 \% \\
11-15 \text { Jahre } 6 \% \\
>16 \text { Jahre } 5 \% \\
\text { nicht näher def. } 5 \%\end{array}$ & 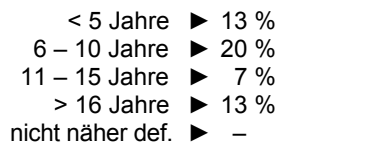 \\
\hline $\begin{array}{l}\text { außerschulische } \\
\text { Erfahrungen mit der } \\
\text { englischen Sprache }\end{array}$ & $75 \%$ & $56 \%$ & $67 \%$ \\
\hline $\begin{array}{l}\text { Art der außerschulischen } \\
\text { Erfahrungen mit der } \\
\text { englischen Sprache }\end{array}$ & $\begin{array}{l}50 \% \text { Kontakt zu anderen Deut- } \\
\text { schen, die Englisch als Zweit- } \\
\text { sprache erwerben, Urlaubsreisen } \\
25 \% \text { längerer Auslands- } \\
\text { aufenthalt }\end{array}$ & $\begin{array}{l}19 \% \text { Auslandsaufenthalte } \\
26 \% \text { Auslandsaufenthalte und } \\
\text { Kommunikationserfahrung mit } \\
\text { englischsprachigen Personen } \\
5 \% \text { nicht näher definiert }\end{array}$ & $\begin{array}{l}50 \% \text { Auslandsaufenthalte } \\
13,5 \% \text { Kontakt zu anderen } \\
\text { englischsprachigen Personen } \\
3,5 \% \text { nicht näher definiert }\end{array}$ \\
\hline $\begin{array}{l}\text { Schulform, an der Eng- } \\
\text { lisch erworben wurde }\end{array}$ & $\begin{array}{ll}\text { Hauptschule } & 43,6 \% \\
\text { Realschule } & 23,0 \% \\
\text { Gymnasium } & 30,8 \% \\
\text { nicht näher definiert } & 2,6 \% \\
\end{array}$ & $\begin{array}{ll}\text { Hauptschule } & 18,5 \% \\
\text { Realschule } & 30,9 \% \\
\text { Gymnasium } & 49,4 \% \\
\text { nicht näher definiert } & 1,2 \% \\
\end{array}$ & $\begin{array}{ll}\text { Hauptschule } & - \\
\text { Realschule } & 17,0 \% \\
\text { Gymnasium } & 83,0 \% \\
\text { nicht näher definiert } & -\end{array}$ \\
\hline Schulabschluß & $\begin{array}{lc}\text { (noch) kein Abschluß } & 20,5 \% \\
\text { Haupt-/Volksschule } & 41,0 \% \\
\text { Mittlere Reife } & 33,3 \% \\
\text { Abitur/Fachabitur } & 5,1 \% \\
\text { sonstige/nicht definiert } & -\end{array}$ & $\begin{array}{lr}\text { (noch) kein Abschluß } & 2,5 \% \\
\text { Haupt-/Volksschule } & 7,4 \% \\
\text { Mittlere Reife } & 40,7 \% \\
\text { Abitur/Fachabitur } & 46,9 \% \\
\text { sonstige/nicht definiert } & 2,5 \%\end{array}$ & $\begin{array}{ll}\text { (noch) kein Abschluß } & - \\
\text { Haupt-/Volksschule } & 3,3 \% \\
\text { Mittlere Reife } & 20,0 \% \\
\text { Abitur/Fachabitur } & 76,7 \% \\
\text { sonstige/nicht definiert } & -\end{array}$ \\
\hline weitere Abschlüsse & $\begin{array}{ll}\text { keine } & 66,7 \% \\
\text { Berufsausbildung } & 28,2 \% \\
\text { Studium } & - \\
\text { sonstige/nicht definiert } & 5,1 \%\end{array}$ & $\begin{array}{ll}\text { keine } & 33,3 \% \\
\text { Berufsausbildung } & 38,3 \% \\
\text { Studium } & 23,5 \% \\
\text { sonstige/nicht definiert } & 4,9 \%\end{array}$ & $\begin{array}{lr}\text { keine } & 6,7 \% \\
\text { Berufsausbildung } & 40,0 \% \\
\text { Studium } & 50,0 \% \\
\text { sonstige/nicht definiert } & -3,3 \%\end{array}$ \\
\hline $\begin{array}{l}\text { Einstellung zur eng- } \\
\text { lischen Sprache }\end{array}$ & $\begin{array}{ll}\text { sehr gut / gut } & 66,7 \% \\
\text { mittelmäßig } & 10,2 \% \\
\text { negativ } & 7,7 \% \\
\text { keine } & 15,4 \%\end{array}$ & $\begin{array}{ll}\text { sehr gut / gut } & 82,7 \% \\
\text { mittelmäßig } & 12,3 \% \\
\text { negativ } & 3,7 \% \\
\text { keine } & 1,2 \%\end{array}$ & $\begin{array}{ll}\text { sehr gut / gut } & 86,7 \% \\
\text { mittelmäßig } & 6,7 \% \\
\text { negativ } & 3,3 \% \\
\text { keine } & 3,3 \%\end{array}$ \\
\hline $\begin{array}{l}\text { Grund für das Erlernen } \\
\text { der englischen Sprache }\end{array}$ & $\begin{array}{l}\text { beruflich und schulisch } 90 \% \\
\text { daneben priv. Interesse } 30 \% \\
\text { keine Angabe }\end{array}$ & $\begin{array}{l}\text { beruflich und schulisch } 100 \% \\
\text { daneben priv. Interesse } \\
\text { keine Angabe }\end{array}$ & $\begin{array}{l}\text { beruflich und schulisch } 100 \% \\
\text { daneben priv. Interesse } \\
\text { keine Angabe }\end{array}$ \\
\hline $\begin{array}{l}\text { Herkunftsland des } \\
\text { Lehrers (deutsch- oder } \\
\text { englischsprachig) }\end{array}$ & 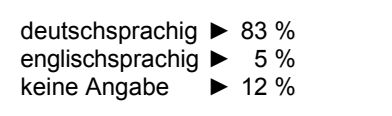 & $\begin{array}{l}\text { deutschsprachig } 81 \% \\
\text { englischsprachig } 13 \% \\
\text { keine Angabe } \quad 6 \%\end{array}$ & $\begin{array}{l}\text { deutschsprachig } 80 \% \\
\text { englischsprachig } 20 \% \\
\text { keine Angabe }\end{array}$ \\
\hline $\begin{array}{l}\text { erlernte Form des Eng- } \\
\text { lischen (BrE oder AE) }\end{array}$ & $\begin{array}{ll}\mathrm{BrE} & 79,5 \% \\
\mathrm{AE} & 7,7 \% \\
\mathrm{BrE}+\mathrm{AE} & 5,1 \% \\
\text { keine Angabe } & 7,7 \% \\
\end{array}$ & $\begin{array}{ll}\mathrm{BrE} & 80 \% \\
\mathrm{AE} & - \\
\mathrm{BrE}+\mathrm{AE} & 33 \% \\
\text { keine Angabe } & 20 \%\end{array}$ & $\begin{array}{ll}\mathrm{BrE} & 71 \% \\
\mathrm{AE} & - \\
\mathrm{BrE}+\mathrm{AE} & 23 \% \\
\text { keine Angabe } & 6 \%\end{array}$ \\
\hline $\begin{array}{l}\text { andere Fremdsprachen } \\
\text { neben Englisch }\end{array}$ & $49 \%$ & $59 \%$ & $93 \%$ \\
\hline
\end{tabular}

Tabelle 35:

Auswertungsbogen der psycho- und soziolinguistischen Fragen / Erwachsenenbildung (Fortsetzung nächste Seite) 


\begin{tabular}{|c|c|c|c|}
\hline & EB - beginners & EB - intermediate & EB - advanced \\
\hline $\begin{array}{l}\text { Art der zusätzlichen } \\
\text { Fremdsprache (Latein, } \\
\text { Französisch, sonstige) }\end{array}$ & $\begin{array}{l}\text { Latein } \\
\text { Französisch } \\
\text { sonstige }\end{array}$ & $\begin{array}{l}\text { Latein } \\
\text { Französisch } \\
\text { sonstige }\end{array}$ & $\begin{array}{l}\text { Latein } \\
\text { Französisch } \\
\text { sonstige }\end{array}$ \\
\hline $\begin{array}{l}\text { Einschätzung der } \\
\text { Sprachkenntnisse in } \\
\text { dieser/diesen } \\
\text { Fremdsprache/n }\end{array}$ & $\begin{array}{l}\text { Note } 1-2>37,0 \% \\
\text { Note } 3-4>42,0 \% \\
\text { Note } 5>21,0 \%\end{array}$ & $\begin{array}{l}\text { Note } 1-2 \longrightarrow 18,8 \% \\
\text { Note } 3-4 \longrightarrow 66,7 \% \\
\text { Note } 5 \longrightarrow 12,5 \%\end{array}$ & $\begin{array}{l}\text { Note } 1-2 \longrightarrow 32,1 \% \\
\text { Note } 3-4 \longrightarrow 42,9 \% \\
\text { Note } 5>14,3 \%\end{array}$ \\
\hline $\begin{array}{l}\text { eigene Einschätzung der } \\
\text { engl. Sprachkenntnisse }\end{array}$ & $\begin{array}{l}\text { Note } 1-2 \longrightarrow 7,7 \% \\
\text { Note } 3-4 \longrightarrow 69,2 \% \\
\text { Note } 5 \longrightarrow 23,1 \%\end{array}$ & $\begin{array}{l}\text { Note } 1-2 \longrightarrow 14,8 \% \\
\text { Note } 3-4 \longrightarrow 65,4 \% \\
\text { Note } 5 \longrightarrow 18,5 \%\end{array}$ & $\begin{array}{l}\text { Note } 1-2 \longrightarrow 23,3 \% \\
\text { Note } 3-4 \longrightarrow 76,7 \% \\
\text { Note } 5 \longrightarrow-\end{array}$ \\
\hline $\begin{array}{l}\text { Einschätzung des } \\
\text { physischen und } \\
\text { psychischen Zustandes }\end{array}$ & $\begin{array}{ll}\text { entspannt / ausgeruht } & 25 \% \\
\text { angespannt / nervös } & 35 \% \\
\text { müde } & 25 \% \\
\text { keine Angabe } & 15 \%\end{array}$ & $\begin{array}{ll}\text { entspannt / ausgeruht } & 45 \% \\
\text { angespannt / nervös } & 35 \% \\
\text { müde } & 20 \% \\
\text { keine Angabe } & -\end{array}$ & $\begin{array}{ll}\text { entspannt / ausgeruht } & 55 \% \\
\text { angespannt / nervös } & 28 \% \\
\text { müde } & 17 \% \\
\text { keine Angabe } & -\end{array}$ \\
\hline $\begin{array}{l}\text { vor dem Test wurden } \\
\text { eingenommen }\end{array}$ & $\begin{array}{ll}\text { Medikamente } & 2,5 \% \\
\text { Alkohol } & - \\
\text { Drogen } & \\
\text { (Nikotin/Koffein) } & 33,3 \%\end{array}$ & $\begin{array}{ll}\text { Medikamente } & 3,7 \% \\
\text { Alkohol } & - \\
\text { Drogen } & \\
\text { (Nikotin/Koffein) } & -38,3 \%\end{array}$ & $\begin{array}{ll}\text { Medikamente } & - \\
\text { Alkohol } & 6,7 \% \\
\text { Drogen } & \\
\text { (Nikotin/Koffein) } & 16,7 \%\end{array}$ \\
\hline $\begin{array}{l}\text { körperlich-seelische } \\
\text { Beschwerden }\end{array}$ & $33,3 \%$ & $26,0 \%$ & $26,7 \%$ \\
\hline Hilfsmittel & keine & keine & keine \\
\hline
\end{tabular}

Fortsetzung Tabelle 35:

Auswertungsbogen der psycho- und soziolinguistischen Fragen / Erwachsenenbildung 
Schreibe bitte einen Brief an einen Briefpartner in englischer Sprache, Der umfassen (zähle bitte am Ende Deine Wörter) und sollte folgendes beinhalten:

1) Euer letzter gemeinsamer Urlaub

2) Vorschläge zur Planung eines nächsten gemeinsamen Urlaubs

3) Schilderung Deiner gegenwärtigen Situation und was $\mathrm{Du}$ in letzter zeit gemacht hast

4) Schilderung des Diebstahls eines Dir gehörenden Gegenstands durch einen Unbekannten

5) Ankündigung eines persönlichen Besuchs beim Briefpartner
Beantworte bitte die folgenden Fragen. Schreibe bitte Deinen Nemen nicht auf den Fragebogen, da Deine Antworten streng vertraulich und anonym behandelt werden. Falls Du eine Frage nicht beantworten kannst schreibe bitte weiß nicht" daneben. Lasse bitte keinen Punkt aus. Beachte bitte, daß manche Fragen ggf. mehrere Antworten zulassen.

1) Wieviel Jahre hast Du Englischunterricht gehabt?

2) Wurde Dein Englischunterricht dabei zeitlich unterbrochen?

Wenn ja, wie lange und wann?

3) Hast Du außerhalb des Unterrichts Erfahrungen mit der englischen Sprache gesammelt (z. B. Auslandsaufenthalt, Arbeit mit englischsprachigen Kollegen, Beziehungen zu englischsprachigen Personen oder Kindern, die Englisch an der Schule lernen)?

4) An welcher Schulform hast du Englisch gelernt (Hauptschule, Realschule, Gymnasium, Privatunterricht, sonstiges)?

5) Welchen Schulabschluß hast Du?

\&) Welche weiteren Abschlüsse hast Du?

7) Wie bist $\mathrm{Du}$ der englischen sprache gegenüber Wie bist Du der englischen sprache gegenuber
eingestellt?

8) Warum lernst Du zur Zeit Englisch?
9) Stammt (e) Dein Lehrer aus einem englischsprachigen Land? Wenn ja, aus welchem?

10) Sprichst Du Britisches Englisch, Amerikanisches Englisch oder eine andere Form der englischen Sprache?

11) Welche Fremdsprachen beherrschst Du?

12) Wie stufst Du Deine Englischkenntnisse ein?
- sehr gut
○ gut
○ befriedigend
$\circ$ ausreichend
○ mangelhaft

13) Falls Du weitere Fremdsprachen beherrschst, beurteile bitte Deine Fähigkeiten entsprechend der o. g. Skala.
- sehr gut
$\circ$ gut
- befriedigend
$\circ$ ausreichend
$\circ$ mangelhaft

14) Beantworte bitte die folgenden Fragen zu Deiner Person:

Alter:

Geschlecht:

Beruf:

15) Wie schätzt Du Dein derzeitiges Befinden ein? (Sei bitte bei der Beantwortung der Fragen aufrichtig.) Kreuze bitte alles Zutreffende an.

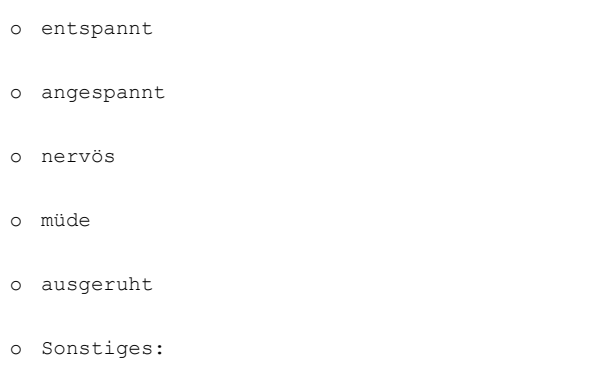

16) Hast Du unmittelbar vor dem Test Medikamente, Alkohol oder sonstige Drogen zu Dir genommen (z. B. Koffein, Nikotin usw.)? Wenn ja, welche?

17) Leidest Du zur zeit unter irgendwelchen körperLeidest Du zur zeit unter irgenawe 

Hiermit versichere ich an Eides statt, daß ich die eingereichte Dissertation ,Die fehleranalytische Relevanz der prädominanten Spracherwerbshypothesen' selbständig und ohne unerlaubte Hilfe verfaßt habe. Anderer als der von mir angegebenen Hilfsmittel und Schriften habe ich mich nicht bedient. Alle wörtlich oder sinngemäß den Schriften anderer Autorinnen oder Autoren entnommenen Stellen habe ich kenntlich gemacht. Die Abhandlung ist noch nicht veröffentlicht worden und noch nicht Gegenstand eines Promotionsverfahrens gewesen. 